\title{
EXPERIMENTAL INVESTIGATION OF CAUSTIC STEAM INJECTION FOR HEAVY OILS
}

\author{
A Thesis \\ by \\ RAJIV MENON MADHAVAN
}

\author{
Submitted to the Office of Graduate Studies of \\ Texas A\&M University \\ in partial fulfillment of the requirements for the degree of \\ MASTER OF SCIENCE
}

May 2009

Major Subject: Petroleum Engineering 


\title{
EXPERIMENTAL INVESTIGATION OF CAUSTIC STEAM INJECTION FOR HEAVY OILS
}

\author{
A Thesis \\ by \\ RAJIV MENON MADHAVAN
}

\begin{abstract}
Submitted to the Office of Graduate Studies of Texas A\&M University

in partial fulfillment of the requirements for the degree of

MASTER OF SCIENCE
\end{abstract}

Approved by:

Chair of Committee, Daulat Mamora

Committee Members, Jerome Schubert

Zoran Sunik

Head of Department, Stephen Holditch

May 2009

Major Subject: Petroleum Engineering 


\begin{abstract}
Experimental Investigation of Caustic Steam Injection for Heavy Oils.
\end{abstract}

(May 2009)

\begin{abstract}
Rajiv Menon Madhavan, B.E. (Hons), Vellore Institute of Technology, India Chair of Advisory Committee: Dr. Daulat Mamora
\end{abstract}

An experimental study has been conducted to compare the effect of steam injection and caustic steam injection in improving the recovery of San Ardo and Duri heavy oils. A 67 cm long x $7.4 \mathrm{~cm}$ O.D (outer diameter), steel injection cell is used in the study. Six thermocouples are placed at specific distances in the injection cell to record temperature profiles and thus the steam front velocity. The injection cell is filled with a mixture of oil, water and sand. Steam is injected at superheated conditions of $238^{\circ} \mathrm{C}$ with the cell outlet pressure set at 200 psig, the cell pressure similar to that found in San Ardo field. The pressure in the separators is kept at 50 psig. The separator liquid is sampled at regular intervals. The liquid is centrifuged to determine the oil and water volumes, and oil viscosity, density and recovery. Acid number measurements are made by the titration method using a pH meter and measuring the EMF values. The interfacial tensions of the oil for different concentrations of $\mathrm{NaOH}$ are also measured using a tensionometer.

Experimental results show that for Duri oil, the addition of caustic results in an increase in recovery of oil from 52\% (steam injection) to $59 \%$ (caustic steam injection). However, caustic has little effect on San Ardo oil where oil recovery is $75 \%$ (steam 
injection) and $76 \%$ (caustic steam injection). Oil production acceleration is seen with steam-caustic injection. With steam caustic injection there is also a decrease in the produced oil viscosity and density for both oils. Sodium hydroxide concentration of 1 wt $\%$ is observed to give the lowest oil-caustic interfacial tension. The acid numbers for San Ardo and Duri oil are measured as 6.2 and 3.57 respectively. 


\section{DEDICATION}

I would like to dedicate my thesis to my parents and my brother who have always given me encouragement and the courage and who have always stood by me and have inspired me in all aspects of my life. I would also like to dedicate my thesis to my teachers and friends at D.A.V. Public School and the Vellore Institute of Technology who helped me during the course of my schooling and undergraduate degree. 


\section{ACKNOWLEDGEMENTS}

I would like to express my gratitude and respect to my advisor, Dr. Mamora, for guiding me through my research and helping me at every step throughout the course of my research. I would like to thank Crisman Institute for Petroleum Research at Texas A\&M University for sponsoring the project.

I would also like to thank my committee members, Dr. Schubert and Dr. Sunik Zoran, for serving on my committee.

I would also like to thank my friends and colleagues and the department faculty and staff for making my time at Texas A\&M University a great experience. Finally, thanks to my family for their encouragement and love. 


\section{TABLE OF CONTENTS}

\section{Page}

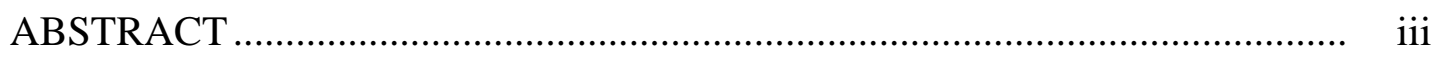

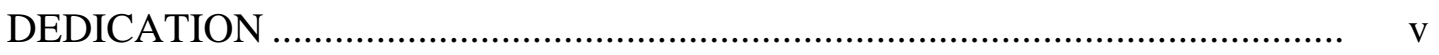

ACKNOWLEDGEMENTS .................................................................. vi

TABLE OF CONTENTS ….................................................................... vii

LIST OF FIGURES.............................................................................. ix

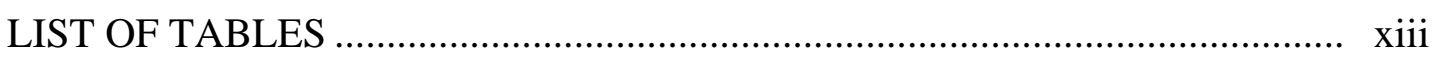

\section{CHAPTER}

I INTRODUCTION ................................................................... 1

1.1 Overview ............................................................................. 1

1.2 Injection Mechanisms ………………………………….......... 2

1.3 Overview of Sodium Hydroxide ................................................. 5

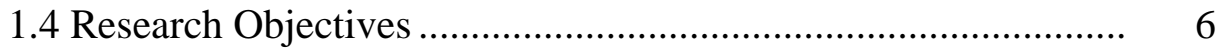

II LITERATURE REVIEW ............................................................. 8

III EXPERIMENTAL APPARATUS AND PROCEDURE ..................... 12

3.1 Apparatus ........................................................................... 12

3.1.1 Fluid Injection System ..................................................... 13

3.1.2 Fluid Production System ................................................... 15

3.1.3 Data Measurement and Recording System ........................ 16

3.2 Experimental Procedure ……………………………………....... 18

IV EXPERIMENTAL RESULTS ....................................................... 21

4.1 Experimental Conditions.............................................................. 21

4.2 Run 1: Base Run for San Ardo Oil Using Pure Steam................. 23

4.3 Run 2: Steam with $\mathrm{NaOH}$ (0.1 wt \%) for San Ardo Oil ............. 27 
CHAPTER Page

4.4 Run 3: Base Run for Duri Oil Using Pure Steam....................... 33

4.5 Run 4: Pure Steam with $\mathrm{NaOH}(0.1$ wt \%) for Duri Oil ............ 37

4.6 Run 5: Pure Steam with $\mathrm{NaOH}(1 \mathrm{wt} \%)$ for Duri Oil ............... 41

4.7 Run 6: Cyclic Injection of $\mathrm{NaOH}(1 \mathrm{wt} \%)$ and Steam for San Ardo Oil.......................................................................... 45

4.8 Run 7: Pure Steam Injection NaOH (1 wt \%) for San Ardo Oil . 50

4.9 Acid Number Measurements................................................. 55

4.10 Interfacial Tension Measurements ...................................... 57

4.11 Comparison and Discussion of Results .................................. 58

4.11.1 Comparison of Runs 3, 4, 5..................................... 59

4.11.2 Comparison of Runs 1, 2, 6, 7 ................................ 63

V SUMMARY, CONCLUSIONS AND RECOMMENDATIONS ....... 67

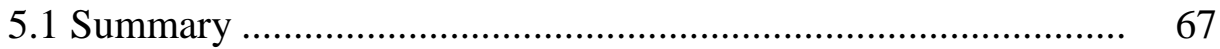

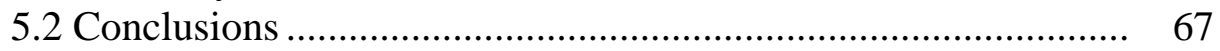

5.3 Recommendations ....................................................... 69

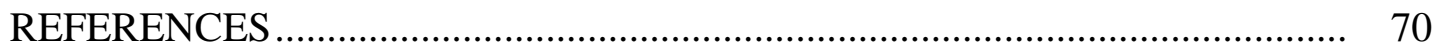

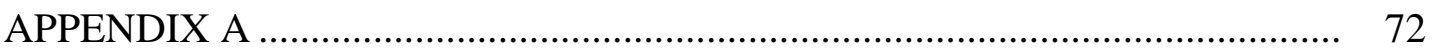

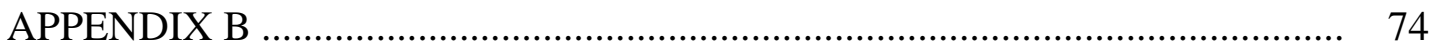

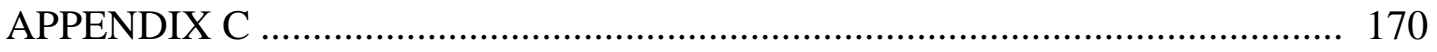

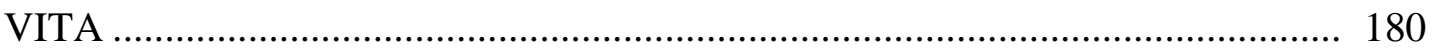




\section{LIST OF FIGURES}

FIGURE Page

1.1 World oil reserves (US Dept of Energy, 2001) ................................... 1

1.2 Temperature and saturation profiles during steam injection (K.C. Hong) 4

3.1 Schematic diagram of the apparatus used for steam injection ................ 12

3.2 Injection cell tamped with mixture ................................................... 14

3.3 Location of the thermocouples ....................................................... 15

$3.4 \quad$ Production separator system............................................................ 16

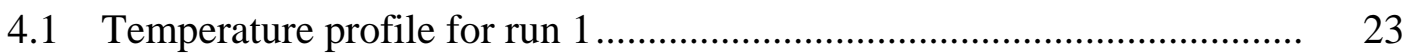

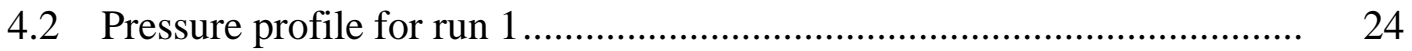

4.3 Liquid production rates for run 1 ................................................. 25

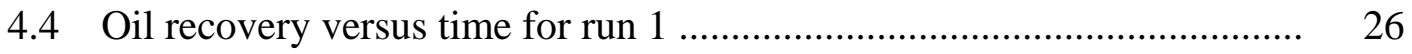

4.5 Oil recovery versus pore volume of steam injected for run 1 ................. 26

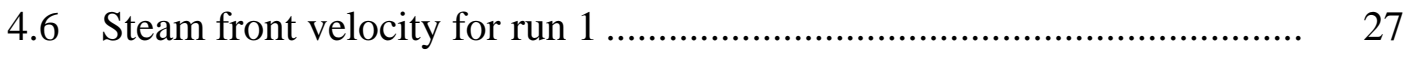

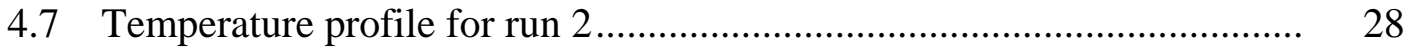

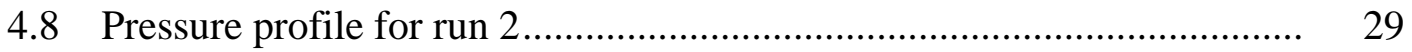

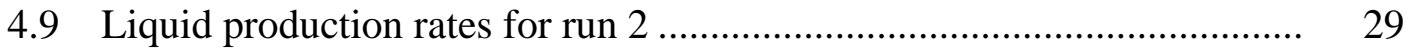

4.10 Oil recovery versus time for run 2 ................................................. 30

4.11 Oil recovery versus pore volume of steam injected for run $2 \ldots \ldots \ldots \ldots \ldots \ldots . . . . . .31$

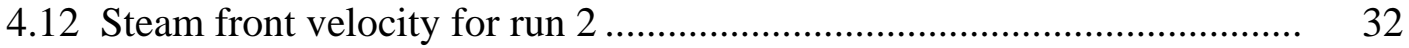

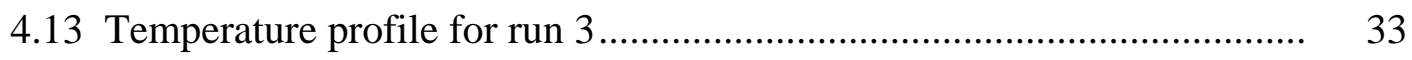


FIGURE Page

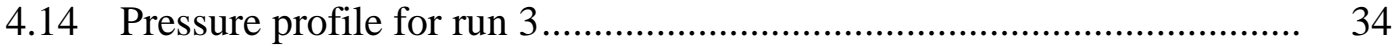

4.15 Liquid production rates for run 3 .............................................. 35

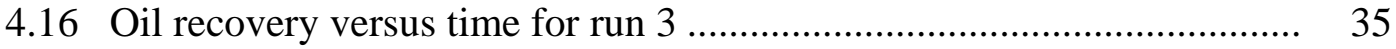

4.17 Oil recovery versus pore volume of steam injected for run 3 ............... 36

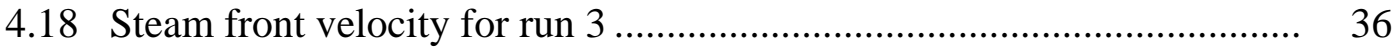

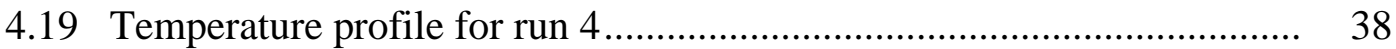

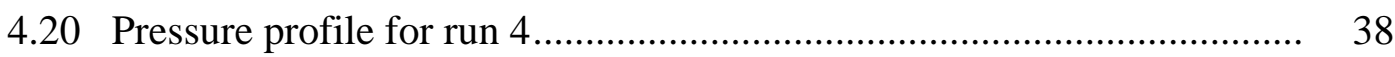

4.21 Liquid production rates for run 4 .................................................. 39

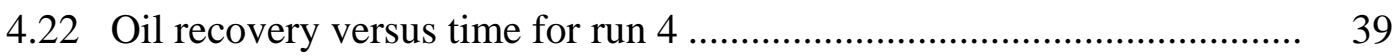

4.23 Oil recovery versus pore volume of steam injected for run 4 ............... 40

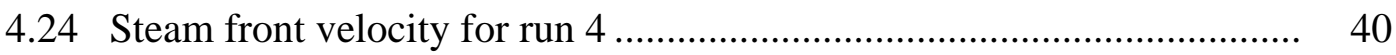

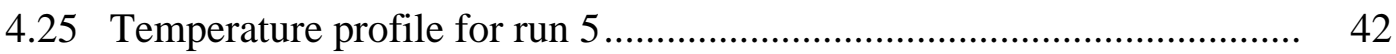

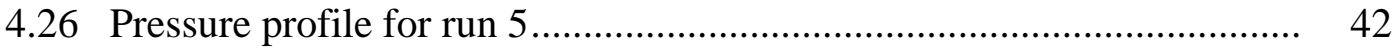

4.27 Liquid production rates for run 5 ................................................. 43

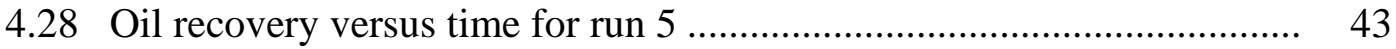

4.29 Oil recovery versus pore volume of steam injected for run 5 ................ 44

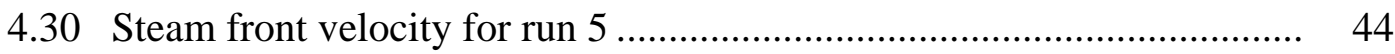

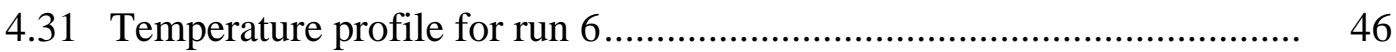

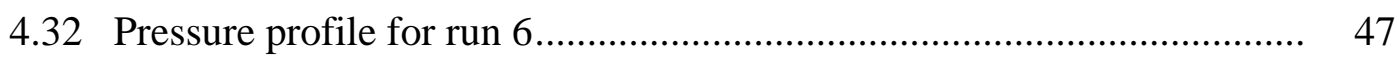

4.33 Liquid production rates for run 6 ................................................ 48

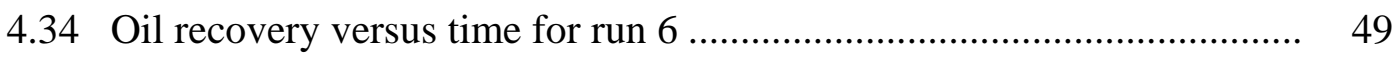


FIGURE Page

4.35 Oil recovery versus pore volume of steam injected for run 6 ................. 49

4.36 Steam front velocity for run 6 .................................................. 50

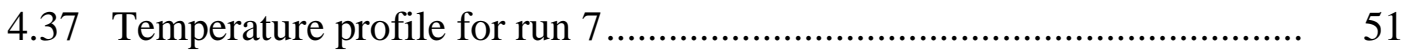

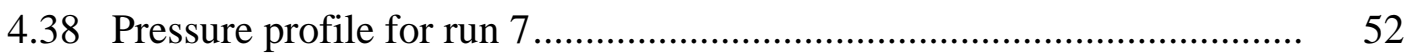

4.39 Liquid production rates for run 7 ................................................. 52

4.40 Oil recovery versus time for run 7 .................................................. 53

4.41 Oil recovery versus pore volume of steam injected for run 7 ............... 53

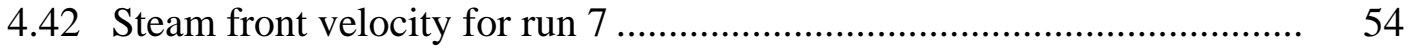

4.43 Blank sample titration ................................................................. 55

4.44 San Ardo oil titration....................................................................... 56

4.45 Duri oil titration....................................................................... 57

4.46 Water production rate versus pore volume of steam injected ................ 59

4.47 Cumulative water volume versus pore volume of steam injected .......... 60

4.48 Oil rate versus pore volume of steam injected .................................. 61

4.49 Oil recovery versus pore volume of steam injected ............................. 62

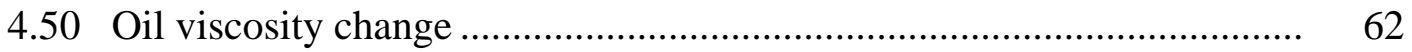

4.51 Water production rate versus pore volume of steam injected ................ 63

4.52 Cumulative water production rate versus pore volume of steam injected.. 64

4.53 Oil rate versus pore volume of steam injected .................................. 65

4.54 Oil recovery versus pore volume of steam injected ............................. 65

4.55 Density change for San Ardo oil ..................................................... 66 
FIGURE $\quad$ Page

4.56 Viscosity change for San Ardo oil ................................................ 66 


\section{LIST OF TABLES}

$\begin{array}{lll}\text { TABLE } & \text { Page }\end{array}$

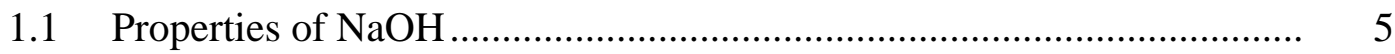

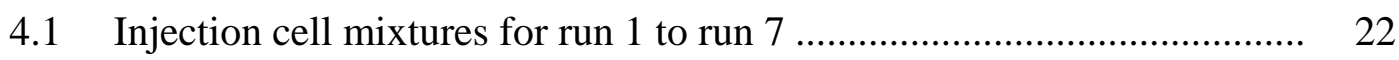

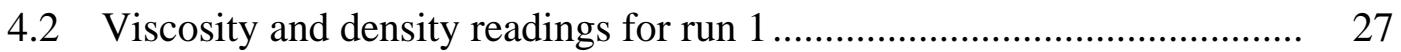

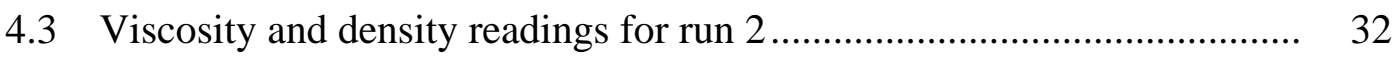

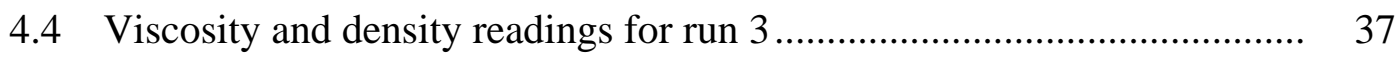

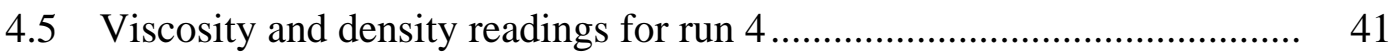

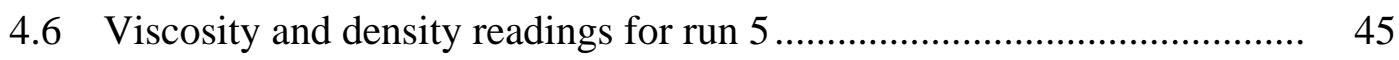

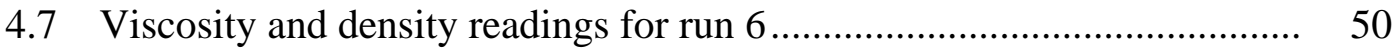

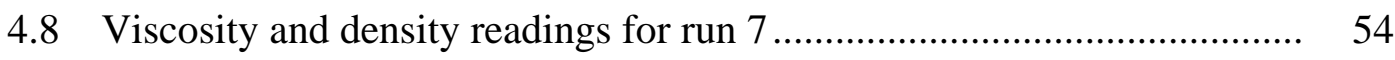

4.9 Interfacial tension measurements for Duri oil................................... 58

4.10 Interfacial tension measurements for San Ardo oil ................................ 58 


\section{CHAPTER I}

\section{INTRODUCTION}

\subsection{Overview}

Heavy oil is a part of the unconventional petroleum reserve. Heavy oil does not flow very easily and is classified as heavy because of its high specific gravity. With increasing demand for oil and with depleting light oil resources it is essential to explore the unconventional petroleum reserve of which heavy oil constitutes a major part. Heavy oil constitutes about 15\% of the world's remaining oil reserves as shown in Fig.1.1.

\section{Total W orld Oil Reserves 9 to 13 Trillion bbls}

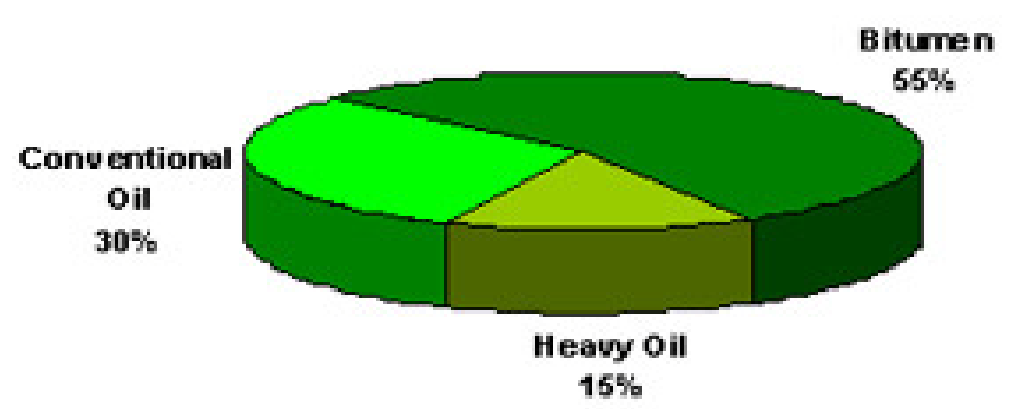

Figure 1.1 - World oil reserves (US Dept of Energy, 2001)

This thesis follows the form and style of the SPE Journal. 
Properties of heavy oil are as follows:

(i) High specific gravity

(ii) High viscosity

(iii) High boiling points

(iv) High content of asphalt, nitrogen and low hydrogen-to-carbon ratio.

Heavy oil has not been fully explored because of the difficulties associated with extracting it, but with improved drilling and enhanced oil recovery techniques there has been a substantial increase in the production of heavy oil. Difficulties associated with the production of heavy oil include the following:

(i) Difficult and expensive to produce

(ii) Requires massive amount of heat to make it more mobile/less viscous.

(iii) It usually contains impurities such as sulphur, heavy metals waxes and residue that must be removed before it is refined.

\subsection{Injection Mechanisms}

Heavy oils are primarily recovered by thermal recovery methods where temperature of the crude is increased so that it can be produced readily. Heating of a reservoir has to be done efficiently. Heat is lost to the under burden and overburden and a part of it is lost through the produced reservoir fluids.

The thermal methods that have been used over the past decade are steam injection and insitu combustion. Steam injection is the most prevalent among the two methods and has been applied effectively in many countries such as Venezuela, Canada and the US. (Prats 1986). 
The factors to be considered for a successful steam flood:

(i) Good quality reservoir with favorable porosity and permeability.

(ii) Lateral continuity of the reservoir over a sufficiently large area.

(iii) Favorable ratio of pay sand to gross interval.

(iv) Adequate oil saturations.

(v) Adequate total pay zone thickness.

Various factors that help increase recovery of oil during steam injection are as follows:

(i) Improve oil mobility by decreasing the viscosity.

(ii) Thermal expansion of the oil.

(iii) Distillation of the lighter hydrocarbons in the steam zone.

Five distinct zones are formed as a result of the injection of steam into the reservoir. The first zone nearest to the injector consists of the water and vapor and the residual oil which is called the steam zone. The second zone consists of the light fractions that are distilled off and condense a little ahead of the steam zone and is called the solvent bank. The solvent bank mixes with the oil thus reducing the interfacial tension and the viscosity. The steam and the volatile oil then condense when they come in contact with the cold matrix and form the hot water zone. As a result of the first three zones an oil bank is formed which comprises the oil that is formed. The fifth zone is formed farthest away from the injector and is composed of the original oil. Fig.1.2 shows the five distinct zones. 


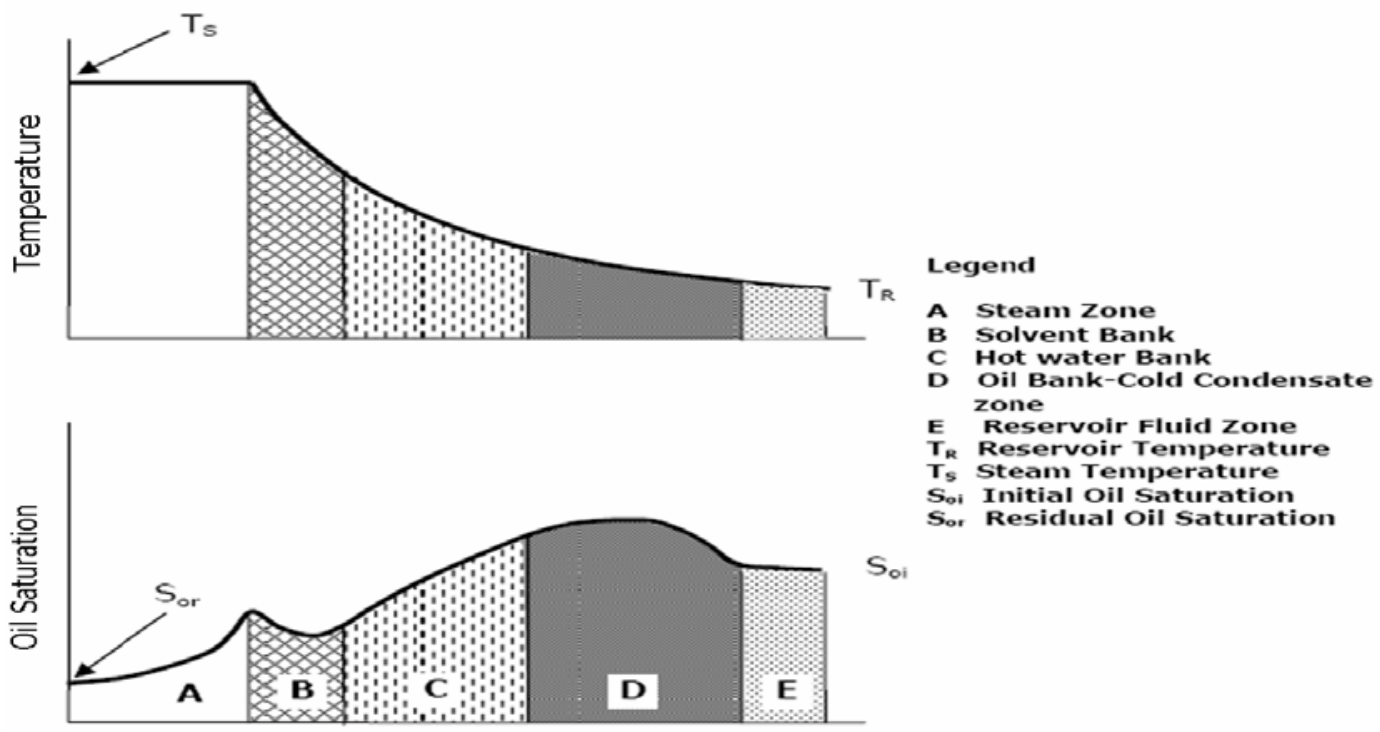

Distance from injection well to producing well

Figure 1.2 - Temperature and Saturation profiles during steam injection (from K. C. Hong 1994).

Although steam flooding enjoys a higher productivity it suffers from the following drawbacks:

(1) Channeling of the steam through high permeability zones.

(2) Gravity override which leads to early breakthrough. This reduces the amount of contacted oil in the reservoir, only heating the upper portion of the reservoir. The oil that is directly below it is not heated. Accumulation of steam on the top portion of the reservoir causes heat losses to the overburden. Therefore the portion of reservoir that is swept by steam has low residual oil saturation whereas the bottom part of the reservoir has significantly higher oil saturation.

To improve oil recovery-particularly residual oil by-passed due to steam override, various steam additives have been studied and tested in the past. Steam additives such as propane and carbondioxide have been shown to increase the recovery factor but the cost 
of the injecting additives with steam is high. A series of experiments conducted in the Ramey Lab at Texas A \& M University has shown that the addition of propane to steam significantly accelerates the start of oil production but not the recovery factor of oil. Therefore there arises a need to improve the heavy oil recovery with the help of a suitable steam additive.

The main purpose of this research is to test the effect of lowering IFT and thus increasing oil recovery by the addition of an alkali to steam. The alkali ( $\mathrm{NaOH})$ would react with organic acids in the oil to form a surfactant that reduces the oil IFT.

\subsection{Overview of Sodium Hydroxide}

Sodium hydroxide is a white solid and is commercially available in pellets, flakes and granules and also as a $50 \%$ saturated solution. Sodium hydroxide is produced in large amounts by the chloralkali process. The basic properties of sodium hydroxide are shown in Table 1.1.

Table 1.1 - Properties of $\mathrm{NaOH}$

\begin{tabular}{|c|c|}
\hline IUPAC name & Sodium hydroxide \\
\hline Other names & Lye, Caustic Soda \\
\hline \multicolumn{2}{|c|}{ Properties } \\
\hline Molecular formula & $\mathrm{NaOH}$ \\
\hline Molar mass & $39.9971 \mathrm{~g} / \mathrm{mol}$ \\
\hline Appearance & White solid \\
\hline Density & $2.1 \mathrm{~g} / \mathrm{cm}^{3}$, solid \\
\hline Melting point & $318^{\circ} \mathrm{C}(591 \mathrm{~K})$ \\
\hline Boiling point & $1390^{\circ} \mathrm{C}(1663 \mathrm{~K})$ \\
\hline Solubility in water & $111 \mathrm{~g} / 100 \mathrm{ml}\left(20^{\circ} \mathrm{C}\right)$ \\
\hline Basicity (pKb) & -2.43 \\
\hline
\end{tabular}


Sodium hydroxide is used as a strong base in the industry. It is also used in:

(i) Production of soap.

(ii) Production of alumina.

(iii) Paper, pulp and textile industry and drain cleaners.

(iv) Aluminum etching and food preparation.

Caustic flood causes wettability reversal and improves the waterflood recovery of heavy oil. The mechanism involved is the drastic reduction of oil-water interfacial tension by the caustic activation of potentially surface active organic acids naturally occurring in the crude oil. The reduction of interfacial tension causes emulsification of the crude oil in situ that tends to lower injected water mobility, damp the tendency toward viscous fingering, slow water channeling caused by reservoir stratification and improve sweep efficiency. The success of a caustic steam flood depends on the amount of acid present in the oil, the reservoir rock pore geometry and the extent to which it consumes the caustic. The acid that is present in the oils reacts with positive sodium ion and form a soap which lowers the interfacial tension. The success of the process also depends on the presence of long chain hydrocarbons.

\subsection{Research Objectives:}

The main objectives of the research are as follows:

(1) To understand the effect of sodium hydroxide on heavy oils such as San Ardo on factors such as recovery factor of oil, interfacial tension, viscosity and density.

(2) To determine the optimal concentration of sodium hydroxide so that maximum amount of surfactant is formed during alkaline steam flooding. 
3) To measure the acid number of the oil in order to determine if sufficient amount of acids are present in the oil to form a significant amount of surfactant.

The alkaline steamflooding experiments are conducted on a one-dimensional cell in the Ramey Laboratory. Although it doesn't represent all the mechanisms like a three dimensional model it gives us valuable information about the process.

The first objective is aimed at understanding the behavior of heavy oils such as San Ardo when subjected to alkaline steam flooding. Alkaline steamflooding is carried out from the start unlike previous processes where the sandpack is waterflooded first and then alkaline steamflooding is carried out.

The second objective is aimed at understanding the optimal concentration at which the sodium hydroxide can be used in the steamflooding process. Since heavy oils like San Ardo have not been studied, the concentration of caustic is an important parameter to be determined.

The third objective is to determine whether there is enough amount of acid in the oil such that it can actually react and form a surfactant. 


\section{CHAPTER II}

\section{LITERATURE REVIEW}

In this section literature reviews of the studies on alkaline flooding are presented. Alkaline flooding processes have always been performed for waterflooded reservoirs.

Jennings (1974) conducted a study of caustic solution and crude oil interfacial tensions and found that a majority of the oils showed a marked surface activity against caustic solutions and the maximum measurable surface activity is obtained for majority of the samples at 0.1 percent by weight and the presence of dissolved solids in the water has a significant effect on the interfacial activity.

Ehrlich et al. (1976) conducted a series of laboratory caustic waterfloods on lighter oils (mostly $>30^{\circ} \mathrm{API}$ ). The caustic depletion measurements were conducted in various oil producing formations and in Berea sandstone and it was found that crude oils with acid numbers greater than 0.1 to $0.2 \mathrm{mg} \mathrm{KOH}$ per gram oil and interfacial tension less than 0.5 dyne/cm gave significant caustic waterflood production and the caustic consumption can be determined from mineral composition methods as determined by X-ray methods.

Goyal and Arora (1978) showed the applicability of caustic flooding for enhancing oil recovery in North Gujarat oil fields. They found that the optimum concentration of caustic that was used for the improvement of recovery was $0.25 \mathrm{wt} \%$. The injection rate affected the ultimate recovery of the oil and a smaller rate of $4 \mathrm{cc} /$ hour gave the best performance. The injection profiles were found to be smooth and the pumping pressures are lower for caustic than water. The breakthrough ultimate recovery with caustic 
injection on natural cores was (in \% OIP): $31.3 \%$ and $55.2 \%$ as against $20.7 \%$ and 41.9 $\%$ with water.

Okoye and Tiab (1982) studied the applicability of alkali as chemical additive to steam in the tertiary recovery of heavy oil. Four different alkalis: Sodium hydroxide, sodium silicate, sodium carbonate and potassium hydroxide were added to steam and tested with a $18{ }^{\circ} \mathrm{API}$.The following results were obtained:

(1) Alkaline solutions of sodium hydroxide and sodium silicate can be employed effectively as a chemical additive in steam flooding.

(2) Alkaline steam flooding can recover between 9 to $14 \%$ more oil than conventional steam flooding.

(3) Optimal temperature range for alkaline steam flooding lies between $250^{\circ} \mathrm{F}$ and $300^{\circ} \mathrm{F}$ above which rapid consumption and deterioration of the alkali begins.

(4) The presence of large $\mathrm{CO}_{3}{ }^{-}$ions are detrimental to alkaline steam flooding and the dissolution of silica by $\mathrm{NaOH}$ will not harm the caustic steam which attributes to the failure and success of the sodium carbonate and sodium silicate floods respectively.

(5) Attainment of very low interfacial tension does not ensure improved oil recovery but a minimum value is necessary for a successful steam alkaline flood.

Tiab, Okoye and Osman (1982) undertook a lab study of the use of caustic soda as a chemical additive in waterflooding and steamflooding in improving tertiary oil recovery. The process was studied using a sandpack saturated with $18^{\circ} \mathrm{API}$ oil. The sand pack was first waterflooded before all the secondary processes were done and the following observations were made: 
(1) Caustic waterflooding is effective when the residual oil saturation after waterflooding is high. When the oil saturation is low, the hot caustic to caustic steam flooding is more effective.

(2) The tendency of steam to override the bottom half of the formation is reduced during caustic steam flooding.

(3) The high recovery from cyclic steam/cool caustic flooding shows more oil can be recovered economically with lower temperature steam or hot water with weak caustic than with high temperature pure steam.

Okoye and Tiab (1985) presented a chemical equilibrium flow model that predicted that the surfactant slug generated insitu increases with temperature and increase in surfactant size recovers the immobile oil leading to higher recovery. They also established that the caustic consumption increases with the increase in the temperature. They predicted a $15 \%$ increase in oil recovery by caustic steam flooding when compared to conventional steam flooding.

Okoye and Haytadavoudi (1990) presented the screening tests that were conducted to determine the feasibility of caustic water flood in Bayou field Louisiana and found that the saponification resulting from $1 \%$ by wt. $\mathrm{NaOH}$ can increase the oil API gravity by 10 at $320^{\circ} \mathrm{F}$.The critical factor for optimal saponification appears to be caustic concentration while elevated temperature merely increase the rate of the reaction. The thermal degradation of caustic oil interaction is severe near the steam temperature. Minimum interfacial tension values are in the temperature range of $120^{\circ} \mathrm{F}$ to $143^{\circ} \mathrm{F}$ which will normally occur ahead of the steam zone and the lower part of the formation over ridden 
by steam. The displacement results conducted show that the caustic steam flood recovered between 10 to $15 \%$ more of OOIP than conventional steamflood.

Shedid and Abbas (2000) conducted flow experiments to study the surfactant alkaline steam flooding process through vertical wells and concluded that addition of surfactant or alkaline to steam drive improves the oil recovery of the process and surfactant steam flood recovers more oil than alkaline steam flood. A new technique for surfactant alkaline steam flooding was proposed which can be applicable to both waterflooded as well as conventional steam projects.

Fan and Buckley (2006) proposed an improved procedure for measuring acid numbers and also illustrated the results by correlating with the oil/ brine interfacial properties. While standard ASTM measurements use $\mathrm{KOH}$ as a titrant for determining acid numbers, substitution of $\mathrm{TBAOH}$ for $\mathrm{KOH}$ conferred additional stability on the titration results. The crude oil and the blank solutions must be spiked with oil soluble acid such as stearic acid to obtain accurate and repeatable titration end points in non aqueous potentiometric titrations Spiking solution ensures clear end points for small samples of oil taken unlike standard ASTM procedures where more quantity of sample is required. 


\section{CHAPTER III}

\section{EXPERIMENTAL APPARATUS AND PROCEDURE}

This chapter explains the experimental methodology used in my research.

\subsection{Apparatus}

The schematic diagram of the apparatus that is used for steam injection in the Ramey Laboratory is shown in Fig. 3.1. The apparatus may be divided into three systems: fluid injection system, fluid production system and the data logger system. A brief description of each system follows:

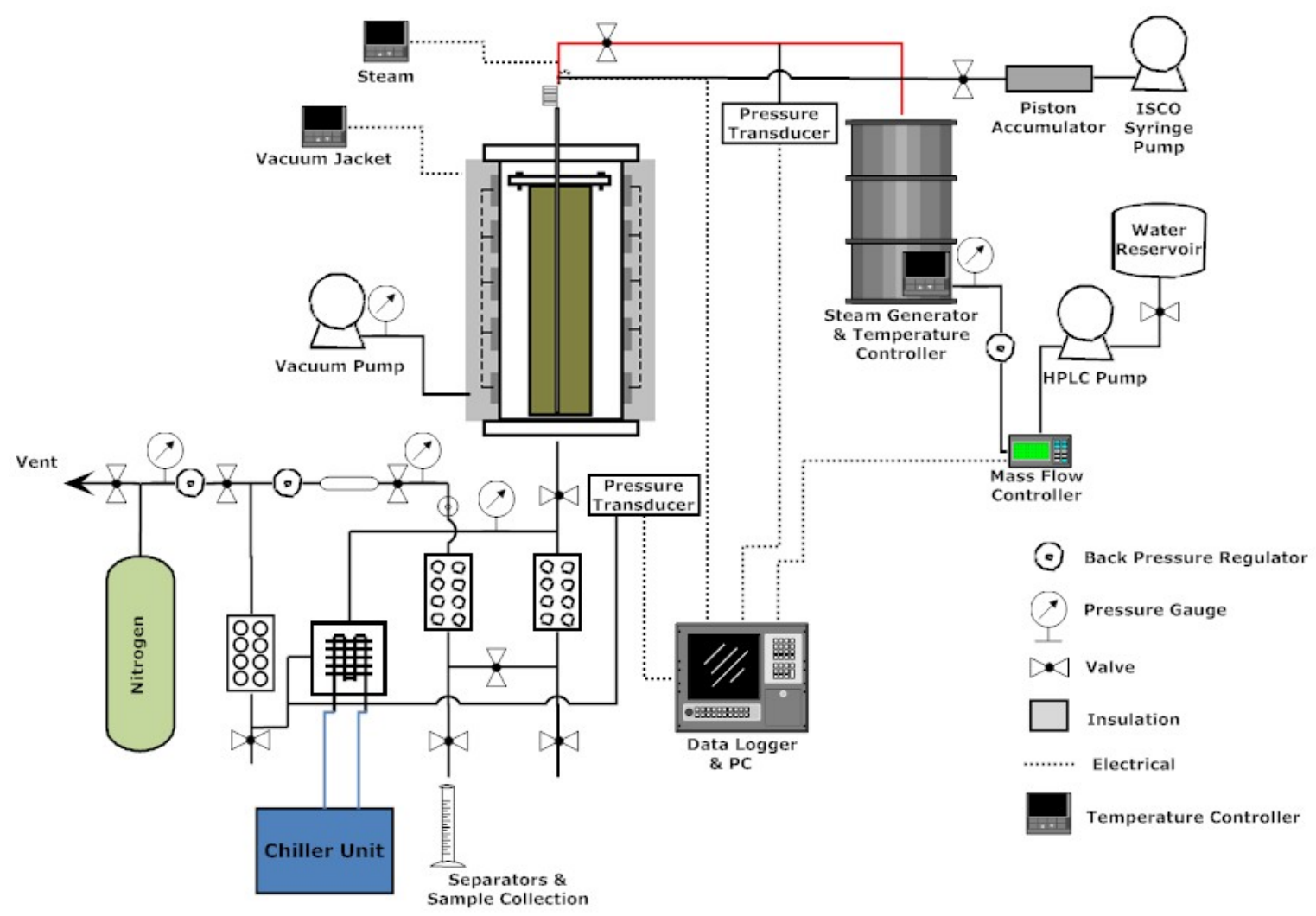

Figure 3.1 - Schematic diagram of the apparatus used for steam injection 


\subsubsection{Fluid Injection System}

The fluid Injection system consists of the following:

(1) Injection cell

(2) Two High Performance Liquid Chromatography Pumps(HPLC)

(3) Steam generator

(4) Accumulator

(5) Water and $\mathrm{NaOH}$ reservoirs

The injection cell contains the mixture of sand, water and oil. The cell is made of stainless steel and has length of $67 \mathrm{~cm}$ and an outer diameter of $7.4 \mathrm{~cm}$ shown in Fig. 3.2. The cell containing the mixture is placed in a vacuum jacket around which are wrapped electric heaters. These electric heaters are set to the reservoir temperature by means of a temperature controller. The vacuum jacket is set to the reservoir temperature and the cell is left overnight to ensure that there is uniform temperature throughout the mixture. A thermowell is placed in the longitudinal axis of the injection cell. The thermowell has a metal mesh attached at the bottom end to prevent any sand production. A thermocouple bundle consisting of six thermocouples is placed inside the thermowell. The thermocouples are placed inside a 1/8” tubing (sheath). The thermocouples measure the temperature throughout the length of the cell shown in Fig. 3.3. The vacuum jacket is connected to a vacuum pump which creates a vacuum in the annulus between the walls of an injection cell and vacuum jacket. The vacuum acts as an insulator and reduces heat losses during steam injection. 


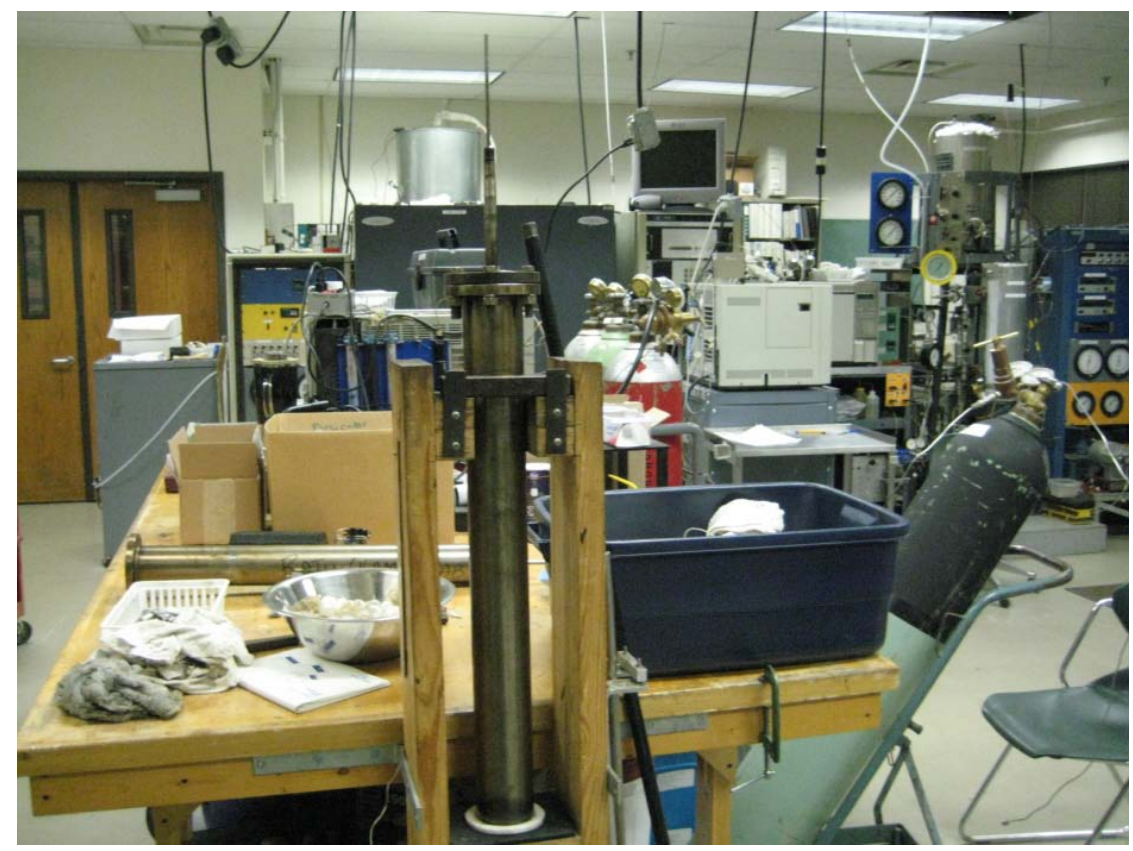

Figure 3.2 - Injection cell tamped with mixture 


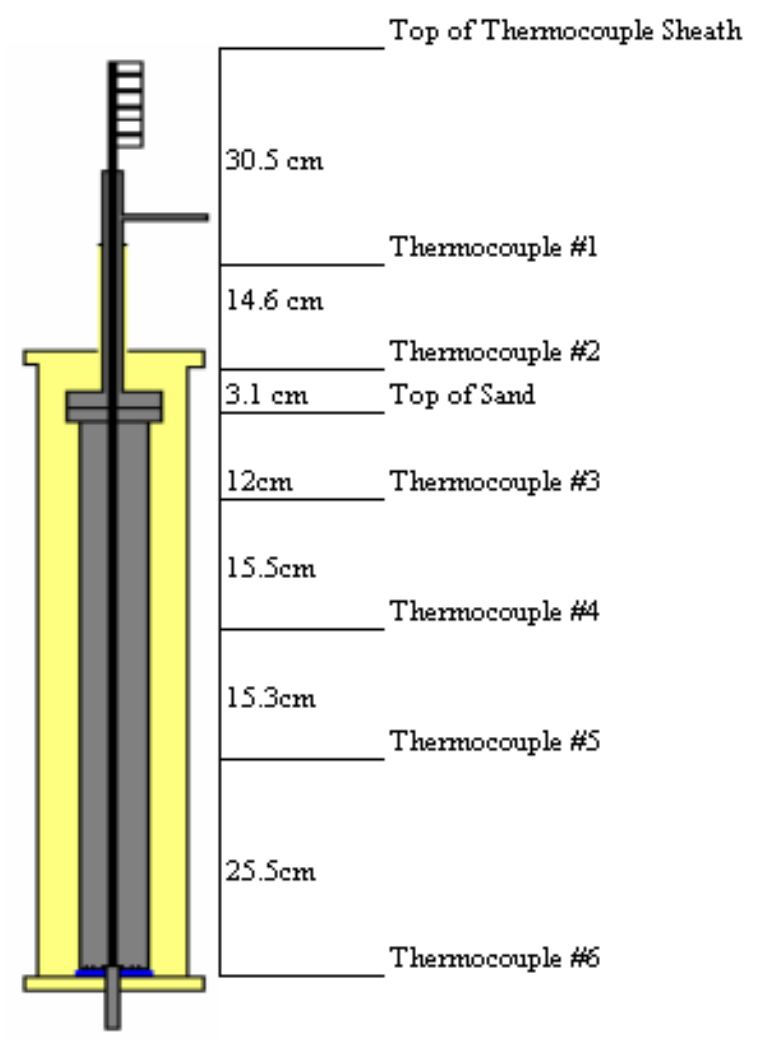

Figure 3.3 - Location of the thermocouples

A constant supply of distilled water and $\mathrm{NaOH}$ solution is supplied to the steam generator using a HPLC pump. The pump is set to a desired flow rate and the readings are directly fed into a data logger system. The second HPLC pump in conjunction with an accumulator is used to inject supplementary fluids like $\mathrm{NaOH}$ solution directly into the cell bypassing the steam generator, as in slug injection. The steam generator generates the steam at the desired temperature by means of a temperature controller.

\subsubsection{Fluid Production System}

The fluid production system consists of two separators and a condenser. Fluids from the cell pass through a tubing that is maintained at a temperature of $50^{\circ} \mathrm{C}$ by means of a 
band heater. The cell is maintained at a constant outlet pressure of 20 Psig by means of a back pressure regulator and the use of nitrogen gas. The first separator is connected to a second separator that is pressure regulated by nitrogen gas at constant pressure of 60 psig. Liquid samples are allowed to pass into the second separator and are collected at the bottom of the second separator in graduated tubes at regular intervals. The condenser unit present can be used to collect any condensate and is kept cool with the help of a chiller unit. The separator system is shown in Fig 3.4.

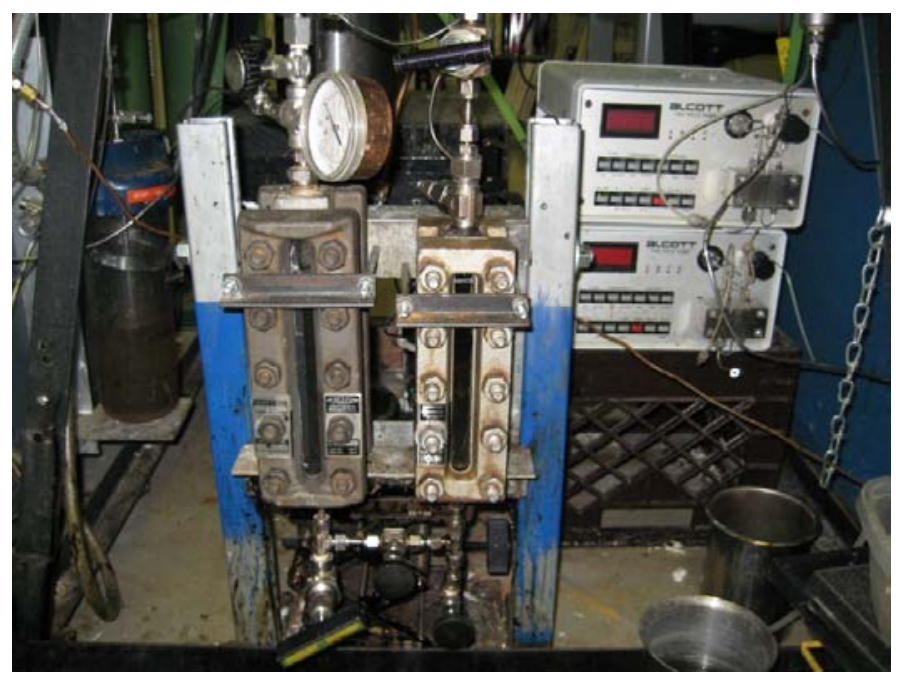

Figure 3.4 - Separator system

\subsubsection{Data Measurement and Recording System}

The various parameters are recorded in a preselected data file .The data logger registers the following

- Injection temperature

- Injection pressure

- Cell profile temperatures 
- Outlet pressure

- Water pump rate

A brief description of the main components of the experimental equipment follows:

(1) HPLC pump: Beckman (model 100A) High Performance Liquid Chromatography pump which ensures water is supplied continuously to the steam generator.

(2) HPLC pump: An Alcott High Performance Liquid Chromatography pump is used to inject the sodium hydroxide solution.

(3) Steam Generator: Custom made by Texaco with a maximum pressure of 2000 psig and a maximum temperature of $1200^{\circ} \mathrm{F}$ that provides the necessary steam of the experiments.

(4) Temperature controllers: A dual circuit temperature controller is used to maintain constant temperature of the steam generator. Other temperature controllers were also used to maintain temperatures for the heating jacket and two band heaters used at the inlet and outlet of the injection cell.

(5) Injection cell: A stainless steel cell that measures $66.5 \mathrm{~cm}$ in length and $7.37 \mathrm{~cm}$ in diameter. And can withstand a maximum pressure of 500 psig at $1000^{\circ} \mathrm{F}$.

(6) Accumulator: A 150 cc stainless steel, high pressure bottle was used to hold the necessary injection fluids.

(7) Heating jacket: A stainless steel cylinder with five steel band heaters and is well insulated to prevent heat loss. It confines the injection cell and the heaters heat the injection cell to reservoir temperature before steam injection and the annulus is 
evacuated with a vacuum pump just before steam injection to minimize heat loss during steam injection.

(8) Vacuum pump: used to evacuate the annulus between the injection cell and the heating jacket to minimize heat loss.

(9) Three-stage separation and sampling system: These are used to separate the liquids from the gases and also to sample the production. They consist of three high temperature high pressures transparent glass level gauges. The first separator in connected to the outlet of the injection cell and the backpressure nitrogen line from the top and connects to the second separator from the bottom The second separator is used to collect the liquid sample at pre-selected intervals. The first separator also connects to a condenser to liquefy the gases before they are collected in third separator.

(10) Chiller unit: used to lower the temperature of the condenser between first and third separator.

(11) Data logger: a Hewlett Packard data acquisition unit was used to log the necessary data such as temperature, pressure and injection rate.

\subsection{Experimental Procedure}

The main steps carried out in a typical experiment are as follows:

First, the steam generator, the pumps, cell and the tubing are cleaned to avoid any contamination and the pressure transducers are calibrated and the data logger system is tested.

Second, the mixture is prepared by mixing for example $5141 \mathrm{~g}$ of sand, $226 \mathrm{~g}$ of water and $671 \mathrm{~g}$ of oil using a predetermined ratio of oil water and sand and is mixed in a 
mixer thoroughly. About a handful of the mixture is then put into the cell and is tamped. The process is repeated until the sand mix fills the cell to the top. The cell is weighed before and after tamping to know the exact amount of mixture in the cell. This information combined with fluid and sand densities are used to calculate the pore volume, fluid volumes and saturations within the cell. The injection cell is then pressure tested and ready to be placed in the vacuum jacket. The cell inlet and outlet are connected to the steam generator and separator respectively.

The temperature of the vacuum jacket is set and left overnight to insure the mixture has reached the required reservoir temperature $\left(60^{\circ} \mathrm{C}\right)$. The annulus between the cell and jacket is evacuated to insure minimal heat loss during the steam injection. To prevent further heat losses one electrical band heater is wrapped around the tubing connecting the cell inlet and to the outlet of the steam generator. One band heater is also wrapped around the first separator. With the thermocouple placed in the thermowell the cell can now be pressurized (200 psig) using nitrogen gas.

The HPLC pump is set to feed water into the steam generator at a constant rate (5.5 $\mathrm{cc} / \mathrm{min}$ ) that is monitored by a mass flowmeter. Injection into the cell begins once the steam generator reaches its preset temperature $\left(237^{\circ} \mathrm{C}\right)$ and pressure $(200 \mathrm{psig})$. The steam injection temperature is maintained by using a temperature controller. Production pressure at the cell outlet is controlled by nitrogen gas supplied through a backpressure regulator.

Water rate, injection pressure, injection temperature, production pressure and cell temperature profile were recorded by the data logger system. The data logger system 
records data every 30 second. The data is displayed real time on the computer display to assist in monitoring experimental conditions during the run.

Periodic sampling was carried out from the second separator by enabling flow from the first separator. The samples are centrifuged for 30 minute at 2000 RPM to insure separation of oil and water for proper measurement of production volumes. The oil production is divided into to equal fractions, one of the first half of the production and the other for the remainder part of the production. Oil density and viscosity for the two production samples are measured using an Anton Parr DMA 4100 density meter and a Brookfield rheometer.

Acid number measurements (AN) were made by using a titration method. The solvents used for the titrations was a mixture of 50\% toluene (HPLC grade), 49.4\% IPA (HPLC Grade) and $0.06 \%$ deionized water. A spiking solution was prepared of 0.02M stearic acid was prepared in the solution above and each AN measurement was spiked with $1 \mathrm{ml}$ of this solution. The titrant used is Tetrabutyl ammonium hydroxide (TBAOH) (Aldrich, $1 \mathrm{M}$ in methanol) diluted to $0.05 \mathrm{M}$ with Ethanol. Titrants were calibrated with a solution of KHP (Aldrich, ACS primary standard) at a concentration of $0.002 \mathrm{M}$ in DDW.Now the EMF readings were measured with a ph meter. A burette was used to deliver titrants at rates of $0.1-0.25 \mathrm{ml} / \mathrm{min}$. Interfacial Tension was measured using a tensionometer for different concentrations of sodium hydroxide and oil. 


\section{CHAPTER IV}

\section{EXPERIMENTAL RESULTS}

\subsection{Experimental Conditions}

A general overview is presented in this section about the conditions at which the experiments were done. A few parameters were kept constant for all the experiments in order to have a comparison between the runs.

(a) Cell Temperature: $60^{\circ} \mathrm{C}$

(b) Water Injection: $5.5 \mathrm{cc} / \mathrm{min}$ (CWE)

(c) Steam Temperature: $237^{\circ} \mathrm{C}\left(\right.$ superheated by $\left.30^{\circ} \mathrm{C}\right)$

(d) Production pressure: 200 psig

(e) Oil saturation

(i) For all experiments involving San Ardo the oil saturation was about $60 \%$.

(ii) For all experiments involving Duri the oil saturation was about $30 \%$.

(f) The time involving simultaneous caustic steam injection was around 4 hrs and time for caustic injected as slug was around 7 hrs.

For all the experiments a standard procedure was followed in preparing the mixture, tamping it into the cell and then conducting the experiments to ensure that the saturations of the oil remain the same. The saturations of air, oil and water for all the runs are shown in Table 4.1.

The following runs will be explained in this chapter:

(a) Run 1: Base run for San Ardo Oil using pure steam 
(b) Run 2: Pure steam with $\mathrm{NaOH}(0.1 \mathrm{wt} \%)$ for San Ardo oil

(c) Run 3: Base run for Duri oil using pure steam

(d) Run 4: Pure steam with $\mathrm{NaOH}(0.1$ wt \%) for Duri oil

(e) Run 5: Pure steam with $\mathrm{NaOH}(1 \mathrm{wt} \%)$ for Duri oil

(f) Run 6: Cyclic Injection of $\mathrm{NaOH}(1 \mathrm{wt} \%)$ and steam for San Ardo oil.

(g) Run 7: Pure steam Injection $\mathrm{NaOH}$ (1 wt \%) for San Ardo oil.

The calculations for the cell mixture are presented in Appendix A. The data-logger results, production data and produced oil analysis data are shown in Appendix B, C and D respectively.

Table 4.1 - Injection cell mixtures for run 1 to run 7

\begin{tabular}{|c|c|c|c|c|c|c|c|}
\hline & Run 1 & Run 2 & Run 3 & Run 4 & Run 5 & Run 6 & Run 7 \\
\hline Sand, g & 5141 & 5141 & 5141 & 5141 & 5141 & 5141 & 5141 \\
\hline Water, g & 226 & 226 & 226 & 226 & 226 & 226 & 226 \\
\hline Oil, g & 671 & 671 & 365 & 365 & 365 & 365 & 671 \\
\hline So, frac & 0.59 & 0.58 & 0.288 & 0.3 & 0.29 & 0.54 & 0.57 \\
\hline Sw, frac & 0.2 & 0.22 & 0.17 & 0.17 & 0.17 & 0.18 & 0.2 \\
\hline
\end{tabular}

For each of the runs the following experiments the following data will be presented:

(a) Temperature profile for each of the thermocouples

(b) Injection and production pressure profiles

(c) Oil and water production

(d) Viscosity and density readings

(e) Average steam front velocity 
For each of the oils the acid number measurements and the interfacial tension measurements with different concentrations of $\mathrm{NaOH}$ are also presented.

\subsection{Run 1: Base Run for San Ardo Oil Using Pure Steam}

For the base run only pure steam was injected by means of the steam generator into the injection cell. The temperature profile for the pure steam is shown in Fig. 4.1.

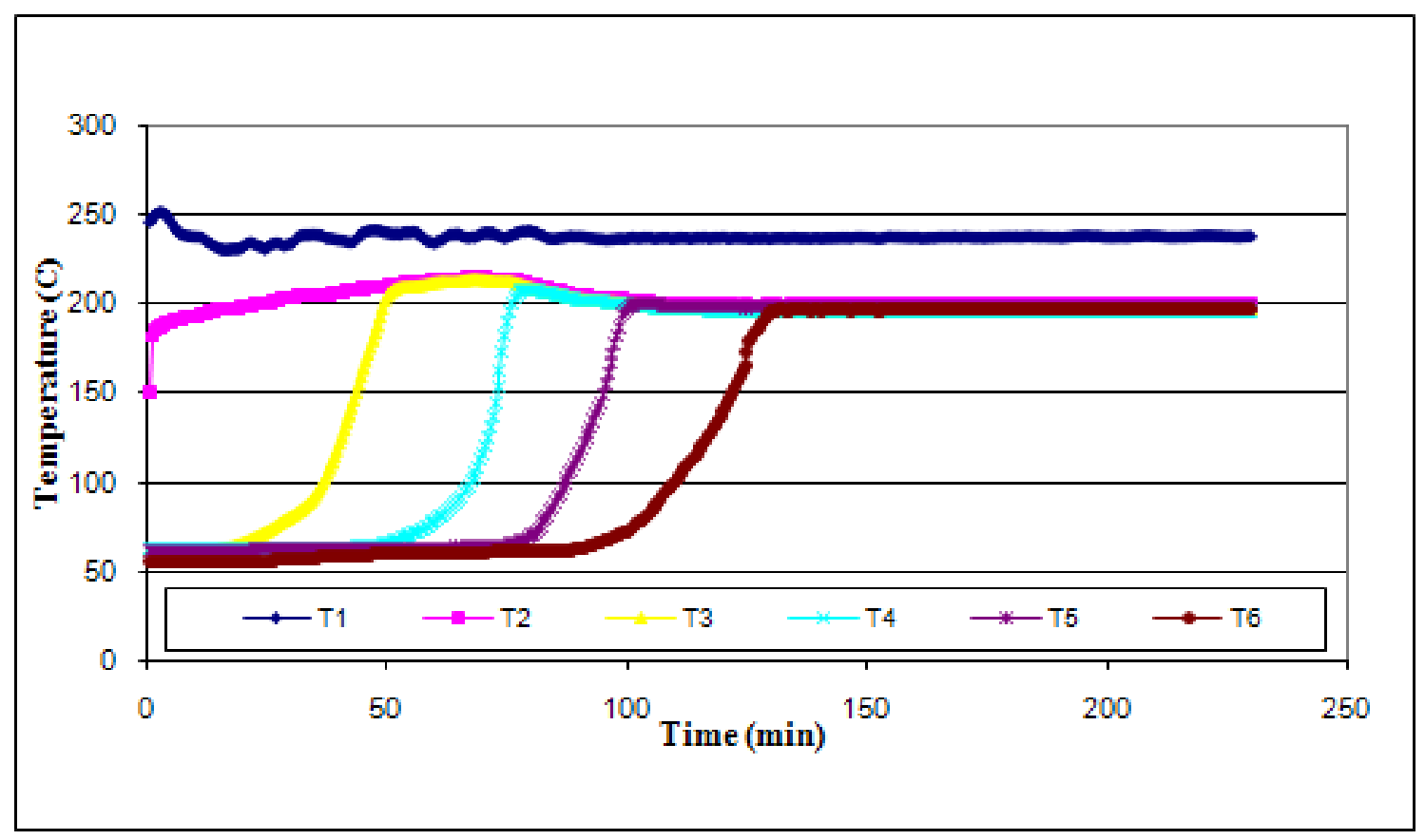

Figure 4.1 - Temperature profile for run 1

From the temperature profile we see that the thermocouples attain a constant temperature after about 135 min indicating the steam front reaching the bottom of the cell. The pressure profiles for run 1 are also plotted and shown in Fig. 4.2. 


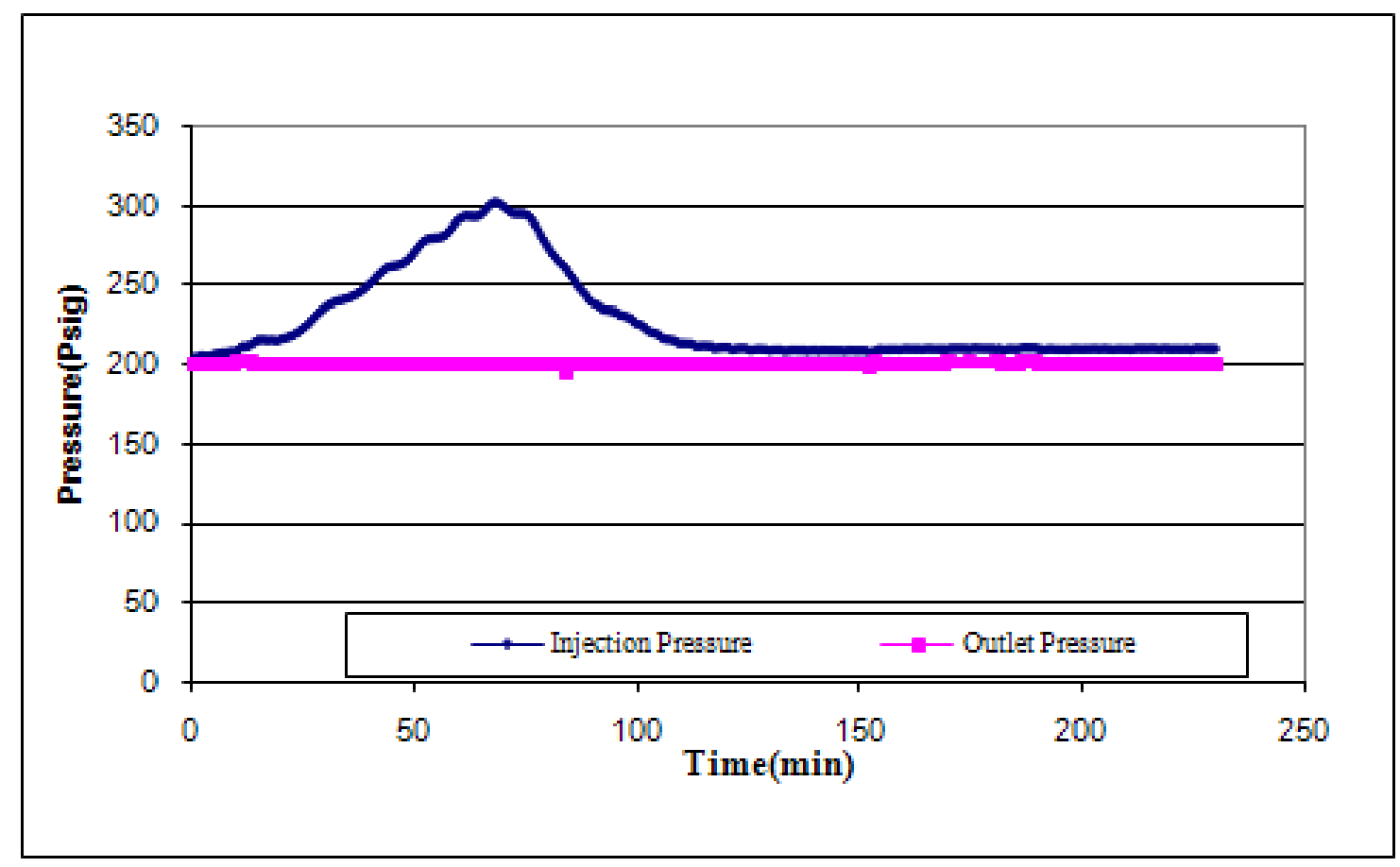

Figure 4.2 - Pressure profile for run 1

From the pressure profile we see that the outlet pressure is maintained at a constant pressure of 200 psi and the injection pressure reaches a maximum value of 306 psi. The increase in the injection pressure is attributed to the oil bank in the cell and the once the oil bank is pushed and starts producing the injection pressure decreases and reaches a minimum value of 206 psi.

The production profiles for oil and water are shown in Fig. 4.3. For the oil production curve we see that the maximum production rate is $9 \mathrm{cc} / \mathrm{min}$ and in the water production the maximum value for the water production is $9.25 \mathrm{cc} / \mathrm{min}$. 


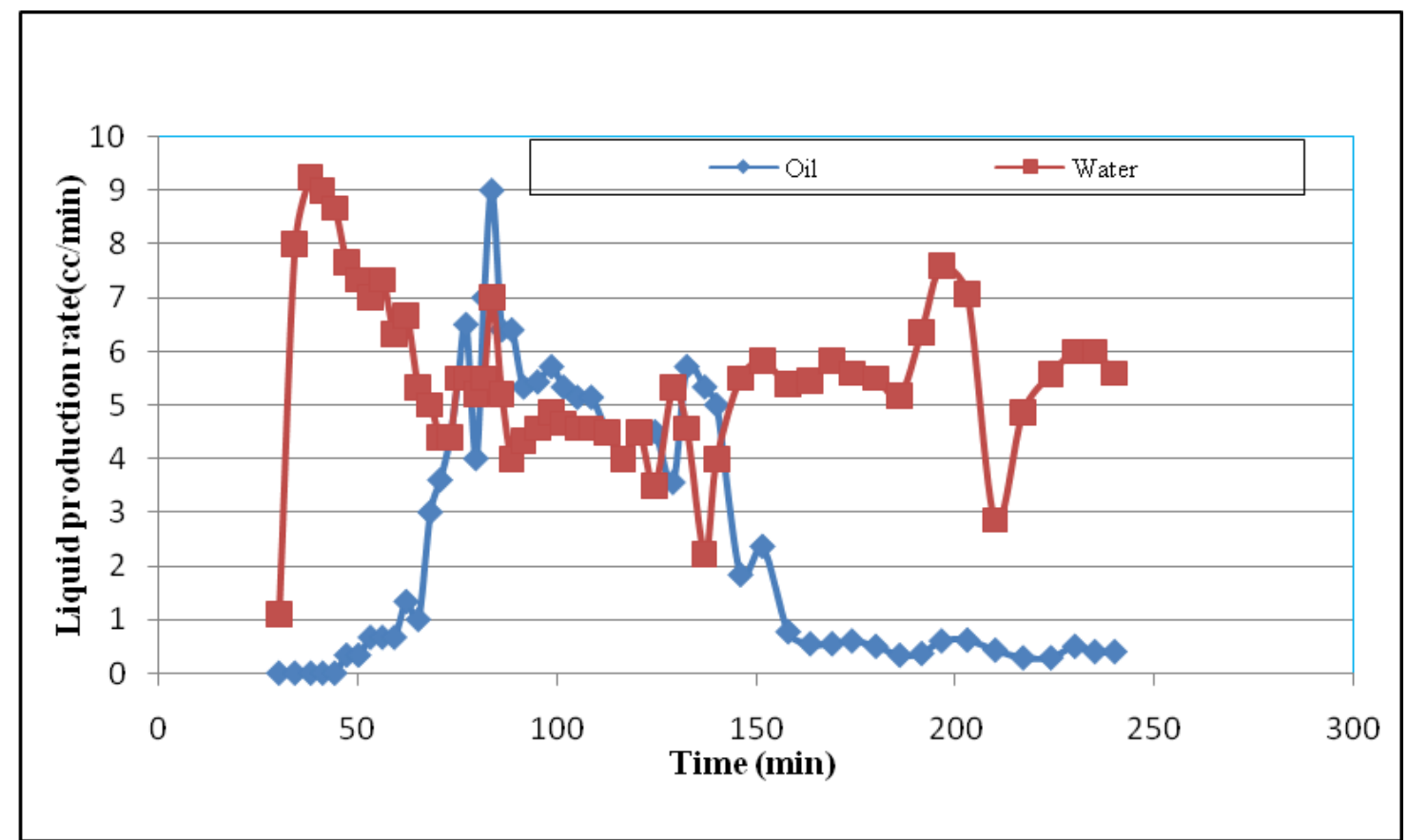

Figure 4.3- Liquid production rates for run 1

The total oil produced in the run was about 460 cc which represents $75.3 \%$ of the original oil in place. The recovery versus time is plotted in Fig. 4.4 and Fig. 4.5 shows the recovery versus pore volume of steam injected. 


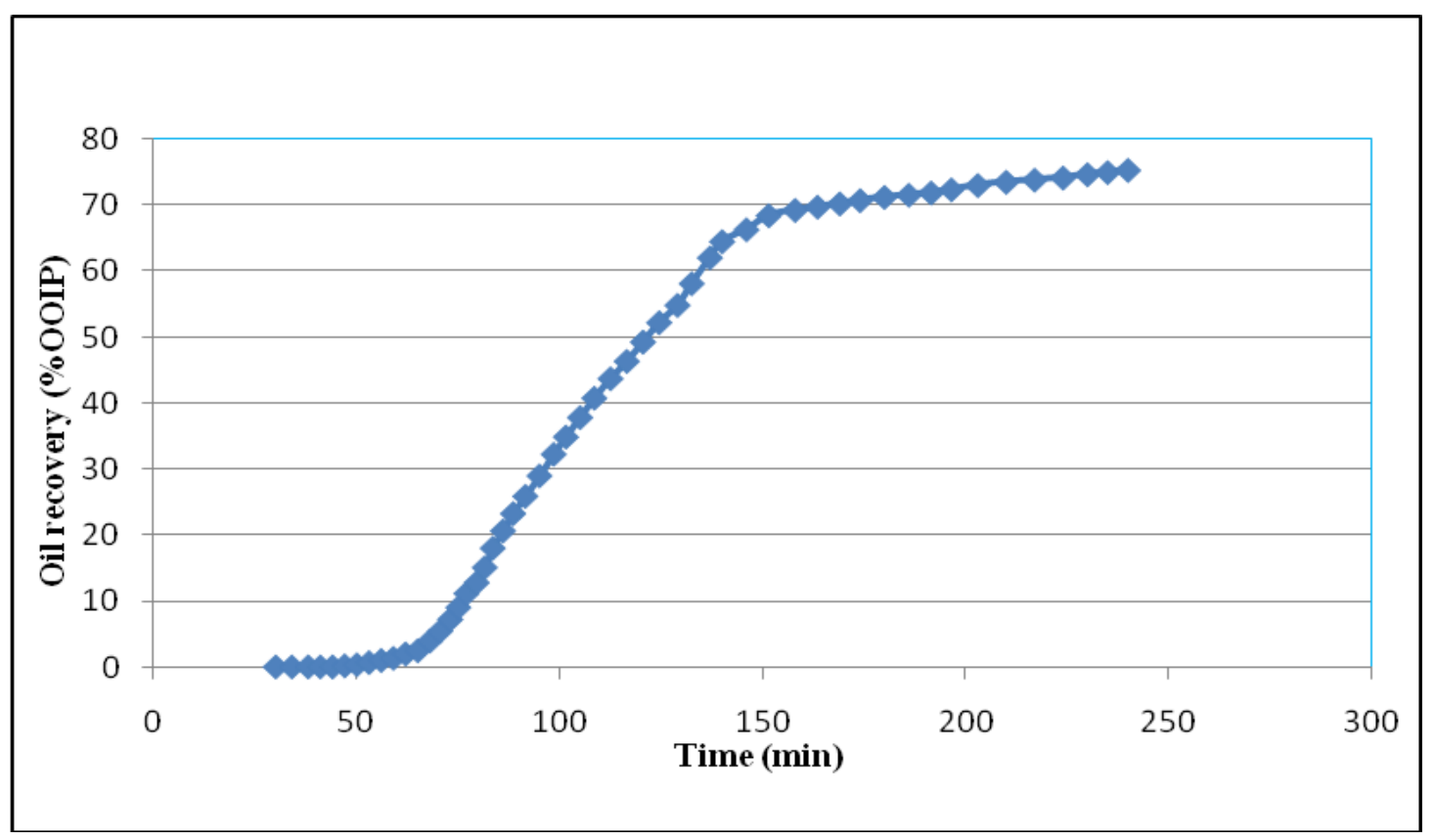

Figure 4.4 - Oil recovery versus time for run 1

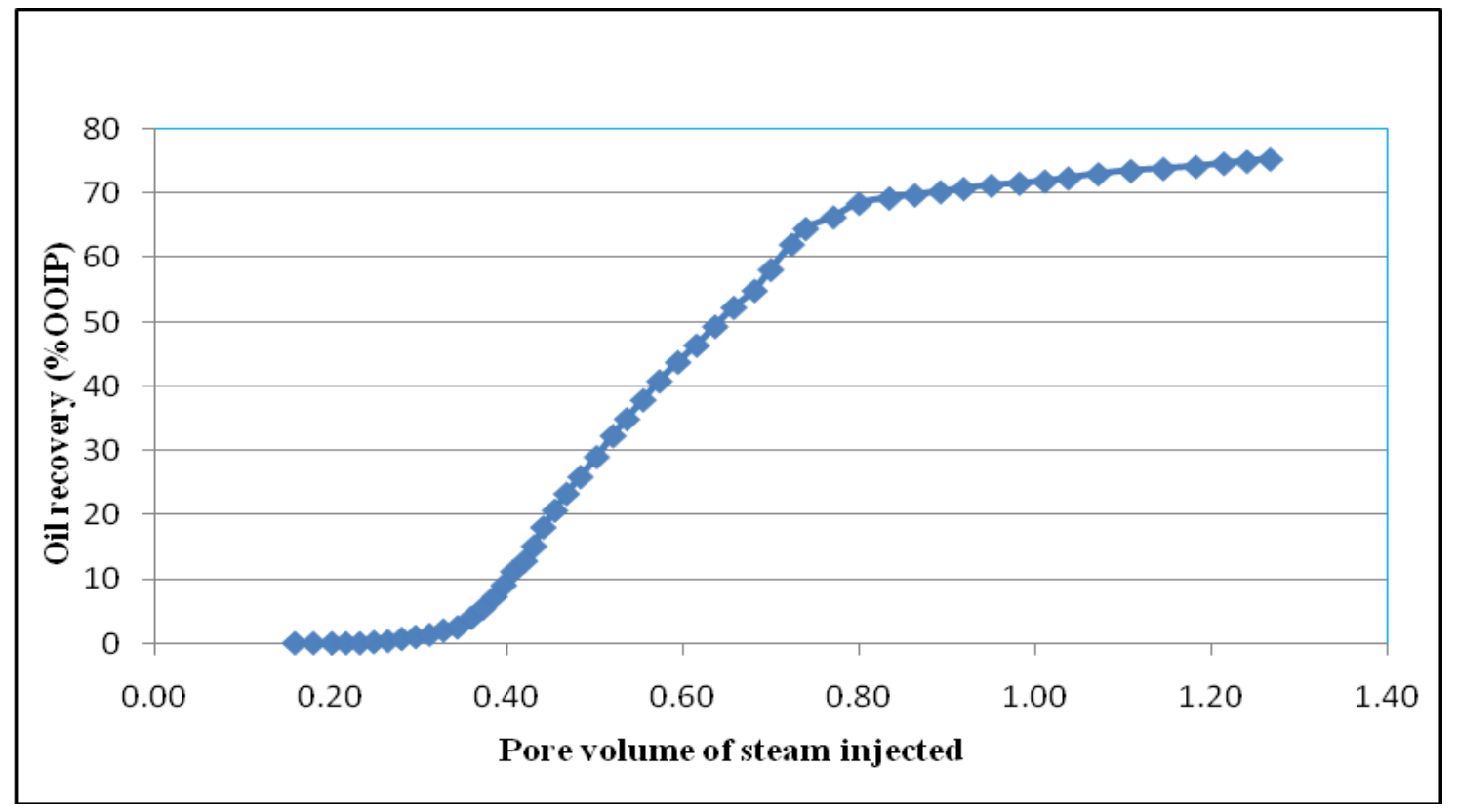

Figure 4.5 - Oil recovery versus pore volume of steam injected for run 1 
The steam front velocity for Run 1 is shown in Fig. 4.6.

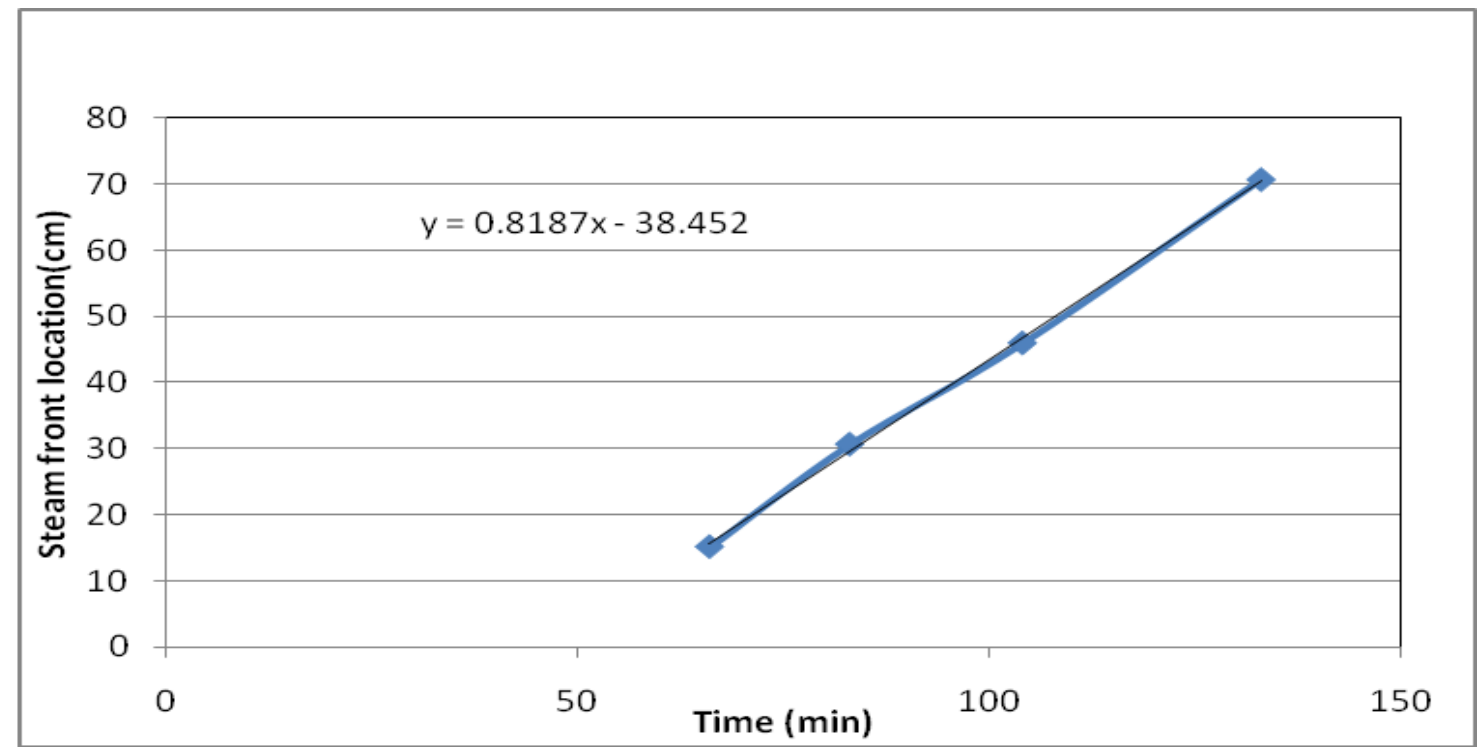

Figure 4.6 - Steam front velocity for run 1

The viscosity and the density readings are shown in Table 4.2.

Table 4.2-Viscosty and density readings for run 1

\begin{tabular}{|c|c|c|c|}
\hline \multicolumn{4}{|c|}{ Run 1 (Pure Steam -San Ardo) } \\
\hline \multirow{2}{*}{ Density $\left({ }^{\circ} \mathrm{API}\right)$} & Initial & Middle & Final \\
\cline { 2 - 4 } & 14 & 14.7 & 16 \\
\hline Temperature $\left({ }^{\circ} \mathrm{C}\right)$ & \multicolumn{3}{|c|}{ Viscosity (cp) } \\
\hline 40 & 5632 & 4400 & 2214 \\
\hline 50 & 2976 & 2106 & 1875 \\
\hline 60 & 1400 & 1189 & 870 \\
\hline
\end{tabular}




\subsection{Run 2: Steam with NaOH (0.1 wt \%) for San Ardo Oil}

For run 2 sodium hydroxide ( $0.1 \mathrm{wt} \%)$ was injected into the cell along with the steam.

The temperature profile for run 2 is shown in Fig. 4.7.

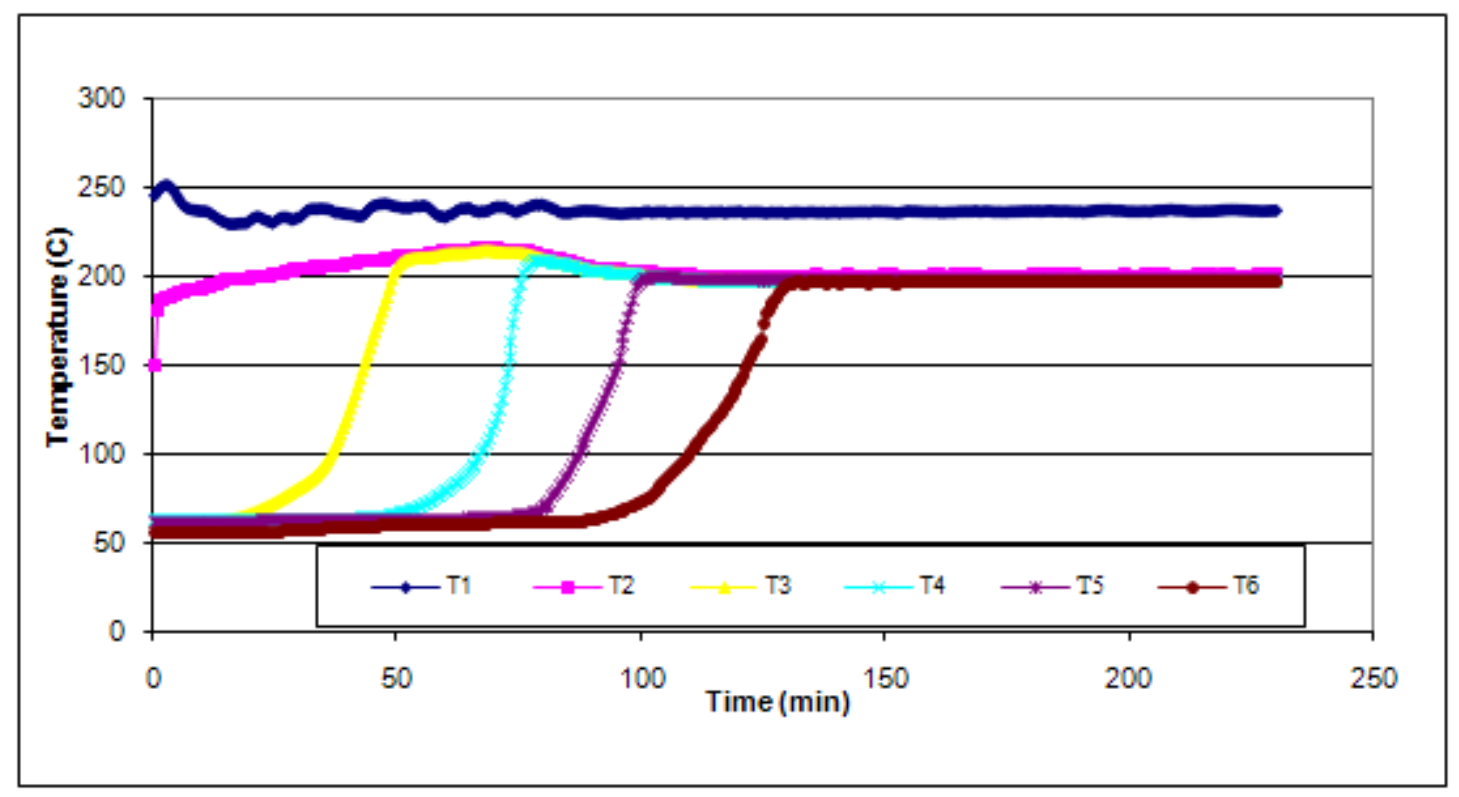

Figure 4.7 - Temperature profile for run 2

The thermocouples reach the constant temperature value at 125 min which is the saturated temperature of $200^{\circ} \mathrm{C}$. The pressure profiles are shown in Fig. 4.8. From the pressure profile it is seen that the maximum pressure is around $310 \mathrm{psig}$ and at the end of the run when the oil is produced it reduces to 206 psig. The oil and water rates are shown in Fig. 4.9 and the maximum oil rate is $7 \mathrm{cc} / \mathrm{min}$ and for maximum water rate is 11.75 $\mathrm{cc} / \mathrm{min}$. 


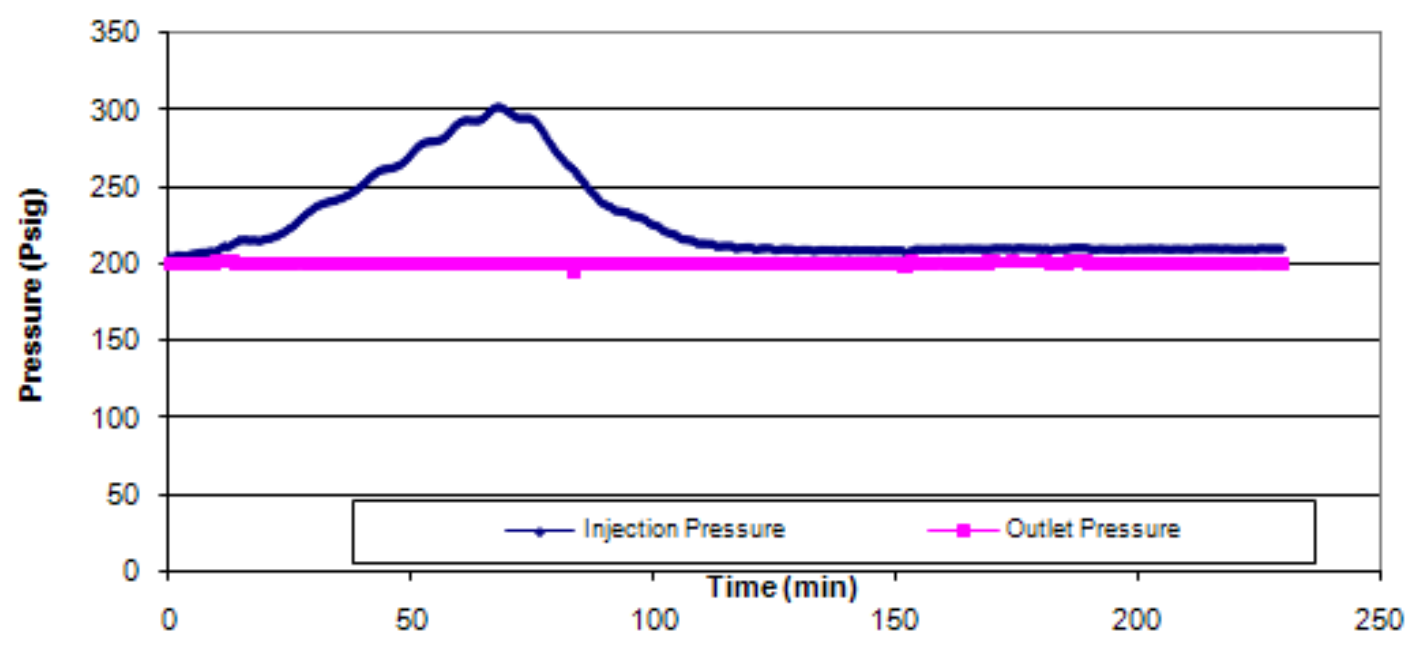

Figure 4.8 - Pressure profile for run 2

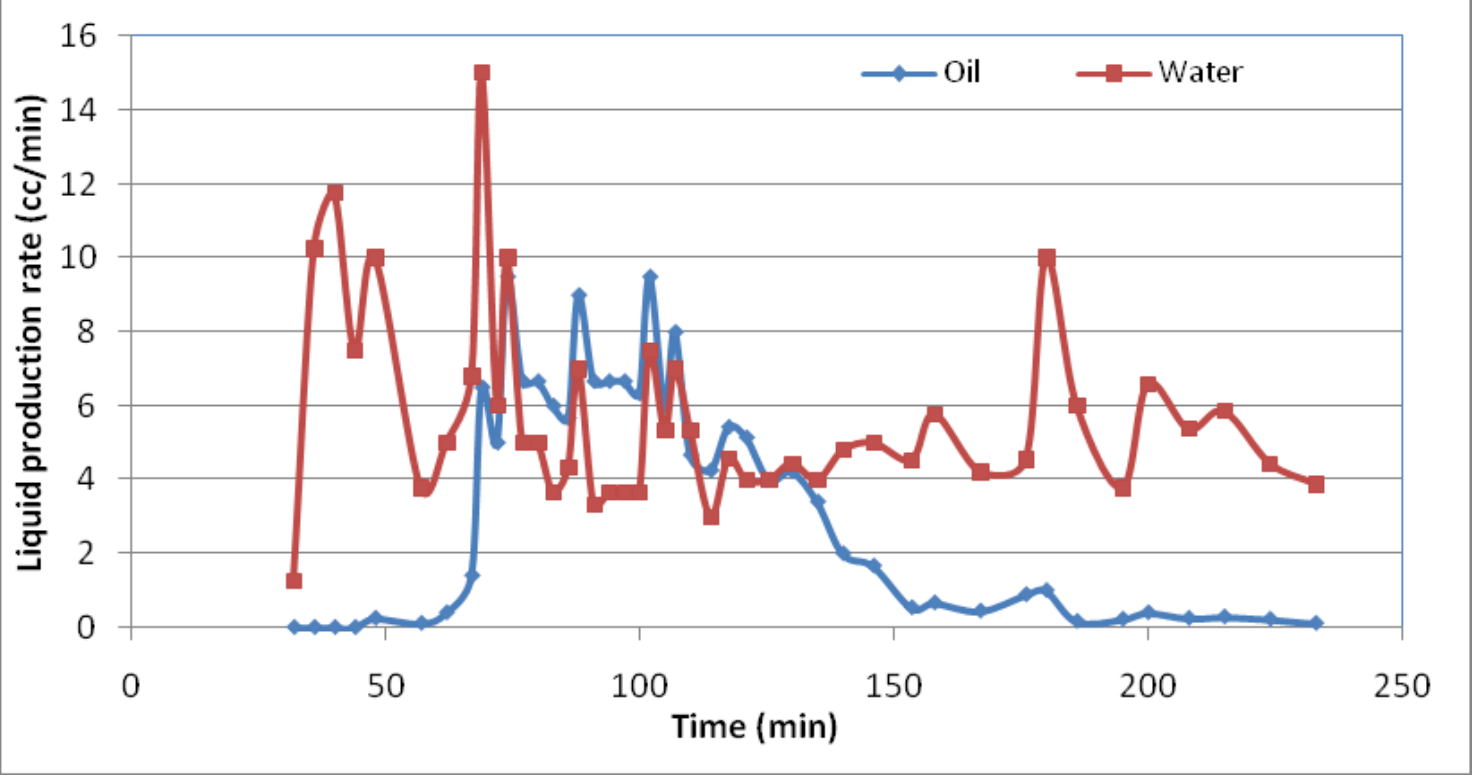

Figure 4.9 - Liquid production rates for run 2 
The total oil recovered from the steam-NaOH injection is 462 cc. Fig. 4.10 showing the oil recovered with respect to time indicates $75.7 \%$ OOIP is recovered. The recovery factor plotted against pore volume is shown in Fig. 4.11.

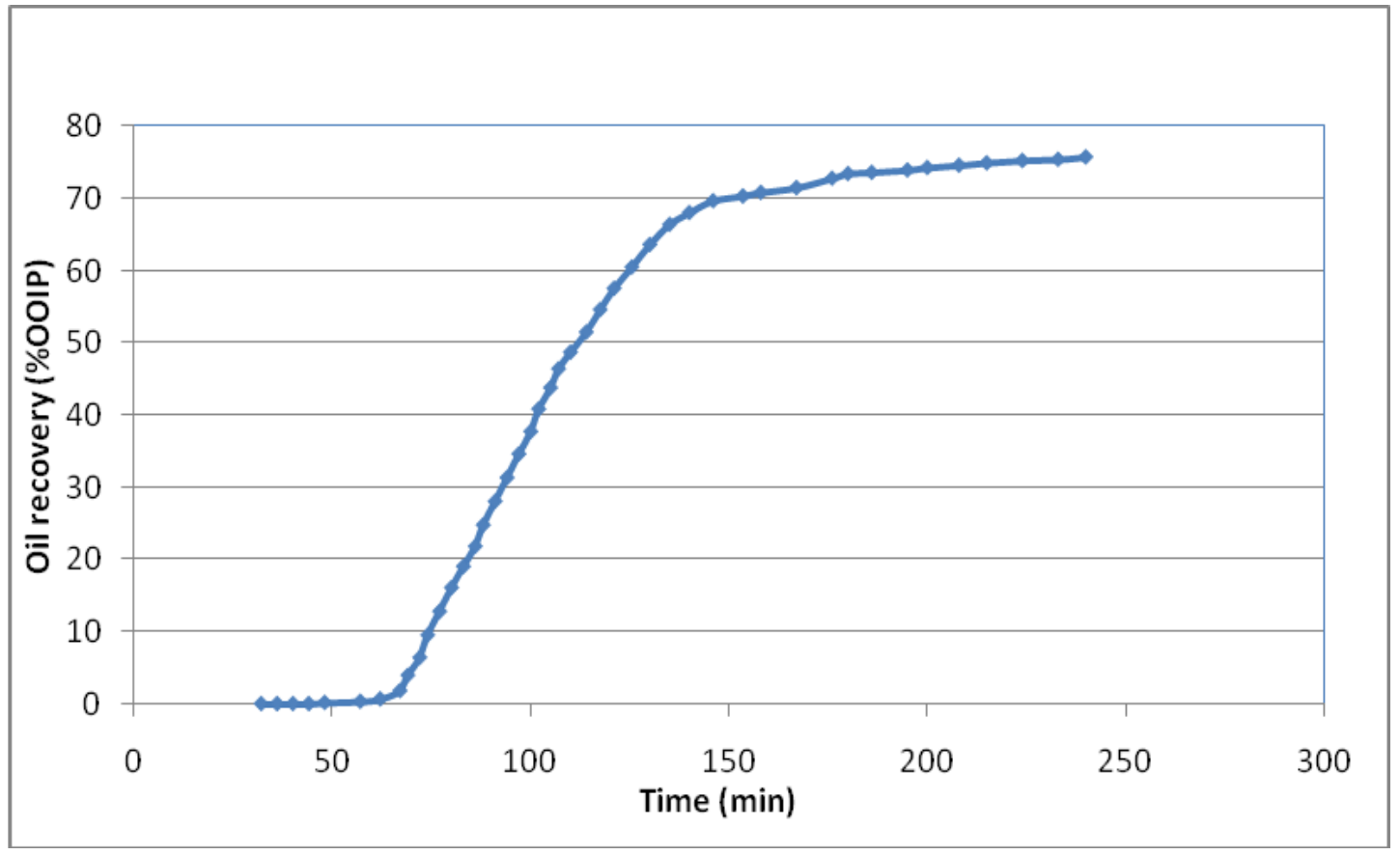

Figure 4.10 - Oil recovery versus time for run 2 


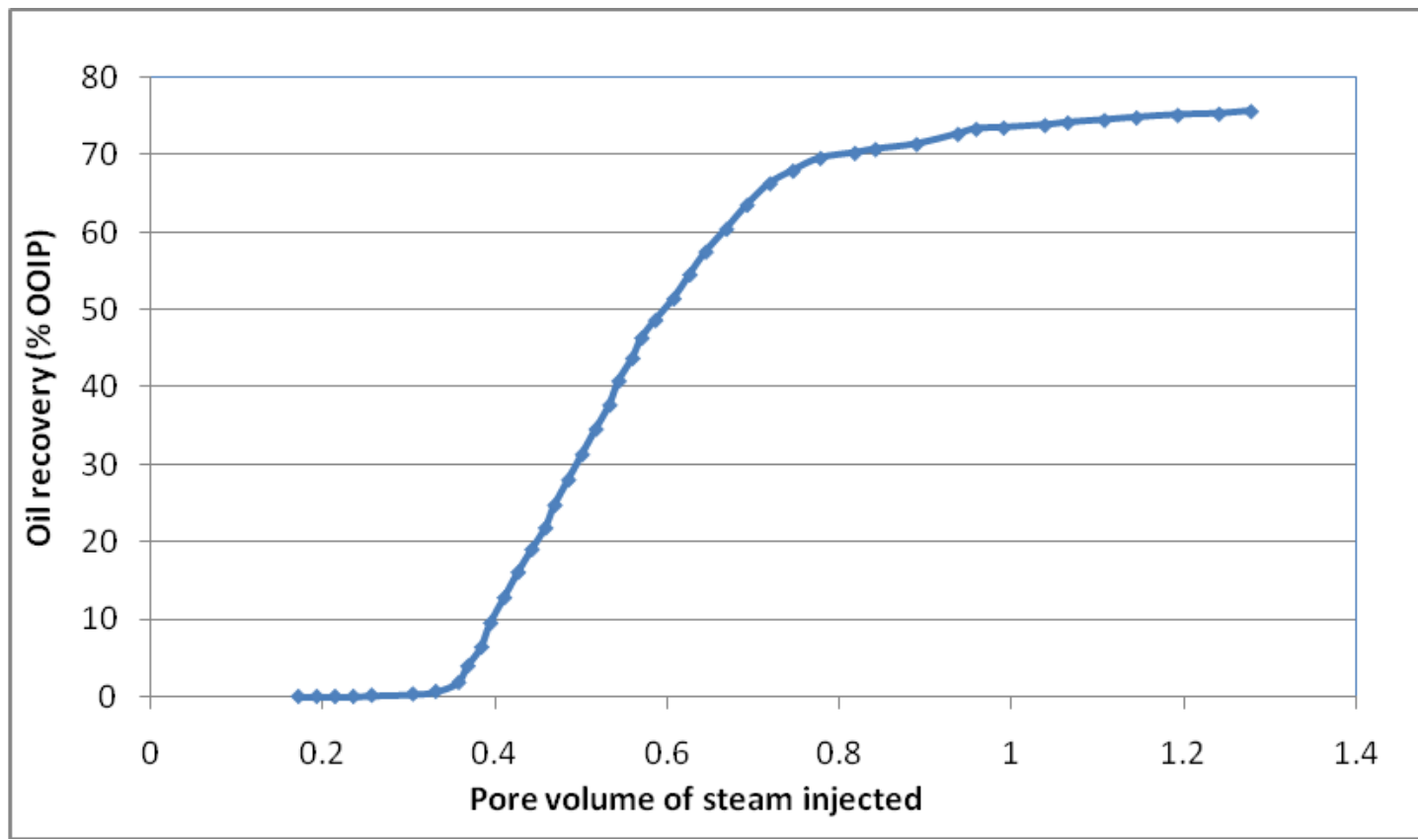

Figure 4.11-Oil Recovery versus pore volume of steam injected for run 2

The steam front velocity for the steam-NaOH is shown in Fig 4.12. 


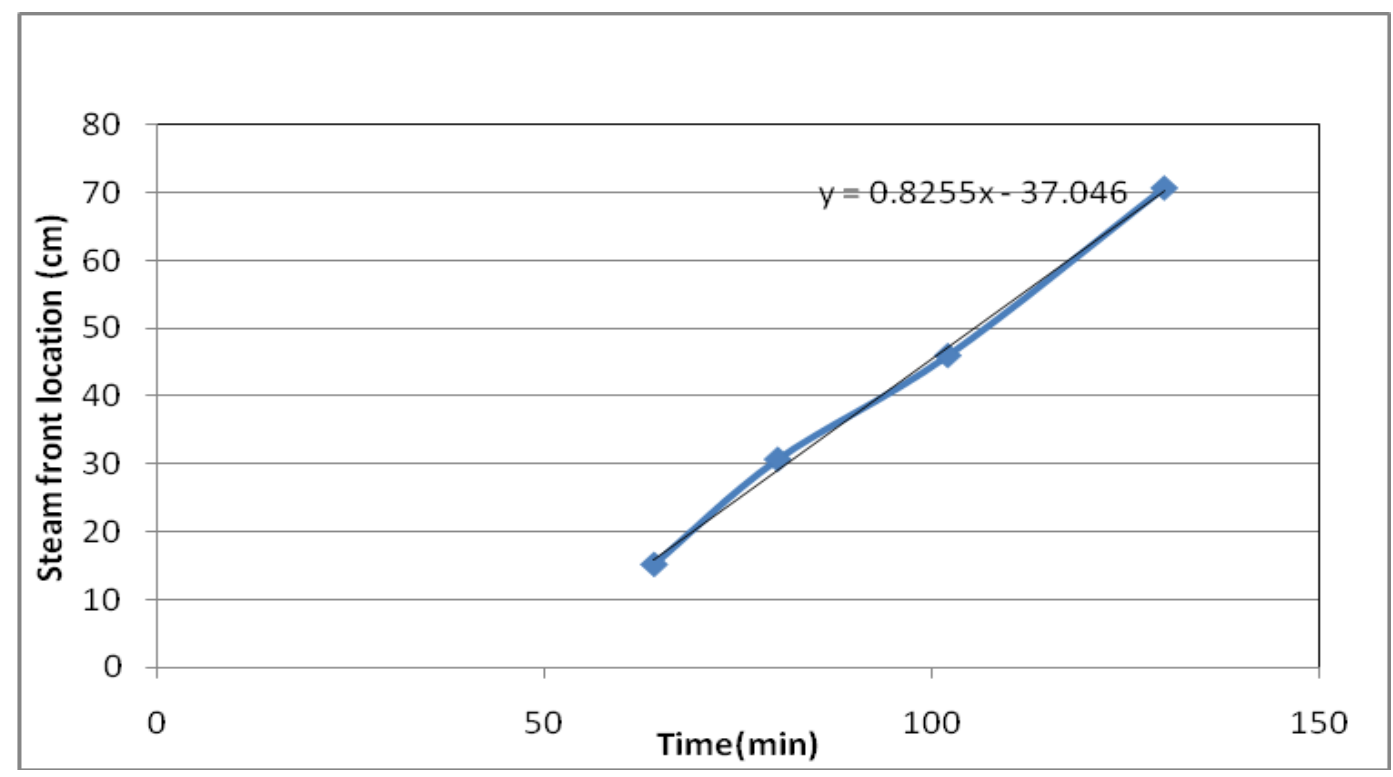

Figure 4.12 - Steam front velocity for run 2

The viscosity and density readings for run 2 are shown in Table 4.3.

Table 4.3-Viscosity and density readings for run 2

\begin{tabular}{|c|c|c|c|}
\hline \multicolumn{4}{|c|}{ Run 2 (Steam+NaOH-San Ardo) } \\
\hline \multirow{2}{*}{ Density $\left({ }^{\circ} \mathrm{API}\right)$} & Initial & Middle & Final \\
\cline { 2 - 4 } & 14.2 & 15 & 16.6 \\
\hline Temperature $\left({ }^{\circ} \mathrm{C}\right)$ & \multicolumn{3}{|c|}{ Viscosity (cp) } \\
\hline 40 & 4812 & 3900 & 2006 \\
\hline 50 & 2700 & 2000 & 1589 \\
\hline 60 & 1346 & 980 & 700 \\
\hline
\end{tabular}




\subsection{Run 3: Base Run for Duri Oil Using Pure Steam}

For Run 3 Duri oil is used which is lighter oil when compared to San Ardo. Pure steam is injected into the injection cell. The mixing ratios for Duri oil are also presented in

\section{Appendix A.}

The temperature profile for run 3 is shown in Fig. 4.13. It is seen that the thermocouple reach a constant temperature at $120 \mathrm{~min}$.

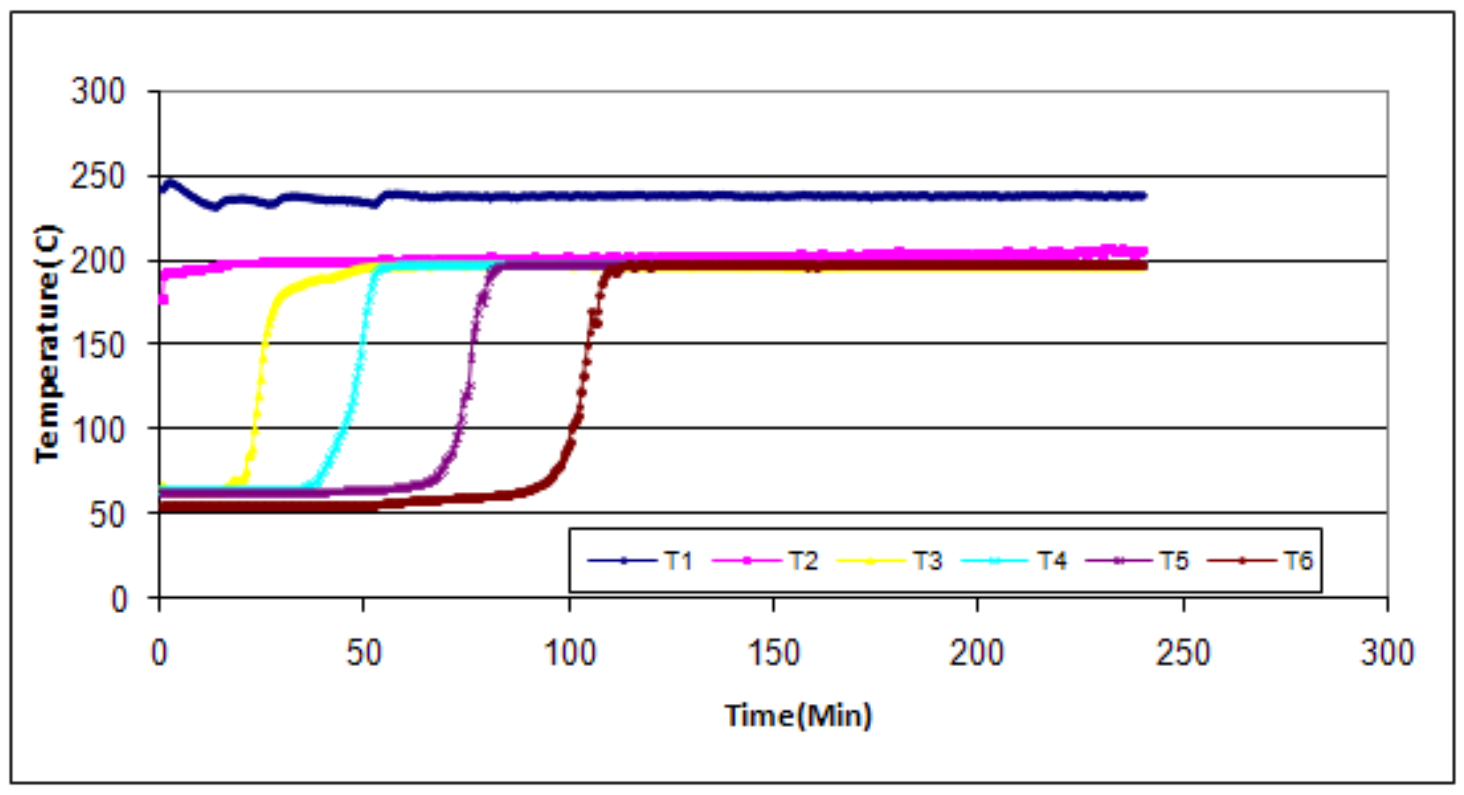

Figure 4.13 - Temperature profile for run 3

The pressure profile for run 3 is shown in Fig. 4.14. From the pressure profile we see that the maximum injection pressure reaches around 210 psi. The outlet pressure is maintained constant at 200 psi. The oil and water production rates are plotted in Fig. 4.15 and the maximum oil production rate is $4 \mathrm{cc} / \mathrm{min}$ and the maximum water production rate is $11.5 \mathrm{cc} / \mathrm{min}$. The oil recovered is $174 \mathrm{cc}$ and accounts for $52 \%$ of the 
original oil in place. Fig. 4.16 shows the recovery of oil with respect of time. Fig. 4.17 shows the oil recovery with respect to the pore volume. The steam front velocity is shown in Fig. 4.18.

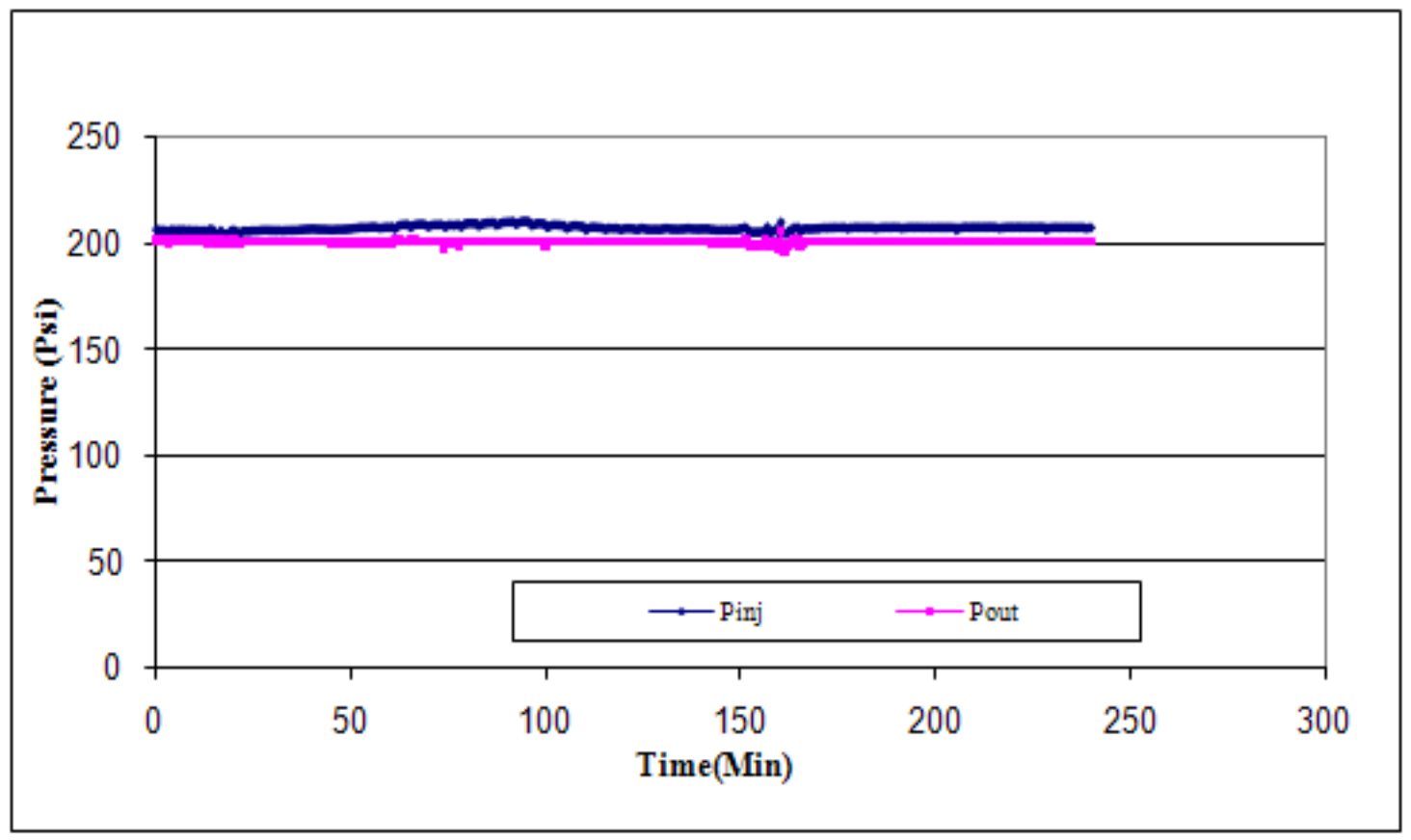

Figure 4.14 - Pressure profile for run 3 


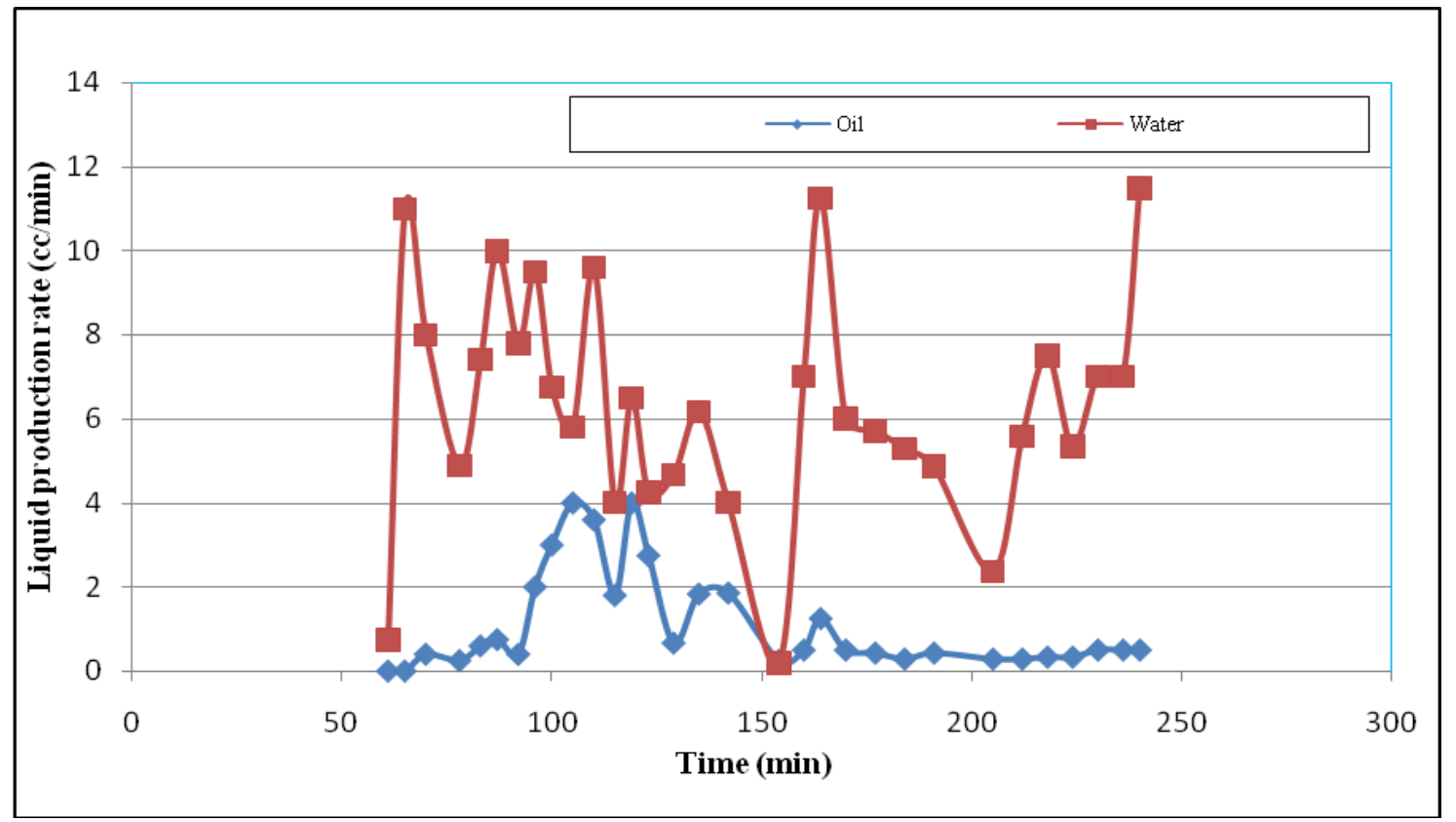

Figure 4.15 - Liquid production rates for run 3

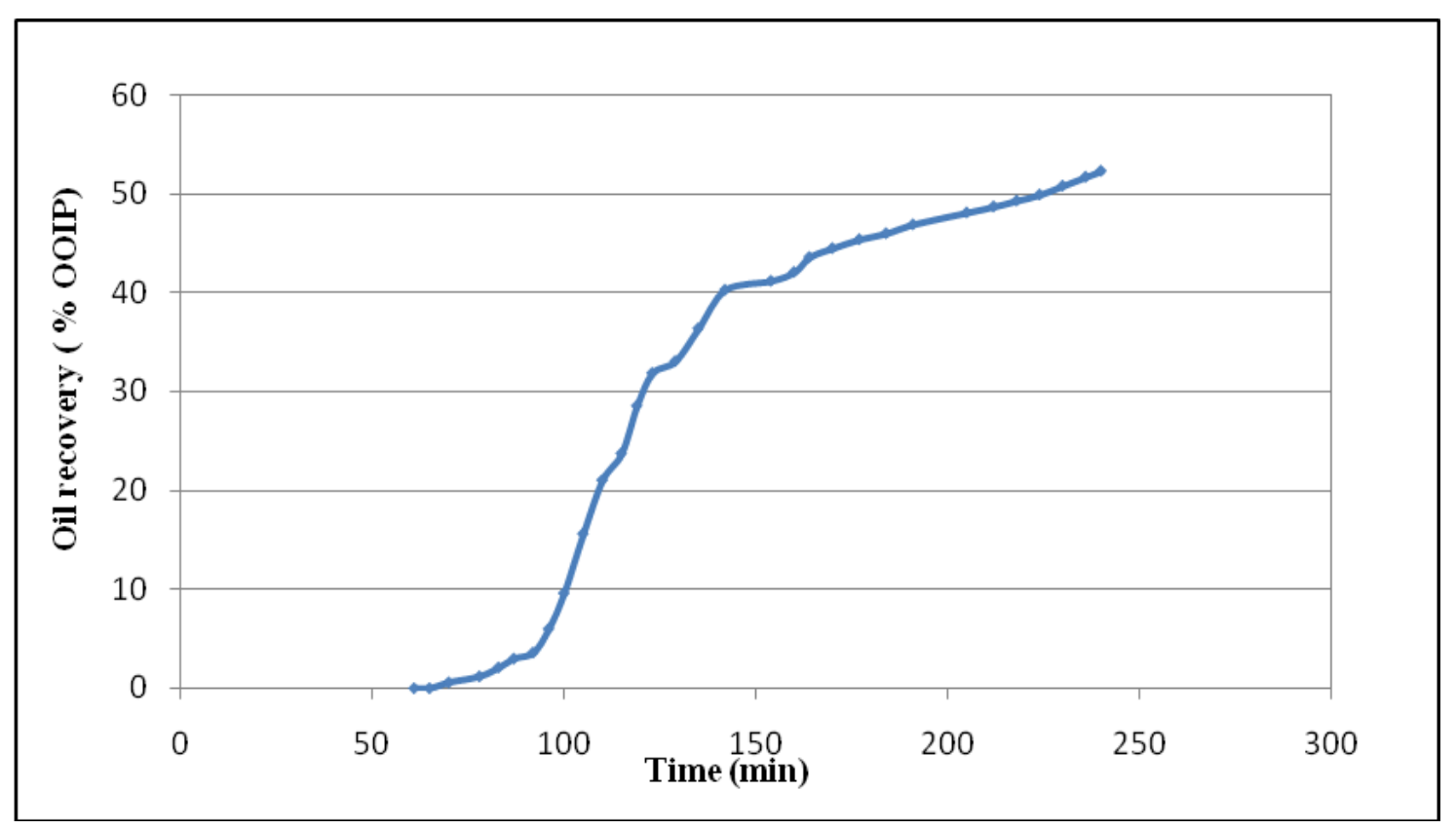

Figure 4.16 - Oil recovery versus time 


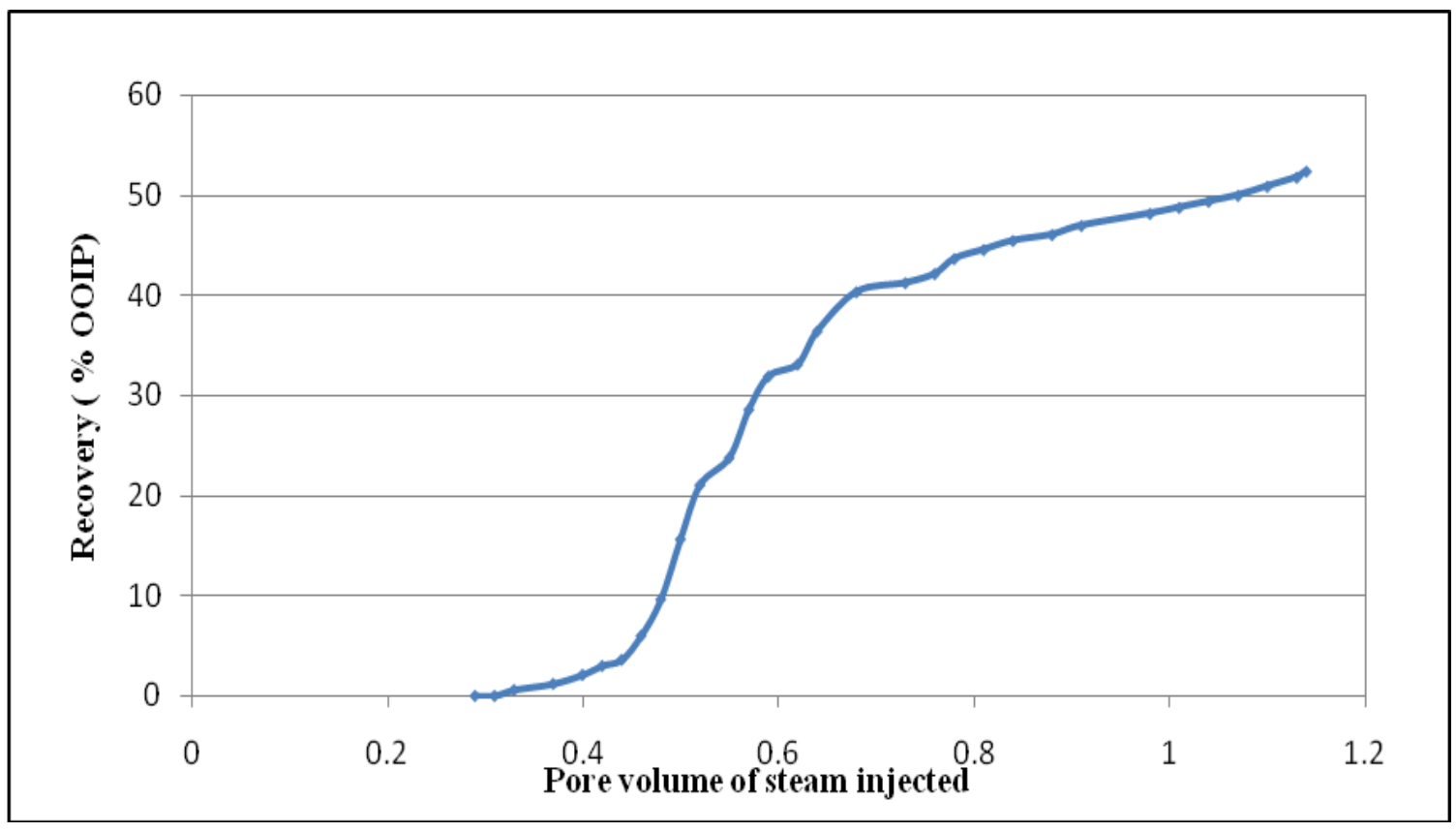

Figure 4.17-Oil recovery versus pore volume for run 3

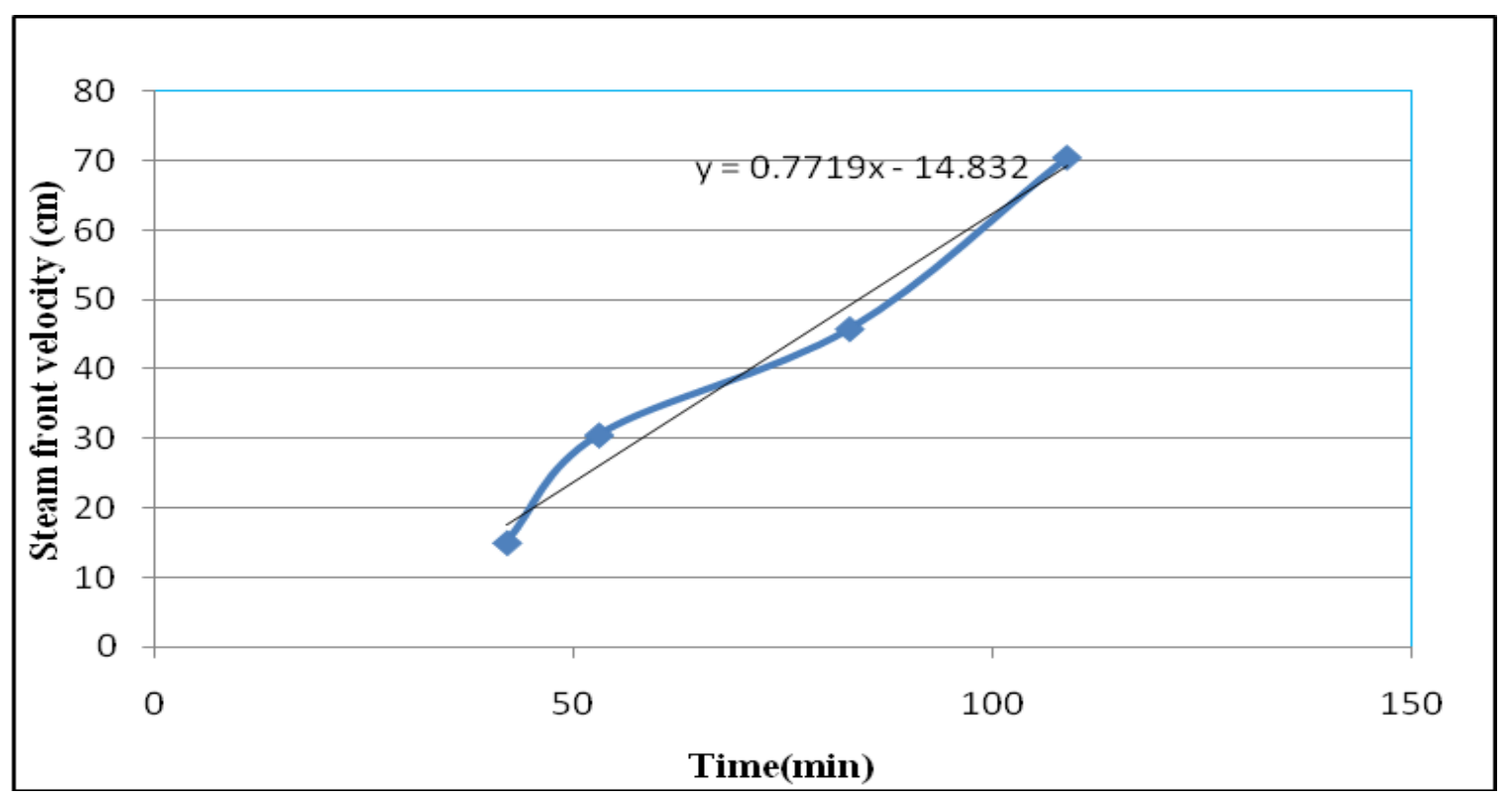

Figure 4.18 - Steam front velocity for run 3

The viscosity and density for Run 3 are shown in Table 4.4. 
Table 4.4-Viscosity and density readings for run 3

\begin{tabular}{|c|c|c|c|}
\hline \multicolumn{4}{|c|}{ Run 3 (Pure Steam -Duri Oil) } \\
\hline \multirow{2}{*}{ Density $\left({ }^{\circ} \mathrm{API}\right)$} & Initial & Middle & Final \\
\cline { 2 - 4 } & 20.41 & 21.6 & 21 \\
\hline Temperature $\left({ }^{\circ} \mathrm{C}\right)$ & \multicolumn{3}{|c|}{ Viscosity (cp) } \\
\hline 40 & 147 & 124 & 100 \\
\hline 50 & 108 & 100 & 70 \\
\hline 60 & 82 & 78 & 63 \\
\hline
\end{tabular}

\subsection{Run 4: Pure Steam with NaOH (0.1 wt \%) for Duri Oil}

For Run 4 steam was injected with 0.1 wt \% $\mathrm{NaOH}$ and the temperature profile is shown in Fig. 4.19. The pressure profiles for run 4 are shown in Fig. 4.20. The production profiles for oil and water are shown in Fig. 4.21. With the $0.1 \mathrm{wt} \% \mathrm{NaOH}$ the quantity of Duri oil recovered is 182 cc which is $53.2 \%$ of the original oil in place. Fig. 4.22 shows the oil recovered with respect to time and Fig. $\mathbf{4 . 2 3}$ shows the oil recovered with respect to pore volume. The steam front velocity is shown in Fig. 4.24. 


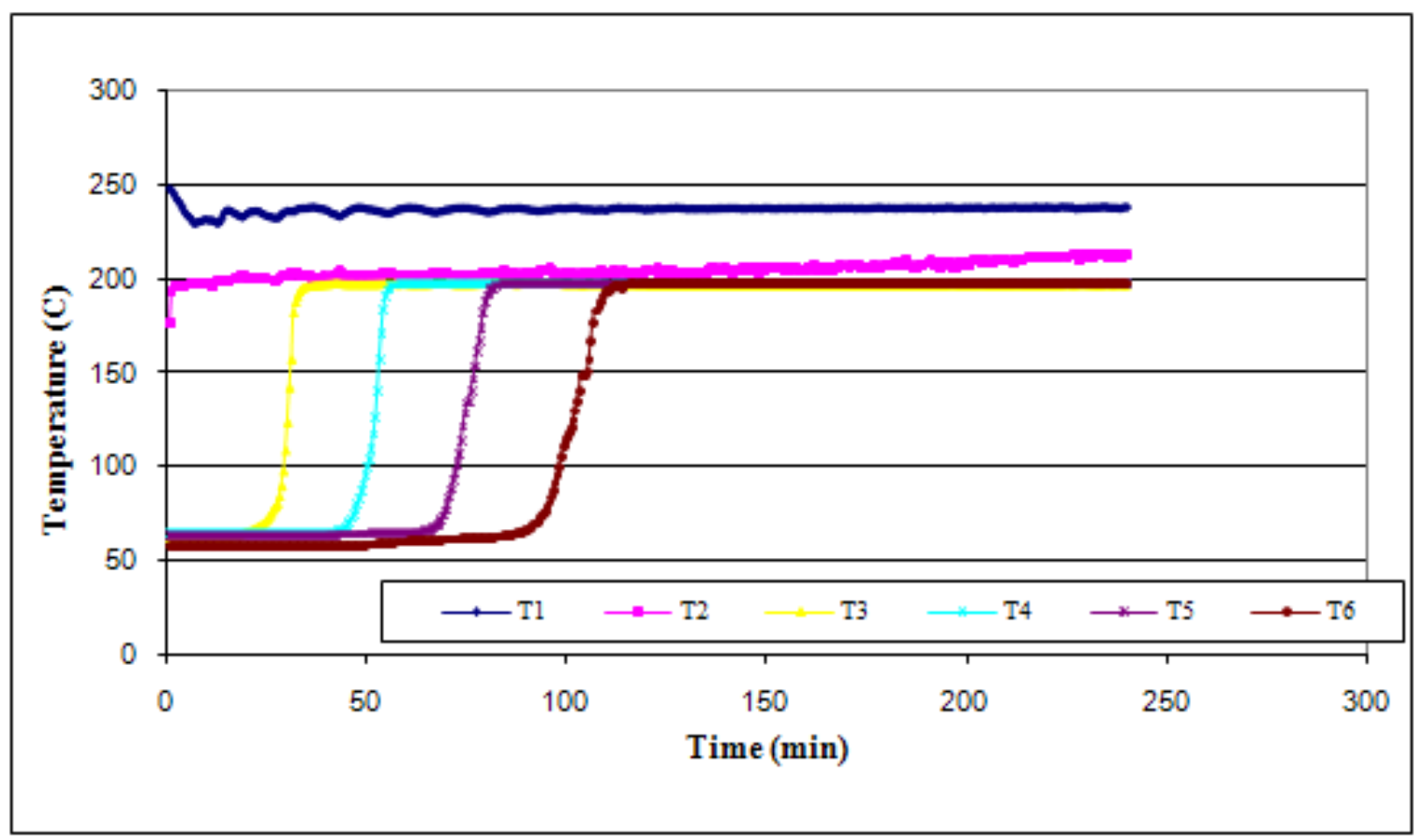

Figure 4.19 - Temperature profile for run 4

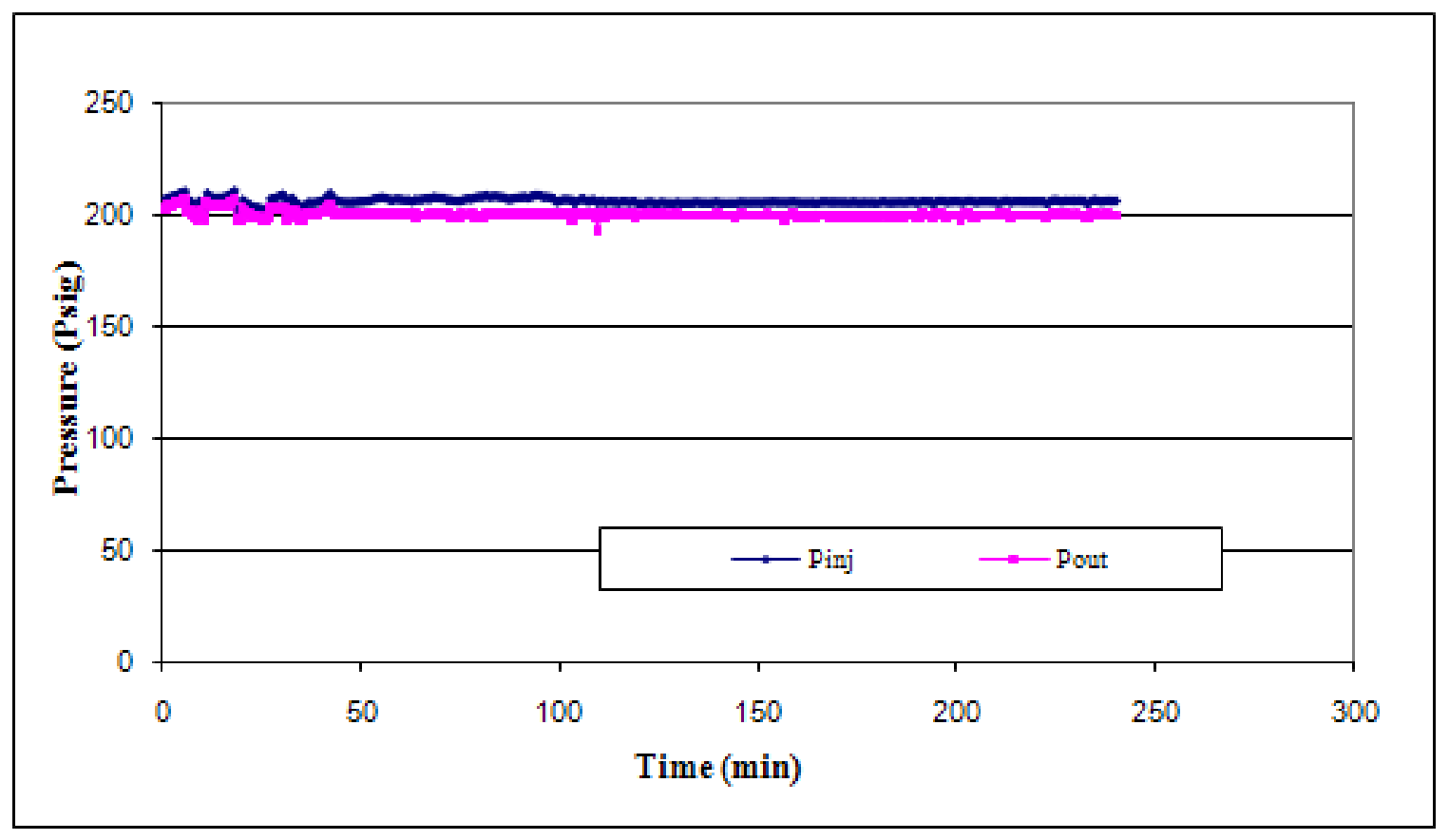

Figure 4.20 - Pressure profile for run 4 


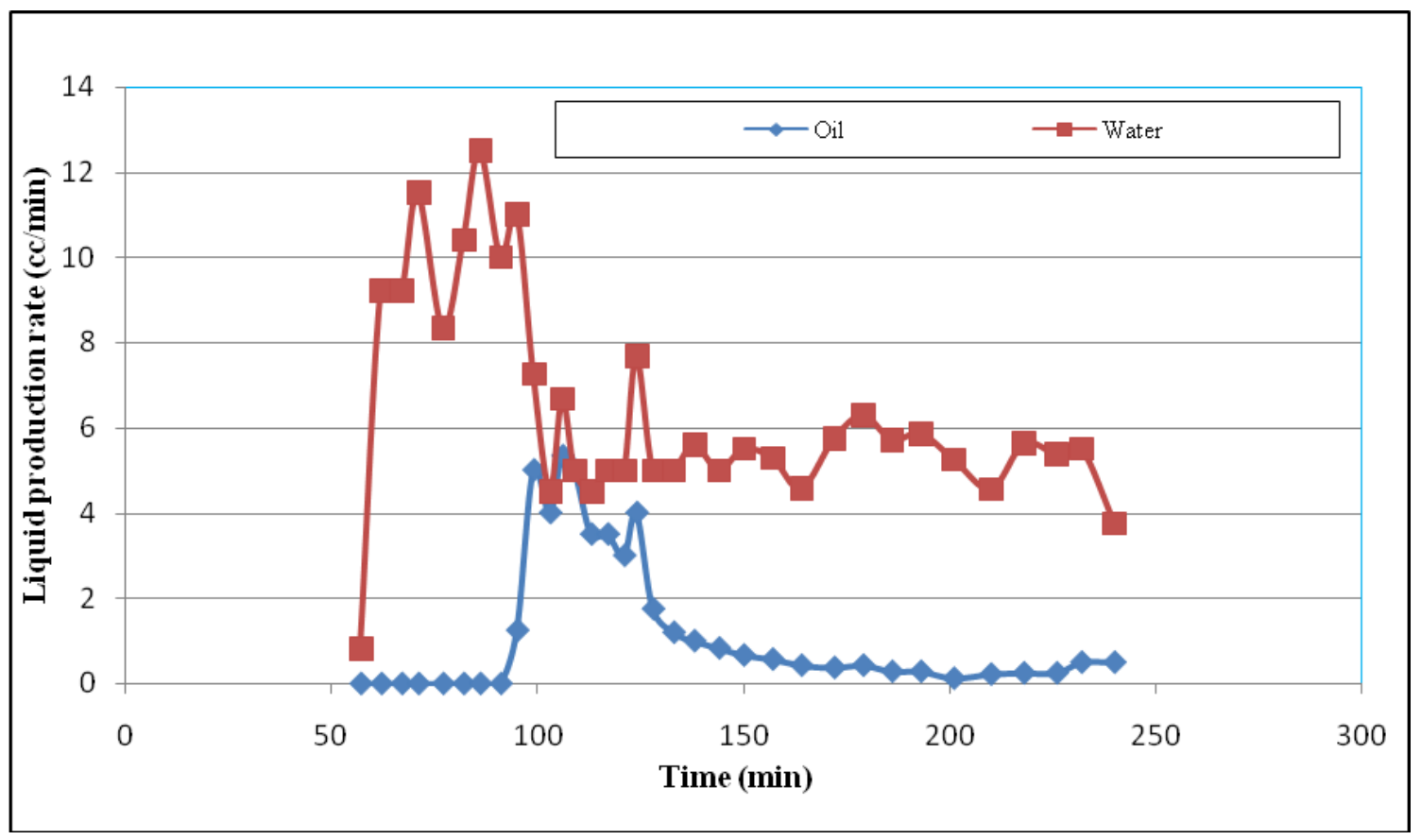

Figure 4.21 - Liquid production rates for run 4

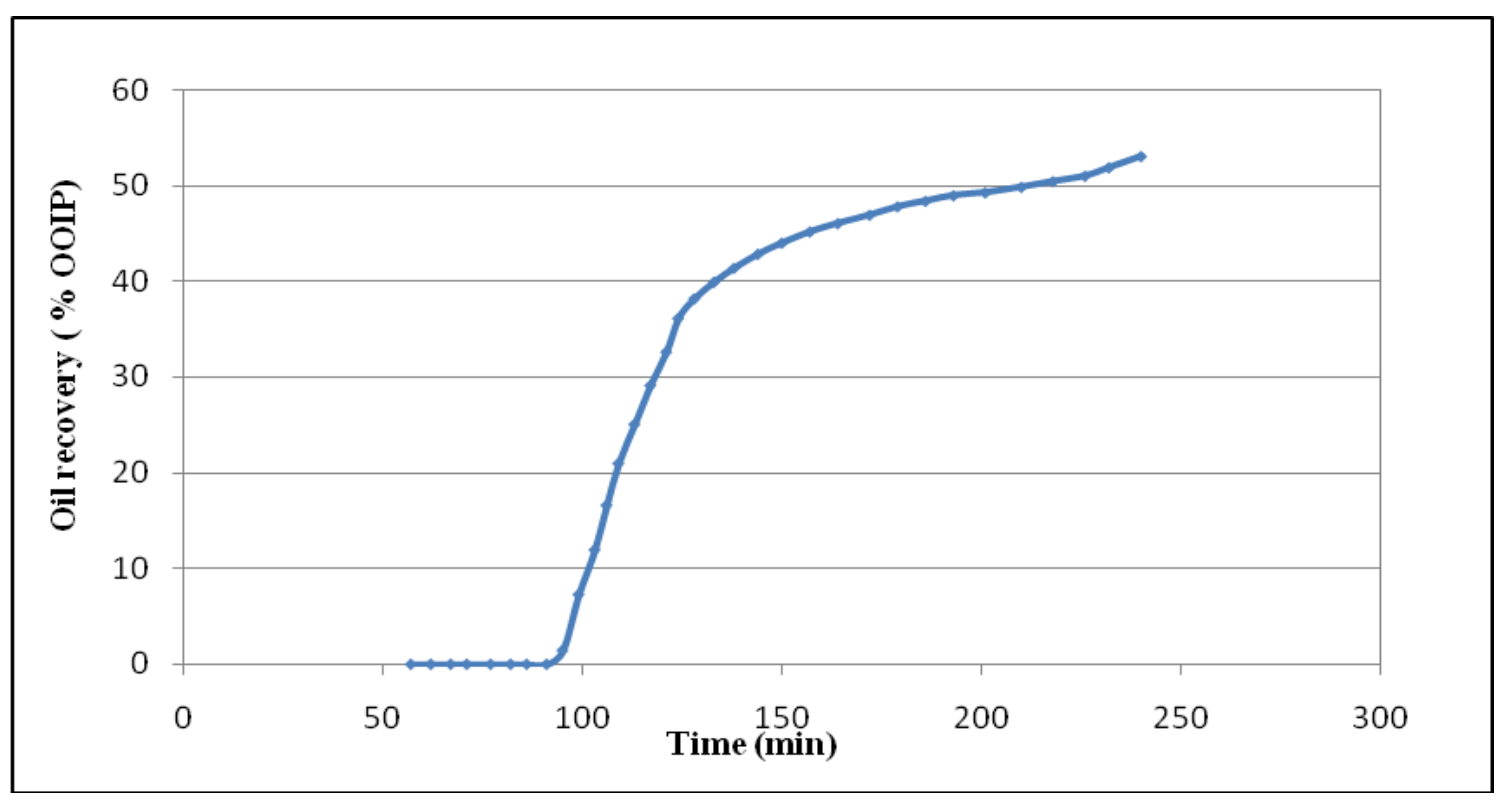

Figure 4.22 - Oil recovery versus time for run 4 


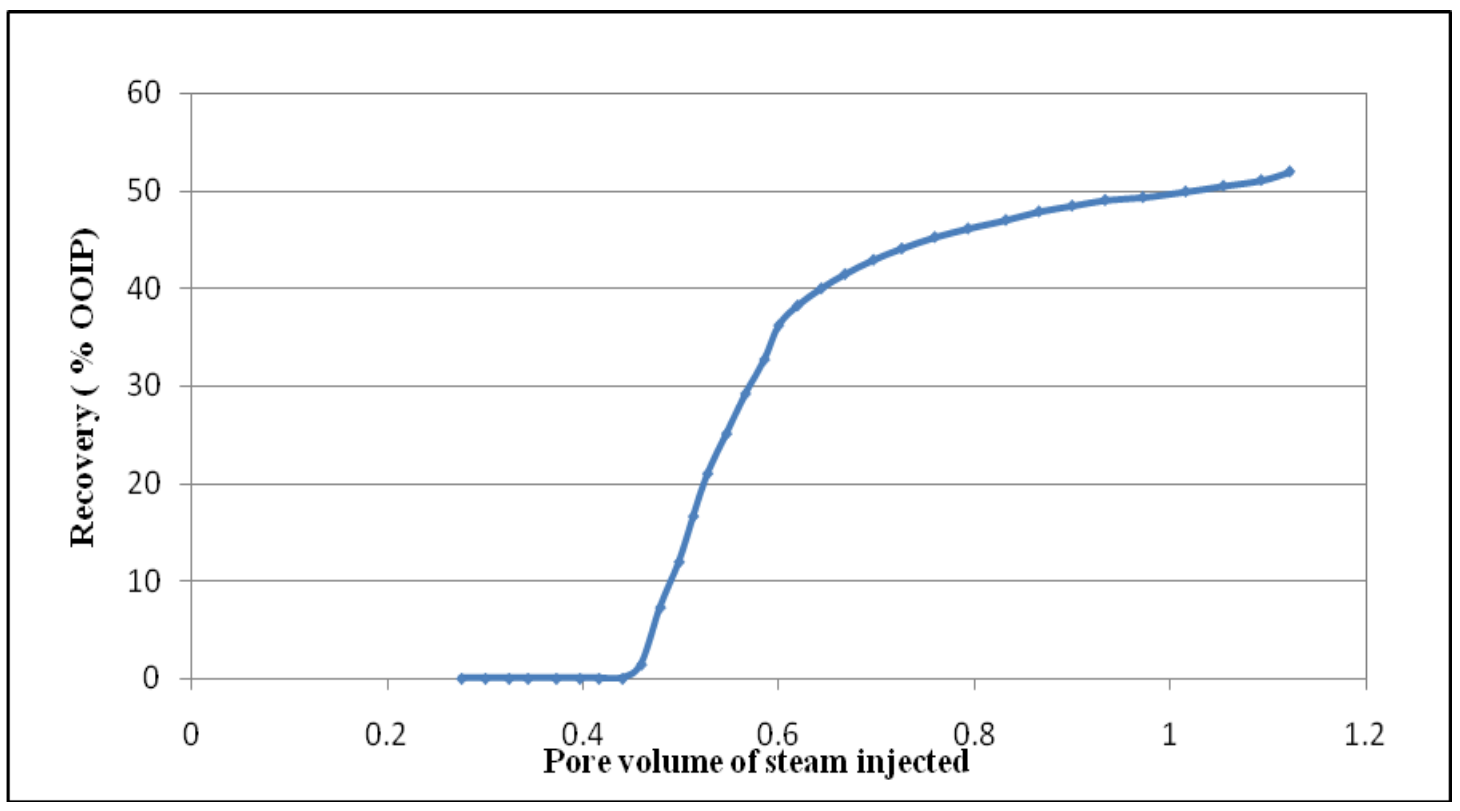

Figure 4.23 - Oil recovery versus pore volume of steam injected for run 4

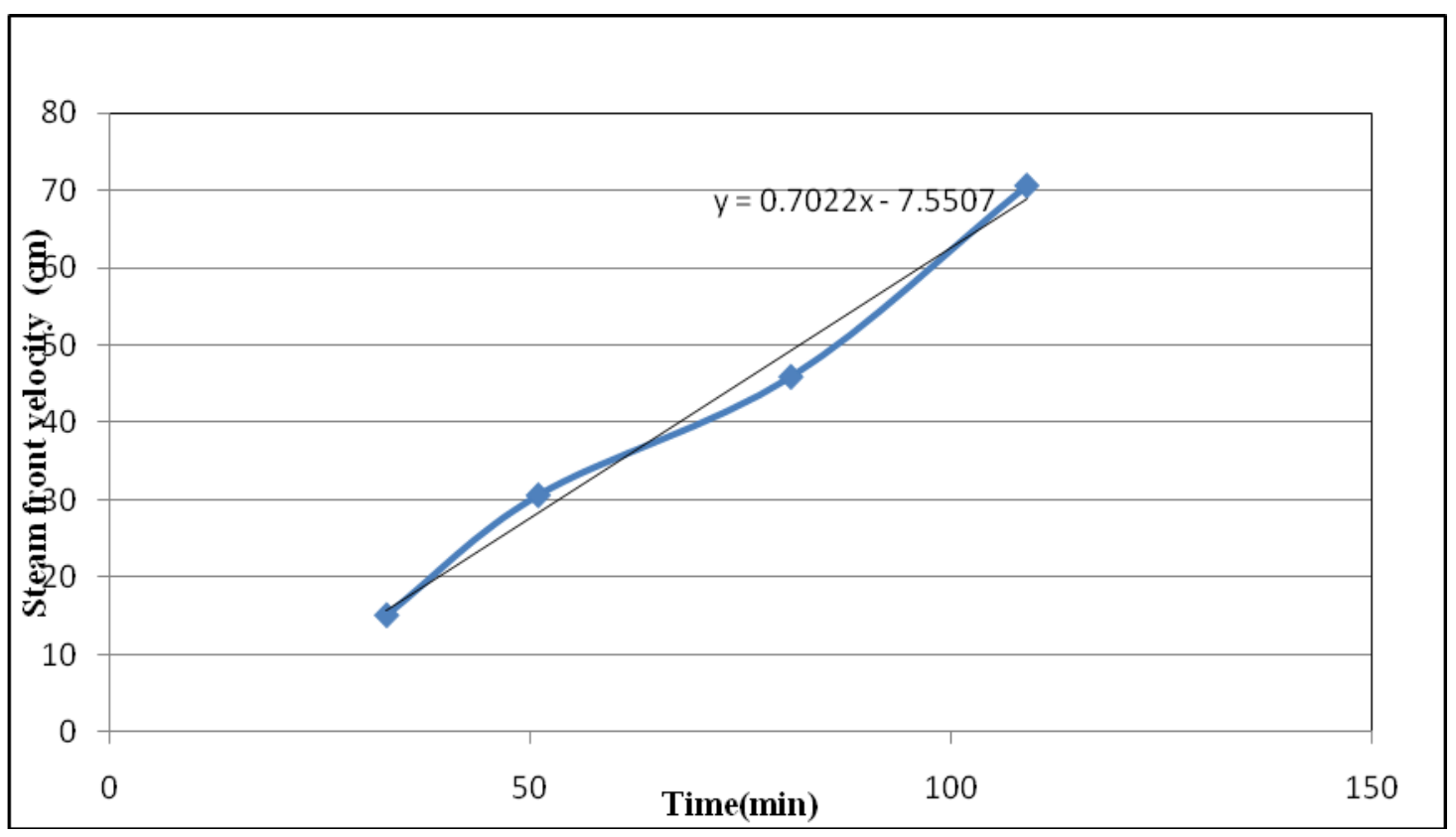

Figure 4.24-Steam front velocity for run 4 
The viscosity and density for Run 4 are shown in Table 4.5.

Table 4.5-Viscosity and density readings for run 4

\begin{tabular}{|c|c|c|c|}
\hline \multicolumn{4}{|c|}{ Run 4 (Steam+NaOH(0.1 wt \%)-Duri Oil) } \\
\hline \multirow{2}{*}{ Density $\left({ }^{\circ} \mathrm{API}\right)$} & Initial & Middle & Final \\
\cline { 2 - 4 } & 20.4 & 21 & 21.3 \\
\hline Temperature $\left({ }^{\circ} \mathrm{C}\right)$ & \multicolumn{3}{|c|}{ Viscosity (cp) } \\
\hline 40 & 150 & 132 & 120 \\
\hline 50 & 132 & 120 & 106 \\
\hline 60 & 110 & 78 & 60 \\
\hline
\end{tabular}

\subsection{Run 5: Pure Steam with NaOH (1 wt \%) for Duri Oil}

For Run 5 steam was injected along with $1 \mathrm{wt} \% \mathrm{NaOH}$ and the temperature profiles are shown in Fig. 4.25. The pressure profiles are shown in Fig. 4.26. The production profiles for both oil and water are plotted in Fig. 4.27. The maximum oil rate is $4.7 \mathrm{cc} / \mathrm{min}$ and the maximum water rate is $13.67 \mathrm{cc} / \mathrm{min}$. The oil recovered is $199 \mathrm{cc}$ which is $59 \%$ of the original oil in place which is shown in Fig. $\mathbf{4 . 2 8}$ and the recovery versus the pore volume is shown in Fig. 4.29. The steam front velocity is shown in Fig. 4.30. 


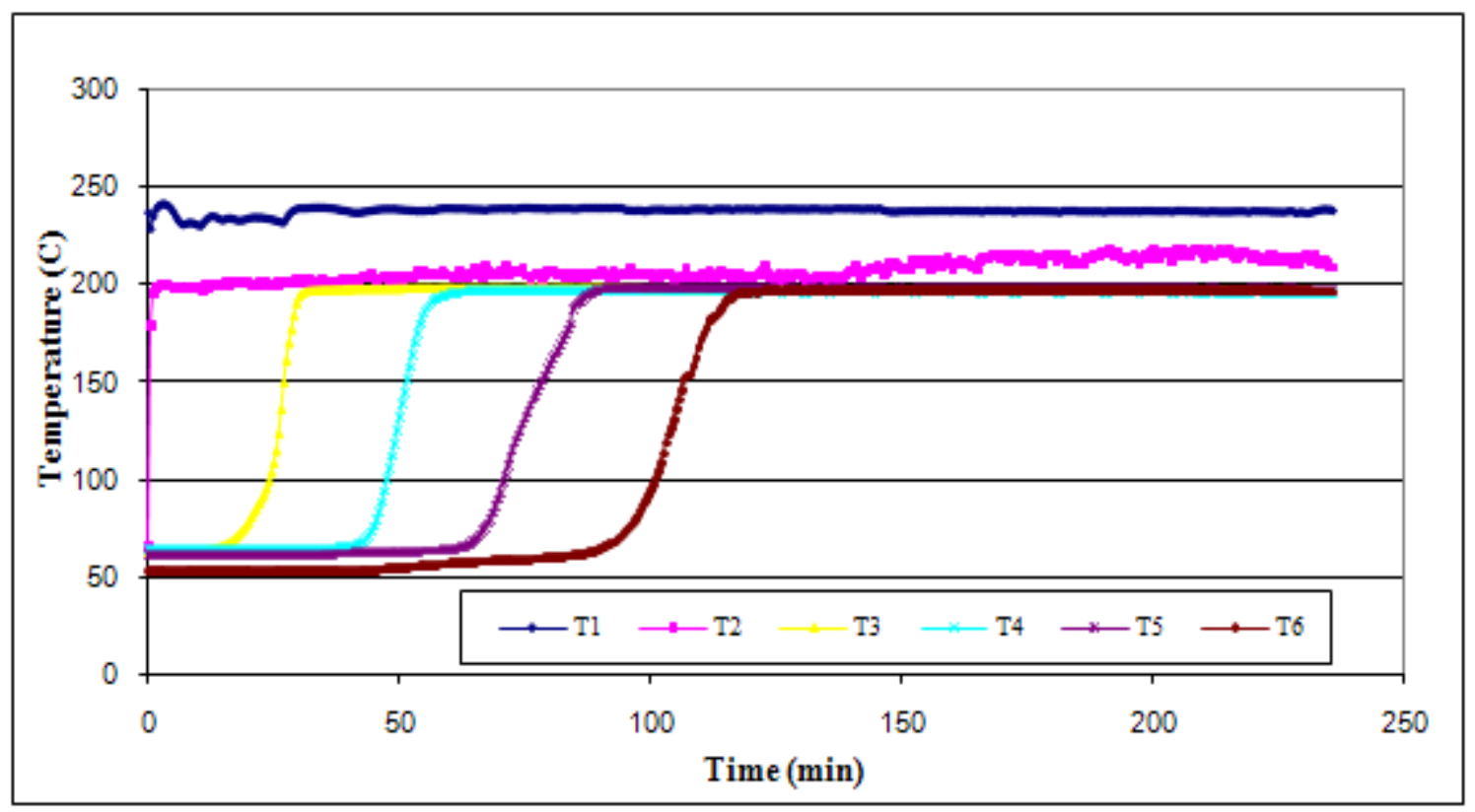

Figure 4.25 - Temperature profile for run 5

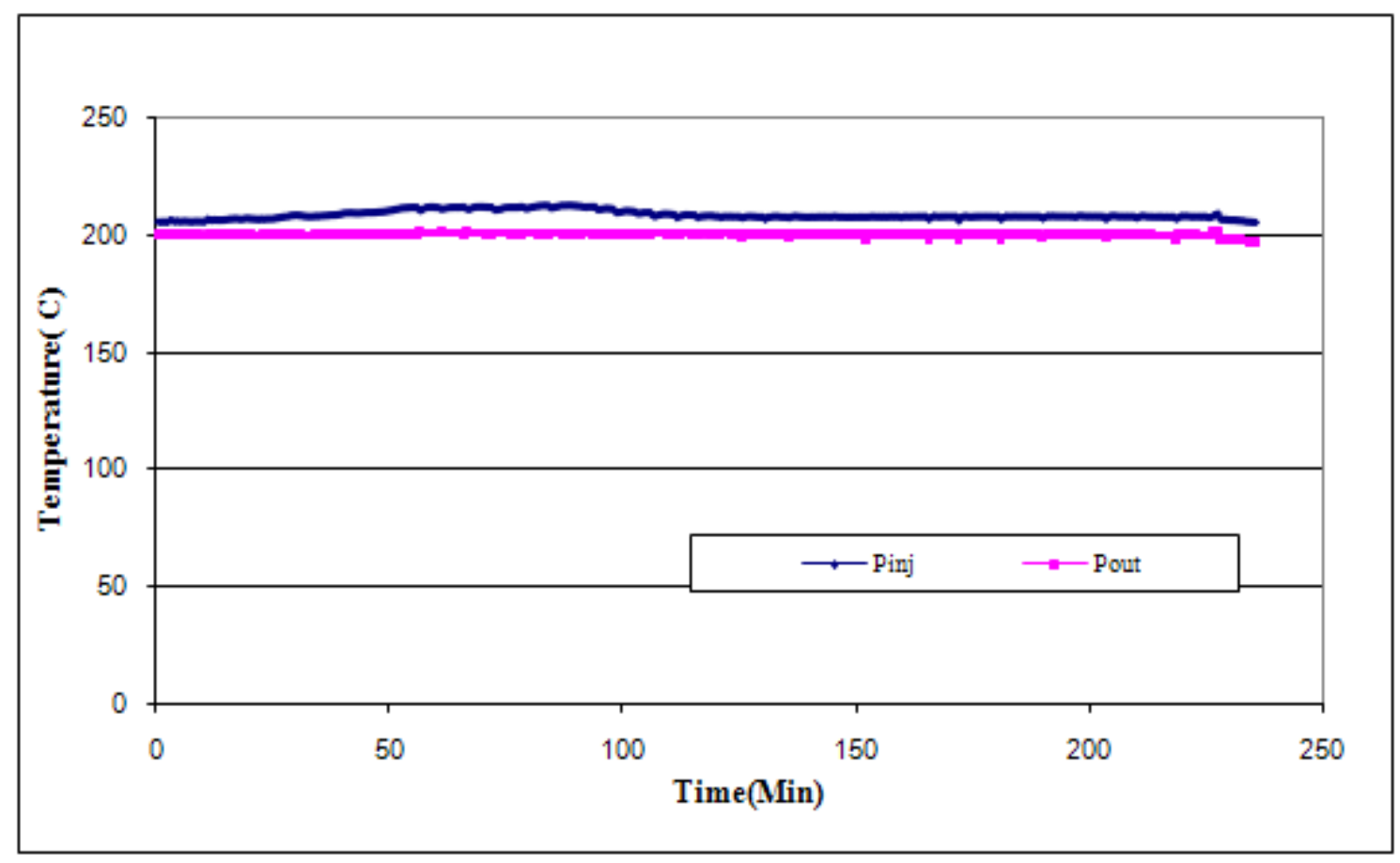

Figure 4.26 - Pressure profile for run 5 


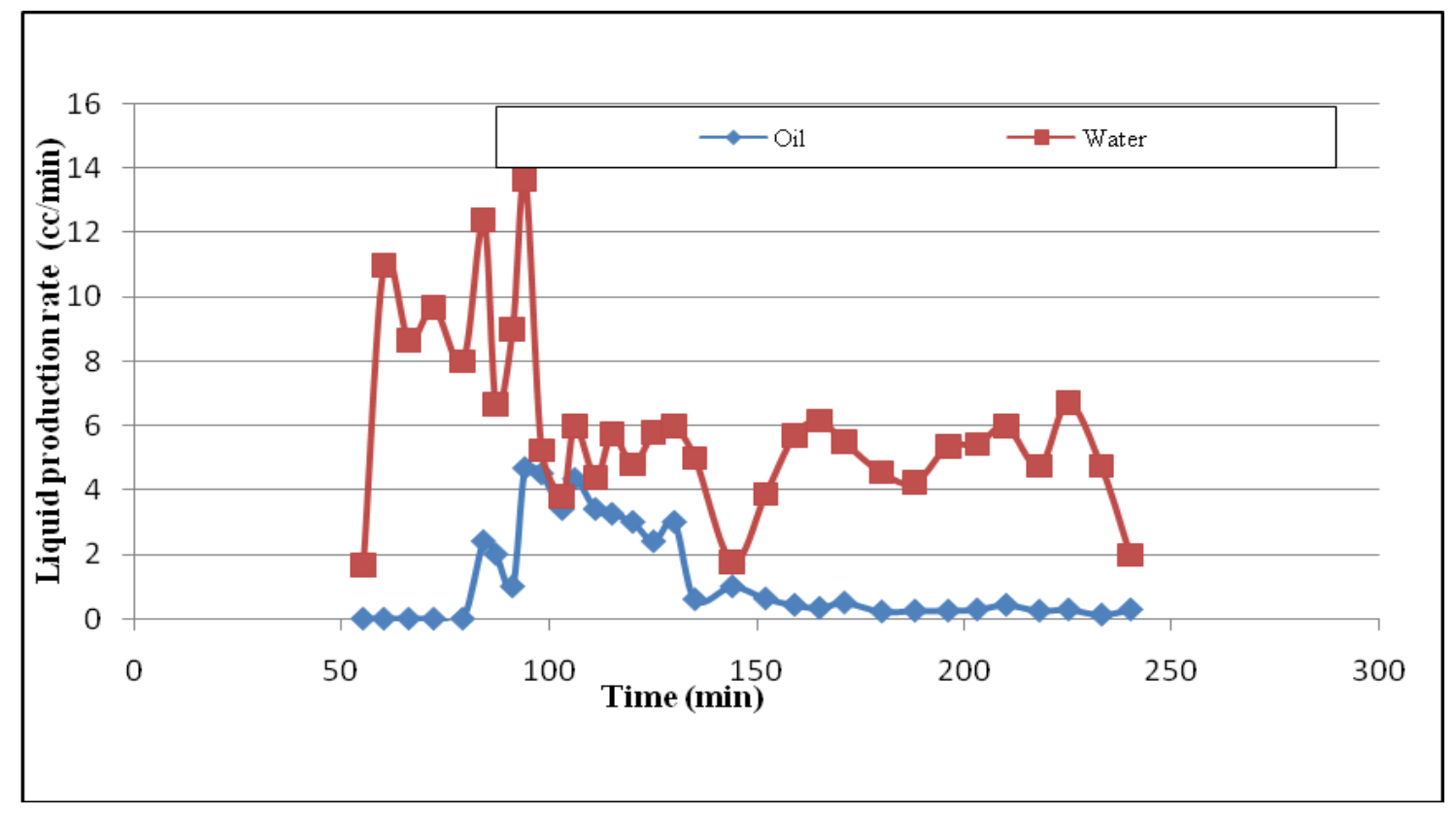

Figure 4.27 - Liquid production rates for run 5

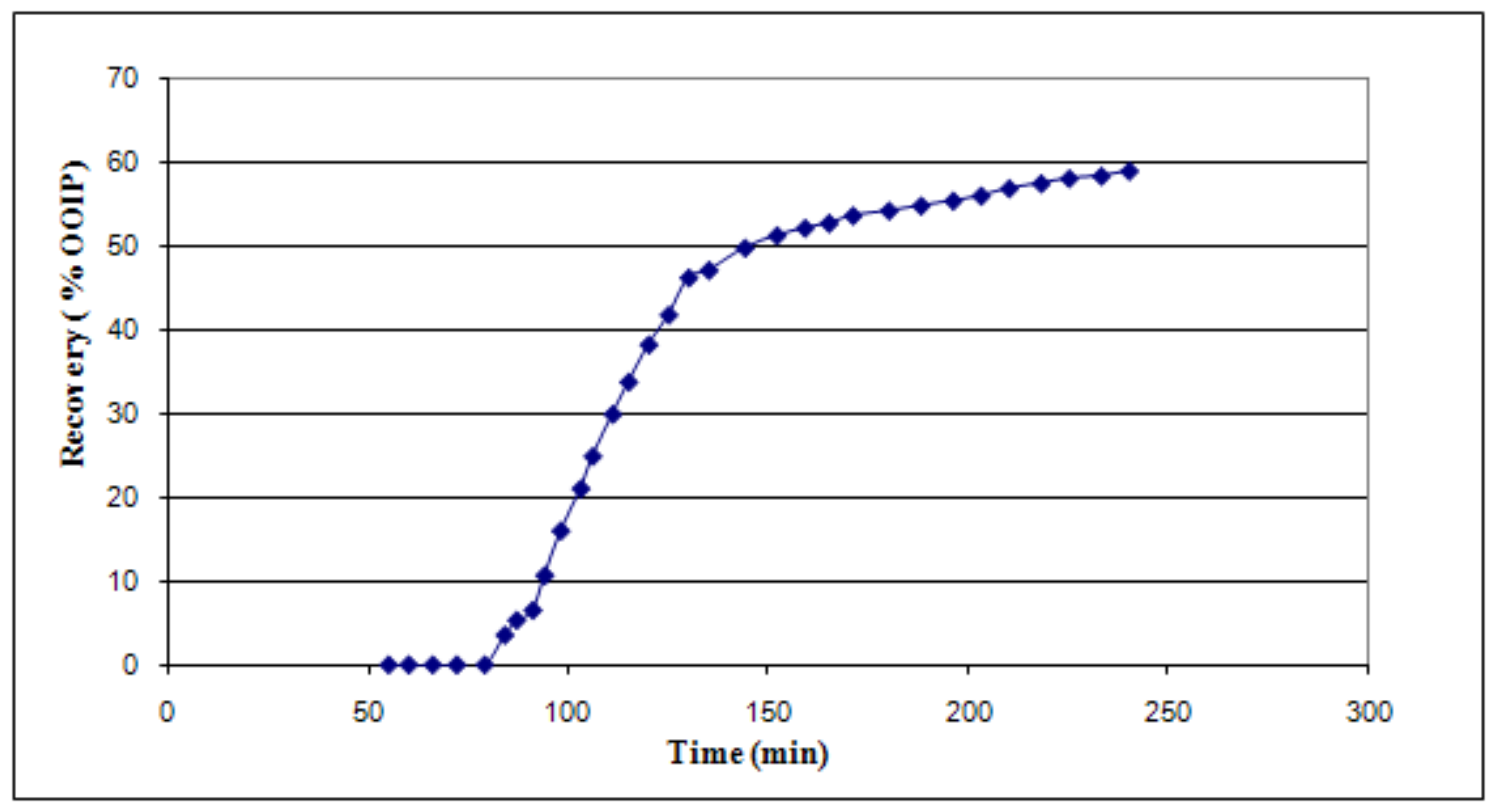

Figure 4.28- Oil recovery versus time for run 5 


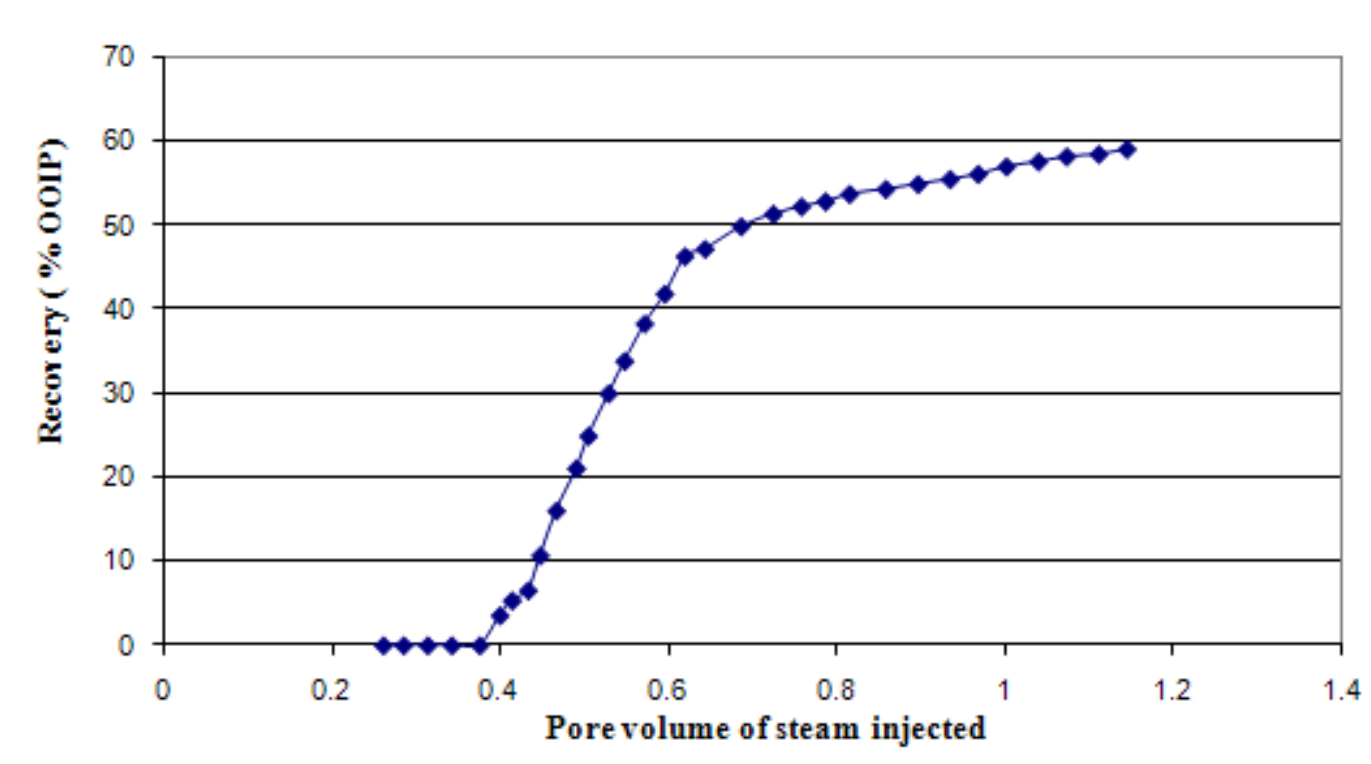

Figure 4.29- Oil recovery versus pore volume of steam injected for run 5

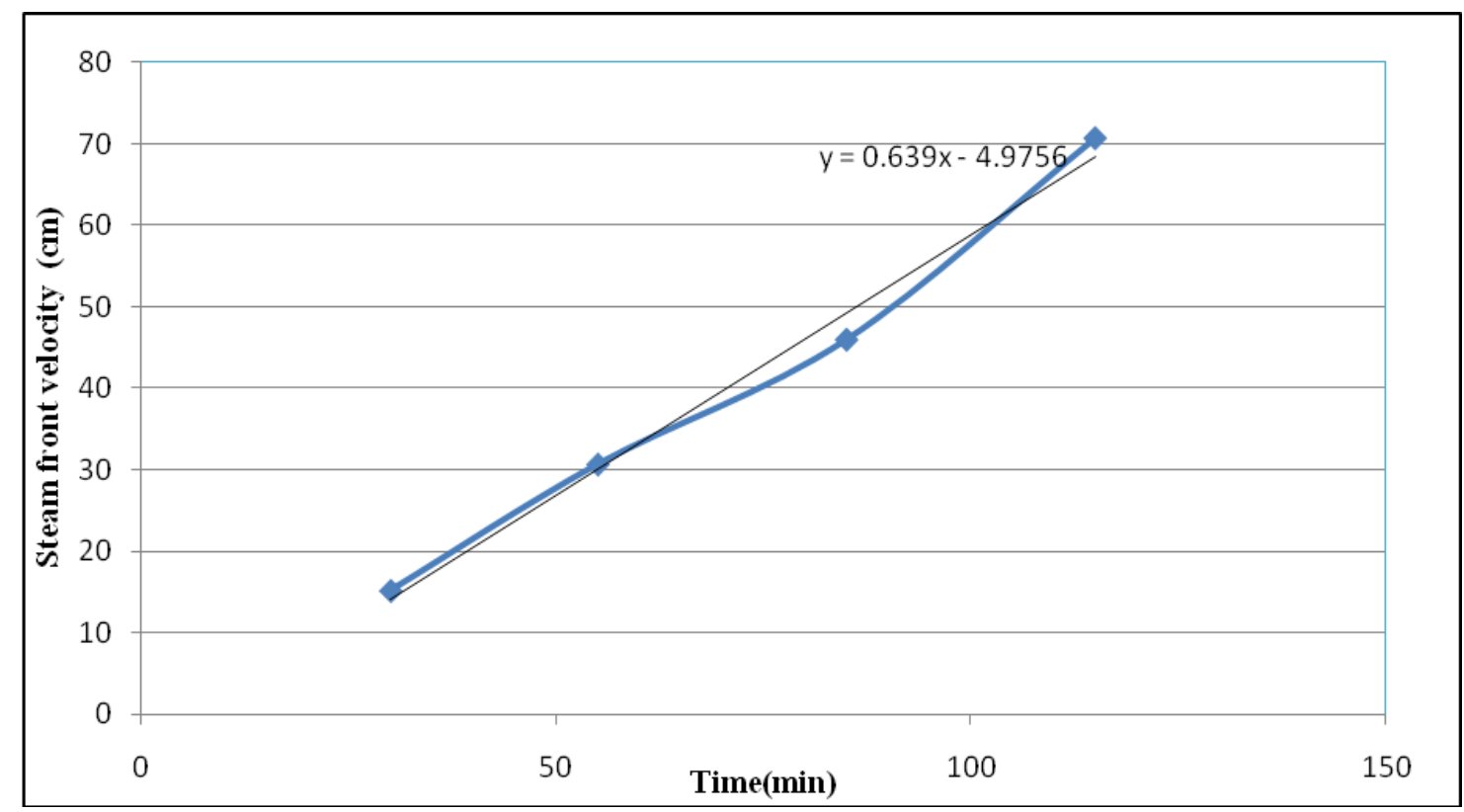

Figure 4.30- Steam front velocity for run 5

The viscosity and density reading for Run 5 are shown in Table 4.6. 
Table 4.6-Viscosity and density readings for run 5

\begin{tabular}{|c|c|c|c|}
\hline \multicolumn{4}{|c|}{ Run 5 (Steam+NaOH(1 wt \%)-Duri Oil) } \\
\hline \multirow{2}{*}{ Density $\left({ }^{\circ} \mathrm{API}\right)$} & Initial & Middle & Final \\
\cline { 2 - 4 } & 20.4 & 21.2 & 21.6 \\
\hline Temperature $\left({ }^{\circ} \mathrm{C}\right)$ & \multicolumn{3}{|c|}{ Viscosity (cp) } \\
\hline 40 & 160 & 130 & 118 \\
\hline 50 & 135 & 118 & 107 \\
\hline 60 & 112 & 80 & 62 \\
\hline
\end{tabular}

\subsection{Run 6: Cyclic Injection of NaOH (1 wt \%) and Steam for San Ardo Oil}

For Run 61 wt \% $\mathrm{NaOH}$ is injected after 4 hrs of steam injection. $\mathrm{NaOH}$ is injected for 25 min and 120 cc of solution is injected and then the sandpack is steamflooded again for an hr. The second cycle of $\mathrm{NaOH}$ is injected for 25 min and the sandpack is steamflooded again for around 1.5 hrs. The temperature profiles that are obtained are shown in Fig. 4.31. The pressure profile is shown in Fig. 4.32. From the temperature and pressure profile we see that the there is a slight discrepancy in the region where the sodium hydroxide was injected and are marked on the graphs. 


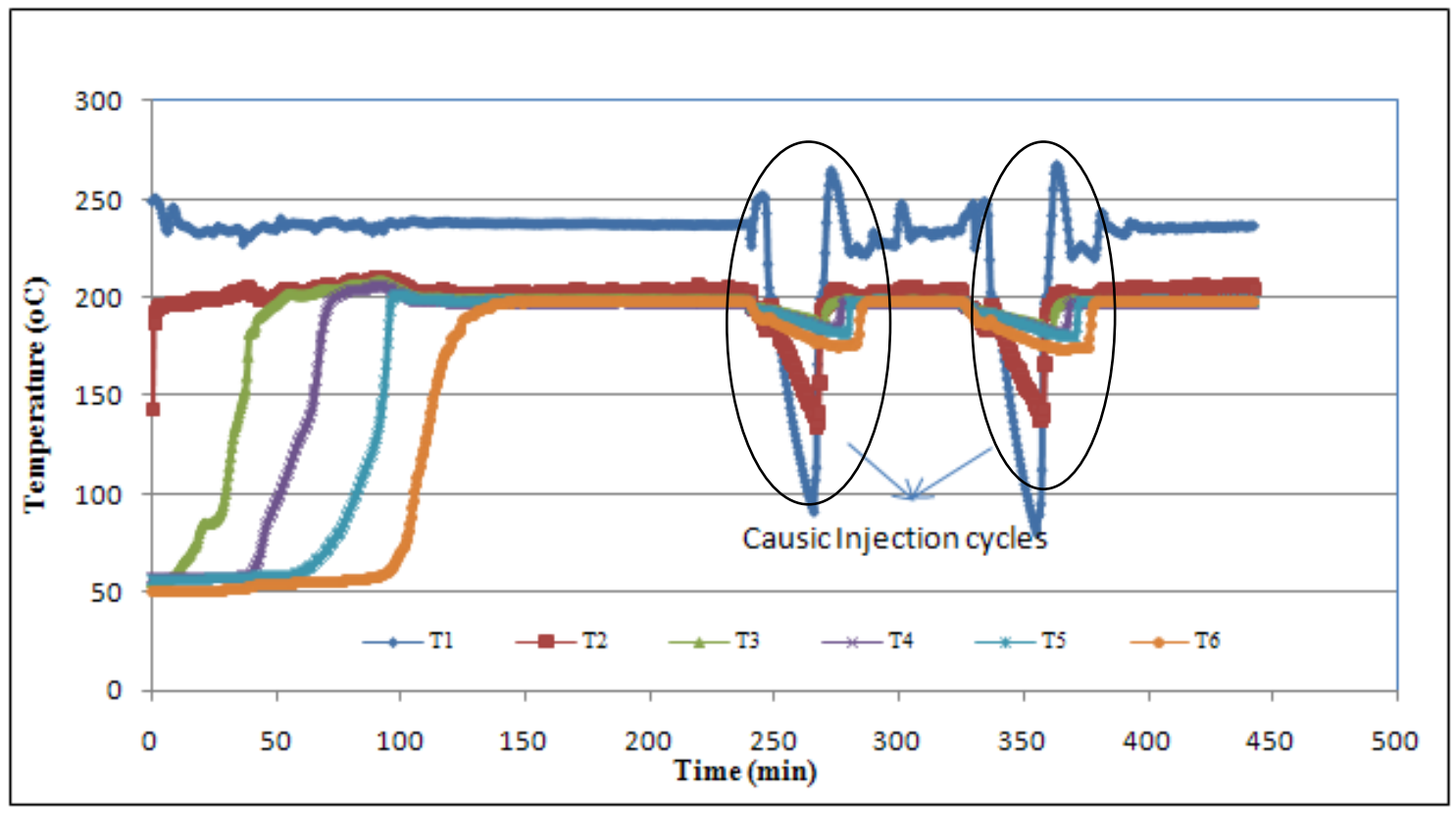

Figure 4.31 - Temperature profile for run 6 


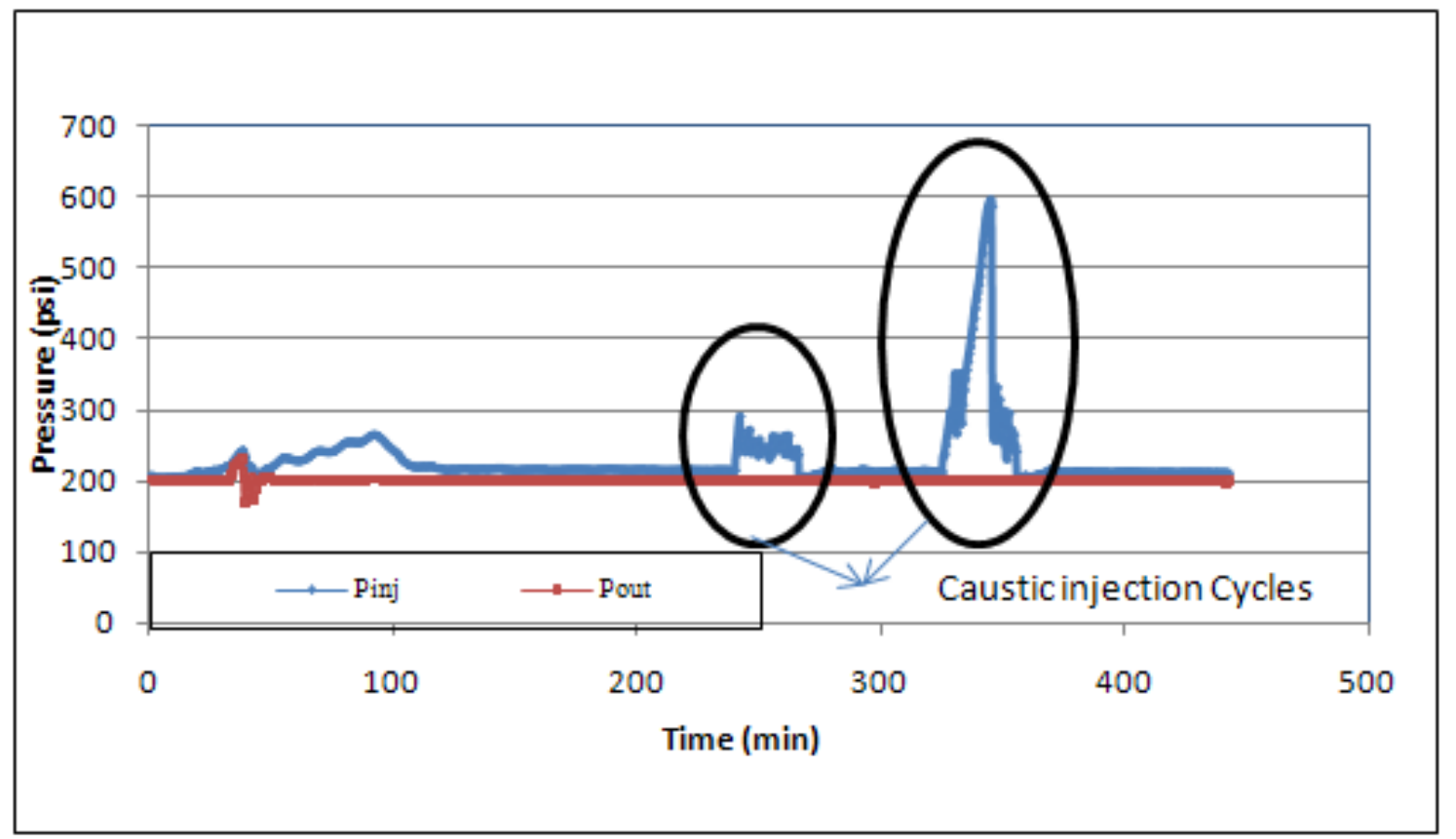

Figure 4.32 - Pressure profile for run 6

The liquid production profiles are plotted in Fig. 4.33. The maximum oil production rate is $6.7 \mathrm{cc} / \mathrm{min}$ and the maximum water production rate $13 \mathrm{cc} / \mathrm{min}$. 


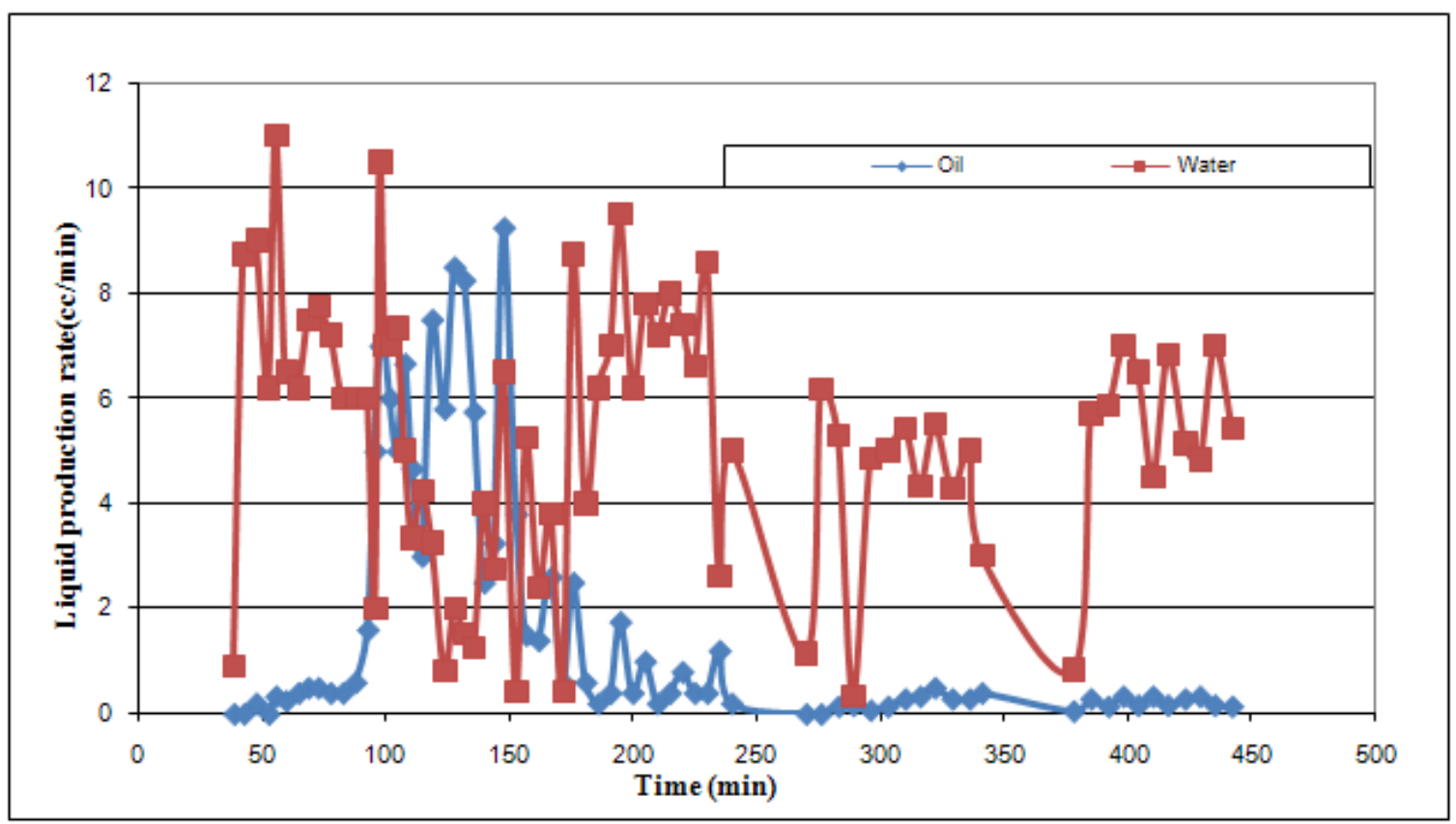

Figure 4.33-Liquid production rates for run 6

Now the total oil that is recovered is $80 \%$ of the original oil in place and is shown in

Fig. 4.34 with respect to time. The percentage of oil recovered plotted against pore volume is shown in Fig. 4.35. The steam front velocity for the cyclic caustic injection is shown in Fig. 4.36. 


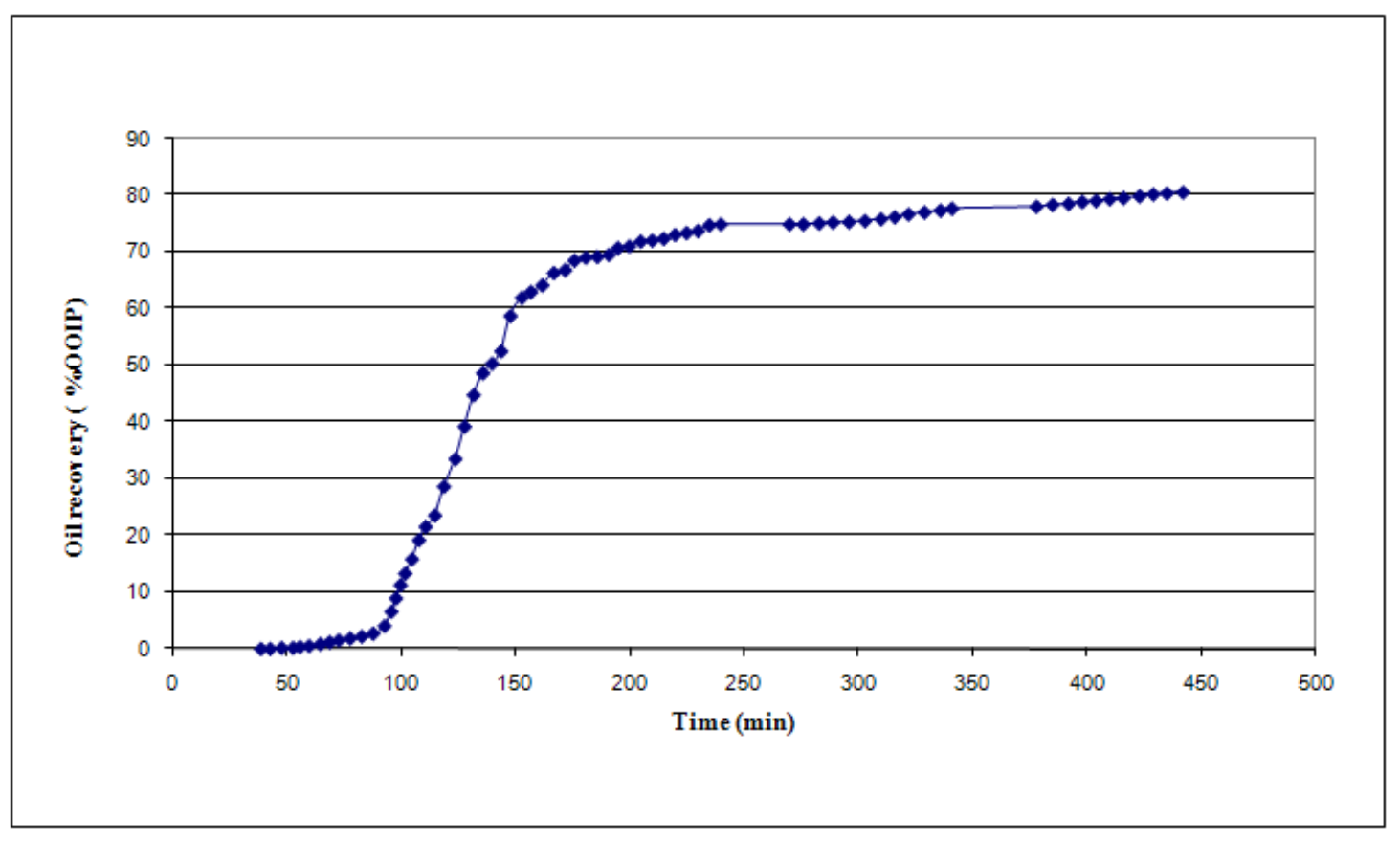

Figure 4.34-Oil recovery versus time for run 6

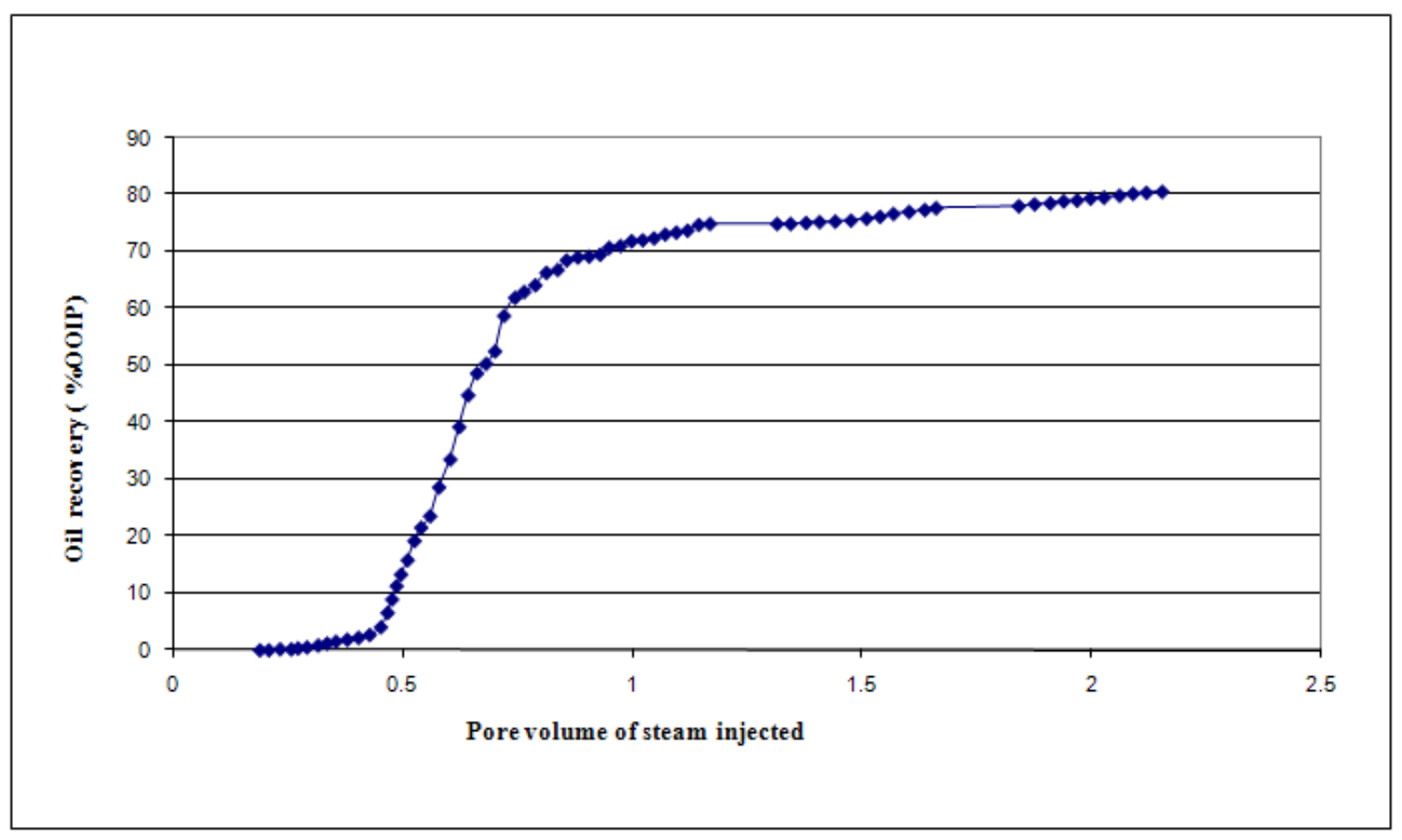

Figure 4.35-Oil recovery versus pore volume of steam injected for run 6 


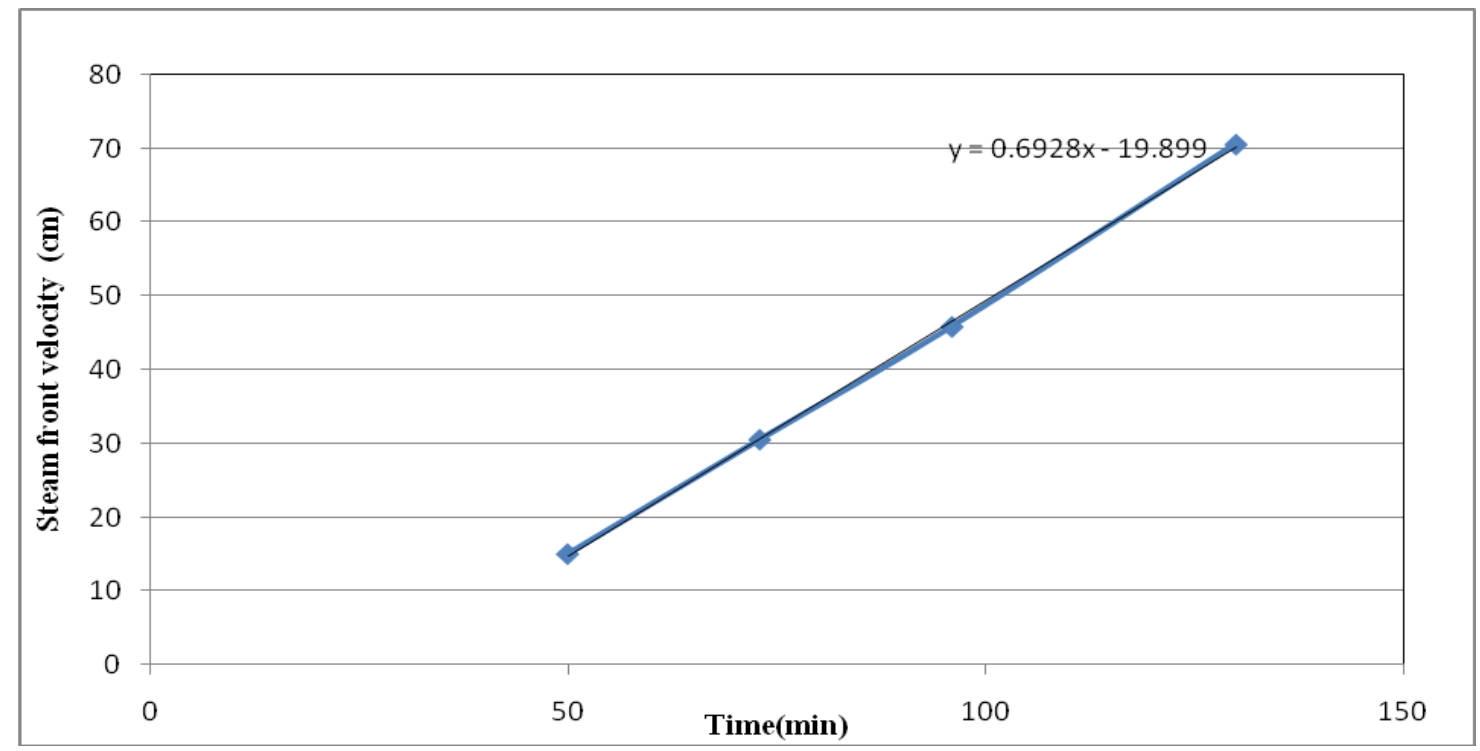

Figure 4.36 - Steam front velocity for Run 6

The viscosity and density for Run 6 are shown in Table 4.7.

Table 4.7- Viscosity and density readings for run 6

\begin{tabular}{|c|c|c|c|}
\hline \multicolumn{4}{|c|}{ Run 6 ( Steam+NaOH( 1 wt \%-as slug) -San Ardo) } \\
\hline \multirow{2}{*}{ Density ( $\left.{ }^{\circ} \mathrm{API}\right)$} & Initial & Middle & Final \\
\cline { 2 - 4 } & 14 & 15.2 & 16.5 \\
\hline Temperature $\left({ }^{\circ} \mathrm{C}\right)$ & \multicolumn{3}{|c|}{ Viscosity (cp) } \\
\hline 40 & 4611 & 3700 & 1895 \\
\hline 50 & 2543 & 1890 & 1500 \\
\hline 60 & 1246 & 900 & 640 \\
\hline
\end{tabular}

\subsection{Run 7: Pure Steam Injection NaOH (1 wt \%) for San Ardo Oil}

In order to compare the recoveries for the $1 \mathrm{wt} \%$ sodium hydroxide injected as a slug a pure steam flood was also conducted for 7 hrs. Fig. 4.37 shows the temperature profile 
for run 7 with pure steam. Pressure Profile for the run is shown in Fig. 4.38. The liquid production rates are shown in Fig. 4.39 and the maximum oil rate is $12 \mathrm{cc} / \mathrm{min}$ and the maximum water rate is.9.75 $\mathrm{cc} / \mathrm{min}$. The oil recovery is shown with respect to time in Fig. 4.40 and with respect to pore volume in Fig. 4.41. The steam front velocity is shown in Fig. 4.42.

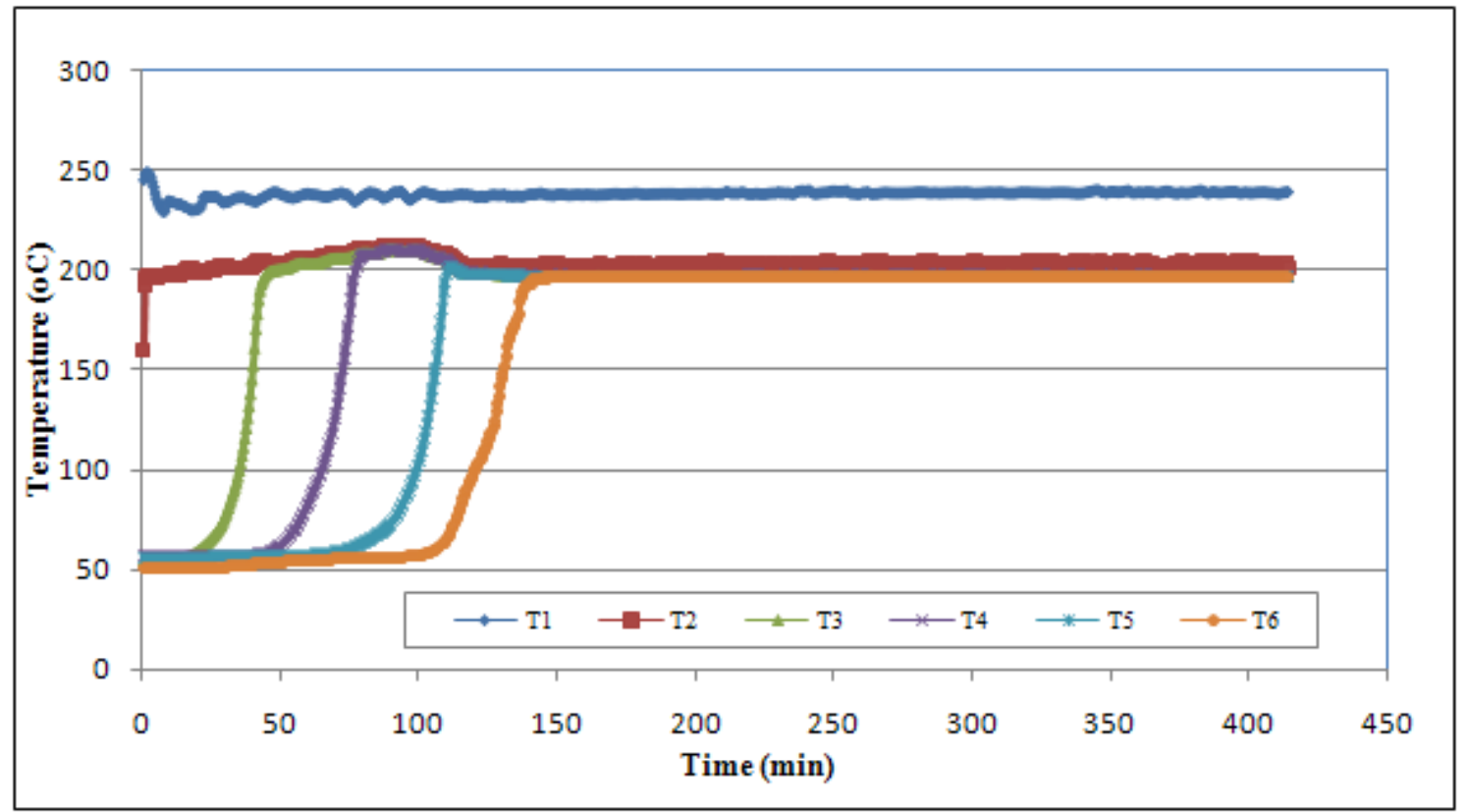

Figure 4.37 - Temperature profile for run 7 


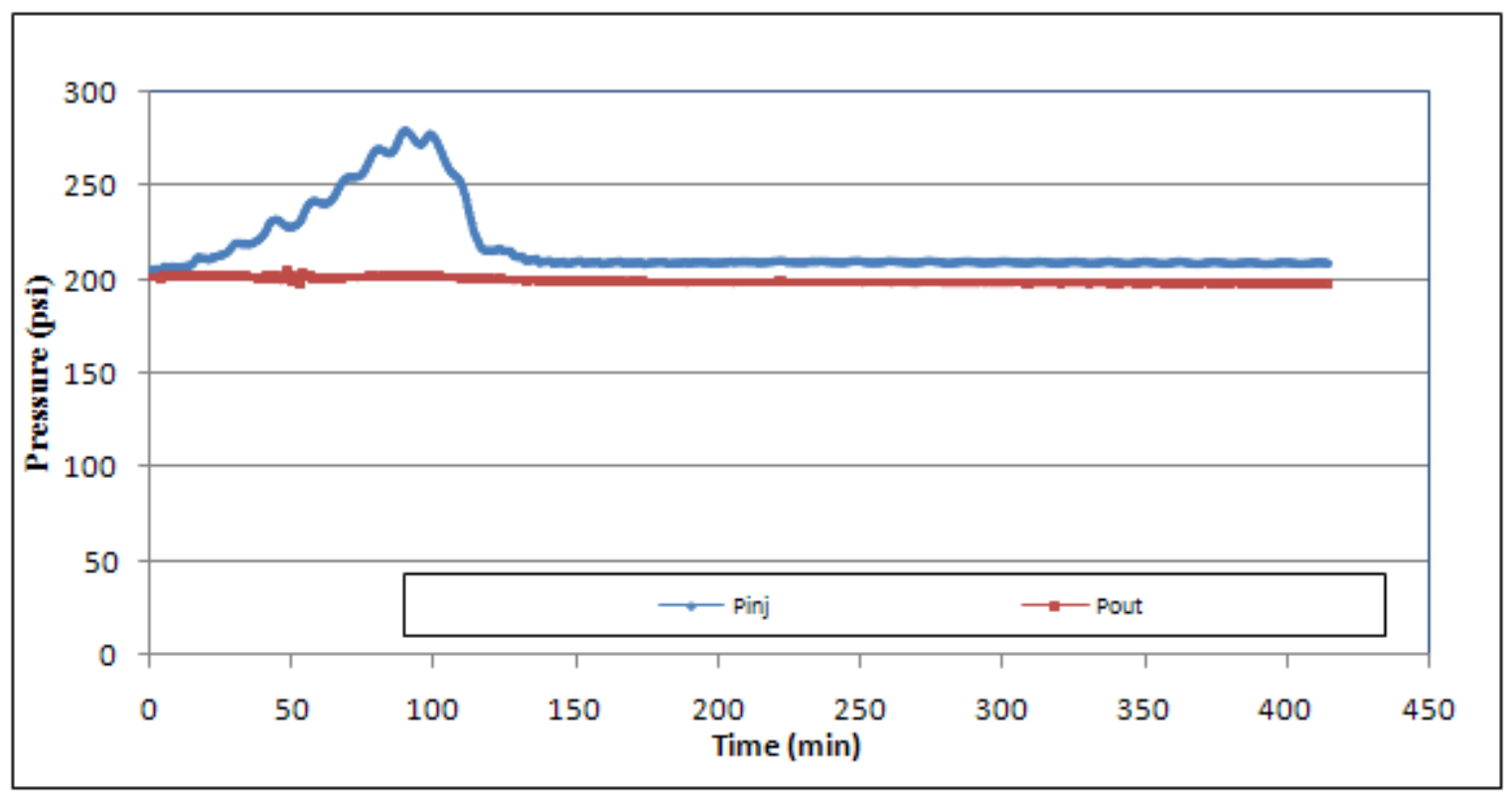

Figure 4.38 - Pressure profile for run 7

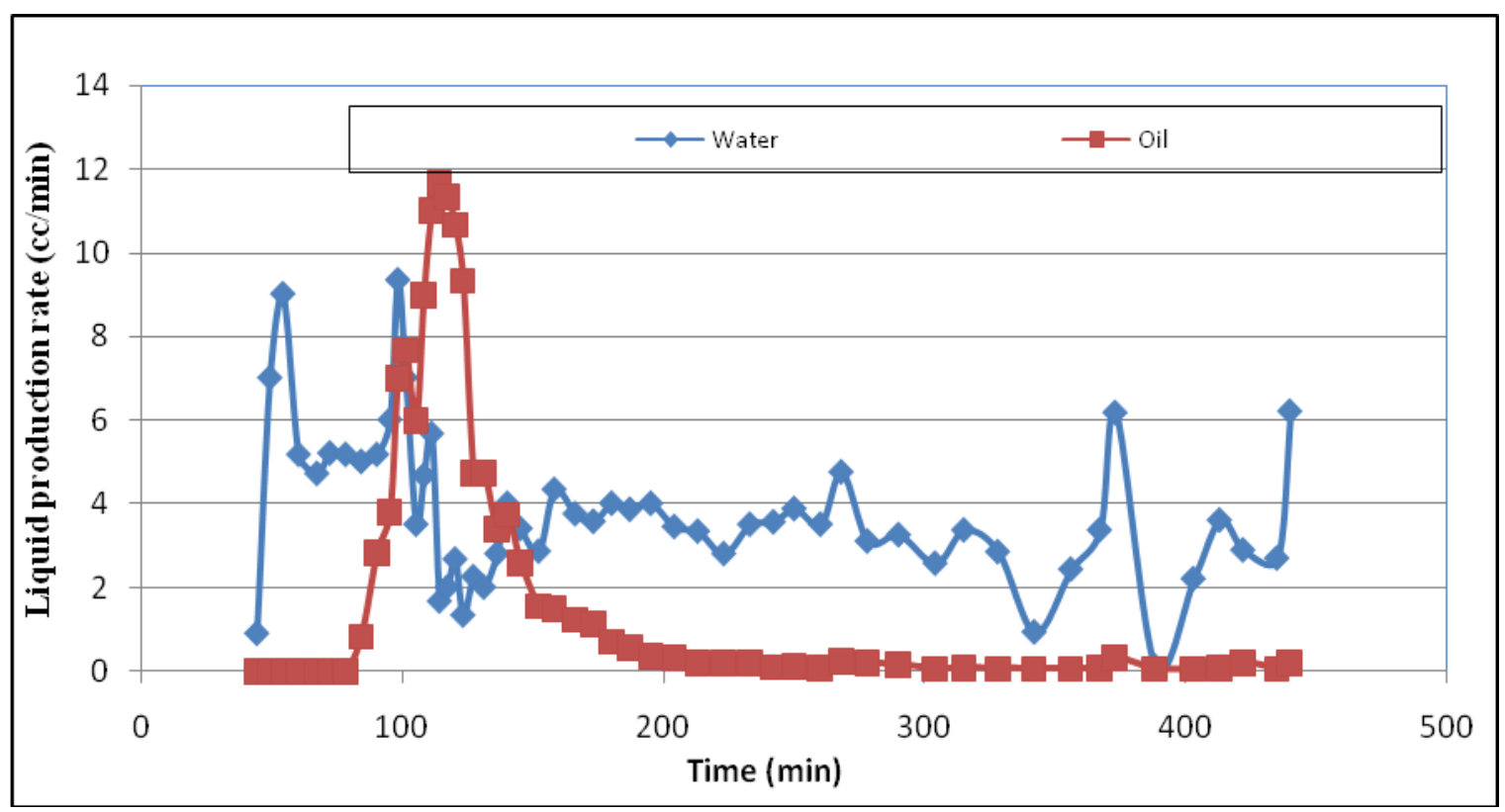

Figure 4.39 - Liquid production rates for run 7 


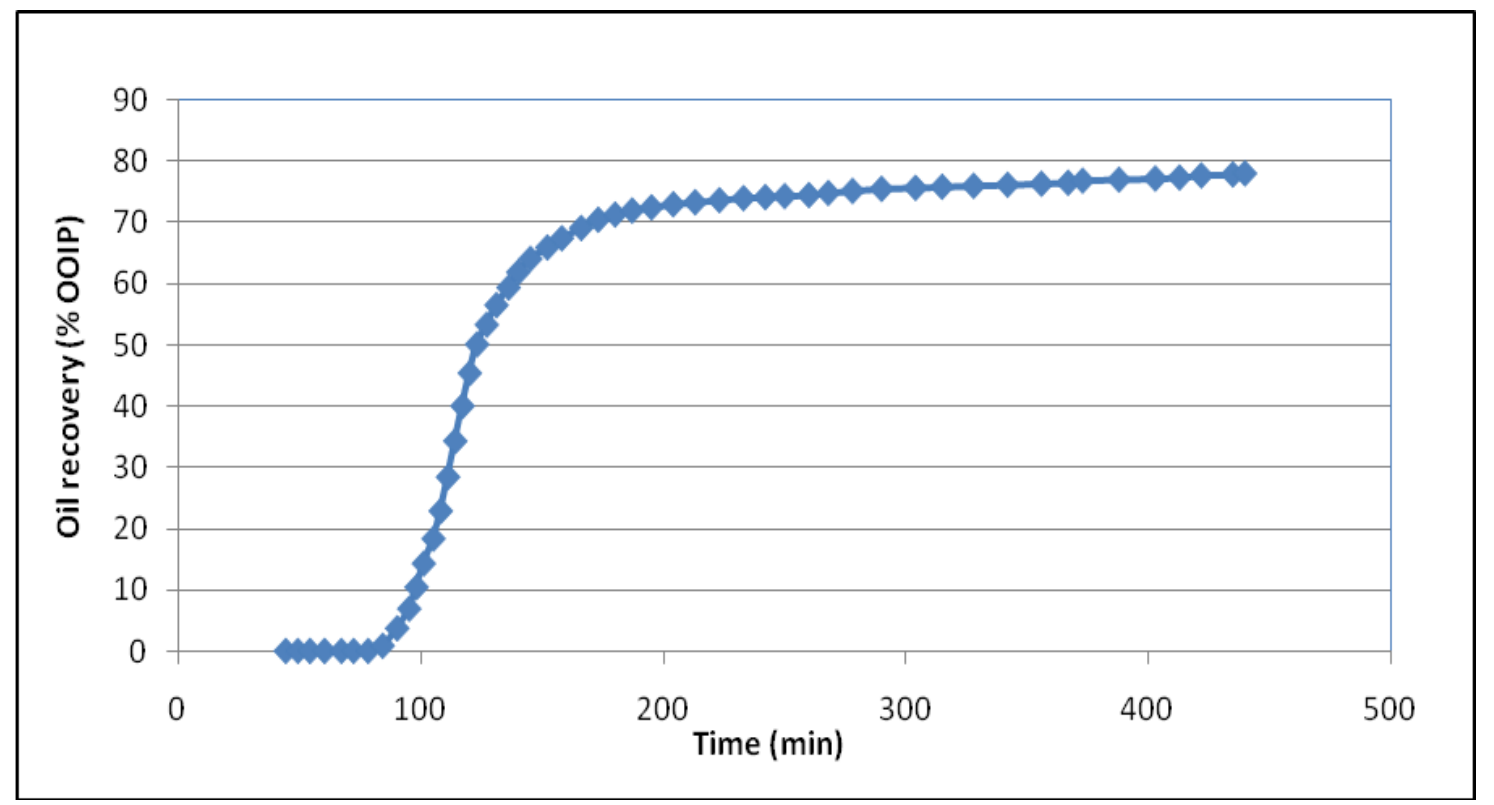

Figure 4.40 - Oil recovery versus time for run 7

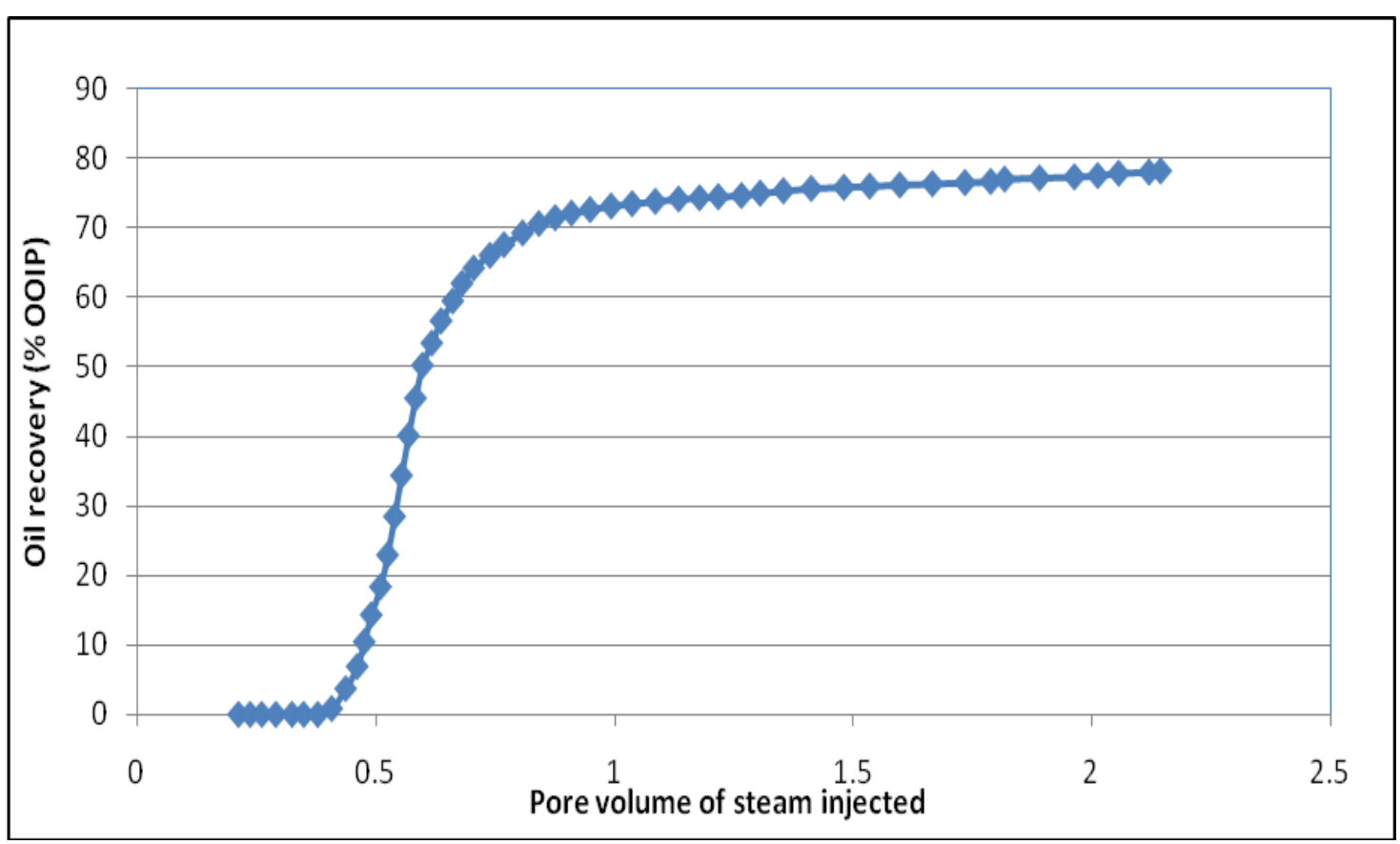

Figure 4.41-Oil recovery versus pore volume of steam injected for run 7 


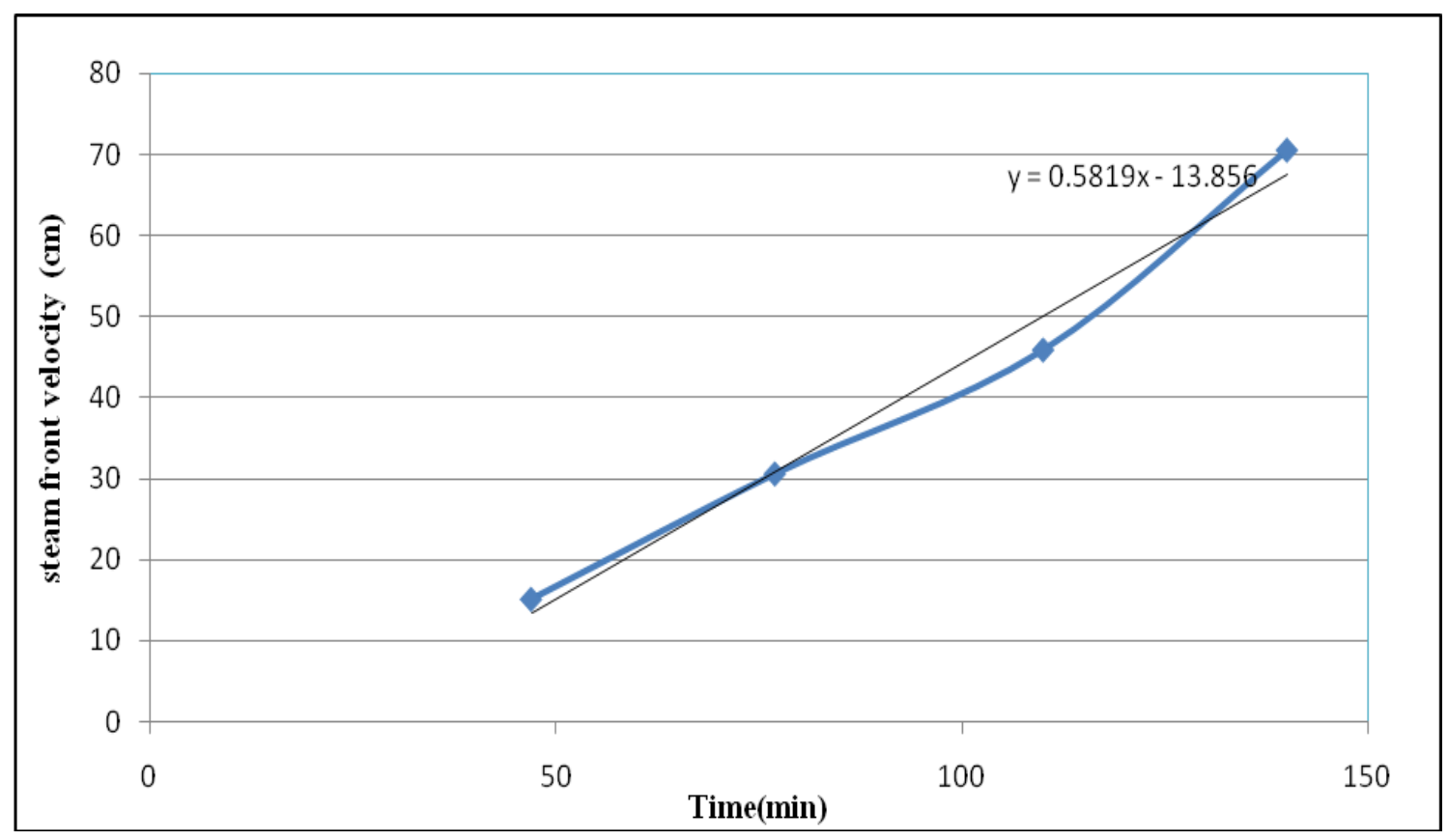

Figure 4.42 - Steam front velocity for run 7

The viscosity and density readings for Run 7 are shown in Table 4.8.

Table 4.8-Viscosity and density readings for run 7

\begin{tabular}{|c|c|c|c|}
\hline \multicolumn{4}{|c|}{ Run 7 (Pure Steam -San Ardo) } \\
\hline \multirow{2}{*}{ Density $\left({ }^{\circ} \mathrm{API}\right)$} & Initial & Middle & Final \\
\cline { 2 - 4 } & 14 & 14.8 & 16.2 \\
\hline Temperature $\left({ }^{\circ} \mathrm{C}\right)$ & \multicolumn{3}{|c|}{ Viscosity (cp) } \\
\hline 40 & 5712 & 4200 & 2000 \\
\hline 50 & 2968 & 2006 & 1756 \\
\hline 60 & 1345 & 1242 & 740 \\
\hline
\end{tabular}




\subsection{Acid Number Measurements}

For the acid number measurements first the solution of $0.05 \mathrm{M}$ TBAOH (tetra butyl ammonium hydroxide) is prepared. In the mixing flask $50 \mathrm{ml}$ of a mixture of $49.4 \%$ IPA (Iso Propyl alcohol), 50 \% Toluene and $0.06 \%$ DDI (deionized distilled water) is taken and a drop of stearic acid is added to spike the mixture. Next the TBAOH is titrated with this blank mixture and the following graph is obtained. From the graph the inflection point (end point) of the titration is measured. Fig. 4.43 shows the titration between the blank sample and the titrant.

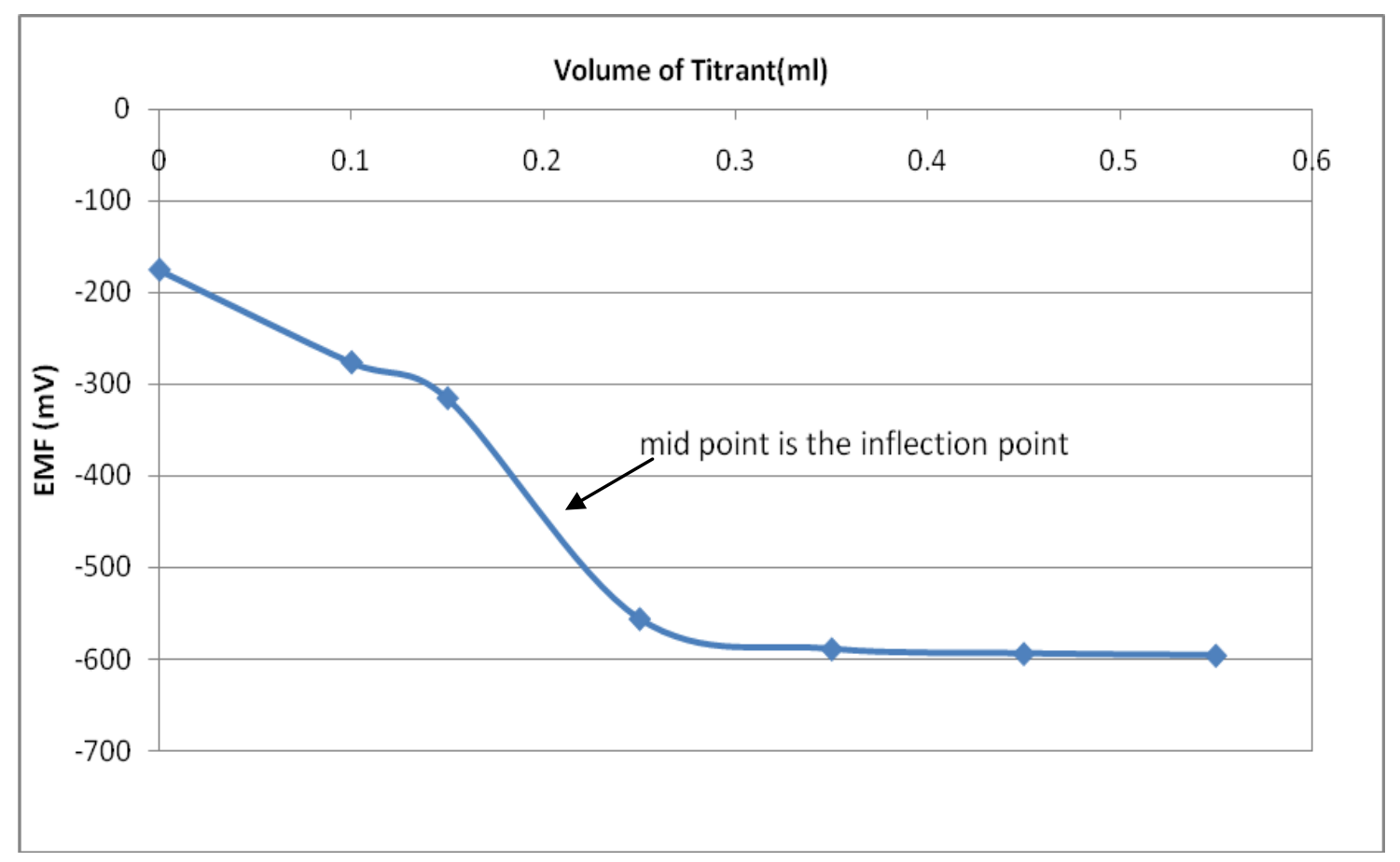

Figure 4.43 - Blank sample titration

Now for San Ardo oil the mixing flask is again filled with the solvent mixture and stearic acid is added to spike the solvent mixture. Next 1 cc of oil is added to the spiked 
solvent mixture and is titrated against TBAOH and Fig. $\mathbf{4 . 4 4}$ shows the result of San Ardo oil. Now after the inflection point is obtained Eqn. 4.1 is used to obtain the value for the acid number.

where

$$
\mathrm{AN}=\left(\mathrm{V}_{\mathrm{i}}-\mathrm{V}_{\mathrm{b}}\right) * \mathrm{M} * \mathrm{~V} /(\mathrm{W})
$$

$\mathrm{AN}$ is the acid number (mg $\mathrm{KOH} / \mathrm{g}$ of oil)

$\mathrm{V}_{\mathrm{i}}$ is the volume of titrant at the sample inflection Point (ml)

$\mathrm{V}_{\mathrm{b}}$ is the volume of titrant at the blank inflection point (ml)

$\mathrm{M}$ is the molar concentration of the TBAOH Titrant (mol/L)

MW is the molecular weight of TBAOH $(\mathrm{gm} / \mathrm{mol})$

$\mathrm{W}$ is weight of oil sample (g).

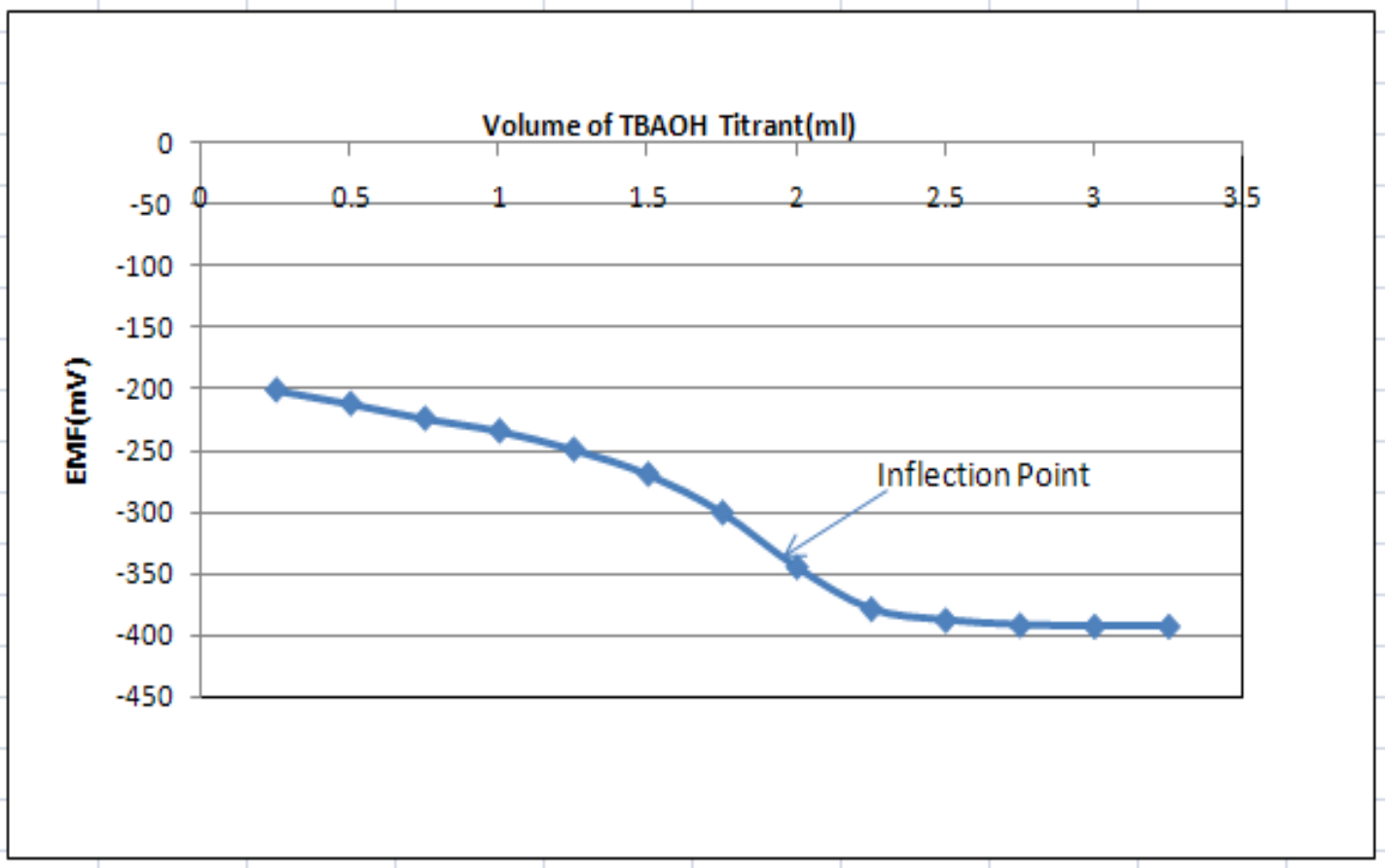

Figure 4.44 - San Ardo oil titration 
For San Ardo oil the value of the acid number obtained is $6.2 \mathrm{mg} \mathrm{KOH} / \mathrm{g}$ of oil. The same measurements are conducted for Duri oil and the acid number value obtained for Duri is $3.6 \mathrm{mg} \mathrm{KOH/g}$ of oil. Fig. $\mathbf{4 . 4 5}$ shows the titration results for Duri oil.

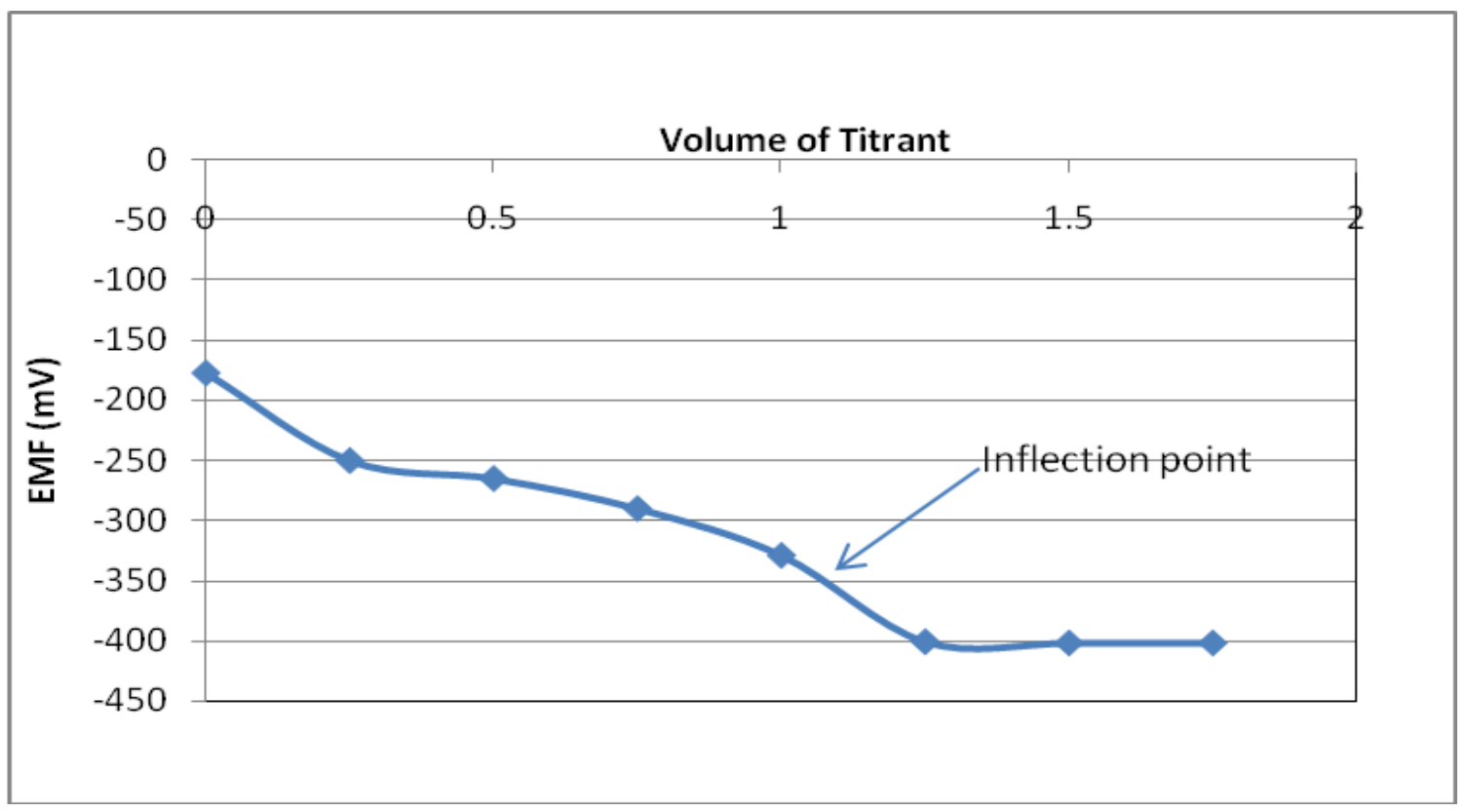

Figure 4.45 - Duri oil titration

\subsection{Interfacial Tension Measurements}

The interfacial tension measurements with different concentrations of $\mathrm{NaOH}$ and San Ardo and Duri oil are shown in Table 4.9 and Table 4.10.From the tables it is observed that for both the oils the lowest interfacial tension is obtained at $1 \mathrm{wt} \% \mathrm{NaOH}$ and the interfacial tension values are lower in case of Duri oil when compared to San Ardo, which can be the reason for Duri oil showing better results with $\mathrm{NaOH}$ than San Ardo. 
Table 4.9-Interfacial tension measurements for Duri oil

\begin{tabular}{|c|c|}
\hline \multicolumn{2}{|c|}{ Duri Oil } \\
\hline $\mathrm{NaOH}($ wt \%) & $\mathrm{IFT}(\mathrm{mN} / \mathrm{m})$ \\
\hline 0.05 & 10.8 \\
\hline 0.1 & 11 \\
\hline 0.5 & 10.2 \\
\hline 1 & $\mathbf{9}$ \\
\hline 5 & 9.5 \\
\hline 10 & 9.8 \\
\hline
\end{tabular}

Table 4.10-Interfacial tension measurements for San Ardo oil

\begin{tabular}{|c|c|}
\hline \multicolumn{2}{|c|}{ San Ardo } \\
\hline $\mathrm{NaOH}$ (wt \%) & $\mathrm{IFT}(\mathrm{mN} / \mathrm{m})$ \\
\hline 0.05 & 26 \\
\hline 0.1 & 26.7 \\
\hline 0.5 & 27 \\
\hline 1 & 25.1 \\
\hline 5 & 26.5 \\
\hline 10 & 27.1 \\
\hline
\end{tabular}

\subsection{Comparison and Discussion of Results}

For the alkaline steamflooding to be effective the acid numbers of the oils should be high and in this case both Duri and San Ardo have a high acid number. The acid number gives an indication of the amount of acids present in the oil which enables it to form a surfactant when it reacts with sodium hydroxide. Although the acid number gives the amount of acids present in the oil, the type of acids present is not known. For the success of alkaline steamflooding the acids present should be long chain carbon molecules which facilitate the formation of soap and thereby reducing the interfacial tension of the oil. For all the experiments the comparison and discussion can be done for the two oils separately. 


\subsubsection{Comparison of Runs (Duri oil)}

For all the Duri oil experiments (Run 3, Run 4, Run 5) water production rates were plotted with respect to pore volume and shown in Fig. 4.46.

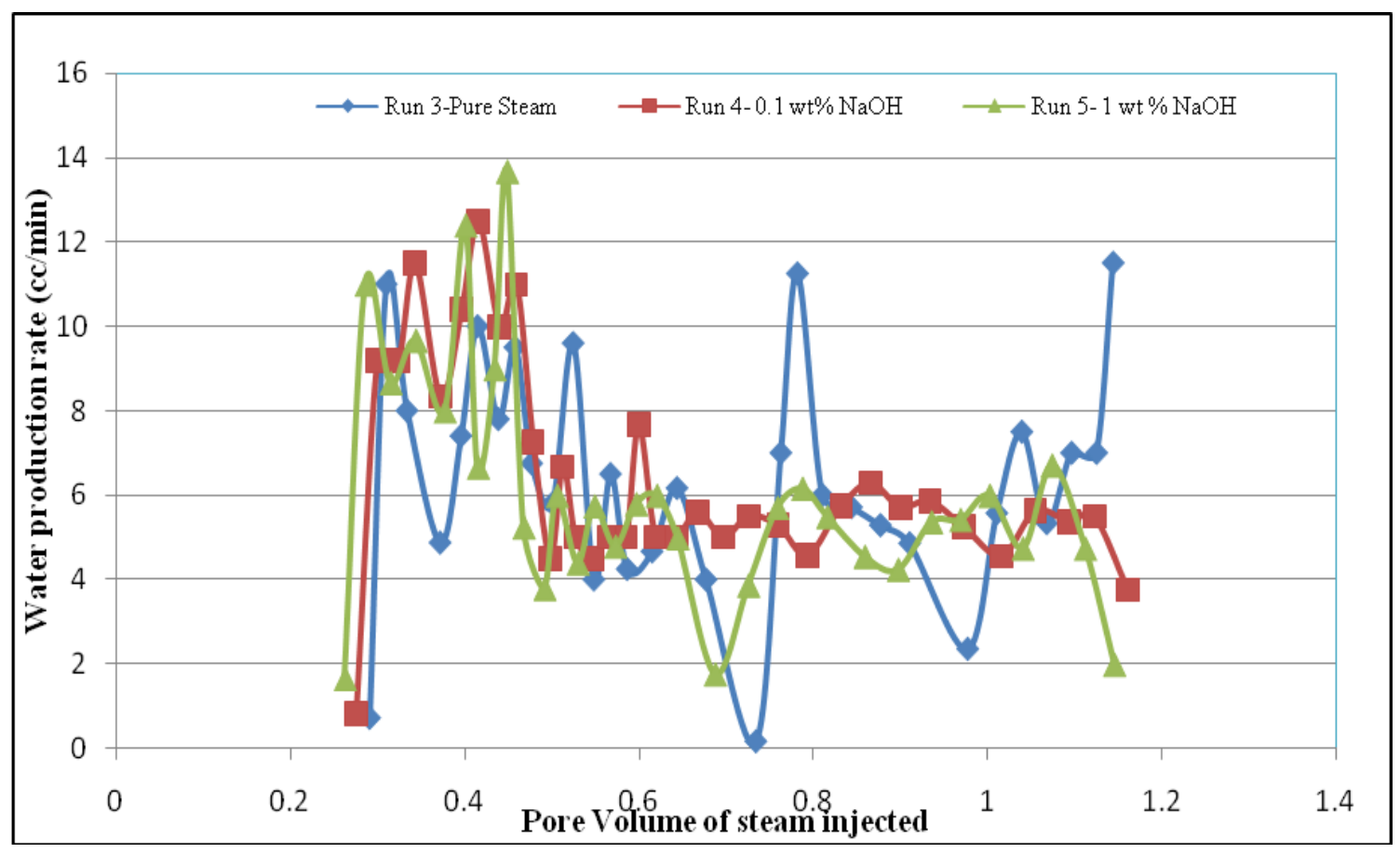

Figure 4.46 - Water production rate versus pore volume of steam injected

In Fig. 4.46, the water production starts faster in both the sodium hydroxide runs and it is fastest in case of Run 5 (0.26 PV) and it production starts late in pure steam run(Run 3-0.29 PV).This shows that there is an acceleration with the addition of sodium hydroxide and the acceleration is higher with $1 \mathrm{wt} \% \mathrm{NaOH}$. 
A plot of cumulative water production for the three different runs is shown in Fig. 4.47. It is seen that the water production in both the runs with sodium hydroxide is higher than the pure steam run.

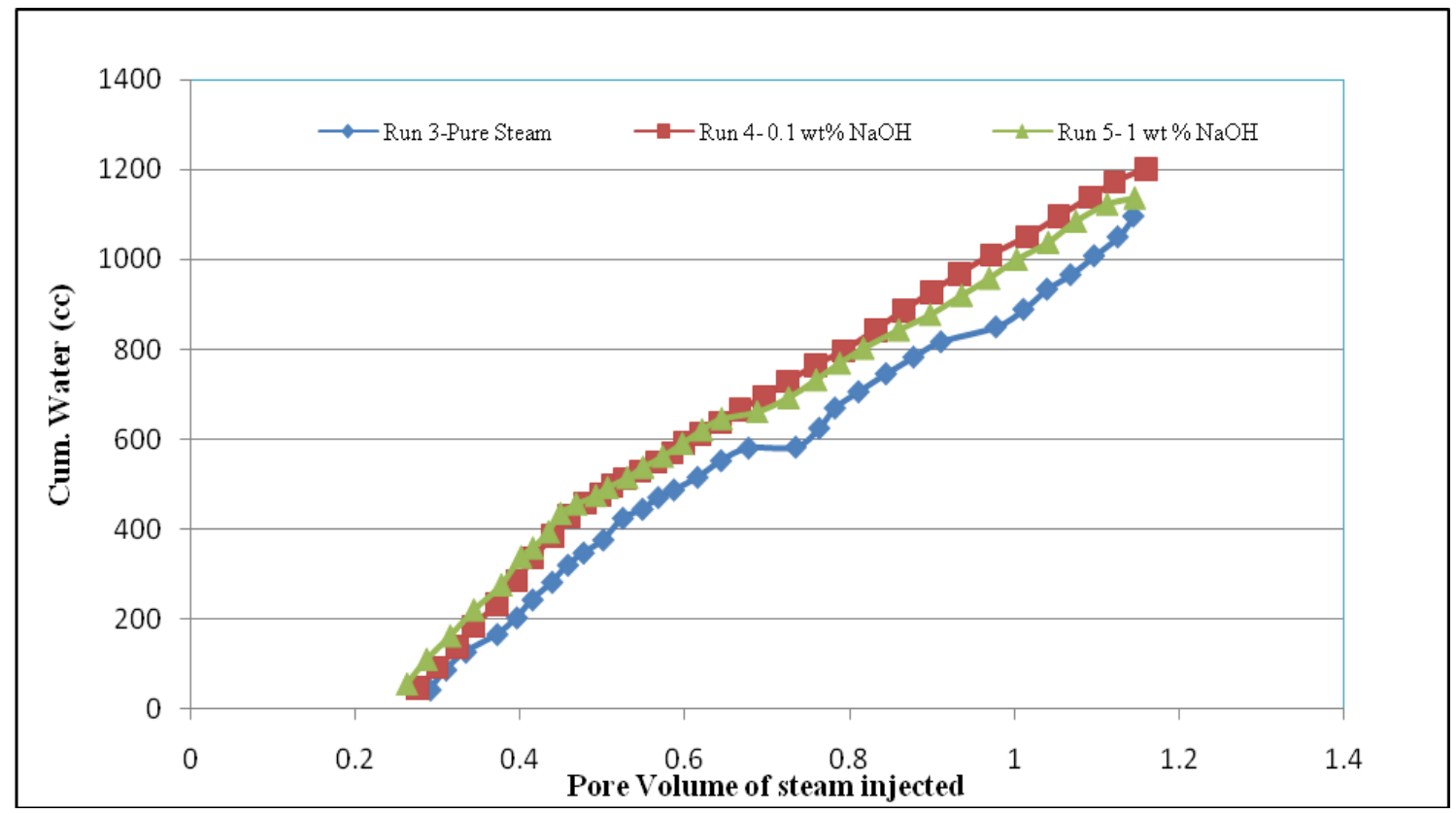

Figure 4.47 - Cumulative water volume versus pore volume of steam injected

A plot of oil rate and pore volume is shown in Fig. 4.48 and it can be seen that production of oil is slower in the sodium hydroxide runs when compared to the pure steam run . The sodium hydroxide run with $1 \mathrm{wt} \% \mathrm{NaOH}$ produces faster that the $0.1 \mathrm{wt}$ \% $\mathrm{NaOH}$ (0.4 PV compared to $0.45 \mathrm{PV})$. Although the production of oil was faster in case of steam we see that the $1 \mathrm{wt} \% \mathrm{NaOH}$ shows $12 \%$ acceleration in the production of oil when compared to the 0.1 wt $\% \mathrm{NaOH}$. 


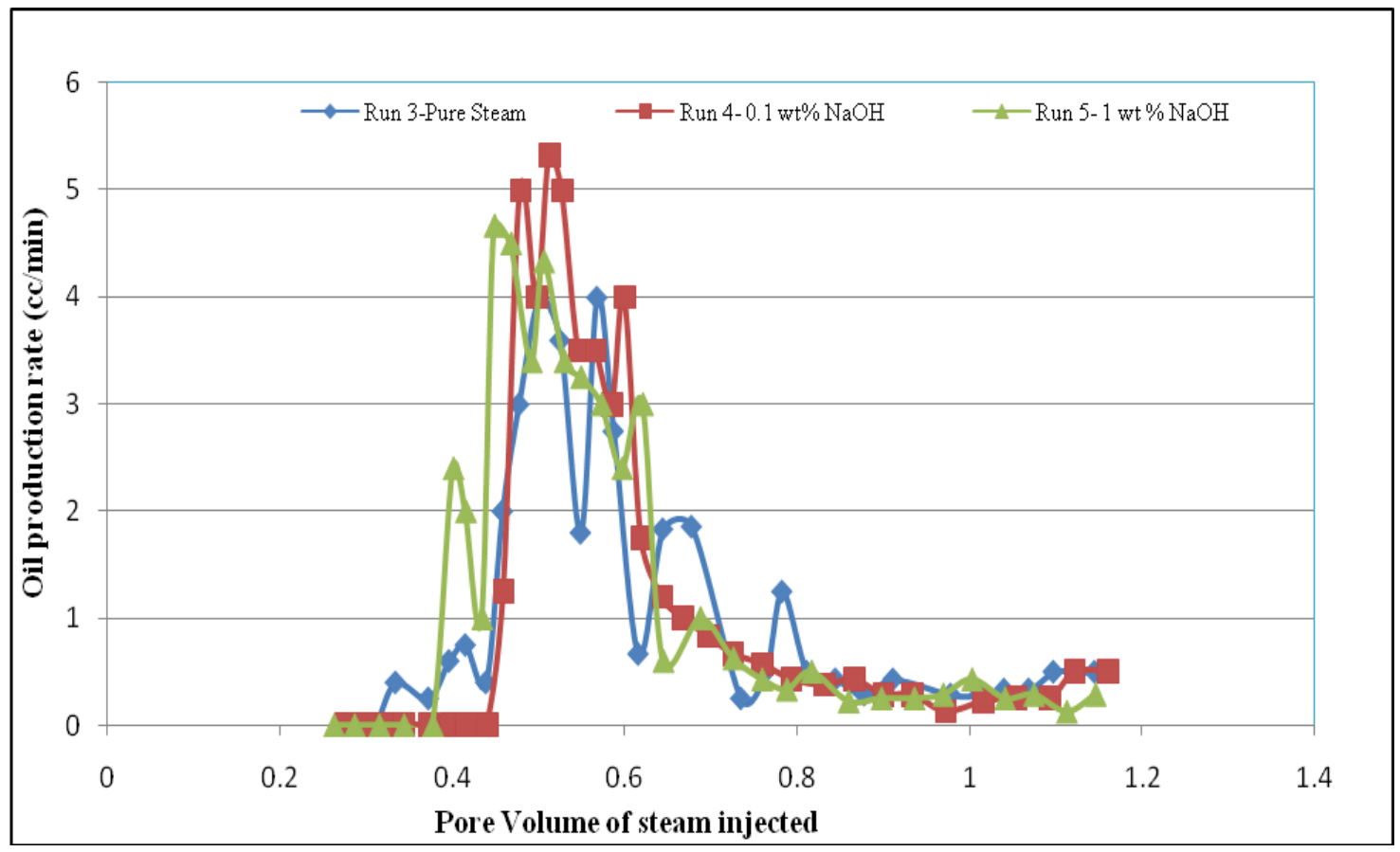

Figure 4.48 - Oil rate versus pore volume of steam injected

The volume of oil is plotted against the pore volume for the three runs, Fig. 4.48 and we see that the recovery is maximum in case of Run 5 and it produces an increase of $7 \%$ in recovery. The increase in recovery is due to the formation of surfactants that reduce the interfacial tension of oil. Run 3 and Run 4 had recovery of $52 \%$ and $53 \%$ respectively and the recovery with 1 wt \% NaOH is $59 \%$.This shows that sodium hydroxide has an effect on Duri and contributes to the higher recovery of the oil.

The change viscosity is also shown in Fig. 4.49. There is a decrease in viscosity and density which also attributes to the higher recovery of oil. 


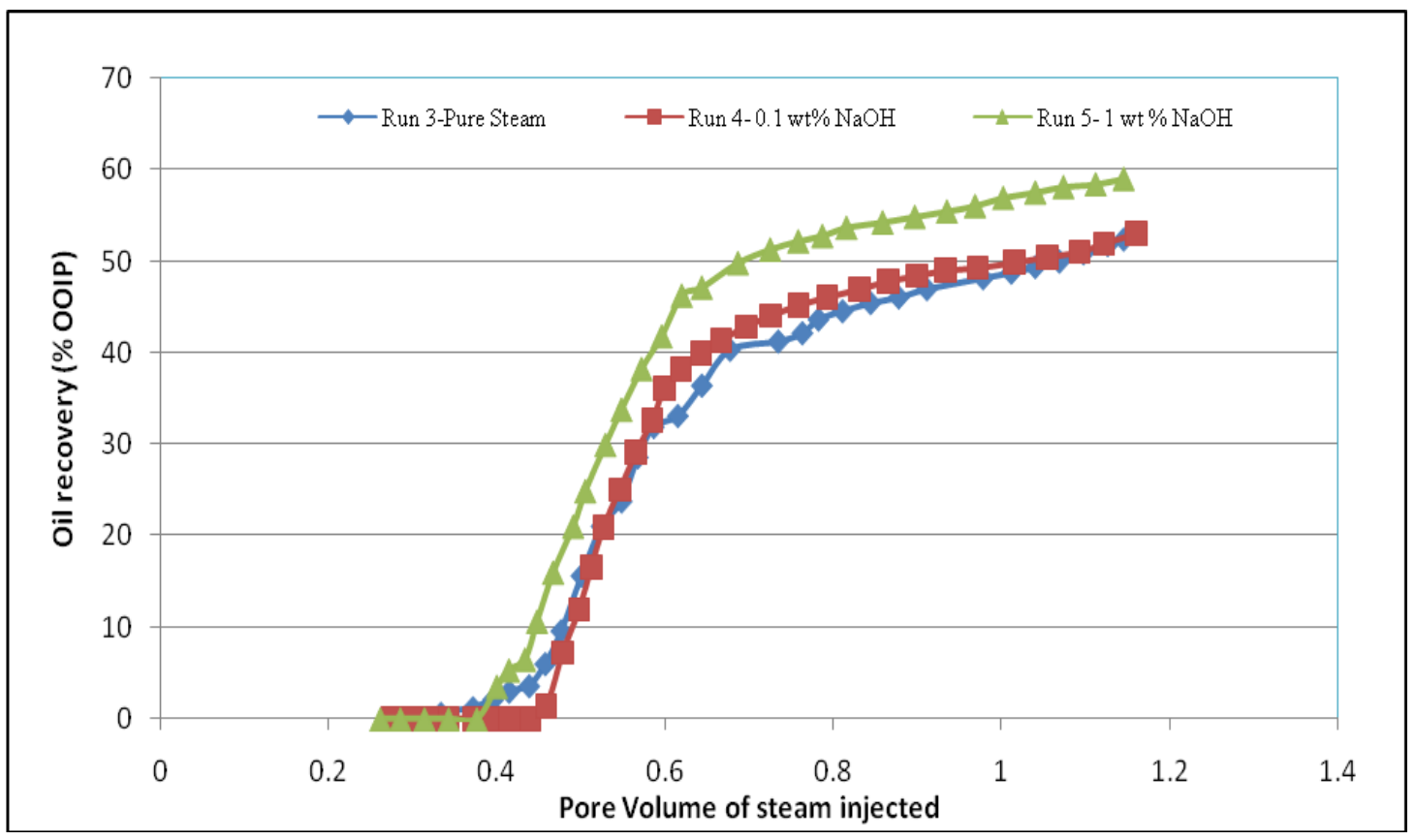

Figure 4.49 - Oil recovery versus pore volume of steam injected

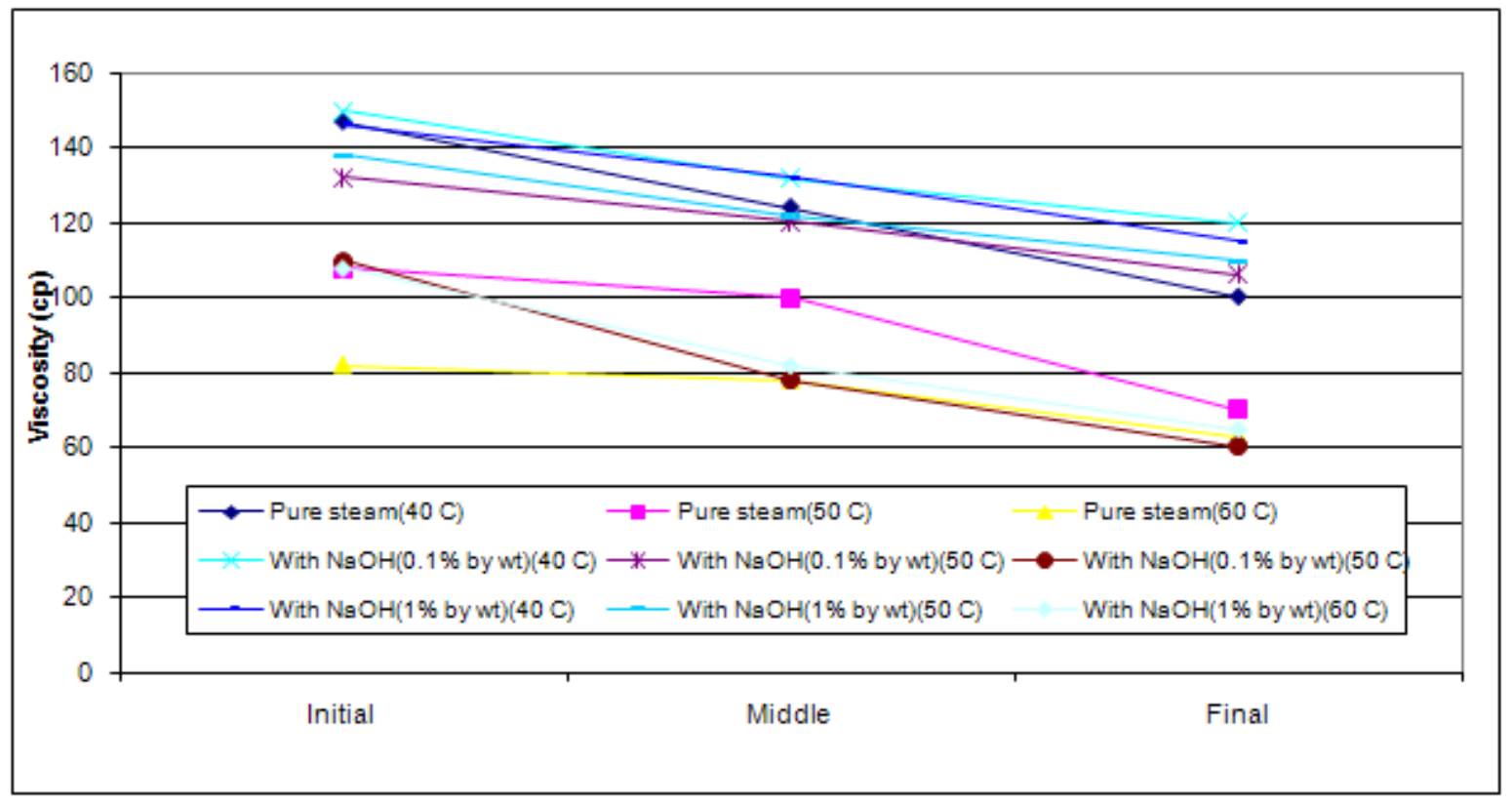

Figure 4.50-Oil viscosity change 


\subsubsection{Comparison of Runs (San Ardo)}

For the comparison of runs involving San Ardo 4 runs 1, 2, 6, 7 are used. Run 2 involve the simultaneous injection of sodium hydroxide with steam whereas in Run 6 the caustic is injected as slug in two cycles.

For all the runs the water production rate was plotted with pore volume shown in Fig. 4.51 and the cumulative water production is shown in Fig. 4.52.The maximum water produced is in the cyclic caustic injection run and maximum water production rate is seen in Run $2(0.1$ wt \% $\mathrm{NaOH})$.

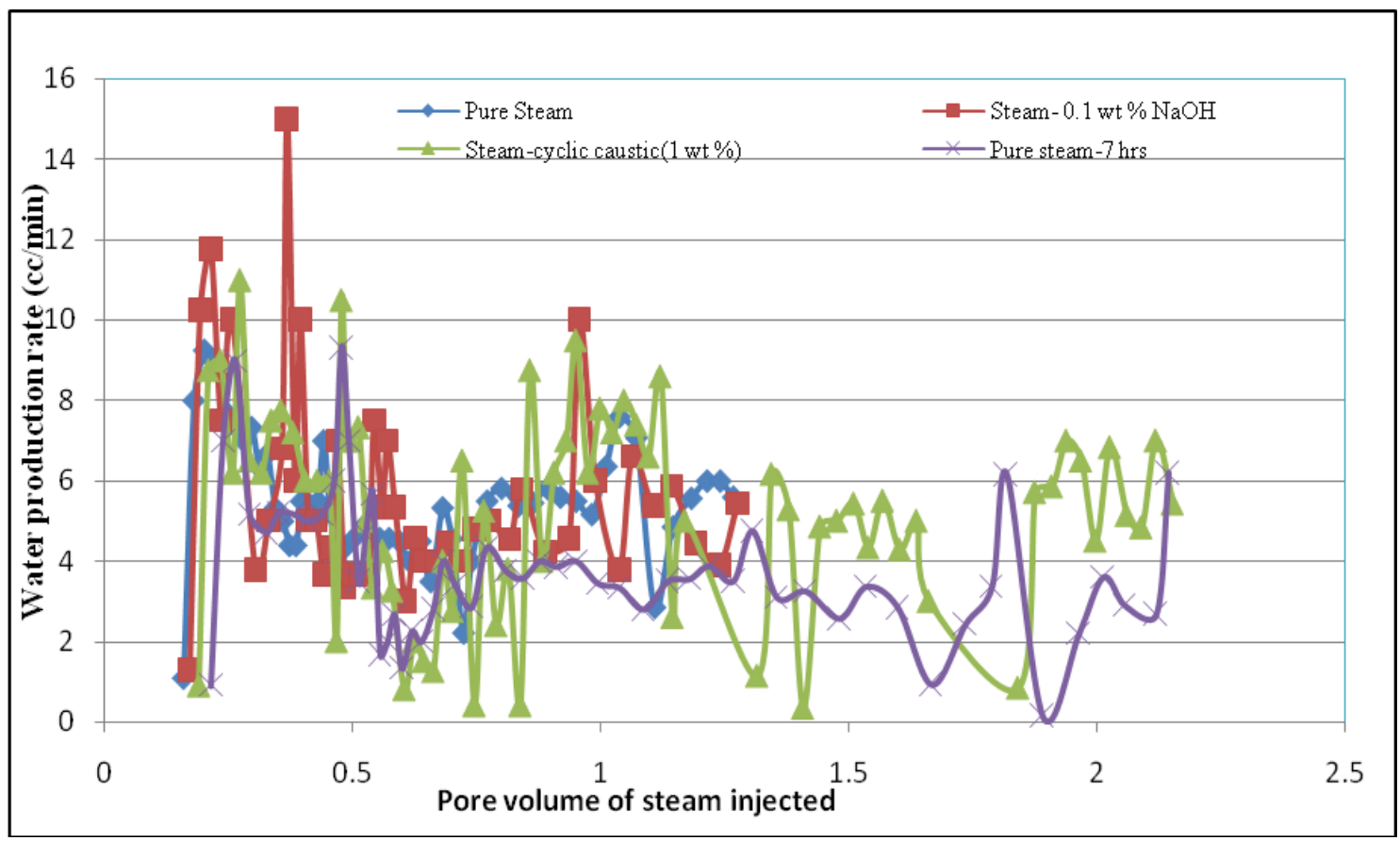

Figure 4.51-Water production rate versus pore volume of steam injected 


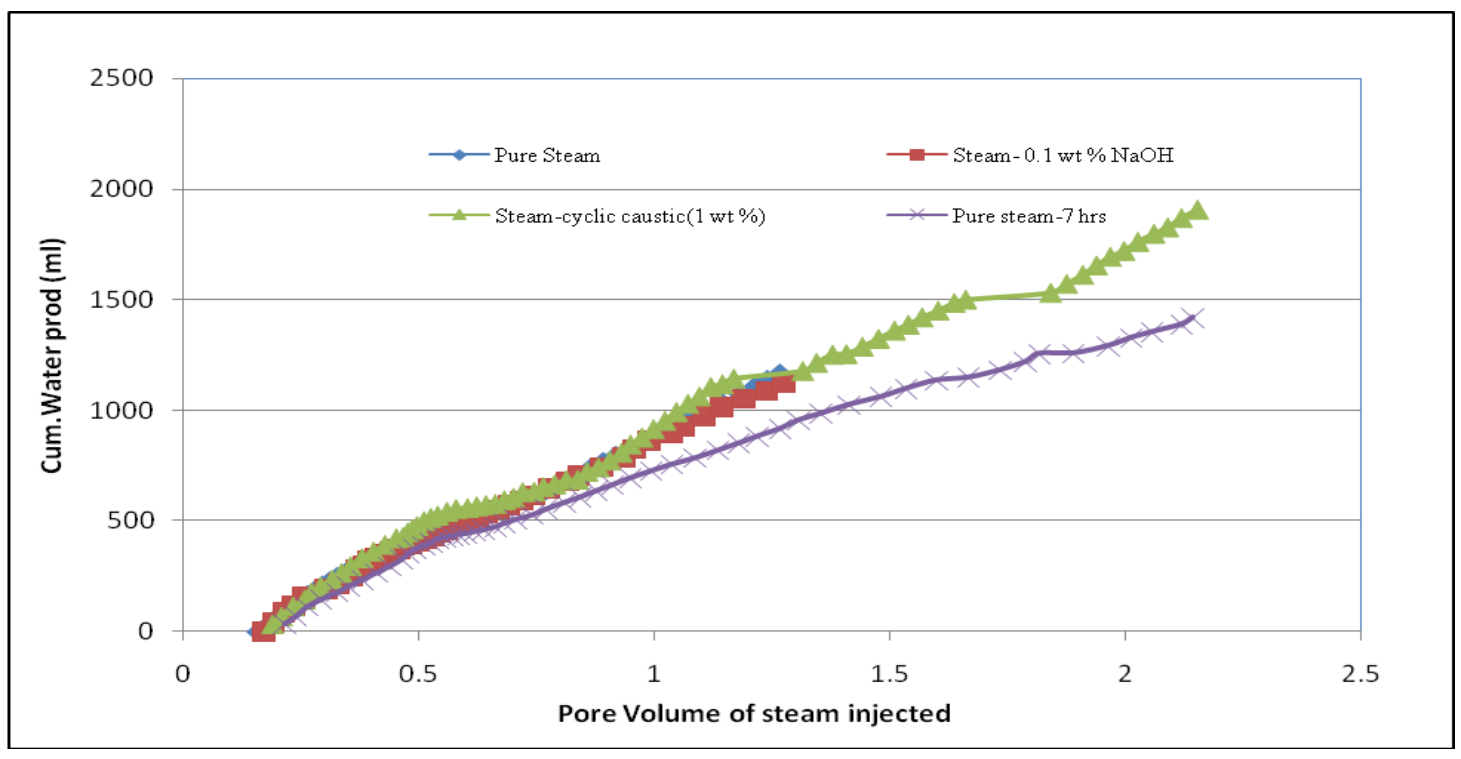

Figure 4.52- Cumulative water production rate versus pore volume of steam injected

The oil rates for all the runs are plotted against pore volume in Fig. 4.53. The oil recovery is plotted against pore volume for all the runs in Fig. 4.54.

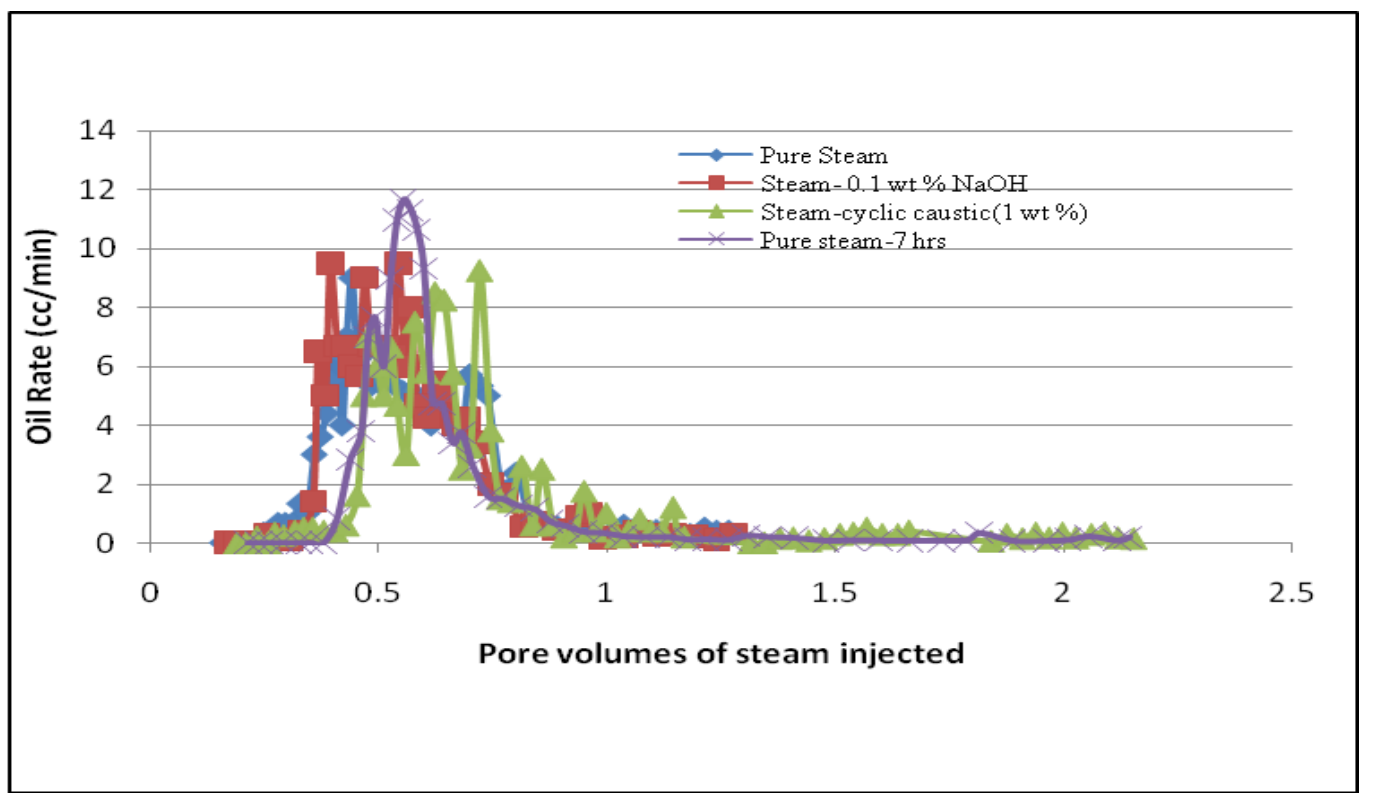

Figure 4.53- Oil rate versus pore volume of steam injected 


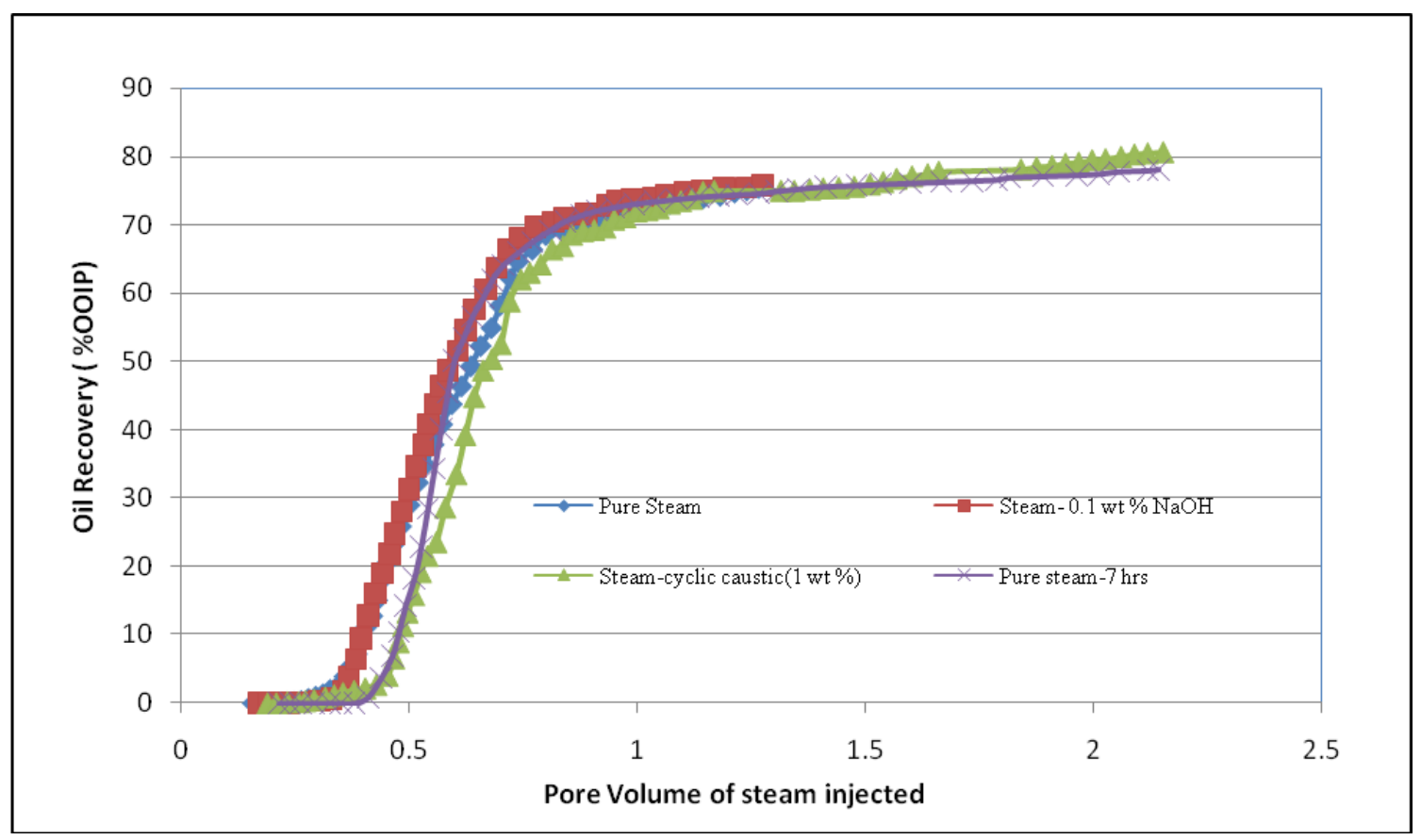

Figure 4.54- Oil rrcovery versus pore volume of steam injected

From the plot it can be seen that there is no difference in oil recovery for Run 1(pure steam) and Run 2 (steam-0.1 wt \% $\mathrm{NaOH}$ ) and even with the cyclic injection of caustic, there is no effect in recovery. The recovery for run 6 and run 7 are $81 \%$ and $78 \%$ respectively..We see that run 6 and run 7 have a higher recovery when compared to Run $1(75.3 \%)$ and Run $2(75.7 \%)$.This is due to the fact that more pore volumes of steam was injected (2.2 PV) when compared to Run 1 and Run 2(1.15 PV).The viscosity and the density are shown in Fig. 4.55 and Fig. 4.56. 


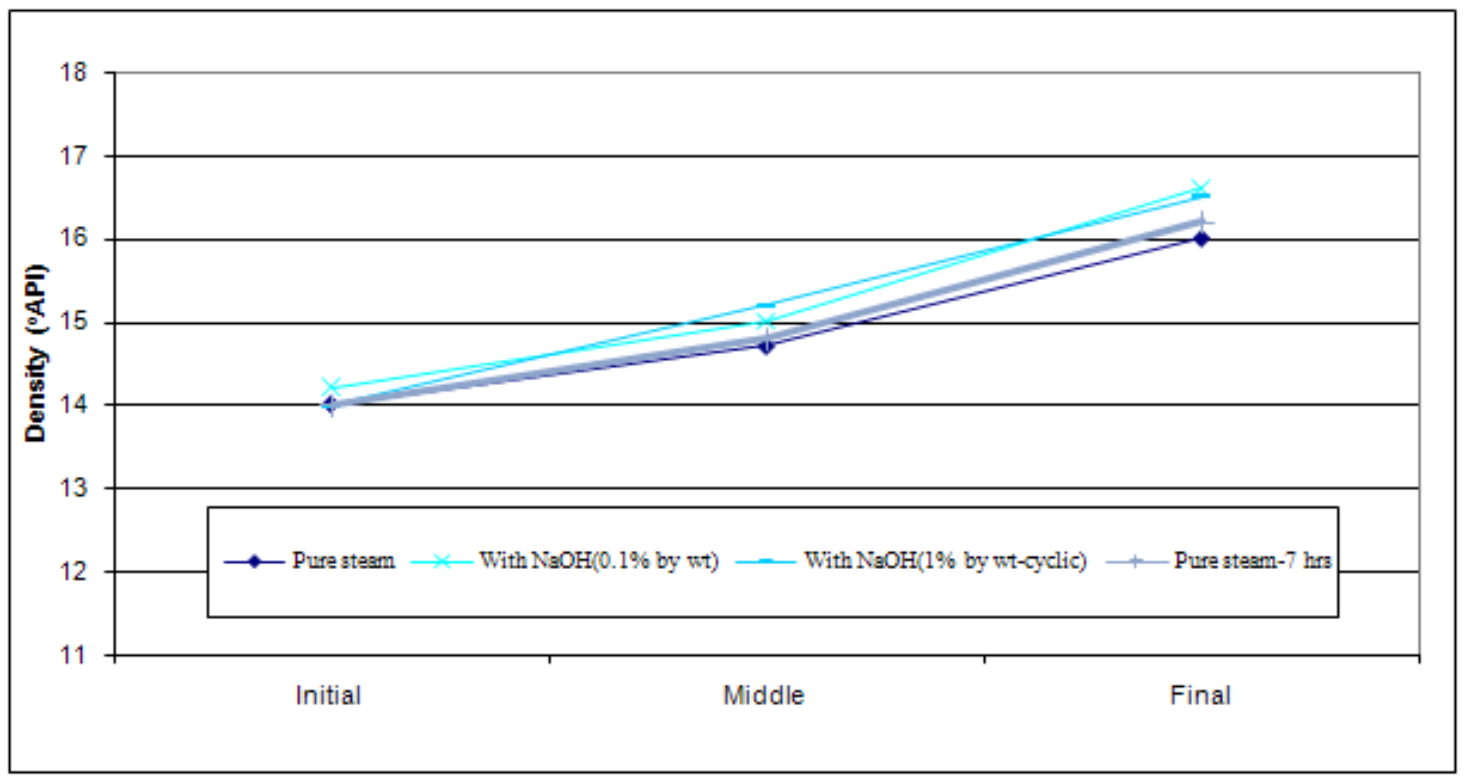

Figure 4.55 - Density change for San Ardo oil

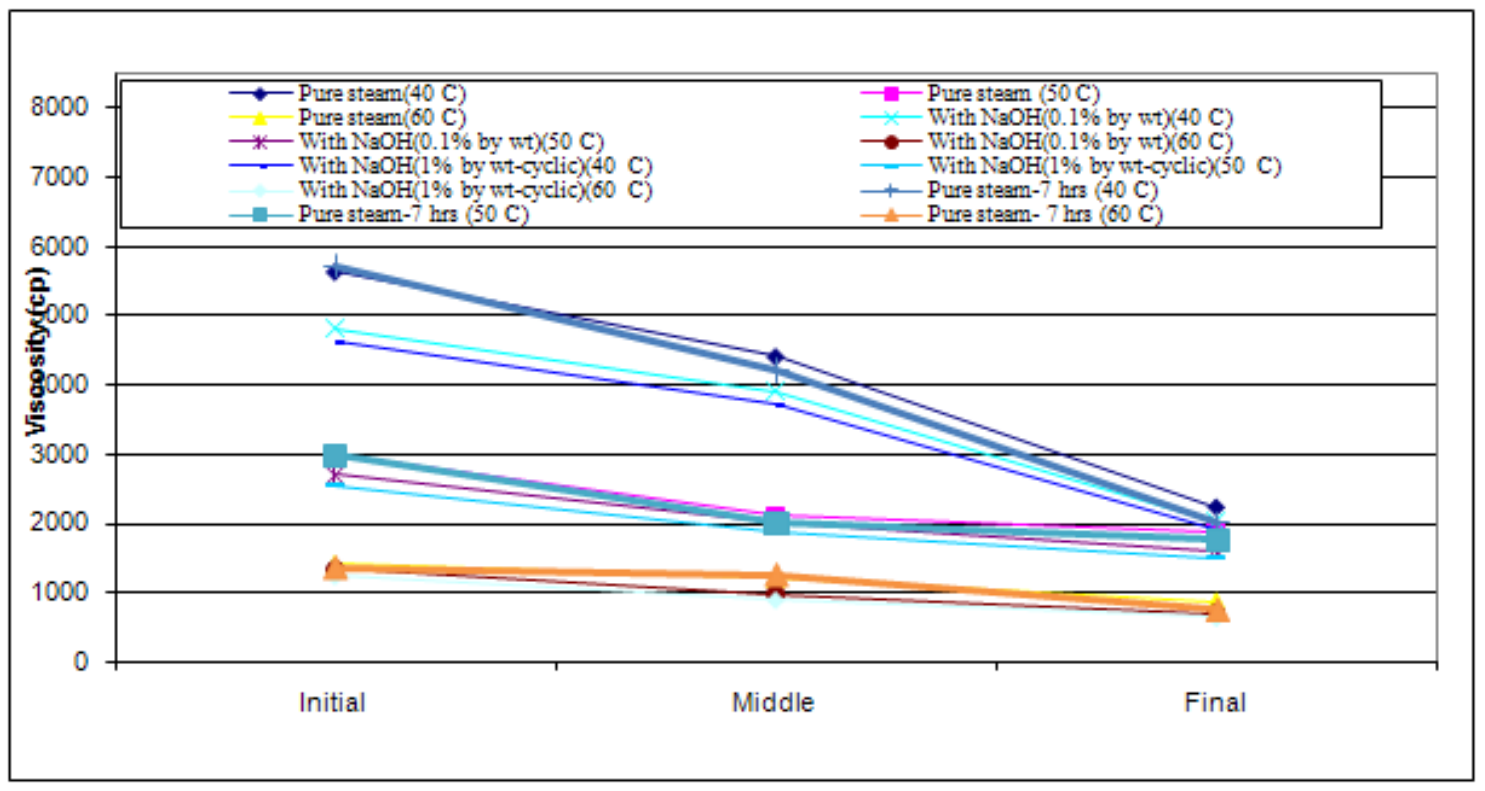

Figure 4.56 - Viscosity change for San Ardo oil 


\section{CHAPTER V}

\section{SUMMARY, CONCLUSIONS AND RECOMMENDATIONS}

\subsection{Summary}

The purpose of the research was to improve the recovery factor of heavy oils by using suitable additives. The additive used was sodium hydroxide which increases improves the secondary oil recovery by reducing the IFT by reacting with the organic acids present in the oil. The experiments were conducted in a one dimensional cell by using a sandpack and testing two heavy oils San Ardo and Duri. The acid number of the oils was also measured by using a titration method and a pH meter to determine the amount of acids present in the oil. The IFT between the oils and different concentrations of $\mathrm{NaOH}$ solution was also measured using a tensionometer.

\subsection{Conclusions}

Main conclusions of the study may be summarized as follows:

(a) San Ardo Oil- With steam-NaOH (0.1 wt \%), oil recovery is 75.7\% OIIP compared to75\% OIIP under steam injection. Oil recovery is practically the same in both cases. For caustic slug injection the recovery was $80 \%$. For pure steam for the exact same pore volumes of steam injected as in the caustic slug run the recovery was $78 \%$. Caustic solution was injected as a slug for San Ardo oil after the sand pack was steamflooded to recover the residual oil, to test it as an injection mechanism because of the failure of simultaneous caustic steam injection but it was not very effective. 
(b) Duri oil- With steam-NaOH (0.1 wt \%), oil recovery is 53\% OIIP compared to 52\% OIIP under steam injection. Oil recovery is practically the same in both cases. With increase in the concentration of $\mathrm{NaOH}$ (from 0.1 to 1 wt \%) oil recovery increased to 59\% OIIP from 52\% OIIP under steam injection. Sodium hydroxide resulted in higher oil recovery with Duri Oil. Although it did not accelerate the process by much there was significant increase in the recovery of oil.

(c) It appears that $\mathrm{NaOH}$ as a steam additive is more effective in the case of Duri oil. This may be due to the formation of more surfactant with Duri oil than San Ardo oil.

(d) The acid number was measured and was found to be $6.25 \mathrm{mg} \mathrm{KOH} / \mathrm{g}$ of oil for San Ardo and $4.2 \mathrm{mg} \mathrm{KOH} / \mathrm{g}$ of oil for Duri, indicating San Ardo to be more acidic than Duri oil.

(d) From the IFT measurements, it is seen that IFT is lower for Duri oil than for San Ardo and the lowest IFT is obtained with $1 \mathrm{wt} \% \mathrm{NaOH}$.The lower value of IFT is an indication that more amount of surfactant is formed in case of Duri oil. The lower values of IFT indicate that the amount of surfactant is sufficient to reduce the residual oil value in the case of Duri oil.

(e) For San Ardo oil there is acceleration in production by $12 \%$ with $\mathrm{NaOH}$-steam injection compared to pure steam injection.

(f) The water volume produced is higher for both oils in cases where sodium hydroxide solution is injected either as a slug or simultaneously with steam.

(g) Higher oil production rates were seen in steam-caustic injection runs when compared to pure steam injection runs. 
The effect of the alkali on San Ardo oil is not seen since the sodium hydroxide is probably not injected in sufficient quantities to bring about a change in interfacial tension and therefore higher recovery. The oil saturation for San Ardo oil used in the experiments is $60 \%$ whereas the oil saturation used for Duri oil is $30 \%$.The success of sodium hydroxide with very heavy oils requires probably large quantities of alkali to be injected over a long period of time.

\subsection{Recommendations}

Following are the main recommendations for future research:

(1) To study further the effect of sodium hydroxide on different kinds of oils and to understand the effect of the acids present in the oil in reducing interfacial tension.

(2) To conduct the experiments on previously waterflooded sandpacks for very heavy oils like San Ardo.

(3) Core flooding would also be helpful in understanding the process of alkaline steam flooding with both heavy and lighter oils.

(4) To test the combination of sodium hydroxide with additives which form the basis for alkaline surfactant process - as in Alkaline Surfactant Polymer (ASP) injection - in improving the recovery of oil.

(5) To test non thermal means of caustic flooding for heavy oils. 


\section{REFERENCES}

Fan, T., Buckley J.S., 2006. Acid Number Measurements Revisited. Paper SPE 99884 presented at the SPE/DOE symposium on Improved Oil Recovery, Tulsa, Oklahoma, 22-26 April.

Goyal, K.L., Arora, P.D., 1978. Enhanced Recovery of Viscous Oil by Caustic Flooding, SPE Paper 7902, Gujarat, India.

Hong, K.C., 1994 Steamflood Reservoir Management: Thermal Enhanced Oil Recovery, PennWell Books, Tulsa, Oklahoma (1994).

Jennings, Y., 1974.A Study of Caustic Solution-Crude Oil Interfacial Tensions. Paper SPE 5049 presented at the 1974 SPE AIME meeting held in Houston, Texas, 6-9 October.

Okoye, C.U., Haytdavoudi, A., 1990.A Laboratory Screening method for a Pilot Steam Caustic Flood. Paper 20068 presented at the 1990 California General Meeting, California, 4-6, April.

Okoye, C.U., Tiab, D., 1982. Enhanced Recovery of Oil by Alkaline Steam Flooding. Paper SPE 11076 presented at the 1982 SPE AIME meeting, New Orleans, and 26-29 September.

Okoye, C.U., Tiab, D., 1985. A Chemical Displacement Model for Alkaline Steamflooding in Linear Systems. Paper SPE 13580 MS presented at the SPE Oilfield and Geothermal Chemistry Symposium, Phoenix, Arizona, 9-11 March.

Prats, M. 1986. Thermal Recovery. Monograph Series, SPE, New York City 7, 1.

Rivero, J: 2007. Experimental studies of steam and steam-propane injection using a novel smart horizontal producer to enhance oil production in the San Ardo field. PhD Dissertation, Texas A \& M University, College Station.

Shedid, A., Abbas, A., 2000.Experimental Study of Surfactant Alkaline Steam Flood through Vertical Wells. Paper SPE 62562 presented at the 2000 SPE/AAGE Western Regional Meeting, California, 19-23, June.

Simangunsong, R: 2005. Experimental studies of steam and steam-propane injection using a novel smart horizontal producer to enhance oil production in the san Ardo field. MS Thesis, Texas A\&M University, College Station. 
Tiab, D., Okoye, U., Osman, M., 1982. Caustic Steam Flooding. Paper SPE 9945 presented at the 1982 California General Meeting, Bakersfield, California, 25-26 March. 


\section{APPENDIX A}

\section{CALCULATION OF FLUID SATURATIONS}

The following is a sample calculation of the fluid saturations and pore volume inside the cell. The calculations are made for Run 1.

1. Cell dimension:

Diameter, $d=7.35$, height, $h=66.5$

Since the cell is cylindrical, the volume of the cell is:

$$
V_{\text {cell }}=h \pi\left(\frac{d}{2}\right)^{2}=2821.54 \mathrm{~cm}^{3}
$$

2. The total weight of mixture $\left(W_{\text {mix }}\right)$ :

Weight of sand, $W_{\text {sand }}=5141 \mathrm{~g}$

Weight of water, $W_{\text {water }}=226 \mathrm{~g}$

Weight of oil, $W_{\text {oil }}=671 \mathrm{~g}$

$W_{\text {mix }}=W_{\text {sand }}+W_{\text {water }}+W_{\text {oil }}$

$W_{\text {mix }}=5141+226+667=6038 \mathrm{~g}$

3. The weight of mixture inside the cell, $W_{\text {mix }}$ in cell is:

Weight of empty cell $=2823.6 \mathrm{~g}$

Weight of cell with mixture inside $=8359 \mathrm{~g}$

Weight of mixture inside the cell, $W_{\text {mixcell }}=8359-2823.6=5535.4 \mathrm{~g}$

4. Since the mixture is homogenous, the proportions of sand, water and oil remain constant before and after packing. The amount of each component inside the cell is then calculated below:

Weight of sand inside the cell, $W_{\text {sandcell }}=\frac{W_{\text {mixcell }}}{W_{\text {mix }}} W_{\text {sand }}=\frac{5535.4}{6032.8} 5141=4717.13 \mathrm{~g}$ 
Weight of water inside the cell, $W_{\text {watercell }}=\frac{W_{\text {mixcell }}}{W_{\text {mix }}} W_{\text {water }}=\frac{5535.3}{6032.8} 226=207.37 \mathrm{~g}$

Weight of oil inside the cell, $W_{\text {oilcell }}=\frac{W_{\text {mixcell }}}{W_{\text {mix }}} W_{\text {oil }}=\frac{5535.3}{6032.8} 443=615.68 \mathrm{~g}$

5. The sand density, $\rho=2.65 \mathrm{~g} / \mathrm{cm}^{3}$. Thus,

Volume of sand inside the cell, $V_{\text {sandcell }}=\frac{W_{\text {sandcell }}}{\rho_{\text {sand }}}=\frac{4717.13}{2.65}=1780.05 \mathrm{~cm}^{3}$

6. The Porosity inside the cell is:

$\phi=\frac{V_{\text {cell }}-V_{\text {sandcell }}}{V_{\text {cell }}}=\frac{2821.54-1780.05}{2821.54}=0.392$

7. The original San Ardo oil has an oil gravity of 12.5 which is equivalent 0.988 $\mathrm{g} / \mathrm{cm}^{3}$. Water and oil volumes inside the cell are calculated as follows:

$V_{\text {watercell }}=\frac{W_{\text {watercell }}}{\rho_{\text {water }}}=\frac{207.37}{1}=207.37 \mathrm{~cm}^{3}$

$V_{\text {oilcell }}=\frac{W_{\text {oilcell }}}{\rho_{\text {water }}}=\frac{615.68}{0.988}=623.15 \mathrm{~cm}^{3}$

8. The pore volume inside the cell is:

$V_{\text {porecell }}=\phi \times$ Vcell $=1041.49 \mathrm{~cm}^{3}$

9. The saturations are calculated as follows:

$S_{w}=\frac{V_{\text {watercell }}}{V_{\text {porecell }}}=\frac{207.17}{1041.49}=0.199$

$S_{o}=\frac{V_{\text {oilcell }}}{V_{\text {porecell }}}=\frac{623.15}{1041.49}=0.598$

$S_{g}=1-S_{o}-S_{w}=0.203$ Where $S_{\mathrm{g}}$ represents the nitrogen saturation. 


\section{APPENDIX B}

\section{TEMPERATURE AND PRESSURE DATA}

The data in the following sets of data are:

T1: TEMPERATURE AT 17.7 CM ABOVE THE SAND MIX FACE.

T2: TEMPERATURE AT 3.1 CM ABOVE THE SAND MIX FACE.

T3: TEMPERATURE AT 12.0 CM INTO THE SAND MIX.

T4: TEMPERATURE AT 27.5 CM INTO THE SAND MIX.

T5: TEMPERATURE AT 42.8 CM INTO THE SAND MIX.

T6: TEMPERATURE AT 68 CM INTO THE SAND MIX.

$P_{\text {INJ: }}$ STEAM INJECTION PRESSURE.

POUT: PRODUCTION PRESSURE.

$\mathrm{V}_{\mathrm{W}}$ : WATER INJECTION RATE.

DATA FOR RUNS ONE TO SEVEN ARE PRESENTED IN TABLES B1 TO B7.

BELOW IS THE LIST OF RUNS AND A BRIEF DESCRIPTION OF EACH:

a) Run 1:Base run for San Ardo Oil using Pure Steam

b) Run 2:Pure steam with $\mathrm{NaOH}(0.1 \mathrm{wt} \%)$ for San Ardo oil

c) Run 3:Base run for Duri oil using Pure steam

d) Run 4:Pure steam with $\mathrm{NaOH}(0.1 \mathrm{wt} \%)$ for Duri oil

e) Run 5:Pure steam with $\mathrm{NaOH}(1 \mathrm{wt} \%)$ for Duri oil

f) Run 6: Cyclic Injection of $\mathrm{NaOH}(1 \mathrm{wt} \%)$ and steam for San Ardo oil.

g) Run 7: Pure steam Injection $\mathrm{NaOH}$ (1 wt \%) for San Ardo oil. 
Table B1-Temperature and Production Data for Run 1

\begin{tabular}{|c|c|c|c|c|c|c|c|c|c|}
\hline $\begin{array}{c}\text { Time } \\
\text { min }\end{array}$ & $\begin{array}{l}\mathrm{T} 1 \\
{ }^{\circ} \mathrm{C}\end{array}$ & $\begin{array}{l}\mathrm{T} 2 \\
{ }^{\circ} \mathrm{C}\end{array}$ & $\begin{array}{l}\mathrm{T} 3 \\
{ }^{\circ} \mathbf{C}\end{array}$ & $\begin{array}{l}\mathrm{T} 4 \\
{ }^{\circ} \mathrm{C}\end{array}$ & $\begin{array}{l}\mathrm{T} 5 \\
{ }^{\circ} \mathbf{C}\end{array}$ & $\begin{array}{l}\mathrm{T} 6 \\
{ }^{\circ} \mathrm{C}\end{array}$ & $\begin{array}{l}\text { Pinj } \\
\text { psig }\end{array}$ & $\begin{array}{l}\text { Pout } \\
\text { psig }\end{array}$ & $\begin{array}{c}\mathrm{Vw} \\
\mathrm{cc} / \mathrm{min}\end{array}$ \\
\hline 0.5455 & 45.71 & 50.72 & 1.32 & 63.36 & 2.02 & 55.8 & 204.33 & 199.73 & .59 \\
\hline 1.045333 & 47.95 & 1.66 & 34 & 3.37 & 04 & 86 & 3.55 & & 62 \\
\hline 1.544167 & 49.64 & 86.17 & 1.37 & 63.37 & 2.05 & 55.83 & 04.88 & 00.39 & .58 \\
\hline 2.044 & 50.67 & 187.11 & 61.39 & 63.38 & 62.07 & 55.81 & 05.04 & 00.48 & 5.54 \\
\hline 2.544833 & 51.23 & 87.68 & 1.37 & 63.38 & 2.07 & 55.83 & & & 54 \\
\hline 3.044667 & 51.32 & 188.35 & 1.38 & 63.38 & 2.06 & 55.86 & 04.67 & & .55 \\
\hline 3.5445 & 50.72 & 88.39 & & 63.41 & & 5.86 & & & 58 \\
\hline 4.045167 & 249.36 & 189.38 & 42 & 63.43 & 62.12 & 55.88 & .72 & & 5.59 \\
\hline 4.545 & 247.62 & 189.65 & 61.44 & 63.43 & 62.15 & 55.89 & & & 5.59 \\
\hline 5.044833 & 45.49 & 189.94 & 61.47 & 63.48 & 62.15 & 55.91 & 205.88 & 200.56 & 5.59 \\
\hline 5.5455 & 243.24 & 190.81 & 61.47 & 63.48 & 2.31 & 55.95 & & 47 & 5.60 \\
\hline 6.0 & 241.25 & & & 63.5 & & 5.98 & & & \\
\hline & 239.8 & & & 63.49 & & 55.96 & & & 60 \\
\hline 7.045 & 238.91 & & & 63.47 & 62.2 & 55.95 & & & 5.54 \\
\hline 7.544833 & 38.32 & 192.31 & 61.34 & 63.49 & 62.23 & 55.97 & & & 5.54 \\
\hline 8.045 & 37.99 & 192.26 & 61.36 & 63.48 & & 55.95 & & 53 & 53 \\
\hline 8.5 & 37.74 & & & 63.5 & & 5.96 & & & \\
\hline & 237.58 & & & 3.49 & & 55.98 & & & 59 \\
\hline 9.5 & 3 & & & 63.49 & & 56 & & & 57 \\
\hline & 237.16 & & & 63.49 & & 56.01 & & & 5.57 \\
\hline 10.54 & 237.12 & 42 & & 63.5 & & 55.99 & & & 5.57 \\
\hline & 236.95 & & & 63.52 & & $\perp$ & & & \\
\hline & 7 & & & 35 & & 6.02 & & & \\
\hline 1 & 235.56 & & & 63.51 & & 56.04 & & & 62 \\
\hline 12.54 & 234.67 & & & 63.51 & & 56.05 & & & 5.56 \\
\hline & 233.81 & & & 63.54 & & 56.09 & & & \\
\hline 13.5 & 232.88 & 196.1 & & 63.52 & & 56.09 & & & 5.53 \\
\hline & 232.01 & .73 & 62 & 63.52 & & 56.1 & & & 5 \\
\hline & 231.49 & & & 63.55 & & 6.1 & & & \\
\hline 15 & 230.85 & 33 & & 63.51 & 4 & 56.12 & & 37 & 59 \\
\hline 15.54567 & 230.25 & & & 63.54 & 62.42 & 56.13 & & 200.71 & 5.57 \\
\hline 16.0455 & 230 & & & 63.5 & 62.4 & 56.13 & & & 5.58 \\
\hline & 2 & & & 3.5 & & 56.11 & & & \\
\hline 17. & 230 & & & 634 & & 561 & & & 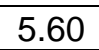 \\
\hline & 230.37 & & & 63.49 & & 6.1 & & & 5.62 \\
\hline 18.04567 & 230.5 & & 64.25 & 63.5 & 62.4 & 56.1 & & & 5.56 \\
\hline 18.54467 & 230.46 & 26 & & 63.48 & & 56.11 & & & 5.53 \\
\hline 19.04433 & 230.52 & 18 & & 63.48 & & 56.13 & & 200.15 & 5.54 \\
\hline 19.5 & 230.97 & 198.43 & 65.61 & 63.45 & 2 & 56.12 & & 200.2 & 5.59 \\
\hline & 232.25 & & & 63.47 & & 56.12 & & 200.25 & \\
\hline 20.54483 & 233.22 & & & 63.46 & & 56.12 & & 200.18 & 5.59 \\
\hline 21.0455 & 233.83 & 199.18 & 67.18 & 63.42 & 2.72 & 56.13 & 16 & 200.12 & 5.60 \\
\hline 21.54533 & 234.23 & 23 & 67 & 63.43 & 62.69 & 56.15 & 21 & 200.16 & 5.60 \\
\hline 22.04517 & 233.73 & 199.67 & 68.32 & 63.45 & 62.6 & 56.12 & 217.2 & 200.1 & 5.62 \\
\hline
\end{tabular}


Table B1. Continued.

\begin{tabular}{|c|c|c|c|c|c|c|c|c|c|}
\hline $\begin{array}{c}\text { Time } \\
\min \end{array}$ & $\begin{array}{l}\mathrm{T} 1 \\
{ }^{\circ} \mathrm{C} \\
\end{array}$ & $\begin{array}{l}\mathrm{T} 2 \\
{ }^{\circ} \mathrm{C} \\
\end{array}$ & $\begin{array}{l}\mathrm{T} 3 \\
{ }^{\circ} \mathrm{C} \\
\end{array}$ & $\begin{array}{l}\mathrm{T} 4 \\
{ }^{\circ} \mathrm{C} \\
\end{array}$ & $\begin{array}{l}\mathrm{T} 5 \\
{ }^{\circ} \mathrm{C} \\
\end{array}$ & $\begin{array}{l}\mathrm{T} 6 \\
{ }^{\circ} \mathrm{C} \\
\end{array}$ & $\begin{array}{l}\text { Pinj } \\
\text { psig }\end{array}$ & $\begin{array}{c}\text { Pout } \\
\text { psig }\end{array}$ & $\begin{array}{c}\mathrm{Vw} \\
\mathrm{cc} / \mathrm{min}\end{array}$ \\
\hline 22.04517 & 233.73 & 199.67 & 68.32 & 63.45 & 62.6 & 56.12 & 217.2 & 200.1 & 5.62 \\
\hline 22.54583 & 233.11 & 199.81 & 68.95 & 63.43 & 62.75 & 56.16 & 218.05 & 200.07 & 5.64 \\
\hline 23.04483 & 232.34 & 199.98 & 69.56 & 63.44 & 62.8 & 56.26 & 218.85 & 200.04 & 5.57 \\
\hline 23.5455 & 231.91 & 200.18 & 70.22 & 63.42 & 62.82 & 56.37 & 219.68 & 200.05 & 5.53 \\
\hline 24.04533 & 231.41 & 200.4 & 70.94 & 63.43 & 62.86 & 56.41 & 220.33 & 200.07 & 5.55 \\
\hline 24.54617 & 230.54 & 200.68 & 71.65 & 63.43 & 62.9 & 56.45 & 221.86 & 200.03 & 5.59 \\
\hline 25.045 & 231.31 & 201.04 & 72.41 & 63.39 & 62.92 & 56.49 & 222.66 & 200.05 & 5.61 \\
\hline 25.544 & 232.43 & 201.19 & 73.19 & 63.4 & 62.94 & 56.5 & 223.8 & 200.06 & 5.59 \\
\hline 26.0465 & 233.4 & 201.41 & 74 & 63.4 & 62.97 & 56.52 & 225.06 & 200.18 & 5.60 \\
\hline 26.54533 & 233.79 & 201.81 & 74.83 & 63.39 & 63.06 & 56.58 & 226.68 & 200.84 & 5.61 \\
\hline 27.04433 & 233.81 & 202.13 & 75.64 & 63.37 & 63.05 & 56.62 & 228.15 & 200.92 & 5.62 \\
\hline 27.545 & 233.71 & 202.37 & 76.53 & 63.37 & 63.09 & 56.72 & 229.53 & 200.72 & 5.61 \\
\hline 28.04483 & 233.28 & 202.84 & 77.35 & 63.38 & 63.15 & 56.8 & 231.13 & 200.76 & 5.55 \\
\hline 28.54467 & 232.28 & 203.21 & 78.2 & 63.34 & 63.17 & 56.86 & 232.37 & 200.67 & 5.53 \\
\hline 29.0455 & 232.74 & 203.34 & 79.03 & 63.34 & 63.21 & 56.94 & 233.58 & 200.65 & 5.53 \\
\hline 29.54533 & 233.44 & 203.54 & 79.82 & 63.33 & 63.25 & 57.04 & 234.63 & 200.65 & 5.55 \\
\hline 30.045 & 233.6 & 203.76 & 80.65 & 63.31 & 63.29 & 57.1 & 236.03 & 200.67 & 5.59 \\
\hline 30.54583 & 234.52 & 204.1 & 81.49 & 63.3 & 63.32 & 57.21 & 237.11 & 198.92 & 5.58 \\
\hline 31.04567 & 236.01 & 204.21 & 82.43 & 63.28 & 63.36 & 57.31 & 237.72 & 200.94 & 5.58 \\
\hline 31.5455 & 237.32 & 204.38 & 83.33 & 63.27 & 63.38 & 57.37 & 238.37 & 200.82 & 5.58 \\
\hline 32.04533 & 238.05 & 204.43 & 84.31 & 63.25 & 63.4 & 57.47 & 238.98 & 200.73 & 5.58 \\
\hline 32.54517 & 238.31 & 204.72 & 85.36 & 63.25 & 63.44 & 57.58 & 239.48 & 200.7 & 5.60 \\
\hline 33.045 & 238.33 & 204.8 & 86.5 & 63.25 & 63.48 & 57.68 & 239.94 & 200.6 & 5.61 \\
\hline 33.54567 & 238.29 & 204.84 & 87.73 & 63.24 & 63.46 & 57.72 & 240.28 & 200.58 & 5.54 \\
\hline 34.0455 & 238.42 & 204.71 & 89.11 & 63.24 & 63.49 & 57.82 & 240.72 & 200.55 & 5.51 \\
\hline 34.54533 & 238.57 & 204.91 & 90.7 & 63.26 & 63.41 & 57.92 & 241 & 200.99 & 5.54 \\
\hline 35.04417 & 238.5 & 205.08 & 92.45 & 63.26 & 63.51 & 58 & 241.79 & 200.81 & 5.54 \\
\hline 35.545 & 238.27 & 205.31 & 94.39 & 63.29 & 63.53 & 58.08 & 242.13 & 200.71 & 5.58 \\
\hline 36.04483 & 238.22 & 205.15 & 96.55 & 63.31 & 63.55 & 58.14 & 242.8 & 200.68 & 5.58 \\
\hline 36.5455 & 237.79 & 205.4 & 98.96 & 63.35 & 63.55 & 58.23 & 243.53 & 200.62 & 5.58 \\
\hline 37.04533 & 236.97 & 205.44 & 101.61 & 63.37 & 63.58 & 58.33 & 244.3 & 200.59 & 5.59 \\
\hline 37.54517 & 236.36 & 205.57 & 104.46 & 63.41 & 63.6 & 58.39 & 245.08 & 200.58 & 5.61 \\
\hline 38.045 & 236.4 & 205.72 & 107.53 & 63.45 & 63.62 & 58.45 & 246.17 & 200.6 & 5.64 \\
\hline 38.54483 & 236.2 & 206 & 110.82 & 63.47 & 63.73 & 58.53 & 247.09 & 200.84 & 5.60 \\
\hline 39.0455 & 235.87 & 206.19 & 114.28 & 63.53 & 63.78 & 58.61 & 248.4 & 200.78 & 5.54 \\
\hline 39.54533 & 235.6 & 206.37 & 117.87 & 63.59 & 63.65 & 58.65 & 249.8 & 200.74 & 5.52 \\
\hline 40.04517 & 235.4 & 206.76 & 121.57 & 63.61 & 63.67 & 58.7 & 251.02 & 200.65 & 5.54 \\
\hline 40.54417 & 235.32 & 207 & 125.4 & 63.67 & 63.71 & 58.76 & 252.33 & 200.62 & 5.57 \\
\hline 41.04383 & 235.05 & 207.29 & 129.37 & 63.73 & 63.69 & 58.78 & 253.84 & 200.56 & 5.60 \\
\hline 41.5455 & 234.71 & 207.57 & 133.48 & 63.79 & 63.71 & 58.88 & 255.54 & 200.84 & 5.57 \\
\hline 42.0445 & 234.32 & 207.9 & 137.69 & 63.86 & 63.62 & 58.96 & 256.86 & 200.72 & 5.60 \\
\hline 42.54517 & 234.23 & 207.94 & 141.92 & 63.94 & 63.75 & 59.04 & 258.07 & 200.64 & 5.61 \\
\hline 43.045 & 234.59 & 208.21 & 146.23 & 64.02 & 63.75 & 59.08 & 259.39 & 200.6 & 5.64 \\
\hline 43.54483 & 235.71 & 208.51 & 150.6 & 64.1 & 63.68 & 59.14 & 260.39 & 200.54 & 5.60 \\
\hline
\end{tabular}


Table B1. Continued.

\begin{tabular}{|c|c|c|c|c|c|c|c|c|c|}
\hline $\begin{array}{l}\text { Time } \\
\text { min }\end{array}$ & $\begin{array}{l}\mathrm{T} 1 \\
{ }^{\circ} \mathrm{C}\end{array}$ & $\begin{array}{l}\mathrm{T} 2 \\
{ }^{\circ} \mathrm{C}\end{array}$ & $\begin{array}{l}\mathrm{T} 3 \\
{ }^{\circ} \mathrm{C}\end{array}$ & $\begin{array}{l}\mathrm{T} 4 \\
{ }^{\circ} \mathrm{C}\end{array}$ & $\begin{array}{l}\mathrm{T} 5 \\
{ }^{\circ} \mathrm{C}\end{array}$ & $\begin{array}{l}\mathrm{T} 6 \\
{ }^{\circ} \mathrm{C}\end{array}$ & $\begin{array}{l}\text { Pinj } \\
\text { psig }\end{array}$ & $\begin{array}{l}\text { Pout } \\
\text { psig }\end{array}$ & $\begin{array}{c}\mathrm{Vw} \\
\mathrm{cc} / \mathrm{min}\end{array}$ \\
\hline 43.54483 & 235.71 & 208.51 & 150.6 & 64.1 & 63.68 & 59.14 & 260.39 & 200.54 & 5.60 \\
\hline 44.04467 & 237.37 & 208.62 & 154.94 & 64.19 & 63.8 & 59.22 & 260.91 & 200.55 & 5.55 \\
\hline 44.54533 & 238.74 & 208.84 & 159.35 & 64.29 & 63.82 & 59.29 & 261.25 & 200.74 & 5.56 \\
\hline 45.04517 & 239.71 & 208.86 & 163.77 & 64.41 & 63.84 & 59.35 & 261.56 & 200.61 & 5.59 \\
\hline 45.545 & 240.21 & 208.75 & 168.02 & 64.52 & 63.86 & 59.43 & 261.78 & 200.51 & 5.61 \\
\hline 46.04483 & 240.66 & 208.92 & 172 & 64.66 & 63.92 & 59.47 & 262.01 & 200.48 & 5.60 \\
\hline 46.54567 & 240.99 & 208.99 & 175.85 & 64.77 & 63.88 & 59.55 & 262.4 & 200.44 & 5.62 \\
\hline 47.0455 & 241.04 & 208.99 & 179.86 & 64.96 & 63.88 & 59.59 & 263.06 & 200.4 & 5.64 \\
\hline 47.54533 & 241.22 & 209.19 & 183.81 & 65.12 & 63.91 & 59.65 & 263.66 & 200.69 & 5.65 \\
\hline 48.045 & 241.04 & 209.43 & 187.93 & 65.31 & 63.93 & 59.69 & 264.62 & 200.57 & 5.58 \\
\hline 48.544 & 240.79 & 209.37 & 192.83 & 65.52 & 63.93 & 59.73 & 265.88 & 200.53 & 5.57 \\
\hline 49.04567 & 240.1 & 209.73 & 197.76 & 65.75 & 63.93 & 59.75 & 267.31 & 200.47 & 5.57 \\
\hline 49.5455 & 239.81 & 209.9 & 201.84 & 66.03 & 63.97 & 59.79 & 268.86 & 200.44 & 5.61 \\
\hline 50.04433 & 239.6 & 210.31 & 204.55 & 66.3 & 63.97 & 59.81 & 270.85 & 200.42 & 5.60 \\
\hline 50.54517 & 239.4 & 210.76 & 206.2 & 66.6 & 63.97 & 59.87 & 272.64 & 200.63 & 5.60 \\
\hline 51.045 & 238.96 & 211.1 & 207.49 & 66.96 & 63.98 & 59.89 & 274.53 & 200.59 & 5.60 \\
\hline 51.54467 & 238.91 & 211.35 & 208.4 & 67.34 & 64 & 59.95 & 276.06 & 200.49 & 5.62 \\
\hline 52.0455 & 239.05 & 211.51 & 209.03 & 67.76 & 63.98 & 59.95 & 277.28 & 200.43 & 5.65 \\
\hline 52.54533 & 238.82 & 211.68 & 209.48 & 68.21 & 64 & 60 & 278.25 & 200.42 & 5.55 \\
\hline 53.04517 & 239.07 & 211.61 & 209.77 & 68.7 & 64.1 & 60.05 & 278.77 & 200.4 & 5.53 \\
\hline 53.545 & 239.66 & 211.93 & 209.93 & 69.23 & 64 & 60.08 & 279.07 & 200.67 & 5.53 \\
\hline 54.04567 & 239.83 & 211.95 & 210.05 & 69.78 & 64.02 & 60.12 & 279.38 & 200.52 & 5.57 \\
\hline 54.5455 & 239.58 & 211.9 & 210.16 & 70.38 & 64.02 & 60.16 & 279.47 & 200.43 & 5.59 \\
\hline 55.04533 & 239.9 & 211.94 & 210.2 & 70.97 & 64.03 & 60.2 & 279.52 & 200.4 & 5.59 \\
\hline 55.54517 & 240.05 & 211.83 & 210.27 & 71.61 & 64.03 & 60.26 & 279.89 & 200.36 & 5.58 \\
\hline 56.045 & 239.76 & 211.97 & 210.36 & 72.27 & 64.03 & 60.28 & 280.34 & 200.31 & 5.61 \\
\hline 56.54483 & 239.24 & 212.14 & 210.44 & 72.97 & 64.05 & 60.32 & 280.87 & 200.57 & 5.58 \\
\hline 57.0455 & 237.88 & 212.19 & 210.62 & 73.67 & 64.05 & 60.34 & 281.98 & 200.49 & 5.64 \\
\hline 57.54533 & 236.52 & 212.48 & 210.85 & 74.44 & 64.07 & 60.36 & 283.56 & 200.45 & 5.57 \\
\hline 58.04517 & 235.47 & 212.66 & 211.14 & 75.21 & 64.05 & 60.4 & 285.29 & 200.41 & 5.54 \\
\hline 58.54417 & 234.53 & 212.93 & 211.44 & 76.02 & 64.07 & 60.44 & 286.98 & 200.37 & 5.53 \\
\hline 59.04483 & 234.36 & 213.29 & 211.8 & 76.92 & 64.07 & 60.46 & 289.04 & 200.4 & 5.56 \\
\hline 59.5455 & 234.2 & 213.48 & 212.07 & 77.82 & 64.13 & 60.46 & 290.43 & 200.64 & 5.61 \\
\hline 60.0445 & 233.64 & 213.77 & 212.3 & 78.8 & 64.1 & 60.48 & 291.56 & 200.48 & 5.58 \\
\hline 60.54517 & 234.77 & 213.97 & 212.5 & 79.81 & 64.19 & 60.52 & 292.51 & 200.44 & 5.58 \\
\hline 61.045 & 235.35 & 214.03 & 212.61 & 80.88 & 64.12 & 60.56 & 292.92 & 200.39 & 5.59 \\
\hline 61.54483 & 235.54 & 214.13 & 212.7 & 81.97 & 64.12 & 60.58 & 293.31 & 200.33 & 5.61 \\
\hline 62.04567 & 237.01 & 214.12 & 212.7 & 83.15 & 64.12 & 60.62 & 293.03 & 200.35 & 5.64 \\
\hline 62.54533 & 237.64 & 214.1 & 212.68 & 84.32 & 64.12 & 60.65 & 292.97 & 200.55 & 5.58 \\
\hline 63.04517 & 238.49 & 214.06 & 212.65 & 85.56 & 64.1 & 60.67 & 292.9 & 200.41 & 5.56 \\
\hline 63.545 & 238.52 & 213.99 & 212.67 & 86.85 & 64.14 & 60.68 & 292.87 & 200.35 & 5.56 \\
\hline 64.04483 & 238.45 & 214.1 & 212.69 & 88.17 & 64.14 & 60.71 & 292.94 & 200.29 & 5.60 \\
\hline 64.54567 & 238.97 & 214.07 & 212.74 & 89.57 & 64.23 & 60.73 & 293.57 & 200.26 & 5.60 \\
\hline 65.0455 & 238.65 & 214.37 & 212.89 & 91.06 & 64.16 & 60.75 & 294.61 & 200.25 & 5.59 \\
\hline
\end{tabular}


Table B1. Continued.

\begin{tabular}{|c|c|c|c|c|c|c|c|c|c|}
\hline $\begin{array}{c}\text { Time } \\
\text { min }\end{array}$ & $\begin{array}{l}\mathrm{T} 1 \\
{ }^{\circ} \mathrm{C}\end{array}$ & $\begin{array}{l}\mathrm{T} 2 \\
{ }^{\circ} \mathrm{C}\end{array}$ & $\begin{array}{l}\mathrm{T} 3 \\
{ }^{\circ} \mathrm{C}\end{array}$ & $\begin{array}{l}\mathrm{T} 4 \\
{ }^{\circ} \mathrm{C} \\
\end{array}$ & $\begin{array}{l}\mathrm{T5} \\
{ }^{\circ} \mathrm{C} \\
\end{array}$ & $\begin{array}{l}\mathrm{T} 6 \\
{ }^{\circ} \mathrm{C} \\
\end{array}$ & $\begin{array}{l}\text { Pinj } \\
\text { psig }\end{array}$ & $\begin{array}{l}\text { Pout } \\
\text { psig }\end{array}$ & $\begin{array}{c}\mathrm{Vw} \\
\mathrm{cc} / \mathrm{min} \\
\end{array}$ \\
\hline 65.54533 & 37.52 & 14.48 & 13.1 & 92.7 & 64.2 & 0.77 & 95.96 & 00.55 & 5.59 \\
\hline 66.04417 & 237.2 & 14.69 & 3.35 & 94.45 & 22 & 01 & 7.62 & & 60 \\
\hline 66.544 & 36.95 & 15.07 & 213.62 & 96.35 & 64.24 & 60.83 & 99.26 & & 5.63 \\
\hline 67.04466 & 36.59 & 15.16 & 213.89 & 98.46 & 64.28 & 60.85 & 300.59 & 00.46 & 5.60 \\
\hline 67.5445 & 37.13 & 15.47 & 214.07 & 100.73 & 64.34 & 60.89 & 01.42 & & 5.54 \\
\hline 68.0 & 236.83 & & & 103.22 & 64.37 & & & & 51 \\
\hline 68.54 & 237.27 & 215.52 & 4.2 & 106 & 64.41 & & & & 53 \\
\hline 69.04501 & 237.76 & 215.58 & 214.13 & 109.1 & 64.49 & & & & 5.58 \\
\hline 69.54 & 238.85 & 215.31 & 214 & 112.53 & & & & 200.04 & 5.60 \\
\hline 70.0 & 239.1 & 215 & & 16 & & & .76 & & \\
\hline 70.54 & 239.53 & 214.93 & & 120.66 & 64.64 & & & & .58 \\
\hline 71.0 & 39.43 & 4 & & 125.52 & & & & & 58 \\
\hline 71.5 & 39.32 & 7 & & 131.09 & & & & & 61 \\
\hline 72.04 & 239.59 & 214 & & 137.41 & 64.87 & & & & 5.62 \\
\hline 72.54467 & 238.69 & 214 & & 144.97 & & & & & 5.57 \\
\hline 73.04 & 238.14 & & & & & & & & 5.53 \\
\hline 73.5 & 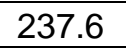 & & & & & & & & 2 \\
\hline 74.0 & 236.85 & & & 173 & & & & & 54 \\
\hline 74.54 & 236.42 & 214 & & 182 & & & & & 5.59 \\
\hline 75.0 & 37.23 & & & 189.88 & & & & & 5.58 \\
\hline 75.54 & 237.92 & 2. & & 195.83 & 65.87 & & & 57 & 5.58 \\
\hline 76.04 & 5 & & & 200.72 & & & & & .58 \\
\hline & & & & & & & & & 57 \\
\hline 77.04 & 239.25 & 2 & & 205 & & & & & 5.60 \\
\hline 77.5 & 240.04 & & & 207 & & & & & 5.62 \\
\hline 78.04 & 240.33 & 212 & & 208.01 & & & & & 5.57 \\
\hline 78.5 & 240.7 & 2 & & 208.3 & & & & & 5.52 \\
\hline 79.0 & 240.29 & & & 208 & & & & & 5.53 \\
\hline 79 . & 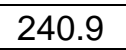 & & & 4 & & & & & 55 \\
\hline & 240.49 & & & & & & & & 5.59 \\
\hline 80.5 & 239.91 & & & 208.12 & & & & & 5.59 \\
\hline 81.0 & 239.73 & 210 & & 207.92 & 9 & & .77 & & 5.58 \\
\hline 81.5 & 38.86 & & & 207 & & & & & 5.58 \\
\hline & 238.46 & & & 207 & & & & & 5.58 \\
\hline 82.5 & & & & 207.29 & & & & & 5.61 \\
\hline 83.0455 & 236.88 & 209.3 & 207.69 & 207.1 & 79.83 & & & 200.24 & 5.62 \\
\hline 83.54533 & 236.42 & 209.14 & & 206.88 & & & & & 5.55 \\
\hline & 236 & & & 206 & & & & & 5.52 \\
\hline 84.54 & 236.1 & 208.5 & & 206. & & & & 200 & 5.52 \\
\hline & 236.1 & & & & & & & & 5.54 \\
\hline 85.54733 & 236.22 & & & & & & .77 & 200.23 & 5.57 \\
\hline 86.04533 & 236.63 & 207.45 & & 205.36 & 9 & & & 200.22 & 5.59 \\
\hline 86.54 & 236.76 & & 20 & 204.89 & & & & 200.38 & 5.58 \\
\hline & 36.92 & 206.56 & 8 & & 99.23 & 61 & 247.44 & 200.29 & 5.57 \\
\hline
\end{tabular}


Table B1. Continued.

\begin{tabular}{|c|c|c|c|c|c|c|c|c|c|}
\hline $\begin{array}{c}\text { Time } \\
\text { min }\end{array}$ & $\begin{array}{l}\mathrm{T} 1 \\
{ }^{\circ} \mathrm{C} \\
\end{array}$ & $\begin{array}{l}\mathrm{T} 2 \\
{ }^{\circ} \mathrm{C} \\
\end{array}$ & $\begin{array}{l}\mathrm{T} 3 \\
{ }^{\circ} \mathrm{C} \\
\end{array}$ & $\begin{array}{l}\mathrm{T} 4 \\
{ }^{\circ} \mathrm{C} \\
\end{array}$ & $\begin{array}{l}\mathrm{T} 5 \\
{ }^{\circ} \mathrm{C} \\
\end{array}$ & $\begin{array}{l}\mathrm{T} 6 \\
{ }^{\circ} \mathrm{C} \\
\end{array}$ & $\begin{array}{l}\text { Pinj } \\
\text { psig }\end{array}$ & $\begin{array}{l}\text { Pout } \\
\text { psig }\end{array}$ & $\begin{array}{l}\mathrm{Vw} \\
\mathrm{cc} / \mathrm{min}\end{array}$ \\
\hline 87.54567 & 237.17 & 206.2 & 204.68 & 204.25 & 101.99 & 62.09 & 245.87 & 200.25 & 5.59 \\
\hline 88.0455 & 237.33 & 206.07 & 204.39 & 203.96 & 104.74 & 62.28 & 244.37 & 200.21 & 5.60 \\
\hline 88.54533 & 237.47 & 205.77 & 204.1 & 203.69 & 107.48 & 62.48 & 242.93 & 200.18 & 5.62 \\
\hline 89.04517 & 237.24 & 205.43 & 203.65 & 203.23 & 110.28 & 62.69 & 240.36 & 200.31 & 5.58 \\
\hline 89.544 & 237.26 & 205.07 & 203.39 & 202.97 & 113.09 & 62.94 & 239.32 & 200.24 & 5.54 \\
\hline 90.04567 & 237.36 & 204.67 & 203.22 & 202.79 & 115.96 & 63.24 & 238.42 & 200.2 & 5.53 \\
\hline 90.5455 & 237.22 & 204.57 & 203.04 & 202.65 & 118.77 & 63.56 & 237.62 & 200.16 & 5.56 \\
\hline 91.04433 & 237.11 & 204.51 & 202.9 & 202.49 & 121.58 & 63.9 & 236.89 & 200.14 & 5.58 \\
\hline 91.54517 & 236.86 & 204.3 & 202.79 & 202.36 & 124.36 & 64.26 & 236.32 & 200.12 & 5.59 \\
\hline 92.04501 & 236.66 & 204.35 & 202.45 & 202.02 & 127.52 & 64.65 & 234.45 & 200.31 & 5.58 \\
\hline 92.54483 & 236.48 & 203.88 & 202.33 & 201.91 & 130.92 & 65.03 & 234.07 & 200.22 & 5.58 \\
\hline 93.0455 & 236.37 & 203.9 & 202.27 & 201.86 & 134.49 & 65.43 & 233.85 & 200.2 & 5.59 \\
\hline 93.54533 & 236.16 & 203.87 & 202.22 & 201.82 & 137.9 & 65.88 & 233.63 & 200.2 & 5.62 \\
\hline 94.04517 & 236 & 203.83 & 202.2 & 201.77 & 141.24 & 66.33 & 233.39 & 200.17 & 5.63 \\
\hline 94.54501 & 235.92 & 203.7 & 202.15 & 201.72 & 144.8 & 66.79 & 233.11 & 200.15 & 5.56 \\
\hline 95.04483 & 235.85 & 203.58 & 202.07 & 201.66 & 148.53 & 67.24 & 232.89 & 200.14 & 5.54 \\
\hline 95.5455 & 235.55 & 203.54 & 201.81 & 201.38 & 154.56 & 67.71 & 231.06 & .33 & 5.56 \\
\hline 96.04533 & 235.58 & 203.31 & 201.66 & 201.25 & 160.88 & 68.24 & 230.71 & 200.22 & 5.58 \\
\hline 96.54517 & 235.74 & 203.31 & 201.59 & 201.18 & 166.14 & 68.74 & 230.42 & 200.21 & 5.61 \\
\hline 97.04417 & 235.9 & 203.25 & 201.53 & 201.12 & 171 & 69.29 & 230 & 200.2 & 5.59 \\
\hline 97.544 & 235.96 & 203.18 & 201.44 & 201.03 & 176.74 & 69.84 & 229.54 & 200.18 & 5.59 \\
\hline 98.04467 & 236.15 & 203.13 & 201.34 & 200.92 & 181.62 & 70.44 & 229.04 & 200.15 & 5.59 \\
\hline 98.54533 & 236.28 & 202.8 & 201.24 & 200.83 & 185.93 & 71.04 & 228.46 & 200.13 & 5.63 \\
\hline 99.04517 & 236.19 & 202.8 & 200.98 & 200.51 & 193.58 & 71.68 & 226.57 & 200.39 & 5.61 \\
\hline 99.54501 & 236.01 & 202.48 & 200.67 & 200.28 & 195.75 & 72.36 & 225.64 & 200.24 & 5.55 \\
\hline 100.0448 & 236.38 & 202.48 & 200.53 & 200.13 & 196.75 & 73.11 & 225.08 & 200.21 & 5.54 \\
\hline 100.5457 & 236.67 & 202.32 & 200.44 & 200.03 & 197.55 & 73.93 & 224.53 & 200.18 & 5.55 \\
\hline 101.0455 & 236.74 & 202.42 & 200.33 & 199.92 & 198.22 & 74.78 & 223.95 & 200.17 & 5.58 \\
\hline 101.5453 & 236.85 & 202.19 & 200.2 & 199.79 & 198.75 & 75.72 & 223.35 & 200.14 & 5.59 \\
\hline 102.045 & 236.4 & 201.92 & 199.84 & 199.4 & 199.16 & 76.75 & 221.23 & 200.3 & 5.58 \\
\hline 102.5458 & 236.45 & 201.72 & 199.63 & 199.22 & 199.38 & 77.86 & 220.54 & 200.23 & 5.58 \\
\hline 103.0457 & 236.61 & 201.65 & 199.54 & 199.11 & 199.49 & 79.06 & 220.08 & 200.17 & 5.57 \\
\hline 103.5455 & 236.81 & 201.45 & 199.43 & 199.02 & 199.39 & 80.37 & 219.57 & 200.15 & 5.61 \\
\hline 104.0462 & 236.73 & 201.45 & 199.3 & 198.89 & 199.36 & 81.75 & 219.05 & 200.11 & 5.63 \\
\hline 104.5452 & 236.77 & 201.42 & 199.21 & 198.8 & 199.3 & 83.21 & 218.56 & 200.1 & 5.57 \\
\hline 105.045 & 236.77 & 201.09 & 199.11 & 198.71 & 199.25 & 84.72 & 218.07 & 200.1 & 5.54 \\
\hline 105.5465 & 236.16 & 201.07 & 198.8 & 198.33 & 198.82 & 86.24 & 216.18 & 200.31 & 5.55 \\
\hline 106.0455 & 236.21 & 200.64 & 198.6 & 198.19 & 198.82 & 87.84 & 215.69 & 200.2 & 5.59 \\
\hline 106.5462 & 236.5 & 200.72 & 198.55 & 198.16 & 198.78 & 89.44 & 215.42 & 200.14 & 5.62 \\
\hline 107.0442 & 236.57 & 200.55 & 198.51 & 198.1 & 198.82 & 91.12 & 215.28 & 200.14 & 5.59 \\
\hline 107.545 & 236.64 & 200.41 & 198.46 & 198.05 & 198.71 & 92.78 & 215 & 200.12 & 5.59 \\
\hline 108.0467 & 236.66 & 200.48 & 198.4 & 198.01 & 198.67 & 94.45 & 214.76 & 200.06 & 5.60 \\
\hline 108.5447 & 236.69 & 200.84 & 198.37 & 197.95 & 198.64 & 96.08 & 214.59 & 200.08 & 5.62 \\
\hline 109.0443 & 236.19 & 200.19 & 198.11 & 197.67 & 198.2 & 97.68 & 213.01 & 200.27 & 5.60 \\
\hline
\end{tabular}


Table B1. Continued.

\begin{tabular}{|c|c|c|c|c|c|c|c|c|c|}
\hline $\begin{array}{c}\text { Time } \\
\text { min }\end{array}$ & $\begin{array}{l}\mathrm{T} 1 \\
{ }^{\circ} \mathrm{C}\end{array}$ & $\begin{array}{l}\mathrm{T} 2 \\
{ }^{\circ} \mathrm{C}\end{array}$ & $\begin{array}{l}\mathrm{T} 3 \\
{ }^{\circ} \mathrm{C}\end{array}$ & $\begin{array}{l}\mathrm{T} 4 \\
{ }^{\circ} \mathrm{C}\end{array}$ & $\begin{array}{l}\mathrm{T} 5 \\
{ }^{\circ} \mathrm{C}\end{array}$ & $\begin{array}{l}\mathrm{T} 6 \\
{ }^{\circ} \mathrm{C}\end{array}$ & $\begin{array}{l}\text { Pinj } \\
\text { psig }\end{array}$ & $\begin{array}{c}\text { Pout } \\
\text { psig }\end{array}$ & $\begin{array}{c}\mathrm{Vw} \\
\mathrm{cc} / \mathrm{min}\end{array}$ \\
\hline 109.5452 & 236.23 & 200.28 & 197.97 & 197.58 & 198.24 & 99.31 & 212.8 & 200.21 & 5.54 \\
\hline 110.045 & 236.44 & 200.62 & 197.95 & 197.56 & 198.26 & 101.01 & 212.77 & 200.14 & 5.53 \\
\hline 110.5448 & 236.53 & 200.21 & 197.97 & 197.57 & 198.26 & 102.71 & 212.76 & 200.15 & 5.54 \\
\hline 111.0455 & 236.64 & 200.42 & 197.95 & 197.56 & 198.24 & 104.43 & 212.71 & 200.12 & 5.57 \\
\hline 111.5453 & 236.69 & 200.28 & 197.93 & 197.56 & 198.24 & 106.17 & 212.61 & 200.09 & 5.58 \\
\hline 112.0452 & 236.6 & 200.44 & 197.93 & 197.54 & 198.2 & 107.88 & 212.53 & 200.1 & 5.58 \\
\hline 112.545 & 236.58 & 200.4 & 197.89 & 197.52 & 198.18 & 109.62 & 212.4 & 200.05 & 5.59 \\
\hline 113.0438 & 236.15 & 200.31 & 197.66 & 197.23 & 197.79 & 111.31 & 210.97 & 200.29 & 5.59 \\
\hline 113.5455 & 236.22 & 199.86 & 197.57 & 197.16 & 197.86 & 113.06 & 210.9 & 200.2 & 5.61 \\
\hline 114.0453 & 236.47 & 200.11 & 197.59 & 197.19 & 197.89 & 114.86 & 210.97 & 200.2 & 5.63 \\
\hline 114.5443 & 236.7 & 199.97 & 197.62 & 197.19 & 197.91 & 116.74 & 211.09 & 0.14 & 5.58 \\
\hline 115.0442 & 236.61 & 200.15 & 197.62 & 197.21 & 197.93 & 118.57 & 211.13 & .14 & 5.54 \\
\hline 115.5448 & 236.63 & 199.75 & 197.62 & 197.21 & 197.93 & 120.41 & 211.1 & 200.14 & 5.54 \\
\hline 116.0447 & 236.72 & 199.98 & 197.62 & 197.23 & 197.91 & 122.28 & 211.08 & 200.1 & 5.57 \\
\hline 116.5445 & 236.79 & 200.22 & 197.62 & 197.23 & 197.91 & 124.12 & 211.06 & 200.11 & 5.59 \\
\hline 117.0452 & 236.25 & 200.05 & 197.35 & 196.92 & 197.51 & 126.03 & 209.46 & 200.28 & 5.60 \\
\hline 117.545 & 236.43 & 199.68 & 197.28 & 196.88 & 197.6 & 128.15 & .59 & .26 & 5.59 \\
\hline 118.0448 & 236.63 & 199.82 & 197.35 & 196.94 & 197.66 & 130.54 & 209.85 & 200.24 & 5.60 \\
\hline 118.5457 & 236.72 & 199.77 & 197.38 & 196.99 & 197.73 & 132.95 & 210 & 200.21 & 5.60 \\
\hline 119.0455 & 236.66 & 200 & 197.42 & 197.03 & 197.74 & 135.24 & 210.19 & 200.2 & 5.64 \\
\hline 119.5453 & 236.77 & 199.87 & 197.44 & 197.05 & 197.74 & 137.48 & 210.2 & 200.18 & 5.57 \\
\hline 120.045 & 236.86 & 199.91 & 197.44 & 197.04 & 197.74 & 139.68 & 210.18 & .16 & 5.53 \\
\hline 120.5448 & 236.91 & 200.09 & 197.42 & 197.02 & 197.71 & 141.9 & 210.13 & 0.15 & 5.54 \\
\hline 121.0457 & 236.21 & 200.21 & 197.19 & 196.74 & 197.36 & 144.78 & 208.79 & 200.25 & 5.57 \\
\hline 121.5455 & 236.26 & 199.51 & 197.13 & 196.74 & 197.45 & 148.23 & 208.95 & 200.26 & 5.59 \\
\hline 122.0453 & 236.53 & 199.91 & 197.22 & 196.81 & 197.56 & 151.36 & 209.28 & 200.26 & 5.59 \\
\hline 122.546 & 236.68 & 199.53 & 197.26 & 196.88 & 197.6 & 154.2 & 209.52 & 200.24 & 5.59 \\
\hline 123.044 & 236.6 & 199.55 & 197.29 & 196.9 & 197.61 & 157.07 & 209.63 & 200.22 & 5.58 \\
\hline 123.5457 & 236.67 & 199.49 & 197.33 & 196.91 & 197.63 & 159.96 & 209.67 & 200.18 & 5.60 \\
\hline 124.0445 & 236.57 & 199.76 & 197.31 & 196.9 & 197.5 & 162.75 & 209.67 & 200.14 & 5.63 \\
\hline 124.5443 & 236.58 & 199.76 & 197.31 & 196.9 & 197.59 & 165.69 & 209.58 & 200.14 & 5.60 \\
\hline 125.0452 & 236.33 & 200.1 & 197.2 & 196.73 & 197.25 & 173.7 & 208.67 & 200.31 & 5.55 \\
\hline 125.545 & 236.08 & 199.1 & 197.04 & 196.64 & 197.32 & 179.27 & 208.37 & 200.18 & 5.54 \\
\hline 126.0457 & 236.28 & 199.69 & 197.07 & 196.68 & 197.43 & 181.44 & 208.71 & 200.26 & 5.56 \\
\hline 126.5455 & 236.47 & 199.31 & 197.14 & 196.75 & 197.48 & 183.63 & 208.98 & 200.24 & 5.62 \\
\hline 127.0453 & 236.51 & 199.76 & 197.18 & 196.78 & 197.59 & 185.85 & 209.17 & 200.22 & 5.61 \\
\hline 127.5452 & 236.38 & 199.31 & 197.2 & 196.82 & 197.5 & 187.63 & 209.17 & 200.15 & 5.61 \\
\hline 128.045 & 236.4 & 199.61 & 197.19 & 196.8 & 197.48 & 189.37 & 209.06 & 200.12 & 5.63 \\
\hline 128.5448 & 236.51 & 199.43 & 197.18 & 196.78 & 197.48 & 191.75 & 209 & 200.06 & 5.64 \\
\hline 129.0455 & 236.49 & 199.06 & 197.18 & 196.78 & 197.48 & 193.13 & 208.96 & 200.06 & 5.61 \\
\hline 129.5453 & 236.04 & 199.77 & 197.01 & 196.62 & 197.28 & 195.26 & 208.22 & 200.21 & 5.56 \\
\hline 130.0452 & 236.15 & 199.31 & 197.01 & 196.6 & 197.32 & 196.37 & 208.22 & 200.18 & 5.57 \\
\hline 130.544 & 236.43 & 198.81 & 197.09 & 196.66 & 197.43 & 196.66 & 208.57 & 200.24 & 5.59 \\
\hline 131.0438 & 236.54 & 199.77 & 197.12 & 196.73 & 197.44 & 196.8 & 208.75 & 200.18 & 5.61 \\
\hline
\end{tabular}


Table B1. Continued.

\begin{tabular}{|c|c|c|c|c|c|c|c|c|c|}
\hline $\begin{array}{c}\text { Time } \\
\text { min }\end{array}$ & $\begin{array}{l}\mathrm{T} 1 \\
{ }^{\circ} \mathrm{C} \\
\end{array}$ & $\begin{array}{l}\mathrm{T} 2 \\
{ }^{\circ} \mathrm{C} \\
\end{array}$ & $\begin{array}{l}\mathrm{T} 3 \\
{ }^{\circ} \mathrm{C} \\
\end{array}$ & $\begin{array}{l}\mathrm{T} 4 \\
{ }^{\circ} \mathrm{C} \\
\end{array}$ & $\begin{array}{l}\mathrm{T} 5 \\
{ }^{\circ} \mathrm{C} \\
\end{array}$ & $\begin{array}{l}\mathrm{T} 6 \\
{ }^{\circ} \mathrm{C} \\
\end{array}$ & $\begin{array}{l}\text { Pinj } \\
\text { psig }\end{array}$ & $\begin{array}{l}\text { Pout } \\
\text { psig }\end{array}$ & $\begin{array}{l}\mathrm{Vw} \\
\mathrm{cc} / \mathrm{min}\end{array}$ \\
\hline 131.5447 & 236.58 & 199.59 & 197.12 & 196.73 & 197.43 & 196.91 & 208.75 & 200.2 & 5.59 \\
\hline 132.0445 & 236.59 & 199.38 & 197.1 & 196.71 & 197.41 & 196.99 & 208.68 & 200.12 & 5.61 \\
\hline 132.5452 & 236.54 & 199.09 & 197.1 & 196.71 & 197.32 & 197.05 & 208.61 & 200.13 & 5.62 \\
\hline 133.045 & 236.36 & 199.18 & 196.98 & 196.58 & 197.21 & 195.58 & 207.86 & 200.23 & 5.65 \\
\hline 133.5448 & 236.36 & 199.46 & 196.96 & 196.58 & 197.3 & 196.72 & 208 & 200.16 & 5.59 \\
\hline 134.0465 & 236.54 & 200.29 & 196.99 & 196.6 & 197.3 & 197.03 & 208.12 & 200.1 & 5.57 \\
\hline 134.5453 & 236.64 & 199.7 & 197.06 & 196.67 & 197.42 & 197.15 & 208.48 & 200.22 & 5.59 \\
\hline 135.0452 & 236.72 & 199.89 & 197.1 & 196.71 & 197.4 & 197.14 & 208.51 & 200.1 & 5.63 \\
\hline 135.545 & 236.52 & 200 & 197.08 & 196.67 & 197.38 & 197.12 & 208.47 & 200.06 & 5.62 \\
\hline 136.0448 & 236.41 & 200.5 & 197.06 & 196.67 & 197.37 & 197.13 & 208.46 & 200.05 & 5.61 \\
\hline 136.5457 & 236.39 & 200.34 & 197.06 & 196.67 & 197.37 & 197.12 & 208.38 & 200.03 & 5.63 \\
\hline 137.0455 & 236.47 & 199.98 & 197.06 & 196.65 & 197.37 & 197.12 & 208.35 & 200.04 & 5.65 \\
\hline 137.5453 & 236.48 & 199.91 & 197.06 & 196.67 & 197.38 & 197.13 & 208.44 & 199.99 & 5.58 \\
\hline 138.045 & 236.21 & 199.91 & 196.95 & 196.56 & 197.29 & 196.22 & 207.93 & 200.16 & 5.55 \\
\hline 138.544 & 236.39 & 199.62 & 196.97 & 196.6 & 197.33 & 197.03 & 208.08 & 200.11 & 5.55 \\
\hline 139.0457 & 236.5 & 199.28 & 197.03 & 196.63 & 197.4 & 197.15 & 208.35 & 200.18 & 5.58 \\
\hline 139.5455 & 236.57 & 200.12 & 197.08 & 196.68 & 197.4 & 197.17 & 208.44 & 200.19 & 5.60 \\
\hline 140.0453 & 236.53 & 200.09 & 197.06 & 196.67 & 197.38 & 197.13 & 208.4 & 200 & 5.59 \\
\hline 140.5442 & 236.25 & 199.68 & 196.95 & 196.58 & 197.29 & 196.74 & 207.96 & 200.1 & 5.59 \\
\hline 141.045 & 236.44 & 199.62 & 197.01 & 196.61 & 197.35 & 197.11 & 208.12 & 200.09 & 5.59 \\
\hline 141.5447 & 236.55 & 199.85 & 197.06 & 196.68 & 197.42 & 197.19 & 208.42 & 200.17 & 5.61 \\
\hline 142.0445 & 236.55 & 200.52 & 197.08 & 196.67 & 197.38 & 197.13 & 208.38 & 200.08 & 5.61 \\
\hline 142.5453 & 236.5 & 200.36 & 197.04 & 196.67 & 197.38 & 197.13 & 208.28 & 200.02 & 5.55 \\
\hline 143.0452 & 236.52 & 199.98 & 197.02 & 196.65 & 197.38 & 197.13 & 208.27 & 200.01 & 5.54 \\
\hline 143.545 & 236.59 & 199.6 & 197.02 & 196.63 & 197.36 & 197.11 & 208.27 & 199.98 & 5.55 \\
\hline 144.0457 & 236.64 & 199.92 & 197.02 & 196.65 & 197.36 & 197.11 & 208.23 & 199.97 & 5.58 \\
\hline 144.5455 & 236.66 & 200.07 & 197.02 & 196.63 & 197.36 & 197.11 & 208.22 & 199.97 & 5.61 \\
\hline 145.0453 & 236.71 & 200.23 & 197.01 & 196.63 & 197.35 & 197.11 & 208.17 & 199.94 & 5.58 \\
\hline 145.5452 & 236.66 & 200.1 & 197.01 & 196.63 & 197.36 & 197.13 & 208.2 & 199.85 & 5.60 \\
\hline 146.045 & 236.71 & 200.14 & 197.02 & 196.65 & 197.38 & 197.13 & 208.19 & 199.91 & 5.61 \\
\hline 146.5438 & 236.5 & 199.98 & 196.93 & 196.56 & 197.33 & 196.49 & 207.86 & 200.14 & 5.64 \\
\hline 147.0455 & 236.69 & 199.82 & 196.99 & 196.61 & 197.38 & 197.15 & 208.08 & 200.07 & 5.61 \\
\hline 147.5445 & 236.89 & 200.3 & 197.04 & 196.67 & 197.43 & 197.2 & 208.32 & 200.23 & 5.56 \\
\hline 148.0443 & 236.91 & 199.64 & 197.08 & 196.68 & 197.45 & 197.2 & 208.35 & 200.08 & 5.56 \\
\hline 148.545 & 236.87 & 199.83 & 197.06 & 196.66 & 197.4 & 197.17 & 208.26 & 200.12 & 5.59 \\
\hline 149.0448 & 236.85 & 200.14 & 197.02 & 196.65 & 197.27 & 197.13 & 208.18 & 199.97 & 5.62 \\
\hline 149.5455 & 236.8 & 200.46 & 197.02 & 196.63 & 197.35 & 197.13 & 208.22 & 199.96 & 5.60 \\
\hline 150.0453 & 236.76 & 199.31 & 197.02 & 196.65 & 197.36 & 197.13 & 208.18 & 200 & 5.60 \\
\hline 150.5452 & 236.69 & 199.92 & 197.02 & 196.65 & 197.38 & 197.13 & 208.2 & 199.95 & 5.61 \\
\hline 151.045 & 236.6 & 199.85 & 197.02 & 196.63 & 197.36 & 197.13 & 208.18 & 199.9 & 5.64 \\
\hline 151.5448 & 236.51 & 200.6 & 197.04 & 196.63 & 197.36 & 197.13 & 208.19 & 199.87 & 5.59 \\
\hline 152.0455 & 236.28 & 199.87 & 196.91 & 196.5 & 197.09 & 196.25 & 207.58 & 197.73 & 5.55 \\
\hline 152.5463 & 236.12 & 200.03 & 196.65 & 196.25 & 196.95 & 196.66 & 206.4 & 198.68 & 5.55 \\
\hline 153.0452 & 236.41 & 199.46 & 196.79 & 196.43 & 197.31 & 197.02 & 207.63 & 199.03 & 5.58 \\
\hline
\end{tabular}


Table B1. Continued.

\begin{tabular}{|c|c|c|c|c|c|c|c|c|c|}
\hline $\begin{array}{c}\text { Time } \\
\text { min }\end{array}$ & $\begin{array}{l}\mathrm{T} 1 \\
{ }^{\circ} \mathrm{C} \\
\end{array}$ & $\begin{array}{l}\mathrm{T} 2 \\
{ }^{\circ} \mathrm{C} \\
\end{array}$ & $\begin{array}{l}\mathrm{T} 3 \\
{ }^{\circ} \mathrm{C} \\
\end{array}$ & $\begin{array}{l}\mathrm{T} 4 \\
{ }^{\circ} \mathrm{C} \\
\end{array}$ & $\begin{array}{l}\mathrm{T} 5 \\
{ }^{\circ} \mathrm{C} \\
\end{array}$ & $\begin{array}{l}\mathrm{T} 6 \\
{ }^{\circ} \mathrm{C} \\
\end{array}$ & $\begin{array}{l}\text { Pinj } \\
\text { psig }\end{array}$ & $\begin{array}{l}\text { Pout } \\
\text { psig }\end{array}$ & $\begin{array}{c}\mathrm{Vw} \\
\mathrm{cc} / \mathrm{min}\end{array}$ \\
\hline 153.546 & 236.62 & 200.3 & 196.92 & 196.54 & 197.33 & 197.08 & 207.78 & 201.15 & 5.61 \\
\hline 154.0448 & 236.89 & 199.73 & 197.09 & 196.72 & 197.65 & 197.33 & 208.74 & 200.91 & 5.58 \\
\hline 154.5457 & 237.16 & 200.05 & 197.2 & 196.84 & 197.61 & 197.4 & 209.14 & 200.93 & 5.59 \\
\hline 155.0455 & 237.18 & 200.41 & 197.24 & 196.86 & 197.56 & 197.31 & 209.05 & 200.38 & 5.60 \\
\hline 155.5453 & 237.05 & 199.89 & 197.18 & 196.77 & 197.52 & 197.27 & 208.86 & 200.57 & 5.65 \\
\hline 156.045 & 237.02 & 200.14 & 197.17 & 196.79 & 197.52 & 197.31 & 208.83 & 200.47 & 5.61 \\
\hline 156.544 & 237.02 & 200.01 & 197.16 & 196.79 & 197.52 & 197.29 & 208.85 & 200.46 & 5.55 \\
\hline 157.0447 & 236.91 & 200.39 & 197.17 & 196.79 & 197.56 & 197.31 & 208.9 & 200.4 & 5.55 \\
\hline 157.5445 & 236.85 & 200.21 & 197.19 & 196.81 & 197.56 & 197.31 & 208.95 & 200.39 & 5.58 \\
\hline 158.0453 & 236.77 & 199.91 & 197.19 & 196.81 & 197.54 & 197.31 & 208.99 & 200.39 & 5.62 \\
\hline 158.5452 & 236.68 & 200 & 197.18 & 196.79 & 197.54 & 197.29 & 208.89 & 200.73 & 5.58 \\
\hline 159.045 & 236.75 & 199.73 & 197.15 & 196.76 & 197.47 & 197.2 & 208.73 & 200.87 & 5.59 \\
\hline 159.5457 & 236.73 & 199.96 & 197.18 & 196.81 & 197.6 & 197.36 & 209.06 & 200.88 & 5.61 \\
\hline 160.0455 & 236.78 & 200.64 & 197.24 & 196.88 & 197.63 & 197.4 & 209.34 & 200.78 & 5.64 \\
\hline 160.5453 & 236.79 & 200.48 & 197.26 & 196.88 & 197.6 & 197.38 & 209.31 & 200.73 & 5.61 \\
\hline 161.0452 & 236.41 & 201.23 & 197.26 & 196.86 & 197.58 & 197.37 & 209.25 & 200.6 & 5.57 \\
\hline 161.545 & 236.44 & 200.39 & 197.22 & 196.85 & 197.6 & 197.35 & 209.23 & .54 & 5.56 \\
\hline 162.0457 & 236.41 & 200.25 & 197.22 & 196.85 & 197.58 & 197.35 & 209.2 & 200.55 & 5.57 \\
\hline 162.5455 & 236.55 & 200.23 & 197.22 & 196.85 & 197.58 & 197.37 & 209.23 & 200.49 & 5.63 \\
\hline 163.0453 & 236.64 & 199.76 & 197.22 & 196.85 & 197.58 & 197.35 & 209.22 & 200.52 & 5.61 \\
\hline 163.5452 & 236.68 & 200.07 & 197.22 & 196.85 & 197.58 & 197.35 & 209.21 & 200.54 & 5.62 \\
\hline 164.0458 & 236.61 & 200.53 & 197.17 & 196.79 & 197.51 & 197.29 & 208.88 & 200.82 & 5.62 \\
\hline 164.5438 & 236.75 & 200.97 & 197.2 & 196.86 & 197.62 & 197.4 & 209.24 & 200.88 & 5.66 \\
\hline 165.0455 & 236.89 & 200.32 & 197.28 & 196.88 & 197.65 & 197.42 & 209.38 & 200.79 & 5.58 \\
\hline 165.5453 & 237 & 199.82 & 197.28 & 196.9 & 197.62 & 197.4 & 209.33 & 200.77 & 5.55 \\
\hline 166.0452 & 236.98 & 200.61 & 197.26 & 196.88 & 197.62 & 197.38 & 209.31 & 200.65 & 5.56 \\
\hline 166.544 & 236.98 & 200.41 & 197.24 & 196.88 & 197.58 & 197.38 & 209.24 & 200.62 & 5.61 \\
\hline 167.0448 & 236.95 & 200.09 & 197.24 & 196.87 & 197.6 & 197.37 & 209.24 & 200.61 & 5.60 \\
\hline 167.5447 & 236.98 & 200.52 & 197.24 & 196.85 & 197.6 & 197.37 & 209.22 & 200.59 & 5.61 \\
\hline 168.0445 & 237.02 & 200.21 & 197.22 & 196.83 & 197.6 & 197.38 & 209.16 & 200.55 & 5.59 \\
\hline 168.5452 & 237.2 & 200.59 & 197.22 & 196.85 & 197.6 & 197.37 & 209.16 & 200.55 & 5.61 \\
\hline 169.045 & 236.93 & 200.25 & 197.22 & 196.85 & 197.6 & 197.37 & 209.15 & 200.55 & 5.63 \\
\hline 169.5448 & 236.8 & 199.96 & 197.17 & 196.79 & 197.6 & 197.28 & 209.01 & 201.24 & 5.58 \\
\hline 170.0455 & 237.04 & 199.78 & 197.24 & 196.88 & 197.67 & 197.46 & 209.33 & 201.15 & 5.55 \\
\hline 170.5453 & 237.16 & 199.84 & 197.3 & 196.92 & 197.69 & 197.47 & 209.52 & 201.12 & 5.56 \\
\hline 171.0452 & 237.07 & 199.98 & 197.3 & 196.94 & 197.71 & 197.48 & 209.61 & 201.05 & 5.60 \\
\hline 171.545 & 236.98 & 199.8 & 197.31 & 196.94 & 197.67 & 197.46 & 209.59 & 200.97 & 5.61 \\
\hline 172.0448 & 236.77 & 200.02 & 197.28 & 196.94 & 197.69 & 197.46 & 209.55 & 200.85 & 5.60 \\
\hline 172.5465 & 236.75 & 199.88 & 197.3 & 196.94 & 197.67 & 197.44 & 209.58 & 200.85 & 5.61 \\
\hline 173.0455 & 236.77 & 199.98 & 197.28 & 196.9 & 197.66 & 197.44 & 209.53 & 200.89 & 5.63 \\
\hline 173.5453 & 236.81 & 199.66 & 197.3 & 196.9 & 197.66 & 197.42 & 209.5 & 200.84 & 5.64 \\
\hline 174.0442 & 236.68 & 200.11 & 197.3 & 196.92 & 197.66 & 197.44 & 209.56 & 200.86 & 5.56 \\
\hline 174.544 & 236.67 & 200.27 & 197.25 & 196.85 & 197.55 & 197.3 & 209.11 & 201.12 & 5.56 \\
\hline 175.0447 & 236.7 & 200.15 & 197.25 & 196.87 & 197.57 & 197.44 & 209.43 & 201.06 & 5.58 \\
\hline
\end{tabular}


Table B1. Continued.

\begin{tabular}{|c|c|c|c|c|c|c|c|c|c|}
\hline $\begin{array}{c}\text { Time } \\
\text { min }\end{array}$ & $\begin{array}{l}\mathrm{T} 1 \\
{ }^{\circ} \mathrm{C} \\
\end{array}$ & $\begin{array}{l}\mathrm{T} 2 \\
{ }^{\circ} \mathrm{C} \\
\end{array}$ & $\begin{array}{l}\mathrm{T} 3 \\
{ }^{\circ} \mathrm{C} \\
\end{array}$ & $\begin{array}{l}\mathrm{T} 4 \\
{ }^{\circ} \mathrm{C} \\
\end{array}$ & $\begin{array}{l}\mathrm{T} 5 \\
{ }^{\circ} \mathrm{C} \\
\end{array}$ & $\begin{array}{l}\mathrm{T} 6 \\
{ }^{\circ} \mathrm{C} \\
\end{array}$ & $\begin{array}{l}\text { Pinj } \\
\text { psig }\end{array}$ & $\begin{array}{l}\text { Pout } \\
\text { psig }\end{array}$ & $\begin{array}{l}\mathrm{Vw} \\
\mathrm{cc} / \mathrm{min}\end{array}$ \\
\hline 175.5445 & 236.83 & 200.08 & 197.3 & 196.94 & 197.71 & 197.5 & 209.69 & 201.02 & 5.60 \\
\hline 176.0453 & 236.88 & 199.83 & 197.32 & 196.96 & 197.71 & 197.48 & 209.76 & 201.01 & 5.61 \\
\hline 176.5452 & 236.83 & 199.76 & 197.32 & 196.96 & 197.68 & 197.46 & 209.64 & 200.88 & 5.59 \\
\hline 177.045 & 236.94 & 200.33 & 197.32 & 196.93 & 197.66 & 197.45 & 209.57 & 200.8 & 5.61 \\
\hline 177.5457 & 236.96 & 200.28 & 197.3 & 196.91 & 197.68 & 197.45 & 209.49 & 200.78 & 5.63 \\
\hline 178.0455 & 237.03 & 199.49 & 197.29 & 196.91 & 197.66 & 197.45 & 209.44 & 200.79 & 5.64 \\
\hline 178.5453 & 237.13 & 199.69 & 197.29 & 196.91 & 197.68 & 197.47 & 209.47 & 200.81 & 5.56 \\
\hline 179.0452 & 237.06 & 200.12 & 197.29 & 196.89 & 197.66 & 197.45 & 209.42 & 200.82 & 5.55 \\
\hline 179.5458 & 237.21 & 200.28 & 197.27 & 196.91 & 197.65 & 197.45 & 209.38 & 200.78 & 5.55 \\
\hline 180.0457 & 237.17 & 200.44 & 197.27 & 196.89 & 197.66 & 197.43 & 209.39 & 200.79 & 5.62 \\
\hline 180.5455 & 237.07 & 200.82 & 197.18 & 196.82 & 197.59 & 197.4 & 209.03 & 201.14 & 5.59 \\
\hline 181.0453 & 237.24 & 200.3 & 197.25 & 196.88 & 197.66 & 197.47 & 9.35 & 201.13 & 5.60 \\
\hline 181.5442 & 237.1 & 199.85 & 197.27 & 196.91 & 197.7 & 197.52 & 209.49 & 200.94 & 5.61 \\
\hline 182.044 & 237.01 & 200.76 & 197.15 & 196.74 & 197.34 & 197.09 & 208.36 & 199.11 & 5.63 \\
\hline 182.5448 & 237.07 & 200.34 & 197.08 & 196.72 & 197.54 & 197.35 & 208.68 & 200.86 & 5.62 \\
\hline 183.0447 & 237.3 & 200.05 & 197.2 & 196.83 & 197.63 & 197.45 & 209.16 & 200.87 & 5.55 \\
\hline 183.5445 & 237.34 & 200.52 & 197.24 & 196.88 & 197.65 & 197.44 & .27 & .73 & 5.55 \\
\hline 184.0452 & 237.38 & 200.12 & 197.24 & 196.88 & 197.65 & 197.44 & 209.29 & 200.7 & 5.58 \\
\hline 184.545 & 237.18 & 200.43 & 197.24 & 196.88 & 197.54 & 197.42 & 209.25 & 200.56 & 5.60 \\
\hline 185.0448 & 237.13 & 200.66 & 197.24 & 196.88 & 197.62 & 197.4 & 209.23 & 200.61 & 5.57 \\
\hline 185.5455 & 237.09 & 200 & 197.22 & 196.87 & 197.62 & 197.37 & 209.24 & 199.08 & 5.56 \\
\hline 186.0453 & 237.06 & 200.52 & 197.24 & 196.88 & 197.71 & 197.46 & 209.45 & 201.59 & 5.59 \\
\hline 186.5452 & 237.18 & 199.84 & 197.33 & 196.99 & 197.78 & 197.6 & 209.9 & 201.54 & 5.58 \\
\hline 187.045 & 237.17 & 200.27 & 197.41 & 197.05 & 197.82 & 197.62 & 210.04 & 201.59 & 5.62 \\
\hline 187.5458 & 237.04 & 199.97 & 197.43 & 197.05 & 197.78 & 197.59 & 210.04 & 201.34 & 5.60 \\
\hline 188.0455 & 236.99 & 200.2 & 197.39 & 197.03 & 197.79 & 197.55 & 209.98 & 201.24 & 5.53 \\
\hline 188.5463 & 236.88 & 200.58 & 197.39 & 197.02 & 197.7 & 197.55 & 209.98 & 201.21 & 5.55 \\
\hline 189.0452 & 236.87 & 200.78 & 197.39 & 197.02 & 197.75 & 197.54 & 209.92 & 201.09 & 5.57 \\
\hline 189.545 & 236.89 & 200.22 & 197.38 & 197 & 197.75 & 197.56 & 209.91 & 201.16 & 5.61 \\
\hline 190.044 & 236.96 & 199.96 & 197.34 & 196.95 & 197.61 & 197.36 & 209.58 & 199.79 & 5.60 \\
\hline 190.5457 & 236.6 & 199.6 & 197.2 & 196.82 & 197.56 & 197.34 & 209.05 & 200.45 & 5.58 \\
\hline 191.0445 & 236.78 & 199.53 & 197.2 & 196.82 & 197.58 & 197.38 & 209.11 & 200.3 & 5.58 \\
\hline 191.5453 & 236.94 & 200.05 & 197.15 & 196.77 & 197.42 & 197.2 & 208.65 & 199.9 & 5.61 \\
\hline 192.045 & 237.11 & 200.18 & 197.15 & 196.79 & 197.58 & 197.4 & 208.95 & 200.7 & 5.64 \\
\hline 192.5448 & 237.32 & 200.18 & 197.2 & 196.86 & 197.65 & 197.45 & 209.24 & 200.74 & 5.57 \\
\hline 193.0447 & 237.47 & 200.43 & 197.24 & 196.86 & 197.63 & 197.44 & 209.26 & 200.69 & 5.56 \\
\hline 193.5455 & 237.61 & 199.77 & 197.22 & 196.86 & 197.56 & 197.39 & 209.15 & 200.48 & 5.54 \\
\hline 194.0453 & 237.68 & 199.89 & 197.19 & 196.83 & 197.58 & 197.39 & 209.02 & 200.44 & 5.58 \\
\hline 194.5452 & 237.74 & 200.34 & 197.17 & 196.83 & 197.6 & 197.39 & 208.96 & 200.4 & 5.60 \\
\hline 195.045 & 237.72 & 199.88 & 197.17 & 196.82 & 197.62 & 197.37 & 208.89 & 200.37 & 5.59 \\
\hline 195.5457 & 237.83 & 199.84 & 197.16 & 196.8 & 197.57 & 197.39 & 208.9 & 200.49 & 5.59 \\
\hline 196.0455 & 237.8 & 200.26 & 197.16 & 196.8 & 197.57 & 197.37 & 208.95 & 200.43 & 5.61 \\
\hline 196.5453 & 237.62 & 200.38 & 197.2 & 196.8 & 197.59 & 197.38 & 208.9 & 200.34 & 5.63 \\
\hline 197.0452 & 237.44 & 199.9 & 197.11 & 196.75 & 197.54 & 197.31 & 208.62 & 200.81 & 5.60 \\
\hline
\end{tabular}


Table B1. Continued.

\begin{tabular}{|c|c|c|c|c|c|c|c|c|c|}
\hline $\begin{array}{c}\text { Time } \\
\text { min }\end{array}$ & $\begin{array}{l}\mathrm{T} 1 \\
{ }^{\circ} \mathrm{C} \\
\end{array}$ & $\begin{array}{l}\mathrm{T} 2 \\
{ }^{\circ} \mathrm{C} \\
\end{array}$ & $\begin{array}{l}\mathrm{T} 3 \\
{ }^{\circ} \mathrm{C} \\
\end{array}$ & $\begin{array}{l}\mathrm{T} 4 \\
{ }^{\circ} \mathrm{C} \\
\end{array}$ & $\begin{array}{l}\mathrm{T5} \\
{ }^{\circ} \mathrm{C} \\
\end{array}$ & $\begin{array}{l}\mathrm{T} 6 \\
{ }^{\circ} \mathrm{C} \\
\end{array}$ & $\begin{array}{l}\text { Pinj } \\
\text { psig }\end{array}$ & $\begin{array}{l}\text { Pout } \\
\text { psig }\end{array}$ & $\begin{array}{l}\mathrm{Vw} \\
\mathrm{cc} / \mathrm{min}\end{array}$ \\
\hline 197.544 & 237.51 & 199.92 & 197.18 & 196.82 & 197.61 & 197.43 & 208.99 & 200.86 & 5.55 \\
\hline 198.0457 & 237.54 & 200.16 & 197.22 & 196.86 & 197.67 & 197.47 & 209.25 & 200.81 & 5.54 \\
\hline 198.5455 & 237.39 & 200.05 & 197.24 & 196.88 & 197.65 & 197.44 & 209.27 & 200.56 & 5.55 \\
\hline 199.0443 & 237.18 & 200.61 & 197.22 & 196.87 & 197.63 & 197.4 & 209.18 & 200.46 & 5.59 \\
\hline 199.5452 & 236.99 & 199.54 & 197.21 & 196.85 & 197.6 & 197.41 & 209.13 & 200.44 & 5.60 \\
\hline 200.045 & 236.97 & 199.99 & 197.23 & 196.85 & 197.59 & 197.41 & 209.12 & 200.41 & 5.58 \\
\hline 200.5448 & 236.95 & 200.06 & 197.23 & 196.85 & 197.61 & 197.39 & 209.22 & 200.46 & 5.58 \\
\hline 201.0447 & 236.9 & 199.42 & 197.23 & 196.85 & 197.63 & 197.43 & 209.22 & 200.55 & 5.60 \\
\hline 201.5453 & 236.89 & 199.88 & 197.25 & 196.86 & 197.63 & 197.41 & 209.23 & 200.43 & 5.64 \\
\hline 202.0452 & 236.96 & 200.37 & 197.25 & 196.86 & 197.61 & 197.41 & 209.25 & 200.56 & 5.60 \\
\hline 202.545 & 237 & 199.8 & 197.24 & 196.88 & 197.63 & 197.42 & 209.34 & 200.45 & 5.56 \\
\hline 203.0448 & 237.09 & 200.64 & 197.26 & 196.88 & 197.61 & 197.42 & 209.3 & 200.4 & 5.56 \\
\hline 203.5455 & 237.07 & 200.28 & 197.22 & 196.86 & 197.6 & 197.38 & 209.29 & 199.15 & 5.58 \\
\hline 204.0453 & 236.86 & 200.75 & 197.15 & 196.81 & 197.51 & 197.39 & 209.01 & 200.86 & 5.61 \\
\hline 204.5452 & 237.04 & 200.29 & 197.23 & 196.89 & 197.67 & 197.49 & 209.34 & 200.99 & 5.60 \\
\hline 205.045 & 237.26 & 200.51 & 197.26 & 196.91 & 197.68 & 197.46 & 209.39 & 200.7 & 5.59 \\
\hline 205.5448 & 237.44 & 200.36 & 197.25 & 196.87 & 197.64 & 197.44 & 209.26 & 200.52 & 5.61 \\
\hline 206.0455 & 237.58 & 199.56 & 197.21 & 196.86 & 197.63 & 197.39 & 209.13 & 200.42 & 5.64 \\
\hline 206.5453 & 237.58 & 199.92 & 197.22 & 196.84 & 197.59 & 197.41 & 209.09 & 200.5 & 5.59 \\
\hline 207.0443 & 237.59 & 200.51 & 197.16 & 196.81 & 197.59 & 197.4 & 208.88 & 200.67 & 5.56 \\
\hline 207.5442 & 237.77 & 200.08 & 197.18 & 196.84 & 197.65 & 197.47 & 209.11 & 200.84 & 5.55 \\
\hline 208.0448 & 237.9 & 199.85 & 197.22 & 196.86 & 197.67 & 197.47 & 209.24 & 200.71 & 5.58 \\
\hline 208.5447 & 237.97 & 199.7 & 197.24 & 196.87 & 197.67 & 197.46 & 209.24 & 200.64 & 5.61 \\
\hline 209.0455 & 237.79 & 200.34 & 197.23 & 196.85 & 197.66 & 197.44 & 209.19 & 200.54 & 5.60 \\
\hline 209.5453 & 237.65 & 200.18 & 197.21 & 196.85 & 197.62 & 197.44 & 209.12 & 200.56 & 5.60 \\
\hline 210.045 & 237.58 & 200.26 & 197.21 & 196.85 & 197.62 & 197.43 & 209.1 & 200.54 & 5.61 \\
\hline 210.5448 & 237.64 & 199.94 & 197.2 & 196.86 & 197.63 & 197.43 & 209.14 & 200.55 & 5.63 \\
\hline 211.0457 & 237.48 & 199.98 & 197.18 & 196.79 & 197.54 & 197.31 & 208.8 & 200.84 & 5.59 \\
\hline 211.5455 & 237.36 & 200.37 & 197.18 & 196.83 & 197.7 & 197.43 & 209.1 & 200.86 & 5.55 \\
\hline 212.0453 & 237.18 & 200.23 & 197.24 & 196.9 & 197.67 & 197.49 & 209.39 & 200.77 & 5.53 \\
\hline 212.5452 & 237.06 & 200.25 & 197.28 & 196.92 & 197.69 & 197.48 & 209.45 & 200.69 & 5.55 \\
\hline 213.044 & 236.97 & 199.93 & 197.26 & 196.91 & 197.66 & 197.46 & 209.43 & 200.59 & 5.58 \\
\hline 213.5457 & 236.97 & 200.33 & 197.27 & 196.89 & 197.66 & 197.45 & 209.38 & 200.59 & 5.60 \\
\hline 214.0455 & 236.99 & 200.74 & 197.25 & 196.89 & 197.64 & 197.43 & 209.41 & 200.53 & 5.58 \\
\hline 214.5443 & 237 & 200.31 & 197.25 & 196.89 & 197.66 & 197.45 & 209.4 & 200.55 & 5.61 \\
\hline 215.0452 & 237.07 & 200.26 & 197.25 & 196.88 & 197.65 & 197.45 & 209.42 & 200.49 & 5.64 \\
\hline 215.545 & 237.14 & 200.41 & 197.28 & 196.86 & 197.65 & 197.45 & 209.42 & 200.62 & 5.63 \\
\hline 216.0448 & 237.09 & 200.75 & 197.26 & 196.88 & 197.67 & 197.46 & 209.48 & 200.5 & 5.56 \\
\hline 216.5447 & 237.11 & 200.9 & 197.26 & 196.9 & 197.66 & 197.46 & 209.42 & 200.51 & 5.54 \\
\hline 217.0453 & 237.22 & 200.2 & 197.25 & 196.89 & 197.64 & 197.43 & 209.38 & 200.57 & 5.55 \\
\hline 217.5452 & 237.24 & 200.11 & 197.16 & 196.8 & 197.57 & 197.37 & 209 & 200.84 & 5.59 \\
\hline 218.045 & 237.46 & 200.08 & 197.21 & 196.88 & 197.61 & 197.48 & 209.34 & 200.81 & 5.60 \\
\hline 218.5448 & 237.57 & 200.23 & 197.27 & 196.91 & 197.7 & 197.49 & 209.47 & 200.89 & 5.59 \\
\hline
\end{tabular}


Table B1. Continued.

\begin{tabular}{|c|c|c|c|c|c|c|c|c|c|}
\hline $\begin{array}{c}\text { Time } \\
\text { min }\end{array}$ & $\begin{array}{c}\text { T1 } \\
{ }^{\circ} \mathbf{C}\end{array}$ & $\begin{array}{c}\text { T2 } \\
{ }^{\circ} \mathbf{C}\end{array}$ & $\begin{array}{c}\text { T3 } \\
{ }^{\circ} \mathbf{C}\end{array}$ & $\begin{array}{c}\mathbf{T} 4 \\
{ }^{\mathbf{}} \mathbf{C}\end{array}$ & $\begin{array}{c}\text { T5 } \\
{ }^{\circ} \mathbf{C}\end{array}$ & $\begin{array}{c}\text { T6 } \\
{ }^{\circ} \mathbf{C}\end{array}$ & $\begin{array}{c}\text { Pinj } \\
\text { psig }\end{array}$ & $\begin{array}{c}\text { Pout } \\
\text { psig }\end{array}$ & $\begin{array}{c}\mathbf{V w} \\
\mathbf{c c / m i n}\end{array}$ \\
\hline 219.0455 & 237.72 & 200.03 & 197.27 & 196.92 & 197.69 & 197.49 & 209.42 & 200.67 & 5.60 \\
\hline 219.5453 & 237.75 & 200.48 & 197.24 & 196.88 & 197.65 & 197.46 & 209.26 & 200.59 & 5.61 \\
\hline 220.0452 & 237.83 & 200.43 & 197.21 & 196.87 & 197.64 & 197.44 & 209.18 & 200.57 & 5.64 \\
\hline 220.545 & 237.87 & 200.5 & 197.21 & 196.83 & 197.64 & 197.43 & 209.16 & 200.51 & 5.57 \\
\hline 221.0438 & 237.87 & 200.19 & 197.2 & 196.87 & 197.64 & 197.45 & 209.21 & 200.54 & 5.54 \\
\hline 221.5455 & 237.87 & 199.83 & 197.2 & 196.86 & 197.63 & 197.43 & 209.18 & 200.56 & 5.54 \\
\hline 222.0445 & 237.82 & 200.16 & 197.22 & 196.86 & 197.65 & 197.43 & 209.16 & 200.5 & 5.58 \\
\hline 222.5443 & 237.73 & 200.19 & 197.2 & 196.86 & 197.63 & 197.44 & 209.17 & 200.57 & 5.60 \\
\hline 223.045 & 237.74 & 199.88 & 197.21 & 196.85 & 197.64 & 197.42 & 209.25 & 200.52 & 5.60 \\
\hline 223.5448 & 237.67 & 200.4 & 197.19 & 196.87 & 197.57 & 197.44 & 209.22 & 200.55 & 5.61 \\
\hline 224.0455 & 237.58 & 199.86 & 197.23 & 196.87 & 197.66 & 197.45 & 209.25 & 200.44 & 5.62 \\
\hline 224.5453 & 237.46 & 200.35 & 197.16 & 196.79 & 197.5 & 197.27 & 208.74 & 200.8 & 5.65 \\
\hline 225.0452 & 237.34 & 199.97 & 197.18 & 196.84 & 197.65 & 197.45 & 209.22 & 200.87 & 5.57 \\
\hline 225.545 & 237.43 & 200.33 & 197.26 & 196.9 & 197.7 & 197.51 & 209.52 & 200.92 & 5.55 \\
\hline 226.0448 & 237.31 & 200.77 & 197.29 & 196.94 & 197.72 & 197.51 & 209.61 & 200.78 & 5.56 \\
\hline 226.5457 & 237.2 & 199.98 & 197.26 & 196.9 & 197.66 & 197.46 & 209.46 & 200.54 & 5.61 \\
\hline 227.0455 & 237.13 & 199.77 & 197.25 & 196.89 & 197.66 & 197.43 & 209.44 & 200.54 & 5.60 \\
\hline 227.5452 & 237.13 & 200.28 & 197.25 & 196.87 & 197.64 & 197.45 & 209.4 & 200.5 & 5.57 \\
\hline 228.045 & 237.17 & 200.1 & 197.23 & 196.88 & 197.72 & 197.43 & 209.4 & 200.47 & 5.58 \\
\hline 228.5448 & 237.28 & 200.78 & 197.22 & 196.88 & 197.65 & 197.45 & 209.4 & 200.52 & 5.61 \\
\hline 229.0457 & 237.38 & 200.19 & 197.24 & 196.88 & 197.67 & 197.45 & 209.42 & 200.55 & 5.63 \\
\hline 229.5455 & 237.45 & 200.36 & 197.22 & 196.87 & 197.65 & 197.46 & 209.42 & 200.48 & 5.60 \\
\hline 230.0443 & 237.49 & 200.67 & 197.23 & 196.87 & 197.64 & 197.44 & 209.38 & 200.59 & 5.56 \\
\hline
\end{tabular}


Table B2-Temperature and production data for run 2

\begin{tabular}{|c|c|c|c|c|c|c|c|c|c|}
\hline $\begin{array}{l}\text { Time } \\
\text { min }\end{array}$ & $\begin{array}{l}\mathrm{T} 1 \\
{ }^{\circ} \mathrm{C} \\
\end{array}$ & $\begin{array}{l}\mathrm{T} 2 \\
{ }^{\circ} \mathrm{C} \\
\end{array}$ & $\begin{array}{l}\mathrm{T} 3 \\
{ }^{\circ} \mathrm{C} \\
\end{array}$ & $\begin{array}{l}\mathrm{T} 4 \\
{ }^{\circ} \mathrm{C} \\
\end{array}$ & $\begin{array}{l}\text { T5 } \\
{ }^{\circ} \mathrm{C} \\
\end{array}$ & $\begin{array}{l}\mathrm{T} 6 \\
{ }^{\circ} \mathrm{C} \\
\end{array}$ & $\begin{array}{l}\text { Pinj } \\
\text { psig }\end{array}$ & $\begin{array}{l}\text { Pout } \\
\text { psig }\end{array}$ & $\begin{array}{c}\mathrm{Vw} \\
\mathrm{cc} / \mathrm{min}\end{array}$ \\
\hline 5.03E-02 & 244.38 & 62.97 & 61.22 & 63.23 & 62.27 & 56.12 & 241.12 & 201.27 & 5.57 \\
\hline 0.5473334 & 235.13 & 177.74 & 61.22 & 63.3 & 62.22 & 56.21 & 206.3 & 200.52 & 5.55 \\
\hline 1.047167 & 235 & 190.44 & 61.22 & 63.3 & 62.24 & 56.21 & 206.1 & 200.42 & 5.55 \\
\hline 1.546 & 232.72 & 191.83 & 61.23 & 63.33 & 62.25 & 56.22 & 205.94 & 200.38 & 5.57 \\
\hline 2.046833 & 233.01 & 191.76 & 61.25 & 63.35 & 62.29 & 56.24 & 205.9 & 200.39 & 5.59 \\
\hline 2.545667 & 233.27 & 191.9 & 61.24 & 63.29 & 62.23 & 56.18 & 206.05 & 200.41 & 5.61 \\
\hline 3.0455 & 233.71 & 192.27 & 61.24 & 63.3 & 62.24 & 56.21 & 206.17 & 200.39 & 5.60 \\
\hline 3.547167 & 233.64 & 192.8 & 61.25 & 63.31 & 62.26 & 56.23 & 206.68 & 200.43 & 5.61 \\
\hline 4.046167 & 233.63 & 193.35 & 61.26 & 63.33 & 62.29 & 56.22 & 206.79 & 200.4 & 5.62 \\
\hline 4.545833 & 233.77 & 193.67 & 61.32 & 63.32 & 62.3 & 56.24 & 206.47 & 200.37 & 5.62 \\
\hline 5.046667 & 233.89 & 193.67 & 61.41 & 63.34 & 62.28 & 56.25 & 206.7 & 200.37 & 5.57 \\
\hline 5.5455 & 233.01 & 193.86 & 61.5 & 63.31 & 62.31 & 56.24 & 206.63 & 200.36 & 5.55 \\
\hline 6.046333 & 233.49 & 193.8 & 61.57 & 63.33 & 62.42 & 56.24 & 206.9 & 200.36 & 5.56 \\
\hline 6.546166 & 232.89 & 194.26 & 61.66 & 63.36 & 62.34 & 56.25 & 207.43 & 200.43 & 5.58 \\
\hline 7.046 & 233.01 & 194.88 & 61.8 & 63.37 & 62.45 & 56.3 & 207.21 & 199.85 & 5.58 \\
\hline 7.546667 & 233.55 & 195.29 & 61.93 & 63.37 & 62.35 & 56.24 & 207.68 & 199.94 & 5.58 \\
\hline 8.045667 & 233.74 & 195.48 & 62.06 & 63.38 & 62.36 & 56.26 & 207.62 & 199.96 & 5.58 \\
\hline 8.546334 & 233.64 & 195.83 & 62.18 & 63.39 & 62.34 & 56.27 & 208.53 & 200.02 & 5.58 \\
\hline 9.046167 & 232.78 & 196.22 & 62.29 & 63.39 & 62.35 & 56.26 & 208.66 & 200.06 & 5.62 \\
\hline 9.546 & 232.54 & 196.58 & 62.32 & 63.4 & 62.29 & 56.26 & 209 & 200.09 & 5.64 \\
\hline 10.04583 & 232.16 & 196.71 & 62.34 & 63.4 & 62.4 & 56.31 & 208.93 & 200.16 & 5.58 \\
\hline 10.5465 & 231.61 & 196.91 & 62.39 & 63.37 & 62.39 & 56.3 & 208.94 & 200.16 & 5.56 \\
\hline 11.04633 & 231.36 & 197.1 & 62.44 & 63.4 & 62.42 & 56.32 & 209.51 & 200.23 & 5.57 \\
\hline 11.54617 & 230.42 & 197.31 & 62.53 & 63.42 & 62.43 & 56.31 & 209.44 & 200.08 & 5.59 \\
\hline 12.046 & 230.34 & 197.35 & 62.64 & 63.43 & 62.45 & 56.34 & 209.43 & 200.03 & 5.60 \\
\hline 12.54583 & 230.52 & 197.44 & 62.74 & 63.42 & 62.46 & 56.35 & 209.52 & 200.68 & 5.59 \\
\hline 13.0465 & 230.58 & 197.5 & 62.87 & 63.42 & 62.45 & 56.35 & 210.39 & 200.91 & 5.58 \\
\hline 13.5455 & 229.84 & 197.73 & 63.05 & 63.43 & 62.46 & 56.34 & 210.51 & 201.41 & 5.61 \\
\hline 14.04533 & 229.69 & 197.71 & 63.25 & 63.42 & 62.46 & 56.35 & 210.99 & 201.26 & 5.65 \\
\hline 14.546 & 229.74 & 197.72 & 63.47 & 63.43 & 62.47 & 56.36 & 211.01 & 200.99 & 5.60 \\
\hline 15.04583 & 229.89 & 198.01 & 63.71 & 63.39 & 62.46 & 56.4 & 211.24 & 200.45 & 5.55 \\
\hline 15.5465 & 231.96 & 198.2 & 63.97 & 63.38 & 62.47 & 56.37 & 211.94 & 200.67 & 5.55 \\
\hline 16.04633 & 234.3 & 198.52 & 64.28 & 63.36 & 62.45 & 56.38 & 212.6 & 200.67 & 5.57 \\
\hline 16.54617 & 235.32 & 199.01 & 64.65 & 63.35 & 62.48 & 56.39 & 213.55 & 200.74 & 5.61 \\
\hline 17.046 & 234.25 & 199.46 & 65.1 & 63.34 & 62.53 & 56.4 & 215.23 & 200.77 & 5.60 \\
\hline 17.54583 & 233.76 & 200.09 & 65.62 & 63.35 & 62.52 & 56.44 & 216.72 & 200.81 & 5.60 \\
\hline 18.04667 & 233.95 & 200.84 & 66.2 & 63.35 & 62.53 & 56.43 & 218.41 & 200.92 & 5.60 \\
\hline 18.5455 & 233.01 & 201.35 & 66.89 & 63.36 & 62.56 & 56.44 & 220.05 & 200.84 & 5.63 \\
\hline 19.04533 & 233.49 & 201.9 & 67.68 & 63.35 & 62.48 & 56.49 & 221.47 & 200.41 & 5.63 \\
\hline 19.546 & 233.7 & 202.43 & 68.67 & 63.34 & 62.62 & 56.5 & 222.26 & 200.37 & 5.58 \\
\hline 20.045 & 233.84 & 202.21 & 69.77 & 63.33 & 62.69 & 56.49 & 221.34 & 200.32 & 5.57 \\
\hline 20.54483 & 233.95 & 201.89 & 70.97 & 63.31 & 62.74 & 56.5 & 220.82 & 200.29 & 5.58 \\
\hline 21.0455 & 234.23 & 201.62 & 72.13 & 63.3 & 62.77 & 56.51 & 220.39 & 200.27 & 5.62 \\
\hline 21.54533 & 234.35 & 201.56 & 73.25 & 63.31 & 62.82 & 56.49 & 220.09 & 200.25 & 5.62 \\
\hline
\end{tabular}


Table B2. Continued.

\begin{tabular}{|c|c|c|c|c|c|c|c|c|c|}
\hline $\begin{array}{l}\text { Time } \\
\text { min }\end{array}$ & $\begin{array}{l}\mathrm{T} 1 \\
{ }^{\circ} \mathrm{C}\end{array}$ & $\begin{array}{l}\mathrm{T} 2 \\
{ }^{\circ} \mathrm{C}\end{array}$ & $\begin{array}{l}\mathrm{T} 3 \\
{ }^{\circ} \mathrm{C}\end{array}$ & $\begin{array}{l}\mathrm{T} 4 \\
{ }^{\circ} \mathrm{C}\end{array}$ & $\begin{array}{l}\mathrm{T} 5 \\
{ }^{\circ} \mathrm{C}\end{array}$ & $\begin{array}{l}\mathrm{T} 6 \\
{ }^{\circ} \mathrm{C}\end{array}$ & $\begin{array}{l}\text { Pinj } \\
\text { psig }\end{array}$ & $\begin{array}{l}\text { Pout } \\
\text { psig }\end{array}$ & $\begin{array}{c}\mathrm{Vw} \\
\mathrm{cc} / \mathrm{min}\end{array}$ \\
\hline 22.04517 & 234.5 & 201.35 & 74.2 & 63.27 & 62.81 & 56.48 & 219.54 & 200.21 & 5.62 \\
\hline 22.54683 & 234.93 & 201.33 & 75.08 & 63.24 & 62.8 & 56.43 & 219.27 & 200.23 & 5.63 \\
\hline 23.04483 & 234.4 & 201.35 & 75.84 & 63.23 & 62.87 & 56.44 & 219.33 & 200.18 & 5.63 \\
\hline 23.5465 & 235.32 & 201.17 & 76.51 & 63.2 & 62.88 & 56.43 & 219.87 & 200.17 & 5.59 \\
\hline 24.04633 & 234.04 & 201.3 & 77.19 & 63.19 & 62.93 & 56.45 & 220.69 & 200.18 & 5.57 \\
\hline 24.54617 & 234.7 & 201.42 & 77.9 & 63.2 & 62.96 & 56.49 & 221.31 & 200.38 & 5.56 \\
\hline 25.046 & 235.12 & 201.56 & 78.67 & 63.18 & 62.99 & 56.58 & 222.08 & 200.16 & 5.59 \\
\hline 25.54483 & 235.16 & 201.71 & 79.47 & 63.19 & 63.02 & 56.65 & 223.01 & 200.15 & 5.61 \\
\hline 26.04567 & 233.9 & 201.84 & 80.25 & 63.18 & 63.06 & 56.72 & 223.52 & 200.14 & 5.59 \\
\hline 26.54633 & 234.47 & 201.92 & 80.99 & 63.17 & 63.09 & 56.78 & 223.98 & 200.14 & 5.59 \\
\hline 27.04617 & 234.28 & 202.06 & 81.67 & 63.16 & 63.12 & 56.85 & 224.87 & 200.16 & 5.61 \\
\hline 27.546 & 233.82 & 202.3 & 82.38 & 63.15 & 63.17 & 56.9 & 226.19 & 200.2 & 5.64 \\
\hline 28.04483 & 233.42 & 202.65 & 83.08 & 63.18 & 63.18 & 57 & 227.97 & 200.16 & 5.62 \\
\hline 28.54567 & 232.39 & 202.93 & 83.87 & 63.19 & 63.23 & 57.07 & 229.78 & 200.23 & 5.58 \\
\hline 29.04633 & 231.31 & 203.37 & 84.74 & 63.16 & 63.25 & 57.13 & 232.31 & 201.02 & 5.57 \\
\hline 29.54617 & 231.35 & 204.07 & 85.72 & 63.17 & 63.25 & 57.22 & 235.42 & 200.84 & 5.59 \\
\hline 30.046 & 230.14 & 204.85 & 86.81 & 63.18 & 63.29 & 57.32 & 239.11 & 200.86 & 5.62 \\
\hline 30.54483 & 230.42 & 205.54 & 88.05 & 63.22 & 63.34 & 57.45 & 243.26 & 200.37 & 5.60 \\
\hline 31.0465 & 230.16 & 206.12 & 89.56 & 63.23 & 63.35 & 57.57 & 246.96 & 200.57 & 5.61 \\
\hline 31.5455 & 230.67 & 206.94 & 91.28 & 63.22 & 63.39 & 57.69 & 250.05 & 200.61 & 5.63 \\
\hline 32.04617 & 230.89 & 207.37 & 93.26 & 63.25 & 63.46 & 57.81 & 251.99 & 200.59 & 5.65 \\
\hline 32.54517 & 231.31 & 207.76 & 96 & 63.28 & 63.51 & 57.94 & 252.95 & 201.1 & 5.61 \\
\hline 33.045 & 232.23 & 207.89 & 100.26 & 63.27 & 63.53 & 58.04 & 253.38 & 201.08 & 5.57 \\
\hline 33.54567 & 232.6 & 207.86 & 104.34 & 63.3 & 63.54 & 58.15 & 253.14 & 200.97 & 5.57 \\
\hline 34.04633 & 232.03 & 207.76 & 107.65 & 63.31 & 63.53 & 58.23 & 252.52 & 200.81 & 5.60 \\
\hline 34.54617 & 232.06 & 207.49 & 110.39 & 63.33 & 63.54 & 58.31 & 251.5 & 200.72 & 5.62 \\
\hline 35.04517 & 233.11 & 207.33 & 112.75 & 63.36 & 63.57 & 58.4 & 250.59 & 200.63 & 5.59 \\
\hline 35.545 & 234.78 & 207.18 & 114.99 & 63.37 & 63.58 & 58.48 & 250.06 & 200.58 & 5.61 \\
\hline 36.04667 & 234.11 & 207.04 & 117.18 & 63.42 & 63.59 & 58.55 & 249.51 & 200.52 & 5.62 \\
\hline 36.5465 & 233.76 & 206.87 & 119.39 & 63.46 & 63.59 & 58.59 & 249.17 & 200.93 & 5.63 \\
\hline 37.04617 & 233.77 & 206.84 & 121.62 & 63.51 & 63.62 & 58.66 & 249.31 & 200.83 & 5.57 \\
\hline 37.546 & 234.15 & 206.91 & 123.92 & 63.57 & 63.63 & 58.72 & 249.09 & 200.78 & 5.56 \\
\hline 38.045 & 234.64 & 207.06 & 126.19 & 63.62 & 63.64 & 58.77 & 249.36 & 200.74 & 5.55 \\
\hline 38.54483 & 233.48 & 207.1 & 128.61 & 63.68 & 63.68 & 58.82 & 249.99 & 200.71 & 5.61 \\
\hline 39.0455 & 234.03 & 207.27 & 131.1 & 63.75 & 63.71 & 58.88 & 250.89 & 200.68 & 5.60 \\
\hline 39.54717 & 233.62 & 207.4 & 133.68 & 63.81 & 63.62 & 58.94 & 252.25 & 200.66 & 5.60 \\
\hline 40.04517 & 232.93 & 207.73 & 136.38 & 63.89 & 63.76 & 59.01 & 254.35 & 200.65 & 5.61 \\
\hline 40.545 & 232.71 & 208.29 & 139.28 & 63.98 & 63.79 & 59.05 & 257.45 & 200.81 & 5.59 \\
\hline 41.04567 & 232.18 & 208.83 & 142.47 & 64.06 & 63.79 & 59.12 & 261.1 & 200.78 & 5.62 \\
\hline 41.5455 & 232.2 & 209.54 & 145.89 & 64.14 & 63.76 & 59.14 & 265.27 & 200.81 & 5.58 \\
\hline 42.04633 & 232.92 & 210.19 & 149.7 & 64.23 & 63.75 & 59.15 & 269.25 & 200.82 & 5.56 \\
\hline 42.54517 & 232.18 & 210.75 & 153.91 & 64.35 & 63.76 & 59.2 & 272.58 & 200.79 & 5.56 \\
\hline 43.045 & 232.81 & 211.1 & 158.43 & 64.47 & 63.77 & 59.24 & 274.95 & 199.68 & 5.57 \\
\hline 43.54583 & 232.91 & 211.36 & 163.18 & 64.63 & 63.79 & 59.34 & 276.21 & 199.69 & 5.61 \\
\hline
\end{tabular}


Table B2. Continued.

\begin{tabular}{|c|c|c|c|c|c|c|c|c|c|}
\hline $\begin{array}{l}\text { Time } \\
\text { min }\end{array}$ & $\begin{array}{l}\mathrm{T} 1 \\
{ }^{\circ} \mathrm{C}\end{array}$ & $\begin{array}{l}\mathrm{T} 2 \\
{ }^{\circ} \mathrm{C}\end{array}$ & $\begin{array}{l}\mathrm{T} 3 \\
{ }^{\circ} \mathrm{C}\end{array}$ & $\begin{array}{l}\mathrm{T} 4 \\
{ }^{\circ} \mathrm{C}\end{array}$ & $\begin{array}{l}\mathrm{T} 5 \\
{ }^{\circ} \mathrm{C}\end{array}$ & $\begin{array}{l}\mathrm{T} 6 \\
{ }^{\circ} \mathrm{C}\end{array}$ & $\begin{array}{l}\text { Pinj } \\
\text { psig }\end{array}$ & $\begin{array}{l}\text { Pout } \\
\text { psig }\end{array}$ & $\begin{array}{c}\mathrm{Vw} \\
\mathrm{cc} / \mathrm{min}\end{array}$ \\
\hline 44.0465 & 233.35 & 211.47 & 168.14 & 64.78 & 63.82 & 59.43 & 276.45 & 200.24 & 5.59 \\
\hline 44.54633 & 234 & 211.44 & 173.44 & 64.94 & 63.83 & 59.45 & 275.83 & 200.85 & 5.60 \\
\hline 45.04517 & 234.63 & 211.36 & 178.48 & 65.16 & 63.85 & 59.54 & 274.98 & 199.88 & 5.60 \\
\hline 45.546 & 234.6 & 211.35 & 183.05 & 65.39 & 63.88 & 59.62 & 274.12 & 200.84 & 5.63 \\
\hline 46.04583 & 236.04 & 211.25 & 187.36 & 65.62 & 63.91 & 59.68 & 273.29 & 200.77 & 5.61 \\
\hline 46.54567 & 234.81 & 211.15 & 191.15 & 65.9 & 63.91 & 59.75 & 272.87 & 200.68 & 5.57 \\
\hline 47.0455 & 236.66 & 211.19 & 194.57 & 66.17 & 63.94 & 59.79 & 272.37 & 200.62 & 5.55 \\
\hline 47.54617 & 238.08 & 211.19 & 197.23 & 66.44 & 63.92 & 59.82 & 271.84 & 200.57 & 5.57 \\
\hline 48.046 & 238.83 & 211.22 & 199.33 & 66.75 & 63.95 & 59.88 & 271.64 & 200.52 & 5.59 \\
\hline 48.54583 & 240.03 & 211.26 & 201.4 & 67.09 & 63.94 & 59.89 & 271.67 & 200.78 & 5.60 \\
\hline 49.04567 & 238.28 & 211.36 & 203.18 & 67.48 & 63.96 & 59.95 & 272.58 & 200.68 & 5.59 \\
\hline 49.5455 & 237.68 & 211.74 & 204.83 & 67.84 & 63.97 & 59.98 & 274.09 & 200.65 & 5.61 \\
\hline 50.04533 & 237.76 & 212.05 & 206.34 & 68.24 & 63.98 & 60.02 & 276.59 & 200.64 & 5.61 \\
\hline 50.54517 & 237.21 & 212.36 & 207.87 & 68.68 & 64 & 60.05 & 279.42 & 200.65 & 5.61 \\
\hline 51.04583 & 236.93 & 213.01 & 209.09 & 69.14 & 64.01 & 60.07 & 282.68 & 200.65 & 5.62 \\
\hline 51.54567 & 237.52 & 213.54 & 210.01 & 69.68 & 63.98 & 60.1 & 285.74 & 200.65 & 5.58 \\
\hline 52.0445 & 237.62 & 213.86 & 210.75 & 70.21 & 64.02 & 60.12 & 288.56 & 200.68 & 5.58 \\
\hline 52.54533 & 237.02 & 214.39 & 211.36 & 70.82 & 64.01 & 60.16 & 290.49 & 200.66 & 5.61 \\
\hline 53.04517 & 237.86 & 214.82 & 211.82 & 71.5 & 64.03 & 60.21 & 291.73 & 200.59 & 5.61 \\
\hline 53.545 & 237.76 & 214.74 & 212.13 & 72.23 & 64.02 & 60.25 & 292.34 & 200.72 & 5.60 \\
\hline 54.04567 & 237.7 & 214.64 & 212.26 & 73 & 64.02 & 60.28 & 292.21 & 200.66 & 5.61 \\
\hline 54.5455 & 238.2 & 214.59 & 212.3 & 73.78 & 64.03 & 60.32 & 291.84 & 200.59 & 5.63 \\
\hline 55.04533 & 238.64 & 214.54 & 212.3 & 74.56 & 64.04 & 60.35 & 291.32 & 200.52 & 5.63 \\
\hline 55.54517 & 237.93 & 214.37 & 212.26 & 75.39 & 64.04 & 60.39 & 290.81 & 200.48 & 5.58 \\
\hline 56.044 & 238.04 & 214.25 & 212.24 & 76.22 & 64.05 & 60.43 & 290.52 & 200.43 & 5.56 \\
\hline 56.54567 & 238.26 & 214.27 & 212.23 & 77.07 & 64.06 & 60.44 & 290.42 & 200.38 & 5.57 \\
\hline 57.0455 & 237.91 & 214.35 & 212.29 & 77.92 & 64.06 & 60.5 & 290.89 & 200.35 & 5.60 \\
\hline 57.54533 & 238.25 & 214.42 & 212.44 & 78.79 & 64.07 & 60.51 & 291.84 & 200.7 & 5.60 \\
\hline 58.04517 & 237.88 & 214.73 & 212.62 & 79.69 & 64.07 & 60.55 & 293.43 & 200.61 & 5.60 \\
\hline 58.54583 & 238.14 & 214.99 & 212.98 & 80.65 & 64.09 & 60.59 & 295.69 & 200.58 & 5.60 \\
\hline 59.04483 & 237.91 & 215.32 & 213.38 & 81.63 & 64.1 & 60.6 & 298.08 & 200.58 & 5.62 \\
\hline 59.5455 & 237.99 & 215.66 & 213.8 & 82.67 & 64.11 & 60.64 & 300.83 & 200.59 & 5.63 \\
\hline 60.04533 & 237.78 & 215.97 & 214.22 & 83.78 & 64.11 & 60.67 & 303.04 & 200.6 & 5.59 \\
\hline 60.54617 & 238.12 & 216.35 & 214.58 & 84.96 & 64.12 & 60.67 & 304.93 & 200.6 & 5.55 \\
\hline 61.04417 & 238.86 & 216.7 & 214.85 & 86.22 & 64.14 & 60.7 & 306.29 & 200.59 & 5.56 \\
\hline 61.54583 & 238.76 & 216.79 & 215.07 & 87.55 & 64.17 & 60.74 & 307.06 & 200.52 & 5.61 \\
\hline 62.04567 & 238.77 & 216.89 & 215.19 & 88.88 & 64.17 & 60.77 & 307.39 & 200.49 & 5.61 \\
\hline 62.54533 & 238.86 & 216.95 & 215.23 & 90.24 & 64.2 & 60.79 & 307.47 & 200.64 & 5.58 \\
\hline 63.04617 & 239.15 & 216.97 & 215.2 & 91.64 & 64.22 & 60.81 & 307.28 & 200.5 & 5.60 \\
\hline 63.54683 & 238.6 & 216.97 & 215.2 & 93.06 & 64.24 & 60.8 & 307.1 & 200.43 & 5.61 \\
\hline 64.04583 & 238.23 & 216.96 & 215.19 & 94.48 & 64.29 & 60.84 & 307.25 & 200.42 & 5.62 \\
\hline 64.5465 & 238.52 & 217.04 & 215.21 & 95.93 & 64.31 & 60.85 & 307.33 & 200.38 & 5.59 \\
\hline 65.0455 & 237.92 & 217.11 & 215.32 & 97.4 & 64.34 & 60.87 & 308.13 & 200.33 & 5.56 \\
\hline 65.54617 & 237.91 & 217.28 & 215.45 & 98.95 & 64.38 & 60.88 & 309.55 & 200.33 & 5.57 \\
\hline
\end{tabular}


Table B2. Continued.

\begin{tabular}{|c|c|c|c|c|c|c|c|c|c|}
\hline $\begin{array}{c}\text { Time } \\
\text { min }\end{array}$ & $\begin{array}{l}\mathrm{T} 1 \\
{ }^{\circ} \mathrm{C} \\
\end{array}$ & $\begin{array}{l}\mathrm{T} 2 \\
{ }^{\circ} \mathrm{C} \\
\end{array}$ & $\begin{array}{l}\mathrm{T} 3 \\
{ }^{\circ} \mathrm{C} \\
\end{array}$ & $\begin{array}{l}\mathrm{T} 4 \\
{ }^{\circ} \mathrm{C} \\
\end{array}$ & $\begin{array}{l}\mathrm{T5} \\
{ }^{\circ} \mathrm{C} \\
\end{array}$ & $\begin{array}{l}\mathrm{T} 6 \\
{ }^{\circ} \mathrm{C} \\
\end{array}$ & $\begin{array}{l}\text { Pinj } \\
\text { psig }\end{array}$ & $\begin{array}{l}\text { Pout } \\
\text { psig }\end{array}$ & $\begin{array}{c}\mathrm{Vw} \\
\mathrm{cc} / \mathrm{min}\end{array}$ \\
\hline 66.046 & 238.36 & 217.66 & 215.69 & 100.55 & 64.4 & 60.86 & 311.05 & 200.33 & 5.58 \\
\hline 66.54483 & 237.13 & 217.75 & 215.98 & 102.18 & 64.48 & 60.89 & 313.01 & 200.36 & 5.61 \\
\hline 67.0465 & 236.97 & 217.92 & 216.27 & 103.97 & 64.55 & 60.91 & 314.62 & 200.37 & 5.63 \\
\hline 67.5455 & 236.89 & 218.32 & 216.57 & 105.8 & 64.57 & 60.92 & 316.28 & 200.44 & 5.60 \\
\hline 68.04433 & 237.45 & 218.54 & 216.79 & 107.78 & 64.63 & 60.92 & 317.42 & 200.51 & 5.64 \\
\hline 68.54517 & 237.61 & 218.69 & 216.95 & 109.83 & 64.69 & 60.93 & 318.27 & 200.5 & 5.65 \\
\hline 69.04583 & 238.39 & 218.85 & 217.06 & 111.99 & 64.66 & 60.97 & 318.81 & 200.5 & 5.59 \\
\hline 69.54567 & 237.84 & 218.84 & 217.14 & 114.3 & 64.91 & 60.99 & 319.16 & 200.49 & 5.56 \\
\hline 70.04633 & 237.64 & 218.84 & 217.16 & 116.69 & 64.93 & 61 & 319 & 200.5 & 5.58 \\
\hline 70.54533 & 238.63 & 218.81 & 217.13 & 119.16 & 65.01 & 61.02 & 318.89 & 200.46 & 5.61 \\
\hline 71.04517 & 238.96 & 218.82 & 217.12 & 121.71 & 65.11 & 61.05 & 318.72 & 200.47 & 5.60 \\
\hline 71.54583 & 238.86 & 218.89 & 217.12 & 124.37 & 65.29 & 61.05 & 318.8 & 200.54 & 5.61 \\
\hline 72.04567 & 238.38 & 218.86 & 217.13 & 127.17 & 65.33 & 61.06 & 318.59 & 200.45 & 5.62 \\
\hline 72.5455 & 238.3 & 218.89 & 217.11 & 130.18 & 65.47 & 61.08 & 318.7 & 200.45 & 5.63 \\
\hline 73.04533 & 239.09 & 218.98 & 217.1 & 133.34 & 65.61 & 61.1 & 318.65 & 200.47 & 5.62 \\
\hline 73.54517 & 238.27 & 219.02 & 217.11 & 136.75 & 65.72 & 61.11 & 318.74 & 200.5 & 5.57 \\
\hline 74.04583 & 238.99 & 218.95 & 217.09 & 140.41 & 65.88 & 61.17 & 318.55 & 200.49 & 5.55 \\
\hline 74.54383 & 239.3 & 218.9 & 217.04 & 144.22 & 66.06 & 61.18 & 318.16 & 199.26 & 5.58 \\
\hline 75.0465 & 239.66 & 218.98 & 216.98 & 148.38 & 66.25 & 61.24 & 317.53 & 200.52 & 5.61 \\
\hline 75.54533 & 240.22 & 218.9 & 216.89 & 152.8 & 66.46 & 61.24 & 317.26 & 200.5 & 5.60 \\
\hline 76.04617 & 239.53 & 218.76 & 216.79 & 157.6 & 66.71 & 61.3 & 316.25 & 200.52 & 5.61 \\
\hline 76.54501 & 238.8 & 218.65 & 216.67 & 162.78 & 66.89 & 61.29 & 315.19 & 200.48 & 5.62 \\
\hline 77.04567 & 238.1 & 218.59 & 216.49 & 168.57 & 67.29 & 61.35 & 314.06 & 200.5 & 5.65 \\
\hline 77.5455 & 239.56 & 218.31 & 216.26 & 175.21 & 67.78 & 61.32 & 312.33 & 200.62 & 5.60 \\
\hline 78.04533 & 239.91 & 218.17 & 216 & 182.88 & 68.11 & 61.32 & 310.61 & 200.45 & 5.57 \\
\hline 78.54517 & 239.1 & 218.08 & 215.76 & 189.24 & 68.6 & 61.35 & 309.21 & 200.41 & 5.58 \\
\hline 79.04501 & 239.97 & 217.89 & 215.51 & 194.51 & 69.18 & 61.37 & 307.63 & 200.38 & 5.61 \\
\hline 79.54483 & 239.92 & 217.68 & 215.26 & 199.28 & 69.8 & 61.38 & 305.97 & 200.29 & 5.61 \\
\hline 80.04567 & 239.48 & 217.45 & 215.06 & 203.35 & 70.52 & 61.42 & 304.73 & 200.26 & 5.60 \\
\hline 80.5445 & 238.73 & 217.37 & 214.86 & 206.38 & 71.3 & 61.43 & 303.48 & 200.53 & 5.60 \\
\hline 81.04617 & 239.84 & 217.19 & 214.65 & 208.48 & 72.11 & 61.45 & 302.25 & 200.38 & 5.63 \\
\hline 81.54501 & 240.42 & 217.54 & 214.46 & 210.04 & 73.02 & 61.49 & 301.23 & 200.35 & 5.62 \\
\hline 82.04483 & 240.43 & 217.45 & 214.3 & 211.06 & 73.99 & 61.5 & 300.26 & 200.31 & 5.57 \\
\hline 82.5465 & 239.23 & 217.08 & 214.16 & 211.68 & 75.02 & 61.52 & 299.14 & 200.31 & 5.57 \\
\hline 83.0455 & 238.68 & 216.92 & 213.97 & 212.04 & 76.1 & 61.54 & 297.93 & 200.27 & 5.58 \\
\hline 83.54617 & 238.14 & 216.54 & 213.74 & 212.19 & 77.27 & 61.59 & 296.49 & 199.86 & 5.61 \\
\hline 84.04501 & 237.52 & 216.5 & 213.53 & 212.24 & 78.49 & 61.61 & 295.13 & 199.76 & 5.60 \\
\hline 84.544 & 237.17 & 216.11 & 213.29 & 212.14 & 79.77 & 61.65 & 293.64 & 199.72 & 5.61 \\
\hline 85.0465 & 237.08 & 215.96 & 213.08 & 212.02 & 81.14 & 61.69 & 292.08 & 199.69 & 5.62 \\
\hline 85.54733 & 237.41 & 215.82 & 212.79 & 211.83 & 82.59 & 61.74 & 290.45 & 199.66 & 5.64 \\
\hline 86.04533 & 237.9 & 215.48 & 212.53 & 211.6 & 84.09 & 61.8 & 288.85 & 199.64 & 5.62 \\
\hline 86.54517 & 238.3 & 215.31 & 212.25 & 211.33 & 85.66 & 61.84 & 286.98 & 199.95 & 5.57 \\
\hline 87.04501 & 237.46 & 215.15 & 211.95 & 211.11 & 87.27 & 61.9 & 285.34 & 199.81 & 5.56 \\
\hline 87.54567 & 237.45 & 214.87 & 211.7 & 210.88 & 88.94 & 61.98 & 283.92 & 199.76 & 5.59 \\
\hline
\end{tabular}


Table B2. Continued.

\begin{tabular}{|c|c|c|c|c|c|c|c|c|c|}
\hline $\begin{array}{c}\text { Time } \\
\text { min }\end{array}$ & $\begin{array}{l}\mathrm{T} 1 \\
{ }^{\circ} \mathrm{C} \\
\end{array}$ & $\begin{array}{l}\mathrm{T} 2 \\
{ }^{\circ} \mathrm{C} \\
\end{array}$ & $\begin{array}{l}\mathrm{T} 3 \\
{ }^{\circ} \mathrm{C} \\
\end{array}$ & $\begin{array}{l}\mathrm{T} 4 \\
{ }^{\circ} \mathrm{C} \\
\end{array}$ & $\begin{array}{l}\mathrm{T} 5 \\
{ }^{\circ} \mathrm{C} \\
\end{array}$ & $\begin{array}{l}\mathrm{T} 6 \\
{ }^{\circ} \mathrm{C} \\
\end{array}$ & $\begin{array}{l}\text { Pinj } \\
\text { psig }\end{array}$ & $\begin{array}{l}\text { Pout } \\
\text { psig }\end{array}$ & $\begin{array}{l}\mathrm{Vw} \\
\mathrm{cc} / \mathrm{min}\end{array}$ \\
\hline 88.0455 & 238.06 & 214.48 & 211.45 & 210.65 & 90.64 & 62.04 & 282.52 & 199.73 & 5.62 \\
\hline 88.54617 & 237.13 & 214.22 & 211.24 & 210.46 & 92.39 & 62.12 & 281.33 & 200.06 & 5.60 \\
\hline 89.04517 & 238.12 & 213.97 & 211.03 & 210.23 & 94.13 & 62.2 & 280.37 & 199.86 & 5.61 \\
\hline 89.54583 & 237.01 & 213.81 & 210.9 & 210.13 & 95.99 & 62.3 & 279.69 & 199.82 & 5.61 \\
\hline 90.04666 & 237.2 & 213.94 & 210.77 & 210.02 & 97.96 & 62.4 & 279.16 & 199.82 & 5.65 \\
\hline 90.5455 & 236.95 & 213.98 & 210.69 & 209.94 & 99.91 & 62.52 & 278.72 & 199.79 & 5.62 \\
\hline 91.04533 & 236.95 & 214.15 & 210.62 & 209.87 & 101.97 & 62.66 & 278.34 & 199.82 & 5.56 \\
\hline 91.54517 & 237.5 & 214.02 & 210.55 & 209.78 & 104.08 & 62.79 & 277.85 & 199.81 & 5.56 \\
\hline 92.044 & 236.71 & 213.94 & 210.43 & 209.66 & 106.32 & 62.97 & 276.98 & 199.93 & 5.58 \\
\hline 92.54483 & 237.83 & 213.89 & 210.26 & 209.52 & 108.63 & 63.14 & 275.9 & 199.84 & 5.60 \\
\hline 93.04467 & 237.27 & 213.79 & 210.07 & 209.31 & 111.15 & 63.34 & 274.57 & 199.82 & 5.60 \\
\hline 93.5445 & 238.32 & 213.52 & 209.84 & 209.07 & 113.76 & 63.57 & 273.05 & 199.44 & 5.61 \\
\hline 94.04517 & 238.07 & 213.44 & 209.57 & 208.82 & 116.62 & 63.83 & 271.42 & 200.04 & 5.62 \\
\hline 94.54501 & 237.65 & 213.26 & 209.25 & 208.5 & 119.65 & 64.09 & 269.6 & 200.49 & 5.64 \\
\hline 95.04483 & 236.77 & 212.44 & 208.94 & 208.2 & 122.94 & 64.41 & 267.7 & 200.32 & 5.60 \\
\hline 95.5455 & 236.87 & 211.88 & 208.62 & 207.92 & 126.42 & 64.76 & 265.95 & 200.27 & 5.57 \\
\hline 96.04533 & 237.12 & 211.88 & 208.32 & 207.62 & 130.02 & 65.12 & 264.36 & 200.24 & 5.58 \\
\hline 96.54517 & 236.75 & 211.94 & 208.02 & 207.32 & 133.78 & 65.52 & 262.66 & 200.2 & 5.61 \\
\hline 97.04501 & 235.87 & 211.33 & 207.75 & 207.05 & 137.65 & 65.96 & 261.19 & 200.17 & 5.61 \\
\hline 97.54483 & 236.24 & 211.37 & 207.47 & 206.74 & 141.7 & 66.44 & 259.68 & 200.25 & 5.61 \\
\hline 98.04567 & 236.37 & 211.04 & 207.22 & 206.51 & 146.1 & 66.93 & 258.49 & 200.16 & 5.62 \\
\hline 98.54533 & 235.89 & 211.15 & 207.01 & 206.31 & 150.6 & 67.46 & 257.49 & 200.16 & 5.63 \\
\hline 99.04517 & 236.07 & 211.2 & 206.84 & 206.12 & 155.18 & 68.07 & 256.67 & 200.17 & 5.61 \\
\hline 99.54501 & 235.7 & 210.92 & 206.66 & 205.96 & 159.86 & 68.73 & 255.95 & 200.18 & 5.56 \\
\hline 100.0448 & 236.42 & 211.02 & 206.52 & 205.82 & 164.59 & 69.42 & 255.25 & 200.18 & 5.57 \\
\hline 100.5457 & 236.08 & 210.84 & 206.29 & 205.56 & 170.24 & 70.18 & 253.73 & 200.49 & 5.59 \\
\hline 101.0455 & 236.98 & 210.68 & 206.08 & 205.39 & 175.34 & 71.01 & 252.66 & 200.48 & 5.61 \\
\hline 101.5453 & 235.82 & 210.65 & 205.85 & 205.16 & 180.3 & 71.95 & 251.36 & 200.46 & 5.59 \\
\hline 102.045 & 236.63 & 210.46 & 205.59 & 204.89 & 184.88 & 72.97 & 249.89 & 200.43 & 5.62 \\
\hline 102.5448 & 236.83 & 210.09 & 205.31 & 204.61 & 189.38 & 74.1 & 248.17 & 200.44 & 5.61 \\
\hline 103.0465 & 236.23 & 209.57 & 204.92 & 204.18 & 193.55 & 75.41 & 245.81 & 200.5 & 5.63 \\
\hline 103.5455 & 235.48 & 209.66 & 204.53 & 203.81 & 195.84 & 76.82 & 243.78 & 200.49 & 5.61 \\
\hline 104.0453 & 235.59 & 209.11 & 204.12 & 203.4 & 197.78 & 78.3 & 241.56 & 200.4 & 5.57 \\
\hline 104.5452 & 236.11 & 208.56 & 203.69 & 202.98 & 199.14 & 79.94 & 239.37 & 200.36 & 5.56 \\
\hline 105.045 & 235.81 & 208.49 & 203.27 & 202.57 & 200.08 & 81.63 & 237.1 & 200.28 & 5.58 \\
\hline 105.5465 & 236.15 & 208.26 & 202.64 & 201.87 & 200.71 & 83.45 & 233.54 & 200.52 & 5.60 \\
\hline 106.0455 & 236.21 & 207.62 & 202.09 & 201.41 & 200.69 & 85.34 & 231.12 & 200.39 & 5.59 \\
\hline 106.5443 & 235.16 & 207.07 & 201.65 & 200.98 & 200.54 & 87.34 & 228.95 & 200.23 & 5.59 \\
\hline 107.0452 & 236.68 & 206.39 & 201.24 & 200.58 & 200.33 & 89.45 & 226.98 & 200.07 & 5.60 \\
\hline 107.545 & 235.84 & 206.38 & 200.74 & 200.01 & 199.81 & 91.61 & 224.14 & 200.4 & 5.63 \\
\hline 108.0448 & 234.9 & 206.22 & 200.3 & 199.65 & 199.76 & 93.85 & 222.46 & 200.26 & 5.61 \\
\hline 108.5455 & 234.79 & 205.03 & 200.03 & 199.42 & 199.57 & 96.16 & 221.15 & 200.1 & 5.56 \\
\hline 109.0453 & 235.64 & 204.91 & 199.78 & 199.19 & 199.39 & 98.44 & 220.08 & 199.94 & 5.56 \\
\hline 109.5452 & 235.25 & 204.46 & 199.59 & 199 & 199.25 & 100.72 & 219.21 & 199.88 & 5.57 \\
\hline
\end{tabular}


Table B2. Continued.

\begin{tabular}{|c|c|c|c|c|c|c|c|c|c|}
\hline $\begin{array}{c}\text { Time } \\
\text { min }\end{array}$ & $\begin{array}{l}\mathrm{T} 1 \\
{ }^{\circ} \mathrm{C} \\
\end{array}$ & $\begin{array}{l}\mathrm{T} 2 \\
{ }^{\circ} \mathrm{C} \\
\end{array}$ & $\begin{array}{l}\mathrm{T} 3 \\
{ }^{\circ} \mathrm{C} \\
\end{array}$ & $\begin{array}{l}\mathrm{T} 4 \\
{ }^{\circ} \mathrm{C} \\
\end{array}$ & $\begin{array}{l}\mathrm{T5} \\
{ }^{\circ} \mathrm{C} \\
\end{array}$ & $\begin{array}{l}\mathrm{T} 6 \\
{ }^{\circ} \mathrm{C} \\
\end{array}$ & $\begin{array}{l}\text { Pinj } \\
\text { psig }\end{array}$ & $\begin{array}{l}\text { Pout } \\
\text { psig }\end{array}$ & $\begin{array}{l}\mathrm{Vw} \\
\mathrm{cc} / \mathrm{min}\end{array}$ \\
\hline 110.045 & 234.97 & 204.36 & 199.42 & 198.86 & 199.15 & 102.88 & 218.55 & 199.79 & 5.60 \\
\hline 110.5448 & 234.84 & 203.93 & 199.22 & 198.56 & 198.75 & 104.91 & 217.08 & 200.25 & 5.59 \\
\hline 111.0455 & 234.9 & 204.06 & 198.97 & 198.42 & 198.76 & 106.93 & 216.38 & 200.15 & 5.59 \\
\hline 111.5453 & 235.37 & 203.38 & 198.89 & 198.35 & 198.73 & 108.94 & 216.08 & 200.16 & 5.61 \\
\hline 112.0452 & 234.67 & 203.76 & 198.85 & 198.32 & 198.71 & 110.92 & 215.82 & 200.12 & 5.61 \\
\hline 112.545 & 235.57 & 203.3 & 198.82 & 198.27 & 198.68 & 112.84 & 215.76 & 200.12 & 5.62 \\
\hline 113.0448 & 236.42 & 203.71 & 198.81 & 198.27 & 198.68 & 114.68 & 215.72 & 200.08 & 5.57 \\
\hline 113.5465 & 236.8 & 203.65 & 198.79 & 198.24 & 198.67 & 116.47 & 215.73 & 200.08 & 5.55 \\
\hline 114.0453 & 235.28 & 203.9 & 198.78 & 198.26 & 198.69 & 118.18 & 215.61 & 200.08 & 5.56 \\
\hline 114.5462 & 236.02 & 204.19 & 198.74 & 198.21 & 198.71 & 119.85 & 215.5 & 200.18 & 5.60 \\
\hline 115.045 & \begin{tabular}{|l|}
236.65 \\
\end{tabular} & 204.51 & 198.75 & 198.21 & 198.64 & 121.54 & 215.52 & 200.16 & 5.60 \\
\hline 115.5458 & 234.93 & 203.5 & 198.75 & 198.23 & 198.75 & 123.21 & 215.49 & 200.17 & 5.59 \\
\hline 116.0455 & 235.76 & 203.38 & 198.76 & 198.22 & 198.67 & 124.88 & 215.56 & 200.16 & 5.60 \\
\hline 116.5453 & 235.62 & 203.52 & 198.76 & 198.24 & 198.67 & 126.6 & 215.58 & 200.13 & 5.62 \\
\hline 117.0443 & 235.42 & 203.58 & 198.76 & 198.23 & 198.69 & 128.31 & 215.66 & 200.15 & 5.63 \\
\hline 117.5442 & 234.3 & 203.28 & 198.78 & 198.25 & 198.68 & 130.03 & 215.67 & 200.16 & 5.58 \\
\hline 118.0448 & 235.55 & 203.35 & 198.61 & 197.96 & 198.32 & 131.91 & 214.56 & 200.22 & 5.58 \\
\hline 118.5465 & 234.93 & 204.12 & 198.48 & 197.95 & 198.39 & 134.21 & 214.28 & 200.21 & 5.57 \\
\hline 119.0445 & 234.2 & 204.05 & 198.49 & 197.93 & 198.47 & 136.84 & 214.35 & 200.2 & 5.60 \\
\hline 119.5453 & 235.7 & 203.86 & 198.49 & 197.93 & 198.38 & 139.36 & 214.25 & 200.11 & 5.61 \\
\hline 120.045 & 235.24 & 203.32 & 198.47 & 197.92 & 198.37 & 141.73 & 214.25 & 200.07 & 5.61 \\
\hline 120.5448 & 236.03 & 203.45 & 198.46 & 197.92 & 198.37 & 144.08 & 214.23 & 200.05 & 5.62 \\
\hline 121.0457 & 235.57 & 203.46 & 198.44 & 197.93 & 198.37 & 146.44 & 214.17 & 200.03 & 5.62 \\
\hline 121.5455 & 234.89 & 203.48 & 198.32 & 197.68 & 198 & 149.12 & 213.07 & 200.13 & 5.62 \\
\hline 122.0443 & 234.54 & 202.86 & 198.17 & 197.61 & 198.07 & 152.8 & 212.74 & 200.11 & 5.57 \\
\hline 122.5442 & 235.56 & 203.11 & 198.15 & 197.61 & 198.09 & 155.98 & 212.72 & 200.16 & 5.56 \\
\hline 123.045 & 235.51 & 202.99 & 198.17 & 197.63 & 198.12 & 158.57 & 212.67 & 200.09 & 5.56 \\
\hline 123.5447 & 235 & 203.19 & 198.15 & 197.62 & 198.1 & 160.76 & 212.72 & 200.04 & 5.60 \\
\hline 124.0455 & 235.25 & 203.21 & 198.14 & 197.62 & 198.1 & 162.83 & 212.57 & 199.99 & 5.58 \\
\hline 124.5453 & 236.15 & 202.98 & 198.12 & 197.6 & 198.09 & 164.76 & 212.58 & 199.96 & 5.59 \\
\hline 125.0452 & 235.61 & 202.93 & 198.11 & 197.59 & 198 & 166.68 & 212.5 & 199.94 & 5.62 \\
\hline 125.545 & 236.83 & 203.02 & 198.11 & 197.59 & 198.08 & 168.59 & 212.48 & 199.94 & 5.61 \\
\hline 126.0457 & 236.12 & 203.2 & 197.95 & 197.33 & 197.7 & 173.57 & 211.37 & 199.95 & 5.61 \\
\hline 126.5465 & 236.16 & 203.44 & 197.83 & 197.29 & 197.81 & 177.64 & 211.25 & 200.07 & 5.55 \\
\hline 127.0453 & 234.39 & 203.74 & 197.85 & 197.33 & 197.87 & 179.83 & 211.31 & 200.11 & 5.55 \\
\hline 127.5452 & 236.2 & 203.01 & 197.85 & 197.35 & 197.86 & 181.49 & 211.36 & 200.16 & 5.56 \\
\hline 128.045 & 234.36 & 202.05 & 197.89 & 197.36 & 197.88 & 182.78 & 211.46 & 200.04 & 5.59 \\
\hline 128.5448 & 236.08 & 202.53 & 197.88 & 197.38 & 197.86 & 184.04 & 211.44 & 199.97 & 5.59 \\
\hline 129.0455 & 235.83 & 202.8 & 197.86 & 197.34 & 197.86 & 185.21 & 211.48 & 199.93 & 5.60 \\
\hline 129.5453 & 235.73 & 202.86 & 197.88 & 197.36 & 197.86 & 186.32 & 211.41 & 199.68 & 5.60 \\
\hline 130.0452 & \begin{tabular}{|l|}
235.69 \\
\end{tabular} & 203.28 & 197.87 & 197.35 & 197.85 & 187.29 & 211.41 & 199.57 & 5.60 \\
\hline 130.545 & 236.54 & 202.87 & 197.78 & 197.19 & 197.67 & 190.65 & 210.86 & 200.02 & 5.63 \\
\hline 131.0457 & 235.46 & 202.53 & 197.75 & 197.25 & 197.78 & 191.75 & 211 & 200.23 & 5.58 \\
\hline 131.5455 & 236.7 & 202.62 & 197.8 & 197.28 & 197.82 & 192.38 & 211.18 & 200.28 & 5.54 \\
\hline
\end{tabular}


Table B2. Continued.

\begin{tabular}{|c|c|c|c|c|c|c|c|c|c|}
\hline $\begin{array}{c}\text { Time } \\
\text { min }\end{array}$ & $\begin{array}{l}\mathrm{T} 1 \\
{ }^{\circ} \mathrm{C} \\
\end{array}$ & $\begin{array}{l}\mathrm{T} 2 \\
{ }^{\circ} \mathrm{C} \\
\end{array}$ & $\begin{array}{l}\mathrm{T} 3 \\
{ }^{\circ} \mathrm{C} \\
\end{array}$ & $\begin{array}{l}\mathrm{T} 4 \\
{ }^{\circ} \mathrm{C} \\
\end{array}$ & $\begin{array}{l}\mathrm{T} 5 \\
{ }^{\circ} \mathrm{C} \\
\end{array}$ & $\begin{array}{l}\mathrm{T} 6 \\
{ }^{\circ} \mathrm{C} \\
\end{array}$ & $\begin{array}{l}\text { Pinj } \\
\text { psig }\end{array}$ & $\begin{array}{l}\text { Pout } \\
\text { psig }\end{array}$ & $\begin{array}{l}\mathrm{Vw} \\
\mathrm{cc} / \mathrm{min}\end{array}$ \\
\hline 132.0453 & 236.13 & 203.16 & 197.82 & 197.32 & 197.84 & 192.92 & 211.34 & 200.3 & 5.54 \\
\hline 132.5452 & 235.45 & 203.29 & 197.88 & 197.32 & 197.86 & 193.38 & 211.43 & 200.29 & 5.56 \\
\hline 133.045 & 236.32 & 203.42 & 197.88 & 197.35 & 197.86 & 193.85 & 211.49 & 200.2 & 5.60 \\
\hline 133.5448 & 236.25 & 203.01 & 197.9 & 197.36 & 197.88 & 194.37 & 211.6 & 200.15 & 5.59 \\
\hline 134.0455 & 236.02 & 203.22 & 197.91 & 197.37 & 197.89 & 194.93 & 211.6 & 200.16 & 5.59 \\
\hline 134.5453 & 235.21 & 202.83 & 197.91 & 197.39 & 197.89 & 195.31 & 211.64 & 200.17 & 5.59 \\
\hline 135.0452 & 235.56 & 202.6 & 197.93 & 197.39 & 197.89 & 195.71 & 211.71 & 200.05 & 5.61 \\
\hline 135.545 & 235.38 & 203.36 & 197.8 & 197.2 & 197.66 & 196.48 & 210.97 & 199.9 & 5.63 \\
\hline 136.0458 & 236.01 & 203.25 & 197.75 & 197.21 & 197.75 & 196.88 & 210.95 & 200.11 & 5.58 \\
\hline 136.5457 & 235.08 & 203.4 & 197.79 & 197.27 & 197.81 & 196.97 & 211.15 & 200.23 & 5.55 \\
\hline 137.0455 & 235.21 & 202.36 & 197.81 & 197.29 & 197.79 & 197.02 & 211.21 & 200.24 & 5.57 \\
\hline 137.5453 & 236.14 & 202.7 & 197.81 & 197.28 & 197.81 & 197.04 & .26 & 200.11 & 5.60 \\
\hline 138.045 & 235.29 & 203.15 & 197.82 & 197.28 & 197.89 & 197.06 & 211.19 & 200.03 & 5.60 \\
\hline 138.5448 & 234.03 & 202.9 & 197.82 & 197.28 & 197.77 & 197.07 & 211.19 & 199.86 & 5.60 \\
\hline 139.0457 & 235.93 & 203.21 & 197.8 & 197.27 & 197.77 & 197.11 & 211.21 & 199.8 & 5.61 \\
\hline 139.5455 & 235.18 & 202.8 & 197.81 & 197.27 & 197.79 & 197.11 & 1.22 & 200.05 & 5.62 \\
\hline 140.0453 & 236.42 & 203.13 & 197.81 & 197.31 & 197.84 & 197.22 & .29 & 45 & 5.62 \\
\hline 140.5452 & 235.96 & 202.74 & 197.76 & 197.17 & 197.65 & 197.01 & 210.81 & 200.15 & 5.57 \\
\hline 141.045 & 235.32 & 202.54 & 197.74 & 197.22 & 197.78 & 197.2 & 210.88 & 200.44 & 5.55 \\
\hline 141.5465 & 235.27 & 202.79 & 197.8 & 197.28 & 197.85 & 197.31 & 211.25 & 200.6 & 5.57 \\
\hline 142.0455 & 236.61 & 202.96 & 197.85 & 197.33 & 197.89 & 197.32 & 211.41 & 200.52 & 5.60 \\
\hline 142.5453 & 235.75 & 203.85 & 197.89 & 197.37 & 197.89 & 197.34 & 211.55 & 200.54 & 5.61 \\
\hline 143.0452 & 236.74 & 203.75 & 197.89 & 197.36 & 197.89 & 197.32 & 211.64 & 200.48 & 5.60 \\
\hline 143.545 & 235.2 & 203.63 & 197.91 & 197.38 & 197.9 & 197.34 & 211.7 & 200.3 & 5.60 \\
\hline 144.0457 & 236.42 & 203.05 & 197.92 & 197.38 & 197.91 & 197.34 & 211.63 & 200.45 & 5.65 \\
\hline 144.5455 & 236.69 & 203.77 & 197.9 & 197.38 & 197.9 & 197.33 & 211.66 & 200.37 & 5.62 \\
\hline 145.0453 & 236.25 & 203.4 & 197.92 & 197.38 & 197.9 & 197.33 & 211.73 & 200.18 & 5.56 \\
\hline 145.546 & 236.04 & 203.46 & 197.94 & 197.4 & 197.91 & 197.37 & 211.87 & 200.16 & 5.57 \\
\hline 146.045 & 234.68 & 203.17 & 197.94 & 197.41 & 197.93 & 197.37 & 211.88 & 200.32 & 5.59 \\
\hline 146.5438 & 235.39 & 202.91 & 197.86 & 197.25 & 197.69 & 197.14 & 211.19 & 199.89 & 5.61 \\
\hline 147.0455 & 235.11 & 203.32 & 197.8 & 197.27 & 197.84 & 197.32 & 211.32 & 200.32 & 5.62 \\
\hline 147.5445 & 235.15 & 203.41 & 197.86 & 197.34 & 197.97 & 197.38 & 211.64 & 200.42 & 5.60 \\
\hline 148.0443 & 236.06 & 203.86 & 197.9 & 197.38 & 197.93 & 197.41 & 211.7 & 200.55 & 5.62 \\
\hline 148.545 & 236.23 & 203.54 & 197.92 & 197.4 & 197.83 & 197.41 & 211.87 & 200.37 & 5.64 \\
\hline 149.0448 & 237.25 & 202.74 & 197.94 & 197.4 & 197.92 & 197.38 & 211.96 & 200.28 & 5.59 \\
\hline 149.5455 & 237.52 & 203.01 & 197.96 & 197.42 & 197.92 & 197.4 & 211.91 & 200.26 & 5.55 \\
\hline 150.0453 & 235.88 & 203.85 & 197.94 & 197.42 & 197.93 & 197.39 & 211.84 & 200.27 & 5.56 \\
\hline 150.5452 & 235.02 & 203.75 & 197.95 & 197.41 & 197.91 & 197.41 & 211.83 & 200.28 & 5.59 \\
\hline 151.045 & 236.53 & 203.62 & 197.93 & 197.39 & 197.91 & 197.37 & 211.79 & 200.16 & 5.60 \\
\hline 151.5448 & 235.72 & 202.89 & 197.93 & 197.39 & 197.91 & 197.39 & 211.77 & 200.3 & 5.60 \\
\hline 152.0455 & 235.11 & 202.89 & 197.93 & 197.4 & 197.9 & 197.4 & 211.87 & 200.13 & 5.62 \\
\hline 152.5453 & 235.76 & 203.79 & 197.94 & 197.38 & 197.9 & 197.4 & 211.76 & 200.21 & 5.62 \\
\hline 153.0452 & 236.48 & 203.85 & 197.92 & 197.4 & 197.93 & 197.45 & 211.81 & 200.31 & 5.63 \\
\hline 153.545 & 236.36 & 203.17 & 197.92 & 197.39 & 197.85 & 197.42 & 211.83 & 200.22 & 5.57 \\
\hline
\end{tabular}


Table B2. Continued.

\begin{tabular}{|c|c|c|c|c|c|c|c|c|c|}
\hline $\begin{array}{l}\text { Time } \\
\text { min }\end{array}$ & $\begin{array}{l}\mathrm{T} 1 \\
{ }^{\circ} \mathrm{C}\end{array}$ & $\begin{array}{l}\mathrm{T} 2 \\
{ }^{\circ} \mathrm{C}\end{array}$ & $\begin{array}{l}\mathrm{T} 3 \\
{ }^{\circ} \mathrm{C}\end{array}$ & $\begin{array}{l}\mathrm{T} 4 \\
{ }^{\circ} \mathrm{C}\end{array}$ & $\begin{array}{l}\mathrm{T} 5 \\
{ }^{\circ} \mathrm{C}\end{array}$ & $\begin{array}{l}\mathrm{T} 6 \\
{ }^{\circ} \mathrm{C}\end{array}$ & $\begin{array}{l}\text { Pinj } \\
\text { psig }\end{array}$ & $\begin{array}{l}\text { Pout } \\
\text { psig }\end{array}$ & $\begin{array}{c}\mathrm{Vw} \\
\mathrm{cc} / \mathrm{min}\end{array}$ \\
\hline 154.044 & 237.15 & 204.16 & 197.83 & 197.25 & 197.73 & 197.28 & 210.35 & 199.85 & 5.57 \\
\hline 154.5457 & 236.09 & 203.35 & 197.82 & 197.28 & 197.82 & 197.35 & 211.34 & 200.22 & 5.57 \\
\hline 155.0445 & 236.31 & 202.1 & 197.86 & 197.34 & 197.89 & 197.45 & 211.17 & 200.47 & 5.60 \\
\hline 155.5443 & 235.17 & 202.94 & 197.9 & 197.38 & 197.95 & 197.5 & 211.62 & 200.57 & 5.60 \\
\hline 156.045 & 235.94 & 203.25 & 197.91 & 197.4 & 197.95 & 197.74 & 211.87 & 200.53 & 5.60 \\
\hline 156.5448 & 235.51 & 202.12 & 197.96 & 197.43 & 197.99 & 197.52 & 212.04 & 200.56 & 5.62 \\
\hline 157.0447 & 234.61 & 202.39 & 197.98 & 197.44 & 197.99 & 197.51 & 212.02 & 200.42 & 5.64 \\
\hline 157.5455 & 236.07 & 203.51 & 197.97 & 197.45 & 197.99 & 197.47 & 212.11 & 200.31 & 5.61 \\
\hline 158.0453 & 235.84 & 204.06 & 197.98 & 197.46 & 197.99 & 197.46 & 212.2 & 200.23 & 5.57 \\
\hline 158.5452 & 235.3 & 204.1 & 197.91 & 197.26 & 197.67 & 196.97 & 211.44 & 197.43 & 5.55 \\
\hline 159.045 & 234.96 & 203.75 & 197.66 & 197.03 & 197.43 & 196.87 & 210.44 & 198.31 & 5.57 \\
\hline 159.5447 & 235.99 & 203.41 & 197.63 & 197.11 & 197.7 & 197.29 & 210.57 & 200.06 & 5.60 \\
\hline 160.0455 & 236.89 & 203.93 & 197.72 & 197.23 & 197.84 & 197.41 & 211.22 & 200.4 & 5.60 \\
\hline 160.5453 & 236.63 & 203.16 & 197.84 & 197.34 & 197.92 & 197.45 & 211.65 & 200.49 & 5.60 \\
\hline 161.0452 & 236.34 & 203.56 & 197.88 & 197.38 & 197.94 & 197.47 & 211.85 & 200.33 & 5.61 \\
\hline 161.545 & 236.66 & 203.56 & 197.94 & 197.4 & 197.97 & 197.49 & 212.05 & 200.56 & 5.61 \\
\hline 162.0457 & 236.45 & 203.31 & 197.96 & 197.44 & 197.98 & 197.47 & 212.14 & 200.35 & 5.63 \\
\hline 162.5455 & 235.55 & 203.5 & 197.96 & 197.44 & 197.96 & 197.48 & 212.04 & 200.22 & 5.57 \\
\hline 163.0453 & 235.29 & 203.67 & 197.96 & 197.43 & 197.96 & 197.48 & 211.89 & 200.11 & 5.56 \\
\hline 163.5442 & 236.36 & 203.68 & 197.95 & 197.42 & 197.93 & 197.43 & 211.97 & 200.08 & 5.58 \\
\hline 164.044 & 237.06 & 202.98 & 197.95 & 197.41 & 197.94 & 197.5 & 211.98 & 200.03 & 5.58 \\
\hline 164.5448 & 236.08 & 203.38 & 197.95 & 197.4 & 197.83 & 197.42 & 212.1 & 200.02 & 5.60 \\
\hline 165.0447 & 236.21 & 204.22 & 197.95 & 197.42 & 197.94 & 197.52 & 212 & 199.9 & 5.61 \\
\hline 165.5453 & 236.66 & 203.45 & 197.94 & 197.4 & 197.92 & 197.4 & 211.98 & 200 & 5.62 \\
\hline 166.0452 & 236.02 & 203.11 & 197.94 & 197.42 & 197.92 & 197.44 & 212.03 & 200.11 & 5.64 \\
\hline 166.545 & 234.58 & 203.21 & 197.96 & 197.42 & 197.96 & 197.46 & 212.07 & 200.14 & 5.61 \\
\hline 167.0448 & 235.68 & 202.99 & 197.94 & 197.44 & 197.94 & 197.46 & 212.07 & 200.08 & 5.57 \\
\hline 167.5455 & 235.61 & 204.19 & 197.96 & 197.43 & 197.95 & 197.45 & 212.13 & 199.89 & 5.56 \\
\hline 168.0453 & 236.45 & 203.78 & 197.97 & 197.41 & 197.93 & 197.39 & 212.05 & 199.63 & 5.60 \\
\hline 168.5452 & 234.61 & 202.82 & 197.86 & 197.27 & 197.75 & 197.27 & 211.44 & 199.88 & 5.60 \\
\hline 169.045 & 235.12 & 203.45 & 197.83 & 197.33 & 197.9 & 197.43 & 211.59 & 200.54 & 5.60 \\
\hline 169.544 & 235.12 & 203.45 & 197.9 & 197.38 & 197.97 & 197.54 & 211.9 & 200.68 & 5.61 \\
\hline 170.0455 & 236.59 & 203.33 & 197.94 & 197.44 & 198.03 & 197.58 & 212.13 & 200.81 & 5.64 \\
\hline 170.5445 & 235.91 & 204.17 & 197.99 & 197.48 & 198.12 & 197.6 & 212.46 & 200.89 & 5.64 \\
\hline 171.0462 & 235.21 & 203.91 & 198.03 & 197.51 & 198.05 & 197.58 & 212.51 & 200.8 & 5.58 \\
\hline 171.5442 & 236.58 & 204.71 & 198.04 & 197.52 & 198.04 & 197.57 & 212.41 & 200.58 & 5.57 \\
\hline 172.0448 & 236.17 & 203.7 & 198.04 & 197.48 & 198 & 197.5 & 212.41 & 200.17 & 5.58 \\
\hline 172.5465 & 236.87 & 203.95 & 198 & 197.47 & 197.86 & 197.47 & 212.2 & 199.95 & 5.61 \\
\hline 173.0445 & 237.21 & 203.9 & 197.99 & 197.43 & 197.95 & 197.45 & 212.25 & 200.04 & 5.60 \\
\hline 173.5453 & 235.66 & 204.26 & 197.97 & 197.44 & 197.97 & 197.47 & 212.06 & 200.03 & 5.61 \\
\hline 174.045 & 236.79 & 204.2 & 197.94 & 197.4 & 197.94 & 197.46 & 212.02 & 199.96 & 5.62 \\
\hline 174.5448 & 235.87 & 204.03 & 197.94 & 197.4 & 197.92 & 197.46 & 212.03 & 200.06 & 5.62 \\
\hline 175.0457 & 235.89 & 204.07 & 197.94 & 197.39 & 197.76 & 197.23 & 212.03 & 196.56 & 5.59 \\
\hline 175.5455 & 236.79 & 203.64 & 197.8 & 197.25 & 197.78 & 197.32 & 211.32 & 199.96 & 5.56 \\
\hline
\end{tabular}


Table B2. Continued.

\begin{tabular}{|c|c|c|c|c|c|c|c|c|c|}
\hline $\begin{array}{c}\text { Time } \\
\text { min }\end{array}$ & $\begin{array}{l}\mathrm{T} 1 \\
{ }^{\circ} \mathrm{C} \\
\end{array}$ & $\begin{array}{l}\mathrm{T} 2 \\
{ }^{\circ} \mathrm{C} \\
\end{array}$ & $\begin{array}{l}\mathrm{T} 3 \\
{ }^{\circ} \mathrm{C} \\
\end{array}$ & $\begin{array}{l}\mathrm{T} 4 \\
{ }^{\circ} \mathrm{C} \\
\end{array}$ & $\begin{array}{l}\mathrm{T} 5 \\
{ }^{\circ} \mathrm{C} \\
\end{array}$ & $\begin{array}{l}\mathrm{T} 6 \\
{ }^{\circ} \mathrm{C} \\
\end{array}$ & $\begin{array}{l}\text { Pinj } \\
\text { psig }\end{array}$ & $\begin{array}{l}\text { Pout } \\
\text { psig }\end{array}$ & $\begin{array}{l}\mathrm{Vw} \\
\mathrm{cc} / \mathrm{min}\end{array}$ \\
\hline 176.0453 & 236.69 & 203.52 & 197.82 & 197.32 & 197.91 & 197.46 & 211.68 & 200.47 & 5.55 \\
\hline 176.5452 & 235.18 & 203.8 & 197.91 & 197.41 & 198.02 & 197.57 & 211.98 & 200.88 & 5.58 \\
\hline 177.045 & 236.3 & 204.07 & 197.99 & 197.47 & 198.06 & 197.63 & 212.23 & 200.94 & 5.59 \\
\hline 177.5457 & 236.12 & 203.88 & 198.03 & 197.51 & 198.1 & 197.65 & 212.43 & 200.94 & 5.60 \\
\hline 178.0455 & 235.87 & 203.72 & 198.03 & 197.52 & 198.1 & 197.65 & 212.5 & 200.87 & 5.62 \\
\hline 178.5453 & 234.98 & 203.56 & 198.05 & 197.55 & 198.1 & 197.63 & 212.68 & 200.73 & 5.62 \\
\hline 179.0442 & 235 & 203.87 & 198.09 & 197.53 & 198.07 & 197.58 & 212.55 & 200.36 & 5.64 \\
\hline 179.545 & 236.41 & 204.01 & 198.02 & 197.5 & 198.03 & 197.53 & 212.49 & 200.19 & 5.61 \\
\hline 180.0457 & 236.83 & 204.61 & 198 & 197.45 & 197.95 & 197.39 & 212.3 & 195.63 & 5.56 \\
\hline 180.5447 & 236.87 & 203.73 & 197.81 & 197.18 & 197.54 & 196.98 & 211.23 & 197.41 & 5.57 \\
\hline 181.0443 & 235.08 & 204.04 & 197.61 & 196.99 & 197.54 & 196.95 & 210.41 & 198.34 & 5.60 \\
\hline 181.5452 & 236.57 & 203.92 & 197.58 & 197.08 & 197.67 & 197.27 & 210.56 & 200 & 5.62 \\
\hline 182.045 & 236.93 & 202.9 & 197.71 & 197.22 & 197.85 & 197.44 & 211.26 & 200.37 & 5.61 \\
\hline 182.5448 & 236.72 & 203.06 & 197.82 & 197.32 & 197.91 & 197.49 & 211.61 & 200.54 & 5.63 \\
\hline 183.0455 & 236 & 202.64 & 197.91 & 197.41 & 198.02 & 197.59 & 211.95 & 200.7 & 5.65 \\
\hline 183.5453 & 235.4 & 202.84 & 197.95 & 197.45 & 198.05 & 197.61 & 212.16 & 200.7 & 5.62 \\
\hline 184.0452 & 235.45 & 203.89 & 197.99 & 197.5 & 198.06 & 197.59 & .26 & .73 & 5.59 \\
\hline 184.545 & 237.05 & 203.74 & 197.99 & 197.49 & 198.04 & 197.59 & 212.31 & 200.58 & 5.60 \\
\hline 185.0448 & 236.8 & 203.34 & 197.99 & 197.47 & 197.94 & 197.54 & 212.28 & 200.25 & 5.62 \\
\hline 185.5455 & 236.03 & 203.72 & 197.98 & 197.46 & 197.98 & 197.51 & 212.27 & 200.01 & 5.61 \\
\hline 186.0453 & 235.93 & 202.86 & 197.94 & 197.4 & 197.94 & 197.46 & 211.97 & 199.78 & 5.63 \\
\hline 186.5443 & 237.18 & 203.94 & 197.91 & 197.39 & 197.94 & 197.46 & 211.95 & 199.97 & 5.65 \\
\hline 187.0442 & 236.69 & 203.1 & 197.93 & 197.41 & 197.98 & 197.5 & 211.98 & 199.95 & 5.65 \\
\hline 187.5448 & 235 & 203.91 & 197.95 & 197.39 & 197.97 & 197.48 & 212.04 & 200.03 & 5.59 \\
\hline 188.0455 & 235.94 & 203.34 & 197.95 & 197.4 & 197.95 & 197.43 & 212.07 & 199.46 & 5.59 \\
\hline 188.5453 & 236.24 & 203.81 & 197.88 & 197.29 & 197.67 & 197.09 & 211.59 & 196.72 & 5.61 \\
\hline 189.0443 & 236.84 & 204.01 & 197.67 & 197.1 & 197.58 & 197.06 & 210.7 & 198.21 & 5.62 \\
\hline 189.545 & 236.95 & 203.35 & 197.62 & 197.04 & 197.53 & 197.03 & 210.59 & 198.02 & 5.60 \\
\hline 190.0448 & 236.14 & 202.62 & 197.58 & 197.07 & 197.64 & 197.17 & 210.53 & 198.98 & 5.63 \\
\hline 190.5447 & 235.95 & 203.32 & 197.66 & 197.12 & 197.82 & 197.34 & 210.9 & 199.81 & 5.65 \\
\hline 191.0455 & 236.45 & 203.59 & 197.73 & 197.25 & 197.86 & 197.45 & 211.16 & 200.03 & 5.63 \\
\hline 191.5453 & 236.46 & 203.84 & 197.81 & 197.29 & 197.9 & 197.48 & 211.52 & 200.27 & 5.59 \\
\hline 192.045 & 235.22 & 203.99 & 197.86 & 197.36 & 197.95 & 197.56 & 211.75 & 200.32 & 5.56 \\
\hline 192.5448 & 236.32 & 204.35 & 197.88 & 197.38 & 197.96 & 197.53 & 211.78 & 200.2 & 5.63 \\
\hline 193.0457 & 235.6 & 203.74 & 197.89 & 197.39 & 197.98 & 197.55 & 211.84 & 200.23 & 5.60 \\
\hline 193.5455 & 235.71 & 203.28 & 197.91 & 197.41 & 198 & 197.53 & 211.9 & 200.2 & 5.62 \\
\hline 194.0453 & 236.7 & 203.25 & 197.93 & 197.41 & 197.98 & 197.53 & 211.92 & 200.21 & 5.65 \\
\hline 194.5452 & 237.37 & 203.52 & 197.91 & 197.39 & 197.97 & 197.52 & 212.03 & 200.16 & 5.65 \\
\hline 195.044 & 236.33 & 203.02 & 197.93 & 197.39 & 197.97 & 197.5 & 211.97 & 200 & 5.59 \\
\hline 195.5465 & 235.4 & 203.81 & 197.9 & 197.38 & 197.92 & 197.45 & 211.84 & 199.82 & 5.57 \\
\hline 196.0463 & 236.53 & 204.69 & 197.9 & 197.36 & 197.94 & 197.45 & 211.78 & 199.8 & 5.60 \\
\hline 196.5443 & 235.14 & 204.12 & 197.89 & 197.38 & 197.94 & 197.48 & 211.81 & 200.01 & 5.61 \\
\hline 197.0442 & 236.36 & 204.25 & 197.89 & 197.37 & 197.96 & 197.51 & 211.86 & 200 & 5.59 \\
\hline 197.545 & 236.52 & 204.41 & 197.91 & 197.39 & 197.96 & 197.5 & 212.05 & 200.03 & 5.60 \\
\hline
\end{tabular}


Table B2. Continued.

\begin{tabular}{|c|c|c|c|c|c|c|c|c|c|}
\hline $\begin{array}{c}\text { Time } \\
\text { min }\end{array}$ & $\begin{array}{l}\mathrm{T} 1 \\
{ }^{\circ} \mathrm{C}\end{array}$ & $\begin{array}{l}\mathrm{T} 2 \\
{ }^{\circ} \mathrm{C}\end{array}$ & $\begin{array}{l}\mathrm{T} 3 \\
{ }^{\circ} \mathrm{C}\end{array}$ & $\begin{array}{l}\mathrm{T} 4 \\
{ }^{\circ} \mathrm{C}\end{array}$ & $\begin{array}{l}\mathrm{T} 5 \\
{ }^{\circ} \mathrm{C}\end{array}$ & $\begin{array}{l}\mathrm{T} 6 \\
{ }^{\circ} \mathrm{C}\end{array}$ & $\begin{array}{l}\text { Pinj } \\
\text { psig }\end{array}$ & $\begin{array}{c}\text { Pout } \\
\text { psig }\end{array}$ & $\begin{array}{c}\mathrm{Vw} \\
\mathbf{c c} / \mathbf{m i n}\end{array}$ \\
\hline 198.0448 & 237.22 & 204.56 & 197.93 & 197.41 & 197.97 & 197.52 & 212 & 200.15 & 5.61 \\
\hline 198.5455 & 235.71 & 204.43 & 197.9 & 197.34 & 197.84 & 197.36 & 211.74 & 199.41 & 5.63 \\
\hline 199.0453 & 236.71 & 204.29 & 197.85 & 197.31 & 197.85 & 197.38 & 211.65 & 199.58 & 5.59 \\
\hline 199.5452 & 236.36 & 204.01 & 197.83 & 197.33 & 197.89 & 197.42 & 211.6 & 199.71 & 5.57 \\
\hline 200.045 & 236.06 & 203.53 & 197.84 & 197.33 & 197.96 & 197.44 & 211.62 & 199.78 & 5.56 \\
\hline 200.5457 & 236.7 & 204.12 & 197.86 & 197.34 & 197.91 & 197.45 & 211.73 & 199.89 & 5.61 \\
\hline 201.0455 & 234.54 & 203.43 & 197.88 & 197.36 & 197.91 & 197.48 & 211.83 & 199.95 & 5.59 \\
\hline 201.5453 & 236.05 & 203.88 & 197.86 & 197.38 & 197.93 & 197.49 & 211.78 & 199.93 & 5.61 \\
\hline 202.0452 & 236.43 & 203.33 & 197.87 & 197.35 & 197.92 & 197.47 & 211.86 & 199.89 & 5.60 \\
\hline 202.5458 & 236.82 & 203.53 & 197.87 & 197.37 & 197.92 & 197.47 & 211.79 & 199.89 & 5.63 \\
\hline 203.0457 & 237.27 & 203.33 & 197.89 & 197.35 & 197.92 & 197.48 & 211.94 & 199.9 & 5.61 \\
\hline 203.5455 & 236.34 & 203.5 & 197.87 & 197.37 & 197.93 & 197.5 & 211.78 & 199.92 & 5.58 \\
\hline 204.0453 & 236.13 & 203.96 & 197.86 & 197.36 & 197.93 & 197.48 & 211.77 & 199.94 & 5.58 \\
\hline 204.5452 & 237.12 & 203.38 & 197.86 & 197.36 & 197.93 & 197.49 & 211.78 & 200.01 & 5.60 \\
\hline 205.045 & 236.6 & 203.16 & 197.88 & 197.38 & 197.97 & 197.56 & 211.87 & 200.56 & 5.60 \\
\hline 205.5448 & 237.18 & 202.74 & 197.92 & 197.42 & 198.05 & 197.64 & 212.11 & 200.91 & 5.61 \\
\hline 206.0455 & 236.88 & 203.37 & 197.98 & 197.49 & 198.1 & 197.71 & 212.27 & 201.17 & 5.60 \\
\hline 206.5463 & 236.51 & 203.91 & 198.02 & 197.53 & 198.18 & 197.77 & 212.51 & 201.4 & 5.61 \\
\hline 207.0452 & 237.22 & 202.89 & 198.07 & 197.59 & 198.22 & 197.82 & 212.79 & 201.62 & 5.62 \\
\hline 207.5442 & 236.4 & 203.29 & 198.11 & 197.65 & 198.26 & 197.86 & 213.03 & 201.83 & 5.57 \\
\hline 208.0448 & 237.18 & 202.93 & 198.17 & 197.7 & 198.31 & 197.92 & 213.32 & 201.94 & 5.56 \\
\hline 208.5457 & 236.12 & 203.19 & 198.17 & 197.58 & 197.92 & 197.35 & 213 & 199.27 & 5.56 \\
\hline 209.0445 & 236.02 & 204.34 & 197.98 & 197.43 & 197.98 & 197.53 & 212.24 & 200.34 & 5.60 \\
\hline 209.5453 & 236.01 & 204.75 & 197.95 & 197.43 & 197.95 & 197.5 & 212.15 & 199.66 & 5.59 \\
\hline 210.045 & 237.05 & 205.06 & 197.92 & 197.36 & 197.86 & 197.36 & 211.98 & 199.05 & 5.59 \\
\hline 210.5448 & 237.28 & 204.65 & 197.83 & 197.27 & 197.79 & 197.31 & 211.7 & 198.92 & 5.59 \\
\hline 211.0457 & 235.66 & 203.88 & 197.78 & 197.24 & 197.78 & 197.31 & 211.5 & 199.1 & 5.61 \\
\hline 211.5455 & 235.79 & 203.69 & 197.76 & 197.23 & 197.75 & 197.3 & 211.43 & 199.04 & 5.62 \\
\hline 212.0453 & 236.61 & 203.84 & 197.75 & 197.23 & 197.78 & 197.34 & 211.42 & 199.35 & 5.58 \\
\hline 212.5452 & 236.35 & 204.98 & 197.75 & 197.23 & 197.82 & 197.39 & 211.51 & 199.45 & 5.56 \\
\hline 213.045 & 235.71 & 204.18 & 197.79 & 197.27 & 197.84 & 197.41 & 211.45 & 199.52 & 5.56 \\
\hline 213.5457 & 236.64 & 203.81 & 197.78 & 197.26 & 197.83 & 197.42 & 211.39 & 199.5 & 5.59 \\
\hline 214.0455 & 235.94 & 204.05 & 197.76 & 197.26 & 197.83 & 197.4 & 211.45 & 199.46 & 5.60 \\
\hline 214.5443 & 236.99 & 203.64 & 197.75 & 197.25 & 197.85 & 197.39 & 211.32 & 199.51 & 5.58 \\
\hline 215.0442 & 237.65 & 204.18 & 197.75 & 197.25 & 197.86 & 197.43 & 211.44 & 199.8 & 5.60 \\
\hline 215.545 & 237.73 & 202.84 & 197.74 & 197.18 & 197.65 & 197.16 & 211.03 & 199.25 & 5.62 \\
\hline 216.0448 & 237.48 & 202.89 & 197.67 & 197.15 & 197.67 & 197.33 & 211 & 200.07 & 5.64 \\
\hline 216.5455 & 237.72 & 203.02 & 197.71 & 197.24 & 197.87 & 197.49 & 211.33 & 200.29 & 5.59 \\
\hline 217.0453 & 236.49 & 202.44 & 197.78 & 197.32 & 197.96 & 197.6 & 211.67 & 200.68 & 5.56 \\
\hline 217.5452 & 236.65 & 203.03 & 197.87 & 197.39 & 198.02 & 197.66 & 211.9 & 200.87 & 5.57 \\
\hline 218.045 & 236.12 & 203.23 & 197.91 & 197.45 & 198.08 & 197.7 & 212.1 & 201.08 & 5.59 \\
\hline 218.5448 & 235.96 & 204.06 & 197.95 & 197.49 & 198.13 & 197.76 & 212.37 & 201.49 & 5.59 \\
\hline 219.0455 & 236.41 & 203.58 & 198.03 & 197.56 & 198.21 & 197.85 & 212.62 & 201.74 & 5.59 \\
\hline
\end{tabular}


Table B2. Continued.

\begin{tabular}{|c|c|c|c|c|c|c|c|c|c|}
\hline $\begin{array}{c}\text { Time } \\
\text { min }\end{array}$ & $\begin{array}{l}\mathrm{T} 1 \\
{ }^{\circ} \mathrm{C} \\
\end{array}$ & $\begin{array}{l}\mathrm{T} 2 \\
{ }^{\circ} \mathrm{C}\end{array}$ & $\begin{array}{l}\mathrm{T} 3 \\
{ }^{\circ} \mathrm{C}\end{array}$ & $\begin{array}{l}\mathrm{T} 4 \\
{ }^{\circ} \mathrm{C}\end{array}$ & $\begin{array}{l}\mathrm{T} 5 \\
{ }^{\circ} \mathrm{C}\end{array}$ & $\begin{array}{l}\mathrm{T} 6 \\
{ }^{\circ} \mathrm{C} \\
\end{array}$ & $\begin{array}{l}\text { Pinj } \\
\text { psig }\end{array}$ & $\begin{array}{l}\text { Pout } \\
\text { psig }\end{array}$ & $\begin{array}{c}\mathrm{Vw} \\
\mathrm{cc} / \mathrm{min}\end{array}$ \\
\hline 219.5453 & 236.14 & 203.49 & 198.09 & 197.62 & 198.28 & 197.92 & 212.97 & 202.17 & 5.59 \\
\hline 220.0452 & 235.9 & 203.69 & 198.16 & 197.7 & 198.34 & 197.98 & 213.31 & 202.56 & 5.63 \\
\hline 220.544 & 236.42 & 202.89 & 198.25 & 197.79 & 198.43 & 198.06 & 213.72 & 202.91 & 5.63 \\
\hline 221.0438 & 236.37 & 203.77 & 198.33 & 197.88 & 198.51 & 198.17 & 214.13 & 203.32 & 5.58 \\
\hline 221.5455 & 236.93 & 203.9 & 198.4 & 197.94 & 198.6 & 198.24 & 214.53 & 203.7 & 5.57 \\
\hline 222.0445 & 237.09 & 204.49 & 198.48 & 198.03 & 198.68 & 198.32 & 214.96 & 204.13 & 5.58 \\
\hline 222.5443 & 236.31 & 203.33 & 198.59 & 198.14 & 198.79 & 198.43 & 215.43 & 204.47 & 5.60 \\
\hline 223.045 & 234.79 & 203.87 & 198.66 & 198.22 & 198.86 & 198.47 & 215.89 & 204.78 & 5.59 \\
\hline 223.5448 & 236.67 & 203.11 & 198.7 & 198.09 & 198.35 & 197.74 & 215.46 & 198.57 & 5.58 \\
\hline 224.0455 & 235.87 & 203.67 & 198.24 & 197.56 & 197.86 & 197.24 & 213.15 & 197.95 & 5.61 \\
\hline 224.5453 & 236.12 & 204.83 & 197.91 & 197.28 & 197.69 & 197.19 & 211.89 & 198.5 & 5.64 \\
\hline 225.0452 & 237.01 & 206.18 & 197.78 & 197.26 & 197.87 & 197.44 & 211.71 & 200.42 & 5.62 \\
\hline 225.545 & 236.35 & 204.86 & 197.88 & 197.41 & 198.07 & 197.68 & 212.28 & 201.29 & 5.57 \\
\hline 226.0448 & 237.15 & 204 & 198.02 & 197.56 & 198.2 & 197.84 & 212.88 & 201.91 & 5.57 \\
\hline 226.5457 & 236.71 & 204.09 & 198.13 & 197.67 & 198.33 & 197.95 & 213.33 & 202.34 & 5.59 \\
\hline 227.0455 & 237.56 & 204.47 & 198.23 & 197.78 & 198.42 & 198.06 & .64 & 202.77 & 5.61 \\
\hline 227.5452 & 237.69 & 203.15 & 198.28 & 197.83 & 198.5 & 198.16 & 214.04 & 203.2 & 5.61 \\
\hline 228.0442 & 235.86 & 203.1 & 198.38 & 197.91 & 198.59 & 198.23 & 214.32 & 203.63 & 5.61 \\
\hline 228.544 & 236.38 & 204.16 & 198.42 & 197.97 & 198.67 & 198.29 & 214.54 & 203.96 & 5.65 \\
\hline 229.0457 & 237.11 & 204.54 & 198.49 & 198.04 & 198.72 & 198.35 & 214.68 & 204.24 & 5.65 \\
\hline 229.5445 & 237.77 & 203.74 & 198.53 & 198.06 & 198.69 & 198.28 & 214.87 & 202.39 & 5.58 \\
\hline 230.0453 & 236.61 & 203.53 & 198.41 & 197.85 & 198.28 & 197.73 & 214 & 199.28 & 5.57 \\
\hline 230.546 & 236.95 & 204.07 & 198.11 & 197.53 & 197.98 & 197.46 & 212.67 & 199.81 & 5.59 \\
\hline 231.5457 & 237.1 & 204.33 & 197.9 & 197.36 & 197.94 & 197.47 & 211.6 & 199.74 & 5.61 \\
\hline 232.0455 & 235.71 & 204.8 & 197.85 & 197.33 & 197.92 & 197.45 & 211.81 & 199.43 & 5.62 \\
\hline 232.5453 & 236.05 & 204.35 & 197.83 & 197.31 & 197.89 & 197.44 & 211.77 & 199.59 & 5.62 \\
\hline 233.0452 & 236.61 & 204.48 & 197.84 & 197.32 & 197.89 & 197.46 & 211.78 & 199.6 & 5.62 \\
\hline 233.545 & 235.72 & 205 & 197.8 & 197.32 & 198 & 197.46 & 211.77 & 199.57 & 5.59 \\
\hline 234.0457 & 236.64 & 205.34 & 197.82 & 197.32 & 197.81 & 197.47 & 211.68 & 199.69 & 5.55 \\
\hline 234.5455 & 236.55 & 205.17 & 197.83 & 197.31 & 197.92 & 197.47 & 211.74 & 199.63 & 5.58 \\
\hline 235.0453 & 236.57 & 205.3 & 197.83 & 197.31 & 197.89 & 197.47 & 211.81 & 199.62 & 5.61 \\
\hline 235.5442 & 236.07 & 205.18 & 197.82 & 197.32 & 197.91 & 197.46 & 211.4 & 199.46 & 5.61 \\
\hline 236.045 & 236.61 & 203.94 & 197.78 & 197.3 & 197.89 & 197.45 & 211.41 & 199.62 & 5.60 \\
\hline 236.5448 & 236.28 & 204.36 & 197.79 & 197.29 & 197.86 & 197.47 & 211.54 & 199.52 & 5.60 \\
\hline 237.0447 & 237.09 & 204.38 & 197.77 & 197.27 & 197.87 & 197.45 & 211.39 & 199.59 & 5.63 \\
\hline 237.5453 & 236.61 & 205.08 & 197.78 & 197.28 & 197.87 & 197.46 & 211.47 & 199.45 & 5.59 \\
\hline 238.0452 & 237.35 & 205.03 & 197.76 & 197.26 & 197.94 & 197.43 & 211 & 199.56 & 5.56 \\
\hline 238.545 & 237.37 & 204.57 & 197.75 & 197.25 & 197.84 & 197.43 & 211.31 & 199.54 & 5.56 \\
\hline 239.0448 & 237.14 & 204.43 & 197.75 & 197.29 & 197.9 & 197.48 & 211.38 & 199.94 & 5.59 \\
\hline 239.5455 & 237.39 & 203.47 & 197.77 & 197.33 & 197.94 & 197.58 & 211.58 & 200.14 & 5.59 \\
\hline 240.0453 & 236.32 & 203.6 & 197.81 & 197.33 & 197.96 & 197.54 & 211.64 & 199.89 & 5.58 \\
\hline
\end{tabular}


Table B3-Temperature and production data for run 3

\begin{tabular}{|c|c|c|c|c|c|c|c|c|c|}
\hline $\begin{array}{l}\text { Time } \\
\text { min }\end{array}$ & $\begin{array}{l}\mathrm{T} 1 \\
{ }^{\circ} \mathrm{C} \\
\end{array}$ & $\begin{array}{l}\mathrm{T} 2 \\
{ }^{\circ} \mathrm{C} \\
\end{array}$ & $\begin{array}{l}\mathrm{T} 3 \\
{ }^{\circ} \mathrm{C} \\
\end{array}$ & $\begin{array}{l}\mathrm{T} 4 \\
{ }^{\circ} \mathrm{C} \\
\end{array}$ & $\begin{array}{l}\mathrm{T} 5 \\
{ }^{\circ} \mathrm{C} \\
\end{array}$ & $\begin{array}{l}\mathrm{T} 6 \\
{ }^{\circ} \mathrm{C} \\
\end{array}$ & $\begin{array}{l}\text { Pinj } \\
\text { psig }\end{array}$ & $\begin{array}{l}\text { Pout } \\
\text { psig }\end{array}$ & $\begin{array}{c}\mathrm{Vw} \\
\mathrm{cc} / \mathrm{min}\end{array}$ \\
\hline 4.93E-02 & 242.48 & 66.26 & 62 & 63.91 & 62.19 & 54.89 & 264.05 & 204.59 & 5.59 \\
\hline 0.5455 & \begin{tabular}{|l|}
242.41 \\
\end{tabular} & 177.23 & 67.41 & 63.93 & 62.02 & 54.93 & 206.45 & 201.62 & 5.60 \\
\hline 1.044333 & 243.9 & 192.05 & 65.92 & 63.97 & 62.14 & 54.91 & 206.22 & 201.34 & 5.62 \\
\hline 1.544167 & 246.02 & 194 & 64.63 & 63.99 & 62.14 & 54.93 & 206.13 & 201.28 & 5.63 \\
\hline 2.045 & \begin{tabular}{|l|}
246.72 \\
\end{tabular} & 193.92 & 63.93 & 63.97 & 62.25 & 54.93 & 206.12 & 201.26 & 5.59 \\
\hline 2.544833 & 246.66 & 193.59 & 63.53 & 63.99 & 62.17 & 54.93 & 206.13 & 201.28 & 5.54 \\
\hline 3.0455 & \begin{tabular}{|l|}
246.02 \\
\end{tabular} & 193.28 & 63.29 & 64.01 & 62.19 & 54.93 & 205.93 & 201.1 & 5.55 \\
\hline 3.545333 & 245.46 & 193.28 & 63.12 & 64.03 & 62.19 & 54.98 & 204.41 & 199.61 & 5.57 \\
\hline 4.045167 & \begin{tabular}{|l|}
244.69 \\
\end{tabular} & 192.76 & 62.99 & 64.03 & 62.19 & 54.95 & 206.31 & 201.48 & 5.62 \\
\hline 4.545 & 243.9 & 192.9 & 62.91 & 64.03 & 62.32 & 54.98 & 206.28 & 201.46 & 5.60 \\
\hline 5.044833 & 242.99 & 193.17 & 62.85 & 64.05 & 62.23 & 54.98 & 206.25 & 201.43 & 5.61 \\
\hline 5.5455 & 241.95 & 193.64 & 62.8 & 64.07 & 62.25 & 55 & 206.08 & 201.27 & 5.62 \\
\hline 6.045333 & 241.12 & 193.89 & 62.78 & 64.08 & 62.27 & 55 & 206.22 & 201.38 & 5.65 \\
\hline 6.545166 & 240.26 & 194.41 & 62.76 & 64.1 & 62.27 & 55.02 & 206.15 & 201.32 & 5.60 \\
\hline 7.044167 & 239.03 & 194.71 & 62.76 & 64.12 & 62.21 & 55.04 & 206.3 & 201.46 & 5.58 \\
\hline 7.544833 & \begin{tabular}{|l|}
238.58 \\
\end{tabular} & 194.95 & 62.76 & 64.14 & 62.29 & 55.06 & 206.25 & 201.41 & 5.57 \\
\hline 8.044666 & 237.83 & 195.02 & 62.78 & 64.12 & 62.31 & 55.04 & 206.12 & 201.28 & 5.59 \\
\hline 8.545333 & 236.98 & 195.14 & 62.8 & 64.16 & 62.33 & 55.06 & 206 & 201.17 & 5.61 \\
\hline 9.045167 & 236.21 & 195.22 & 62.82 & 64.16 & 62.34 & 55.08 & 206.28 & 201.43 & 5.61 \\
\hline 9.545 & 235.64 & 195.11 & 62.86 & 64.18 & 62.34 & 55.08 & 206.05 & 201.2 & 5.62 \\
\hline 10.04483 & 234.57 & 195.27 & 62.86 & 64.16 & 62.36 & 55.08 & 205.99 & 201.14 & 5.64 \\
\hline 10.54567 & 233.99 & 195.36 & 62.89 & 64.18 & 62.25 & 55.12 & 206.15 & 201.3 & 5.64 \\
\hline 11.0455 & \begin{tabular}{|l|}
233.78 \\
\end{tabular} & 195.6 & 62.95 & 64.22 & 62.37 & 55.1 & 205.96 & 201.11 & 5.59 \\
\hline 11.54533 & \begin{tabular}{|l|}
233.56 \\
\end{tabular} & 195.72 & 62.99 & 64.18 & 62.37 & 55.1 & 205.98 & \begin{tabular}{|l|}
201.08 \\
\end{tabular} & 5.56 \\
\hline 12.045 & 232.53 & 195.7 & 63.1 & 64.22 & 62.4 & 55.12 & 205.96 & 201.1 & 5.58 \\
\hline 12.54483 & 232.4 & 195.67 & 63.16 & 64.22 & 62.39 & 55.1 & 205.96 & 201.1 & 5.60 \\
\hline 13.0465 & \begin{tabular}{|l|}
232.13 \\
\end{tabular} & 195.88 & 63.24 & 64.24 & 62.42 & 55.14 & 205.93 & 201.1 & 5.60 \\
\hline 13.5455 & 231.58 & 196.21 & 63.33 & 64.24 & 62.43 & 55.16 & 204.06 & 199.21 & 5.60 \\
\hline 14.04433 & 232.6 & 196.35 & 63.43 & 64.26 & 62.43 & 55.18 & 206.56 & 201.68 & 5.62 \\
\hline 14.546 & \begin{tabular}{|l|}
233.39 \\
\end{tabular} & 197 & 63.54 & 64.22 & 62.6 & 55.16 & 206.37 & 201.4 & 5.64 \\
\hline 15.044 & \begin{tabular}{|l|}
234.93 \\
\end{tabular} & 197.62 & 63.73 & 64.19 & 62.48 & 55.18 & 206.38 & 201.38 & 5.63 \\
\hline 15.54467 & 235.47 & 197.86 & 65 & 64.21 & 62.47 & 55.18 & 204.36 & \begin{tabular}{|l|}
199.41 \\
\end{tabular} & 5.57 \\
\hline 16.04633 & 236.09 & 198.04 & 66.53 & 64.21 & 62.47 & 55.16 & 204.58 & 199.51 & 5.55 \\
\hline 16.54533 & 236.29 & 198.43 & 66.83 & 64.21 & 62.47 & 55.18 & 204.59 & 199.51 & 5.56 \\
\hline 17.04517 & \begin{tabular}{|l|}
236.52 \\
\end{tabular} & 198.7 & 67.19 & 64.23 & 62.49 & 55.2 & 206 & \begin{tabular}{|l|}
201.08 \\
\end{tabular} & 5.60 \\
\hline 17.545 & 236.38 & 198.74 & 69.81 & 64.26 & 62.47 & 55.2 & 204.36 & 199.43 & 5.59 \\
\hline 18.04567 & 236.4 & 198.7 & 70.93 & 64.25 & 62.47 & 55.19 & 204.55 & 199.5 & 5.59 \\
\hline 18.5455 & \begin{tabular}{|l|}
236.49 \\
\end{tabular} & 198.67 & 70.31 & 64.27 & 62.41 & 55.21 & 204.55 & 199.53 & 5.59 \\
\hline 19.04533 & 236.74 & 198.79 & 70.23 & 64.25 & 62.49 & 55.25 & 204.71 & 199.57 & 5.61 \\
\hline 19.54517 & 236.83 & 198.67 & 70.61 & 64.27 & 62.49 & 55.21 & 205.87 & 200.99 & 5.63 \\
\hline 20.044 & \begin{tabular}{|l|}
236.92 \\
\end{tabular} & 198.45 & 71.01 & 64.29 & 62.47 & 55.21 & 206.19 & 201.16 & 5.61 \\
\hline 20.54383 & \begin{tabular}{|l|}
236.67 \\
\end{tabular} & 198.69 & 72.66 & 64.31 & 62.49 & 55.25 & 206.13 & \begin{tabular}{|l|}
201.09 \\
\end{tabular} & 5.56 \\
\hline 21.0455 & 236.55 & 198.72 & 75.97 & 64.31 & 62.61 & 55.25 & 205.58 & 200.01 & 5.55 \\
\hline 21.5445 & 236.21 & 198.51 & 84.81 & 64.33 & 62.49 & 55.23 & 204.75 & 199.66 & 5.57 \\
\hline
\end{tabular}


Table B3. Continued.

\begin{tabular}{|c|c|c|c|c|c|c|c|c|c|}
\hline $\begin{array}{c}\text { Time } \\
\min \end{array}$ & $\begin{array}{l}\mathrm{T} 1 \\
{ }^{\circ} \mathrm{C} \\
\end{array}$ & $\begin{array}{l}\mathrm{T} 2 \\
{ }^{\circ} \mathrm{C} \\
\end{array}$ & $\begin{array}{l}\mathrm{T} 3 \\
{ }^{\circ} \mathrm{C} \\
\end{array}$ & $\begin{array}{l}\mathrm{T} 4 \\
{ }^{\circ} \mathrm{C} \\
\end{array}$ & $\begin{array}{l}\mathrm{T} 5 \\
{ }^{\circ} \mathrm{C} \\
\end{array}$ & $\begin{array}{l}\mathrm{T} 6 \\
{ }^{\circ} \mathrm{C} \\
\end{array}$ & $\begin{array}{l}\text { Pinj } \\
\text { psig }\end{array}$ & $\begin{array}{c}\text { Pout } \\
\text { psig }\end{array}$ & $\begin{array}{c}\mathrm{Vw} \\
\mathrm{cc} / \mathrm{min}\end{array}$ \\
\hline 22.04517 & 236.16 & 198.58 & 85.99 & 64.35 & 62.49 & 55.18 & 204.93 & 199.98 & 5.60 \\
\hline 22.545 & 236.12 & 198.8 & 89.01 & 64.37 & 62.48 & 55.23 & 205.71 & 200.54 & 5.59 \\
\hline 23.04483 & 235.85 & 198.98 & 100.37 & 64.39 & 62.5 & 55.25 & 205.87 & 200.51 & 5.58 \\
\hline 23.5455 & 235.55 & 198.91 & 110.57 & 64.39 & 62.44 & 55.25 & 205.7 & 200.5 & 5.61 \\
\hline 24.04533 & 234.78 & 198.78 & 120.28 & 64.41 & 62.42 & 55.24 & 205.69 & 200.48 & 5.62 \\
\hline 24.54617 & 234.75 & 198.89 & 130.32 & 64.45 & 62.52 & 55.27 & 205.79 & 200.49 & 5.64 \\
\hline 25.045 & 234.62 & 199.09 & 142.83 & 64.45 & 62.42 & 55.22 & 205.96 & 200.49 & 5.58 \\
\hline 25.54483 & 234.44 & 199.18 & 151.65 & 64.43 & 62.43 & 55.18 & 205.87 & 200.46 & 5.57 \\
\hline 26.04567 & 233.37 & 199.31 & 157.76 & 64.41 & 62.45 & 55.18 & 205.99 & 200.51 & 5.56 \\
\hline 26.54533 & 233.66 & 199.38 & 163.23 & 64.4 & 62.48 & 55.22 & 205.98 & 200.48 & 5.60 \\
\hline 27.04433 & 233.77 & 199.4 & 167.38 & 64.38 & 62.48 & 55.18 & 206.12 & 200.48 & 5.61 \\
\hline 27.54417 & 233.69 & 199.38 & 171.14 & 64.4 & 62.51 & 55.18 & 206.13 & 200.47 & 5.60 \\
\hline 28.04483 & 234.05 & 199.59 & 174.65 & 64.42 & 62.51 & 55.2 & 206.28 & 200.47 & 5.61 \\
\hline 28.54467 & 235.31 & 199.77 & 177.15 & 64.4 & 62.47 & 55.17 & 206.24 & 200.46 & 5.63 \\
\hline 29.0455 & 236.35 & 200.06 & 178.94 & 64.42 & 62.51 & 55.17 & 206.19 & 200.45 & 5.65 \\
\hline 29.54533 & 236.96 & 200.03 & 180.09 & 64.44 & 62.53 & 55.13 & 206.16 & 200.44 & 5.60 \\
\hline 30.045 & 237.59 & 200.12 & 181.03 & 64.44 & 62.53 & 55.17 & 206.08 & 200.42 & 5.57 \\
\hline 30.54483 & 237.97 & 199.87 & 181.86 & 64.44 & 62.53 & 55.12 & 206.09 & 200.42 & 5.56 \\
\hline 31.04567 & 237.86 & 200.01 & 182.63 & 64.5 & 62.55 & 55.14 & 206.09 & 200.39 & 5.59 \\
\hline 31.5455 & 238.09 & 200.12 & 183.33 & 64.5 & 62.66 & 55.14 & 206.04 & 200.39 & 5.60 \\
\hline 32.04533 & 238.4 & 199.77 & 183.8 & 64.56 & 62.55 & 55.14 & 206.17 & 200.41 & 5.59 \\
\hline 32.54417 & 238.36 & 199.71 & 184.37 & 64.58 & 62.54 & 55.12 & 205.96 & 200.36 & 5.60 \\
\hline 33.044 & 238.37 & 199.91 & 184.8 & 64.65 & 62.56 & 55.16 & 206.01 & 200.35 & 5.60 \\
\hline 33.54467 & 238.22 & 199.46 & 184.99 & 64.71 & 62.52 & 55.14 & 206.13 & 200.35 & 5.66 \\
\hline 34.0455 & 238.06 & 199.32 & 185.65 & 64.81 & 62.54 & 55.16 & 206.1 & 200.34 & 5.61 \\
\hline 34.54533 & 237.87 & 199.32 & 186.08 & 64.98 & 62.54 & 55.18 & 206.11 & 200.35 & 5.56 \\
\hline 35.04517 & 237.85 & 199.95 & 186.94 & 65.21 & 62.56 & 55.2 & 206.14 & 200.33 & 5.58 \\
\hline 35.545 & 237.8 & 199.75 & 187.32 & 65.47 & 62.58 & 55.24 & 206.18 & 200.33 & 5.62 \\
\hline 36.04567 & 237.53 & 199.54 & 187.84 & 65.83 & 62.58 & 55.22 & 206.21 & 200.34 & 5.63 \\
\hline 36.5455 & 237.37 & 199.27 & 188.11 & 66.34 & 62.6 & 55.3 & 206.19 & 200.33 & 5.60 \\
\hline 37.04533 & 237.19 & 199.56 & 188.24 & 66.97 & 62.57 & 55.25 & 206.35 & 200.35 & 5.60 \\
\hline 37.54417 & 236.87 & 199.45 & 188.6 & 67.86 & 62.58 & 55.25 & 206.37 & 200.34 & 5.62 \\
\hline 38.044 & 236.8 & 199.51 & 188.83 & 68.99 & 62.62 & 55.29 & 206.44 & 200.34 & 5.63 \\
\hline 38.54483 & 236.73 & 199.82 & 189.26 & 70.48 & 62.62 & 55.27 & 206.56 & 200.36 & 5.59 \\
\hline 39.0455 & 236.59 & 199.74 & 189.68 & 72.38 & 62.76 & 55.29 & 206.59 & 200.36 & 5.57 \\
\hline 39.54533 & 236.5 & 199.53 & 189.53 & 74.4 & 62.68 & 55.29 & 206.61 & 200.35 & 5.57 \\
\hline 40.04517 & 236.34 & 199.75 & 189.32 & 76.54 & 62.76 & 55.31 & 206.65 & 200.35 & 5.59 \\
\hline 40.545 & 235.71 & 199.8 & 189.34 & 78.81 & 62.84 & 55.33 & 206.79 & 200.37 & 5.59 \\
\hline 41.04483 & 236.13 & 199.64 & 189.4 & 81.19 & 62.92 & 55.33 & 206.69 & 200.34 & 5.58 \\
\hline 41.5455 & 236.29 & 200.23 & 189.93 & 83.46 & 62.97 & 55.33 & 206.6 & 200.33 & 5.59 \\
\hline 42.04533 & 235.95 & 200.09 & 190.31 & 85.98 & 62.97 & 55.31 & 206.63 & 200.33 & 5.60 \\
\hline 42.54517 & 235.92 & 199.76 & 190.87 & 88.5 & 63.07 & 55.32 & 206.52 & 200.31 & 5.63 \\
\hline 43.04417 & 236.26 & 199.2 & 191.23 & 90.85 & 63.11 & 55.33 & 206.54 & 200.32 & 5.60 \\
\hline 43.54483 & 235.95 & 199.58 & 191.61 & 93.59 & 63.17 & 55.32 & 206.46 & 200.31 & 5.57 \\
\hline
\end{tabular}


Table B3. Continued.

\begin{tabular}{|c|c|c|c|c|c|c|c|c|c|}
\hline $\begin{array}{c}\text { Time } \\
\text { min }\end{array}$ & $\begin{array}{l}\mathrm{T} 1 \\
{ }^{\circ} \mathrm{C} \\
\end{array}$ & $\begin{array}{l}\mathrm{T} 2 \\
{ }^{\circ} \mathrm{C} \\
\end{array}$ & $\begin{array}{l}\mathrm{T} 3 \\
{ }^{\circ} \mathrm{C} \\
\end{array}$ & $\begin{array}{l}\mathrm{T} 4 \\
{ }^{\circ} \mathrm{C} \\
\end{array}$ & $\begin{array}{l}\mathrm{T} 5 \\
{ }^{\circ} \mathrm{C} \\
\end{array}$ & $\begin{array}{l}\mathrm{T} 6 \\
{ }^{\circ} \mathrm{C} \\
\end{array}$ & $\begin{array}{l}\text { Pinj } \\
\text { psig }\end{array}$ & $\begin{array}{l}\text { Pout } \\
\text { psig }\end{array}$ & $\begin{array}{c}\mathrm{Vw} \\
\mathrm{cc} / \mathrm{min}\end{array}$ \\
\hline 44.0465 & 236.12 & 199.42 & 191.9 & 96.26 & 63.21 & 55.3 & 206.54 & 200.29 & 5.57 \\
\hline 44.5445 & 236.24 & 199.62 & 192.29 & 99.31 & 63.28 & 55.3 & 206.41 & 200.27 & 5.56 \\
\hline 45.04433 & 236.14 & 199.26 & 192.76 & 102.38 & 63.31 & 55.3 & 206.36 & 200.28 & 5.60 \\
\hline 45.545 & 235.28 & 199.32 & 193.15 & 105.31 & 63.37 & 55.29 & 206.32 & 200.25 & 5.60 \\
\hline 46.04483 & 235.89 & 199.57 & 193.57 & 108.48 & 63.4 & 55.29 & 206.41 & 200.26 & 5.59 \\
\hline 46.54567 & 236 & 200.11 & 194.03 & 112.76 & 63.42 & 55.27 & 206.49 & 200.24 & 5.59 \\
\hline 47.0455 & 235.21 & 199.98 & 194.47 & 117.63 & 63.48 & 55.29 & 206.53 & 200.28 & 5.60 \\
\hline 47.54533 & 235.41 & 199.55 & 194.79 & 123.19 & 63.52 & 55.29 & 206.49 & 200.25 & 5.62 \\
\hline 48.045 & 235.37 & 199.73 & 195.11 & 129.57 & 63.56 & 55.27 & 206.55 & 200.26 & 5.60 \\
\hline 48.544 & 235.41 & 200.15 & 195.42 & 136.68 & 63.58 & 55.28 & 206.54 & 200.25 & 5.56 \\
\hline 49.04567 & 234.55 & 199.52 & 195.65 & 144.26 & 63.64 & 55.26 & 206.58 & 200.26 & 5.56 \\
\hline 49.5445 & 234.59 & 199.92 & 195.83 & 152.85 & 63.66 & 55.26 & 206.71 & 200.25 & 5.55 \\
\hline 50.04433 & 234.7 & 199.83 & 195.98 & 161.76 & 63.68 & 55.26 & 206.71 & 200.26 & 5.59 \\
\hline 50.54517 & 234.67 & 199.67 & 196.12 & 169.61 & 63.72 & 55.26 & 206.82 & 200.25 & 5.59 \\
\hline 51.045 & 234.13 & 199.42 & 196.27 & 177.17 & 63.78 & 55.28 & 206.97 & 200.27 & 5.59 \\
\hline 51.54567 & 233.61 & 200.03 & 196.38 & 183 & 63.85 & 55.32 & 207.08 & 200.27 & 5.59 \\
\hline 52.0455 & 233.79 & 199.85 & 196.47 & 187.22 & 63.91 & 55.44 & 207.21 & 200.25 & 5.61 \\
\hline 52.54533 & 233.29 & 199.82 & 196.58 & 191.17 & 63.97 & 55.57 & 207.32 & 200.3 & 5.63 \\
\hline 53.04517 & 235.05 & 199.95 & 196.61 & 193.17 & 64.05 & 55.71 & 207.38 & 200.18 & 5.60 \\
\hline 53.545 & 236.43 & 200.07 & 196.69 & 194.52 & 64.13 & 55.88 & 207.39 & 200.18 & 5.56 \\
\hline 54.04567 & 237.74 & 200.59 & 196.73 & 195.27 & 64.22 & 56.02 & 207.37 & 200.18 & 5.56 \\
\hline 54.5455 & 238.82 & 200.43 & 196.76 & 195.69 & 64.3 & 56.13 & 207.35 & 200.16 & 5.57 \\
\hline 55.04433 & 239.55 & 200.58 & 196.78 & 196.01 & 64.39 & 56.27 & 207.45 & 200.17 & 5.60 \\
\hline 55.54517 & 239.5 & 200.9 & 196.84 & 196.26 & 64.49 & 56.38 & 207.52 & 200.15 & 5.60 \\
\hline 56.045 & 239.7 & 200.29 & 196.84 & 196.41 & 64.59 & 56.51 & 207.54 & 200.13 & 5.60 \\
\hline 56.54483 & 239.2 & 200.26 & 196.86 & 196.48 & 64.68 & 56.61 & 207.59 & 200.15 & 5.60 \\
\hline 57.0455 & 239.74 & 199.98 & 196.86 & 196.52 & 64.82 & 56.75 & 207.41 & 200.15 & 5.62 \\
\hline 57.54533 & 239.74 & 200.17 & 196.84 & 196.52 & 64.91 & 56.84 & 207.15 & 200.12 & 5.63 \\
\hline 58.04617 & 239.81 & 199.92 & 196.81 & 196.54 & 65.05 & 56.94 & 207.13 & 200.08 & 5.58 \\
\hline 58.545 & 239.63 & 200.36 & 196.85 & 196.58 & 65.18 & 57.04 & 207.34 & 200.12 & 5.55 \\
\hline 59.04483 & 239.58 & 200.3 & 196.87 & 196.61 & 65.35 & 57.17 & 207.39 & 200.12 & 5.54 \\
\hline 59.5455 & 239.47 & 200.54 & 196.88 & 196.65 & 65.56 & 57.31 & 207.45 & 200.11 & 5.57 \\
\hline 60.04533 & 239.1 & 200.82 & 196.9 & 196.67 & 65.73 & 57.4 & 207.42 & 200.1 & 5.61 \\
\hline 60.54517 & 239.26 & 200.2 & 196.92 & 196.69 & 65.92 & 57.52 & 207.49 & 200.1 & 5.59 \\
\hline 61.04417 & 238.78 & 200.4 & 196.94 & 196.71 & 66.09 & 57.61 & 207.61 & 200.11 & 5.60 \\
\hline 61.54483 & 238.6 & 201.22 & 196.82 & 196.55 & 66.38 & 57.77 & 206.65 & 201.01 & 5.61 \\
\hline 62.04467 & 238.89 & 200.74 & 196.86 & 196.7 & 66.57 & 57.83 & 207.35 & 200.95 & 5.66 \\
\hline 62.54533 & 239.09 & 200.67 & 197.06 & 196.86 & 66.8 & 57.92 & 208.13 & 200.99 & 5.61 \\
\hline 63.04517 & 238.57 & 201.8 & 197.15 & 196.95 & 67.03 & 57.98 & 208.42 & 200.95 & 5.54 \\
\hline 63.545 & 237.82 & 201.28 & 197.18 & 196.97 & 67.33 & 58.06 & 208.51 & 200.91 & 5.53 \\
\hline 64.04483 & 238.13 & 200.84 & 197.2 & 196.99 & 67.65 & 58.15 & 208.58 & 200.9 & 5.54 \\
\hline 64.54567 & 238.42 & 200.71 & 197.19 & 196.99 & 68.01 & 58.23 & 208.62 & 200.87 & 5.57 \\
\hline 65.0455 & 238.22 & 201.02 & 197.19 & 196.99 & 68.45 & 58.33 & 208.68 & 200.87 & 5.60 \\
\hline 65.54533 & 237.61 & 200.59 & 196.96 & 196.72 & 68.98 & 58.44 & 207.34 & 201.01 & 5.63 \\
\hline
\end{tabular}


Table B3. Continued.

\begin{tabular}{|c|c|c|c|c|c|c|c|c|c|}
\hline $\begin{array}{c}\text { Time } \\
\text { min }\end{array}$ & $\begin{array}{l}\mathrm{T} 1 \\
{ }^{\circ} \mathrm{C} \\
\end{array}$ & $\begin{array}{l}\mathrm{T} 2 \\
{ }^{\circ} \mathrm{C} \\
\end{array}$ & $\begin{array}{l}\mathrm{T} 3 \\
{ }^{\circ} \mathrm{C} \\
\end{array}$ & $\begin{array}{l}\mathrm{T} 4 \\
{ }^{\circ} \mathrm{C} \\
\end{array}$ & $\begin{array}{l}\mathrm{T} 5 \\
{ }^{\circ} \mathrm{C} \\
\end{array}$ & $\begin{array}{l}\mathrm{T} 6 \\
{ }^{\circ} \mathrm{C} \\
\end{array}$ & $\begin{array}{l}\text { Pinj } \\
\text { psig }\end{array}$ & $\begin{array}{l}\text { Pout } \\
\text { psig }\end{array}$ & $\begin{array}{l}\mathrm{Vw} \\
\mathrm{cc} / \mathrm{min}\end{array}$ \\
\hline 66.04417 & 238.06 & 200.32 & 197.01 & 196.85 & 69.47 & 58.5 & 207.94 & 200.98 & 5.63 \\
\hline 66.544 & 237.72 & 200.41 & 197.12 & 196.96 & 70 & 58.58 & 208.5 & 201.02 & 5.64 \\
\hline 67.04567 & 237.69 & 200.36 & 197.19 & 197 & 70.79 & 58.66 & 208.57 & 200.94 & 5.64 \\
\hline 67.5445 & 238.19 & 200.67 & 197.21 & 197.02 & 71.77 & 58.73 & 208.72 & 200.91 & 5.59 \\
\hline 68.04533 & 237.78 & 200.4 & 197.23 & 197.05 & 73.22 & 58.77 & 208.76 & 200.9 & 5.58 \\
\hline 68.54517 & 238.07 & 200.6 & 197.23 & 197.05 & 74.92 & 58.87 & 208.75 & 200.89 & 5.61 \\
\hline 69.04501 & 238.52 & 201.01 & 197.23 & 197.04 & 76.81 & 58.93 & 208.81 & 200.88 & 5.60 \\
\hline 69.54567 & 238.5 & 201.41 & 197.24 & 197 & 79.16 & 59.03 & 208.39 & 200.92 & 5.59 \\
\hline 70.0455 & 238.66 & 200.8 & 197.02 & 196.81 & 82.08 & 59.12 & 207.66 & 200.98 & 5.60 \\
\hline 70.54533 & 238.72 & 200.75 & 197.04 & 196.86 & 83.36 & 59.18 & 207.98 & 200.95 & 5.62 \\
\hline 71.04417 & 237.97 & 201 & 197.13 & 196.96 & 85.11 & 59.24 & 208.37 & 200.97 & 5.64 \\
\hline 71.544 & 238.51 & 200.61 & 197.17 & 196.99 & 87.75 & 59.3 & 208.51 & 200.93 & 5.62 \\
\hline 72.04567 & 238.49 & 200.95 & 197.19 & 197.01 & 91.23 & 59.37 & 208.56 & 200.92 & 5.57 \\
\hline 72.54467 & 238.6 & 200.43 & 197.19 & 197.01 & 95.48 & 59.45 & 208.57 & 200.9 & 5.56 \\
\hline 73.04533 & 238.47 & 200.95 & 197.19 & 197.01 & 100.45 & 59.51 & 208.6 & 200.88 & 5.59 \\
\hline 73.54517 & 238.4 & 200.79 & 197.21 & 197.01 & 106.29 & 59.6 & 208.66 & 200.86 & 5.60 \\
\hline 74.04501 & 238.41 & 200.08 & 197.19 & 196.98 & 115.1 & 59.7 & 208.13 & .35 & 5.58 \\
\hline 74.54483 & 238.05 & 199.72 & 197 & 196.8 & 120.3 & 59.78 & 207.51 & 200.93 & 5.60 \\
\hline 75.0455 & 238.43 & 200.15 & 197.07 & 196.89 & 120.06 & 59.84 & 208.1 & 200.94 & 5.59 \\
\hline 75.54533 & 238.55 & 200.87 & 197.18 & 197.02 & 126.33 & 59.88 & 208.6 & 200.96 & 5.63 \\
\hline 76.04517 & 238.63 & 201.36 & 197.2 & 197 & 142.96 & 59.97 & 208.43 & 199.45 & 5.61 \\
\hline 76.54417 & 238.34 & 201.52 & 197.15 & 196.93 & 154.99 & 60.05 & 208.21 & 200.3 & 5.56 \\
\hline 77.04483 & 238.49 & 201.36 & 197.13 & 196.95 & 161.49 & 60.13 & 208.39 & 200.29 & 5.55 \\
\hline 77.5455 & 238.11 & 201.22 & 197.19 & 196.99 & 169.24 & 60.2 & 208.52 & 200.31 & 5.56 \\
\hline 78.0445 & 238.24 & 201.15 & 197.2 & 197.03 & 177.03 & 60.32 & 208.57 & 198.05 & 5.59 \\
\hline 78.54517 & 237.7 & 200.73 & 196.99 & 196.78 & 178.52 & 60.4 & 207.52 & 200.88 & 5.60 \\
\hline 79.04501 & 238.35 & 201.11 & 197.07 & 196.9 & 175.63 & 60.44 & 208.17 & 200.87 & 5.59 \\
\hline 79.54483 & 238.53 & 201.42 & 197.21 & 197.07 & 180.41 & 60.49 & 208.86 & 200.91 & 5.61 \\
\hline 80.04567 & 238.49 & 201.4 & 197.28 & 197.12 & 187.96 & 60.55 & 209.12 & 200.88 & 5.64 \\
\hline 80.54533 & 236.99 & 201.8 & 197.34 & 197.14 & 191.89 & 60.65 & 209.24 & 200.88 & 5.63 \\
\hline 81.04517 & 237.9 & 202.32 & 197.34 & 197.16 & 193.61 & 60.74 & 209.17 & 200.83 & 5.59 \\
\hline 81.54417 & 238.41 & 201.39 & 197.34 & 197.16 & 194.85 & 60.84 & 209.21 & 200.81 & 5.56 \\
\hline 82.044 & 238.45 & 201.14 & 197.34 & 197.14 & 195.73 & 60.92 & 209.18 & 200.79 & 5.57 \\
\hline 82.54567 & 238.39 & 200.67 & 197.32 & 197.15 & 196.32 & 61.05 & 209.14 & 200.78 & 5.56 \\
\hline 83.0445 & 238.39 & 200.83 & 197.09 & 196.89 & 196.91 & 61.18 & 207.93 & 200.9 & 5.60 \\
\hline 83.54533 & 238.57 & 201.59 & 197.15 & 196.98 & 196.99 & 61.24 & 208.41 & 200.91 & 5.58 \\
\hline 84.04501 & 238.38 & 201.5 & 197.26 & 197.08 & 197.15 & 61.36 & 208.84 & 200.92 & 5.59 \\
\hline 84.54483 & 238.54 & 201.57 & 197.33 & 197.15 & 197.36 & 61.47 & 209.17 & 200.91 & 5.60 \\
\hline 85.04567 & 238.5 & 201.22 & 197.37 & 197.2 & 197.6 & 61.61 & 209.27 & 200.87 & 5.61 \\
\hline 85.5455 & 237.52 & 200.7 & 197.37 & 197.19 & 197.64 & 61.8 & 209.34 & 200.86 & 5.63 \\
\hline 86.04533 & 238.18 & 200.32 & 197.4 & 197.21 & 197.71 & 62.01 & 209.44 & 200.84 & 5.57 \\
\hline 86.54517 & 238.13 & 201.43 & 197.4 & 197.22 & 197.76 & 62.25 & 209.51 & 200.84 & 5.56 \\
\hline 87.044 & 237.4 & 201.2 & 197.42 & 197.23 & 197.82 & 62.5 & 209.54 & 200.83 & 5.57 \\
\hline 87.54567 & 237.85 & 201.13 & 197.21 & 196.98 & 197.6 & 62.88 & 208.31 & 200.91 & 5.61 \\
\hline
\end{tabular}


Table B3. Continued.

\begin{tabular}{|c|c|c|c|c|c|c|c|c|c|}
\hline $\begin{array}{c}\text { Time } \\
\text { min }\end{array}$ & $\begin{array}{l}\mathrm{T} 1 \\
{ }^{\circ} \mathrm{C} \\
\end{array}$ & $\begin{array}{l}\mathrm{T} 2 \\
{ }^{\circ} \mathrm{C} \\
\end{array}$ & $\begin{array}{l}\mathrm{T} 3 \\
{ }^{\circ} \mathrm{C} \\
\end{array}$ & $\begin{array}{l}\mathrm{T} 4 \\
{ }^{\circ} \mathrm{C} \\
\end{array}$ & $\begin{array}{l}\mathrm{T} 5 \\
{ }^{\circ} \mathrm{C} \\
\end{array}$ & $\begin{array}{l}\mathrm{T} 6 \\
{ }^{\circ} \mathrm{C} \\
\end{array}$ & $\begin{array}{l}\text { Pinj } \\
\text { psig }\end{array}$ & $\begin{array}{l}\text { Pout } \\
\text { psig }\end{array}$ & $\begin{array}{c}\mathrm{Vw} \\
\mathrm{cc} / \mathrm{min}\end{array}$ \\
\hline 88.0455 & 238.33 & 201.54 & 197.19 & 196.98 & 197.69 & 63.07 & 208.53 & 200.87 & 5.61 \\
\hline 88.54433 & \begin{tabular}{|l|}
238.37 \\
\end{tabular} & 201.2 & 197.28 & 197.12 & 197.82 & 63.28 & 209.14 & 200.87 & 5.60 \\
\hline 89.046 & 238.66 & 201.96 & 197.37 & 197.19 & 197.89 & 63.56 & 209.4 & 200.87 & 5.61 \\
\hline 89.54501 & \begin{tabular}{|l|}
238.32 \\
\end{tabular} & 201.49 & 197.43 & 197.23 & 197.93 & 63.88 & 209.58 & 200.86 & 5.63 \\
\hline 90.04483 & 238.35 & 201.53 & 197.45 & 197.25 & 197.95 & 64.28 & 209.64 & 200.86 & 5.62 \\
\hline 90.5455 & \begin{tabular}{|l|}
238.73 \\
\end{tabular} & 201.37 & 197.45 & 197.27 & 197.98 & 64.68 & 209.7 & 200.86 & 5.58 \\
\hline 91.04533 & 238.71 & 201.89 & 197.47 & 197.27 & 198 & 65.12 & 209.76 & 200.86 & 5.58 \\
\hline 91.54517 & \begin{tabular}{|l|}
238.52 \\
\end{tabular} & 202.18 & 197.47 & 197.29 & 198.02 & 65.61 & 209.85 & 200.85 & 5.59 \\
\hline 92.04501 & 238.13 & 202.12 & 197.49 & 197.31 & 197.95 & 66.14 & 209.88 & 200.84 & 5.63 \\
\hline 92.54383 & 238.43 & 201.5 & 197.27 & 197.06 & 197.81 & 66.97 & 208.67 & 200.9 & 5.62 \\
\hline 93.0455 & 238.31 & 201.8 & 197.27 & 197.09 & 197.88 & 67.46 & 209.14 & 200.85 & 5.59 \\
\hline 93.54533 & 238.7 & 201.12 & 197.36 & 197.2 & 198.01 & 67.99 & 209.49 & 200.89 & 5.60 \\
\hline 94.04433 & 238.54 & 201.11 & 197.47 & 197.27 & 198.1 & 68.75 & 209.85 & 200.91 & 5.62 \\
\hline 94.54501 & 238.86 & 201.56 & 197.51 & 197.33 & 198.14 & 69.73 & 209.99 & 200.9 & 5.61 \\
\hline 95.04483 & \begin{tabular}{|l|}
239.03 \\
\end{tabular} & 202.04 & 197.53 & 197.33 & 198.14 & 70.86 & 209.99 & 200.87 & 5.56 \\
\hline 95.54467 & 238.94 & 201.45 & 197.53 & 197.35 & 198.16 & 72.12 & 209.99 & 200.85 & 5.56 \\
\hline 96.04533 & 238.98 & 201.25 & 197.51 & 197.32 & 198.19 & 73.48 & 209.86 & 200.84 & 5.59 \\
\hline 96.54517 & 238.22 & 201.38 & 197.3 & 197.03 & 197.76 & 75.58 & 208.25 & 200.79 & 5.62 \\
\hline 97.046 & \begin{tabular}{|l|}
238.67 \\
\end{tabular} & 201.29 & 197.14 & 196.96 & 197.78 & 77.18 & 208.39 & 200.72 & 5.61 \\
\hline 97.54583 & 238.48 & 200.61 & 197.21 & 197.05 & 197.89 & 78.63 & 208.76 & 200.74 & 5.60 \\
\hline 98.04567 & 238.82 & 200.56 & 197.28 & 197.1 & 197.95 & 80.59 & 209.09 & 200.74 & 5.62 \\
\hline 98.54533 & 238.66 & 201.06 & 197.34 & 197.16 & 198.02 & 83.07 & 209.2 & 200.73 & 5.64 \\
\hline 99.04617 & 238.85 & 201.81 & 197.32 & 197.16 & 197.98 & 85.89 & 209.09 & 200.72 & 5.61 \\
\hline 99.54501 & 238.48 & 202.19 & 197.32 & 197.13 & 197.97 & 89.16 & 209.01 & 200.69 & 5.57 \\
\hline 100.044 & 237.91 & 202.09 & 197.29 & 197.11 & 197.91 & 92.92 & 208.84 & 198.3 & 5.56 \\
\hline 100.5457 & \begin{tabular}{|l|}
238.41 \\
\end{tabular} & 201.62 & 197 & 196.79 & 197.63 & 100.18 & 207.43 & 200.75 & 5.58 \\
\hline 101.0445 & 238.7 & 201.8 & 197 & 196.84 & 197.72 & 102.66 & 207.73 & 200.74 & 5.60 \\
\hline 101.5453 & 239 & 201.19 & 197.07 & 196.91 & 197.79 & 104.92 & 208.14 & 200.74 & 5.61 \\
\hline 102.045 & 239.2 & 201.59 & 197.17 & 196.97 & 197.86 & 108.43 & 208.43 & 200.74 & 5.61 \\
\hline 102.5448 & \begin{tabular}{|l|}
238.66 \\
\end{tabular} & 201.93 & 197.22 & 197.04 & 197.9 & 113.68 & 208.6 & 200.76 & 5.63 \\
\hline 103.0457 & 238.54 & 201.75 & 197.2 & 197.01 & 197.87 & 122.07 & 208.46 & 200.71 & 5.65 \\
\hline 103.5455 & 238.81 & 202.04 & 197.18 & 196.99 & 197.87 & 131.3 & 208.45 & 200.7 & 5.59 \\
\hline 104.0453 & \begin{tabular}{|l|}
238.99 \\
\end{tabular} & 201.45 & 197.17 & 196.97 & 197.83 & 140.48 & 208.33 & 200.69 & 5.58 \\
\hline 104.5452 & 238.33 & 200.79 & 197.15 & 196.95 & 197.81 & 149.61 & 208.23 & 200.68 & 5.60 \\
\hline 105.0458 & 238.58 & 201.34 & 197.15 & 196.95 & 197.82 & 157.63 & 208.22 & 200.67 & 5.63 \\
\hline 105.5457 & 238.37 & 201.9 & 196.89 & 196.65 & 197.49 & 170.07 & 206.95 & 200.74 & 5.62 \\
\hline 106.0455 & \begin{tabular}{|l|}
238.37 \\
\end{tabular} & 202.24 & 196.87 & 196.69 & 197.58 & 162.67 & 207.2 & 200.74 & 5.61 \\
\hline 106.5453 & 238.87 & 202.01 & 196.96 & 196.8 & 197.69 & 163.34 & 207.67 & 200.77 & 5.63 \\
\hline 107.0442 & 239.03 & 201.45 & 197.07 & 196.89 & 197.78 & 169.72 & 208.06 & 200.78 & 5.65 \\
\hline 107.545 & 238.92 & 202.01 & 197.12 & 196.92 & 197.8 & 179.42 & 208.18 & 200.76 & 5.61 \\
\hline 108.0457 & 238.58 & 201.96 & 197.12 & 196.92 & 197.8 & 186.17 & 208.13 & 200.74 & 5.56 \\
\hline 108.5447 & 238.98 & 201.76 & 197.09 & 196.89 & 197.75 & 190.06 & 208.06 & 200.68 & 5.58 \\
\hline 109.0453 & 238.6 & 202.07 & 197.05 & 196.86 & 197.72 & 192.25 & 207.87 & 200.66 & 5.63 \\
\hline 109.5452 & 238.68 & 201.82 & 197.05 & 196.84 & 197.73 & 193.67 & 207.82 & 200.66 & 5.61 \\
\hline
\end{tabular}


Table B3. Continued.

\begin{tabular}{|c|c|c|c|c|c|c|c|c|c|}
\hline $\begin{array}{c}\text { Time } \\
\min \end{array}$ & $\begin{array}{l}\mathrm{T} 1 \\
{ }^{\circ} \mathrm{C} \\
\end{array}$ & $\begin{array}{l}\mathrm{T} 2 \\
{ }^{\circ} \mathrm{C} \\
\end{array}$ & $\begin{array}{l}\mathrm{T} 3 \\
{ }^{\circ} \mathrm{C}\end{array}$ & $\begin{array}{l}\mathrm{T} 4 \\
{ }^{\circ} \mathrm{C}\end{array}$ & $\begin{array}{l}\mathrm{T} 5 \\
{ }^{\circ} \mathrm{C} \\
\end{array}$ & $\begin{array}{l}\mathrm{T} 6 \\
{ }^{\circ} \mathrm{C}\end{array}$ & $\begin{array}{l}\text { Pinj } \\
\text { psig }\end{array}$ & $\begin{array}{l}\text { Pout } \\
\text { psig }\end{array}$ & $\begin{array}{c}\mathrm{Vw} \\
\mathrm{cc} / \mathrm{min}\end{array}$ \\
\hline 110.0458 & 238.73 & 202.64 & 197 & 196.82 & 197.72 & 194.62 & 207.7 & 200.59 & 5.61 \\
\hline 110.5448 & 238.82 & 202.93 & 196.64 & 196.45 & 197.32 & 194.3 & 206.12 & 200.75 & 5.61 \\
\hline 111.0455 & 238.61 & 202.86 & 196.77 & 196.63 & 197.54 & 191.68 & 206.92 & 200.75 & 5.64 \\
\hline 111.5453 & 238.99 & 201.91 & 196.91 & 196.75 & 197.63 & 192.63 & 207.36 & 200.8 & 5.60 \\
\hline 112.0452 & 239.11 & 201.03 & 196.97 & 196.75 & 197.67 & 194.64 & 207.48 & 200.8 & 5.58 \\
\hline 112.545 & 239.22 & 200.51 & 196.99 & 196.77 & 197.67 & 195.68 & 207.52 & 200.78 & 5.58 \\
\hline 113.0448 & 238.65 & 200.82 & 196.97 & 196.79 & 197.65 & 196.29 & 207.45 & 200.73 & 5.61 \\
\hline 113.5455 & 239.04 & 201.25 & 196.95 & 196.74 & 197.62 & 196.56 & 207.31 & 200.67 & 5.62 \\
\hline 114.0453 & 238.95 & 202.36 & 196.93 & 196.74 & 197.62 & 196.68 & 207.34 & 200.62 & 5.61 \\
\hline 114.5443 & 238.83 & 202.45 & 196.92 & 196.72 & 197.62 & 196.77 & 207.19 & 200.62 & 5.62 \\
\hline 115.045 & 238.97 & 201.61 & 196.9 & 196.69 & 197.58 & 196.85 & 207.1 & 200.57 & 5.63 \\
\hline 115.5448 & 238.92 & 201.77 & 196.65 & 196.45 & 197.44 & 195.43 & 206.34 & 200.77 & 5.63 \\
\hline 116.0447 & 239.19 & 201.83 & 196.81 & 196.63 & 197.53 & 195.88 & 206.89 & 200.78 & 5.58 \\
\hline 116.5453 & 239.19 & 201.4 & 196.88 & 196.71 & 197.49 & 196.71 & 207.16 & 200.8 & 5.56 \\
\hline 117.0452 & 239.32 & 201.38 & 196.87 & 196.67 & 197.55 & 196.92 & 206.99 & 200.77 & 5.58 \\
\hline 117.545 & 239.21 & 202.4 & 196.87 & 196.67 & 197.55 & 197.01 & 206.95 & 200.76 & 5.60 \\
\hline 118.0448 & 239.21 & 202.62 & 196.87 & 196.67 & 197.57 & 197.08 & 207.05 & 200.78 & 5.60 \\
\hline 118.5457 & 238.24 & 201.29 & 196.87 & 196.65 & 197.55 & 197.1 & 206.96 & 200.7 & 5.60 \\
\hline 119.0455 & 238.76 & 201.72 & 196.83 & 196.64 & 197.43 & 197.1 & 206.84 & 200.64 & 5.62 \\
\hline 119.5453 & 238.69 & 202.4 & 196.58 & 196.42 & 197.28 & 195.69 & 206.31 & 200.79 & 5.65 \\
\hline 120.0442 & 238.73 & 200.83 & 196.76 & 196.6 & 197.5 & 195.96 & 206.7 & 200.69 & 5.63 \\
\hline 120.544 & 238.94 & 200.31 & 196.8 & 196.62 & 197.52 & 197.02 & 206.84 & 200.69 & 5.58 \\
\hline 121.0457 & 238.64 & 201.1 & 196.84 & 196.66 & 197.54 & 197.16 & 206.97 & 200.73 & 5.57 \\
\hline 121.5445 & 238.91 & 201.03 & 196.86 & 196.66 & 197.55 & 197.2 & 206.95 & 200.85 & 5.59 \\
\hline 122.0453 & 239 & 202.32 & 196.84 & 196.64 & 197.63 & 197.2 & 206.9 & 200.79 & 5.60 \\
\hline 122.5452 & 238.77 & 202.3 & 196.82 & 196.64 & 197.52 & 197.16 & 206.82 & 200.64 & 5.59 \\
\hline 123.045 & 239.02 & 201.62 & 196.79 & 196.59 & 197.45 & 197.16 & 206.63 & 200.5 & 5.60 \\
\hline 123.5457 & 238.81 & 202.5 & 196.68 & 196.5 & 197.41 & 196.93 & 206.46 & 200.63 & 5.62 \\
\hline 124.0455 & 238.57 & 202.09 & 196.68 & 196.52 & 197.42 & 197 & 206.46 & 200.75 & 5.64 \\
\hline 124.5453 & 238.88 & 201.79 & 196.81 & 196.64 & 197.54 & 197.24 & 207.04 & 200.87 & 5.60 \\
\hline 125.0442 & 239.17 & 202.7 & 196.84 & 196.66 & 197.54 & 197.26 & 206.99 & 200.81 & 5.57 \\
\hline 125.544 & 238.86 & 202.13 & 196.84 & 196.65 & 197.54 & 197.26 & 206.88 & 200.65 & 5.56 \\
\hline 126.0457 & 238.58 & 201.07 & 196.77 & 196.58 & 197.47 & 197.19 & 206.59 & 200.41 & 5.59 \\
\hline 126.5447 & 238.74 & 200.66 & 196.74 & 196.54 & 197.44 & 197.17 & 206.47 & 200.39 & 5.63 \\
\hline 127.0453 & 238.79 & 201.59 & 196.74 & 196.56 & 197.44 & 197.17 & 206.53 & 200.36 & 5.60 \\
\hline 127.5452 & 239.04 & 202.72 & 196.72 & 196.54 & 197.44 & 197.19 & 206.5 & 200.39 & 5.59 \\
\hline 128.045 & 238.51 & 202.53 & 196.74 & 196.56 & 197.46 & 197.21 & 206.54 & 200.31 & 5.61 \\
\hline 128.5448 & 238.94 & 202.65 & 196.74 & 196.56 & 197.46 & 197.19 & 206.57 & 200.42 & 5.64 \\
\hline 129.0455 & 238.76 & 202.76 & 196.74 & 196.55 & 197.46 & 197.21 & 206.5 & 200.33 & 5.60 \\
\hline 129.5463 & 238.94 & 203.51 & 196.58 & 196.44 & 197.34 & 196.37 & 206.43 & 200.71 & 5.57 \\
\hline 130.0452 & 239.12 & 202.67 & 196.78 & 196.58 & 197.48 & 196.85 & 206.66 & 200.61 & 5.56 \\
\hline 130.545 & 238.96 & 202.78 & 196.78 & 196.6 & 197.52 & 197.23 & 206.78 & 200.61 & 5.59 \\
\hline 131.0448 & 239.1 & 202.28 & 196.8 & 196.64 & 197.53 & 197.3 & 206.95 & 200.65 & 5.61 \\
\hline 131.5455 & 239.32 & 202.66 & 196.84 & 196.66 & 197.57 & 197.32 & 206.98 & 200.76 & 5.61 \\
\hline
\end{tabular}


Table B3. Continued.

\begin{tabular}{|c|c|c|c|c|c|c|c|c|c|}
\hline $\begin{array}{c}\text { Time } \\
\text { min }\end{array}$ & $\begin{array}{l}\mathrm{T} 1 \\
{ }^{\circ} \mathrm{C} \\
\end{array}$ & $\begin{array}{l}\mathrm{T} 2 \\
{ }^{\circ} \mathrm{C} \\
\end{array}$ & $\begin{array}{l}\mathrm{T} 3 \\
{ }^{\circ} \mathrm{C} \\
\end{array}$ & $\begin{array}{l}\mathrm{T} 4 \\
{ }^{\circ} \mathrm{C} \\
\end{array}$ & $\begin{array}{l}\mathrm{T} 5 \\
{ }^{\circ} \mathrm{C} \\
\end{array}$ & $\begin{array}{l}\mathrm{T} 6 \\
{ }^{\circ} \mathrm{C} \\
\end{array}$ & $\begin{array}{l}\text { Pinj } \\
\text { psig }\end{array}$ & $\begin{array}{l}\text { Pout } \\
\text { psig }\end{array}$ & $\begin{array}{c}\mathrm{Vw} \\
\mathrm{cc} / \mathrm{min}\end{array}$ \\
\hline 132.0453 & 239.34 & 202.03 & 196.8 & 196.6 & 197.5 & 197.27 & 206.74 & 200.54 & 5.62 \\
\hline 132.5452 & 238.46 & 202.3 & 196.79 & 196.59 & 197.48 & 197.27 & 206.67 & 200.48 & 5.62 \\
\hline 133.045 & \begin{tabular}{|l|}
239.07 \\
\end{tabular} & 202.25 & 196.77 & 196.57 & 197.47 & 197.23 & 206.62 & 200.36 & 5.65 \\
\hline 133.544 & 239 & 201.8 & 196.75 & 196.57 & 197.47 & 197.25 & 206.61 & 200.45 & 5.60 \\
\hline 134.0455 & 238.05 & 203.06 & 196.75 & 196.56 & 197.47 & 197.24 & 206.57 & 200.47 & 5.55 \\
\hline 134.5445 & 238.88 & 203.18 & 196.77 & 196.59 & 197.49 & 197.26 & 206.6 & 200.42 & 5.56 \\
\hline 135.0452 & \begin{tabular}{|l|}
238.72 \\
\end{tabular} & 202.45 & 196.77 & 196.59 & 197.47 & 197.27 & 206.69 & 200.37 & 5.59 \\
\hline 135.545 & 239.02 & 202.81 & 196.65 & 196.51 & 197.45 & 196.74 & 206.66 & 200.69 & 5.61 \\
\hline 136.0448 & 239.31 & 202.52 & 196.79 & 196.59 & 197.51 & 197.2 & 206.81 & 200.62 & 5.60 \\
\hline 136.5457 & 239.15 & 201.54 & 196.81 & 196.62 & 197.53 & 197.31 & 206.89 & 200.42 & 5.61 \\
\hline 137.0455 & 239.1 & 202.79 & 196.83 & 196.65 & 197.56 & 197.35 & 207.02 & 200.66 & 5.62 \\
\hline 137.5453 & 239.24 & 202.22 & 196.81 & 196.63 & 197.51 & 197.3 & 206.79 & 200.5 & 5.63 \\
\hline 138.045 & 238.96 & 202.62 & 196.78 & 196.6 & 197.51 & 197.3 & 206.71 & 200.52 & 5.59 \\
\hline 138.5448 & 238.94 & 202.1 & 196.78 & 196.6 & 197.5 & 197.26 & 206.7 & 200.37 & 5.56 \\
\hline 139.0457 & 239.19 & 202.64 & 196.76 & 196.58 & 197.48 & 197.28 & 206.65 & 200.42 & 5.57 \\
\hline 139.5455 & 239.14 & 202.67 & 196.76 & 196.57 & 197.48 & 197.28 & 206.66 & 200.4 & 5.61 \\
\hline 140.0443 & 238.52 & 203.48 & 196.78 & 196.6 & 197.5 & 197.3 & 206.72 & 200.36 & 5.61 \\
\hline 140.5452 & 238.53 & 203.19 & 196.77 & 196.59 & 197.5 & 197.29 & 206.74 & 200.41 & 5.60 \\
\hline 141.045 & 238.59 & 201.62 & 196.78 & 196.59 & 197.48 & 197.3 & 206.72 & 200.47 & 5.61 \\
\hline 141.5447 & 238.43 & 201.76 & 196.79 & 196.59 & 197.52 & 197.32 & 206.74 & 200.5 & 5.62 \\
\hline 142.0455 & \begin{tabular}{|l|}
238.09 \\
\end{tabular} & 202.39 & 196.79 & 196.59 & 197.49 & 197.31 & 206.74 & 200.33 & 5.64 \\
\hline 142.5453 & \begin{tabular}{|l|}
238.36 \\
\end{tabular} & 203.22 & 196.61 & 196.45 & 197.38 & 196.93 & 206.44 & 200.56 & 5.58 \\
\hline 143.0452 & 238.7 & 203.52 & 196.74 & 196.54 & 197.41 & 197.2 & 206.33 & 199.77 & 5.57 \\
\hline 143.545 & 238.41 & 203.61 & 196.74 & 196.54 & 197.42 & 197.25 & 206.48 & 200.04 & 5.57 \\
\hline 144.0457 & 238.54 & 202.61 & 196.68 & 196.49 & 197.38 & 197.18 & 206.17 & 199.92 & 5.61 \\
\hline 144.5455 & 238.65 & 203.18 & 196.67 & 196.47 & 197.4 & 197.2 & 206.21 & 200.05 & 5.60 \\
\hline 145.0453 & 238.06 & 202.65 & 196.68 & 196.49 & 197.38 & 197.2 & 206.26 & 200.02 & 5.60 \\
\hline 145.5442 & 238.36 & 202.83 & 196.69 & 196.49 & 197.38 & 197.22 & 206.34 & 200.11 & 5.59 \\
\hline 146.044 & 238.6 & 202.86 & 196.67 & 196.49 & 197.49 & 197.23 & 206.24 & 199.97 & 5.63 \\
\hline 146.5448 & 237.93 & 202.83 & 196.71 & 196.49 & 197.4 & 197.23 & 206.29 & 200.04 & 5.63 \\
\hline 147.0447 & 238.46 & 202.67 & 196.69 & 196.49 & 197.39 & 197.23 & 206.28 & 199.97 & 5.58 \\
\hline 147.5453 & 238.49 & 203.33 & 196.69 & 196.51 & 197.41 & 197.21 & 206.21 & 200.04 & 5.56 \\
\hline 148.0452 & 238.33 & 202.39 & 196.69 & 196.51 & 197.41 & 197.23 & 206.29 & 199.98 & 5.58 \\
\hline 148.545 & 237.8 & 203.25 & 196.69 & 196.49 & 197.43 & 197.26 & 206.29 & 200.01 & 5.61 \\
\hline 149.0448 & 238.41 & 203.32 & 196.71 & 196.51 & 197.41 & 197.23 & 206.29 & 200.02 & 5.60 \\
\hline 149.5455 & 238.12 & 202.94 & 196.71 & 196.51 & 197.43 & 197.25 & 206.33 & 199.99 & 5.60 \\
\hline 150.0453 & 238.64 & 201.14 & 196.77 & 196.59 & 197.52 & 197.34 & 206.85 & 200.87 & 5.61 \\
\hline 150.5452 & 238.75 & 202.46 & 196.69 & 196.53 & 197.5 & 197.2 & 206.87 & 200.91 & 5.62 \\
\hline 151.045 & 238.82 & 202.89 & 196.86 & 196.7 & 197.61 & 197.43 & 207.24 & 200.8 & 5.62 \\
\hline 151.544 & 238.62 & 202.34 & 196.91 & 196.73 & 197.63 & 197.45 & 207.32 & 201.05 & 5.57 \\
\hline 152.0455 & 238.48 & 201.94 & 196.88 & 196.64 & 197.5 & 197.36 & 206.64 & 199.1 & 5.55 \\
\hline 152.5445 & 238.27 & 203.66 & 196.48 & 196.23 & 197.13 & 196.99 & 204.99 & 198.51 & 5.56 \\
\hline 153.0452 & 238.29 & 202.27 & 196.45 & 196.25 & 197.07 & 197 & 205.23 & 198.87 & 5.59 \\
\hline 153.545 & 238.43 & 202.48 & 196.47 & 196.29 & 197.16 & 197 & 205.28 & 199 & 5.60 \\
\hline
\end{tabular}


Table B3. Continued.

\begin{tabular}{|c|c|c|c|c|c|c|c|c|c|}
\hline $\begin{array}{c}\text { Time } \\
\text { min }\end{array}$ & $\begin{array}{l}\mathrm{T} 1 \\
{ }^{\circ} \mathrm{C} \\
\end{array}$ & $\begin{array}{l}\mathrm{T} 2 \\
{ }^{\circ} \mathrm{C} \\
\end{array}$ & $\begin{array}{l}\mathrm{T} 3 \\
{ }^{\circ} \mathrm{C} \\
\end{array}$ & $\begin{array}{l}\mathrm{T} 4 \\
{ }^{\circ} \mathrm{C} \\
\end{array}$ & $\begin{array}{l}\mathrm{T} 5 \\
{ }^{\circ} \mathrm{C} \\
\end{array}$ & $\begin{array}{l}\mathrm{T} 6 \\
{ }^{\circ} \mathrm{C} \\
\end{array}$ & $\begin{array}{l}\text { Pinj } \\
\text { psig }\end{array}$ & $\begin{array}{l}\text { Pout } \\
\text { psig }\end{array}$ & $\begin{array}{c}\mathrm{Vw} \\
\mathrm{cc} / \mathrm{min} \\
\end{array}$ \\
\hline 154.0448 & 238.45 & 202.64 & 196.47 & 196.27 & 197.17 & 197.04 & 205.26 & 198.92 & 5.58 \\
\hline 154.5457 & 238.59 & 202.74 & 196.49 & 196.29 & 197.18 & 197.04 & 205.36 & 198.96 & 5.60 \\
\hline 155.0455 & 238.63 & 203.17 & 196.49 & 196.29 & 197.19 & 197.04 & 205.32 & 198.88 & 5.61 \\
\hline 155.5453 & 238.85 & 203.54 & 196.49 & 196.29 & 197.19 & 197.02 & 205.31 & 198.93 & 5.64 \\
\hline 156.045 & 238.65 & 203.19 & 196.49 & 196.29 & 197.19 & 197.04 & 205.39 & 198.92 & 5.58 \\
\hline 156.5448 & 238.9 & 203.85 & 196.45 & 196.31 & 197.28 & 197.03 & 205.79 & 201.37 & 5.57 \\
\hline 157.0457 & 239.35 & 202.35 & 196.88 & 196.74 & 197.67 & 196.94 & 207.6 & 199.37 & 5.56 \\
\hline 157.5455 & 238.9 & 202.97 & 196.6 & 196.31 & 197.1 & 196.94 & 204.85 & 197.53 & 5.58 \\
\hline 158.0453 & 238.83 & 203.05 & 196.35 & 196.21 & 197.16 & 196.19 & 205.42 & 199.59 & 5.62 \\
\hline 158.5442 & 239 & 203.39 & 196.55 & 196.37 & 197.28 & 196.91 & 205.72 & 199.41 & 5.60 \\
\hline 159.045 & 238.82 & 203.08 & 196.66 & 196.48 & 197.43 & 197.25 & 206.38 & 200.09 & 5.61 \\
\hline 159.5457 & 238.53 & 203.21 & 196.75 & 196.55 & 197.48 & 197.32 & 206.66 & 200.14 & 5.61 \\
\hline 160.0445 & 238.42 & 203.12 & 196.51 & 196.25 & 197.07 & 196.94 & 204.63 & 197.46 & 5.63 \\
\hline 160.5453 & 239.12 & 202.19 & 196.69 & 196.51 & 197.07 & 195.76 & 209.76 & 205.77 & 5.60 \\
\hline 161.0452 & 238.66 & 203.41 & 196.73 & 196.34 & 197.04 & 196.37 & 204.84 & 197.33 & 5.57 \\
\hline 161.545 & 238.41 & 203.8 & 196.41 & 196.18 & 197 & 196.82 & 204.54 & 195.47 & 5.56 \\
\hline 162.0467 & 238.37 & 203.56 & 196.27 & 196.11 & 197.04 & 196.68 & 204.81 & 198.65 & 5.57 \\
\hline 162.5455 & 238.75 & 202.61 & 196.52 & 196.34 & 197.29 & 197.09 & 205.9 & 199.53 & 5.60 \\
\hline 163.0453 & 238.65 & 202.55 & 196.66 & 196.49 & 197.4 & 197.26 & 206.32 & 199.74 & 5.59 \\
\hline 163.5452 & 238.49 & 203.36 & 196.66 & 196.49 & 197.38 & 197.24 & 206.23 & 199.55 & 5.61 \\
\hline 164.045 & 238.9 & 202.38 & 196.83 & 196.7 & 197.65 & 197.44 & 207.43 & 201.6 & 5.62 \\
\hline 164.5457 & 238.79 & 202.7 & 196.88 & 196.69 & 197.69 & 197.44 & 207.11 & 200.44 & 5.64 \\
\hline 165.0455 & 238.63 & 202.99 & 196.79 & 196.65 & 197.58 & 196.94 & 207.35 & 201.29 & 5.61 \\
\hline 165.5453 & 238.69 & 203.44 & 196.62 & 196.35 & 197.19 & 196.99 & 205.22 & 198.12 & 5.57 \\
\hline 166.0452 & 238.89 & 202.99 & 196.62 & 196.46 & 197.42 & 197.12 & 206.47 & 200.24 & 5.56 \\
\hline 166.545 & 238.48 & 203.1 & 196.75 & 196.57 & 197.5 & 197.34 & 206.79 & 200.26 & 5.60 \\
\hline 167.0438 & 238.35 & 203.14 & 196.82 & 196.6 & 197.5 & 197.37 & 206.83 & 200.29 & 5.61 \\
\hline 167.5455 & 238.69 & 202.1 & 196.78 & 196.59 & 197.48 & 197.34 & 206.74 & 200.28 & 5.61 \\
\hline 168.0445 & 238.78 & 202.23 & 196.75 & 196.57 & 197.48 & 197.34 & 206.71 & 200.37 & 5.60 \\
\hline 168.5443 & 238.52 & 203.91 & 196.77 & 196.55 & 197.49 & 197.33 & 206.74 & 200.31 & 5.62 \\
\hline 169.045 & 238.66 & 203.65 & 196.77 & 196.57 & 197.49 & 197.34 & 206.77 & 200.32 & 5.63 \\
\hline 169.5448 & 238.81 & 202.11 & 196.75 & 196.58 & 197.49 & 197.33 & 206.93 & 200.26 & 5.58 \\
\hline 170.0455 & 238.58 & 201.91 & 196.77 & 196.58 & 197.58 & 197.35 & 206.79 & 200.26 & 5.56 \\
\hline 170.5453 & 237.74 & 202.77 & 196.74 & 196.58 & 197.53 & 197.38 & 206.99 & 200.76 & 5.57 \\
\hline 171.0452 & 238.26 & 203.1 & 196.83 & 196.65 & 197.56 & 197.42 & 207.15 & 200.73 & 5.58 \\
\hline 171.545 & 238.62 & 203.55 & 196.87 & 196.67 & 197.57 & 197.46 & 207.24 & 200.74 & 5.60 \\
\hline 172.0448 & 238.33 & 201.97 & 196.87 & 196.67 & 197.59 & 197.44 & 207.18 & 200.8 & 5.59 \\
\hline 172.5457 & 238.8 & 203.85 & 196.85 & 196.66 & 197.57 & 197.44 & 207.14 & 200.72 & 5.59 \\
\hline 173.0455 & 238.37 & 202.8 & 196.86 & 196.68 & 197.59 & 197.45 & 207.23 & 200.75 & 5.60 \\
\hline 173.5443 & 237.24 & 203.77 & 196.89 & 196.7 & 197.59 & 197.47 & 207.24 & 200.71 & 5.62 \\
\hline 174.045 & 238.2 & 204.4 & 196.88 & 196.7 & 197.59 & 197.47 & 207.24 & 200.7 & 5.61 \\
\hline 174.5448 & 238.73 & 203.93 & 196.89 & 196.68 & 197.61 & 197.49 & 207.34 & 200.74 & 5.57 \\
\hline 175.0447 & 238.11 & 204.22 & 196.9 & 196.7 & 197.61 & 197.51 & 207.29 & 200.68 & 5.56 \\
\hline 175.5455 & 238.52 & 204.22 & 196.9 & 196.7 & 197.7 & 197.49 & 207.29 & 200.7 & 5.55 \\
\hline
\end{tabular}


Table B3. Continued.

\begin{tabular}{|c|c|c|c|c|c|c|c|c|c|}
\hline $\begin{array}{c}\text { Time } \\
\text { min }\end{array}$ & $\begin{array}{l}\mathrm{T} 1 \\
{ }^{\circ} \mathrm{C} \\
{ }^{2}\end{array}$ & $\begin{array}{l}\mathrm{T} 2 \\
{ }^{\circ} \mathrm{C}\end{array}$ & $\begin{array}{l}\mathrm{T} 3 \\
{ }^{\circ} \mathrm{C}\end{array}$ & $\begin{array}{l}\mathrm{T} 4 \\
{ }^{\circ} \mathrm{C} \\
\end{array}$ & $\begin{array}{l}\mathrm{T} 5 \\
{ }^{\circ} \mathrm{C} \\
\end{array}$ & $\begin{array}{l}\mathrm{T} 6 \\
{ }^{\circ} \mathrm{C}\end{array}$ & $\begin{array}{l}\text { Pinj } \\
\text { psig }\end{array}$ & $\begin{array}{l}\text { Pout } \\
\text { psig }\end{array}$ & $\begin{array}{c}\mathrm{Vw} \\
\mathrm{cc} / \mathrm{min}\end{array}$ \\
\hline 176.0453 & 238.08 & 204.89 & 196.9 & 196.72 & 197.62 & 197.49 & 207.33 & 200.72 & 5.60 \\
\hline 176.5452 & 238.54 & 202.42 & 196.92 & 196.72 & 197.62 & 197.49 & 207.36 & 200.69 & 5.59 \\
\hline 177.045 & 238.76 & 203.87 & 196.9 & 196.71 & 197.64 & 197.5 & 207.34 & 200.65 & 5.60 \\
\hline 177.5457 & 238.73 & 203.06 & 196.76 & 196.55 & 197.5 & 197.34 & 206.78 & 200.68 & 5.61 \\
\hline 178.0455 & 238.73 & 202.35 & 196.85 & 196.68 & 197.59 & 197.45 & 207.16 & 200.7 & 5.63 \\
\hline 178.5462 & 238.23 & 204 & 196.87 & 196.68 & 197.59 & 197.45 & 207.2 & 200.69 & 5.64 \\
\hline 179.0452 & 238.77 & 204.25 & 196.89 & 196.69 & 197.54 & 197.48 & 207.28 & 200.82 & 5.57 \\
\hline 179.544 & 238.26 & 204.93 & 196.91 & 196.71 & 197.65 & 197.52 & 207.42 & 200.77 & 5.55 \\
\hline 180.0457 & 238.77 & 203.88 & 196.93 & 196.73 & 197.63 & 197.52 & 207.39 & 200.65 & 5.55 \\
\hline 180.5455 & 238.97 & 205.53 & 196.93 & 196.72 & 197.63 & 197.5 & 207.34 & 200.66 & 5.57 \\
\hline 181.0443 & 239.06 & 203.83 & 196.92 & 196.72 & 197.61 & 197.49 & 207.34 & 200.7 & 5.61 \\
\hline 181.5452 & 238.04 & 204.44 & 196.92 & 196.7 & 197.63 & 197.51 & 207.37 & 200.68 & 5.61 \\
\hline 182.045 & 238.58 & 204.04 & 196.92 & 196.7 & 197.64 & 197.49 & 207.36 & 200.72 & 5.60 \\
\hline 182.5448 & 238.85 & 204.28 & 196.92 & 196.72 & 197.62 & 197.51 & 207.36 & 200.69 & 5.61 \\
\hline 183.0455 & 238.76 & 204.17 & 196.92 & 196.72 & 197.62 & 197.51 & 207.39 & 200.68 & 5.64 \\
\hline 183.5453 & 238.44 & 204.62 & 196.92 & 196.71 & 197.62 & 197.5 & 207.33 & 200.72 & 5.61 \\
\hline 184.0452 & 238.8 & 204.57 & 196.92 & 196.71 & 197.64 & 197.5 & 207.33 & 200.63 & 5.56 \\
\hline 184.545 & 238.44 & 204.23 & 196.75 & 196.57 & 197.61 & 197.37 & 206.94 & 200.67 & 5.58 \\
\hline 185.0457 & 238.6 & 203.54 & 196.87 & 196.68 & 197.57 & 197.45 & 207.14 & 200.7 & 5.59 \\
\hline 185.5455 & 238.98 & 204.61 & 196.86 & 196.66 & 197.57 & 197.45 & 207.13 & 200.73 & 5.60 \\
\hline 186.0453 & 239.02 & 203.95 & 196.9 & 196.7 & 197.61 & 197.49 & 207.29 & 200.73 & 5.60 \\
\hline 186.5452 & 239.04 & 203.92 & 196.9 & 196.68 & 197.61 & 197.51 & 207.33 & 200.69 & 5.58 \\
\hline 187.0442 & 239.08 & 204.88 & 196.92 & 196.7 & 197.63 & 197.51 & 207.38 & 200.67 & 5.62 \\
\hline 187.544 & 238.4 & 204.9 & 196.92 & 196.72 & 197.61 & 197.51 & 207.36 & 200.64 & 5.65 \\
\hline 188.0447 & 237.7 & 204.31 & 196.9 & 196.72 & 197.64 & 197.53 & 207.37 & 200.68 & 5.60 \\
\hline 188.5445 & 237.74 & 203.26 & 196.9 & 196.71 & 197.62 & 197.49 & 207.33 & 200.64 & 5.57 \\
\hline 189.0452 & 238.64 & 203.42 & 196.9 & 196.69 & 197.62 & 197.5 & 207.37 & 200.61 & 5.57 \\
\hline 189.545 & 238.65 & 203.57 & 196.91 & 196.71 & 197.62 & 197.51 & 207.46 & 200.74 & 5.61 \\
\hline 190.0448 & 238.55 & 204.3 & 196.92 & 196.71 & 197.62 & 197.5 & 207.4 & 200.69 & 5.61 \\
\hline 190.5457 & 238.71 & 204.16 & 196.91 & 196.71 & 197.55 & 197.52 & 207.34 & 200.62 & 5.60 \\
\hline 191.0455 & 238.66 & 203.75 & 196.91 & 196.71 & 197.61 & 197.5 & 207.34 & 200.64 & 5.62 \\
\hline 191.5453 & 238.16 & 203.97 & 196.75 & 196.57 & 197.52 & 197.38 & 206.95 & 200.67 & 5.64 \\
\hline 192.045 & 238.75 & 204.49 & 196.86 & 196.66 & 197.57 & 197.45 & 207.19 & 200.69 & 5.62 \\
\hline 192.544 & 238.5 & 204.15 & 196.86 & 196.68 & 197.59 & 197.45 & 207.22 & 200.7 & 5.58 \\
\hline 193.0457 & 238.79 & 203.34 & 196.88 & 196.72 & 197.54 & 197.51 & 207.37 & 200.75 & 5.55 \\
\hline 193.5445 & 238.88 & 205.26 & 196.9 & 196.72 & 197.63 & 197.52 & 207.41 & 200.62 & 5.57 \\
\hline 194.0443 & 238.74 & 204.29 & 196.92 & 196.7 & 197.62 & 197.49 & 207.39 & 200.72 & 5.60 \\
\hline 194.5452 & 238.76 & 205.14 & 196.9 & 196.7 & 197.62 & 197.47 & 207.33 & 200.64 & 5.59 \\
\hline 195.045 & 238.49 & 203.24 & 196.9 & 196.71 & 197.62 & 197.49 & 207.39 & 200.65 & 5.60 \\
\hline 195.5447 & 238.85 & 202.26 & 196.91 & 196.71 & 197.6 & 197.5 & 207.4 & 200.63 & 5.61 \\
\hline 196.0455 & 238.44 & 203.85 & 196.91 & 196.73 & 197.68 & 197.52 & 207.36 & 200.59 & 5.64 \\
\hline 196.5453 & 238.93 & 202.87 & 196.89 & 196.71 & 197.62 & 197.48 & 207.33 & 200.68 & 5.64 \\
\hline 197.0452 & 239.03 & 204.05 & 196.89 & 196.69 & 197.63 & 197.48 & 207.34 & 200.58 & 5.57 \\
\hline 197.545 & 238.82 & 202.8 & 196.89 & 196.71 & 197.61 & 197.48 & 207.34 & 200.57 & 5.56 \\
\hline
\end{tabular}


Table B3. Continued.

\begin{tabular}{|c|c|c|c|c|c|c|c|c|c|}
\hline $\begin{array}{l}\text { Time } \\
\text { min }\end{array}$ & $\begin{array}{l}\mathrm{T} 1 \\
{ }^{\circ} \mathrm{C}\end{array}$ & $\begin{array}{l}\mathrm{T} 2 \\
{ }^{\circ} \mathrm{C}\end{array}$ & $\begin{array}{l}\mathrm{T} 3 \\
{ }^{\circ} \mathrm{C}\end{array}$ & $\begin{array}{l}\mathrm{T} 4 \\
{ }^{\circ} \mathrm{C}\end{array}$ & $\begin{array}{l}\mathrm{T} 5 \\
{ }^{\circ} \mathrm{C}\end{array}$ & $\begin{array}{l}\mathrm{T} 6 \\
{ }^{\circ} \mathrm{C}\end{array}$ & $\begin{array}{l}\text { Pinj } \\
\text { psig }\end{array}$ & $\begin{array}{l}\text { Pout } \\
\text { psig }\end{array}$ & $\begin{array}{c}\mathrm{Vw} \\
\mathrm{cc} / \mathrm{min}\end{array}$ \\
\hline 198.0457 & 238.77 & 203.45 & 196.89 & 196.72 & 197.61 & 197.5 & 207.33 & 200.59 & 5.59 \\
\hline 198.5455 & 238.72 & 203.47 & 196.9 & 196.7 & 197.6 & 197.49 & 207.29 & 200.58 & 5.61 \\
\hline 199.0453 & 239.13 & 203.04 & 196.9 & 196.68 & 197.6 & 197.51 & 207.37 & 200.62 & 5.60 \\
\hline 199.5452 & 238.54 & 204.38 & 196.92 & 196.72 & 197.62 & 197.49 & 207.36 & 200.62 & 5.61 \\
\hline 200.045 & 238.29 & 204.21 & 196.9 & 196.7 & 197.6 & 197.49 & 207.29 & 200.53 & 5.60 \\
\hline 200.5438 & 238.88 & 204.62 & 196.9 & 196.7 & 197.6 & 197.49 & 207.38 & 200.6 & 5.62 \\
\hline 201.0455 & 239.15 & 203.96 & 196.9 & 196.69 & 197.6 & 197.49 & 207.33 & 200.53 & 5.61 \\
\hline 201.5445 & 238.4 & 204.09 & 196.9 & 196.69 & 197.6 & 197.48 & 207.31 & 200.57 & 5.56 \\
\hline 202.0452 & 238.94 & 204.44 & 196.89 & 196.69 & 197.6 & 197.5 & 207.3 & 200.65 & 5.56 \\
\hline 202.545 & 238.51 & 204.71 & 196.91 & 196.69 & 197.61 & 197.5 & 207.32 & 200.56 & 5.57 \\
\hline 203.0448 & 238.96 & 202.98 & 196.89 & 196.69 & 197.61 & 197.5 & 207.35 & 200.53 & 5.59 \\
\hline 203.5455 & 239.11 & 203.46 & 196.89 & 196.7 & 197.61 & 197.48 & 207.37 & 200.52 & 5.59 \\
\hline 204.0453 & 239.02 & 204.74 & 196.89 & 196.68 & 197.59 & 197.5 & 207.35 & 200.52 & 5.59 \\
\hline 204.5452 & 238.72 & 204.74 & 196.9 & 196.68 & 197.61 & 197.51 & 207.33 & 200.5 & 5.61 \\
\hline 205.045 & 237.64 & 204.31 & 196.9 & 196.7 & 197.61 & 197.51 & 207.34 & 200.51 & 5.62 \\
\hline 205.5448 & 238.31 & 204.53 & 196.69 & 196.45 & 197.37 & 197.2 & 206.13 & 200.6 & 5.65 \\
\hline 206.0455 & 238.33 & 204.32 & 196.83 & 196.63 & 197.57 & 197.4 & 207.19 & 200.66 & 5.59 \\
\hline 206.5453 & 238.65 & 205.01 & 196.87 & 196.65 & 197.59 & 197.46 & 207.2 & 200.68 & 5.55 \\
\hline 207.0452 & 238.82 & 205.61 & 196.87 & 196.67 & 197.59 & 197.5 & 207.3 & 200.7 & 5.55 \\
\hline 207.5442 & 238.53 & 204.97 & 196.93 & 196.71 & 197.62 & 197.52 & 207.5 & 200.8 & 5.59 \\
\hline 208.0448 & 238.32 & 203.57 & 196.93 & 196.75 & 197.64 & 197.54 & 207.52 & 200.73 & 5.60 \\
\hline 208.5447 & 238.36 & 204.32 & 196.91 & 196.71 & 197.63 & 197.52 & 207.43 & 200.69 & 5.59 \\
\hline 209.0445 & 238.88 & 204.41 & 196.9 & 196.72 & 197.61 & 197.52 & 207.47 & 200.7 & 5.60 \\
\hline 209.5453 & 238.25 & 203.07 & 196.9 & 196.72 & 197.63 & 197.51 & 207.47 & 200.64 & 5.62 \\
\hline 210.045 & 238.72 & 204.22 & 196.92 & 196.72 & 197.63 & 197.51 & 207.52 & 200.63 & 5.65 \\
\hline 210.5448 & 238.9 & 205.03 & 196.92 & 196.72 & 197.63 & 197.53 & 207.44 & 200.63 & 5.60 \\
\hline 211.0457 & 238.92 & 205.14 & 196.9 & 196.71 & 197.62 & 197.51 & 207.54 & 200.65 & 5.57 \\
\hline 211.5455 & 238.94 & 205.27 & 196.92 & 196.71 & 197.64 & 197.53 & 207.44 & 200.61 & 5.59 \\
\hline 212.0453 & 238.64 & 203.53 & 196.92 & 196.73 & 197.64 & 197.51 & 207.5 & 200.62 & 5.63 \\
\hline 212.5452 & 238.87 & 204.55 & 196.91 & 196.69 & 197.62 & 197.5 & 207.45 & 200.66 & 5.62 \\
\hline 213.045 & 238.85 & 205.72 & 196.91 & 196.71 & 197.63 & 197.52 & 207.47 & 200.61 & 5.63 \\
\hline 213.5457 & 238.96 & 203.98 & 196.91 & 196.71 & 197.63 & 197.52 & 207.47 & 200.66 & 5.64 \\
\hline 214.0455 & 239.05 & 203.39 & 196.93 & 196.73 & 197.66 & 197.52 & 207.52 & 200.64 & 5.67 \\
\hline 214.5443 & 238.79 & 203.77 & 196.91 & 196.72 & 197.61 & 197.52 & 207.49 & 200.6 & 5.59 \\
\hline 215.0442 & 238.95 & 204.36 & 196.9 & 196.7 & 197.63 & 197.52 & 207.45 & 200.59 & 5.58 \\
\hline 215.545 & 239.04 & 205.01 & 196.92 & 196.7 & 197.62 & 197.53 & 207.44 & 200.57 & 5.58 \\
\hline 216.0448 & 239.12 & 204.96 & 196.9 & 196.7 & 197.62 & 197.53 & 207.43 & 200.71 & 5.59 \\
\hline 216.5447 & 238.78 & 205.23 & 196.71 & 196.51 & 197.48 & 197.26 & 206.91 & 200.67 & 5.62 \\
\hline 217.0453 & 238.35 & 204.41 & 196.85 & 196.64 & 197.57 & 197.46 & 207.25 & 200.61 & 5.62 \\
\hline 217.5452 & 238.78 & 205.32 & 196.87 & 196.67 & 197.57 & 197.48 & 207.26 & 200.67 & 5.63 \\
\hline 218.045 & 238.19 & 204.43 & 196.87 & 196.69 & 197.6 & 197.5 & 207.39 & 200.68 & 5.65 \\
\hline 218.5448 & 238.85 & 205.02 & 196.89 & 196.71 & 197.62 & 197.52 & 207.46 & 200.64 & 5.60 \\
\hline 219.0455 & 238.84 & 205.9 & 196.91 & 196.69 & 197.61 & 197.52 & 207.46 & 200.64 & 5.56 \\
\hline
\end{tabular}


Table B3. Continued.

\begin{tabular}{|c|c|c|c|c|c|c|c|c|c|}
\hline $\begin{array}{c}\text { Time } \\
\text { min }\end{array}$ & $\begin{array}{l}\mathrm{T} 1 \\
{ }^{\circ} \mathrm{C} \\
\end{array}$ & $\begin{array}{l}\mathrm{T} 2 \\
{ }^{\circ} \mathrm{C} \\
\end{array}$ & $\begin{array}{l}\mathrm{T} 3 \\
{ }^{\circ} \mathrm{C} \\
\end{array}$ & $\begin{array}{l}\mathrm{T} 4 \\
{ }^{\circ} \mathrm{C} \\
\end{array}$ & $\begin{array}{l}\mathrm{T5} \\
{ }^{\circ} \mathrm{C} \\
\end{array}$ & $\begin{array}{l}\mathrm{T} 6 \\
{ }^{\circ} \mathrm{C} \\
\end{array}$ & $\begin{array}{l}\text { Pinj } \\
\text { psig }\end{array}$ & $\begin{array}{l}\text { Pout } \\
\text { psig }\end{array}$ & $\begin{array}{c}\mathrm{Vw} \\
\mathrm{cc} / \mathrm{min}\end{array}$ \\
\hline 219.5453 & 238.57 & 206.71 & 196.89 & 196.7 & 197.61 & 197.52 & 207.49 & 200.6 & 5.57 \\
\hline 220.0452 & 238.64 & 204.13 & 196.89 & 196.7 & 197.63 & 197.52 & 207.44 & 200.61 & 5.61 \\
\hline 220.544 & 239.11 & 204.74 & 196.9 & 196.7 & 197.61 & 197.5 & 207.42 & 200.65 & 5.60 \\
\hline 221.0438 & 238.5 & 204.58 & 196.9 & 196.7 & 197.61 & 197.52 & 207.49 & 200.64 & 5.60 \\
\hline 221.5447 & 238.95 & 204.56 & 196.9 & 196.72 & 197.72 & 197.53 & 207.47 & 200.63 & 5.61 \\
\hline 222.0445 & 238.58 & 203.97 & 196.9 & 196.7 & 197.62 & 197.53 & 207.47 & 200.57 & 5.64 \\
\hline 222.5452 & 238.85 & 204.17 & 196.9 & 196.69 & 197.62 & 197.51 & 207.43 & 200.64 & 5.63 \\
\hline 223.045 & 239.17 & 205.05 & 196.89 & 196.69 & 197.6 & 197.51 & 207.45 & 200.58 & 5.58 \\
\hline 223.5448 & 239.41 & 204.59 & 196.87 & 196.69 & 197.6 & 197.5 & 207.44 & 200.56 & 5.55 \\
\hline 224.0455 & 239.32 & 205.07 & 196.91 & 196.71 & 197.62 & 197.52 & 207.44 & 200.66 & 5.58 \\
\hline 224.5453 & 238.89 & 205.7 & 196.91 & 196.69 & 197.61 & 197.54 & 207.46 & 200.63 & 5.61 \\
\hline 225.0452 & 239.13 & 205.56 & 196.89 & 196.71 & 197.63 & 197.52 & 207.45 & 200.63 & 5.59 \\
\hline 225.545 & 239.04 & 204.97 & 196.91 & 196.7 & 197.61 & 197.52 & 207.46 & 200.6 & 5.60 \\
\hline 226.044 & 238.66 & 203.63 & 196.91 & 196.72 & 197.61 & 197.52 & 207.44 & 200.61 & 5.60 \\
\hline 226.5465 & 238.81 & 204.13 & 196.9 & 196.72 & 197.61 & 197.52 & 207.49 & 200.54 & 5.63 \\
\hline 227.0455 & 238.18 & 205.97 & 196.9 & 196.7 & 197.61 & 197.51 & 207.48 & 200.66 & 5.62 \\
\hline 227.5443 & 238.86 & 205.87 & 196.9 & 196.7 & 197.62 & 197.53 & 207.42 & .55 & 5.56 \\
\hline 228.045 & 238.92 & 206.53 & 196.9 & 196.7 & 197.6 & 197.53 & 207.57 & 200.6 & 5.57 \\
\hline 228.5448 & 238.81 & 205.5 & 196.65 & 196.44 & 197.37 & 197.21 & 206.38 & 200.55 & 5.59 \\
\hline 229.0447 & 238.54 & 206.43 & 196.81 & 196.63 & 197.55 & 197.42 & 207.16 & 200.54 & 5.63 \\
\hline 229.5455 & 238.92 & 205.96 & 196.83 & 196.64 & 197.55 & 197.46 & 207.22 & 200.65 & 5.61 \\
\hline 230.0453 & 238.64 & 204.69 & 196.87 & 196.69 & 197.6 & 197.5 & 207.36 & 200.55 & 5.61 \\
\hline 230.5452 & 238.05 & 206.52 & 196.89 & 196.71 & 197.62 & 197.52 & 207.43 & 200.69 & 5.62 \\
\hline 231.045 & 238.05 & 207.08 & 196.91 & 196.69 & 197.61 & 197.52 & 207.4 & 200.58 & 5.65 \\
\hline 231.5457 & 238.84 & 207.63 & 196.89 & 196.68 & 197.59 & 197.48 & 207.35 & 200.53 & 5.59 \\
\hline 232.0455 & 239.27 & 207.6 & 196.89 & 196.68 & 197.61 & 197.5 & 207.35 & 200.57 & 5.56 \\
\hline 232.5453 & 238.79 & 206.98 & 196.91 & 196.7 & 197.6 & 197.52 & 207.37 & 200.51 & 5.58 \\
\hline 233.0442 & 238.68 & 206.82 & 196.88 & 196.68 & 197.6 & 197.52 & 207.37 & 200.55 & 5.61 \\
\hline 233.544 & 239.24 & 204.94 & 196.9 & 196.68 & 197.6 & 197.51 & 207.38 & 200.53 & 5.61 \\
\hline 234.0457 & 237.98 & 205.98 & 196.9 & 196.7 & 197.62 & 197.53 & 207.39 & 200.53 & 5.61 \\
\hline 234.5447 & 238.74 & 206.35 & 196.9 & 196.7 & 197.6 & 197.51 & 207.37 & 200.51 & 5.61 \\
\hline 235.0453 & 239.06 & 206.89 & 196.88 & 196.69 & 197.6 & 197.53 & 207.38 & 200.55 & 5.64 \\
\hline 235.5452 & 238.85 & 205.39 & 196.9 & 196.67 & 197.58 & 197.51 & 207.37 & 200.49 & 5.61 \\
\hline 236.045 & 238.9 & 206.02 & 196.9 & 196.69 & 197.6 & 197.51 & 207.36 & 200.54 & 5.57 \\
\hline 236.5448 & 239.08 & 206.43 & 196.91 & 196.69 & 197.51 & 197.52 & 207.37 & 200.5 & 5.58 \\
\hline 237.0455 & 237.83 & 204.8 & 196.89 & 196.69 & 197.61 & 197.52 & 207.37 & 200.49 & 5.59 \\
\hline 237.5453 & 238.51 & 205.97 & 196.91 & 196.69 & 197.62 & 197.52 & 207.39 & 200.54 & 5.61 \\
\hline 238.0452 & 238.94 & 203.98 & 196.89 & 196.68 & 197.61 & 197.5 & 207.38 & 200.55 & 5.60 \\
\hline 238.544 & 238.68 & 205.45 & 196.7 & 196.51 & 197.46 & 197.23 & 206.92 & 200.48 & 5.60 \\
\hline 239.0438 & 238.82 & 205.74 & 196.79 & 196.59 & 197.5 & 197.41 & 207.01 & 200.41 & 5.63 \\
\hline 239.5455 & 238.52 & 205.85 & 196.84 & 196.64 & 197.56 & 197.45 & 207.23 & 200.66 & 5.65 \\
\hline
\end{tabular}


Table B4-Temperature and production data for run 4

\begin{tabular}{|c|c|c|c|c|c|c|c|c|c|}
\hline $\begin{array}{c}\text { Time } \\
\text { min }\end{array}$ & $\begin{array}{l}\mathrm{T} 1 \\
{ }^{\circ} \mathrm{C}\end{array}$ & $\begin{array}{l}\mathrm{T} 2 \\
{ }^{\circ} \mathrm{C}\end{array}$ & $\begin{array}{l}\mathrm{T} 3 \\
{ }^{\circ} \mathrm{C}\end{array}$ & $\begin{array}{l}\mathrm{T} 4 \\
{ }^{\circ} \mathrm{C}\end{array}$ & $\begin{array}{l}\mathrm{T} 5 \\
{ }^{\circ} \mathrm{C}\end{array}$ & $\begin{array}{l}\mathrm{T} 6 \\
{ }^{\circ} \mathrm{C}\end{array}$ & $\begin{array}{l}\text { Pinj } \\
\text { psig }\end{array}$ & $\begin{array}{c}\text { Pout } \\
\text { psig }\end{array}$ & $\begin{array}{c}\mathrm{Vw} \\
\mathrm{cc} / \mathrm{min}\end{array}$ \\
\hline 0.5455 & 247.89 & 176.06 & 62.21 & 64.43 & 63.61 & 57.5 & 207.19 & 203.66 & 5.57 \\
\hline 1.046167 & 246.48 & 193.18 & 62.24 & 64.45 & 63.64 & 57.54 & 207.59 & 203.98 & 5.55 \\
\hline 1.545167 & 245.05 & 195.29 & 62.27 & 64.48 & 63.69 & 57.57 & 207.97 & 204.45 & 5.55 \\
\hline 2.045 & 242.94 & 195.74 & 62.3 & 64.51 & 63.7 & 57.6 & 208.29 & 204.8 & 5.57 \\
\hline 2.544833 & 241.37 & 195.91 & 62.26 & 64.48 & 63.67 & 57.56 & 208.58 & 205.08 & 5.59 \\
\hline 3.0455 & 239.87 & 195.92 & 62.27 & 64.5 & 63.67 & 57.57 & 208.9 & 205.37 & 5.61 \\
\hline 3.545333 & 238.07 & 196.05 & 62.3 & 64.51 & 63.72 & 57.62 & 209.22 & 205.72 & 5.60 \\
\hline 4.044333 & 235.98 & 196.29 & 62.32 & 64.54 & 63.72 & 57.64 & 209.57 & 206.07 & 5.61 \\
\hline 4.545833 & 234.27 & 196.46 & 62.35 & 64.55 & 63.73 & 57.63 & 209.94 & 206.45 & 5.62 \\
\hline 5.045667 & 233.2 & 196.64 & 62.36 & 64.57 & 63.76 & 57.64 & 210.35 & 206.85 & 5.62 \\
\hline 5.5455 & 232.25 & 196.78 & 62.36 & 64.54 & 63.74 & 57.66 & 210.74 & 207.23 & 5.57 \\
\hline 6.046333 & 230.86 & 197.37 & 62.39 & 64.62 & 63.94 & 57.69 & 205.7 & 202.24 & 5.55 \\
\hline 6.545166 & 228.99 & 196.86 & 62.38 & 64.61 & 63.82 & 57.72 & 206.32 & 202.8 & 5.56 \\
\hline 7.045 & 229.16 & 196.78 & 62.38 & 64.64 & 63.84 & 57.72 & 206.25 & 202.57 & 5.58 \\
\hline 7.544833 & 229.92 & 197.14 & 62.41 & 64.66 & 63.87 & 57.75 & 4.71 & 201.02 & 5.58 \\
\hline 8.045667 & 230.21 & 197.17 & 62.38 & 64.65 & 63.87 & 57.74 & 203.17 & 199.47 & 5.58 \\
\hline 8.545333 & 230.5 & 197.19 & 62.42 & 64.64 & 63.88 & 57.74 & 201.53 & 197.88 & 5.58 \\
\hline 9.046167 & 231.04 & 196.48 & 62.39 & 64.62 & 63.87 & 57.73 & 206.43 & 202.68 & 5.58 \\
\hline 9.545 & 231.26 & 197.56 & 62.38 & 64.63 & 63.85 & 57.74 & 204.85 & 201.16 & 5.62 \\
\hline 10.04483 & 230.97 & 197.64 & 62.42 & 64.6 & 63.84 & 57.74 & 203.32 & 199.62 & 5.64 \\
\hline 10.5465 & 230.74 & 197.36 & 62.43 & 64.58 & 63.85 & 57.73 & 201.85 & 198.13 & 5.58 \\
\hline 11.04633 & 231 & 195.03 & 62.47 & 64.63 & 63.89 & 57.74 & 209.84 & 206.03 & 5.56 \\
\hline 11.54617 & 230.54 & 197.64 & 62.5 & 64.6 & 63.86 & 57.76 & 208.56 & 205.12 & 5.57 \\
\hline 12.045 & 229.38 & 198.14 & 62.52 & 64.58 & 63.85 & 57.73 & 208.46 & 204.89 & 5.59 \\
\hline 12.54483 & 229.22 & 198.27 & 62.58 & 64.61 & 63.85 & 57.71 & 207.92 & 204.28 & 5.60 \\
\hline 13.04567 & 229.87 & 198.21 & 62.63 & 64.59 & 63.84 & 57.74 & 207.79 & 204.18 & 5.59 \\
\hline 13.5455 & 232.04 & 198.44 & 62.69 & 64.58 & 63.84 & 57.73 & 207.75 & 204.08 & 5.58 \\
\hline 14.04533 & 234.59 & 198.7 & 62.77 & 64.57 & 63.83 & 57.71 & 207.72 & 204.1 & 5.61 \\
\hline 14.54517 & 236.01 & 198.92 & 62.85 & 64.55 & 63.84 & 57.7 & 207.82 & 204.14 & 5.65 \\
\hline 15.045 & 236.25 & 198.83 & 62.97 & 64.56 & 63.84 & 57.72 & 208.04 & 204.26 & 5.60 \\
\hline 15.54567 & 236 & 198.97 & 63.11 & 64.58 & 63.83 & 57.73 & 208.28 & 204.43 & 5.55 \\
\hline 16.0455 & 235.38 & 199.54 & 63.26 & 64.59 & 63.81 & 57.73 & 208.53 & 204.71 & 5.55 \\
\hline 16.54533 & 234.83 & 199.62 & 63.48 & 64.59 & 63.84 & 57.74 & 208.88 & 205.09 & 5.57 \\
\hline 17.04517 & 234.03 & 199.86 & 63.69 & 64.6 & 63.84 & 57.74 & 209.46 & 205.6 & 5.61 \\
\hline 17.545 & 233.51 & 200.72 & 63.96 & 64.6 & 63.86 & 57.75 & 210.16 & 206.26 & 5.60 \\
\hline 18.04483 & 233.18 & 200.89 & 64.25 & 64.62 & 63.89 & 57.75 & 210.92 & 206.97 & 5.60 \\
\hline 18.5455 & 232.39 & 201.37 & 64.69 & 64.61 & 63.91 & 57.79 & 202.28 & 198.46 & 5.60 \\
\hline 19.04533 & 232.93 & 201.16 & 65.07 & 64.61 & 63.91 & 57.78 & 201.98 & 198.12 & 5.63 \\
\hline 19.54517 & 233.67 & 200.45 & 65.41 & 64.58 & 63.9 & 57.74 & 201.68 & 197.76 & 5.63 \\
\hline 20.045 & 234.88 & 199.43 & 65.7 & 64.59 & 63.9 & 57.77 & 207.1 & 203.21 & 5.58 \\
\hline 20.54483 & 235.38 & 199.98 & 66.14 & 64.57 & 63.91 & 57.75 & 206.2 & 202.31 & 5.57 \\
\hline 21.0455 & 235.53 & 199.73 & 66.58 & 64.55 & 63.93 & 57.77 & 205.5 & 201.53 & 5.58 \\
\hline 21.54533 & 235.6 & 199.73 & 67.09 & 64.54 & 63.92 & 57.74 & 204.89 & 200.91 & 5.62 \\
\hline 22.04517 & 235.73 & 199.43 & 67.62 & 64.54 & 63.9 & 57.72 & 204.37 & 200.35 & 5.62 \\
\hline
\end{tabular}


Table B4. Continued.

\begin{tabular}{|c|c|c|c|c|c|c|c|c|c|}
\hline $\begin{array}{c}\text { Time } \\
\min \end{array}$ & $\begin{array}{l}\mathrm{T} 1 \\
{ }^{\circ} \mathrm{C} \\
\end{array}$ & $\begin{array}{l}\mathrm{T} 2 \\
{ }^{\circ} \mathrm{C} \\
\end{array}$ & $\begin{array}{l}\mathrm{T} 3 \\
{ }^{\circ} \mathrm{C} \\
\end{array}$ & $\begin{array}{l}\mathrm{T} 4 \\
{ }^{\circ} \mathrm{C} \\
\end{array}$ & $\begin{array}{l}\mathrm{T} 5 \\
{ }^{\circ} \mathrm{C} \\
\end{array}$ & $\begin{array}{l}\mathrm{T} 6 \\
{ }^{\circ} \mathrm{C} \\
\end{array}$ & $\begin{array}{l}\text { Pinj } \\
\text { psig }\end{array}$ & $\begin{array}{l}\text { Pout } \\
\text { psig }\end{array}$ & $\begin{array}{c}\mathrm{Vw} \\
\mathrm{cc} / \mathrm{min}\end{array}$ \\
\hline 22.545 & 235.54 & 199.49 & 68.21 & 64.55 & 63.92 & 57.73 & 203.9 & 199.95 & 5.62 \\
\hline 23.04483 & 235.27 & 199.31 & 68.85 & 64.59 & 63.94 & 57.75 & 203.57 & 199.51 & 5.63 \\
\hline 23.5455 & 234.47 & 199.81 & 69.53 & 64.59 & 63.93 & 57.73 & 203.32 & 199.22 & 5.63 \\
\hline 24.04533 & 233.93 & 200.32 & 70.31 & 64.61 & 63.87 & 57.74 & 203.4 & 199.02 & 5.59 \\
\hline 24.54517 & 233.42 & 199.94 & 71.29 & 64.61 & 63.86 & 57.74 & 203.01 & 198.79 & 5.57 \\
\hline 25.045 & 233.03 & 199.46 & 72.5 & 64.62 & 63.81 & 57.74 & 202.81 & 198.57 & 5.56 \\
\hline 25.54483 & 232.72 & 199.27 & 73.95 & 64.62 & 63.92 & 57.75 & 202.68 & 198.41 & 5.59 \\
\hline 26.04567 & 232.44 & 199.36 & 75.78 & 64.62 & 63.81 & 57.71 & 202.62 & 198.2 & 5.61 \\
\hline 26.54533 & 232.01 & 198.74 & 77.97 & 64.62 & 63.81 & 57.71 & 202.85 & 199.59 & 5.59 \\
\hline 27.04517 & 232.3 & 198.95 & 79.25 & 64.63 & 63.81 & 57.71 & 207.21 & 203.27 & 5.59 \\
\hline 27.545 & 231.96 & 199.51 & 80.58 & 64.63 & 63.84 & 57.76 & 207.54 & 202.99 & 5.61 \\
\hline 28.04483 & 232.56 & 199.78 & 84.5 & 64.63 & 63.84 & 57.72 & 207.64 & 202.96 & 5.64 \\
\hline 28.54567 & 234.64 & 201.04 & 90.04 & 64.63 & 63.82 & 57.72 & 207.84 & 203.02 & 5.62 \\
\hline 29.0455 & 234.64 & 201.06 & 97.83 & 64.62 & 63.8 & 57.74 & 208.22 & 203.28 & 5.58 \\
\hline 29.54533 & 235.43 & 201.27 & 108.88 & 64.58 & 63.77 & 57.74 & 208.76 & 203.63 & 5.57 \\
\hline 30.045 & 235.9 & 201.04 & 123.74 & 64.58 & 63.71 & 57.73 & 209.27 & 204.08 & 5.59 \\
\hline 30.54583 & 235.61 & 201.71 & 142.12 & 64.58 & 63.75 & 57.73 & 208.59 & 203.75 & 5.62 \\
\hline 31.04567 & 235.86 & 202.62 & 157.14 & 64.57 & 63.7 & 57.71 & 206.42 & 198.03 & 5.60 \\
\hline 31.5455 & 235.4 & 203.82 & 182.03 & 64.53 & 63.7 & 57.67 & 205.59 & 201.04 & 5.61 \\
\hline 32.04617 & 236.31 & 203.09 & 187.54 & 64.5 & 63.7 & 57.62 & 206.92 & 202.46 & 5.63 \\
\hline 32.54517 & 236.94 & 202.55 & 190.7 & 64.46 & 63.68 & 57.6 & 207.74 & 202.62 & 5.65 \\
\hline 33.045 & 237.05 & 201.66 & 193.35 & 64.4 & 63.69 & 57.55 & 206.69 & 201.77 & 5.61 \\
\hline 33.54567 & 237.29 & 201.21 & 194.7 & 64.42 & 63.71 & 57.53 & 205.69 & 200.75 & 5.57 \\
\hline 34.04633 & 237.18 & 201.77 & 195.38 & 64.41 & 63.69 & 57.51 & 204.79 & 199.77 & 5.57 \\
\hline 34.54533 & 237.31 & 201.41 & 195.68 & 64.41 & 63.71 & 57.51 & 203.89 & 198.69 & 5.60 \\
\hline 35.04417 & 237.41 & 201.54 & 195.9 & 64.41 & 63.71 & 57.51 & 204.21 & 200.15 & 5.62 \\
\hline 35.545 & 237.72 & 201.13 & 196.02 & 64.43 & 63.73 & 57.51 & 203.9 & 198.73 & 5.59 \\
\hline 36.04483 & 237.81 & 200.56 & 196.29 & 64.49 & 63.73 & 57.5 & 205.1 & 201.24 & 5.61 \\
\hline 36.54467 & 237.88 & 200.47 & 196.42 & 64.49 & 63.71 & 57.48 & 205.72 & 200.88 & 5.62 \\
\hline 37.04533 & 237.58 & 200.36 & 196.6 & 64.51 & 63.77 & 57.52 & 205.6 & 200.82 & 5.63 \\
\hline 37.54517 & 237.31 & 200.27 & 196.71 & 64.54 & 63.79 & 57.5 & 205.58 & 200.86 & 5.57 \\
\hline 38.045 & 237.06 & 200.52 & 196.78 & 64.58 & 63.9 & 57.46 & 205.64 & 200.91 & 5.56 \\
\hline 38.54483 & 236.94 & 200.79 & 196.87 & 64.64 & 63.87 & 57.48 & 205.81 & 200.97 & 5.55 \\
\hline 39.04467 & 236.67 & 201.26 & 196.93 & 64.7 & 63.91 & 57.46 & 205.98 & 201.09 & 5.61 \\
\hline 39.54533 & 236.33 & 201.01 & 197.02 & 64.78 & 63.94 & 57.47 & 206.21 & 201.24 & 5.60 \\
\hline 40.04517 & 236.08 & 200.8 & 197.07 & 64.83 & 64.04 & 57.47 & 206.68 & 201.49 & 5.60 \\
\hline 40.545 & 235.22 & 200.74 & 197.18 & 64.93 & 64.06 & 57.49 & 207.02 & 201.92 & 5.61 \\
\hline 41.04483 & 234.52 & 200.84 & 197.33 & 65.08 & 64.14 & 57.53 & 207.69 & 202.53 & 5.59 \\
\hline 41.5455 & 234.1 & 201.71 & 197.51 & 65.18 & 64.2 & 57.53 & 208.68 & 203.34 & 5.62 \\
\hline 42.04633 & 233.9 & 201.86 & 197.72 & 65.35 & 64.25 & 57.53 & 209.73 & 204.34 & 5.58 \\
\hline 42.54617 & 233.26 & 202.86 & 197.72 & 65.56 & 64.33 & 57.49 & 207.51 & 201.64 & 5.56 \\
\hline 43.045 & 232.83 & 203.95 & 197.38 & 65.82 & 64.43 & 57.51 & 207.48 & 201.54 & 5.56 \\
\hline 43.54483 & 233.54 & 202.47 & 197.28 & 66.15 & 64.43 & 57.49 & 206.72 & 200.46 & 5.57 \\
\hline 44.04567 & 234.1 & 201.83 & 197.15 & 66.64 & 64.43 & 57.49 & 206.36 & 200.37 & 5.61 \\
\hline
\end{tabular}


Table B4. Continued.

\begin{tabular}{|c|c|c|c|c|c|c|c|c|c|}
\hline $\begin{array}{l}\text { Time } \\
\text { min }\end{array}$ & $\begin{array}{l}\mathrm{T} 1 \\
{ }^{\circ} \mathrm{C}\end{array}$ & $\begin{array}{l}\mathrm{T} 2 \\
{ }^{\circ} \mathrm{C}\end{array}$ & $\begin{array}{l}\mathrm{T} 3 \\
{ }^{\circ} \mathrm{C}\end{array}$ & $\begin{array}{l}\mathrm{T} 4 \\
{ }^{\circ} \mathrm{C}\end{array}$ & $\begin{array}{l}\mathrm{T} 5 \\
{ }^{\circ} \mathrm{C}\end{array}$ & $\begin{array}{l}\mathrm{T} 6 \\
{ }^{\circ} \mathrm{C}\end{array}$ & $\begin{array}{l}\text { Pinj } \\
\text { psig }\end{array}$ & $\begin{array}{c}\text { Pout } \\
\text { psig }\end{array}$ & $\begin{array}{c}\mathrm{Vw} \\
\mathrm{cc} / \mathrm{min}\end{array}$ \\
\hline 44.54533 & 234.84 & 201.74 & 197.08 & 67.39 & 64.49 & 57.5 & 206.11 & 200.41 & 5.59 \\
\hline 45.04517 & 235.52 & 202.03 & 197.03 & 68.55 & 64.52 & 57.48 & 205.97 & 200.41 & 5.60 \\
\hline 45.545 & 236.25 & 201.94 & 197.03 & 70.13 & 64.56 & 57.48 & 205.82 & 200.43 & 5.60 \\
\hline 46.04583 & 236.63 & 202.06 & 197.01 & 72.11 & 64.6 & 57.52 & 205.76 & 200.43 & 5.63 \\
\hline 46.54467 & 236.97 & 201.19 & 197 & 74.46 & 64.64 & 57.61 & 205.67 & 200.44 & 5.61 \\
\hline 47.0445 & 237.44 & 201.47 & 196.96 & 77.07 & 64.64 & 57.67 & 205.72 & 200.46 & 5.57 \\
\hline 47.54533 & 237.64 & 201.62 & 196.96 & 79.96 & 64.68 & 57.77 & 205.77 & 200.43 & 5.55 \\
\hline 48.045 & 237.71 & 201.32 & 196.98 & 83.18 & 64.7 & 57.85 & 205.79 & 200.47 & 5.57 \\
\hline 48.54483 & 237.52 & 201.12 & 197 & 86.66 & 64.72 & 57.94 & 205.86 & 200.45 & 5.59 \\
\hline 49.04567 & 237.39 & 201.48 & 197.02 & 90.45 & 64.74 & 58.04 & 205.96 & 200.46 & 5.60 \\
\hline 49.5455 & 236.93 & 200.94 & 197.04 & 94.6 & 64.74 & 58.14 & 205.99 & 200.46 & 5.59 \\
\hline 50.04533 & 236.66 & 201.3 & 197.06 & 99.02 & 64.75 & 58.23 & 206 & 200.47 & 5.61 \\
\hline 50.54517 & 236.7 & 200.95 & 197.04 & 103.83 & 64.75 & 58.27 & 206.11 & 200.49 & 5.61 \\
\hline 51.04583 & 236.54 & 200.66 & 197.08 & 109.67 & 64.79 & 58.37 & 206.24 & 200.47 & 5.61 \\
\hline 51.5475 & 236.2 & 201.63 & 197.12 & 116.94 & 64.77 & 58.47 & 206.34 & 200.47 & 5.62 \\
\hline 52.0455 & 236 & 201.13 & 197.14 & 125.9 & 64.81 & 58.56 & 206.53 & 200.49 & 5.58 \\
\hline 52.54533 & 235.85 & 201.17 & 197.2 & 139.71 & 64.81 & 58.68 & 206.8 & 200.51 & 5.58 \\
\hline 53.04517 & 235.51 & 201.19 & 197.25 & 156.74 & 64.79 & 58.76 & 207.15 & 200.5 & 5.61 \\
\hline 53.54583 & 235.2 & 201.5 & 197.32 & 171 & 64.82 & 58.84 & 207.45 & 200.63 & 5.61 \\
\hline 54.04567 & 234.9 & 202.47 & 197.34 & 183.38 & 64.82 & 58.97 & 207.53 & 200.64 & 5.60 \\
\hline 54.5455 & 234.69 & 202 & 197.38 & 190.2 & 64.88 & 59.1 & 207.75 & 200.82 & 5.61 \\
\hline 55.04433 & 234.55 & 202.54 & 197.44 & 193.62 & 64.9 & 59.2 & 207.9 & 200.83 & 5.63 \\
\hline 55.54417 & 234.64 & 202.38 & 197.42 & 195.4 & 64.94 & 59.32 & 207.79 & 200.71 & 5.63 \\
\hline 56.045 & 234.98 & 202.73 & 197.41 & 196.19 & 64.98 & 59.43 & 207.57 & 200.66 & 5.58 \\
\hline 56.54483 & 235.42 & 202.09 & 197.34 & 196.44 & 65.02 & 59.51 & 207.33 & 200.56 & 5.56 \\
\hline 57.0465 & 236.04 & 202 & 197.29 & 196.54 & 65.06 & 59.63 & 207.15 & 200.57 & 5.57 \\
\hline 57.54633 & 236.57 & 201.55 & 197.13 & 196.45 & 65.08 & 59.71 & 206.47 & 201.27 & 5.60 \\
\hline 58.04517 & 236.68 & 202.07 & 197.19 & 196.58 & 65.11 & 59.75 & 206.92 & 201.29 & 5.60 \\
\hline 58.54417 & 237 & 201.65 & 197.29 & 196.69 & 64.99 & 59.81 & 207.21 & 201.31 & 5.60 \\
\hline 59.04567 & 237.2 & 201.15 & 197.3 & 196.71 & 65.17 & 59.87 & 207.15 & 201.2 & 5.60 \\
\hline 59.5465 & 237.44 & 201.67 & 197.28 & 196.73 & 65.19 & 59.93 & 207.11 & 201.12 & 5.62 \\
\hline 60.04533 & 237.62 & 201.31 & 197.25 & 196.68 & 65.23 & 59.97 & 206.93 & 200.97 & 5.63 \\
\hline 60.54517 & 237.6 & 201.48 & 197.22 & 196.68 & 65.29 & 60.03 & 206.84 & 200.92 & 5.59 \\
\hline 61.045 & 237.46 & 200.94 & 197.22 & 196.66 & 65.35 & 60.09 & 206.76 & 200.82 & 5.55 \\
\hline 61.54583 & 237.43 & 200.93 & 197.2 & 196.67 & 65.39 & 60.13 & 206.74 & 200.79 & 5.56 \\
\hline 62.04567 & 237.47 & 200.65 & 197.19 & 196.65 & 65.47 & 60.17 & 206.76 & 200.75 & 5.61 \\
\hline 62.54533 & 237.11 & 201.87 & 197.07 & 196.55 & 65.6 & 60.25 & 206.1 & 201.3 & 5.61 \\
\hline 63.04517 & 236.97 & 201.24 & 197.11 & 196.62 & 65.68 & 60.29 & 206.53 & 201.33 & 5.58 \\
\hline 63.54417 & 236.92 & 201.32 & 197.22 & 196.75 & 65.8 & 60.31 & 207.05 & 199.53 & 5.60 \\
\hline 64.044 & 236.46 & 200.71 & 197.2 & 196.74 & 65.97 & 60.39 & 206.86 & 201.24 & 5.61 \\
\hline 64.54567 & 236.07 & 201.31 & 197.22 & 196.76 & 66.2 & 60.41 & 206.94 & 200.33 & 5.62 \\
\hline 65.0445 & 235.82 & 201.45 & 197.23 & 196.74 & 66.49 & 60.51 & 206.93 & 200.33 & 5.59 \\
\hline 65.54533 & 235.66 & 202.44 & 197.25 & 196.78 & 66.93 & 60.55 & 207.14 & 200.32 & 5.56 \\
\hline 66.045 & 235.25 & 202.7 & 197.29 & 196.86 & 67.31 & 60.65 & 207.35 & 200.36 & 5.57 \\
\hline
\end{tabular}


Table B4. Continued.

\begin{tabular}{|c|c|c|c|c|c|c|c|c|c|}
\hline $\begin{array}{l}\text { Time } \\
\text { min }\end{array}$ & $\begin{array}{l}\mathrm{T} 1 \\
{ }^{\circ} \mathrm{C}\end{array}$ & $\begin{array}{l}\mathrm{T} 2 \\
{ }^{\circ} \mathrm{C}\end{array}$ & $\begin{array}{l}\mathrm{T} 3 \\
{ }^{\circ} \mathrm{C}\end{array}$ & $\begin{array}{l}\mathrm{T} 4 \\
{ }^{\circ} \mathrm{C}\end{array}$ & $\begin{array}{l}\mathrm{T} 5 \\
{ }^{\circ} \mathrm{C}\end{array}$ & $\begin{array}{l}\mathrm{T} 6 \\
{ }^{\circ} \mathrm{C}\end{array}$ & $\begin{array}{l}\text { Pinj } \\
\text { psig }\end{array}$ & $\begin{array}{l}\text { Pout } \\
\text { psig }\end{array}$ & $\begin{array}{c}\mathrm{Vw} \\
\mathrm{cc} / \mathrm{min}\end{array}$ \\
\hline 66.544 & 235.13 & 202.2 & 197.33 & 196.88 & 67.9 & 60.69 & 207.53 & 200.35 & 5.58 \\
\hline 67.04567 & 234.97 & 202.84 & 197.28 & 196.79 & 68.69 & 60.77 & 206.99 & 201.02 & 5.61 \\
\hline 67.5445 & 235.16 & 202.35 & 197.3 & 196.88 & 69.54 & 60.83 & 207.63 & 201.02 & 5.63 \\
\hline 68.04533 & 235.47 & 203.48 & 197.43 & 197 & 70.66 & 60.91 & 208.2 & 201.08 & 5.60 \\
\hline 68.54417 & 235.54 & 202.64 & 197.43 & 197 & 72.23 & 60.94 & 207.95 & 200.86 & 5.64 \\
\hline 69.04501 & 235.87 & 202.22 & 197.44 & 197 & 74.34 & 61.02 & 207.85 & 200.78 & 5.65 \\
\hline 69.54567 & 236.34 & 202.85 & 197.4 & 196.97 & 76.97 & 61.08 & 207.8 & 200.68 & 5.59 \\
\hline 70.0455 & 236.41 & 202.87 & 197.37 & 196.98 & 80.1 & 61.12 & 207.64 & 200.6 & 5.56 \\
\hline 70.54433 & 236.58 & 202.39 & 197.37 & 196.98 & 83.95 & 61.2 & 207.58 & 200.55 & 5.58 \\
\hline 71.04517 & 236.94 & 201.94 & 197.34 & 196.93 & 88.28 & 61.24 & 207.4 & 200.49 & 5.61 \\
\hline 71.54501 & 237.14 & 201.45 & 197.22 & 196.79 & 93.35 & 61.28 & 206.72 & 200.88 & 5.60 \\
\hline 72.04483 & 237.36 & 201.6 & 197.17 & 196.8 & 97.05 & 61.33 & 206.87 & 200.9 & 5.61 \\
\hline 72.5455 & 237.42 & 201.89 & 197.19 & 196.82 & 101.3 & 61.37 & 206.79 & 199.84 & 5.62 \\
\hline 73.04533 & 237.34 & 201.5 & 197.16 & 196.8 & 107.37 & 61.41 & 206.62 & 199.76 & 5.63 \\
\hline 73.54517 & 237.47 & 201.72 & 197.13 & 196.76 & 114.23 & 61.47 & 206.48 & 199.72 & 5.62 \\
\hline 74.04501 & 237.43 & 200.93 & 197.1 & 196.74 & 121.95 & 61.51 & 206.34 & 199.69 & 5.57 \\
\hline 74.54483 & 237.42 & 200.74 & 197.09 & 196.71 & 128.68 & 61.56 & 206.36 & 199.66 & 5.55 \\
\hline 75.0455 & 237.39 & 200.76 & 197.11 & 196.73 & 134.1 & 61.58 & 206.46 & 201.4 & 5.58 \\
\hline 75.54533 & 237.25 & 201.2 & 197.17 & 196.83 & 134.91 & 61.62 & 206.94 & 200.23 & 5.61 \\
\hline 76.04617 & 236.79 & 201.4 & 197.22 & 196.88 & 140.44 & 61.63 & 207.1 & 200.27 & 5.60 \\
\hline 76.54501 & 236.7 & 201.65 & 197.26 & 196.92 & 146.85 & 61.71 & 207.29 & 200.25 & 5.61 \\
\hline 77.04483 & 236.69 & 201.51 & 197.3 & 196.96 & 153.69 & 61.71 & 207.47 & 200.23 & 5.62 \\
\hline 77.5455 & 236.5 & 201.59 & 197.26 & 196.88 & 161.76 & 61.77 & 207.13 & 200.78 & 5.65 \\
\hline 78.04533 & 236.25 & 201.63 & 197.3 & 196.94 & 166.65 & 61.8 & 207.43 & 200.87 & 5.60 \\
\hline 78.54517 & 236.02 & 201.81 & 197.37 & 197.05 & 173.75 & 61.86 & 207.91 & 199.39 & 5.57 \\
\hline 79.04501 & 235.6 & 202.71 & 197.45 & 197.07 & 182.19 & 61.92 & 208.06 & 199.36 & 5.58 \\
\hline 79.54483 & 235.37 & 201.96 & 197.45 & 197.09 & 187.94 & 61.98 & 208.22 & 199.37 & 5.61 \\
\hline 80.04567 & 235.1 & 202.35 & 197.49 & 197.12 & 191.39 & 62.04 & 208.22 & 199.39 & 5.61 \\
\hline 80.54633 & 235.23 & 203.39 & 197.48 & 197.12 & 193.68 & 62.15 & 208.26 & 199.39 & 5.60 \\
\hline 81.04517 & 235.52 & 202.69 & 197.52 & 197.18 & 194.58 & 62.2 & 208.64 & 201.12 & 5.60 \\
\hline 81.54501 & 235.6 & 202.93 & 197.6 & 197.26 & 195.41 & 62.27 & 208.89 & 200.01 & 5.63 \\
\hline 82.04483 & 235.91 & 202.83 & 197.5 & 197.12 & 196.53 & 62.39 & 208.11 & 200.54 & 5.62 \\
\hline 82.54467 & 236.31 & 203.07 & 197.46 & 197.12 & 196.91 & 62.5 & 208.21 & 200.64 & 5.57 \\
\hline 83.0455 & 236.75 & 203.11 & 197.5 & 197.17 & 197.11 & 62.62 & 208.41 & 200.63 & 5.57 \\
\hline 83.54433 & 236.82 & 203.74 & 197.53 & 197.19 & 197.28 & 62.76 & 208.49 & 200.61 & 5.58 \\
\hline 84.04501 & 236.99 & 204.59 & 197.53 & 197.19 & 197.37 & 62.92 & 208.42 & 200.56 & 5.61 \\
\hline 84.54483 & 237.15 & 203.23 & 197.5 & 197.16 & 197.43 & 63.09 & 208.26 & 200.51 & 5.60 \\
\hline 85.04467 & 237 & 202.84 & 197.47 & 197.13 & 197.47 & 63.25 & 208.19 & 200.46 & 5.61 \\
\hline 85.5455 & 237.24 & 203.81 & 197.46 & 197.12 & 197.49 & 63.44 & 208.01 & 200.41 & 5.62 \\
\hline 86.04533 & 237.17 & 203.52 & 197.43 & 197.09 & 197.62 & 63.62 & 207.92 & 200.36 & 5.64 \\
\hline 86.54517 & 237.05 & 202.52 & 197.33 & 196.97 & 197.45 & 63.87 & 207.25 & 200.6 & 5.62 \\
\hline 87.04583 & 237.25 & 203.53 & 197.22 & 196.88 & 197.44 & 64.07 & 207.07 & 200.55 & 5.57 \\
\hline 87.54567 & 237.33 & 202.08 & 197.23 & 196.92 & 197.48 & 64.28 & 207.25 & 200.52 & 5.56 \\
\hline 88.0455 & 237.41 & 202.34 & 197.27 & 197 & 197.54 & 64.53 & 207.37 & 200.52 & 5.59 \\
\hline
\end{tabular}


Table B4. Continued.

\begin{tabular}{|c|c|c|c|c|c|c|c|c|c|}
\hline $\begin{array}{c}\text { Time } \\
\text { min }\end{array}$ & $\begin{array}{l}\mathrm{T} 1 \\
{ }^{\circ} \mathrm{C} \\
\end{array}$ & $\begin{array}{l}\mathrm{T} 2 \\
{ }^{\circ} \mathrm{C} \\
\end{array}$ & $\begin{array}{l}\mathrm{T} 3 \\
{ }^{\circ} \mathrm{C} \\
\end{array}$ & $\begin{array}{l}\mathrm{T} 4 \\
{ }^{\circ} \mathrm{C} \\
\end{array}$ & $\begin{array}{l}\mathrm{T5} \\
{ }^{\circ} \mathrm{C} \\
\end{array}$ & $\begin{array}{l}\mathrm{T} 6 \\
{ }^{\circ} \mathrm{C} \\
\end{array}$ & $\begin{array}{l}\text { Pinj } \\
\text { psig }\end{array}$ & $\begin{array}{l}\text { Pout } \\
\text { psig }\end{array}$ & $\begin{array}{l}\mathrm{Vw} \\
\mathrm{cc} / \mathrm{min}\end{array}$ \\
\hline 88.54533 & 237.09 & 202.43 & 197.31 & 197.02 & 197.61 & 64.89 & 207.63 & 200.5 & 5.62 \\
\hline 89.04517 & 236.98 & 202.49 & 197.35 & 197.05 & 197.65 & 65.28 & 207.71 & 200.49 & 5.60 \\
\hline 89.54501 & 236.76 & 202.4 & 197.37 & 197.07 & 197.68 & 65.77 & 207.85 & 200.47 & 5.61 \\
\hline 90.04567 & 236.74 & 202.5 & 197.4 & 197.07 & 197.7 & 66.33 & 207.94 & 200.45 & 5.61 \\
\hline 90.5455 & 236.41 & 202.15 & 197.44 & 197.11 & 197.76 & 66.96 & 208.12 & 200.45 & 5.65 \\
\hline 91.04617 & 236.25 & 202.13 & 197.44 & 197.14 & 197.8 & 67.66 & 208.25 & 200.44 & 5.62 \\
\hline 91.546 & 236.17 & 202.59 & 197.45 & 197.09 & 197.73 & 68.55 & 207.84 & 200.66 & 5.56 \\
\hline 92.04501 & 235.96 & 203.67 & 197.35 & 197.04 & 197.72 & 69.48 & 207.74 & 200.59 & 5.56 \\
\hline 92.54483 & 235.78 & 203.17 & 197.39 & 197.1 & 197.78 & 70.33 & 208.14 & 200.56 & 5.58 \\
\hline 93.0455 & 235.88 & 203.07 & 197.48 & 197.18 & 197.8 & 71.43 & 208.52 & 200.59 & 5.60 \\
\hline 93.54533 & 236.05 & 203.99 & 197.56 & 197.25 & 197.95 & 72.76 & 208.84 & 200.61 & 5.60 \\
\hline 94.04433 & 236.21 & 203.76 & 197.6 & 197.3 & 197.99 & 74.27 & 208.96 & 200.6 & 5.61 \\
\hline 94.54417 & 236.33 & 203.96 & 197.61 & 197.28 & 198.02 & 76.08 & 208.96 & 200.59 & 5.62 \\
\hline 95.04483 & 236.46 & 203.66 & 197.61 & 197.25 & 198 & 78.18 & 208.83 & 200.56 & 5.64 \\
\hline 95.54467 & 236.39 & 205.49 & 197.51 & 197.17 & 197.87 & 80.91 & 208.17 & 200.63 & 5.60 \\
\hline 96.04533 & 236.63 & 204.71 & 197.42 & 197.1 & 197.83 & 83.75 & 208.06 & 200.59 & 5.57 \\
\hline 96.54517 & 236.9 & 203.34 & 197.41 & 197.12 & 197.86 & 87.03 & 208.23 & 200.57 & 5.58 \\
\hline 97.04501 & 236.92 & 203.25 & 197.42 & 197.09 & 197.85 & 90.93 & 208.06 & 200.53 & 5.61 \\
\hline 97.54483 & 237.2 & 202.29 & 197.37 & 197.06 & 197.83 & 95.29 & 207.92 & 200.48 & 5.61 \\
\hline 98.04467 & 237.2 & 202.28 & 197.35 & 197.03 & 197.8 & 100.01 & 207.67 & 200.43 & 5.61 \\
\hline 98.5445 & 237.28 & 202.2 & 197.29 & 196.97 & 197.63 & 104.92 & 207.22 & 200.04 & 5.62 \\
\hline 99.04517 & 236.97 & 202.81 & 197.01 & 196.65 & 197.4 & 110.46 & 205.85 & 200.72 & 5.63 \\
\hline 99.54501 & 237.02 & 202.08 & 196.98 & 196.69 & 197.46 & 112.73 & 206.22 & 200.75 & 5.61 \\
\hline 100.0448 & 237.14 & 203.25 & 197.05 & 196.79 & 197.56 & 114.81 & 206.61 & 200.74 & 5.56 \\
\hline 100.5457 & 237.39 & 202.72 & 197.11 & 196.83 & 197.63 & 117.54 & 206.87 & 200.72 & 5.57 \\
\hline 101.0455 & 237.33 & 202.92 & 197.17 & 196.87 & 197.69 & 120.97 & 207 & 200.76 & 5.59 \\
\hline 101.5453 & 237.47 & 202.08 & 197.2 & 196.87 & 197.68 & 125.18 & 207.01 & 200.73 & 5.61 \\
\hline 102.045 & 237.57 & 202.16 & 197.18 & 196.88 & 197.67 & 129.75 & 206.99 & 200.67 & 5.59 \\
\hline 102.5448 & 237.34 & 201.72 & 197.17 & 196.87 & 197.65 & 134.84 & 206.86 & 200.61 & 5.62 \\
\hline 103.0447 & 237.13 & 201.74 & 197.16 & 196.85 & 197.66 & 139.98 & 206.84 & 198.01 & 5.61 \\
\hline 103.5455 & 236.69 & 201.86 & 196.84 & 196.52 & 197.31 & 148.4 & 205.27 & 200.77 & 5.63 \\
\hline 104.0453 & 236.75 & 202.51 & 196.86 & 196.6 & 197.42 & 147.74 & 205.79 & 200.77 & 5.61 \\
\hline 104.5452 & 236.67 & 203.73 & 197 & 196.73 & 197.55 & 147.89 & 206.46 & 200.83 & 5.57 \\
\hline 105.045 & 236.67 & 202.68 & 197.13 & 196.86 & 197.75 & 150.36 & 206.99 & 200.85 & 5.56 \\
\hline 105.5457 & 236.62 & 202.7 & 197.19 & 196.92 & 197.72 & 156.89 & 207.18 & 200.86 & 5.58 \\
\hline 106.0455 & 236.58 & 203.01 & 197.23 & 196.92 & 197.73 & 166.73 & 207.28 & 200.82 & 5.60 \\
\hline 106.5453 & 236.38 & 202.91 & 197.18 & 196.82 & 197.55 & 176.64 & 206.44 & 200.45 & 5.59 \\
\hline 107.0442 & 236.3 & 203.22 & 196.93 & 196.61 & 197.45 & 182.68 & 205.98 & 200.85 & 5.59 \\
\hline 107.544 & 236.45 & 201.79 & 197.03 & 196.74 & 197.57 & 182.54 & 206.56 & 200.88 & 5.60 \\
\hline 108.0467 & 236.49 & 203.34 & 197.1 & 196.82 & 197.64 & 184.75 & 206.83 & 200.93 & 5.63 \\
\hline 108.5455 & 236.58 & 203.88 & 197.15 & 196.86 & 197.68 & 187.41 & 207.02 & 200.93 & 5.61 \\
\hline 109.0453 & 236.55 & 203.47 & 197.1 & 196.74 & 197.51 & 190.95 & 206.12 & 199.28 & 5.56 \\
\hline 109.5452 & 236.07 & 203.16 & 196.91 & 196.6 & 197.46 & 193.14 & 205.58 & 193.14 & 5.56 \\
\hline 110.044 & 236.33 & 202.27 & 196.86 & 196.59 & 197.43 & 192.42 & 205.91 & 201.06 & 5.57 \\
\hline
\end{tabular}


Table B4. Continued.

\begin{tabular}{|c|c|c|c|c|c|c|c|c|c|}
\hline $\begin{array}{c}\text { Time } \\
\text { min }\end{array}$ & $\begin{array}{l}\mathrm{T} 1 \\
{ }^{\circ} \mathrm{C} \\
\end{array}$ & $\begin{array}{l}\mathrm{T} 2 \\
{ }^{\circ} \mathrm{C} \\
\end{array}$ & $\begin{array}{l}\mathrm{T} 3 \\
{ }^{\circ} \mathrm{C} \\
\end{array}$ & $\begin{array}{l}\mathrm{T} 4 \\
{ }^{\circ} \mathrm{C} \\
\end{array}$ & $\begin{array}{l}\mathrm{T5} \\
{ }^{\circ} \mathrm{C} \\
\end{array}$ & $\begin{array}{l}\mathrm{T} 6 \\
{ }^{\circ} \mathrm{C} \\
\end{array}$ & $\begin{array}{l}\text { Pinj } \\
\text { psig }\end{array}$ & $\begin{array}{l}\text { Pout } \\
\text { psig }\end{array}$ & $\begin{array}{l}\mathrm{Vw} \\
\mathrm{cc} / \mathrm{min}\end{array}$ \\
\hline 110.5448 & 236.87 & 202.65 & 196.99 & 196.72 & 197.54 & 193.12 & 206.39 & 201.06 & 5.60 \\
\hline 111.0447 & 236.97 & 202.94 & 196.92 & 196.6 & 197.41 & 194.93 & 205.65 & 199.58 & 5.59 \\
\hline 111.5453 & 237.22 & 204.95 & 196.84 & 196.57 & 197.41 & 195.69 & 205.74 & 200.81 & 5.59 \\
\hline 112.0452 & 237.26 & 203.43 & 196.91 & 196.63 & 197.49 & 195.93 & 205.99 & 200.85 & 5.61 \\
\hline 112.545 & 237.47 & 202.67 & 196.94 & 196.67 & 197.51 & 196.13 & 206.13 & 200.86 & 5.61 \\
\hline 113.0448 & 237.69 & 203.41 & 196.96 & 196.67 & 197.5 & 196.32 & 206.08 & 200.79 & 5.62 \\
\hline 113.5455 & 237.17 & 203.41 & 196.86 & 196.59 & 197.5 & 194.89 & 205.85 & 201.18 & 5.57 \\
\hline 114.0453 & 237.27 & 204.1 & 196.95 & 196.67 & 197.51 & 195.99 & 206.1 & 199.99 & 5.55 \\
\hline 114.5452 & 237.26 & 203.74 & 196.82 & 196.53 & 197.35 & 196.56 & 205.45 & 200.7 & 5.56 \\
\hline 115.045 & 237.14 & 202.44 & 196.86 & 196.6 & 197.54 & 196.8 & 205.79 & 200.93 & 5.60 \\
\hline 115.5448 & 237.41 & 202.66 & 196.95 & 196.67 & 197.52 & 196.92 & 206.17 & 201.04 & 5.60 \\
\hline 116.0455 & 237.24 & 202.52 & 196.96 & 196.69 & 197.53 & 196.97 & 206.12 & 200.9 & 5.59 \\
\hline 116.5453 & 237.31 & 202.91 & 196.94 & 196.66 & 197.5 & 197 & 206.06 & 200.74 & 5.60 \\
\hline 117.0452 & 237.25 & 202.5 & 196.93 & 196.64 & 197.49 & 197.02 & 205.93 & 200.64 & 5.62 \\
\hline 117.5442 & 236.95 & 202.86 & 196.81 & 196.58 & 197.4 & 196.56 & 205.74 & 200.91 & 5.63 \\
\hline 118.044 & 236.97 & 203.17 & 196.91 & 196.64 & 197.5 & 197.05 & 206.03 & 200.91 & 5.58 \\
\hline 118.5457 & 236.87 & 202.53 & 196.95 & 196.68 & 197.54 & 197.14 & 206.19 & 201.05 & 5.58 \\
\hline 119.0455 & 236.52 & 203.06 & 197 & 196.7 & 197.51 & 197.13 & 206.01 & 199.23 & 5.57 \\
\hline 119.5453 & 236.41 & 205.18 & 196.74 & 196.44 & 197.26 & 196.94 & 205.03 & 200.13 & 5.60 \\
\hline 120.045 & 236.76 & 204.66 & 196.75 & 196.48 & 197.3 & 197 & 205.25 & 200.15 & 5.61 \\
\hline 120.5448 & 236.66 & 203.97 & 196.81 & 196.52 & 197.36 & 197.06 & 205.46 & 200.12 & 5.61 \\
\hline 121.0447 & 236.64 & 203.65 & 196.72 & 196.4 & 197.24 & 196.62 & 204.99 & 200.46 & 5.62 \\
\hline 121.5455 & 236.92 & 203.62 & 196.82 & 196.53 & 197.39 & 196.89 & 205.65 & 200.45 & 5.62 \\
\hline 122.0453 & 236.9 & 203.63 & 196.86 & 196.57 & 197.5 & 197.14 & 205.73 & 200.45 & 5.62 \\
\hline 122.5452 & 237.21 & 205.31 & 196.88 & 196.6 & 197.45 & 197.2 & 205.88 & 200.56 & 5.57 \\
\hline 123.045 & 237.08 & 202.97 & 196.9 & 196.6 & 197.46 & 197.19 & 205.79 & 200.4 & 5.56 \\
\hline 123.5457 & 237.01 & 202.93 & 196.84 & 196.55 & 197.3 & 197.14 & 205.5 & 200.17 & 5.56 \\
\hline 124.0455 & 237 & 203.63 & 196.81 & 196.52 & 197.36 & 197.11 & 205.43 & 200.13 & 5.60 \\
\hline 124.5453 & 236.98 & 204.71 & 196.72 & 196.44 & 197.3 & 196.88 & 205.21 & 200.42 & 5.58 \\
\hline 125.0452 & 237.19 & 204.25 & 196.8 & 196.55 & 197.39 & 197.1 & 205.6 & 200.39 & 5.59 \\
\hline 125.544 & 237.28 & 202.84 & 196.86 & 196.59 & 197.45 & 197.22 & 205.76 & 200.49 & 5.62 \\
\hline 126.0457 & 237.45 & 204.19 & 196.86 & 196.59 & 197.44 & 197.22 & 205.77 & 200.46 & 5.61 \\
\hline 126.5447 & 237.26 & 201.69 & 196.83 & 196.56 & 197.41 & 197.19 & 205.49 & 200.36 & 5.61 \\
\hline 127.0443 & 237.58 & 202.37 & 196.8 & 196.52 & 197.36 & 197.16 & 205.46 & 200.2 & 5.55 \\
\hline 127.5452 & 237.59 & 201.98 & 196.79 & 196.5 & 197.36 & 197.15 & 205.43 & 200.14 & 5.55 \\
\hline 128.045 & 237.36 & 201.43 & 196.78 & 196.51 & 197.39 & 197.17 & 205.34 & 200.15 & 5.56 \\
\hline 128.5448 & 237.24 & 203.1 & 196.73 & 196.44 & 197.34 & 196.98 & 205.38 & 200.41 & 5.59 \\
\hline 129.0455 & 237.25 & 203.04 & 196.8 & 196.54 & 197.4 & 197.18 & 205.57 & 200.31 & 5.59 \\
\hline 129.5453 & 236.96 & 202.29 & 196.86 & 196.58 & 197.46 & 197.26 & 205.78 & 200.43 & 5.60 \\
\hline 130.0452 & 236.85 & 203.44 & 196.87 & 196.58 & 197.43 & 197.25 & 205.65 & 200.3 & 5.60 \\
\hline 130.545 & 236.9 & 202.89 & 196.82 & 196.53 & 197.39 & 197.21 & 205.42 & 200.22 & 5.60 \\
\hline 131.0438 & 237.02 & 201.23 & 196.79 & 196.52 & 197.38 & 197.18 & 205.5 & 200.19 & 5.63 \\
\hline 131.5455 & 236.97 & 202.22 & 196.8 & 196.53 & 197.37 & 197.21 & 205.44 & 200.21 & 5.58 \\
\hline 132.0445 & 237.01 & 203.5 & 196.8 & 196.53 & 197.39 & 197.19 & 205.48 & 200.12 & 5.54 \\
\hline
\end{tabular}


Table B4. Continued.

\begin{tabular}{|c|c|c|c|c|c|c|c|c|c|}
\hline $\begin{array}{c}\text { Time } \\
\text { min }\end{array}$ & $\begin{array}{l}\mathrm{T} 1 \\
{ }^{\circ} \mathrm{C} \\
\end{array}$ & $\begin{array}{l}\mathrm{T} 2 \\
{ }^{\circ} \mathrm{C} \\
\end{array}$ & $\begin{array}{l}\mathrm{T} 3 \\
{ }^{\circ} \mathrm{C} \\
\end{array}$ & $\begin{array}{l}\mathrm{T} 4 \\
{ }^{\circ} \mathrm{C} \\
\end{array}$ & $\begin{array}{l}\mathrm{T} 5 \\
{ }^{\circ} \mathrm{C} \\
\end{array}$ & $\begin{array}{l}\mathrm{T} 6 \\
{ }^{\circ} \mathrm{C} \\
\end{array}$ & $\begin{array}{l}\text { Pinj } \\
\text { psig }\end{array}$ & $\begin{array}{l}\text { Pout } \\
\text { psig }\end{array}$ & $\begin{array}{c}\mathrm{Vw} \\
\mathrm{cc} / \mathrm{min} \\
\end{array}$ \\
\hline 132.5443 & 236.92 & 202.68 & 196.81 & 196.54 & 197.4 & 197.2 & 205.5 & 200.34 & 5.54 \\
\hline 133.045 & 236.95 & 203.79 & 196.81 & 196.52 & 197.38 & 197.2 & 205.57 & 200.1 & 5.56 \\
\hline 133.5448 & 236.97 & 203.37 & 196.74 & 196.48 & 197.35 & 197.14 & 205.45 & 200.34 & 5.60 \\
\hline 134.0465 & 236.96 & 201.82 & 196.84 & 196.57 & 197.41 & 197.25 & 205.78 & 200.33 & 5.59 \\
\hline 134.5453 & 236.96 & 201.44 & 196.86 & 196.61 & 197.49 & 197.29 & 205.85 & 200.37 & 5.59 \\
\hline 135.0452 & 236.8 & 204.76 & 196.85 & 196.58 & 197.44 & 197.24 & 205.65 & 200.23 & 5.59 \\
\hline 135.545 & 236.95 & 204.66 & 196.82 & 196.55 & 197.39 & 197.25 & 205.55 & 200.23 & 5.61 \\
\hline 136.0448 & 236.96 & 205.49 & 196.82 & 196.54 & 197.39 & 197.22 & 205.56 & 200.25 & 5.63 \\
\hline 136.5457 & 237.11 & 205.94 & 196.81 & 196.54 & 197.4 & 197.2 & 205.57 & 200.27 & 5.58 \\
\hline 137.0455 & 237.02 & 204.39 & 196.82 & 196.53 & 197.39 & 197.21 & 205.5 & 200.17 & 5.55 \\
\hline 137.5453 & 237.17 & 204.38 & 196.8 & 196.53 & 197.29 & 197.23 & 205.5 & 200.19 & 5.57 \\
\hline 138.045 & 237.18 & 204.43 & 196.81 & 196.54 & 197.4 & 197.24 & 205.57 & 200.18 & 5.60 \\
\hline 138.544 & 237.2 & 204.13 & 196.74 & 196.47 & 197.35 & 197.19 & 205.51 & 200.36 & 5.60 \\
\hline 139.0465 & 237.26 & 203.71 & 196.85 & 196.6 & 197.46 & 197.3 & 205.85 & 200.37 & 5.60 \\
\hline 139.5455 & 237.19 & 205.36 & 196.87 & 196.61 & 197.47 & 197.32 & 205.86 & 200.47 & 5.61 \\
\hline 140.0462 & 237.36 & 205.03 & 196.83 & 196.56 & 197.4 & 197.26 & 205.55 & 200.16 & 5.62 \\
\hline 140.5452 & 237.31 & 203.94 & 196.8 & 196.53 & 197.37 & 197.23 & 205.49 & 200.13 & 5.62 \\
\hline 141.044 & 237.3 & 201.94 & 196.76 & 196.51 & 197.28 & 197.21 & 205.4 & 200.14 & 5.57 \\
\hline 141.5457 & 237.34 & 202.84 & 196.79 & 196.52 & 197.36 & 197.22 & 205.39 & 200.05 & 5.55 \\
\hline 142.0445 & 237.32 & 204.29 & 196.76 & 196.49 & 197.37 & 197.19 & 205.45 & 200.1 & 5.57 \\
\hline 142.5443 & 237.13 & 205.14 & 196.78 & 196.49 & 197.37 & 197.23 & 205.43 & 200.06 & 5.60 \\
\hline 143.0452 & 237.28 & 204.68 & 196.77 & 196.5 & 197.29 & 197.21 & 205.38 & 200.03 & 5.61 \\
\hline 143.545 & 237 & 205.55 & 196.81 & 196.52 & 197.38 & 197.24 & 205.52 & 200.02 & 5.60 \\
\hline 144.0457 & 237.04 & 204.31 & 196.76 & 196.49 & 197.39 & 197.22 & 205.41 & 199.92 & 5.60 \\
\hline 144.5455 & 236.86 & 205.52 & 196.74 & 196.48 & 197.39 & 197.23 & 205.58 & 200.34 & 5.65 \\
\hline 145.0453 & 237.01 & 204.63 & 196.86 & 196.59 & 197.48 & 197.32 & 205.87 & 200.32 & 5.62 \\
\hline 145.5452 & 237.28 & 204.47 & 196.86 & 196.59 & 197.45 & 197.29 & 205.79 & 200.43 & 5.56 \\
\hline 146.045 & 237.27 & 203.58 & 196.85 & 196.58 & 197.44 & 197.3 & 205.75 & 200.3 & 5.57 \\
\hline 146.5438 & 237.26 & 205.84 & 196.84 & 196.57 & 197.44 & 197.28 & 205.71 & 200.28 & 5.59 \\
\hline 147.0455 & 237.08 & 205.27 & 196.8 & 196.55 & 197.41 & 197.27 & 205.67 & 200.15 & 5.61 \\
\hline 147.5445 & 237.21 & 204.81 & 196.81 & 196.56 & 197.42 & 197.26 & 205.63 & 200.12 & 5.62 \\
\hline 148.0443 & 236.97 & 202.79 & 196.83 & 196.55 & 197.42 & 197.28 & 205.69 & 200.13 & 5.60 \\
\hline 148.545 & 237.06 & 205.09 & 196.82 & 196.55 & 197.43 & 197.27 & 205.7 & 200.11 & 5.62 \\
\hline 149.0448 & 237.19 & 206.01 & 196.82 & 196.55 & 197.41 & 197.27 & 205.67 & 200.08 & 5.64 \\
\hline 149.5455 & 237.43 & 204.28 & 196.83 & 196.58 & 197.44 & 197.28 & 205.74 & 200.03 & 5.59 \\
\hline 150.0453 & 237.35 & 204.94 & 196.85 & 196.56 & 197.44 & 197.3 & 205.72 & 200.06 & 5.55 \\
\hline 150.5452 & 237.33 & 205.88 & 196.84 & 196.57 & 197.45 & 197.3 & 205.72 & 200.04 & 5.56 \\
\hline 151.045 & 237.02 & 205.24 & 196.75 & 196.49 & 197.33 & 197.2 & 205.47 & 200.29 & 5.59 \\
\hline 151.5448 & 237.09 & 205.91 & 196.85 & 196.58 & 197.48 & 197.32 & 205.84 & 200.3 & 5.60 \\
\hline 152.0455 & 236.99 & 205.11 & 196.87 & 196.62 & 197.5 & 197.34 & 205.9 & 200.39 & 5.60 \\
\hline 152.5453 & 237.08 & 205.38 & 196.88 & 196.59 & 197.47 & 197.34 & 205.82 & 200.36 & 5.62 \\
\hline 153.0443 & 237.27 & 204.87 & 196.86 & 196.59 & 197.56 & 197.33 & 205.95 & 200.34 & 5.62 \\
\hline 153.5442 & 237.27 & 205.02 & 196.89 & 196.62 & 197.49 & 197.35 & 205.95 & 200.27 & 5.63 \\
\hline 154.0448 & 237.19 & 204.54 & 196.87 & 196.6 & 197.48 & 197.34 & 205.94 & 200.2 & 5.57 \\
\hline
\end{tabular}


Table B4. Continued.

\begin{tabular}{|c|c|c|c|c|c|c|c|c|c|}
\hline $\begin{array}{c}\text { Time } \\
\text { min }\end{array}$ & $\begin{array}{l}\mathrm{T} 1 \\
{ }^{\circ} \mathrm{C} \\
\end{array}$ & $\begin{array}{l}\mathrm{T} 2 \\
{ }^{\circ} \mathrm{C} \\
\end{array}$ & $\begin{array}{l}\mathrm{T} 3 \\
{ }^{\circ} \mathrm{C} \\
\end{array}$ & $\begin{array}{l}\mathrm{T} 4 \\
{ }^{\circ} \mathrm{C} \\
\end{array}$ & $\begin{array}{l}\mathrm{T5} \\
{ }^{\circ} \mathrm{C} \\
\end{array}$ & $\begin{array}{l}\mathrm{T} 6 \\
{ }^{\circ} \mathrm{C} \\
\end{array}$ & $\begin{array}{l}\text { Pinj } \\
\text { psig }\end{array}$ & $\begin{array}{l}\text { Pout } \\
\text { psig }\end{array}$ & $\begin{array}{c}\mathrm{Vw} \\
\mathrm{cc} / \mathrm{min}\end{array}$ \\
\hline 154.5447 & 237.07 & 205.94 & 196.88 & 196.61 & 197.49 & 197.34 & 205.86 & 200.13 & 5.57 \\
\hline 155.0455 & 237.31 & 203.6 & 196.85 & 196.56 & 197.46 & 197.31 & 205.77 & 200.07 & 5.57 \\
\hline 155.5453 & 237.26 & 203.67 & 196.83 & 196.58 & 197.46 & 197.32 & 205.79 & 200.08 & 5.60 \\
\hline 156.045 & 237.21 & 205.31 & 196.86 & 196.57 & 197.45 & 197.32 & 205.79 & 200.1 & 5.60 \\
\hline 156.5448 & 237.23 & 205.04 & 196.83 & 196.57 & 197.43 & 197.29 & 205.82 & 198.41 & 5.60 \\
\hline 157.0457 & 236.95 & 205.75 & 196.78 & 196.53 & 197.31 & 197.3 & 205.69 & 200.28 & 5.62 \\
\hline 157.5455 & 237.22 & 203.5 & 196.83 & 196.6 & 197.46 & 197.32 & 205.87 & 200.3 & 5.64 \\
\hline 158.0453 & 237.33 & 204.41 & 196.86 & 196.59 & 197.48 & 197.34 & 205.84 & 200.31 & 5.61 \\
\hline 158.5452 & 237.37 & 204.09 & 196.86 & 196.61 & 197.49 & 197.35 & 205.99 & 200.43 & 5.57 \\
\hline 159.045 & 237.32 & 204.35 & 196.87 & 196.62 & 197.49 & 197.33 & 205.98 & 200.19 & 5.55 \\
\hline 159.5457 & 237.13 & 204.39 & 196.83 & 196.57 & 197.44 & 197.32 & 205.8 & 199.91 & 5.57 \\
\hline 160.0445 & 237.24 & 205.52 & 196.8 & 196.55 & 197.43 & 197.27 & 205.71 & 199.99 & 5.60 \\
\hline 160.5443 & 237.16 & 205.53 & 196.81 & 196.54 & 197.42 & 197.27 & 205.61 & 199.95 & 5.60 \\
\hline 161.0452 & 237.22 & 206.2 & 196.8 & 196.53 & 197.44 & 197.28 & 205.64 & 199.96 & 5.60 \\
\hline 161.545 & 237.28 & 207.52 & 196.8 & 196.53 & 197.41 & 197.27 & 205.62 & 199.93 & 5.61 \\
\hline 162.0457 & 237.43 & 206.24 & 196.81 & 196.54 & 197.43 & 197.29 & 205.58 & 199.89 & 5.61 \\
\hline 162.5455 & 237.29 & 204.4 & 196.81 & 196.54 & 197.44 & 197.29 & 205.69 & 199.86 & 5.63 \\
\hline 163.0453 & 237.35 & 203.69 & 196.81 & 196.56 & 197.42 & 197.3 & 205.64 & 199.93 & 5.57 \\
\hline 163.5452 & 237.51 & 205.27 & 196.78 & 196.55 & 197.43 & 197.28 & 205.61 & 199.91 & 5.56 \\
\hline 164.045 & 237.21 & 206.28 & 196.8 & 196.56 & 197.41 & 197.29 & 205.66 & 199.89 & 5.58 \\
\hline 164.5448 & 237.07 & 205.66 & 196.7 & 196.43 & 197.31 & 197.17 & 205.28 & 200.27 & 5.58 \\
\hline 165.0455 & 237.27 & 204.42 & 196.78 & 196.53 & 197.41 & 197.28 & 205.57 & 200.09 & 5.60 \\
\hline 165.5453 & 237.1 & 205.58 & 196.85 & 196.57 & 197.46 & 197.34 & 205.92 & 200.38 & 5.61 \\
\hline 166.0443 & 237.05 & 203.95 & 196.86 & 196.59 & 197.47 & 197.34 & 205.87 & 200.29 & 5.62 \\
\hline 166.545 & 237.34 & 204.58 & 196.85 & 196.58 & 197.49 & 197.37 & 205.92 & 200.23 & 5.64 \\
\hline 167.0448 & 237.27 & 205.73 & 196.85 & 196.6 & 197.48 & 197.35 & 205.94 & 200.21 & 5.61 \\
\hline 167.5455 & 237.35 & 205.22 & 196.86 & 196.59 & 197.47 & 197.32 & 205.8 & 200.05 & 5.57 \\
\hline 168.0453 & 237.37 & 206.85 & 196.84 & 196.58 & 197.45 & 197.33 & 205.8 & 199.97 & 5.56 \\
\hline 168.5452 & 237.29 & 207.68 & 196.83 & 196.58 & 197.46 & 197.33 & 205.78 & 200 & 5.60 \\
\hline 169.045 & 237.45 & 206.25 & 196.83 & 196.55 & 197.44 & 197.3 & 205.75 & 199.99 & 5.60 \\
\hline 169.5448 & 237.37 & 206.61 & 196.82 & 196.57 & 197.45 & 197.32 & 205.79 & 199.94 & 5.60 \\
\hline 170.0455 & 237.3 & 206.28 & 196.82 & 196.57 & 197.45 & 197.31 & 205.75 & 199.98 & 5.61 \\
\hline 170.5453 & 237.38 & 205.41 & 196.83 & 196.56 & 197.46 & 197.31 & 205.75 & 199.94 & 5.64 \\
\hline 171.0452 & 237.49 & 206.91 & 196.83 & 196.55 & 197.46 & 197.34 & 205.71 & 199.97 & 5.64 \\
\hline 171.545 & 237.14 & 207.31 & 196.82 & 196.55 & 197.45 & 197.32 & 205.76 & 199.99 & 5.58 \\
\hline 172.044 & 237.03 & 207.03 & 196.84 & 196.56 & 197.45 & 197.31 & 205.73 & 199.89 & 5.57 \\
\hline 172.5457 & 237.18 & 206.5 & 196.74 & 196.49 & 197.4 & 197.24 & 205.62 & 199.75 & 5.58 \\
\hline 173.0455 & 237.2 & 206.84 & 196.78 & 196.53 & 197.44 & 197.32 & 205.7 & 199.77 & 5.61 \\
\hline 173.5443 & 237.44 & 206.08 & 196.84 & 196.57 & 197.47 & 197.34 & 205.8 & 200.29 & 5.60 \\
\hline 174.045 & 237.11 & 205.8 & 196.88 & 196.61 & 197.42 & 197.36 & 205.98 & 200.15 & 5.61 \\
\hline 174.5448 & 237.07 & 205.73 & 196.85 & 196.58 & 197.46 & 197.35 & 205.73 & 199.91 & 5.62 \\
\hline 175.0457 & 237.1 & 205.89 & 196.8 & 196.53 & 197.41 & 197.3 & 205.61 & 199.8 & 5.62 \\
\hline 175.5455 & 237.33 & 206.36 & 196.77 & 196.52 & 197.41 & 197.29 & 205.66 & 199.81 & 5.59 \\
\hline 176.0453 & 237.19 & 206.24 & 196.79 & 196.54 & 197.43 & 197.31 & 205.65 & 199.89 & 5.56 \\
\hline
\end{tabular}


Table B4. Continued.

\begin{tabular}{|c|c|c|c|c|c|c|c|c|c|}
\hline $\begin{array}{c}\text { Time } \\
\text { min }\end{array}$ & $\begin{array}{l}\mathrm{T} 1 \\
{ }^{\circ} \mathrm{C} \\
\end{array}$ & $\begin{array}{l}\mathrm{T} 2 \\
{ }^{\circ} \mathrm{C} \\
\end{array}$ & $\begin{array}{l}\mathrm{T} 3 \\
{ }^{\circ} \mathrm{C} \\
\end{array}$ & $\begin{array}{l}\mathrm{T} 4 \\
{ }^{\circ} \mathrm{C} \\
\end{array}$ & $\begin{array}{l}\mathrm{T5} \\
{ }^{\circ} \mathrm{C} \\
\end{array}$ & $\begin{array}{l}\mathrm{T} 6 \\
{ }^{\circ} \mathrm{C} \\
\end{array}$ & $\begin{array}{l}\text { Pinj } \\
\text { psig }\end{array}$ & $\begin{array}{l}\text { Pout } \\
\text { psig }\end{array}$ & $\begin{array}{l}\mathrm{Vw} \\
\mathrm{cc} / \mathrm{min}\end{array}$ \\
\hline 176.5452 & 237.47 & 206.16 & 196.79 & 196.54 & 197.44 & 197.31 & 205.69 & 199.9 & 5.55 \\
\hline 177.045 & 237.51 & 207.38 & 196.78 & 196.53 & 197.43 & 197.3 & 205.73 & 199.88 & 5.58 \\
\hline 177.5457 & 237.62 & 205.31 & 196.8 & 196.54 & 197.41 & 197.29 & 205.62 & 199.86 & 5.59 \\
\hline 178.0455 & 237.68 & 204.99 & 196.81 & 196.54 & 197.43 & 197.31 & 205.65 & 199.88 & 5.60 \\
\hline 178.5453 & 237.59 & 206.39 & 196.8 & 196.55 & 197.42 & 197.3 & 205.68 & 199.81 & 5.62 \\
\hline 179.0442 & 237.44 & 206.34 & 196.8 & 196.53 & 197.44 & 197.3 & 205.6 & 199.84 & 5.62 \\
\hline 179.544 & 237.15 & 207.55 & 196.71 & 196.46 & 197.36 & 197.22 & 205.45 & 199.9 & 5.64 \\
\hline 180.0448 & 237.34 & 206.19 & 196.75 & 196.5 & 197.4 & 197.27 & 205.54 & 199.88 & 5.61 \\
\hline 180.5447 & 237.52 & 206.95 & 196.79 & 196.56 & 197.44 & 197.33 & 205.9 & 200.17 & 5.56 \\
\hline 181.0453 & 237.35 & 207.45 & 196.85 & 196.6 & 197.48 & 197.37 & 205.98 & 200.23 & 5.57 \\
\hline 181.5452 & 237.32 & 207.33 & 196.87 & 196.62 & 197.5 & 197.38 & 206 & 200.19 & 5.60 \\
\hline 182.045 & 237.28 & 208.16 & 196.84 & 196.57 & 197.49 & 197.35 & 205.83 & 199.92 & 5.62 \\
\hline 182.5448 & 237.18 & 207.29 & 196.83 & 196.56 & 197.44 & 197.33 & 205.74 & 199.83 & 5.61 \\
\hline 183.0455 & 237.35 & 206.47 & 196.8 & 196.51 & 197.43 & 197.3 & 205.64 & 199.9 & 5.63 \\
\hline 183.5453 & 237.24 & 207.37 & 196.82 & 196.55 & 197.45 & 197.34 & 205.76 & 199.91 & 5.65 \\
\hline 184.0452 & 237.44 & 208.8 & 196.81 & 196.57 & 197.45 & 197.34 & 205.74 & 199.95 & 5.62 \\
\hline 184.544 & 237.36 & 209.63 & 196.83 & 196.56 & 197.47 & 197.35 & 205.82 & 199.93 & 5.59 \\
\hline 185.0448 & 237.47 & 207.27 & 196.82 & 196.55 & 197.46 & 197.33 & 205.84 & 199.87 & 5.60 \\
\hline 185.5447 & 237.49 & 207.2 & 196.82 & 196.55 & 197.45 & 197.32 & 205.87 & 199.93 & 5.62 \\
\hline 186.0445 & 237.3 & 206.46 & 196.84 & 196.56 & 197.45 & 197.32 & 205.78 & 199.89 & 5.61 \\
\hline 186.5452 & 237.52 & 205.82 & 196.79 & 196.54 & 197.44 & 197.33 & 205.75 & 199.92 & 5.63 \\
\hline 187.045 & 237.34 & 205.68 & 196.72 & 196.49 & 197.39 & 197.28 & 205.65 & 200.19 & 5.65 \\
\hline 187.5448 & 237.44 & 205.11 & 196.84 & 196.57 & 197.46 & 197.36 & 205.92 & 200.19 & 5.65 \\
\hline 188.0455 & 237.35 & 206.94 & 196.84 & 196.59 & 197.49 & 197.38 & 205.96 & 200.26 & 5.59 \\
\hline 188.5453 & 237.45 & 207.35 & 196.86 & 196.59 & 197.51 & 197.4 & 206.12 & 200.19 & 5.59 \\
\hline 189.0452 & 237.18 & 207.02 & 196.87 & 196.6 & 197.48 & 197.37 & 205.97 & 200.05 & 5.61 \\
\hline 189.545 & 237.29 & 207.86 & 196.82 & 196.55 & 197.43 & 197.3 & 205.67 & 199.8 & 5.62 \\
\hline 190.044 & 237.44 & 207.83 & 196.78 & 196.5 & 197.41 & 197.3 & 205.62 & 199.81 & 5.60 \\
\hline 190.5465 & 237.57 & 209.09 & 196.79 & 196.54 & 197.45 & 197.31 & 205.75 & 199.96 & 5.63 \\
\hline 191.0455 & 237.52 & 209.52 & 196.83 & 196.6 & 197.49 & 197.38 & 206.05 & 200.58 & 5.65 \\
\hline 191.5443 & 237.02 & 206.91 & 196.9 & 196.65 & 197.55 & 197.42 & 206.25 & 200.43 & 5.63 \\
\hline 192.045 & 237.26 & 207.85 & 196.89 & 196.6 & 197.46 & 197.41 & 206.12 & 200.21 & 5.59 \\
\hline 192.5448 & 237.41 & 208.55 & 196.88 & 196.59 & 197.5 & 197.38 & 205.93 & 200.08 & 5.56 \\
\hline 193.0447 & 237.5 & 205.83 & 196.84 & 196.59 & 197.51 & 197.37 & 206.06 & 200.13 & 5.63 \\
\hline 193.5455 & 237.4 & 206 & 196.87 & 196.6 & 197.49 & 197.39 & 205.99 & 200.12 & 5.60 \\
\hline 194.0453 & 237.4 & 208.38 & 196.8 & 196.48 & 197.23 & 197.19 & 204.86 & 199.17 & 5.62 \\
\hline 194.5452 & 237.57 & 209.34 & 196.8 & 196.55 & 197.48 & 197.36 & 205.98 & 200.4 & 5.65 \\
\hline 195.045 & 237.82 & 209.13 & 196.86 & 196.61 & 197.53 & 197.42 & 206.21 & 200.43 & 5.65 \\
\hline 195.5457 & 237.65 & 208.34 & 196.9 & 196.67 & 197.55 & 197.44 & 206.32 & 200.6 & 5.59 \\
\hline 196.0455 & 237.79 & 206.31 & 196.92 & 196.67 & 197.57 & 197.46 & 206.33 & 200.4 & 5.57 \\
\hline 196.5453 & 237.4 & 205.16 & 196.93 & 196.68 & 197.64 & 197.46 & 206.3 & 200.36 & 5.60 \\
\hline 197.0442 & 237.35 & 205.61 & 196.88 & 196.61 & 197.5 & 197.38 & 205.98 & 199.93 & 5.61 \\
\hline 197.545 & 237.23 & 207.73 & 196.83 & 196.56 & 197.47 & 197.35 & 205.84 & 200.08 & 5.59 \\
\hline 198.0448 & 237.27 & 209.33 & 196.83 & 196.6 & 197.48 & 197.37 & 205.99 & 200.21 & 5.60 \\
\hline
\end{tabular}


Table B4. Continued.

\begin{tabular}{|c|c|c|c|c|c|c|c|c|c|}
\hline $\begin{array}{c}\text { Time } \\
\text { min }\end{array}$ & $\begin{array}{l}\mathrm{T} 1 \\
{ }^{\circ} \mathrm{C}\end{array}$ & $\begin{array}{l}\mathrm{T} 2 \\
{ }^{\circ} \mathrm{C}\end{array}$ & $\begin{array}{l}\mathrm{T} 3 \\
{ }^{\circ} \mathrm{C}\end{array}$ & $\begin{array}{l}\mathrm{T} 4 \\
{ }^{\circ} \mathrm{C}\end{array}$ & $\begin{array}{l}\mathrm{T} 5 \\
{ }^{\circ} \mathrm{C}\end{array}$ & $\begin{array}{l}\mathrm{T} 6 \\
{ }^{\circ} \mathrm{C}\end{array}$ & $\begin{array}{l}\text { Pinj } \\
\text { psig }\end{array}$ & $\begin{array}{c}\text { Pout } \\
\text { psig }\end{array}$ & $\begin{array}{c}\mathrm{Vw} \\
\mathbf{c c} / \mathbf{m i n}\end{array}$ \\
\hline 198.5455 & 237.31 & 207.02 & 196.87 & 196.62 & 197.48 & 197.41 & 206.16 & 200.31 & 5.61 \\
\hline 199.0453 & 237.42 & 206.45 & 196.87 & 196.62 & 197.52 & 197.41 & 206.11 & 200.27 & 5.63 \\
\hline 199.5452 & 237.62 & 208.48 & 196.88 & 196.63 & 197.5 & 197.4 & 206.26 & 200.21 & 5.59 \\
\hline 200.045 & 237.39 & 207.93 & 196.88 & 196.63 & 197.47 & 197.42 & 206.11 & 200.22 & 5.57 \\
\hline 200.5448 & 237.67 & 207.11 & 196.85 & 196.58 & 197.49 & 197.4 & 206.08 & 200.1 & 5.56 \\
\hline 201.0455 & 237.47 & 209.38 & 196.87 & 196.58 & 197.46 & 197.36 & 205.86 & 198.33 & 5.61 \\
\hline 201.5453 & 237.48 & 210.23 & 196.77 & 196.52 & 197.43 & 197.31 & 205.72 & 200.31 & 5.59 \\
\hline 202.0452 & 237.57 & 210.13 & 196.84 & 196.61 & 197.51 & 197.42 & 206.08 & 200.4 & 5.61 \\
\hline 202.544 & 237.72 & 209.92 & 196.9 & 196.65 & 197.53 & 197.42 & 206.29 & 200.52 & 5.60 \\
\hline 203.0438 & 237.7 & 209.78 & 196.9 & 196.67 & 197.57 & 197.48 & 206.43 & 200.5 & 5.63 \\
\hline 203.5447 & 237.65 & 208.49 & 196.93 & 196.66 & 197.59 & 197.45 & 206.34 & 200.34 & 5.61 \\
\hline 204.0445 & 237.1 & 209.43 & 196.88 & 196.63 & 197.5 & 197.4 & 206.05 & 199.95 & 5.58 \\
\hline 204.5452 & 237.18 & 209.91 & 196.84 & 196.56 & 197.45 & 197.36 & 205.83 & 199.91 & 5.58 \\
\hline 205.045 & 237.36 & 208.13 & 196.81 & 196.56 & 197.44 & 197.37 & 205.87 & 200.04 & 5.60 \\
\hline 205.5448 & 237.49 & 208.4 & 196.85 & 196.58 & 197.5 & 197.39 & 206.06 & 200.25 & 5.60 \\
\hline 206.0455 & 237.69 & 208.01 & 196.87 & 196.62 & 197.5 & 197.41 & 206.12 & 200.27 & 5.61 \\
\hline 206.5453 & 237.78 & 208.69 & 196.88 & 196.63 & 197.54 & 197.42 & 206.17 & 200.19 & 5.60 \\
\hline 207.0452 & 237.52 & 210.11 & 196.86 & 196.61 & 197.51 & 197.42 & 206.08 & 200.17 & 5.61 \\
\hline 207.545 & 237.38 & 209.74 & 196.87 & 196.62 & 197.49 & 197.42 & 206.08 & 200.15 & 5.62 \\
\hline 208.044 & 237.47 & 209.06 & 196.87 & 196.6 & 197.59 & 197.41 & 206.07 & 200.13 & 5.57 \\
\hline 208.5457 & 237.62 & 209.5 & 196.84 & 196.59 & 197.5 & 197.4 & 206.01 & 200.18 & 5.56 \\
\hline 209.0445 & 237.61 & 210.11 & 196.86 & 196.61 & 197.42 & 197.38 & 206.06 & 200.05 & 5.56 \\
\hline 209.5453 & 237.41 & 210.08 & 196.85 & 196.6 & 197.49 & 197.4 & 205.98 & 200.02 & 5.60 \\
\hline 210.045 & 237.52 & 209.24 & 196.83 & 196.58 & 197.48 & 197.37 & 205.94 & 200.01 & 5.59 \\
\hline 210.5448 & 237.58 & 209.21 & 196.71 & 196.42 & 197.36 & 197.21 & 205.52 & 200.41 & 5.59 \\
\hline 211.0457 & 237.73 & 209.42 & 196.82 & 196.57 & 197.47 & 197.36 & 205.9 & 200.05 & 5.59 \\
\hline 211.5455 & 237.68 & 208.12 & 196.84 & 196.59 & 197.49 & 197.4 & 206.1 & 200.42 & 5.61 \\
\hline 212.0453 & 237.88 & 209.58 & 196.88 & 196.65 & 197.65 & 197.46 & 206.38 & 200.56 & 5.62 \\
\hline 212.5452 & 237.59 & 210.15 & 196.94 & 196.69 & 197.6 & 197.49 & 206.44 & 200.44 & 5.58 \\
\hline 213.045 & 237.58 & 210.48 & 196.89 & 196.62 & 197.5 & 197.41 & 206.06 & 199.85 & 5.56 \\
\hline 213.5457 & 237.4 & 210.18 & 196.8 & 196.53 & 197.43 & 197.34 & 205.73 & 199.85 & 5.56 \\
\hline 214.0455 & 237.5 & 210.48 & 196.83 & 196.57 & 197.47 & 197.38 & 206.04 & 200.28 & 5.59 \\
\hline 214.5443 & 237.61 & 210.49 & 196.88 & 196.61 & 197.53 & 197.42 & 206.21 & 200.21 & 5.60 \\
\hline 215.0452 & 237.67 & 210.69 & 196.89 & 196.63 & 197.55 & 197.42 & 206.22 & 200.29 & 5.58 \\
\hline 215.545 & 237.33 & 211.09 & 196.89 & 196.62 & 197.53 & 197.43 & 206.16 & 200.27 & 5.60 \\
\hline 216.0448 & 237.67 & 211.29 & 196.86 & 196.62 & 197.52 & 197.41 & 206.24 & 200.17 & 5.62 \\
\hline 216.5455 & 237.66 & 211.42 & 196.88 & 196.63 & 197.52 & 197.43 & 206.15 & 200.12 & 5.64 \\
\hline 217.0453 & 237.93 & 211.28 & 196.86 & 196.61 & 197.53 & 197.42 & 206.15 & 200.19 & 5.59 \\
\hline 217.5452 & 237.63 & 210.87 & 196.89 & 196.63 & 197.53 & 197.42 & 206.16 & 200.19 & 5.56 \\
\hline 218.045 & 237.67 & 210.94 & 196.87 & 196.62 & 197.5 & 197.41 & 206.11 & 200.11 & 5.57 \\
\hline 218.5448 & 237.64 & 211.09 & 196.86 & 196.61 & 197.52 & 197.41 & 206.08 & 200.15 & 5.59 \\
\hline 219.0455 & 237.6 & 211.23 & 196.86 & 196.59 & 197.43 & 197.41 & 206.09 & 200.1 & 5.59 \\
\hline 219.5453 & 237.73 & 211.43 & 196.86 & 196.59 & 197.51 & 197.4 & 206.11 & 200.17 & 5.59 \\
\hline
\end{tabular}


Table B4. Continued.

\begin{tabular}{|c|c|c|c|c|c|c|c|c|c|}
\hline $\begin{array}{c}\text { Time } \\
\text { min }\end{array}$ & $\begin{array}{l}\mathrm{T} 1 \\
{ }^{\circ} \mathrm{C} \\
\end{array}$ & $\begin{array}{l}\mathrm{T} 2 \\
{ }^{\circ} \mathrm{C} \\
\end{array}$ & $\begin{array}{l}\mathrm{T} 3 \\
{ }^{\circ} \mathrm{C} \\
\end{array}$ & $\begin{array}{l}\mathrm{T} 4 \\
{ }^{\circ} \mathrm{C} \\
\end{array}$ & $\begin{array}{l}\mathrm{T} 5 \\
{ }^{\circ} \mathrm{C} \\
\end{array}$ & $\begin{array}{l}\mathrm{T} 6 \\
{ }^{\circ} \mathrm{C} \\
\end{array}$ & $\begin{array}{l}\text { Pinj } \\
\text { psig }\end{array}$ & $\begin{array}{l}\text { Pout } \\
\text { psig }\end{array}$ & $\begin{array}{c}\mathrm{Vw} \\
\mathrm{cc} / \mathrm{min}\end{array}$ \\
\hline 220.0443 & 237.83 & 211.3 & 196.87 & 196.62 & 197.51 & 197.4 & 206.09 & 200.08 & 5.59 \\
\hline 220.545 & 237.7 & 211.39 & 196.87 & 196.6 & 197.5 & 197.41 & 206.07 & 200.1 & 5.63 \\
\hline 221.0448 & 237.71 & 211.27 & 196.85 & 196.59 & 197.5 & 197.39 & 206.09 & 200.01 & 5.63 \\
\hline 221.5455 & 237.53 & 211.59 & 196.84 & 196.57 & 197.47 & 197.38 & 206.01 & 200.03 & 5.58 \\
\hline 222.0453 & 237.3 & 211.52 & 196.84 & 196.58 & 197.49 & 197.38 & 206.04 & 199.96 & 5.57 \\
\hline 222.5452 & 237.47 & 211.81 & 196.67 & 196.38 & 197.33 & 197.21 & 205.34 & 199.88 & 5.58 \\
\hline 223.045 & 237.68 & 211.74 & 196.73 & 196.47 & 197.39 & 197.28 & 205.59 & 200.11 & 5.60 \\
\hline 223.5448 & 238.01 & 211.89 & 196.82 & 196.57 & 197.48 & 197.39 & 206.15 & 200.37 & 5.59 \\
\hline 224.0455 & 237.93 & 212.11 & 196.86 & 196.66 & 197.57 & 197.49 & 206.55 & 200.8 & 5.58 \\
\hline 224.5453 & 237.77 & 211.79 & 196.97 & 196.74 & 197.65 & 197.54 & 206.79 & 200.91 & 5.61 \\
\hline 225.0443 & 237.86 & 211.76 & 196.99 & 196.72 & 197.63 & 197.53 & 206.65 & 200.59 & 5.64 \\
\hline 225.5442 & 237.68 & 212.03 & 196.94 & 196.67 & 197.58 & 197.48 & 206.44 & 200.33 & 5.62 \\
\hline 226.0448 & 237.81 & 212.11 & 196.87 & 196.6 & 197.52 & 197.41 & 206.1 & 200.08 & 5.57 \\
\hline 226.5447 & 237.78 & 212.29 & 196.86 & 196.61 & 197.52 & 197.41 & 206.13 & 200.23 & 5.57 \\
\hline 227.0455 & 236.94 & 212.25 & 196.9 & 196.61 & 197.56 & 197.45 & 206.32 & .39 & 5.59 \\
\hline 227.5452 & 237.09 & 212.33 & 196.92 & 196.65 & 197.56 & 197.47 & 206.48 & 200.45 & 5.61 \\
\hline 228.045 & 237.22 & 210.22 & 196.92 & 196.69 & 197.58 & 197.48 & 206.41 & 200.37 & 5.61 \\
\hline 228.5448 & 237.2 & 210.76 & 196.92 & 196.69 & 197.59 & 197.48 & 206.46 & 200.33 & 5.61 \\
\hline 229.0457 & 237.33 & 211.84 & 196.95 & 196.68 & 197.57 & 197.46 & 206.42 & 200.38 & 5.65 \\
\hline 229.5455 & 237.32 & 212.31 & 196.93 & 196.68 & 197.58 & 197.47 & 206.43 & 200.32 & 5.65 \\
\hline 230.0443 & 237.46 & 212.26 & 196.93 & 196.67 & 197.58 & 197.47 & 206.38 & 200.47 & 5.58 \\
\hline 230.5442 & 237.77 & 212.37 & 196.9 & 196.65 & 197.55 & 197.46 & 206.5 & 200.33 & 5.57 \\
\hline 231.045 & 237.42 & 211.06 & 196.92 & 196.65 & 197.57 & 197.48 & 206.41 & 200.32 & 5.59 \\
\hline 231.5447 & 237.63 & 211.57 & 196.91 & 196.62 & 197.55 & 197.44 & 206.35 & 200.26 & 5.61 \\
\hline 232.0455 & 237.71 & 212.56 & 196.91 & 196.64 & 197.56 & 197.47 & 206.34 & 200.26 & 5.61 \\
\hline 232.5453 & 237.61 & 212.88 & 196.77 & 196.47 & 197.36 & 197.24 & 205.29 & 199.04 & 5.62 \\
\hline 233.0452 & 237.72 & 212.85 & 196.67 & 196.4 & 197.31 & 197.22 & 205.36 & 199.67 & 5.62 \\
\hline 233.545 & 237.83 & 212.71 & 196.76 & 196.55 & 197.44 & 197.37 & 206.03 & 200.36 & 5.62 \\
\hline 234.0457 & 237.96 & 212.52 & 196.89 & 196.69 & 197.57 & 197.48 & 206.52 & 200.82 & 5.59 \\
\hline 234.5455 & 237.96 & 212.57 & 197 & 196.73 & 197.65 & 197.55 & 206.83 & 200.91 & 5.55 \\
\hline 235.0453 & 237.75 & 212.7 & 197 & 196.74 & 197.63 & 197.56 & 206.73 & 200.62 & 5.58 \\
\hline 235.5442 & 237.61 & 211.4 & 196.95 & 196.67 & 197.56 & 197.49 & 206.36 & 200.2 & 5.61 \\
\hline 236.045 & 237.4 & 211.76 & 196.85 & 196.6 & 197.48 & 197.4 & 206.02 & 200.02 & 5.61 \\
\hline 236.5448 & 237.51 & 212.48 & 196.85 & 196.6 & 197.51 & 197.41 & 206.16 & 200.2 & 5.60 \\
\hline 237.0455 & 237.49 & 212.54 & 196.89 & 196.64 & 197.5 & 197.43 & 206.29 & 200.35 & 5.60 \\
\hline 237.5453 & 237.46 & 212.77 & 196.93 & 196.66 & 197.59 & 197.48 & 206.44 & 200.47 & 5.63 \\
\hline 238.0452 & 237.16 & 211.56 & 196.95 & 196.66 & 197.58 & 197.49 & 206.51 & 200.45 & 5.59 \\
\hline 238.545 & 237.18 & 210.86 & 196.92 & 196.65 & 197.47 & 197.47 & 206.32 & 200.27 & 5.56 \\
\hline 239.0448 & 237.65 & 212.25 & 196.87 & 196.63 & 197.53 & 197.44 & 206.34 & 200.26 & 5.56 \\
\hline 239.5455 & 237.97 & 212.78 & 196.89 & 196.62 & 197.46 & 197.46 & 206.3 & 200.37 & 5.59 \\
\hline 240.0453 & 237.89 & 212.95 & 196.89 & 196.64 & 197.55 & 197.46 & 206.4 & 200.3 & 5.59 \\
\hline
\end{tabular}


Table B5-Temperature and production data for run 5

\begin{tabular}{|c|c|c|c|c|c|c|c|c|c|}
\hline $\begin{array}{c}\text { Time } \\
\text { min }\end{array}$ & $\begin{array}{l}\mathrm{T} 1 \\
{ }^{\circ} \mathrm{C}\end{array}$ & $\begin{array}{l}\mathrm{T} 2 \\
{ }^{\circ} \mathrm{C}\end{array}$ & $\begin{array}{l}\mathrm{T} 3 \\
{ }^{\circ} \mathrm{C}\end{array}$ & $\begin{array}{l}\mathrm{T} 4 \\
{ }^{\circ} \mathrm{C}\end{array}$ & $\begin{array}{l}\mathrm{T} 5 \\
{ }^{\circ} \mathrm{C}\end{array}$ & $\begin{array}{l}\mathrm{T} 6 \\
{ }^{\circ} \mathrm{C}\end{array}$ & $\begin{array}{l}\text { Pinj } \\
\text { psig }\end{array}$ & $\begin{array}{c}\text { Pout } \\
\text { psig }\end{array}$ & $\begin{array}{c}\mathrm{Vw} \\
\mathrm{cc} / \mathrm{min}\end{array}$ \\
\hline 4.93E-02 & 236.77 & 65.75 & 62.44 & 64.01 & 62.12 & 53.22 & 298.01 & 199.8 & 5.61 \\
\hline 0.5455 & 228.38 & 179.04 & 62.45 & 64.19 & 62.13 & 53.29 & 205.27 & 200.25 & 5.62 \\
\hline 1.045333 & 234.59 & 195.72 & 62.42 & 64.15 & 62.1 & 53.28 & 205.05 & 200.06 & 5.61 \\
\hline 1.545167 & 238.16 & 199.24 & 62.45 & 64.16 & 62.13 & 53.31 & 205.28 & 200.04 & 5.56 \\
\hline 2.045 & 240.03 & 199.34 & 62.39 & 64.11 & 62.07 & 53.28 & 205.19 & 199.9 & 5.54 \\
\hline 2.544833 & 240.83 & 200 & 62.38 & 64.06 & 62.1 & 53.31 & 205.14 & 200.5 & 5.55 \\
\hline 3.0455 & 241.15 & 199.68 & 62.37 & 64.07 & 62.07 & 53.28 & 205.96 & 200.7 & 5.58 \\
\hline 3.546333 & 240.87 & 200.26 & 62.38 & 64.05 & 62.06 & 53.29 & 205.63 & 200.44 & 5.59 \\
\hline 4.045167 & 240.13 & 199.84 & 62.35 & 64.04 & 62.05 & 53.28 & 205.35 & 200.3 & 5.59 \\
\hline 4.544167 & 238.86 & 199.5 & 62.36 & 64.03 & 62.04 & 53.27 & 205.51 & 200.32 & 5.60 \\
\hline 5.044833 & 237.01 & 199.02 & 62.37 & 64.06 & 62.07 & 53.28 & 205.36 & 200.23 & 5.62 \\
\hline 5.544667 & 234.65 & 198.69 & 62.38 & 64.07 & 62.08 & 53.29 & 205.39 & 200.21 & 5.60 \\
\hline 6.045333 & 232.53 & 198.47 & 62.41 & 64.08 & 62.07 & 53.32 & 205.48 & 200.14 & 5.56 \\
\hline 6.545166 & 230.95 & 198.35 & 62.44 & 64.09 & 62.1 & 53.33 & 205.41 & 200.12 & 5.56 \\
\hline 7.045 & 230.63 & 198.02 & 62.49 & 64.08 & 62.11 & 53.34 & 205.35 & 200.11 & 5.58 \\
\hline 7.544833 & 231.04 & 198.3 & 62.5 & 64.1 & 62.08 & 53.33 & 205.2 & 200.04 & 5.60 \\
\hline 8.045667 & 231.48 & 198.92 & 62.56 & 64.1 & 62.11 & 53.36 & 205.37 & 200.07 & 5.58 \\
\hline 8.545333 & 231.54 & 199.14 & 62.63 & 64.14 & 62.06 & 53.37 & 205.37 & 200.1 & 5.58 \\
\hline 9.045167 & 231.17 & 199.18 & 62.71 & 64.1 & 62.11 & 53.36 & 205.41 & 200.05 & 5.60 \\
\hline 9.544168 & 230.63 & 198.92 & 62.82 & 64.09 & 62.06 & 53.39 & 205.35 & 199.99 & 5.61 \\
\hline 10.04483 & 230.1 & 197.95 & 62.96 & 64.11 & 62.13 & 53.4 & 205.28 & 199.94 & 5.57 \\
\hline 10.54567 & 230.05 & 198.44 & 63.1 & 64.09 & 62.14 & 53.39 & 205.38 & 200 & 5.57 \\
\hline 11.0445 & 231.64 & 197.84 & 63.26 & 64.08 & 62.11 & 53.34 & 206.4 & 200.93 & 5.56 \\
\hline 11.54533 & 233.17 & 199.8 & 63.5 & 64.08 & 62.02 & 53.39 & 206.11 & 200.76 & 5.58 \\
\hline 12.045 & 234.21 & 199.24 & 63.71 & 64.07 & 62.11 & 53.36 & 205.91 & 200.56 & 5.59 \\
\hline 12.54483 & 234.74 & 200.12 & 64.01 & 64.08 & 62.1 & 53.37 & 206.13 & 200.52 & 5.60 \\
\hline 13.04567 & 234.66 & 200.72 & 64.38 & 64.09 & 61.99 & 53.4 & 205.93 & 200.5 & 5.61 \\
\hline 13.5455 & 234.38 & 199.33 & 64.71 & 64.08 & 62.1 & 53.37 & 205.97 & 200.41 & 5.65 \\
\hline 14.04533 & 233.67 & 200.04 & 65.11 & 64.09 & 62.13 & 53.41 & 205.95 & 200.49 & 5.61 \\
\hline 14.54417 & 232.93 & 199.6 & 65.52 & 64.1 & 61.96 & 53.38 & 206.07 & 200.36 & 5.57 \\
\hline 15.044 & 232.9 & 200.27 & 65.96 & 64.09 & 62.09 & 53.41 & 206.22 & 200.38 & 5.56 \\
\hline 15.54467 & 233.54 & 201.35 & 66.56 & 64.1 & 62.11 & 53.4 & 206.39 & 200.42 & 5.60 \\
\hline 16.0445 & 233.83 & 200.59 & 67.21 & 64.11 & 62.1 & 53.37 & 206.49 & 200.44 & 5.59 \\
\hline 16.54533 & 233.7 & 201.68 & 68.03 & 64.12 & 62.11 & 53.4 & 206.54 & 200.45 & 5.60 \\
\hline 17.04517 & 233.35 & 202.47 & 69.01 & 64.11 & 62.06 & 53.39 & 206.69 & 200.42 & 5.60 \\
\hline 17.545 & 232.96 & 202.27 & 70.27 & 64.13 & 62.05 & 53.42 & 206.6 & 200.32 & 5.64 \\
\hline 18.04567 & 232.65 & 201.59 & 71.61 & 64.14 & 62.08 & 53.43 & 206.4 & 200.18 & 5.60 \\
\hline 18.5455 & 232.69 & 202.48 & 72.96 & 64.17 & 62.09 & 53.46 & 206.47 & 200.09 & 5.57 \\
\hline 19.04533 & 232.97 & 202 & 74.41 & 64.18 & 62.1 & 53.48 & 206.66 & 200.13 & 5.56 \\
\hline 19.54417 & 233.39 & 202.42 & 76.13 & 64.11 & 62.03 & 53.4 & 206.76 & 200.18 & 5.59 \\
\hline 20.045 & 233.77 & 200.91 & 78.22 & 64.14 & 61.98 & 53.42 & 206.73 & 200.13 & 5.60 \\
\hline 20.54483 & 234.09 & 200.47 & 80.44 & 64.13 & 62.01 & 53.39 & 206.57 & 200.05 & 5.60 \\
\hline 21.04467 & 234.25 & 200.28 & 82.75 & 64.14 & 62.02 & 53.44 & 206.55 & 200.01 & 5.60 \\
\hline 21.54533 & 234.19 & 200.05 & 85.04 & 64.14 & 61.99 & 53.43 & 206.43 & 199.96 & 5.64 \\
\hline
\end{tabular}


Table B5. Continued.

\begin{tabular}{|c|c|c|c|c|c|c|c|c|c|}
\hline $\begin{array}{l}\text { Time } \\
\text { min }\end{array}$ & $\begin{array}{l}\mathrm{T} 1 \\
{ }^{\circ} \mathrm{C}\end{array}$ & $\begin{array}{l}\mathrm{T} 2 \\
{ }^{\circ} \mathrm{C}\end{array}$ & $\begin{array}{l}\mathrm{T} 3 \\
{ }^{\circ} \mathrm{C}\end{array}$ & $\begin{array}{l}\mathrm{T} 4 \\
{ }^{\circ} \mathrm{C}\end{array}$ & $\begin{array}{l}\mathrm{T} 5 \\
{ }^{\circ} \mathrm{C}\end{array}$ & $\begin{array}{l}\mathrm{T} 6 \\
{ }^{\circ} \mathrm{C}\end{array}$ & $\begin{array}{l}\text { Pinj } \\
\text { psig }\end{array}$ & $\begin{array}{l}\text { Pout } \\
\text { psig }\end{array}$ & $\begin{array}{c}\mathrm{Vw} \\
\mathrm{cc} / \mathrm{min}\end{array}$ \\
\hline 22.04517 & 234.14 & 201.37 & 87.25 & 64.13 & 62.01 & 53.4 & 206.39 & 199.94 & 5.61 \\
\hline 22.545 & 234.12 & 200.14 & 89.48 & 64.16 & 62.02 & 53.45 & 206.54 & 200.02 & 5.56 \\
\hline 23.04483 & 233.93 & 201.47 & 92 & 64.17 & 62.16 & 53.47 & 206.42 & 200.05 & 5.56 \\
\hline 23.5455 & 233.72 & 201.21 & 95.11 & 64.2 & 62.06 & 53.48 & 206.53 & 200.06 & 5.58 \\
\hline 24.04633 & 233.51 & 199.47 & 98.91 & 64.2 & 62.08 & 53.49 & 206.55 & 200.05 & 5.60 \\
\hline 24.54517 & 233.23 & 199.85 & 103.25 & 64.19 & 61.87 & 53.46 & 206.6 & 200.08 & 5.60 \\
\hline 25.04417 & 232.86 & 200.07 & 108.25 & 64.18 & 61.97 & 53.47 & 206.65 & 200.08 & 5.60 \\
\hline 25.544 & 232.51 & 201.23 & 114.46 & 64.17 & 61.96 & 53.47 & 206.77 & 200.06 & 5.62 \\
\hline 26.04567 & 232.14 & 200.5 & 123.24 & 64.23 & 62.08 & 53.5 & 206.96 & 200.08 & 5.62 \\
\hline 26.5445 & 231.61 & 201.63 & 135.59 & 64.24 & 61.97 & 53.51 & 207.17 & 200.09 & 5.57 \\
\hline 27.04517 & 231.8 & 200.94 & 149.34 & 64.25 & 61.96 & 53.52 & 207.43 & 200.15 & 5.55 \\
\hline 27.545 & 233.78 & 201.65 & 160.67 & 64.26 & 61.95 & 53.53 & 207.56 & 200.14 & 5.56 \\
\hline 28.04483 & 235.81 & 201.05 & 169.67 & 64.28 & 62.11 & 53.55 & 207.72 & 200.13 & 5.60 \\
\hline 28.54567 & 237.21 & 202.27 & 176.54 & 64.27 & 61.97 & 53.48 & 207.85 & 200.09 & 5.59 \\
\hline 29.0455 & 238.06 & 201.81 & 183.53 & 64.28 & 61.97 & 53.47 & 208.15 & 200.07 & 5.60 \\
\hline 29.54533 & 238.54 & 203.13 & 190.01 & 64.19 & 61.92 & 53.34 & 208.23 & 200.08 & 5.61 \\
\hline 30.045 & 238.85 & 202.02 & 193.39 & 64.18 & 61.93 & 53.33 & 208.26 & 200.06 & 5.63 \\
\hline 30.544 & 238.87 & 202.62 & 194.99 & 64.15 & 61.94 & 53.3 & 208.18 & 200.03 & 5.57 \\
\hline 31.04567 & 238.86 & 201.73 & 195.77 & 64.2 & 61.91 & 53.31 & 208.07 & 200.04 & 5.54 \\
\hline 31.5455 & 238.92 & 202.24 & 196.22 & 64.2 & 61.95 & 53.34 & 207.91 & 200.02 & 5.55 \\
\hline 32.04433 & 239.02 & 203.11 & 196.46 & 64.27 & 61.96 & 53.32 & 207.84 & 200.01 & 5.58 \\
\hline 32.54517 & 239.1 & 202.47 & 196.61 & 64.27 & 61.95 & 53.31 & 207.68 & 199.99 & 5.59 \\
\hline 33.04583 & 239.19 & 201.72 & 196.76 & 64.34 & 62.03 & 53.32 & 207.7 & 200 & 5.59 \\
\hline 33.54467 & 239.27 & 200.91 & 196.79 & 64.4 & 62.09 & 53.33 & 207.67 & 199.98 & 5.59 \\
\hline 34.0455 & 239.26 & 201.04 & 196.9 & 64.47 & 62.19 & 53.35 & 207.82 & 200.18 & 5.60 \\
\hline 34.54533 & 239.25 & 203.28 & 196.94 & 64.57 & 62.28 & 53.42 & 207.86 & 200.16 & 5.61 \\
\hline 35.04517 & 239.18 & 202.23 & 196.97 & 64.63 & 62.25 & 53.4 & 207.85 & 200.17 & 5.56 \\
\hline 35.545 & 239.08 & 201.42 & 197.01 & 64.69 & 62.39 & 53.41 & 207.96 & 200.16 & 5.56 \\
\hline 36.04567 & 238.95 & 201.23 & 197.04 & 64.78 & 62.43 & 53.4 & 208.05 & 200.14 & 5.57 \\
\hline 36.5455 & 238.85 & 201.61 & 197.04 & 64.9 & 62.5 & 53.39 & 208.09 & 200.14 & 5.59 \\
\hline 37.04533 & 238.62 & 200.76 & 197.07 & 65 & 62.56 & 53.41 & 208.17 & 200.14 & 5.59 \\
\hline 37.54417 & 238.43 & 202.29 & 197.09 & 65.12 & 62.64 & 53.44 & 208.25 & 200.14 & 5.59 \\
\hline 38.044 & 238.17 & 201.65 & 197.13 & 65.26 & 62.69 & 53.41 & 208.37 & 200.15 & 5.60 \\
\hline 38.54483 & 237.9 & 203.5 & 197.14 & 65.38 & 62.62 & 53.42 & 208.46 & 200.13 & 5.61 \\
\hline 39.04467 & 237.7 & 202.57 & 197.2 & 65.55 & 62.76 & 53.38 & 208.64 & 200.14 & 5.61 \\
\hline 39.54633 & 237.47 & 202.83 & 197.22 & 65.77 & 62.88 & 53.39 & 208.82 & 200.12 & 5.56 \\
\hline 40.04517 & 237.19 & 202.89 & 197.3 & 65.98 & 62.94 & 53.4 & 209.03 & 200.14 & 5.56 \\
\hline 40.545 & 236.94 & 202.14 & 197.31 & 66.24 & 62.96 & 53.37 & 209.24 & 200.14 & 5.57 \\
\hline 41.04567 & 236.73 & 203.76 & 197.35 & 66.53 & 63.03 & 53.39 & 209.29 & 200.13 & 5.60 \\
\hline 41.5455 & 236.67 & 203.03 & 197.38 & 66.87 & 63.06 & 53.38 & 209.3 & 200.14 & 5.60 \\
\hline 42.04533 & 236.75 & 202.22 & 197.36 & 67.29 & 63.08 & 53.37 & 209.29 & 200.11 & 5.61 \\
\hline 42.54517 & 236.91 & 205.03 & 197.35 & 67.88 & 63.1 & 53.37 & 209.26 & 200.11 & 5.63 \\
\hline 43.045 & 237.14 & 203.52 & 197.34 & 68.68 & 63.04 & 53.38 & 209.19 & 200.14 & 5.63 \\
\hline 43.54483 & 237.32 & 204.29 & 197.34 & 69.8 & 63.21 & 53.4 & 209.21 & 200.1 & 5.58 \\
\hline
\end{tabular}


Table B5. Continued.

\begin{tabular}{|c|c|c|c|c|c|c|c|c|c|}
\hline $\begin{array}{l}\text { Time } \\
\text { min }\end{array}$ & $\begin{array}{l}\mathrm{T} 1 \\
{ }^{\circ} \mathrm{C}\end{array}$ & $\begin{array}{l}\mathrm{T} 2 \\
{ }^{\circ} \mathrm{C}\end{array}$ & $\begin{array}{l}\mathrm{T} 3 \\
{ }^{\circ} \mathrm{C}\end{array}$ & $\begin{array}{l}\mathrm{T} 4 \\
{ }^{\circ} \mathrm{C}\end{array}$ & $\begin{array}{l}\mathrm{T} 5 \\
{ }^{\circ} \mathrm{C}\end{array}$ & $\begin{array}{l}\mathrm{T} 6 \\
{ }^{\circ} \mathrm{C}\end{array}$ & $\begin{array}{l}\text { Pinj } \\
\text { psig }\end{array}$ & $\begin{array}{c}\text { Pout } \\
\text { psig }\end{array}$ & $\begin{array}{c}\mathrm{Vw} \\
\mathrm{cc} / \mathrm{min}\end{array}$ \\
\hline 44.04567 & 237.58 & 203.85 & 197.35 & 71.29 & 63.26 & 53.45 & 209.25 & 200.09 & 5.56 \\
\hline 44.5445 & 237.83 & 205.38 & 197.38 & 73.32 & 63.3 & 53.51 & 209.35 & 200.09 & 5.57 \\
\hline 45.04433 & 238.04 & 203.56 & 197.38 & 75.95 & 63.36 & 53.67 & 209.44 & 200.09 & 5.59 \\
\hline 45.545 & 238.2 & 203.06 & 197.39 & 79.39 & 63.39 & 53.83 & 209.5 & 200.11 & 5.58 \\
\hline 46.04483 & 238.33 & 203.78 & 197.39 & 83.53 & 63.43 & 53.99 & 209.5 & 200.12 & 5.60 \\
\hline 46.54567 & 238.5 & 203.41 & 197.4 & 88.51 & 63.48 & 54.13 & 209.53 & 200.2 & 5.60 \\
\hline 47.0455 & 238.56 & 203.94 & 197.44 & 94.01 & 63.5 & 54.25 & 209.62 & 200.33 & 5.62 \\
\hline 47.54533 & 238.53 & 203.86 & 197.45 & 100.06 & 63.55 & 54.39 & 209.75 & 200.47 & 5.57 \\
\hline 48.045 & 238.55 & 202.46 & 197.49 & 106.08 & 63.57 & 54.51 & 209.86 & 200.61 & 5.55 \\
\hline 48.544 & 238.58 & 203.92 & 197.51 & 112.25 & 63.6 & 54.65 & 209.97 & 200.72 & 5.55 \\
\hline 49.04567 & 238.53 & 202.57 & 197.53 & 118.65 & 63.62 & 54.73 & 210.1 & 200.73 & 5.59 \\
\hline 49.5445 & 238.43 & 204.07 & 197.58 & 125.32 & 63.63 & 54.81 & 210.2 & 200.71 & 5.60 \\
\hline 50.04533 & 238.29 & 203.04 & 197.62 & 132.42 & 63.54 & 54.93 & 210.37 & 200.68 & 5.60 \\
\hline 50.54517 & 238.23 & 203.19 & 197.62 & 138.89 & 63.68 & 55.05 & 210.54 & 200.65 & 5.61 \\
\hline 51.045 & 238.09 & 205.11 & 197.68 & 145.08 & 63.7 & 55.15 & 210.85 & 200.61 & 5.63 \\
\hline 51.54567 & 237.97 & 204.96 & 197.76 & 151.31 & 63.75 & 55.25 & 211.12 & 200.71 & 5.61 \\
\hline 52.0455 & 237.9 & 204.66 & 197.8 & 157.95 & 63.75 & 55.37 & 211.3 & 200.63 & 5.56 \\
\hline 52.54533 & 237.76 & 204.68 & 197.83 & 164.45 & 63.79 & 55.47 & 211.49 & 200.61 & 5.55 \\
\hline 53.04517 & 237.66 & 204.89 & 197.85 & 170.34 & 63.84 & 55.61 & 211.64 & 200.57 & 5.56 \\
\hline 53.544 & 237.53 & 206.84 & 197.91 & 175.63 & 63.77 & 55.75 & 211.74 & 200.54 & 5.60 \\
\hline 54.04567 & 237.58 & 205.97 & 197.91 & 180.09 & 63.93 & 55.85 & 211.81 & 200.53 & 5.59 \\
\hline 54.54467 & 237.57 & 205.2 & 197.92 & 183.47 & 63.97 & 55.97 & 211.86 & 200.52 & 5.60 \\
\hline 55.04433 & 237.61 & 206.66 & 197.92 & 185.99 & 64.03 & 56.09 & 211.85 & 200.49 & 5.60 \\
\hline 55.54517 & 237.73 & 206.42 & 197.91 & 188.29 & 64.08 & 56.21 & 211.87 & 200.47 & 5.63 \\
\hline 56.045 & 237.95 & 205.47 & 197.9 & 189.71 & 64.1 & 56.33 & 211.83 & 200.65 & 5.58 \\
\hline 56.54483 & 237.67 & 206.3 & 197.69 & 192.31 & 64.14 & 56.47 & 210.51 & 201.2 & 5.56 \\
\hline 57.0455 & 237.98 & 205.57 & 197.7 & 193.52 & 64.19 & 56.61 & 210.92 & 201.07 & 5.57 \\
\hline 57.54533 & 238.39 & 205.08 & 197.81 & 193.65 & 64.23 & 56.67 & 211.58 & 201.02 & 5.60 \\
\hline 58.04617 & 238.63 & 205.24 & 197.92 & 194.02 & 64.31 & 56.77 & 211.97 & 201 & 5.61 \\
\hline 58.545 & 238.76 & 207.13 & 197.96 & 194.54 & 64.39 & 56.87 & 212.09 & 200.97 & 5.61 \\
\hline 59.04383 & 238.77 & 205.38 & 197.97 & 194.99 & 64.49 & 56.97 & 212.09 & 200.95 & 5.61 \\
\hline 59.54467 & 238.83 & 205.06 & 197.96 & 195.38 & 64.61 & 57.06 & 212.01 & 200.9 & 5.64 \\
\hline 60.04533 & 238.8 & 205.03 & 197.96 & 195.69 & 64.77 & 57.17 & 211.9 & 200.89 & 5.59 \\
\hline 60.54517 & 238.78 & 205.2 & 197.91 & 195.98 & 64.92 & 57.24 & 211.83 & 200.86 & 5.56 \\
\hline 61.045 & 238.63 & 204.59 & 197.81 & 196.25 & 65.16 & 57.37 & 211.22 & 201.13 & 5.57 \\
\hline 61.54483 & 238.67 & 204.98 & 197.8 & 196.47 & 65.39 & 57.45 & 211.29 & 201.03 & 5.61 \\
\hline 62.04567 & 238.73 & 206.68 & 197.82 & 196.67 & 65.7 & 57.54 & 211.46 & 200.96 & 5.60 \\
\hline 62.54533 & 238.71 & 205 & 197.86 & 196.8 & 66.04 & 57.6 & 211.68 & 200.97 & 5.60 \\
\hline 63.04517 & 238.65 & 204.6 & 197.9 & 196.93 & 66.48 & 57.68 & 211.85 & 200.94 & 5.61 \\
\hline 63.545 & 238.56 & 204.69 & 197.92 & 197.01 & 67.04 & 57.76 & 211.9 & 200.92 & 5.63 \\
\hline 64.044 & 238.46 & 206.47 & 197.95 & 197.11 & 67.76 & 57.84 & 211.94 & 200.9 & 5.60 \\
\hline 64.54567 & 238.46 & 205.31 & 197.91 & 197.16 & 68.63 & 57.94 & 211.95 & 200.9 & 5.57 \\
\hline 65.0455 & 238.38 & 208.48 & 197.96 & 197.19 & 69.67 & 58.02 & 211.96 & 200.85 & 5.58 \\
\hline 65.54533 & 238.31 & 206.47 & 197.94 & 197.24 & 70.9 & 58.12 & 212 & 200.84 & 5.61 \\
\hline
\end{tabular}


Table B5. Continued.

\begin{tabular}{|c|c|c|c|c|c|c|c|c|c|}
\hline $\begin{array}{l}\text { Time } \\
\text { min }\end{array}$ & $\begin{array}{l}\mathrm{T} 1 \\
{ }^{\circ} \mathrm{C}\end{array}$ & $\begin{array}{l}\mathrm{T} 2 \\
{ }^{\circ} \mathrm{C}\end{array}$ & $\begin{array}{l}\mathrm{T} 3 \\
{ }^{\circ} \mathrm{C}\end{array}$ & $\begin{array}{l}\mathrm{T} 4 \\
{ }^{\circ} \mathrm{C}\end{array}$ & $\begin{array}{l}\mathrm{T} 5 \\
{ }^{\circ} \mathrm{C}\end{array}$ & $\begin{array}{l}\mathrm{T} 6 \\
{ }^{\circ} \mathrm{C}\end{array}$ & $\begin{array}{l}\text { Pinj } \\
\text { psig }\end{array}$ & $\begin{array}{l}\text { Pout } \\
\text { psig }\end{array}$ & $\begin{array}{c}\mathrm{Vw} \\
\mathrm{cc} / \mathrm{min}\end{array}$ \\
\hline 66.045 & 238.34 & 205.76 & 197.97 & 197.27 & 72.38 & 58.21 & 212.03 & 200.81 & 5.60 \\
\hline 66.54483 & 238.22 & 204.52 & 197.92 & 197.22 & 74.2 & 58.32 & 211.3 & 201.18 & 5.61 \\
\hline 67.04567 & 238.06 & 209.54 & 197.76 & 197.13 & 75.79 & 58.39 & 211.04 & 200.95 & 5.63 \\
\hline 67.5455 & 238.26 & 207 & 197.8 & 197.21 & 77.86 & 58.45 & 211.49 & 200.96 & 5.63 \\
\hline 68.04533 & 238.5 & 205.88 & 197.91 & 197.34 & 80.32 & 58.51 & 211.91 & 200.93 & 5.58 \\
\hline 68.54517 & 238.61 & 206.6 & 197.99 & 197.4 & 83.24 & 58.55 & 212.17 & 200.88 & 5.57 \\
\hline 69.04501 & 238.63 & 206.36 & 197.98 & 197.46 & 86.59 & 58.62 & 212.28 & 200.87 & 5.59 \\
\hline 69.54567 & 238.64 & 205.77 & 198 & 197.48 & 90.25 & 58.72 & 212.24 & 200.84 & 5.59 \\
\hline 70.04633 & 238.68 & 207.37 & 197.99 & 197.47 & 94.07 & 58.8 & 212.16 & 200.82 & 5.59 \\
\hline 70.54433 & 238.68 & 206.21 & 197.98 & 197.47 & 98.31 & 58.86 & 212.08 & 200.79 & 5.61 \\
\hline 71.04417 & 238.71 & 209.97 & 197.94 & 197.44 & 102.74 & 58.92 & 212 & 200.76 & 5.61 \\
\hline 71.54501 & 238.78 & 205 & 197.93 & 197.46 & 107.13 & 58.98 & 211.93 & 200.75 & 5.62 \\
\hline 72.04483 & 238.79 & 206.21 & 197.93 & 197.45 & 111.28 & 59.04 & 211.91 & 200.73 & 5.57 \\
\hline 72.5455 & 238.51 & 207.21 & 197.74 & 197.26 & 115.98 & 59.12 & 210.74 & 201.08 & 5.57 \\
\hline 73.04533 & 238.56 & 206.63 & 197.69 & 197.21 & 119.34 & 59.15 & 210.83 & 200.94 & 5.59 \\
\hline 73.54517 & 238.77 & 207.08 & 197.73 & 197.29 & 122.34 & 59.19 & 211.08 & 200.9 & 5.60 \\
\hline 74.04501 & 238.97 & 208.02 & 197.79 & 197.33 & 125.47 & 59.2 & 211.37 & 200.89 & 5.60 \\
\hline 74.54383 & 239.01 & 208.41 & 197.85 & 197.42 & 128.78 & 59.26 & 211.68 & 200.9 & 5.60 \\
\hline 75.0455 & 239.01 & 205.2 & 197.87 & 197.46 & 132.06 & 59.32 & 211.83 & 200.85 & 5.62 \\
\hline 75.5445 & 239.02 & 204.84 & 197.95 & 197.5 & 135.16 & 59.34 & 211.91 & 200.83 & 5.62 \\
\hline 76.04517 & 238.97 & 203.11 & 197.92 & 197.49 & 138.09 & 59.42 & 211.94 & 200.81 & 5.58 \\
\hline 76.54501 & 238.88 & 204.9 & 197.92 & 197.53 & 140.81 & 59.49 & 211.96 & 200.77 & 5.57 \\
\hline 77.04483 & 238.87 & 206.5 & 197.93 & 197.51 & 143.25 & 59.57 & 211.96 & 200.77 & 5.57 \\
\hline 77.5455 & 238.86 & 206.34 & 197.95 & 197.52 & 145.81 & 59.65 & 211.97 & 200.74 & 5.59 \\
\hline 78.04533 & 238.82 & 205.54 & 197.95 & 197.54 & 148.09 & 59.76 & 212.08 & 200.7 & 5.60 \\
\hline 78.54517 & 238.79 & 203.15 & 197.97 & 197.54 & 150.47 & 59.84 & 212.16 & 200.67 & 5.60 \\
\hline 79.04501 & 238.64 & 205.48 & 197.91 & 197.5 & 153.54 & 59.94 & 211.52 & 201.06 & 5.62 \\
\hline 79.54483 & 238.55 & 207.38 & 197.84 & 197.43 & 157.01 & 60.04 & 211.44 & 200.92 & 5.64 \\
\hline 80.04567 & 238.63 & 207.51 & 197.86 & 197.45 & 159.56 & 60.12 & 211.68 & 200.89 & 5.58 \\
\hline 80.5445 & 238.67 & 207.41 & 197.92 & 197.53 & 161.87 & 60.2 & 212 & 200.85 & 5.57 \\
\hline 81.04433 & 238.76 & 206.61 & 197.98 & 197.58 & 164.2 & 60.32 & 212.26 & 200.84 & 5.59 \\
\hline 81.54501 & 238.83 & 205.73 & 198.03 & 197.64 & 166.59 & 60.42 & 212.5 & 200.83 & 5.60 \\
\hline 82.04483 & 238.82 & 206.44 & 198.06 & 197.68 & 168.93 & 60.56 & 212.64 & 200.79 & 5.61 \\
\hline 82.54567 & 238.84 & 205.38 & 198.1 & 197.7 & 171.42 & 60.71 & 212.73 & 200.81 & 5.60 \\
\hline 83.0455 & 238.81 & 204.69 & 198.08 & 197.73 & 173.83 & 60.85 & 212.79 & 200.76 & 5.63 \\
\hline 83.54533 & 238.75 & 206.4 & 198.09 & 197.73 & 176.5 & 61.01 & 212.77 & 200.76 & 5.63 \\
\hline 84.04501 & 238.82 & 204.56 & 198.09 & 197.73 & 179.39 & 61.14 & 212.78 & 200.74 & 5.57 \\
\hline 84.544 & 238.48 & 205.49 & 197.92 & 197.54 & 187.2 & 61.36 & 211.63 & 201.01 & 5.56 \\
\hline 85.04567 & 238.54 & 203.58 & 197.89 & 197.51 & 189.05 & 61.51 & 211.76 & 200.87 & 5.58 \\
\hline 85.5445 & 238.72 & 204.76 & 197.93 & 197.53 & 190.37 & 61.69 & 212.07 & 200.87 & 5.61 \\
\hline 86.04433 & 238.87 & 205.99 & 198 & 197.64 & 191.1 & 61.92 & 212.41 & 200.84 & 5.61 \\
\hline 86.54517 & 238.97 & 206.08 & 198.06 & 197.7 & 192.33 & 62.17 & 212.63 & 200.82 & 5.61 \\
\hline 87.04501 & 239.01 & 204.54 & 198.1 & 197.71 & 193.67 & 62.51 & 212.76 & 200.78 & 5.63 \\
\hline 87.54567 & 238.97 & 205.32 & 198.12 & 197.74 & 194.66 & 62.82 & 212.78 & 200.77 & 5.60 \\
\hline
\end{tabular}


Table B5. Continued.

\begin{tabular}{|c|c|c|c|c|c|c|c|c|c|}
\hline $\begin{array}{l}\text { Time } \\
\text { min }\end{array}$ & $\begin{array}{l}\mathrm{T} 1 \\
{ }^{\circ} \mathrm{C}\end{array}$ & $\begin{array}{l}\mathrm{T} 2 \\
{ }^{\circ} \mathrm{C}\end{array}$ & $\begin{array}{l}\mathrm{T} 3 \\
{ }^{\circ} \mathrm{C}\end{array}$ & $\begin{array}{l}\mathrm{T} 4 \\
{ }^{\circ} \mathrm{C}\end{array}$ & $\begin{array}{l}\mathrm{T} 5 \\
{ }^{\circ} \mathrm{C}\end{array}$ & $\begin{array}{l}\mathrm{T} 6 \\
{ }^{\circ} \mathrm{C}\end{array}$ & $\begin{array}{l}\text { Pinj } \\
\text { psig }\end{array}$ & $\begin{array}{l}\text { Pout } \\
\text { psig }\end{array}$ & $\begin{array}{c}\mathrm{Vw} \\
\mathrm{cc} / \mathrm{min}\end{array}$ \\
\hline 88.0455 & 239.01 & 205.63 & 198.09 & 197.77 & 195.3 & 63.17 & 212.81 & 200.77 & 5.56 \\
\hline 88.54533 & 239 & 205.7 & 198.11 & 197.72 & 195.93 & 63.55 & 212.81 & 200.73 & 5.58 \\
\hline 89.04517 & 239.02 & 205.35 & 198.1 & 197.74 & 196.34 & 63.97 & 212.77 & 200.72 & 5.60 \\
\hline 89.544 & 238.99 & 205.96 & 198.06 & 197.74 & 196.77 & 64.44 & 212.7 & 200.71 & 5.61 \\
\hline 90.04567 & 238.99 & 207.09 & 198.05 & 197.73 & 197.17 & 64.96 & 212.62 & 200.71 & 5.62 \\
\hline 90.54467 & 239 & 207.99 & 198.04 & 197.71 & 197.46 & 65.53 & 212.57 & 200.66 & 5.62 \\
\hline 91.04533 & 239.02 & 205.08 & 198.04 & 197.7 & 197.68 & 66.14 & 212.54 & 200.65 & 5.65 \\
\hline 91.54517 & 238.93 & 204.61 & 197.97 & 197.63 & 197.79 & 66.86 & 212.19 & 200.94 & 5.59 \\
\hline 92.04501 & 238.96 & 205.41 & 197.96 & 197.62 & 197.92 & 67.52 & 212.12 & 200.86 & 5.56 \\
\hline 92.54483 & 239.05 & 206.79 & 197.94 & 197.62 & 198 & 68.21 & 212.17 & 200.84 & 5.58 \\
\hline 93.0455 & 239.03 & 203.5 & 197.97 & 197.63 & 198.07 & 69.02 & 212.27 & 200.82 & 5.61 \\
\hline 93.54533 & 239 & 205.04 & 197.97 & 197.65 & 198.13 & 69.95 & 212.27 & 200.8 & 5.62 \\
\hline 94.04517 & 239.01 & 205.96 & 197.97 & 197.63 & 198.15 & 70.95 & 212.23 & 200.76 & 5.61 \\
\hline 94.54417 & 238.54 & 205.05 & 197.82 & 197.46 & 197.96 & 72.24 & 211.02 & 200.84 & 5.63 \\
\hline 95.04483 & 238.14 & 205.95 & 197.73 & 197.37 & 197.98 & 73.43 & 210.93 & 200.75 & 5.62 \\
\hline 95.54467 & 237.91 & 206.35 & 197.72 & 197.41 & 198.06 & 74.69 & 211.18 & 200.77 & 5.57 \\
\hline 96.04533 & 237.82 & 208.08 & 197.77 & 197.45 & 198.1 & 76.16 & 211.35 & 200.76 & 5.56 \\
\hline 96.54517 & 237.8 & 205.37 & 197.79 & 197.47 & 198.13 & 77.81 & 211.42 & 200.73 & 5.57 \\
\hline 97.04501 & 237.81 & 206.28 & 197.78 & 197.48 & 198.16 & 79.8 & 211.41 & 200.68 & 5.59 \\
\hline 97.54483 & 237.83 & 206.34 & 197.77 & 197.46 & 198.16 & 82.04 & 211.29 & 200.67 & 5.58 \\
\hline 98.04567 & 237.85 & 206.49 & 197.75 & 197.43 & 198.11 & 84.55 & 211.12 & 200.66 & 5.59 \\
\hline 98.54533 & 237.44 & 205.63 & 197.5 & 197.16 & 197.79 & 87.57 & 209.57 & 200.83 & 5.60 \\
\hline 99.04517 & 237.61 & 206.59 & 197.4 & 197.08 & 197.8 & 89.82 & 209.57 & 200.72 & 5.62 \\
\hline 99.54417 & 237.85 & 205.55 & 197.42 & 197.12 & 197.85 & 92.13 & 209.84 & 200.79 & 5.59 \\
\hline 100.0448 & 238.15 & 206.14 & 197.5 & 197.17 & 197.93 & 94.79 & 210.08 & 200.81 & 5.57 \\
\hline 100.5447 & 238.25 & 204.68 & 197.52 & 197.21 & 197.97 & 97.78 & 210.24 & 200.79 & 5.56 \\
\hline 101.0463 & 238.25 & 203.9 & 197.54 & 197.24 & 197.99 & 101.2 & 210.28 & 200.77 & 5.60 \\
\hline 101.5453 & 238.31 & 204.1 & 197.53 & 197.22 & 197.96 & 105.01 & 210.21 & 200.76 & 5.60 \\
\hline 102.045 & 238.31 & 205.46 & 197.51 & 197.19 & 197.94 & 109.24 & 210.1 & 200.73 & 5.60 \\
\hline 102.5448 & 238.23 & 206.04 & 197.5 & 197.17 & 197.94 & 113.78 & 209.98 & 200.71 & 5.60 \\
\hline 103.0457 & 238.01 & 205.22 & 197.36 & 197.04 & 197.73 & 119.33 & 209.14 & 200.82 & 5.63 \\
\hline 103.5455 & 238.02 & 205.79 & 197.27 & 196.97 & 197.74 & 123.29 & 209.08 & 200.74 & 5.59 \\
\hline 104.0453 & 238.02 & 205.12 & 197.31 & 196.99 & 197.78 & 126.81 & 209.22 & 200.76 & 5.56 \\
\hline 104.5452 & 238.01 & 203.4 & 197.33 & 197.03 & 197.8 & 131.16 & 209.42 & 200.74 & 5.57 \\
\hline 105.044 & 238.1 & 203.67 & 197.35 & 197.05 & 197.82 & 136.28 & 209.44 & 200.74 & 5.61 \\
\hline 105.5457 & 238.16 & 203.71 & 197.34 & 197.05 & 197.8 & 141.4 & 209.42 & 200.75 & 5.60 \\
\hline 106.0445 & 238.23 & 204.09 & 197.32 & 197.04 & 197.81 & 146.34 & 209.33 & 200.7 & 5.61 \\
\hline 106.5453 & 238.13 & 205.03 & 197.24 & 196.9 & 197.51 & 151.63 & 207.9 & 200.96 & 5.60 \\
\hline 107.0452 & 237.9 & 208.45 & 197.03 & 196.72 & 197.49 & 152.76 & 207.93 & 200.84 & 5.63 \\
\hline 107.545 & 238.15 & 203.94 & 197.08 & 196.76 & 197.57 & 153.72 & 208.24 & 200.85 & 5.59 \\
\hline 108.0467 & 238.37 & 204.47 & 197.14 & 196.84 & 197.64 & 154.75 & 208.62 & 200.85 & 5.57 \\
\hline 108.5455 & 238.52 & 203.81 & 197.2 & 196.91 & 197.72 & 158.22 & 208.87 & 200.87 & 5.58 \\
\hline 109.0453 & 238.57 & 205.12 & 197.24 & 196.95 & 197.74 & 163.12 & 208.97 & 200.84 & 5.60 \\
\hline 109.5452 & 238.54 & 203.76 & 197.24 & 196.96 & 197.71 & 168.31 & 208.91 & 200.8 & 5.60 \\
\hline
\end{tabular}


Table B5. Continued.

\begin{tabular}{|c|c|c|c|c|c|c|c|c|c|}
\hline $\begin{array}{l}\text { Time } \\
\text { min }\end{array}$ & $\begin{array}{l}\mathrm{T} 1 \\
{ }^{\circ} \mathrm{C}\end{array}$ & $\begin{array}{l}\mathrm{T} 2 \\
{ }^{\circ} \mathrm{C}\end{array}$ & $\begin{array}{l}\mathrm{T} 3 \\
{ }^{\circ} \mathrm{C}\end{array}$ & $\begin{array}{l}\mathrm{T} 4 \\
{ }^{\circ} \mathrm{C}\end{array}$ & $\begin{array}{l}\text { T5 } \\
{ }^{\circ} \mathrm{C}\end{array}$ & $\begin{array}{l}\mathrm{T} 6 \\
{ }^{\circ} \mathrm{C}\end{array}$ & $\begin{array}{l}\text { Pinj } \\
\text { psig }\end{array}$ & $\begin{array}{l}\text { Pout } \\
\text { psig }\end{array}$ & $\begin{array}{c}\mathrm{Vw} \\
\mathrm{cc} / \mathrm{min}\end{array}$ \\
\hline 110.044 & 238.49 & 205.59 & 197.21 & 196.92 & 197.69 & 172.22 & 208.79 & 200.76 & 5.61 \\
\hline 110.5448 & 238.46 & 206.47 & 197.18 & 196.89 & 197.66 & 175.44 & 208.67 & 200.74 & 5.63 \\
\hline 111.0447 & 238.37 & 204 & 197.16 & 196.86 & 197.66 & 178.23 & 208.59 & 200.71 & 5.62 \\
\hline 111.5445 & 237.97 & 207.34 & 196.93 & 196.61 & 197.36 & 182.07 & 207.19 & 200.87 & 5.58 \\
\hline 112.0452 & 238.09 & 206.37 & 196.92 & 196.63 & 197.44 & 182.38 & 207.58 & 200.8 & 5.59 \\
\hline 112.545 & 238.33 & 204.96 & 196.99 & 196.71 & 197.53 & 183.21 & 207.98 & 200.88 & 5.58 \\
\hline 113.0448 & 238.51 & 204.05 & 197.05 & 196.76 & 197.57 & 184.2 & 208.2 & 200.84 & 5.62 \\
\hline 113.5455 & 238.53 & 205.11 & 197.09 & 196.82 & 197.63 & 185.69 & 208.44 & 200.87 & 5.60 \\
\hline 114.0453 & 238.55 & 207.99 & 197.13 & 196.84 & 197.65 & 187.86 & 208.47 & 200.83 & 5.61 \\
\hline 114.5443 & 238.52 & 205.87 & 197.11 & 196.86 & 197.63 & 189.89 & 208.39 & 200.79 & 5.62 \\
\hline 115.0442 & 238.45 & 206.62 & 197.1 & 196.81 & 197.6 & 191.63 & 208.26 & 200.74 & 5.62 \\
\hline 115.5448 & 238.46 & 206.3 & 197.06 & 196.78 & 197.58 & 192.94 & 208.17 & 200.71 & 5.59 \\
\hline 116.0447 & 238.1 & 204.48 & 196.87 & 196.57 & 197.37 & 193.13 & 207.23 & 200.83 & 5.56 \\
\hline 116.5453 & 238.32 & 204.75 & 196.93 & 196.64 & 197.47 & 194.65 & 207.65 & 200.81 & 5.59 \\
\hline 117.0452 & 238.5 & 205.35 & 196.99 & 196.7 & 197.52 & 195.32 & 207.86 & 200.84 & 5.60 \\
\hline 117.545 & 238.51 & 206.21 & 197.01 & 196.7 & 197.53 & 195.68 & 207.89 & 200.82 & 5.60 \\
\hline 118.0448 & 238.44 & 205.16 & 197.01 & 196.72 & 197.55 & 195.9 & 207.97 & 200.86 & 5.60 \\
\hline 118.5457 & 238.51 & 204.68 & 197.01 & 196.74 & 197.53 & 196.13 & 207.97 & 200.81 & 5.63 \\
\hline 119.0455 & 238.53 & 204.77 & 197.02 & 196.73 & 197.54 & 196.33 & 207.96 & 200.77 & 5.60 \\
\hline 119.5453 & 238.5 & 203.61 & 197 & 196.71 & 197.54 & 196.46 & 207.86 & 200.74 & 5.56 \\
\hline 120.045 & 238.47 & 204.4 & 196.97 & 196.7 & 197.52 & 196.57 & 207.77 & 200.7 & 5.56 \\
\hline 120.544 & 238.49 & 204.87 & 196.99 & 196.69 & 197.49 & 196.67 & 207.76 & 200.66 & 5.59 \\
\hline 121.0457 & 238.19 & 204.96 & 196.83 & 196.56 & 197.26 & 195.6 & 207.23 & 200.82 & 5.61 \\
\hline 121.5455 & 238.39 & 205.43 & 196.91 & 196.64 & 197.48 & 196.66 & 207.6 & 200.82 & 5.60 \\
\hline 122.0453 & 238.53 & 207.6 & 196.95 & 196.69 & 197.48 & 196.95 & 207.7 & 200.82 & 5.61 \\
\hline 122.5452 & 238.64 & 209.57 & 196.97 & 196.7 & 197.52 & 197.04 & 207.71 & 200.85 & 5.64 \\
\hline 123.045 & 238.68 & 205.45 & 196.95 & 196.66 & 197.49 & 197.09 & 207.69 & 200.78 & 5.65 \\
\hline 123.5457 & 238.59 & 204.76 & 196.95 & 196.67 & 197.49 & 197.1 & 207.62 & 200.78 & 5.56 \\
\hline 124.0455 & 238.58 & 204.4 & 196.95 & 196.67 & 197.51 & 197.15 & 207.64 & 200.77 & 5.55 \\
\hline 124.5453 & 238.58 & 202.4 & 196.96 & 196.67 & 197.48 & 197.15 & 207.61 & 200.73 & 5.57 \\
\hline 125.0452 & 238.53 & 203 & 196.93 & 196.66 & 197.46 & 197.16 & 207.56 & 200.69 & 5.59 \\
\hline 125.544 & 238.51 & 205.79 & 196.91 & 196.6 & 197.32 & 197.02 & 207.04 & 199.6 & 5.60 \\
\hline 126.0457 & 238.39 & 202.54 & 196.86 & 196.59 & 197.43 & 196.82 & 207.38 & 200.77 & 5.61 \\
\hline 126.5447 & 238.52 & 201.95 & 196.93 & 196.65 & 197.4 & 197.2 & 207.65 & 200.82 & 5.62 \\
\hline 127.0453 & 238.52 & 205.58 & 196.97 & 196.68 & 197.51 & 197.26 & 207.76 & 200.81 & 5.63 \\
\hline 127.5452 & 238.56 & 205.1 & 196.97 & 196.67 & 197.51 & 197.26 & 207.69 & 200.81 & 5.56 \\
\hline 128.045 & 238.55 & 203.48 & 196.96 & 196.67 & 197.48 & 197.26 & 207.61 & 200.77 & 5.57 \\
\hline 128.5448 & 238.55 & 205.41 & 196.92 & 196.64 & 197.48 & 197.27 & 207.54 & 200.71 & 5.60 \\
\hline 129.0455 & 238.46 & 205.7 & 196.93 & 196.64 & 197.47 & 197.23 & 207.44 & 200.67 & 5.61 \\
\hline 129.5453 & 238.43 & 205.6 & 196.91 & 196.63 & 197.45 & 197.24 & 207.44 & 200.61 & 5.61 \\
\hline 130.047 & 238.52 & 206.15 & 196.92 & 196.61 & 197.45 & 197.24 & 207.43 & 200.62 & 5.62 \\
\hline 130.545 & 238.43 & 204.51 & 196.83 & 196.52 & 197.26 & 196.97 & 206.33 & 200.8 & 5.65 \\
\hline 131.0448 & 238.4 & 203.35 & 196.83 & 196.58 & 197.44 & 196.69 & 207.36 & 200.74 & 5.60 \\
\hline 131.5465 & 238.53 & 203.62 & 196.89 & 196.62 & 197.32 & 197.21 & 207.42 & 200.69 & 5.57 \\
\hline
\end{tabular}


Table B5. Continued.

\begin{tabular}{|c|c|c|c|c|c|c|c|c|c|}
\hline $\begin{array}{c}\text { Time } \\
\text { min }\end{array}$ & $\begin{array}{l}\mathrm{T} 1 \\
{ }^{\circ} \mathrm{C} \\
\end{array}$ & $\begin{array}{l}\mathrm{T} 2 \\
{ }^{\circ} \mathrm{C} \\
\end{array}$ & $\begin{array}{l}\mathrm{T3} \\
{ }^{\circ} \mathrm{C} \\
\end{array}$ & $\begin{array}{l}\mathrm{T} 4 \\
{ }^{\circ} \mathrm{C} \\
\end{array}$ & $\begin{array}{l}\mathrm{T} 5 \\
{ }^{\circ} \mathrm{C}\end{array}$ & $\begin{array}{l}\mathrm{T} 6 \\
{ }^{\circ} \mathrm{C} \\
\end{array}$ & $\begin{array}{l}\text { Pinj } \\
\text { psig }\end{array}$ & $\begin{array}{l}\text { Pout } \\
\text { psig }\end{array}$ & $\begin{array}{c}\mathrm{Vw} \\
\mathrm{cc} / \mathrm{min}\end{array}$ \\
\hline 132.0453 & 238.59 & 203.17 & 196.93 & 196.66 & 197.5 & 197.3 & 207.67 & 200.78 & 5.59 \\
\hline 132.5443 & 238.64 & 203.55 & 196.95 & 196.7 & 197.52 & 197.3 & 207.75 & 200.82 & 5.61 \\
\hline 133.045 & 238.57 & 204.04 & 196.95 & 196.68 & 197.51 & 197.31 & 207.73 & 200.76 & 5.60 \\
\hline 133.5448 & 238.47 & 203.7 & 196.93 & 196.67 & 197.49 & 197.31 & 207.59 & 200.7 & 5.59 \\
\hline 134.0447 & 238.34 & 204.6 & 196.92 & 196.63 & 197.47 & 197.29 & 207.49 & 200.56 & 5.60 \\
\hline 134.5453 & 238.4 & 203.51 & 196.89 & 196.62 & 197.44 & 197.26 & 207.44 & 200.51 & 5.62 \\
\hline 135.0452 & 238.48 & 202.92 & 196.89 & 196.62 & 197.44 & 197.28 & 207.43 & 200.61 & 5.58 \\
\hline 135.545 & 238.23 & 204.68 & 196.8 & 196.52 & 197.32 & 196.77 & 206.97 & 198.98 & 5.56 \\
\hline 136.0448 & 238.46 & 204.31 & 196.88 & 196.61 & 197.48 & 197.22 & 207.52 & 200.74 & 5.57 \\
\hline 136.5457 & 238.63 & 203.81 & 196.91 & 196.65 & 197.49 & 197.31 & 207.64 & 200.7 & 5.59 \\
\hline 137.0455 & 238.74 & 202.64 & 196.97 & 196.68 & 197.54 & 197.4 & 207.8 & 200.78 & 5.60 \\
\hline 137.5462 & 238.69 & 202.86 & 196.95 & 196.67 & 197.51 & 197.33 & 207.68 & 200.74 & 5.60 \\
\hline 138.0442 & 238.6 & 204.14 & 196.92 & 196.63 & 197.48 & 197.32 & 207.54 & 200.73 & 5.61 \\
\hline 138.5448 & 238.64 & 205.2 & 196.89 & 196.62 & 197.46 & 197.32 & 207.49 & 200.61 & 5.64 \\
\hline 139.0457 & 238.59 & 206.42 & 196.87 & 196.62 & 197.45 & 197.3 & 207.42 & 200.57 & 5.57 \\
\hline 139.5445 & 238.7 & 206.89 & 196.89 & 196.61 & 197.47 & 197.29 & 207.41 & 200.5 & 5.55 \\
\hline 140.0453 & 238.65 & 207.41 & 196.88 & 196.61 & 197.45 & 197.29 & 207.45 & 200.51 & 5.58 \\
\hline 140.5452 & 238.61 & 209.83 & 196.88 & 196.61 & 197.45 & 197.29 & 207.44 & 200.53 & 5.60 \\
\hline 141.045 & 238.58 & 206.73 & 196.89 & 196.62 & 197.48 & 197.31 & 207.46 & 200.49 & 5.58 \\
\hline 141.5457 & 238.47 & 204.67 & 196.9 & 196.64 & 197.46 & 197.32 & 207.47 & 200.47 & 5.60 \\
\hline 142.0455 & 238.46 & 204.23 & 196.89 & 196.64 & 197.48 & 197.32 & 207.5 & 200.44 & 5.61 \\
\hline 142.5453 & 238.53 & 207.83 & 196.87 & 196.62 & 197.46 & 197.3 & 207.5 & 200.43 & 5.63 \\
\hline 143.0452 & 238.59 & 206.51 & 196.91 & 196.62 & 197.48 & 197.32 & 207.51 & 200.42 & 5.58 \\
\hline 143.544 & 238.57 & 208.68 & 196.9 & 196.63 & 197.45 & 197.31 & 207.52 & 200.5 & 5.54 \\
\hline 144.0457 & 238.61 & 207.62 & 196.92 & 196.63 & 197.49 & 197.33 & 207.54 & 200.39 & 5.56 \\
\hline 144.5447 & 238.4 & 208.38 & 196.81 & 196.54 & 197.44 & 197.28 & 207.39 & 200.66 & 5.58 \\
\hline 145.0453 & 238.62 & 209.76 & 196.88 & 196.62 & 197.48 & 197.35 & 207.58 & 200.65 & 5.59 \\
\hline 145.5452 & 238.64 & 207.77 & 196.96 & 196.67 & 197.53 & 197.39 & 207.77 & 200.65 & 5.59 \\
\hline 146.045 & 238.05 & 208.15 & 196.93 & 196.62 & 197.48 & 197.34 & 207.52 & 200.43 & 5.59 \\
\hline 146.5448 & 237.57 & 205.99 & 196.89 & 196.62 & 197.47 & 197.31 & 207.46 & 200.47 & 5.61 \\
\hline 147.0455 & 237.3 & 207.05 & 196.88 & 196.61 & 197.45 & 197.31 & 207.43 & 200.39 & 5.61 \\
\hline 147.5453 & 237.12 & 210.81 & 196.86 & 196.61 & 197.45 & 197.31 & 207.41 & 200.47 & 5.56 \\
\hline 148.0452 & 237.13 & 211.29 & 196.9 & 196.61 & 197.46 & 197.33 & 207.4 & 200.38 & 5.55 \\
\hline 148.544 & 237.11 & 210.94 & 196.86 & 196.6 & 197.46 & 197.31 & 207.38 & 200.37 & 5.57 \\
\hline 149.0448 & 237.19 & 209.81 & 196.87 & 196.6 & 197.46 & 197.3 & 207.4 & 200.42 & 5.60 \\
\hline 149.5447 & 237.29 & 209.37 & 196.87 & 196.62 & 197.48 & 197.32 & 207.4 & 200.34 & 5.59 \\
\hline 150.0453 & 237.3 & 208.33 & 196.89 & 196.62 & 197.47 & 197.32 & 207.42 & 200.36 & 5.61 \\
\hline 150.5452 & 237.32 & 209 & 196.86 & 196.63 & 197.45 & 197.32 & 207.42 & 200.3 & 5.62 \\
\hline 151.045 & 237.37 & 209.11 & 196.86 & 196.61 & 197.45 & 197.33 & 207.43 & 200.35 & 5.63 \\
\hline 151.5448 & 237.45 & 208.68 & 196.88 & 196.63 & 197.47 & 197.33 & 207.46 & 200.33 & 5.57 \\
\hline 152.0455 & 237.47 & 212.28 & 196.88 & 196.61 & 197.46 & 197.29 & 207.39 & 198.53 & 5.56 \\
\hline 152.5453 & 237.31 & 211.46 & 196.81 & 196.58 & 197.46 & 197.3 & 207.35 & 200.27 & 5.57 \\
\hline 153.0452 & 237.4 & 211.24 & 196.89 & 196.62 & 197.48 & 197.35 & 207.52 & 200.64 & 5.59 \\
\hline 153.545 & 237.44 & 212.5 & 196.93 & 196.67 & 197.52 & 197.39 & 207.69 & 200.55 & 5.60 \\
\hline
\end{tabular}


Table B5. Continued.

\begin{tabular}{|c|c|c|c|c|c|c|c|c|c|}
\hline $\begin{array}{c}\text { Time } \\
\text { min }\end{array}$ & $\begin{array}{l}\mathrm{T} 1 \\
{ }^{\circ} \mathrm{C}\end{array}$ & $\begin{array}{l}\mathrm{T} 2 \\
{ }^{\circ} \mathrm{C}\end{array}$ & $\begin{array}{l}\mathrm{T} 3 \\
{ }^{\circ} \mathrm{C}\end{array}$ & $\begin{array}{l}\mathrm{T} 4 \\
{ }^{\circ} \mathrm{C}\end{array}$ & $\begin{array}{l}\mathrm{T} 5 \\
{ }^{\circ} \mathrm{C}\end{array}$ & $\begin{array}{l}\mathrm{T} 6 \\
{ }^{\circ} \mathrm{C}\end{array}$ & $\begin{array}{l}\text { Pinj } \\
\text { psig }\end{array}$ & $\begin{array}{l}\text { Pout } \\
\text { psig }\end{array}$ & $\begin{array}{c}\mathrm{Vw} \\
\mathrm{cc} / \mathrm{min}\end{array}$ \\
\hline 154.0448 & 237.4 & 211.55 & 196.91 & 196.64 & 197.5 & 197.36 & 207.56 & 200.45 & 5.58 \\
\hline 154.5447 & 237.46 & 212.2 & 196.9 & 196.63 & 197.47 & 197.36 & 207.54 & 200.43 & 5.60 \\
\hline 155.0455 & 237.55 & 212.63 & 196.9 & 196.63 & 197.47 & 197.34 & 207.52 & 200.45 & 5.63 \\
\hline 155.5453 & 237.5 & 207.78 & 196.9 & 196.63 & 197.47 & 197.35 & 207.53 & 200.46 & 5.60 \\
\hline 156.045 & 237.56 & 210.04 & 196.9 & 196.63 & 197.48 & 197.37 & 207.52 & 200.42 & 5.56 \\
\hline 156.5448 & 237.52 & 212.87 & 196.9 & 196.62 & 197.48 & 197.35 & 207.56 & 200.39 & 5.55 \\
\hline 157.0457 & 237.51 & 213.14 & 196.89 & 196.66 & 197.5 & 197.37 & 207.56 & 200.41 & 5.59 \\
\hline 157.5455 & 237.58 & 211.68 & 196.89 & 196.64 & 197.48 & 197.36 & 207.61 & 200.44 & 5.60 \\
\hline 158.0443 & 237.55 & 210.1 & 196.89 & 196.64 & 197.5 & 197.36 & 207.6 & 200.37 & 5.58 \\
\hline 158.5452 & 237.5 & 210.93 & 196.89 & 196.64 & 197.5 & 197.36 & 207.59 & 200.41 & 5.60 \\
\hline 159.045 & 237.59 & 213.56 & 196.9 & 196.65 & 197.51 & 197.38 & 207.64 & 200.31 & 5.63 \\
\hline 159.5457 & 237.34 & 211.52 & 196.83 & 196.54 & 197.42 & 197.28 & 207.22 & 200.58 & 5.62 \\
\hline 160.0455 & 237.52 & 208.41 & 196.87 & 196.62 & 197.49 & 197.37 & 207.63 & 200.63 & 5.56 \\
\hline 160.5453 & 237.61 & 211.39 & 196.94 & 196.67 & 197.51 & 197.39 & 207.7 & 200.53 & 5.56 \\
\hline 161.0452 & 237.58 & 210.53 & 196.91 & 196.66 & 197.5 & 197.35 & 207.58 & 200.44 & 5.58 \\
\hline 161.545 & 237.53 & 212.23 & 196.89 & 196.62 & 197.5 & 197.36 & 207.6 & 200.47 & 5.60 \\
\hline 162.0457 & 237.55 & 212.97 & 196.89 & 196.64 & 197.5 & 197.38 & 207.61 & 200.44 & 5.59 \\
\hline 162.5455 & 237.53 & 211.2 & 196.93 & 196.64 & 197.5 & 197.36 & 207.64 & 200.38 & 5.60 \\
\hline 163.0443 & 237.5 & 211.47 & 196.92 & 196.63 & 197.51 & 197.38 & 207.66 & 200.36 & 5.61 \\
\hline 163.5452 & 237.47 & 209.45 & 196.92 & 196.65 & 197.53 & 197.38 & 207.67 & 200.34 & 5.63 \\
\hline 164.045 & 237.41 & 210.61 & 196.92 & 196.65 & 197.51 & 197.39 & 207.67 & 200.34 & 5.58 \\
\hline 164.5448 & 237.51 & 209.54 & 196.91 & 196.65 & 197.51 & 197.39 & 207.67 & 200.3 & 5.56 \\
\hline 165.0455 & 237.51 & 207.94 & 196.92 & 196.66 & 197.52 & 197.39 & 207.68 & 200.32 & 5.57 \\
\hline 165.5453 & 237.12 & 212.89 & 196.73 & 196.44 & 197.25 & 197.12 & 206.36 & 198.65 & 5.60 \\
\hline 166.0452 & 237.24 & 214.18 & 196.68 & 196.43 & 197.34 & 197.23 & 207.03 & 200.55 & 5.60 \\
\hline 166.544 & 237.64 & 214.49 & 196.89 & 196.64 & 197.52 & 197.4 & 207.8 & 200.61 & 5.60 \\
\hline 167.0438 & 237.66 & 213.6 & 196.95 & 196.68 & 197.52 & 197.42 & 207.77 & 200.48 & 5.61 \\
\hline 167.5447 & 237.54 & 214.69 & 196.9 & 196.65 & 197.49 & 197.38 & 207.6 & 200.43 & 5.63 \\
\hline 168.0453 & 237.56 & 215.27 & 196.88 & 196.63 & 197.47 & 197.37 & 207.58 & 200.41 & 5.59 \\
\hline 168.5452 & 237.59 & 215.57 & 196.9 & 196.63 & 197.51 & 197.39 & 207.65 & 200.41 & 5.57 \\
\hline 169.045 & 237.65 & 215.65 & 196.91 & 196.65 & 197.51 & 197.39 & 207.7 & 200.38 & 5.58 \\
\hline 169.5448 & 237.58 & 214.29 & 196.92 & 196.66 & 197.52 & 197.41 & 207.7 & 200.31 & 5.62 \\
\hline 170.0455 & 237.53 & 214.49 & 196.91 & 196.66 & 197.52 & 197.39 & 207.72 & 200.27 & 5.59 \\
\hline 170.5453 & 237.48 & 215.24 & 196.89 & 196.66 & 197.52 & 197.39 & 207.68 & 200.34 & 5.59 \\
\hline 171.0452 & 237.55 & 213.04 & 196.91 & 196.64 & 197.52 & 197.4 & 207.71 & 200.31 & 5.60 \\
\hline 171.5442 & 237.48 & 213.83 & 196.91 & 196.65 & 197.52 & 197.43 & 207.68 & 200.27 & 5.63 \\
\hline 172.0448 & 236.93 & 215.09 & 196.63 & 196.34 & 197.1 & 197.01 & 205.65 & 198.11 & 5.58 \\
\hline 172.5457 & 237.24 & 215.3 & 196.67 & 196.44 & 197.39 & 197.15 & 207.28 & 200.6 & 5.55 \\
\hline 173.0445 & 237.52 & 215.7 & 196.89 & 196.6 & 197.51 & 197.37 & 207.69 & 200.52 & 5.57 \\
\hline 173.5453 & 237.56 & 215.7 & 196.94 & 196.67 & 197.53 & 197.43 & 207.83 & 200.51 & 5.60 \\
\hline 174.045 & 237.49 & 212.68 & 196.91 & 196.66 & 197.5 & 197.39 & 207.59 & 200.34 & 5.58 \\
\hline 174.5448 & 237.44 & 212.66 & 196.89 & 196.61 & 197.47 & 197.36 & 207.55 & 200.35 & 5.59 \\
\hline 175.0457 & 237.53 & 213.85 & 196.88 & 196.63 & 197.49 & 197.38 & 207.58 & 200.34 & 5.61 \\
\hline 175.5455 & 237.55 & 216.05 & 196.88 & 196.63 & 197.5 & 197.38 & 207.62 & 200.3 & 5.64 \\
\hline
\end{tabular}


Table B5. Continued.

\begin{tabular}{|c|c|c|c|c|c|c|c|c|c|}
\hline $\begin{array}{c}\text { Time } \\
\text { min }\end{array}$ & $\begin{array}{l}\mathrm{T} 1 \\
{ }^{\circ} \mathrm{C}\end{array}$ & $\begin{array}{l}\mathrm{T} 2 \\
{ }^{\circ} \mathrm{C}\end{array}$ & $\begin{array}{l}\mathrm{T} 3 \\
{ }^{\circ} \mathrm{C}\end{array}$ & $\begin{array}{l}\mathrm{T} 4 \\
{ }^{\circ} \mathrm{C}\end{array}$ & $\begin{array}{l}\mathrm{T} 5 \\
{ }^{\circ} \mathrm{C}\end{array}$ & $\begin{array}{l}\mathrm{T} 6 \\
{ }^{\circ} \mathrm{C}\end{array}$ & $\begin{array}{l}\text { Pinj } \\
\text { psig }\end{array}$ & $\begin{array}{l}\text { Pout } \\
\text { psig }\end{array}$ & $\begin{array}{c}\mathrm{Vw} \\
\mathrm{cc} / \mathrm{min}\end{array}$ \\
\hline 176.0453 & 237.5 & 214.6 & 196.9 & 196.65 & 197.51 & 197.38 & 207.65 & 200.28 & 5.60 \\
\hline 176.5442 & 237.54 & 212.42 & 196.9 & 196.61 & 197.49 & 197.39 & 207.64 & 200.25 & 5.57 \\
\hline 177.045 & 237.49 & 214.36 & 196.92 & 196.63 & 197.49 & 197.39 & 207.69 & 200.24 & 5.58 \\
\hline 177.5447 & 237.43 & 211.32 & 196.91 & 196.63 & 197.5 & 197.41 & 207.72 & 200.21 & 5.61 \\
\hline 178.0455 & 237.38 & 211.41 & 196.89 & 196.64 & 197.5 & 197.39 & 207.67 & 200.24 & 5.59 \\
\hline 178.5453 & 237.35 & 215.15 & 196.87 & 196.62 & 197.5 & 197.39 & 207.66 & 200.21 & 5.60 \\
\hline 179.0452 & 237.35 & 215.73 & 196.89 & 196.64 & 197.52 & 197.4 & 207.68 & 200.17 & 5.61 \\
\hline 179.545 & 237.32 & 215.92 & 196.89 & 196.63 & 197.47 & 197.4 & 207.64 & 200.11 & 5.62 \\
\hline 180.0457 & 237.28 & 215.39 & 196.9 & 196.63 & 197.49 & 197.4 & 207.65 & 200.11 & 5.57 \\
\hline 180.5455 & 237.21 & 212.92 & 196.9 & 196.63 & 197.51 & 197.4 & 207.65 & 200.01 & 5.55 \\
\hline 181.0443 & 236.91 & 215.05 & 196.69 & 196.38 & 197.21 & 197.12 & 206.28 & 198.67 & 5.54 \\
\hline 181.5442 & 237.22 & 213.39 & 196.67 & 196.4 & 197.37 & 197.24 & 207.09 & 200.51 & 5.59 \\
\hline 182.045 & 237.62 & 213.18 & 196.87 & 196.64 & 197.51 & 197.41 & 207.83 & 200.64 & 5.60 \\
\hline 182.5448 & 237.51 & 215.33 & 196.93 & 196.66 & 197.52 & 197.41 & 207.71 & 200.37 & 5.60 \\
\hline 183.0455 & 237.55 & 211.3 & 196.89 & 196.64 & 197.5 & 197.39 & 207.68 & 200.42 & 5.59 \\
\hline 183.5453 & 237.6 & 210.95 & 196.89 & 196.63 & 197.5 & 197.4 & 207.74 & 200.34 & 5.63 \\
\hline 184.0462 & 237.59 & 212.72 & 196.91 & 196.61 & 197.52 & 197.4 & 207.74 & 200.3 & 5.60 \\
\hline 184.5458 & 237.54 & 211.02 & 196.9 & 196.63 & 197.51 & 197.4 & 207.73 & 200.25 & 5.57 \\
\hline 185.0448 & 237.36 & 210.78 & 196.92 & 196.65 & 197.53 & 197.42 & 207.71 & 200.21 & 5.56 \\
\hline 185.5455 & 237.36 & 210.96 & 196.88 & 196.63 & 197.49 & 197.39 & 207.69 & 200.18 & 5.57 \\
\hline 186.0453 & 237.27 & 211.32 & 196.91 & 196.64 & 197.5 & 197.39 & 207.67 & 200.17 & 5.59 \\
\hline 186.5452 & 237.3 & 213.75 & 196.89 & 196.64 & 197.5 & 197.39 & 207.66 & 200.18 & 5.59 \\
\hline 187.045 & 237.31 & 214.49 & 196.87 & 196.64 & 197.61 & 197.41 & 207.66 & 200.16 & 5.60 \\
\hline 187.5448 & 237.28 & 213.24 & 196.89 & 196.63 & 197.5 & 197.39 & 207.68 & 200.1 & 5.62 \\
\hline 188.0455 & 237.34 & 216.19 & 196.88 & 196.63 & 197.49 & 197.38 & 207.67 & 200.1 & 5.62 \\
\hline 188.5453 & 237.36 & 215.27 & 196.88 & 196.63 & 197.49 & 197.4 & 207.68 & 200.06 & 5.56 \\
\hline 189.0452 & 237.34 & 214.95 & 196.88 & 196.62 & 197.62 & 197.4 & 207.68 & 200.18 & 5.56 \\
\hline 189.545 & 237.29 & 216.7 & 196.89 & 196.6 & 197.5 & 197.39 & 207.55 & 199.73 & 5.57 \\
\hline 190.0467 & 237.01 & 217.21 & 196.71 & 196.44 & 197.28 & 197.18 & 206.73 & 198.92 & 5.60 \\
\hline 190.5447 & 237.26 & 217.17 & 196.77 & 196.53 & 197.45 & 197.34 & 207.49 & 200.41 & 5.59 \\
\hline 191.0455 & 237.5 & 217.84 & 196.91 & 196.66 & 197.52 & 197.45 & 207.82 & 200.44 & 5.59 \\
\hline 191.5453 & 237.39 & 217.14 & 196.91 & 196.64 & 197.61 & 197.43 & 207.77 & 200.44 & 5.59 \\
\hline 192.045 & 237.45 & 215.64 & 196.9 & 196.66 & 197.52 & 197.42 & 207.83 & 200.42 & 5.64 \\
\hline 192.5448 & 237.41 & 215.48 & 196.9 & 196.65 & 197.51 & 197.42 & 207.83 & 200.29 & 5.59 \\
\hline 193.0457 & 237.4 & 217.49 & 196.92 & 196.65 & 197.53 & 197.42 & 207.78 & 200.27 & 5.56 \\
\hline 193.5455 & 237.4 & 214.2 & 196.89 & 196.65 & 197.51 & 197.41 & 207.76 & 200.25 & 5.58 \\
\hline 194.0453 & 237.33 & 214.11 & 196.89 & 196.62 & 197.48 & 197.41 & 207.73 & 200.2 & 5.60 \\
\hline 194.5442 & 237.42 & 212.18 & 196.89 & 196.62 & 197.5 & 197.39 & 207.73 & 200.18 & 5.61 \\
\hline 195.045 & 237.42 & 214.28 & 196.89 & 196.64 & 197.5 & 197.4 & 207.74 & 200.18 & 5.60 \\
\hline 195.5457 & 237.37 & 213.65 & 196.91 & 196.63 & 197.52 & 197.42 & 207.72 & 200.11 & 5.62 \\
\hline 196.0455 & 237.43 & 215.85 & 196.9 & 196.63 & 197.51 & 197.4 & 207.71 & 200.1 & 5.63 \\
\hline 196.5453 & 237.45 & 214.21 & 196.88 & 196.63 & 197.49 & 197.42 & 207.71 & 200.14 & 5.57 \\
\hline 197.0452 & 237.27 & 211.44 & 196.83 & 196.56 & 197.42 & 197.32 & 207.38 & 200.35 & 5.57 \\
\hline 197.545 & 237.42 & 213.36 & 196.89 & 196.64 & 197.53 & 197.44 & 207.88 & 200.46 & 5.60 \\
\hline
\end{tabular}


Table B5. Continued.

\begin{tabular}{|c|c|c|c|c|c|c|c|c|c|}
\hline $\begin{array}{c}\text { Time } \\
\text { min }\end{array}$ & $\begin{array}{l}\mathrm{T} 1 \\
{ }^{\circ} \mathrm{C}\end{array}$ & $\begin{array}{l}\mathrm{T} 2 \\
{ }^{\circ} \mathrm{C}\end{array}$ & $\begin{array}{l}\mathrm{T} 3 \\
{ }^{\circ} \mathrm{C}\end{array}$ & $\begin{array}{l}\mathrm{T} 4 \\
{ }^{\circ} \mathrm{C}\end{array}$ & $\begin{array}{l}\mathrm{T} 5 \\
{ }^{\circ} \mathrm{C}\end{array}$ & $\begin{array}{l}\mathrm{T} 6 \\
{ }^{\circ} \mathrm{C}\end{array}$ & $\begin{array}{l}\text { Pinj } \\
\text { psig }\end{array}$ & $\begin{array}{c}\text { Pout } \\
\text { psig }\end{array}$ & $\begin{array}{c}\mathrm{Vw} \\
\mathrm{cc} / \mathrm{min}\end{array}$ \\
\hline 198.0457 & 237.47 & 215.02 & 196.91 & 196.66 & 197.55 & 197.45 & 207.92 & 200.4 & 5.62 \\
\hline 198.5465 & 237.53 & 215.04 & 196.91 & 196.66 & 197.52 & 197.43 & 207.86 & 200.24 & 5.62 \\
\hline 199.0453 & 237.48 & 214.17 & 196.9 & 196.64 & 197.5 & 197.41 & 207.78 & 200.25 & 5.62 \\
\hline 199.5442 & 237.48 & 216.86 & 196.88 & 196.63 & 197.52 & 197.42 & 207.77 & 200.23 & 5.64 \\
\hline 200.045 & 237.45 & 217.73 & 196.86 & 196.63 & 197.49 & 197.42 & 207.77 & 200.14 & 5.59 \\
\hline 200.5448 & 237.4 & 214.32 & 196.88 & 196.63 & 197.49 & 197.41 & 207.76 & 200.13 & 5.57 \\
\hline 201.0455 & 237.36 & 213.41 & 196.89 & 196.64 & 197.5 & 197.41 & 207.76 & 200.15 & 5.58 \\
\hline 201.5463 & 237.35 & 215.94 & 196.89 & 196.62 & 197.5 & 197.41 & 207.73 & 200.15 & 5.61 \\
\hline 202.0452 & 237.42 & 216.51 & 196.87 & 196.61 & 197.48 & 197.39 & 207.73 & 200.08 & 5.59 \\
\hline 202.545 & 237.44 & 216.28 & 196.88 & 196.61 & 197.49 & 197.38 & 207.71 & 200.12 & 5.60 \\
\hline 203.0448 & 237.48 & 216.98 & 196.9 & 196.61 & 197.52 & 197.4 & 207.71 & 200.06 & 5.61 \\
\hline 203.5455 & 237.07 & 213.05 & 196.68 & 196.38 & 197.22 & 197.11 & 206.35 & 199 & 5.63 \\
\hline 204.0453 & 237.45 & 216.99 & 196.76 & 196.49 & 197.44 & 197.33 & 207.52 & 200.43 & 5.57 \\
\hline 204.5443 & 237.65 & 217.78 & 196.9 & 196.64 & 197.51 & 197.46 & 207.9 & 200.43 & 5.58 \\
\hline 205.0442 & 237.6 & 215.68 & 196.93 & 196.66 & 197.53 & 197.44 & 207.88 & 200.42 & 5.59 \\
\hline 205.5448 & 237.57 & 217.94 & 196.91 & 196.64 & 197.55 & 197.45 & 207.89 & 200.34 & 5.61 \\
\hline 206.0447 & 237.57 & 216.55 & 196.89 & 196.63 & 197.52 & 197.43 & 207.78 & 200.16 & 5.60 \\
\hline 206.5453 & 237.48 & 216.73 & 196.9 & 196.63 & 197.5 & 197.43 & 207.71 & 200.12 & 5.61 \\
\hline 207.0452 & 237.45 & 215.07 & 196.86 & 196.59 & 197.49 & 197.4 & 207.67 & 200.16 & 5.64 \\
\hline 207.545 & 237.39 & 217.34 & 196.86 & 196.61 & 197.49 & 197.4 & 207.69 & 200.04 & 5.62 \\
\hline 208.0448 & 237.38 & 217.93 & 196.88 & 196.6 & 197.47 & 197.42 & 207.69 & 200.09 & 5.57 \\
\hline 208.5457 & 237.4 & 218.28 & 196.87 & 196.6 & 197.39 & 197.37 & 207.67 & 200.04 & 5.56 \\
\hline 209.0455 & 237.29 & 218.35 & 196.85 & 196.58 & 197.35 & 197.37 & 207.7 & 199.99 & 5.60 \\
\hline 209.5462 & 237.23 & 216.67 & 196.86 & 196.59 & 197.47 & 197.38 & 207.66 & 200.06 & 5.60 \\
\hline 210.0442 & 237.12 & 214.17 & 196.8 & 196.5 & 197.34 & 197.27 & 206.92 & 200.19 & 5.59 \\
\hline 210.5458 & 237.11 & 217.64 & 196.83 & 196.56 & 197.45 & 197.34 & 207.53 & 200.03 & 5.61 \\
\hline 211.0457 & 237.25 & 218.4 & 196.85 & 196.61 & 197.49 & 197.4 & 207.82 & 200.47 & 5.63 \\
\hline 211.5445 & 237.34 & 214.8 & 196.9 & 196.65 & 197.53 & 197.44 & 207.94 & 200.33 & 5.61 \\
\hline 212.0453 & 237.29 & 214.54 & 196.9 & 196.62 & 197.48 & 197.39 & 207.73 & 200 & 5.56 \\
\hline 212.5452 & 237.22 & 216.42 & 196.82 & 196.58 & 197.44 & 197.35 & 207.59 & 199.98 & 5.57 \\
\hline 213.045 & 237.19 & 217.73 & 196.82 & 196.59 & 197.45 & 197.38 & 207.62 & 200.03 & 5.61 \\
\hline 213.5457 & 237.17 & 217.1 & 196.82 & 196.59 & 197.45 & 197.36 & 207.62 & 200.02 & 5.60 \\
\hline 214.0455 & 237.18 & 217.41 & 196.83 & 196.57 & 197.45 & 197.36 & 207.57 & 199.98 & 5.59 \\
\hline 214.5462 & 237.16 & 217.77 & 196.83 & 196.58 & 197.44 & 197.35 & 207.6 & 199.96 & 5.60 \\
\hline 215.0452 & 237.18 & 218.33 & 196.81 & 196.58 & 197.46 & 197.39 & 207.58 & 200 & 5.62 \\
\hline 215.544 & 237.22 & 216.9 & 196.81 & 196.56 & 197.44 & 197.35 & 207.57 & 199.95 & 5.60 \\
\hline 216.0457 & 237.26 & 215.61 & 196.82 & 196.58 & 197.44 & 197.34 & 207.56 & 199.9 & 5.56 \\
\hline 216.5447 & 237.3 & 214.2 & 196.82 & 196.55 & 197.46 & 197.34 & 207.52 & 199.91 & 5.56 \\
\hline 217.0453 & 237.37 & 215.81 & 196.82 & 196.57 & 197.45 & 197.36 & 207.55 & 199.96 & 5.59 \\
\hline 217.5452 & 237.32 & 216.48 & 196.83 & 196.57 & 197.45 & 197.36 & 207.55 & 199.91 & 5.60 \\
\hline 218.045 & 237.29 & 217.02 & 196.81 & 196.56 & 197.44 & 197.35 & 207.53 & 199.85 & 5.60 \\
\hline 218.5448 & 236.91 & 216.31 & 196.67 & 196.4 & 197.22 & 197.13 & 206.47 & 198.3 & 5.61 \\
\hline 219.0455 & 237.04 & 216.09 & 196.62 & 196.38 & 197.33 & 197.23 & 207.07 & 200.32 & 5.64 \\
\hline
\end{tabular}


Table B5. Continued.

\begin{tabular}{|c|c|c|c|c|c|c|c|c|c|}
\hline $\begin{array}{c}\text { Time } \\
\text { min }\end{array}$ & $\begin{array}{l}\mathrm{T} 1 \\
{ }^{\circ} \mathrm{C} \\
\end{array}$ & $\begin{array}{l}\mathrm{T} 2 \\
{ }^{\circ} \mathrm{C}\end{array}$ & $\begin{array}{l}\mathrm{T} 3 \\
{ }^{\circ} \mathrm{C} \\
\end{array}$ & $\begin{array}{l}\mathrm{T} 4 \\
{ }^{\circ} \mathrm{C} \\
\end{array}$ & $\begin{array}{l}\mathrm{T} 5 \\
{ }^{\circ} \mathrm{C} \\
\end{array}$ & $\begin{array}{l}\mathrm{T} 6 \\
{ }^{\circ} \mathrm{C} \\
\end{array}$ & $\begin{array}{l}\text { Pinj } \\
\text { psig }\end{array}$ & $\begin{array}{l}\text { Pout } \\
\text { psig }\end{array}$ & $\begin{array}{l}\mathrm{Vw} \\
\mathrm{cc} / \mathrm{min}\end{array}$ \\
\hline 219.5453 & 237.49 & 213.98 & 196.83 & 196.6 & 197.51 & 197.42 & 207.87 & 200.44 & 5.61 \\
\hline 220.0452 & 237.38 & 216.15 & 196.87 & 196.62 & 197.5 & 197.41 & 207.79 & 200.39 & 5.57 \\
\hline 220.544 & 237.42 & 213.2 & 196.87 & 196.62 & 197.5 & 197.41 & 207.85 & 200.29 & 5.57 \\
\hline 221.0438 & 237.37 & 214.2 & 196.88 & 196.62 & 197.63 & 197.43 & 207.81 & 200.21 & 5.61 \\
\hline 221.5447 & 237.32 & 212.13 & 196.86 & 196.61 & 197.49 & 197.4 & 207.7 & 200.07 & 5.60 \\
\hline 222.0453 & 237.3 & 212.87 & 196.85 & 196.59 & 197.47 & 197.37 & 207.63 & 199.96 & 5.60 \\
\hline 222.5452 & 237.29 & 212.89 & 196.85 & 196.58 & 197.48 & 197.37 & 207.63 & 200.01 & 5.62 \\
\hline 223.045 & 237.18 & 215.83 & 196.83 & 196.58 & 197.46 & 197.37 & 207.64 & 200.04 & 5.65 \\
\hline 223.5458 & 237.15 & 215.92 & 196.83 & 196.57 & 197.46 & 197.37 & 207.62 & 199.89 & 5.58 \\
\hline 224.0455 & 237.1 & 212 & 196.84 & 196.59 & 197.46 & 197.38 & 207.63 & 199.92 & 5.56 \\
\hline 224.5453 & 237.07 & 212.52 & 196.82 & 196.59 & 197.45 & 197.36 & 207.64 & 199.94 & 5.57 \\
\hline 225.0452 & 237.09 & 214.83 & 196.84 & 196.56 & 197.45 & 197.38 & 207.59 & 199.89 & 5.59 \\
\hline 225.545 & 236.89 & 212.12 & 196.72 & 196.47 & 197.35 & 197.26 & 207.18 & 200.01 & 5.59 \\
\hline 226.044 & 237 & 212.23 & 196.76 & 196.47 & 197.4 & 197.31 & 207.37 & 199.82 & 5.59 \\
\hline 226.5447 & 237.26 & 214.57 & 196.83 & 196.58 & 197.53 & 197.44 & 207.91 & 201.45 & 5.61 \\
\hline 227.0445 & 237.46 & 213.3 & 197.03 & 196.78 & 197.68 & 197.6 & 208.69 & 201.28 & 5.63 \\
\hline 227.5452 & 237.42 & 211.3 & 197.11 & 196.86 & 197.75 & 197.66 & 208.95 & 201.61 & 5.58 \\
\hline 228.045 & 236.69 & 214.74 & 196.79 & 196.48 & 197.23 & 197.16 & 206.38 & 198.29 & 5.56 \\
\hline 228.5448 & 236.57 & 214.13 & 196.54 & 196.27 & 197.15 & 197.06 & 206.26 & 198.74 & 5.58 \\
\hline 229.0457 & 236.96 & 211.27 & 196.56 & 196.31 & 197.19 & 197.1 & 206.37 & 198.48 & 5.62 \\
\hline 229.5455 & 236.93 & 214.07 & 196.51 & 196.26 & 197.12 & 197.03 & 206.08 & 198.24 & 5.60 \\
\hline 230.0453 & 236.54 & 215.23 & 196.47 & 196.21 & 197.06 & 197.01 & 205.98 & 198.24 & 5.62 \\
\hline 230.5442 & 236.43 & 215.77 & 196.46 & 196.19 & 197.07 & 197 & 205.97 & 198.16 & 5.64 \\
\hline 231.044 & 236.67 & 212.36 & 196.44 & 196.19 & 197.07 & 196.98 & 205.93 & 198.09 & 5.63 \\
\hline 231.5447 & 237.08 & 214.47 & 196.44 & 196.18 & 197.05 & 196.96 & 205.84 & 198.02 & 5.57 \\
\hline 232.0455 & 237.46 & 215.65 & 196.41 & 196.14 & 197 & 196.95 & 205.71 & 197.94 & 5.58 \\
\hline 232.5453 & 237.88 & 211.13 & 196.36 & 196.11 & 196.88 & 196.93 & 205.65 & 197.96 & 5.62 \\
\hline 233.0452 & 238.11 & 212.21 & 196.36 & 196.09 & 196.99 & 196.9 & 205.53 & 197.72 & 5.61 \\
\hline 233.545 & 238.29 & 212.96 & 196.35 & 196.08 & 196.96 & 196.87 & 205.39 & 197.73 & 5.61 \\
\hline 234.0457 & 238.39 & 212.34 & 196.31 & 196.04 & 196.9 & 196.85 & 205.3 & 197.48 & 5.64 \\
\hline 234.5455 & 238.3 & 211.28 & 196.28 & 196.01 & 196.89 & 196.82 & 205.19 & 197.32 & 5.64 \\
\hline 235.0443 & 238.23 & 209.35 & 196.27 & 196 & 197.02 & 196.82 & 205.09 & 197.29 & 5.58 \\
\hline
\end{tabular}


Table B6-Temperature and production data for run 6

\begin{tabular}{|c|c|c|c|c|c|c|c|c|c|}
\hline $\begin{array}{l}\text { Time } \\
\text { min }\end{array}$ & $\begin{array}{l}\mathrm{T} 1 \\
{ }^{\circ} \mathrm{C}\end{array}$ & $\begin{array}{l}\mathrm{T} 2 \\
{ }^{\circ} \mathrm{C}\end{array}$ & $\begin{array}{l}\mathrm{T} 3 \\
{ }^{\circ} \mathrm{C}\end{array}$ & $\begin{array}{l}\mathrm{T} 4 \\
{ }^{\circ} \mathrm{C}\end{array}$ & $\begin{array}{l}\text { T5 } \\
{ }^{\circ} \mathrm{C}\end{array}$ & $\begin{array}{l}\mathrm{T} 6 \\
{ }^{\circ} \mathrm{C}\end{array}$ & $\begin{array}{l}\text { Pinj } \\
\text { psig }\end{array}$ & $\begin{array}{l}\text { Pout } \\
\text { psig }\end{array}$ & $\begin{array}{c}\mathrm{Vw} \\
\mathrm{cc} / \mathrm{min}\end{array}$ \\
\hline 0.5465 & 48.42 & 42.88 & 55.58 & 58.24 & 56.72 & 50.27 & 207.83 & 202.86 & 5.57 \\
\hline 1.046167 & 248.98 & 187.26 & 55.54 & 58.28 & 56.76 & 50.33 & 206.3 & 202.458 & 5.55 \\
\hline 1.546 & 249.95 & 192.86 & 55.56 & 58.24 & 56.78 & 50.33 & 205.1 & 201.282 & 5.55 \\
\hline 2.046833 & 250.03 & 194.31 & 55.53 & 58.2 & 56.71 & 50.29 & 204.3 & 200.508 & 5.57 \\
\hline 2.545667 & 249.33 & 195.17 & 55.53 & 58.21 & 56.71 & 50.28 & 204.28 & 200.511 & 5.59 \\
\hline 3.0465 & 248.42 & 195.79 & 55.57 & 58.19 & 56.73 & 50.3 & 204.15 & 200.373 & 5.61 \\
\hline 3.546333 & 246.97 & 195.88 & 55.57 & 58.21 & 56.73 & 50.32 & 204.24 & 200.457 & 5.60 \\
\hline 4.046167 & 244.99 & 196.2 & 55.57 & 58.23 & 56.75 & 50.34 & 204.2 & 200.406 & 5.61 \\
\hline 4.545833 & 242.61 & 196.29 & 55.61 & 58.25 & 56.79 & 50.34 & 204.11 & 200.388 & 5.62 \\
\hline 5.045667 & 240.12 & 196.54 & 55.62 & 58.29 & 56.81 & 50.35 & 204.12 & 200.364 & 5.62 \\
\hline 5.547333 & 237.35 & 196.28 & 55.66 & 58.3 & 56.82 & 50.35 & 204.17 & 200.445 & 5.57 \\
\hline 6.046333 & 234.66 & 196.26 & 55.66 & 58.32 & 56.78 & 50.37 & 204.35 & 334 & 5.55 \\
\hline 6.547 & 233.18 & 196.12 & 55.87 & 58.3 & 56.8 & 50.37 & 204.56 & .52 & 5.56 \\
\hline 7.046 & 233.39 & 196.63 & 56.56 & 58.32 & 56.86 & 50.39 & 204.97 & 200.553 & 5.58 \\
\hline 7.546667 & 236.53 & 196.84 & 57.15 & 58.29 & 56.84 & 50.38 & 204.89 & 200.502 & 5.58 \\
\hline 8.0465 & 241.57 & 197.37 & 57.85 & 58.27 & 56.81 & 50.34 & 204.7 & 403 & 5.58 \\
\hline 8.546334 & 244.73 & 197.83 & 58.46 & 58.23 & 56.83 & 50.34 & 204.87 & 277 & 5.58 \\
\hline 9.046167 & 245.56 & 197.96 & 58.54 & 58.26 & 56.83 & 50.36 & 92 & 403 & 5.58 \\
\hline 9.546 & 244.77 & 197.28 & 58.83 & 58.26 & 56.85 & 50.33 & 205.02 & 253 & 5.62 \\
\hline 10.04667 & 242.8 & 197.25 & 59.76 & 58.3 & 56.85 & 50.39 & 205.4 & .376 & 5.64 \\
\hline 10.5465 & 240.29 & 197.46 & 60.82 & 58.3 & 56.9 & 50.39 & 205.16 & 196 & 5.58 \\
\hline 11.04633 & 237.92 & 197.25 & 61.75 & 58.28 & 56.88 & 50.3 & 3 & 81 & 5.56 \\
\hline 11.54717 & 23 & 197.32 & 62.7 & 58.28 & 56.88 & 50.37 & 57 & 75 & 5.57 \\
\hline 12.046 & 236.87 & 197.33 & 63.89 & 58.29 & 56.92 & 50.4 & 205.93 & 337 & 5.59 \\
\hline 12.54583 & 236.71 & 197.02 & 65.03 & 58.29 & 56.92 & 50.42 & 205.94 & 200.193 & 5.60 \\
\hline 13.0475 & 236.03 & 197.46 & 65.86 & 58.31 & 56.94 & 50.34 & 205 & 076 & 5.59 \\
\hline 13.54633 & 235.89 & 196.94 & 66.36 & 58.31 & 56.95 & 50.42 & 205.96 & 043 & 5.58 \\
\hline 14.04617 & 235.81 & 197.12 & 67.02 & 58.32 & 56.93 & 50.41 & 206.38 & 109 & 5.61 \\
\hline 14.547 & 235.74 & 197.41 & 67.85 & 58.32 & 56.97 & 50.45 & 206.71 & 84 & 5.65 \\
\hline 15.04583 & 235.45 & 197.81 & 68.57 & 58.3 & 56.97 & 50.45 & 206.91 & 175 & 5.60 \\
\hline 15.5465 & 234.99 & 198.02 & 69.12 & 58.27 & 56.98 & 50.43 & 207.39 & 200.331 & 5.55 \\
\hline 16.04733 & 234.51 & 197.7 & 70.16 & 58.29 & 56.92 & 50.45 & 208.53 & 200.523 & 5.55 \\
\hline 16.54617 & 233.94 & 198.19 & 71.86 & 58.27 & 56.98 & 50.46 & 20 & 308 & 5.57 \\
\hline 17.04783 & 233.4 & 198.59 & 73.12 & 58.25 & 56.94 & 50.44 & $20 s$ & 308 & 5.61 \\
\hline 17.54683 & 232.9 & 199.25 & 73.27 & 58.26 & 56.98 & 50.46 & 211.09 & 200.79 & 5.60 \\
\hline 18.04667 & 232.53 & 199.83 & 73.86 & 58.24 & 57.02 & 50.48 & 212.01 & 200.733 & 5.60 \\
\hline 18.5465 & 232.32 & 199.77 & 75.29 & 58.24 & 57.05 & 50.52 & 212.56 & 200.775 & 5.60 \\
\hline 19.04717 & 232.26 & 200.29 & 77.65 & 58.22 & 57.05 & 50.5 & 212.99 & 200.805 & 5.63 \\
\hline 19.546 & 232.25 & 200.17 & 80.47 & 58.23 & 57.07 & 50.51 & 212.69 & 200.658 & 5.63 \\
\hline 20.04583 & 232.23 & 199.71 & 82.77 & 58.21 & 57.07 & 50.53 & 212.12 & 200.409 & 5.58 \\
\hline 20.54567 & 232.45 & 199.19 & 84.21 & 58.13 & 56.99 & 50.45 & 211.61 & 200.184 & 5.57 \\
\hline 21.0465 & 232.68 & 199.62 & 85.15 & 58.14 & 57.01 & 50.45 & 211.06 & 199.017 & 5.58 \\
\hline 21.54633 & 232.97 & 198.94 & 85.58 & 58.14 & 57.09 & 50.45 & 210.79 & 201.318 & 5.62 \\
\hline 22.047 & 233.29 & 198.85 & 85.17 & 58.08 & 57.15 & 50.44 & 211.23 & 201.351 & 5.62 \\
\hline
\end{tabular}


Table B6. Continued.

\begin{tabular}{|c|c|c|c|c|c|c|c|c|c|}
\hline $\begin{array}{c}\text { Time } \\
\text { min }\end{array}$ & $\begin{array}{l}\mathrm{T} 1 \\
{ }^{\circ} \mathrm{C} \\
\end{array}$ & $\begin{array}{l}\mathrm{T} 2 \\
{ }^{\circ} \mathrm{C} \\
\end{array}$ & $\begin{array}{l}\mathrm{T} 3 \\
{ }^{\circ} \mathrm{C} \\
\end{array}$ & $\begin{array}{l}\mathrm{T} 4 \\
{ }^{\circ} \mathrm{C} \\
\end{array}$ & $\begin{array}{l}\mathrm{T5} \\
{ }^{\circ} \mathrm{C} \\
\end{array}$ & $\begin{array}{l}\mathrm{T} 6 \\
{ }^{\circ} \mathrm{C} \\
\end{array}$ & $\begin{array}{l}\text { Pinj } \\
\text { psig }\end{array}$ & $\begin{array}{l}\text { Pout } \\
\text { psig }\end{array}$ & $\begin{array}{c}\mathrm{Vw} \\
\mathrm{cc} / \mathrm{min}\end{array}$ \\
\hline 22.54583 & 233.58 & 198.75 & 84.91 & 58.09 & 57.19 & 50.46 & 211.81 & 201.366 & 5.62 \\
\hline 23.04567 & 233.66 & 199 & 84.89 & 58.07 & 57.23 & 50.44 & 212.05 & 201.447 & 5.63 \\
\hline 23.5465 & 233.21 & 199.15 & 85.1 & 58.05 & 57.31 & 50.48 & 212.86 & 201.495 & 5.63 \\
\hline 24.04633 & 232.78 & 198.99 & 85.4 & 58.07 & 57.35 & 50.46 & 213.08 & 201.585 & 5.59 \\
\hline 24.547 & 232.46 & 199.56 & 85.76 & 58.05 & 57.37 & 50.48 & 213.47 & 201.63 & 5.57 \\
\hline 25.046 & 232.3 & 199.13 & 86.13 & 58.03 & 57.39 & 50.45 & 213.57 & 201.423 & 5.56 \\
\hline 25.54583 & 232.28 & 199.17 & 86.54 & 58.03 & 57.41 & 50.43 & 214.13 & 201.747 & 5.59 \\
\hline 26.0465 & 233.66 & 199.67 & 87.05 & 58.03 & 57.45 & 50.43 & 214.83 & 201.726 & 5.61 \\
\hline 26.54633 & 235.22 & 199.67 & 87.8 & 58.02 & 57.48 & 50.43 & 214.79 & 201.459 & 5.59 \\
\hline 27.04617 & 235.62 & 199.97 & 88.62 & 58 & 57.52 & 50.45 & 214.51 & 201.441 & 5.59 \\
\hline 27.54683 & 235.2 & 199.9 & 89.47 & 58.02 & 57.58 & 50.54 & 214.72 & 201.378 & 5.61 \\
\hline 28.04667 & 234.56 & 199.94 & 90.55 & 58.04 & 57.6 & 50.64 & 215.1 & 200.421 & 5.64 \\
\hline 28.5475 & 234.51 & 200.03 & 92.14 & 58.06 & 57.64 & 50.74 & 215.63 & 200.568 & 5.62 \\
\hline 29.04633 & 234.4 & 200.07 & 94.26 & 58.06 & 57.68 & 50.83 & 216.12 & 200.517 & 5.58 \\
\hline 29.54617 & 234.13 & 200.41 & 96.93 & 58.06 & 57.7 & 50.89 & 216.79 & 682 & 5.57 \\
\hline 30.046 & 233.81 & 200.53 & 100.25 & 58.06 & 57.74 & 50.97 & 217.76 & 201.138 & 5.59 \\
\hline 30.54583 & 233.49 & 201.16 & 104.47 & 58.08 & 57.72 & 51.04 & 218.86 & 201.225 & 5.62 \\
\hline 31.0475 & 233.25 & 201.12 & 109.35 & 58.12 & 57.79 & 51.14 & 220.02 & 201.825 & 5.60 \\
\hline 31.54633 & 233.43 & 202.06 & 113.93 & 58.15 & 57.81 & 51.31 & 220.9 & 200.925 & 5.61 \\
\hline 32.04617 & 233.49 & 202.18 & 118.79 & 58.19 & 57.87 & 51.37 & 221.15 & 201.441 & 5.63 \\
\hline 32.546 & 233.47 & 201.93 & 123.77 & 58.25 & 57.91 & 51.48 & 221.46 & 204.198 & 5.65 \\
\hline 33.04583 & 233.56 & 202.63 & 128.25 & 58.29 & 57.96 & 51.58 & 222.57 & 207.855 & 5.61 \\
\hline 33.5465 & 233.81 & 203.2 & 131.79 & 58.32 & 57.96 & 51.59 & 224.17 & 210.66 & 5.57 \\
\hline 34.04733 & 234.24 & 202.88 & 134.42 & 58.38 & 57.96 & 51.54 & 227.15 & 221.343 & 5.57 \\
\hline 34.54617 & 234.56 & 202.8 & 136.04 & 58.4 & 57.94 & 51.54 & 230.18 & 221.4 & 5.60 \\
\hline 35.046 & 234.41 & 203.64 & 138.38 & 58.47 & 57.96 & 51.53 & 232.55 & 222.567 & 5.62 \\
\hline 35.54583 & 234.09 & 203.87 & 141.12 & 58.53 & 57.96 & 51.57 & 234.32 & 224.298 & 5.59 \\
\hline 36.04667 & 234.07 & 203.48 & 143.72 & 58.58 & 57.99 & 51.57 & 236.15 & 226.041 & 5.61 \\
\hline 36.5465 & 231.69 & 203.78 & 146.13 & 58.6 & 57.94 & 51.53 & 239.19 & 227.898 & 5.62 \\
\hline 37.04617 & 226.83 & 204.73 & 148.8 & 58.66 & 57.96 & 51.57 & 240.56 & 229.911 & 5.63 \\
\hline 37.546 & 229.7 & 204.41 & 151.13 & 58.73 & 57.97 & 51.63 & 241.92 & 231.864 & 5.57 \\
\hline 38.04583 & 231.6 & 205.07 & 153.16 & 58.79 & 57.99 & 51.64 & 243.13 & 211.281 & 5.56 \\
\hline 38.54567 & 231.61 & 205.41 & 159.44 & 58.92 & 58.07 & 52 & 238.35 & 202.824 & 5.55 \\
\hline 39.0465 & 231.02 & 205.1 & 169.75 & 59.09 & 58.2 & 52.36 & 232.41 & 169.362 & 5.61 \\
\hline 39.54717 & 230.11 & 203.8 & 181.43 & 59.35 & 58.33 & 52.73 & 223.45 & 185.217 & 5.60 \\
\hline 40.04617 & 230.48 & 202.85 & 182.27 & 59.6 & 58.39 & 52.9 & 220.24 & 192.462 & 5.60 \\
\hline 40.54683 & 231.4 & 202.4 & 181.86 & 60.2 & 58.42 & 52.99 & 219.28 & 197.265 & 5.61 \\
\hline 41.04567 & 232.57 & 201.88 & 182.07 & 61.19 & 58.44 & 53.06 & 219.39 & 200.643 & 5.59 \\
\hline 41.5465 & 233.31 & 201.91 & 182.82 & 62.23 & 58.44 & 53.08 & 219.98 & 203.568 & 5.62 \\
\hline 42.04633 & 233.5 & 201.82 & 185.06 & 63.42 & 58.49 & 53.27 & 216.67 & 174.096 & 5.58 \\
\hline 42.54617 & 233.86 & 201.28 & 188.79 & 64.85 & 58.47 & 53.34 & 211.47 & 182.598 & 5.56 \\
\hline 43.046 & 234.18 & 200.78 & 189.51 & 66.23 & 58.53 & 53.43 & 209.11 & 186.768 & 5.56 \\
\hline 43.54583 & 234.62 & 199.54 & 189.92 & 67.96 & 58.52 & 53.47 & 208.06 & 189.852 & 5.57 \\
\hline 44.0465 & 235.18 & 199.22 & 190.31 & 70.49 & 58.54 & 53.49 & 207.66 & 203.019 & 5.61 \\
\hline
\end{tabular}


Table B6. Continued.

\begin{tabular}{|c|c|c|c|c|c|c|c|c|c|}
\hline $\begin{array}{c}\text { Time } \\
\text { min }\end{array}$ & $\begin{array}{l}\mathrm{T} 1 \\
{ }^{\circ} \mathrm{C} \\
\end{array}$ & $\begin{array}{l}\mathrm{T} 2 \\
{ }^{\circ} \mathrm{C} \\
\end{array}$ & $\begin{array}{l}\mathrm{T} 3 \\
{ }^{\circ} \mathrm{C} \\
\end{array}$ & $\begin{array}{l}\mathrm{T} 4 \\
{ }^{\circ} \mathrm{C} \\
\end{array}$ & $\begin{array}{l}\mathrm{T} 5 \\
{ }^{\circ} \mathrm{C} \\
\end{array}$ & $\begin{array}{l}\mathrm{T} 6 \\
{ }^{\circ} \mathrm{C} \\
\end{array}$ & $\begin{array}{l}\text { Pinj } \\
\text { psig }\end{array}$ & $\begin{array}{l}\text { Pout } \\
\text { psig }\end{array}$ & $\begin{array}{c}\mathrm{Vw} \\
\mathrm{cc} / \mathrm{min}\end{array}$ \\
\hline 44.54633 & 235.8 & 199.18 & 190.83 & 73.59 & 58.54 & 53.41 & 208.4 & 201.126 & 5.59 \\
\hline 45.047 & 235.87 & 199.16 & 191.42 & 76.78 & 58.54 & 53.43 & 209.71 & 201.303 & 5.60 \\
\hline 45.54683 & 235.98 & 199.16 & 191.95 & 79.73 & 58.53 & 53.42 & 211.05 & 201.768 & 5.60 \\
\hline 46.04583 & 235.67 & 199.1 & 192.56 & 82.4 & 58.53 & 53.4 & 212.64 & 202.446 & 5.63 \\
\hline 46.5465 & 235.19 & 199.9 & 193.13 & 84.74 & 58.53 & 53.42 & 214.15 & 203.412 & 5.61 \\
\hline 47.04633 & 234.83 & 199.62 & 193.71 & 86.75 & 58.53 & 53.42 & 215.55 & 202.614 & 5.57 \\
\hline 47.54717 & 234.68 & 200.11 & 194.19 & 88.45 & 58.53 & 53.46 & 216.59 & 203.688 & 5.55 \\
\hline 48.04683 & 234.57 & 200.35 & 194.72 & 89.99 & 58.52 & 53.47 & 217.21 & 202.95 & 5.57 \\
\hline 48.54667 & 234.6 & 200.36 & 195.24 & 91.38 & 58.54 & 53.49 & 217.7 & 204.111 & 5.59 \\
\hline 49.0465 & 234.58 & 200.41 & 195.56 & 92.68 & 58.54 & 53.53 & 218.16 & 203.253 & 5.60 \\
\hline 49.54633 & 234.55 & 200.66 & 195.99 & 93.98 & 58.54 & 53.56 & 218.78 & 203.607 & 5.59 \\
\hline 50.04717 & 234.42 & 201.1 & 196.41 & 95.23 & 58.57 & 53.59 & 219.6 & 202.026 & 5.61 \\
\hline 50.546 & 233.91 & 200.82 & 196.86 & 96.54 & 58.57 & 53.63 & 220.55 & 201.969 & 5.61 \\
\hline 51.04683 & 234.68 & 201.17 & 197.55 & 97.86 & 58.59 & 53.68 & 222.25 & 201.567 & 5.61 \\
\hline 51.5465 & 237.58 & 201.8 & 198.18 & 99.26 & 58.56 & 53.7 & 223.58 & .474 & 62 \\
\hline 52.04633 & 239.71 & 202.24 & 198.77 & 100.78 & 58.6 & 53.77 & 25.1 & 201.48 & .58 \\
\hline 52.54617 & 239.33 & 203.03 & 199.36 & 102.41 & 58.62 & 53.79 & 226.71 & 201.501 & 5.58 \\
\hline 53.046 & 238 & 203.62 & 199.93 & 104.13 & 58.65 & 53.86 & 228.12 & 202.539 & 5.61 \\
\hline 53.54683 & 236.05 & 204.19 & 200.46 & 105.92 & 58.69 & 53.92 & 230.13 & 202.257 & 5.61 \\
\hline 54.04667 & 235.26 & 205.02 & 200.91 & 107.71 & 58.72 & 53.97 & 231.63 & 201.483 & 5.60 \\
\hline 54.5465 & 235.81 & 204.93 & 201.26 & 109.56 & 58.79 & 54.05 & 232.42 & 201.477 & 61 \\
\hline 55.04717 & 236.63 & 204.84 & 201.44 & 111.46 & 58.85 & 54.1 & 232.47 & 201.432 & 5.63 \\
\hline 55.547 & 236.92 & 204.53 & 201.51 & 113.39 & 58.92 & 54.17 & 232.3 & 201.417 & 5.63 \\
\hline 56.04583 & 236.98 & 204.87 & 201.5 & 115.34 & 59.01 & 54.23 & 232.04 & 201.366 & 5.58 \\
\hline 56.54567 & 237.11 & 203.67 & 201.45 & 117.26 & 59.1 & 54.3 & 231.36 & 202.428 & 5.56 \\
\hline 57.0465 & 237.43 & 204.06 & 201.39 & 119.11 & 59.23 & 54.33 & 230.82 & 218 & 5.57 \\
\hline 57.54633 & 237.15 & 203.86 & 201.3 & 120.93 & 59.34 & 54.39 & 230.36 & 202.062 & 5.60 \\
\hline 58.04617 & 237.12 & 203.46 & 201.2 & 122.64 & 59.47 & 54.4 & 229.83 & 201.867 & 5.60 \\
\hline 58.54583 & 236.99 & 203.39 & 201.13 & 124.29 & 59.68 & 54.46 & 229.27 & 201.63 & 5.60 \\
\hline 59.04567 & 237.11 & 203.13 & 201.04 & 125.86 & 59.88 & 54.49 & 228.8 & 201.453 & 5.60 \\
\hline 59.54734 & 237.03 & 203.18 & 201 & 127.37 & 60.13 & 54.55 & 228.55 & 201.33 & 5.62 \\
\hline 60.04633 & 236.91 & 202.77 & 200.96 & 128.85 & 60.41 & 54.56 & 228.29 & 201.252 & 5.63 \\
\hline 60.54617 & 236.9 & 203.11 & 200.92 & 130.27 & 60.71 & 54.6 & 228.05 & 202.221 & 5.59 \\
\hline 61.04683 & 237.01 & 203 & 200.92 & 131.66 & 61.01 & 54.63 & 228.28 & 202.131 & 5.55 \\
\hline 61.54583 & 236.75 & 203.28 & 200.97 & 133.06 & 61.33 & 54.65 & 228.4 & 202.026 & 5.56 \\
\hline 62.0465 & 236.73 & 203.38 & 201.02 & 134.44 & 61.68 & 54.66 & 228.69 & 201.906 & 5.61 \\
\hline 62.54633 & 236.69 & 203.25 & 201.11 & 135.88 & 62.06 & 54.72 & 229.16 & 201.75 & 5.61 \\
\hline 63.047 & 236.46 & 203.41 & 201.21 & 137.37 & 62.45 & 54.79 & 229.74 & 201.711 & 5.58 \\
\hline 63.546 & 236.3 & 203.38 & 201.35 & 138.96 & 62.87 & 54.77 & 230.44 & 201.615 & 5.60 \\
\hline 64.04767 & 236.56 & 203.51 & 201.49 & 140.74 & 63.32 & 54.78 & 231.29 & 201.54 & 5.61 \\
\hline 64.5465 & 236.59 & 204.12 & 201.7 & 142.85 & 63.79 & 54.82 & 232.26 & 201.576 & 5.62 \\
\hline 65.04633 & 236.34 & 204.67 & 201.93 & 145.4 & 64.31 & 54.83 & 234.03 & 201.474 & 5.59 \\
\hline 65.54617 & 234.24 & 204.58 & 202.23 & 148.84 & 64.88 & 54.87 & 235.06 & 202.242 & 5.56 \\
\hline 66.046 & 234.38 & 204.65 & 202.52 & 153.32 & 65.44 & 54.9 & 236.5 & 202.227 & 5.57 \\
\hline
\end{tabular}


Table B6. Continued.

\begin{tabular}{|c|c|c|c|c|c|c|c|c|c|}
\hline $\begin{array}{l}\text { Time } \\
\text { min }\end{array}$ & $\begin{array}{l}\mathrm{T} 1 \\
{ }^{\circ} \mathrm{C}\end{array}$ & $\begin{array}{l}\mathrm{T} 2 \\
{ }^{\circ} \mathrm{C}\end{array}$ & $\begin{array}{l}\mathrm{T} 3 \\
{ }^{\circ} \mathrm{C}\end{array}$ & $\begin{array}{l}\mathrm{T} 4 \\
{ }^{\circ} \mathrm{C}\end{array}$ & $\begin{array}{l}\mathrm{T} 5 \\
{ }^{\circ} \mathrm{C}\end{array}$ & $\begin{array}{l}\mathrm{T} 6 \\
{ }^{\circ} \mathrm{C}\end{array}$ & $\begin{array}{l}\text { Pinj } \\
\text { psig }\end{array}$ & $\begin{array}{l}\text { Pout } \\
\text { psig }\end{array}$ & $\begin{array}{c}\mathrm{Vw} \\
\mathrm{cc} / \mathrm{min}\end{array}$ \\
\hline 66.54583 & 234.43 & 205.11 & 202.8 & 158.69 & 66.08 & 54.96 & 237.94 & 202.125 & 5.58 \\
\hline 67.0465 & 234.66 & 205.97 & 203.07 & 164.49 & 66.7 & 54.91 & 239.25 & 202.215 & 5.61 \\
\hline 67.54733 & 235.71 & 205.85 & 203.29 & 170.63 & 67.34 & 55.01 & 240.26 & 202.227 & 5.63 \\
\hline 68.04716 & 236.75 & 206.08 & 203.47 & 176.24 & 68.01 & 55.06 & 240.92 & 202.11 & 5.60 \\
\hline 68.546 & 237.1 & 205.79 & 203.59 & 181.15 & 68.71 & 55.11 & 241.37 & 202.071 & 5.64 \\
\hline 69.04867 & 237.35 & 206.06 & 203.7 & 185.33 & 69.44 & 55.13 & 241.72 & 201.888 & 5.65 \\
\hline 69.5465 & 237.48 & 205.84 & 203.73 & 188.97 & 70.17 & 55.17 & 241.68 & 202.422 & 5.59 \\
\hline 70.04633 & 237.6 & 206.73 & 203.76 & 191.41 & 70.94 & 55.22 & 241.8 & 200.988 & 5.56 \\
\hline 70.54617 & 237.72 & 206.77 & 203.76 & 193.5 & 71.73 & 55.2 & 241.67 & 200.877 & 5.58 \\
\hline 71.046 & 237.57 & 206.12 & 203.7 & 195.09 & 72.5 & 55.29 & 241.32 & 200.802 & 5.61 \\
\hline 71.54583 & 237.77 & 206.24 & 203.67 & 196.7 & 73.36 & 55.29 & 241.07 & 200.79 & 5.60 \\
\hline 72.04666 & 237.96 & 205.88 & 203.61 & 197.9 & 74.2 & 55.34 & 240.8 & 38 & 5.61 \\
\hline 72.5465 & 238.08 & 205.25 & 203.59 & 198.61 & 75.06 & 55.37 & 240.67 & 834 & 5.62 \\
\hline 73.04617 & 238.21 & 205.57 & 203.57 & 199.16 & 75.96 & 55.39 & 240.63 & 201.75 & 5.63 \\
\hline 73.546 & 238.33 & 205.51 & 203.58 & 199.84 & 76.84 & 55.41 & 240.62 & 202.074 & 5.62 \\
\hline 74.04766 & 238.27 & 205.35 & 203.58 & 200.28 & 77.81 & 55.44 & 240.68 & 942 & 5.57 \\
\hline 74.54567 & 238.12 & 205.22 & 203.63 & 200.64 & 78.71 & 55.46 & 96 & 792 & 5.55 \\
\hline 75.0465 & 237.89 & 205.77 & 203.68 & 201.03 & 79.7 & 55.45 & 241.75 & 201.786 & 5.58 \\
\hline 75.54633 & 237.08 & 205.75 & 203.84 & 201.37 & 80.71 & 55.47 & 242.25 & 201.69 & 5.61 \\
\hline 76.04617 & 236.92 & 206.27 & 203.98 & 201.69 & 81.75 & 55.52 & 243.07 & 201.609 & 5.60 \\
\hline 76.54583 & 236.5 & 205.85 & 204.15 & 201.99 & 82.83 & 55.54 & 244.17 & 201.528 & 5.61 \\
\hline 77.04567 & 236.23 & 206.16 & 204.38 & 202.34 & 83.99 & 55.56 & 245.56 & 201.495 & 5.62 \\
\hline 77.5465 & 235.9 & 206.51 & 204.67 & 202.68 & 85.19 & 55.57 & 24 & 46 & 5.65 \\
\hline 78.04633 & 235.7 & 207.12 & 204.97 & 203.02 & 86.45 & 55.61 & 248.65 & 201.585 & 5.60 \\
\hline 78.547 & 235.83 & 207.71 & 205.25 & 203.36 & 87.83 & 55.64 & 250.06 & 202.113 & 5.57 \\
\hline 79.04867 & 235.84 & 207.6 & 205.5 & 203.67 & 89.28 & 55.68 & 251.36 & 202.056 & 5.58 \\
\hline 79.54583 & 236.16 & 208.29 & 205.75 & 203.98 & 90.77 & 55.69 & 252.52 & 202.065 & 5.61 \\
\hline 80.0465 & 236.73 & 207.98 & 205.94 & 204.19 & 92.31 & 55.75 & 253.43 & 889 & 5.61 \\
\hline 80.54633 & 236.12 & 208.87 & 206.1 & 204.36 & 93.91 & 55.8 & 253.98 & 200.916 & 5.60 \\
\hline 81.04617 & 236.26 & 208.5 & 206.19 & 204.49 & 95.56 & 55.87 & 254.35 & 200.892 & 5.60 \\
\hline 81.546 & 237.26 & 208.46 & 206.24 & 204.57 & 97.19 & 55.91 & 254.59 & 200.856 & 5.63 \\
\hline 82.04583 & 236.63 & 208.5 & 206.27 & 204.66 & 98.85 & 55.96 & 254.65 & 200.862 & 5.62 \\
\hline 82.5475 & 236.99 & 207.89 & 206.27 & 204.67 & 100.57 & 56.03 & 254.58 & 200.862 & 5.57 \\
\hline 83.04633 & 236.48 & 207.98 & 206.26 & 204.69 & 102.16 & 56.09 & 254.37 & 200.769 & 5.57 \\
\hline 83.54617 & 237.41 & 208.26 & 206.22 & 204.67 & 103.86 & 56.14 & 254.13 & 201.732 & 5.58 \\
\hline 84.046 & 237.82 & 207.74 & 206.17 & 204.67 & 105.52 & 56.21 & 253.84 & 201.927 & 5.61 \\
\hline 84.54583 & 237.73 & 207.69 & 206.15 & 204.66 & 107.11 & 56.29 & 253.7 & 201.906 & 5.60 \\
\hline 85.0465 & 235.93 & 208.01 & 206.16 & 204.66 & 108.69 & 56.34 & 253.71 & 201.939 & 5.61 \\
\hline 85.54633 & 234.94 & 208.58 & 206.2 & 204.71 & 110.28 & 56.43 & 254.07 & 201.78 & 5.62 \\
\hline 86.04617 & 235.35 & 207.84 & 206.21 & 204.74 & 111.88 & 56.49 & 254.13 & 201.6 & 5.64 \\
\hline 86.546 & 234.31 & 208.57 & 206.26 & 204.81 & 113.48 & 56.64 & 254.55 & 201.597 & 5.62 \\
\hline 87.04583 & 234.96 & 208.53 & 206.35 & 204.88 & 115.09 & 56.63 & 255.22 & 201.531 & 5.57 \\
\hline 87.5465 & 235.06 & 209.03 & 206.49 & 204.99 & 116.72 & 56.75 & 256.02 & 201.495 & 5.56 \\
\hline 88.04733 & 234.81 & 208.53 & 206.67 & 205.18 & 118.36 & 56.84 & 257.14 & 201.498 & 5.59 \\
\hline
\end{tabular}


Table B6. Continued.

\begin{tabular}{|c|c|c|c|c|c|c|c|c|c|}
\hline $\begin{array}{c}\text { Time } \\
\text { min }\end{array}$ & $\begin{array}{l}\mathrm{T} 1 \\
{ }^{\circ} \mathrm{C} \\
\end{array}$ & $\begin{array}{l}\mathrm{T} 2 \\
{ }^{\circ} \mathrm{C}\end{array}$ & $\begin{array}{l}\mathrm{T} 3 \\
{ }^{\circ} \mathrm{C} \\
\end{array}$ & $\begin{array}{l}\mathrm{T} 4 \\
{ }^{\circ} \mathrm{C} \\
\end{array}$ & $\begin{array}{l}\mathrm{T5} \\
{ }^{\circ} \mathrm{C} \\
\end{array}$ & $\begin{array}{l}\mathrm{T} 6 \\
{ }^{\circ} \mathrm{C} \\
\end{array}$ & $\begin{array}{l}\text { Pinj } \\
\text { psig }\end{array}$ & $\begin{array}{l}\text { Pout } \\
\text { psig }\end{array}$ & $\begin{array}{c}\mathrm{Vw} \\
\mathrm{cc} / \mathrm{min}\end{array}$ \\
\hline 88.54617 & 235.2 & 208.67 & 206.9 & 205.34 & 120.1 & 56.93 & 258.51 & 201.945 & 5.62 \\
\hline 89.04784 & 232.69 & 209.65 & 207.14 & 205.61 & 121.91 & 57.04 & 259.9 & 202.026 & 5.60 \\
\hline 89.54583 & 234.85 & 210.11 & 207.39 & 205.84 & 123.87 & 57.15 & 261.33 & 201.903 & 5.61 \\
\hline 90.04666 & 235.71 & 210.4 & 207.66 & 206.1 & 125.95 & 57.23 & 262.62 & 201.96 & 5.61 \\
\hline 90.5465 & 233.93 & 210.29 & 207.87 & 206.33 & 128.21 & 57.45 & 263.59 & 201.96 & 5.65 \\
\hline 91.04617 & 234.7 & 210.55 & 208.01 & 206.49 & 130.77 & 57.62 & 264.19 & 201.978 & 5.62 \\
\hline 91.546 & 235.52 & 210.34 & 208.1 & 206.59 & 133.66 & 57.84 & 264.54 & 201.984 & 5.56 \\
\hline 92.04583 & 236.38 & 210.64 & 208.13 & 206.63 & 137.01 & 57.99 & 264.55 & 201.99 & 5.56 \\
\hline 92.54666 & 233.87 & 210.24 & 208.11 & 206.62 & 140.97 & 58.2 & 264.18 & 202.017 & 5.58 \\
\hline 93.0465 & 234.22 & 210.42 & 208.02 & 206.53 & 145.71 & 58.46 & 263.57 & 202.047 & 5.60 \\
\hline 93.54716 & 233.51 & 210.04 & 207.93 & 206.4 & 151.39 & 58.68 & 262.86 & 202.209 & 5.60 \\
\hline 94.048 & 236.17 & 209.7 & 207.69 & 206.19 & 158.06 & 59.04 & 261.55 & 202.188 & 5.61 \\
\hline 94.54583 & 237.28 & 209.42 & 207.46 & 205.97 & 165.83 & 59.36 & 260.19 & 202.176 & 5.62 \\
\hline 95.04567 & 237.22 & 208.97 & 207.2 & 205.72 & 176.1 & 59.81 & 258.69 & 202.221 & 5.64 \\
\hline 95.5465 & 237.61 & 209.04 & 206.9 & 205.37 & 185.61 & 60.34 & 257.01 & 308 & 5.60 \\
\hline 96.04633 & 237.79 & 208.74 & 206.61 & 205.03 & 193.32 & 60.97 & 255.24 & 200.631 & 5.57 \\
\hline 96.54617 & 237.72 & 208.61 & 206.23 & 204.64 & 197.15 & 61.72 & 253.29 & 201.636 & 5.58 \\
\hline 97.046 & 237.5 & 208.91 & 205.87 & 204.29 & 198.6 & 62.61 & 251.37 & 201.6 & 5.61 \\
\hline 97.54583 & 237.3 & 207.74 & 205.52 & 203.93 & 199.47 & 63.57 & 249.56 & 201.576 & 5.61 \\
\hline 98.0465 & 237.15 & 208.29 & 205.18 & 203.59 & 200.08 & 64.58 & 247.88 & 201.528 & 5.61 \\
\hline 98.54716 & 237.04 & 208.42 & 204.87 & 203.28 & 200.43 & 65.58 & 246.43 & 201.918 & 5.62 \\
\hline 99.047 & 236.38 & 207.43 & 204.57 & 202.99 & 200.93 & 66.6 & 244.81 & 201.909 & 5.63 \\
\hline 99.546 & 236.95 & 207.05 & 204.3 & 202.74 & 201 & 67.6 & 243.38 & 201.522 & 5.61 \\
\hline 100.0458 & 237.34 & 207.1 & 204.04 & 202.52 & 201 & 68.71 & 242.05 & 201.837 & 5.56 \\
\hline 100.5465 & 237.48 & 206.98 & 203.75 & 202.23 & 200.96 & 69.91 & 240.49 & 201.945 & 5.57 \\
\hline 101.0463 & 237.55 & 206.33 & 203.48 & 201.98 & .91 & 71.27 & 239.09 & 954 & 5.59 \\
\hline 101.5462 & 237.28 & 206.17 & 203.21 & 201.73 & 200.74 & 72.71 & 237.66 & 201.894 & 5.61 \\
\hline 102.046 & 237.06 & 206.4 & 202.91 & 201.44 & 200.56 & 74.37 & 235.97 & 201.729 & 5.59 \\
\hline 102.5467 & 237.27 & 205.41 & 202.44 & 200.83 & 200.09 & 76.3 & 232.94 & 201.525 & 5.62 \\
\hline 103.0475 & 237.68 & 204.78 & 201.92 & 200.5 & 199.98 & 78.57 & 230.69 & 201.42 & 5.61 \\
\hline 103.5463 & 238.04 & 204.74 & 201.52 & 200.2 & 199.77 & 81.34 & 228.95 & 201.294 & 5.63 \\
\hline 104.049 & 238.31 & 204.56 & 201.21 & 199.92 & 199.56 & 84.76 & 227.46 & 201.177 & 5.61 \\
\hline 104.546 & 238.41 & 203.8 & 200.92 & 199.69 & 199.38 & 88.52 & 226.14 & 201.102 & 5.57 \\
\hline 105.0458 & 238.57 & 203.64 & 200.67 & 199.45 & 199.22 & 92.47 & 224.86 & 201.03 & 5.56 \\
\hline 105.5465 & 238.51 & 203.76 & 200.43 & 199.18 & 198.89 & 96.55 & 223.59 & 201.537 & 5.58 \\
\hline 106.0463 & 238.55 & 203.15 & 200.14 & 198.96 & 198.86 & 100.65 & 222.47 & 201.351 & 5.60 \\
\hline 106.5472 & 238.36 & 203.1 & 199.98 & 198.87 & 198.78 & 104.48 & 221.74 & 201.348 & 5.59 \\
\hline 107.046 & 238.22 & 202.25 & 199.85 & 198.76 & 198.69 & 107.82 & 221.19 & 201.321 & 5.59 \\
\hline 107.5458 & 238.05 & 202.72 & 199.76 & 198.67 & 198.61 & 110.82 & 220.78 & 201.369 & 5.60 \\
\hline 108.0467 & 237.94 & 203.55 & 199.63 & 198.47 & 198.34 & 113.78 & 220 & 201.624 & 5.63 \\
\hline 108.5465 & 237.96 & 202.57 & 199.49 & 198.41 & 198.41 & 116.91 & 219.5 & 201.354 & 5.61 \\
\hline 109.0472 & 237.96 & 202.31 & 199.43 & 198.39 & 198.41 & 119.77 & 219.35 & 201.285 & 5.56 \\
\hline 109.547 & 237.79 & 203.12 & 199.43 & 198.35 & 198.46 & 122.42 & 219.4 & 201.324 & 5.56 \\
\hline 110.0458 & 237.52 & 202.51 & 199.43 & 198.37 & 198.44 & 125.13 & 219.45 & 201.345 & 5.57 \\
\hline
\end{tabular}


Table B6. Continued.

\begin{tabular}{|c|c|c|c|c|c|c|c|c|c|}
\hline $\begin{array}{c}\text { Time } \\
\text { min }\end{array}$ & $\begin{array}{l}\mathrm{T} 1 \\
{ }^{\circ} \mathrm{C} \\
\end{array}$ & $\begin{array}{l}\mathrm{T} 2 \\
{ }^{\circ} \mathrm{C} \\
\end{array}$ & $\begin{array}{l}\mathrm{T} 3 \\
{ }^{\circ} \mathrm{C} \\
\end{array}$ & $\begin{array}{l}\mathrm{T} 4 \\
{ }^{\circ} \mathrm{C} \\
\end{array}$ & $\begin{array}{l}\mathrm{T5} \\
{ }^{\circ} \mathrm{C} \\
\end{array}$ & $\begin{array}{l}\mathrm{T} 6 \\
{ }^{\circ} \mathrm{C} \\
\end{array}$ & $\begin{array}{l}\text { Pinj } \\
\text { psig }\end{array}$ & $\begin{array}{l}\text { Pout } \\
\text { psig }\end{array}$ & $\begin{array}{c}\mathrm{Vw} \\
\mathrm{cc} / \mathrm{min}\end{array}$ \\
\hline 110.5457 & 237.2 & 202.67 & 199.46 & 198.42 & 198.51 & 128 & 219.63 & 201.342 & 5.60 \\
\hline 111.0465 & 237.25 & 202.39 & 199.49 & 198.44 & 198.51 & 131.01 & 219.78 & 201.348 & 5.59 \\
\hline 111.5463 & 237.24 & 203.55 & 199.54 & 198.47 & 198.52 & 134.2 & 220.08 & 201.306 & 5.59 \\
\hline 112.0462 & 237.31 & 202.73 & 199.6 & 198.52 & 198.54 & 137.45 & 220.42 & 201.258 & 5.61 \\
\hline 112.5458 & 237.24 & 203.08 & 199.54 & 198.23 & 198.11 & 140.98 & 219.63 & 201.474 & 5.61 \\
\hline 113.0467 & 237.36 & 203.13 & 199.43 & 198.28 & 198.34 & 144.61 & 219.52 & 201.546 & 5.62 \\
\hline 113.5465 & 237.54 & 202.59 & 199.46 & 198.37 & 198.44 & 148.35 & 219.82 & 201.552 & 5.57 \\
\hline 114.0472 & 237.75 & 202.88 & 199.55 & 198.46 & 198.53 & 151.9 & 220.09 & 201.6 & 5.55 \\
\hline 114.5462 & 237.66 & 202.97 & 199.58 & 198.51 & 198.56 & 155.21 & 220.43 & 201.654 & 5.56 \\
\hline 115.046 & 237.73 & 203 & 199.63 & 198.54 & 198.59 & 157.99 & .42 & 201.735 & 5.60 \\
\hline 115.5458 & 237.89 & 203.32 & 199.63 & 198.52 & 198.56 & 160.37 & 220.34 & 201.702 & 5.60 \\
\hline 116.0473 & 238.08 & 203.26 & 199.59 & 198.5 & 198.54 & 162.46 & 220.15 & 201.486 & 5.59 \\
\hline 116.5472 & 237.92 & 204.65 & 199.41 & 198.08 & 198 & 164.63 & 218.7 & 201.537 & 5.60 \\
\hline 117.0462 & 237.95 & 204.28 & 199.19 & 198.05 & 198.12 & 167.08 & 217.93 & 201.687 & 5.62 \\
\hline 546 & 238 & 202.65 & 199.12 & 198.03 & 198.15 & 168.95 & .78 & .69 & 5.63 \\
\hline 118.0458 & 238.11 & 202.18 & 199.08 & 198.02 & 198.18 & 170.39 & 217.62 & 201.564 & 5.58 \\
\hline 118.5465 & 238.1 & 202.28 & 199.04 & 198 & 198.15 & 171.56 & 217.44 & .522 & 5.58 \\
\hline 119.0463 & 238.12 & 202.21 & 199.02 & 197.98 & 198.14 & 172.54 & 217.28 & 201.48 & 5.57 \\
\hline 119.5472 & 238.1 & 202.89 & 198.97 & 197.94 & 198.09 & 173.35 & 217.06 & 201.381 & 5.60 \\
\hline 120.046 & 237.96 & 202.8 & 198.93 & 197.91 & 198.07 & 174 & 5.94 & .327 & 5.61 \\
\hline 120.5458 & 237.63 & 202.29 & 198.8 & 197.6 & 197.58 & 175.41 & .98 & 609 & 5.61 \\
\hline 121.0475 & 237.45 & 202.81 & 198.64 & 197.56 & 197.69 & 178.52 & 215.44 & 201.567 & 5.62 \\
\hline 121.5463 & 237.48 & 201.77 & 198.58 & 197.58 & 197.79 & 179.67 & 215.3 & 201.636 & 5.62 \\
\hline 122.0472 & 237.66 & 201.58 & 198.6 & 197.59 & 197.86 & 180.51 & 215.43 & 201.654 & 5.62 \\
\hline 122.547 & 237.69 & 202 & 198.63 & 197.64 & 197.86 & 180.84 & 215.63 & 201.714 & 5.57 \\
\hline 123.0468 & 237.69 & 202.22 & 198.68 & 197.69 & 197.91 & 181.15 & .91 & 564 & 5.56 \\
\hline 123.5465 & 237.63 & 202.67 & 198.73 & 197.75 & 197.96 & 181.49 & 216.12 & 201.54 & 5.56 \\
\hline 124.0463 & 237.47 & 202.42 & 198.76 & 197.78 & 197.99 & 181.91 & 216.35 & 201.546 & 5.60 \\
\hline 124.5472 & 237.29 & 202.38 & 198.8 & 197.6 & 197.53 & 184.03 & 216 & 201.627 & 5.58 \\
\hline 125.046 & 237.34 & 202.61 & 198.65 & 197.56 & 197.68 & 187.79 & 215.62 & 201.483 & 5.59 \\
\hline 125.5458 & 237.37 & 202.75 & 198.67 & 197.61 & 197.79 & 188.51 & 215.83 & 201.741 & 5.62 \\
\hline 126.0467 & 237.46 & 202.82 & 198.7 & 197.66 & 197.86 & 188.85 & 216.14 & 201.669 & 5.61 \\
\hline 126.5473 & 237.35 & 202.07 & 198.77 & 197.73 & 197.89 & 189.13 & 216.45 & 201.51 & 5.61 \\
\hline 127.0472 & 237.28 & 202.85 & 198.82 & 197.78 & 197.93 & 189.4 & 216.67 & 201.414 & 5.55 \\
\hline 127.546 & 237.2 & 202.6 & 198.87 & 197.82 & 197.98 & 189.68 & 216.86 & 201.426 & 5.55 \\
\hline 128.0458 & 237.31 & 202.45 & 198.91 & 197.87 & 198.01 & 189.93 & 216.99 & 201.414 & 5.56 \\
\hline 128.5467 & 237.41 & 201.99 & 198.94 & 197.88 & 198.03 & 190.02 & 217.06 & 201.291 & 5.59 \\
\hline 129.0465 & 237.41 & 202.13 & 198.96 & 197.88 & 198.04 & 190.36 & 217.14 & 201.285 & 5.59 \\
\hline 129.5463 & 237.42 & 202.82 & 198.95 & 197.9 & 198.04 & 190.53 & 217.19 & 201.315 & 5.60 \\
\hline 130.047 & 237.48 & 202.55 & 198.95 & 197.9 & 198.04 & 190.85 & 217.17 & 201.132 & 5.60 \\
\hline 130.5468 & 237.47 & 202.78 & 198.9 & 197.7 & 197.69 & 192.77 & 216.51 & 201.429 & 5.60 \\
\hline 131.0457 & 237.51 & 202.65 & 198.79 & 197.71 & 197.86 & 192.93 & 216.22 & 201.57 & 5.63 \\
\hline 131.5473 & 237.49 & 202.78 & 198.78 & 197.73 & 197.89 & 192.73 & 216.23 & 201.51 & 5.58 \\
\hline 132.0463 & 237.47 & 201.68 & 198.78 & 197.76 & 197.94 & 192.71 & 216.36 & 201.378 & 5.54 \\
\hline
\end{tabular}


Table B6. Continued.

\begin{tabular}{|c|c|c|c|c|c|c|c|c|c|}
\hline $\begin{array}{c}\text { Time } \\
\text { min }\end{array}$ & $\begin{array}{l}\mathrm{T} 1 \\
{ }^{\circ} \mathrm{C} \\
\end{array}$ & $\begin{array}{l}\mathrm{T} 2 \\
{ }^{\circ} \mathrm{C} \\
\end{array}$ & $\begin{array}{l}\mathrm{T} 3 \\
{ }^{\circ} \mathrm{C} \\
\end{array}$ & $\begin{array}{l}\mathrm{T} 4 \\
{ }^{\circ} \mathrm{C} \\
\end{array}$ & $\begin{array}{l}\mathrm{T} 5 \\
{ }^{\circ} \mathrm{C} \\
\end{array}$ & $\begin{array}{l}\mathrm{T} 6 \\
{ }^{\circ} \mathrm{C} \\
\end{array}$ & $\begin{array}{l}\text { Pinj } \\
\text { psig }\end{array}$ & $\begin{array}{l}\text { Pout } \\
\text { psig }\end{array}$ & $\begin{array}{c}\mathrm{Vw} \\
\mathrm{cc} / \mathrm{min}\end{array}$ \\
\hline 132.5462 & 237.48 & 202.04 & 198.78 & 197.72 & 197.88 & 192.87 & 216.18 & 201.504 & 5.54 \\
\hline 133.046 & 237.48 & 201.84 & 198.78 & 197.74 & 197.92 & 193.13 & 216.15 & 201.483 & 5.56 \\
\hline 133.5458 & 237.43 & 202.45 & 198.77 & 197.77 & 197.95 & 193.46 & 216.24 & 201.507 & 5.60 \\
\hline 134.0465 & 237.41 & 202.68 & 198.77 & 197.77 & 197.97 & 193.63 & 216.3 & 201.525 & 5.59 \\
\hline 134.5463 & 237.33 & 202.19 & 198.79 & 197.8 & 197.98 & 193.79 & 216.32 & 201.429 & 5.59 \\
\hline 135.0462 & 237.35 & 201.87 & 198.79 & 197.8 & 198 & 193.97 & 216.41 & 201.39 & 5.59 \\
\hline 135.546 & 237.36 & 202.55 & 198.8 & 197.82 & 198.02 & 194.17 & 216.48 & 363 & 5.61 \\
\hline 136.0458 & 237.26 & 201.97 & 198.86 & 197.85 & 198.05 & 194.36 & 216.59 & 201.342 & 5.63 \\
\hline 136.5475 & 237.27 & 202.1 & 198.87 & 197.87 & 198.07 & 194.52 & 216.71 & 201.402 & 5.58 \\
\hline 137.0463 & 237.25 & 201.81 & 198.89 & 197.9 & 198.12 & 194.68 & 216.83 & 201.252 & 5.55 \\
\hline 137.5462 & 237.2 & 201.7 & 198.85 & 197.72 & 197.72 & 195.99 & .31 & 135 & 5.57 \\
\hline 138. & 237.18 & 202.7 & 198.78 & 197.7 & 197.85 & 196.31 & 13 & 73 & 5.60 \\
\hline 138.5458 & 237.14 & 202.84 & 198.76 & 197.74 & 197.88 & 196.43 & .26 & 369 & 5.60 \\
\hline 139.0465 & 237.15 & 202.19 & 198.79 & 197.75 & 197.89 & 196.48 & 216.4 & 534 & 5.60 \\
\hline 139.5463 & 237.21 & 202.87 & 198.82 & 197.8 & 197.95 & 196.55 & 216.57 & 645 & 5.61 \\
\hline 140. & 237.22 & 203.33 & 198.86 & 197.82 & 197.98 & 196.6 & 216.74 & 513 & 5.62 \\
\hline 46 & 237.22 & 202.72 & 198.89 & 197.85 & 198.01 & 196.65 & 0.9 & 26 & 5.62 \\
\hline 141. & 237.25 & 203.45 & 198.93 & 197.87 & 198.05 & 196.76 & 7.06 & 147 & 5.57 \\
\hline 141.5465 & 237.25 & 203.7 & 198.96 & 197.92 & 198.06 & 196.83 & 7.17 & 339 & 5.55 \\
\hline 142.0473 & 237.27 & 202.57 & 198.97 & 197.92 & 198.06 & 196.86 & 217.27 & 426 & 5.57 \\
\hline 142.5462 & 237.25 & 203.32 & 198.99 & 197.97 & 198.1 & 197 & 217.4 & 201.21 & 5.60 \\
\hline 143.046 & 237.28 & 203.63 & 199.01 & 197.97 & 198.1 & 196.98 & 217.42 & 201 & 5.61 \\
\hline 143 & 237.41 & 203.22 & 199.02 & 198 & 198.13 & 197.06 & .42 & 12 & 5.60 \\
\hline 144.0467 & 237.39 & 202.37 & 199.02 & 197.98 & 198.11 & 197.09 & 217.47 & 075 & 5.60 \\
\hline 144.5465 & 237.28 & 203 & 198.97 & 197.77 & 197.77 & 196.85 & 216.66 & 333 & 5.65 \\
\hline 145.0462 & 237.13 & 202.98 & 198.84 & 197.73 & 197.82 & 197.01 & 216.25 & 561 & 5.62 \\
\hline 5.547 & 237.24 & 202.85 & 198.77 & 197.73 & 197.85 & 197.08 & 216.17 & 585 & 5.56 \\
\hline 146 & 237.27 & 202.56 & 198.76 & 197.73 & 197.85 & 197.08 & .16 & 141 & 5.57 \\
\hline 146.5457 & 237.41 & 202.36 & 198.74 & 197.69 & 197.8 & 197.06 & .97 & 85 & 5.59 \\
\hline 147.0473 & 237.38 & 201.84 & 198.69 & 197.65 & 197.78 & 197.06 & 215.79 & 943 & 5.61 \\
\hline 147.5463 & 237.3 & 201.23 & 198.67 & 197.63 & 197.85 & 197.08 & 215.71 & .018 & 5.62 \\
\hline 148.047 & 237.26 & 201.93 & 198.65 & 197.65 & 197.79 & 197.09 & 215.6 & 200.823 & 5.60 \\
\hline 148.5468 & 237.25 & 201.62 & 198.63 & 197.63 & 197.79 & 197.11 & 215.57 & 952 & 5.62 \\
\hline 149.0457 & 237.24 & 202.26 & 198.63 & 197.64 & 197.82 & 197.13 & 215.52 & 793 & 5.64 \\
\hline 149.5465 & 237.21 & 202.93 & 198.58 & 197.48 & 197.59 & 196.89 & 214.98 & 201.234 & 5.59 \\
\hline 150.0463 & 237.12 & 202.26 & 198.52 & 197.52 & 197.73 & 197.09 & 215 & 201.357 & 5.55 \\
\hline 150.5462 & 237.17 & 201.65 & 198.54 & 197.59 & 197.84 & 197.19 & 215.16 & 201.339 & 5.56 \\
\hline 151.046 & 237.17 & 201.79 & 198.57 & 197.62 & 197.84 & 197.23 & 215.3 & 201.429 & 5.59 \\
\hline 151.5458 & 237.1 & 201.61 & 198.61 & 197.64 & 197.85 & 197.23 & 215.5 & 201.204 & 5.60 \\
\hline 152.0465 & 237.08 & 202.21 & 198.62 & 197.64 & 197.85 & 197.19 & 215.54 & 201.207 & 5.60 \\
\hline 152.5472 & 237.08 & 201.94 & 198.64 & 197.66 & 197.83 & 197.23 & 215.56 & 201.042 & 5.62 \\
\hline 153.0462 & 237.11 & 202.33 & 198.64 & 197.65 & 197.85 & 197.23 & 215.62 & 201.12 & 5.62 \\
\hline 153.546 & 237.02 & 202.38 & 198.66 & 197.67 & 197.85 & 197.22 & 215.68 & 201.153 & 5.63 \\
\hline 154.0458 & 237 & 202.47 & 198.67 & 197.67 & 197.87 & 197.24 & 215.85 & 201.201 & 5.57 \\
\hline
\end{tabular}


Table B6. Continued.

\begin{tabular}{|c|c|c|c|c|c|c|c|c|c|}
\hline $\begin{array}{c}\text { Time } \\
\text { min }\end{array}$ & $\begin{array}{l}\mathrm{T} 1 \\
{ }^{\circ} \mathrm{C} \\
\end{array}$ & $\begin{array}{l}\mathrm{T} 2 \\
{ }^{\circ} \mathrm{C}\end{array}$ & $\begin{array}{l}\mathrm{T} 3 \\
{ }^{\circ} \mathrm{C} \\
\end{array}$ & $\begin{array}{l}\mathrm{T} 4 \\
{ }^{\circ} \mathrm{C} \\
\end{array}$ & $\begin{array}{l}\mathrm{T5} \\
{ }^{\circ} \mathrm{C} \\
\end{array}$ & $\begin{array}{l}\mathrm{T} 6 \\
{ }^{\circ} \mathrm{C} \\
\end{array}$ & $\begin{array}{l}\text { Pinj } \\
\text { psig }\end{array}$ & $\begin{array}{l}\text { Pout } \\
\text { psig }\end{array}$ & $\begin{array}{c}\mathrm{Vw} \\
\mathrm{cc} / \mathrm{min}\end{array}$ \\
\hline 154.5475 & 237 & 202.47 & 198.71 & 197.69 & 197.86 & 197.26 & 215.93 & 201.138 & 5.57 \\
\hline 155.0473 & 237.07 & 201.71 & 198.72 & 197.72 & 197.88 & 197.27 & 216.05 & 201.129 & 5.57 \\
\hline 155.5462 & 237.05 & 202.32 & 198.74 & 197.7 & 197.64 & 196.98 & 216.05 & 201.153 & 5.60 \\
\hline 156.046 & 236.99 & 203.14 & 198.68 & 197.63 & 197.77 & 197.18 & 215.71 & 201.42 & 5.60 \\
\hline 156.5458 & 236.94 & 202.6 & 198.68 & 197.68 & 197.91 & 197.3 & 215.92 & 201.549 & 5.60 \\
\hline 157.0465 & 237.02 & 202.44 & 198.72 & 197.75 & 197.93 & 197.34 & 216.16 & 201.327 & 5.62 \\
\hline 157.5473 & 237.08 & 202.06 & 198.77 & 197.75 & 197.91 & 197.28 & 216.27 & 201.288 & 5.64 \\
\hline 158.0472 & 237.11 & 202.46 & 198.78 & 197.76 & 197.91 & 197.28 & 216.29 & 201.147 & 5.61 \\
\hline 158.546 & 237.15 & 203.83 & 198.78 & 197.75 & 197.91 & 197.28 & 216.27 & 201.129 & 5.57 \\
\hline 159.0468 & 237.15 & 204.03 & 198.8 & 197.76 & 197.89 & 197.28 & .28 & 201.189 & 5.55 \\
\hline 159.5475 & 237.15 & 203.9 & 198.78 & 197.78 & 197.91 & 197.3 & 216.28 & 200.979 & 5.57 \\
\hline 160.0473 & 237.11 & 203.26 & 198.76 & 197.76 & 197.89 & 197.28 & 216.17 & 201.264 & 5.60 \\
\hline 160.5462 & 237.14 & 203.26 & 198.75 & 197.74 & 197.9 & 197.26 & 216.13 & 201.051 & 5.60 \\
\hline 161.046 & 237.16 & 202.43 & 198.74 & 197.74 & 197.88 & 197.28 & 216.08 & 201.009 & 5.60 \\
\hline 161. & 237.25 & 203.27 & 198.74 & 197.72 & 197.88 & 197.31 & 97 & 066 & 5.61 \\
\hline 162.0467 & 237.27 & 203.02 & 198.72 & 197.72 & 197.9 & 197.29 & .91 & .94 & 5.61 \\
\hline 162.5465 & 237.26 & 202.7 & 198.7 & 197.7 & 197.88 & 197.27 & 215.87 & 200.934 & 5.63 \\
\hline 163.0462 & 237.21 & 202.45 & 198.67 & 197.59 & 197.61 & 197 & 215.43 & 201.102 & 5.57 \\
\hline 163.546 & 237.19 & 202.66 & 198.54 & 197.5 & 197.59 & 197.02 & 215 & 200.922 & 5.56 \\
\hline 164.0458 & 237.26 & 202.76 & 198.5 & 197.52 & 197.72 & 197.18 & 214.88 & 408 & 5.58 \\
\hline 164 & 237.22 & 202.55 & 198.52 & 197.57 & 197.86 & 197.32 & 5.1 & 339 & .58 \\
\hline 165.0465 & 237.33 & 203.18 & 198.56 & 197.61 & 197.91 & 197.37 & 215.21 & 201.471 & 5.60 \\
\hline 165.5472 & 237.26 & 201.76 & 198.57 & 197.64 & 197.87 & 197.34 & 215.3 & 200.886 & 5.61 \\
\hline 166.047 & 237.17 & 201.99 & 198.61 & 197.64 & 197.84 & 197.28 & 215.29 & 200.757 & 5.62 \\
\hline 166.5458 & 237.04 & 201.88 & 198.59 & 197.61 & 197.82 & 197.25 & 215.23 & 865 & 5.64 \\
\hline 167.0457 & 237.02 & 202.53 & 198.57 & 197.62 & 197.84 & 197.28 & 5.2 & 979 & 5.61 \\
\hline 167.5465 & 236.99 & 202.33 & 198.57 & 197.62 & 197.85 & 197.28 & 215.27 & 200.922 & 5.57 \\
\hline 168.0463 & 237.02 & 202.44 & 198.59 & 197.64 & 197.82 & 197.3 & 215.31 & 200.853 & 5.56 \\
\hline 168.5462 & 237.04 & 202.12 & 198.61 & 197.66 & 197.85 & 197.28 & 215.52 & 200.94 & 5.60 \\
\hline 169.046 & 237 & 201.83 & 198.62 & 197.66 & 197.85 & 197.28 & 215.5 & 200.781 & 5.60 \\
\hline 169.5467 & 237.06 & 202.52 & 198.62 & 197.66 & 197.85 & 197.3 & 215.58 & 200.829 & 5.60 \\
\hline 170.0473 & 237.04 & 204.26 & 198.66 & 197.67 & 197.87 & 197.3 & 215.64 & 200.802 & 5.61 \\
\hline 170.5463 & 237.02 & 203.46 & 198.66 & 197.71 & 197.87 & 197.3 & 215.72 & 200.889 & 5.64 \\
\hline 171.0462 & 237.07 & 202.59 & 198.69 & 197.72 & 197.89 & 197.31 & 215.84 & 200.913 & 5.64 \\
\hline 171.546 & 237.05 & 204.35 & 198.71 & 197.72 & 197.9 & 197.29 & 215.88 & 200.796 & 5.58 \\
\hline 172.0458 & 236.98 & 203.9 & 198.71 & 197.72 & 197.88 & 197.24 & 216.01 & 200.793 & 5.57 \\
\hline 172.5465 & 237.07 & 203.25 & 198.74 & 197.74 & 197.92 & 197.31 & 216.12 & 200.772 & 5.58 \\
\hline 173.0463 & 237.14 & 203.43 & 198.74 & 197.76 & 197.95 & 197.33 & 216.11 & 200.913 & 5.61 \\
\hline 173.5462 & 237.14 & 203.31 & 198.76 & 197.77 & 197.95 & 197.36 & 216.16 & 200.793 & 5.60 \\
\hline 174.0497 & 237.14 & 203.75 & 198.78 & 197.77 & 197.95 & 197.34 & 216.19 & 200.847 & 5.61 \\
\hline 174.5458 & 237.17 & 202.86 & 198.76 & 197.72 & 197.86 & 197.31 & 215.98 & 201.342 & 5.62 \\
\hline 175.0465 & 237.23 & 202.66 & 198.74 & 197.75 & 197.95 & 197.43 & 216.01 & 201.321 & 5.62 \\
\hline 175.5463 & 237.17 & 202.37 & 198.76 & 197.77 & 197.95 & 197.41 & 216.12 & 201.183 & 5.59 \\
\hline 176.0462 & 237.21 & 202.52 & 198.74 & 197.77 & 197.95 & 197.38 & 216.04 & 201.126 & 5.56 \\
\hline
\end{tabular}


Table B6. Continued.

\begin{tabular}{|c|c|c|c|c|c|c|c|c|c|}
\hline $\begin{array}{c}\text { Time } \\
\text { min }\end{array}$ & $\begin{array}{l}\mathrm{T} 1 \\
{ }^{\circ} \mathrm{C} \\
\end{array}$ & $\begin{array}{l}\mathrm{T} 2 \\
{ }^{\circ} \mathrm{C} \\
\end{array}$ & $\begin{array}{l}\mathrm{T} 3 \\
{ }^{\circ} \mathrm{C} \\
\end{array}$ & $\begin{array}{l}\mathrm{T} 4 \\
{ }^{\circ} \mathrm{C} \\
\end{array}$ & $\begin{array}{l}\mathrm{T5} \\
{ }^{\circ} \mathrm{C} \\
\end{array}$ & $\begin{array}{l}\mathrm{T} 6 \\
{ }^{\circ} \mathrm{C} \\
\end{array}$ & $\begin{array}{l}\text { Pinj } \\
\text { psig }\end{array}$ & $\begin{array}{l}\text { Pout } \\
\text { psig }\end{array}$ & $\begin{array}{c}\mathrm{Vw} \\
\mathrm{cc} / \mathrm{min}\end{array}$ \\
\hline 176.546 & 237.26 & 202.94 & 198.74 & 197.77 & 197.93 & 197.39 & 216 & 201.27 & 5.55 \\
\hline 177.0458 & 237.24 & 202.82 & 198.74 & 197.77 & 197.95 & 197.38 & 215.97 & 201 & 5.58 \\
\hline 177.5465 & 237.23 & 202.39 & 198.72 & 197.73 & 197.91 & 197.36 & 215.88 & 201.033 & 5.59 \\
\hline 178.0463 & 237.24 & 202.69 & 198.7 & 197.73 & 197.93 & 197.37 & 215.77 & 201 & 5.60 \\
\hline 178.5462 & 237.19 & 202.67 & 198.68 & 197.71 & 197.89 & 197.35 & 215.72 & 200.802 & 5.62 \\
\hline 179.046 & 237.17 & 202.21 & 198.66 & 197.71 & 197.93 & 197.37 & 215.64 & 200.97 & 5.62 \\
\hline 179.5458 & 237.11 & 202.44 & 198.66 & 197.69 & 197.91 & 197.37 & 215.6 & 201.033 & 5.64 \\
\hline 180.0475 & 237.1 & 202.62 & 198.64 & 197.7 & 197.89 & 197.35 & 215.55 & 201.06 & 5.61 \\
\hline 180.5465 & 237.02 & 203.01 & 198.63 & 197.69 & 197.89 & 197.35 & 215.51 & 200.829 & 5.56 \\
\hline 181.0462 & 237.02 & 202.62 & 198.64 & 197.69 & 197.89 & 197.35 & 215.49 & 201.075 & 5.57 \\
\hline 181.546 & 237.04 & 202.67 & 198.61 & 197.64 & 197.82 & 197.32 & 215.28 & 200.691 & 5.60 \\
\hline 182.0458 & 237.06 & 203.89 & 198.59 & 197.64 & 197.86 & 197.32 & 215.28 & 200.904 & 5.62 \\
\hline 182.5457 & 237.04 & 203.62 & 198.57 & 197.66 & 197.89 & 197.37 & 215.3 & 201.18 & 5.61 \\
\hline 183.0465 & 237.02 & 202.65 & 198.62 & 197.69 & 197.91 & 197.39 & 215.53 & 201.123 & 5.63 \\
\hline 183 & 236.97 & 202.53 & 198.64 & 197.71 & 197.93 & 197.39 & 63 & 051 & 5.65 \\
\hline 184 & 236.93 & 201.97 & 198.64 & 197.73 & 197.94 & 197.4 & 215.69 & 201.177 & 5.62 \\
\hline 184.5458 & 236.84 & 203.06 & 198.68 & 197.74 & 197.96 & 197.42 & 215.83 & 201.177 & 5.59 \\
\hline 185.0457 & 236.88 & 204.53 & 198.69 & 197.74 & 197.92 & 197.46 & 215.92 & 201.234 & 5.60 \\
\hline 185.5473 & 236.86 & 203.99 & 198.69 & 197.76 & 197.99 & 197.4 & 216.05 & 201.153 & 5.62 \\
\hline 186 & 236.86 & 203.19 & 198.75 & 197.8 & 197.98 & 197.42 & 216.12 & 201.123 & 5.61 \\
\hline 186 & 236.82 & 203.22 & 198.76 & 197.8 & 197.99 & 197.42 & 216.25 & 201.069 & 5.63 \\
\hline 187.046 & 236.86 & 202.74 & 198.8 & 197.81 & 198.01 & 197.49 & 216.29 & 201.093 & 5.65 \\
\hline 187.5458 & 236.82 & 202.52 & 198.8 & 197.81 & 197.99 & 197.42 & 216.41 & 201.054 & 5.65 \\
\hline 188.0465 & 236.91 & 203.45 & 198.78 & 197.85 & 198.03 & 197.45 & 216.4 & 201.051 & 5.59 \\
\hline 188.5463 & 236.95 & 203.51 & 198.8 & 197.83 & 198.03 & 197.44 & 216.47 & 201.084 & 5.59 \\
\hline 189.047 & 236.91 & 203.22 & 198.81 & 197.85 & 198.03 & 197.44 & 216.45 & 048 & 5.61 \\
\hline 189.546 & 236.89 & 203.22 & 198.81 & 197.85 & 197.99 & 197.43 & 216.47 & 201.051 & 5.62 \\
\hline 190.0458 & 236.82 & 202.88 & 198.8 & 197.85 & 197.99 & 197.43 & 216.45 & 200.94 & 5.60 \\
\hline 190.5465 & 236.8 & 203.02 & 198.78 & 197.85 & 197.99 & 197.42 & 216.34 & 200.961 & 5.63 \\
\hline 191.0473 & 236.84 & 202.74 & 198.78 & 197.83 & 198.01 & 197.42 & 216.29 & 200.931 & 5.65 \\
\hline 191.547 & 236.91 & 203.13 & 198.78 & 197.83 & 197.99 & 197.42 & 216.26 & 200.934 & 5.63 \\
\hline 192.046 & 236.98 & 202.91 & 198.74 & 197.76 & 197.85 & 197.31 & 215.93 & 201.219 & 5.59 \\
\hline 192.5458 & 236.89 & 203 & 198.69 & 197.74 & 197.88 & 197.35 & 215.76 & 200.634 & 5.56 \\
\hline 193.0465 & 236.82 & 202.57 & 198.65 & 197.7 & 197.86 & 197.31 & 215.66 & 200.523 & 5.63 \\
\hline 193.5463 & 236.8 & 203.4 & 198.63 & 197.68 & 197.86 & 197.33 & 215.52 & 200.784 & 5.60 \\
\hline 194.0462 & 236.8 & 202.98 & 198.63 & 197.68 & 197.86 & 197.34 & 215.49 & 200.967 & 5.62 \\
\hline 194.546 & 236.78 & 203.16 & 198.6 & 197.68 & 197.9 & 197.38 & 215.49 & 201.081 & 5.65 \\
\hline 195.0458 & 236.85 & 203.5 & 198.6 & 197.7 & 197.92 & 197.4 & 215.45 & 201.132 & 5.65 \\
\hline 195.5465 & 236.83 & 203.59 & 198.6 & 197.7 & 197.92 & 197.4 & 215.48 & 201.078 & 5.59 \\
\hline 196.0473 & 236.82 & 203.02 & 198.6 & 197.72 & 197.93 & 197.4 & 215.43 & 201.078 & 5.57 \\
\hline 196.5472 & 236.8 & 203.11 & 198.58 & 197.7 & 197.92 & 197.4 & 215.41 & 201.024 & 5.60 \\
\hline 197.0478 & 236.76 & 203.23 & 198.58 & 197.68 & 197.92 & 197.38 & 215.41 & 200.988 & 5.61 \\
\hline 197.5458 & 236.76 & 203.61 & 198.6 & 197.68 & 197.92 & 197.4 & 215.44 & 201.087 & 5.59 \\
\hline 198.0467 & 236.74 & 203.63 & 198.6 & 197.7 & 197.9 & 197.4 & 215.51 & 201.039 & 5.60 \\
\hline
\end{tabular}


Table B6. Continued.

\begin{tabular}{|c|c|c|c|c|c|c|c|c|c|}
\hline $\begin{array}{c}\text { Time } \\
\text { min }\end{array}$ & $\begin{array}{l}\mathrm{T} 1 \\
{ }^{\circ} \mathrm{C} \\
\end{array}$ & $\begin{array}{l}\mathrm{T} 2 \\
{ }^{\circ} \mathrm{C} \\
\end{array}$ & $\begin{array}{l}\mathrm{T} 3 \\
{ }^{\circ} \mathrm{C} \\
\end{array}$ & $\begin{array}{l}\mathrm{T} 4 \\
{ }^{\circ} \mathrm{C} \\
\end{array}$ & $\begin{array}{l}\mathrm{T5} \\
{ }^{\circ} \mathrm{C} \\
\end{array}$ & $\begin{array}{l}\mathrm{T} 6 \\
{ }^{\circ} \mathrm{C} \\
\end{array}$ & $\begin{array}{l}\text { Pinj } \\
\text { psig }\end{array}$ & $\begin{array}{l}\text { Pout } \\
\text { psig }\end{array}$ & $\begin{array}{c}\mathrm{Vw} \\
\mathrm{cc} / \mathrm{min}\end{array}$ \\
\hline 198.5465 & 236.73 & 204.86 & 198.6 & 197.7 & 197.95 & 197.38 & 215.55 & 200.976 & 5.61 \\
\hline 199.0462 & 236.65 & 203.32 & 198.6 & 197.74 & 197.93 & 197.4 & 215.65 & 201.042 & 5.63 \\
\hline 199.547 & 236.71 & 202.98 & 198.63 & 197.74 & 197.95 & 197.41 & 215.71 & 201.018 & 5.59 \\
\hline 200.0458 & 236.64 & 203.14 & 198.65 & 197.74 & 197.97 & 197.41 & 215.75 & 200.967 & 5.57 \\
\hline 200.5457 & 236.62 & 203.43 & 198.67 & 197.75 & 197.93 & 197.41 & 215.83 & 201.048 & 5.56 \\
\hline 201.0473 & 236.56 & 203.37 & 198.68 & 197.77 & 197.97 & 197.41 & 215.9 & 201.03 & 5.61 \\
\hline 201.5463 & 236.29 & 204.74 & 198.7 & 197.77 & 197.98 & 197.41 & 215.97 & 201.027 & 5.59 \\
\hline 202.0462 & 236.44 & 203.62 & 198.7 & 197.79 & 198 & 197.43 & 216.06 & 200.919 & 5.61 \\
\hline 202.5458 & 236.47 & 203.14 & 198.72 & 197.81 & 197.98 & 197.45 & 216.12 & 201.006 & 5.60 \\
\hline 203.0457 & 236.6 & 203 & 198.72 & 197.7 & 197.7 & 197.12 & 215.89 & 199.518 & 5.63 \\
\hline 203.5483 & 236.62 & 203.37 & 198.61 & 197.59 & 197.73 & 197.2 & 5.27 & 136 & 5.61 \\
\hline 204.0463 & 236.67 & 203.5 & 198.56 & 197.63 & 197.82 & 197.25 & 5.27 & 147 & 5.58 \\
\hline 204.5462 & 236.81 & 203.3 & 198.57 & 197.66 & 197.93 & 197.43 & 215.42 & 186 & 5.58 \\
\hline 205.046 & 236.85 & 203.32 & 198.59 & 197.7 & 197.95 & 197.45 & 215.59 & 201.261 & 5.60 \\
\hline 205.5458 & 236.83 & 204.38 & 198.63 & 197.73 & 197.98 & 197.48 & 215.71 & 201.255 & 5.60 \\
\hline 206.0465 & 236.83 & 202.98 & 198.63 & 197.75 & 197.97 & 197.5 & 5.73 & 012 & 5.61 \\
\hline 206.5482 & 236.83 & 202.8 & 198.65 & 197.75 & 197.95 & 197.34 & .71 & 718 & 5.60 \\
\hline 207.047 & 236.78 & 202.85 & 198.63 & 197.71 & 197.89 & 197.36 & 215.58 & 673 & 5.61 \\
\hline 207.546 & 236.74 & 203.46 & 198.61 & 197.7 & 197.89 & 197.36 & 215.44 & 200.535 & 5.62 \\
\hline 208.0467 & 236.54 & 203.68 & 198.59 & 197.68 & 197.88 & 197.34 & 215.31 & 200.568 & 5.57 \\
\hline 208.5465 & 236.76 & 203.95 & 198.56 & 197.66 & 197.88 & 197.36 & 215.16 & 200.67 & 5.56 \\
\hline 209.0463 & 236.85 & 202.92 & 198.52 & 197.64 & 197.84 & 197.32 & 5.06 & 499 & 5.56 \\
\hline 209.5462 & 236.78 & 202.48 & 198.5 & 197.62 & 197.84 & 197.32 & 4.97 & 505 & 5.60 \\
\hline 210.046 & 236.7 & 203.35 & 198.5 & 197.61 & 197.84 & 197.3 & 214.93 & 526 & 5.59 \\
\hline 210.5458 & 236.81 & 203.82 & 198.48 & 197.59 & 197.84 & 197.32 & 214.82 & 200.517 & 5.59 \\
\hline 211.0465 & 236.85 & 203.21 & 198.47 & 197.59 & 197.82 & 197.3 & 214.83 & 200.511 & 5.59 \\
\hline 211.5463 & 236.8 & 203.71 & 198.45 & 197.57 & 197.82 & 197.32 & 214.8 & 604 & 5.61 \\
\hline 212.0462 & 236.76 & 204 & 198.47 & 197.59 & 197.84 & 197.32 & 214.8 & 532 & 5.62 \\
\hline 212.547 & 236.74 & 204.2 & 198.47 & 197.59 & 197.82 & 197.32 & 214.86 & 200.472 & 5.58 \\
\hline 213.0458 & 236.78 & 203.37 & 198.47 & 197.61 & 197.84 & 197.32 & 214.9 & 200.409 & 5.56 \\
\hline 213.5465 & 236.76 & 202.67 & 198.49 & 197.61 & 197.86 & 197.32 & 214.9 & 200.454 & 5.56 \\
\hline 214.0473 & 236.69 & 203.05 & 198.49 & 197.61 & 197.84 & 197.32 & 215.02 & 200.409 & 5.59 \\
\hline 214.5472 & 236.65 & 202.55 & 198.5 & 197.64 & 197.84 & 197.34 & 215.06 & 200.595 & 5.60 \\
\hline 215.047 & 236.74 & 203.21 & 198.54 & 197.64 & 197.88 & 197.34 & 215.08 & 200.688 & 5.58 \\
\hline 215.5458 & 236.72 & 204.21 & 198.52 & 197.64 & 197.89 & 197.41 & 215.17 & 200.529 & 5.60 \\
\hline 216.0475 & 236.72 & 204.25 & 198.54 & 197.66 & 197.86 & 197.41 & 215.33 & 200.529 & 5.62 \\
\hline 216.5473 & 236.6 & 204.07 & 198.56 & 197.68 & 197.88 & 197.36 & 215.36 & 200.436 & 5.64 \\
\hline 217.0462 & 236.54 & 203.84 & 198.59 & 197.68 & 197.89 & 197.34 & 215.43 & 200.553 & 5.59 \\
\hline 217.546 & 236.58 & 203.37 & 198.59 & 197.64 & 197.8 & 197.28 & 215.37 & 201.156 & 5.56 \\
\hline 218.0458 & 236.26 & 203.71 & 198.56 & 197.62 & 197.82 & 197.32 & 215.15 & 200.814 & 5.57 \\
\hline 218.5457 & 236.44 & 204.75 & 198.54 & 197.66 & 197.91 & 197.41 & 215.35 & 201.291 & 5.59 \\
\hline 219.0465 & 236.65 & 206.49 & 198.59 & 197.71 & 197.96 & 197.39 & 215.51 & 201.12 & 5.59 \\
\hline 219.5463 & 236.7 & 206.09 & 198.63 & 197.73 & 197.96 & 197.44 & 215.67 & 201.054 & 5.59 \\
\hline
\end{tabular}


Table B6. Continued.

\begin{tabular}{|c|c|c|c|c|c|c|c|c|c|}
\hline $\begin{array}{c}\text { Time } \\
\text { min }\end{array}$ & $\begin{array}{l}\mathrm{T} 1 \\
{ }^{\circ} \mathrm{C} \\
\end{array}$ & $\begin{array}{l}\mathrm{T} 2 \\
{ }^{\circ} \mathrm{C} \\
\end{array}$ & $\begin{array}{l}\mathrm{T} 3 \\
{ }^{\circ} \mathrm{C} \\
\end{array}$ & $\begin{array}{l}\mathrm{T} 4 \\
{ }^{\circ} \mathrm{C} \\
\end{array}$ & $\begin{array}{l}\mathrm{T5} \\
{ }^{\circ} \mathrm{C} \\
\end{array}$ & $\begin{array}{l}\mathrm{T} 6 \\
{ }^{\circ} \mathrm{C} \\
\end{array}$ & $\begin{array}{l}\text { Pinj } \\
\text { psig }\end{array}$ & $\begin{array}{c}\text { Pout } \\
\text { psig }\end{array}$ & $\begin{array}{c}\mathrm{Vw} \\
\mathrm{cc} / \mathrm{min}\end{array}$ \\
\hline 220.0462 & 236.7 & 204.57 & 198.64 & 197.75 & 197.98 & 197.41 & 215.73 & 200.802 & 5.59 \\
\hline 220.5458 & 236.65 & 203.87 & 198.65 & 197.73 & 197.95 & 197.39 & 215.63 & 200.769 & 5.63 \\
\hline 221.0457 & 236.72 & 203.51 & 198.63 & 197.71 & 197.91 & 197.37 & 215.47 & 200.64 & 5.63 \\
\hline 221.5473 & 236.76 & 204.64 & 198.59 & 197.68 & 197.89 & 197.35 & 215.4 & 200.706 & 5.58 \\
\hline 222.0472 & 236.78 & 204.36 & 198.57 & 197.68 & 197.89 & 197.36 & 215.32 & 200.616 & 5.57 \\
\hline 222.547 & 236.72 & 204.03 & 198.56 & 197.66 & 197.93 & 197.35 & 215.27 & 200.571 & 5.58 \\
\hline 223.0468 & 236.83 & 204.34 & 198.56 & 197.66 & 197.88 & 197.34 & 215.14 & 200.538 & 5.60 \\
\hline 223.5458 & 236.7 & 203.66 & 198.54 & 197.66 & 197.89 & 197.32 & 215.1 & 200.484 & 5.59 \\
\hline 224.0465 & 236.72 & 203.64 & 198.52 & 197.62 & 197.86 & 197.34 & 214.85 & 200.484 & 5.58 \\
\hline 224.5463 & 236.72 & 203.69 & 198.5 & 197.64 & 197.86 & 197.34 & 4.97 & 200.511 & 5.61 \\
\hline 225.0462 & 236.58 & 203.64 & 198.49 & 197.61 & 197.88 & 197.34 & 214.86 & 200.496 & 5.64 \\
\hline 225.546 & 236.58 & 203.18 & 198.49 & 197.63 & 197.86 & 197.28 & 214.81 & 200.76 & 5.62 \\
\hline 226.0458 & 236.53 & 203.12 & 198.49 & 197.63 & 197.86 & 197.36 & 214.79 & 200.544 & 5.57 \\
\hline 226.5465 & 236.49 & 203.68 & 198.49 & 197.61 & 197.86 & 197.34 & 214.72 & 200.562 & 5.57 \\
\hline 227. & 236.49 & 204.05 & 198.47 & 197.59 & 197.86 & 197.37 & .78 & .49 & 5.59 \\
\hline 227.5462 & 236.42 & 204.72 & 198.49 & 197.61 & 197.88 & 197.32 & .74 & 502 & 5.61 \\
\hline 228.0468 & 236.47 & 204.5 & 198.48 & 197.61 & 197.88 & 197.37 & 214.83 & 200.529 & 5.61 \\
\hline 228.5467 & 236.58 & 204.02 & 198.48 & 197.62 & 197.88 & 197.37 & 214.88 & 200.475 & 5.61 \\
\hline 229.0465 & 236.7 & 204.77 & 198.48 & 197.62 & 197.82 & 197.36 & 214.89 & 200.541 & 5.65 \\
\hline 229.5463 & 236.62 & 204.46 & 198.49 & 197.55 & 197.71 & 197.21 & 214.63 & 199.524 & 5.65 \\
\hline 230.0462 & 236.53 & 203.93 & 198.43 & 197.54 & 197.79 & 197.32 & 214.6 & 201.177 & .58 \\
\hline 230.546 & 236.58 & 204.36 & 198.45 & 197.59 & 197.86 & 197.39 & 214.84 & 201.402 & 5.57 \\
\hline 231.0458 & 236.6 & 205.32 & 198.47 & 197.64 & 197.93 & 197.46 & 215.08 & 200.901 & 5.59 \\
\hline 231.5465 & 236.67 & 205.16 & 198.54 & 197.68 & 197.93 & 197.43 & 215.27 & 200.697 & 5.61 \\
\hline 232.0463 & 236.69 & 204.21 & 198.56 & 197.68 & 197.91 & 197.39 & 215.32 & 200.502 & 5.61 \\
\hline 232 & 236.63 & 204 & 198.57 & 197.68 & 197.89 & 197.36 & .31 & 478 & 5.62 \\
\hline 233.046 & 236.54 & 204.3 & 198.57 & 197.68 & 197.89 & 197.35 & 215.35 & 200.538 & 5.62 \\
\hline 233.5477 & 236.67 & 203.25 & 198.59 & 197.68 & 197.89 & 197.37 & 215.31 & 200.58 & 5.62 \\
\hline 234.0475 & 236.78 & 202.62 & 198.57 & 197.68 & 197.86 & 197.39 & 215.33 & 200.598 & 5.59 \\
\hline 234.5473 & 236.79 & 203.91 & 198.57 & 197.7 & 197.89 & 197.37 & 215.35 & 200.538 & 5.55 \\
\hline 235.0462 & 236.83 & 204.5 & 198.57 & 197.68 & 197.88 & 197.36 & 215.31 & 200.553 & 5.58 \\
\hline 235.546 & 236.87 & 204.5 & 198.56 & 197.68 & 197.89 & 197.36 & 215.29 & 200.487 & 5.61 \\
\hline 236.0458 & 236.97 & 205 & 198.56 & 197.68 & 197.88 & 197.36 & 215.24 & 200.526 & 5.61 \\
\hline 236.5457 & 236.99 & 204.14 & 198.54 & 197.68 & 197.88 & 197.36 & 215.18 & 200.544 & 5.60 \\
\hline 237.0473 & 236.94 & 203.21 & 198.54 & 197.66 & 197.88 & 197.36 & 215.18 & 200.427 & 5.60 \\
\hline 237.5463 & 236.94 & 203.41 & 198.52 & 197.66 & 197.88 & 197.36 & 215.08 & 200.397 & 5.63 \\
\hline 238.0462 & 237.04 & 202.66 & 198.47 & 197.54 & 197.7 & 197.18 & 214.59 & 200.64 & 5.59 \\
\hline 238.5458 & 237.08 & 203.19 & 198.41 & 197.54 & 197.79 & 197.3 & 214.45 & 201.039 & 5.56 \\
\hline 239.0457 & 237.13 & 203.37 & 198.4 & 197.57 & 197.86 & 197.46 & 214.65 & 201.297 & 5.56 \\
\hline 239.5465 & 237.06 & 203.77 & 198.43 & 197.62 & 197.93 & 197.48 & 214.78 & 201.333 & 5.59 \\
\hline 240.0463 & 237.12 & 203.43 & 198.47 & 197.64 & 197.95 & 197.46 & 214.91 & 201.219 & 5.59 \\
\hline 240.5462 & 229.68 & 200.47 & 197.53 & 197 & 197.54 & 197.41 & 237.15 & 200.235 & 5.58 \\
\hline 241.046 & 225.8 & 194.96 & 194.8 & 194.81 & 195.41 & 195.84 & 257.7 & 200.007 & 5.57 \\
\hline 241.5458 & 233.37 & 194.47 & 194.56 & 194.1 & 194.22 & 193.65 & 275.72 & 200.856 & 5.55 \\
\hline
\end{tabular}


Table B6. Continued.

\begin{tabular}{|c|c|c|c|c|c|c|c|c|c|}
\hline $\begin{array}{c}\text { Time } \\
\text { min }\end{array}$ & $\begin{array}{l}\mathrm{T} 1 \\
{ }^{\circ} \mathrm{C} \\
\end{array}$ & $\begin{array}{l}\mathrm{T} 2 \\
{ }^{\circ} \mathrm{C} \\
\end{array}$ & $\begin{array}{l}\mathrm{T} 3 \\
{ }^{\circ} \mathrm{C} \\
\end{array}$ & $\begin{array}{l}\mathrm{T} 4 \\
{ }^{\circ} \mathrm{C} \\
\end{array}$ & $\begin{array}{l}\text { T5 } \\
{ }^{\circ} \mathrm{C} \\
\end{array}$ & $\begin{array}{l}\mathrm{T} 6 \\
{ }^{\circ} \mathrm{C} \\
\end{array}$ & $\begin{array}{l}\text { Pinj } \\
\text { psig }\end{array}$ & $\begin{array}{l}\text { Pout } \\
\text { psig }\end{array}$ & $\begin{array}{c}\mathrm{Vw} \\
\mathrm{cc} / \mathrm{min}\end{array}$ \\
\hline 241.5458 & 233.37 & 194.47 & 194.56 & 194.1 & 194.22 & 193.65 & 275.72 & 200.856 & 5.55 \\
\hline 242.0465 & 240.06 & 194.39 & 194.58 & 193.79 & 193.69 & 192.13 & 290.5 & 201.066 & 5.55 \\
\hline 242.5472 & 244.9 & 194.08 & 194.55 & 193.6 & 193.4 & 191.04 & 255.42 & 201.153 & 5.57 \\
\hline 243.0462 & 248.09 & 193.78 & 194.49 & 193.47 & 193.22 & 190.23 & 248.27 & 201.177 & 5.59 \\
\hline 243.546 & 249.99 & 193.1 & 194.48 & 193.42 & 193.1 & 189.66 & 267.33 & 201.291 & 5.61 \\
\hline 244.0458 & 249.8 & 192.22 & 194.49 & 193.46 & 193.22 & 189.41 & 240.15 & 201.138 & 5.60 \\
\hline 244.5465 & 250.37 & 190.12 & 194.42 & 193.39 & 193.19 & 189.16 & 251.32 & 200.901 & 5.61 \\
\hline 245.0473 & 251.29 & 188.24 & 194.32 & 193.28 & 193.1 & 188.78 & 254.84 & 200.877 & 5.62 \\
\hline 245.547 & 251.59 & 186.63 & 194.23 & 193.19 & 192.99 & 188.42 & 250.26 & 200.85 & 5.62 \\
\hline 246.0468 & 251.06 & 185.2 & 194.14 & 193.1 & 192.87 & 188.06 & 271.81 & 200.982 & 5.57 \\
\hline 246.5458 & 249.96 & 183.83 & 194.03 & 192.99 & 192.73 & 187.78 & 239.61 & 997 & 5.55 \\
\hline 247.0465 & 242.4 & 190.77 & 193.14 & 193.12 & 192.83 & 189.05 & 252.06 & 394 & 5.56 \\
\hline 247.5463 & 216.66 & 194.64 & 193.3 & 193.09 & 192.89 & 189.7 & 255.68 & 349 & 5.58 \\
\hline 248.0462 & 204.53 & 195.44 & 193.63 & 192.98 & 192.8 & 189.45 & 255.6 & 200.409 & 5.58 \\
\hline 248.546 & 200.43 & 195.74 & 193.73 & 192.87 & 192.68 & 189.11 & 245.57 & 200.163 & 5.58 \\
\hline 249.0467 & 199.58 & 195.03 & 193.45 & 192.62 & 192.36 & 188.11 & 242.93 & 235 & 5.58 \\
\hline 249 & 199.04 & 193.25 & 193.29 & 192.41 & 192.07 & 187.46 & 09 & 23 & 5.58 \\
\hline 250.0463 & 196.66 & 190.98 & 193.16 & 192.23 & 191.82 & 187.02 & 258.09 & .28 & 5.62 \\
\hline 250.5462 & 191.95 & 186.98 & 193.04 & 192.07 & 191.61 & 186.68 & 237.47 & 200.367 & 5.64 \\
\hline 251.046 & 186.97 & 183.32 & 192.94 & 191.93 & 191.43 & 186.39 & 239.74 & 200.406 & 5.58 \\
\hline 251.5468 & 183.4 & 180.9 & 192.81 & 191.75 & 191.22 & 186.11 & 245.37 & 427 & 5.56 \\
\hline 252.0467 & 179.49 & 179.27 & 192.67 & 191.59 & 191.02 & 185.8 & 247.32 & 373 & 5.57 \\
\hline 252.5465 & 175.18 & 178.03 & 192.54 & 191.43 & 190.82 & 185.52 & 243.25 & .52 & 5.59 \\
\hline 253.0462 & 171.26 & 176.99 & 192.39 & 191.27 & 190.65 & 185.23 & 245.54 & 200.526 & 5.60 \\
\hline 253.546 & 167.41 & 175.97 & 192.24 & 191.1 & 190.43 & 184.95 & 247.23 & 200.436 & 5.59 \\
\hline 254.0458 & 162.78 & 174.87 & 192.1 & 190.96 & 190.26 & 184.66 & 230.42 & 200.4 & 5.58 \\
\hline 254.5457 & 159.3 & 173.73 & 191.96 & 190.8 & 190.04 & 184.38 & 233.98 & 200.424 & 5.61 \\
\hline 255.0465 & 155.45 & 172.43 & 191.8 & 190.62 & 189.83 & 184.09 & 248.65 & .403 & 5.65 \\
\hline 255.5472 & 151.28 & 171.09 & 191.66 & 190.46 & 189.63 & 183.81 & 263.23 & 200.397 & 5.60 \\
\hline 256.0462 & 147.8 & 169.65 & 191.52 & 190.3 & 189.47 & 183.54 & 240.16 & 200.355 & 5.55 \\
\hline 256.5458 & 143.6 & 168.12 & 191.38 & 190.12 & 189.26 & 183.23 & 254.34 & 200.316 & 5.55 \\
\hline 257.0467 & 140 & 166.61 & 191.22 & 189.96 & 189.07 & 182.95 & 246 & .361 & 5.57 \\
\hline 257.5465 & 136.44 & 165.06 & 191.04 & 189.76 & 188.78 & 182.41 & 261.46 & 200.688 & 5.61 \\
\hline 258.0463 & 133.18 & 163.48 & 190.91 & 189.61 & 188.6 & 182.23 & 245.88 & 200.946 & 5.60 \\
\hline 258.5462 & 129.9 & 161.89 & 190.77 & 189.45 & 188.41 & 182.04 & 245.95 & 201.051 & 5.60 \\
\hline 259.0468 & 126.68 & 160.29 & 190.63 & 189.29 & 188.23 & 181.82 & 247.16 & 201.12 & 5.60 \\
\hline 259.5467 & 123.7 & 158.72 & 190.49 & 189.11 & 188.03 & 181.56 & 262.25 & 201.267 & 5.63 \\
\hline 260.0465 & 120.44 & 157.11 & 190.37 & 188.93 & 187.84 & 181.31 & 248.62 & 201.27 & 5.63 \\
\hline 260.5472 & 117.78 & 155.62 & 190.19 & 188.75 & 187.64 & 181.02 & 236.59 & 201.306 & 5.58 \\
\hline 261.0462 & 115.09 & 154.03 & 190.06 & 188.59 & 187.45 & 180.77 & 264.05 & 201.219 & 5.57 \\
\hline 261.546 & 111.72 & 152.53 & 189.91 & 188.43 & 187.25 & 180.52 & 261.54 & 201.333 & 5.58 \\
\hline 262.0458 & 109.11 & 151.1 & 189.76 & 188.26 & 187.04 & 180.24 & 263.92 & 201.312 & 5.62 \\
\hline 262.5475 & 106.12 & 149.56 & 189.62 & 188.08 & 186.84 & 179.95 & 236.4 & 201.156 & 5.62 \\
\hline
\end{tabular}


Table B6. Continued.

\begin{tabular}{|c|c|c|c|c|c|c|c|c|c|}
\hline $\begin{array}{c}\text { Time } \\
\text { min }\end{array}$ & $\begin{array}{l}\mathrm{T} 1 \\
{ }^{\circ} \mathrm{C}\end{array}$ & $\begin{array}{l}\mathrm{T} 2 \\
{ }^{\circ} \mathrm{C}\end{array}$ & $\begin{array}{l}\mathrm{T} 3 \\
{ }^{\circ} \mathrm{C}\end{array}$ & $\begin{array}{l}\mathrm{T} 4 \\
{ }^{\circ} \mathrm{C}\end{array}$ & $\begin{array}{l}\mathrm{T} 5 \\
{ }^{\circ} \mathrm{C}\end{array}$ & $\begin{array}{l}\mathrm{T} 6 \\
{ }^{\circ} \mathrm{C} \\
\end{array}$ & $\begin{array}{l}\text { Pinj } \\
\text { psig }\end{array}$ & $\begin{array}{l}\text { Pout } \\
\text { psig }\end{array}$ & $\begin{array}{c}\mathrm{Vw} \\
\mathrm{cc} / \mathrm{min}\end{array}$ \\
\hline 263.0463 & 103.51 & 148.03 & 189.48 & 187.9 & 186.63 & 179.65 & 234.84 & 201.168 & 5.62 \\
\hline 263.5462 & 101.26 & 146.58 & 189.34 & 187.74 & 186.43 & 179.36 & 236.73 & 201.12 & 5.63 \\
\hline 264.046 & 98.81 & 145.11 & 189.18 & 187.56 & 186.24 & 179.08 & 237.09 & 201.138 & 5.63 \\
\hline 264.5467 & 96.89 & 143.64 & 189.04 & 187.39 & 186.02 & 178.81 & 244.29 & 201.111 & 5.59 \\
\hline 265.0465 & 94.84 & 142.25 & 188.89 & 187.21 & 185.81 & 178.52 & 233.08 & 201.087 & 5.57 \\
\hline 265.5473 & 92.63 & 140.94 & 188.75 & 187.03 & 185.63 & 178.24 & 245.19 & 201.117 & 5.56 \\
\hline 266.0462 & 90.55 & 139.67 & 188.59 & 186.87 & 185.4 & 177.94 & 237.08 & 201.066 & 5.59 \\
\hline 266.547 & 106.46 & 133.34 & 188.31 & 186.57 & 185.08 & 177.38 & 203.33 & 200.085 & 5.61 \\
\hline 267.0458 & 113.14 & 135.56 & 187.97 & 186.32 & 184.8 & 176.73 & 205.3 & 201.219 & 5.59 \\
\hline 267.5465 & 165.58 & 140.89 & 187.77 & 186.05 & 184.53 & 176.79 & 205.74 & 811 & 5.59 \\
\hline 268.0463 & 194.66 & 156.33 & 186.86 & 185.55 & 184.13 & 176.99 & 204.47 & 548 & 5.61 \\
\hline 268.5462 & 198.92 & 189.12 & 185.35 & 185.48 & 183.99 & 177.13 & 206.73 & 397 & 5.64 \\
\hline 269.046 & 199.82 & 194.23 & 184.82 & 185.38 & 183.89 & 177.26 & 206.15 & 200.496 & 5.62 \\
\hline 269.5458 & 202.13 & 195.43 & 185.11 & 185.25 & 183.73 & 177.03 & 205.43 & 200.631 & 5.58 \\
\hline 270.0467 & 207.39 & 195.86 & 185.52 & 185.09 & 183.53 & 176.62 & 205.58 & 895 & 5.57 \\
\hline 270.5473 & 222.16 & 196.39 & 189.43 & 184.93 & 183.32 & 176.26 & 22 & 266 & 5.59 \\
\hline 271.0462 & 237.71 & 196.73 & 193.98 & 184.73 & 183.08 & 175.97 & 206.68 & 207 & 5.62 \\
\hline 271.547 & 250.53 & 197.89 & 195.6 & 184.7 & 182.87 & 175.72 & 207.29 & 201.408 & 5.60 \\
\hline 272.0468 & 256.92 & 199.65 & 196.16 & 184.63 & 182.67 & 175.49 & 208.12 & 201.276 & 5.61 \\
\hline 272.5467 & 262.01 & 201.76 & 196.46 & 184.51 & 182.5 & 175.35 & 208.86 & 201.336 & 5.63 \\
\hline 273.0473 & 264.35 & 202.93 & 196.7 & 184.51 & 182.35 & 175.22 & 209.34 & 201.369 & 5.65 \\
\hline 273.5463 & 261.7 & 202.87 & 196.93 & 184.58 & 182.3 & 175.12 & 209.87 & 1.33 & 5.61 \\
\hline 274.0462 & 261.96 & 204.09 & 197.15 & 184.76 & 182.25 & 174.99 & 210.28 & 201.357 & 5.57 \\
\hline 274.5458 & 258.78 & 204.78 & 197.33 & 185.07 & 182.16 & 174.89 & 210.54 & 201.327 & 5.57 \\
\hline 275.0457 & 257.23 & 204.44 & 197.42 & 185.37 & 182.11 & 174.76 & 210.77 & 201.336 & 5.60 \\
\hline 275.5465 & 255.03 & 204.53 & 197.54 & 185.75 & 182 & 174.62 & 211.23 & 201.324 & 5.62 \\
\hline 276.0472 & 252.19 & 204.17 & 197.67 & 186.2 & 181.95 & 174.5 & 211.86 & 333 & 5.59 \\
\hline 276.5462 & 249.09 & 203.28 & 197.82 & 187.28 & 181.84 & 174.57 & 212.5 & 201.459 & 5.61 \\
\hline 277.046 & 245.83 & 202.67 & 197.98 & 191.98 & 181.76 & 174.64 & 213.38 & 201.477 & 5.62 \\
\hline 277.5467 & 242.44 & 202.15 & 198.2 & 196.12 & 181.67 & 174.72 & 214.41 & 201.462 & 5.63 \\
\hline 278.0465 & 238.51 & 202.37 & 198.43 & 197.19 & 181.6 & 174.83 & 215.59 & 201.609 & 5.57 \\
\hline 278.5463 & 234.66 & 203.05 & 198.66 & 197.57 & 181.53 & 175.01 & 216.49 & .852 & 5.56 \\
\hline 279.0462 & 231.67 & 202.55 & 198.84 & 197.79 & 181.46 & 175.13 & 217.08 & 201.615 & 5.55 \\
\hline 279.546 & 229.04 & 202.07 & 198.94 & 197.9 & 181.39 & 175.26 & 217.18 & 201.726 & 5.61 \\
\hline 280.0458 & 224.55 & 202.04 & 198.97 & 197.97 & 183.42 & 175.41 & 216.91 & 201.582 & 5.60 \\
\hline 280.5465 & 222.28 & 202.07 & 198.87 & 197.81 & 196 & 175.61 & 215.74 & 201.555 & 5.60 \\
\hline 281.0463 & 222.33 & 201.41 & 198.58 & 197.65 & 197.61 & 175.7 & 214.33 & 201.426 & 5.61 \\
\hline 281.5462 & 222.98 & 200.64 & 198.35 & 197.51 & 197.7 & 175.77 & 213.42 & 200.769 & 5.59 \\
\hline 282.046 & 223.53 & 200.18 & 198.19 & 197.38 & 197.67 & 175.72 & 212.72 & 200.664 & 5.62 \\
\hline 282.5467 & 224.54 & 200.03 & 198.05 & 197.29 & 197.65 & 175.68 & 212.21 & 200.517 & 5.58 \\
\hline 283.0465 & 225.33 & 200.11 & 197.96 & 197.26 & 197.64 & 175.72 & 211.96 & 200.367 & 5.56 \\
\hline 283.5463 & 225.44 & 200.27 & 197.92 & 197.24 & 197.66 & 176.87 & 211.76 & 200.424 & 5.56 \\
\hline 284.0462 & 223.47 & 200.7 & 197.91 & 197.24 & 197.68 & 181.59 & 211.76 & 200.436 & 5.57 \\
\hline
\end{tabular}


Table B6. Continued.

\begin{tabular}{|c|c|c|c|c|c|c|c|c|c|}
\hline $\begin{array}{c}\text { Time } \\
\text { min }\end{array}$ & $\begin{array}{l}\mathrm{T} 1 \\
{ }^{\circ} \mathrm{C} \\
\end{array}$ & $\begin{array}{l}\mathrm{T} 2 \\
{ }^{\circ} \mathrm{C} \\
\end{array}$ & $\begin{array}{l}\mathrm{T} 3 \\
{ }^{\circ} \mathrm{C} \\
\end{array}$ & $\begin{array}{l}\mathrm{T} 4 \\
{ }^{\circ} \mathrm{C} \\
\end{array}$ & $\begin{array}{l}\mathrm{T} 5 \\
{ }^{\circ} \mathrm{C} \\
\end{array}$ & $\begin{array}{l}\mathrm{T} 6 \\
{ }^{\circ} \mathrm{C} \\
\end{array}$ & $\begin{array}{l}\text { Pinj } \\
\text { psig }\end{array}$ & $\begin{array}{l}\text { Pout } \\
\text { psig }\end{array}$ & $\begin{array}{c}\mathrm{Vw} \\
\mathrm{cc} / \mathrm{min}\end{array}$ \\
\hline 284.547 & 221.89 & 199.95 & 197.91 & 197.28 & 197.71 & 188.02 & 211.78 & 200.397 & 5.61 \\
\hline 285.0458 & 222.15 & 199.86 & 197.91 & 197.21 & 197.5 & 192.59 & 211.58 & 201.084 & 5.59 \\
\hline 285.5465 & 221.61 & 199.9 & 197.86 & 197.23 & 197.73 & 195.05 & 211.62 & 201.396 & 5.60 \\
\hline 286.0463 & 222.08 & 200.02 & 197.91 & 197.3 & 197.8 & 196.23 & 211.97 & 201.15 & 5.60 \\
\hline 286.5462 & 222.04 & 200.31 & 197.97 & 197.34 & 197.81 & 196.77 & 212.06 & 200.802 & 5.63 \\
\hline 287.046 & 221.38 & 200.76 & 197.99 & 197.36 & 197.79 & 196.93 & 212.05 & 200.808 & 5.61 \\
\hline 287.5458 & 223.06 & 200.67 & 197.97 & 197.36 & 197.81 & 197.07 & 212.17 & 200.853 & 5.57 \\
\hline 288.0467 & 223.76 & 201 & 198.01 & 197.38 & 197.84 & 197.18 & 212.4 & 200.838 & 5.55 \\
\hline 288.5465 & 224.5 & 200.84 & 198.08 & 197.42 & 197.9 & 197.25 & 212.81 & 200.877 & 5.57 \\
\hline 289.0462 & 226.39 & 201.44 & 198.15 & 197.49 & 197.92 & 197.27 & 213.3 & 201.027 & 5.59 \\
\hline 289.547 & 230.93 & 202.59 & 198.19 & 197.44 & 197.78 & 197.17 & 3.13 & 201.147 & 5.60 \\
\hline 290.0458 & 232.86 & 203.6 & 198.17 & 197.45 & 197.87 & 197.28 & .42 & 354 & 5.59 \\
\hline 290.5457 & 231.72 & 203.54 & 198.24 & 197.54 & 197.94 & 197.33 & 4.09 & 201.372 & 5.61 \\
\hline 291.0465 & 229.77 & 203.19 & 198.41 & 197.65 & 198.01 & 197.37 & 214.64 & 201.201 & 5.61 \\
\hline 291.5463 & 227.35 & 203.08 & 198.51 & 197.74 & 198.05 & 197.28 & 215.23 & 200.922 & 5.61 \\
\hline 292.0462 & 226.3 & 203.24 & 198.64 & 197.82 & 198.09 & 197.32 & .75 & .88 & 5.62 \\
\hline 292.5458 & 227.01 & 203.24 & 198.7 & 197.86 & 198.1 & 197.34 & .81 & 91 & 5.58 \\
\hline 293.0457 & 226.96 & 203.6 & 198.66 & 197.78 & 198 & 197.17 & 5.26 & 159 & 5.58 \\
\hline 293.5465 & 227.89 & 203.07 & 198.59 & 197.77 & 198.07 & 197.37 & 215.06 & 201.111 & 5.61 \\
\hline 294.0482 & 227.21 & 203.05 & 198.54 & 197.77 & 198.05 & 197.36 & 1.74 & 201.123 & 5.61 \\
\hline 294.547 & 227.32 & 201.91 & 198.45 & 197.71 & 198.02 & 197.36 & 4.29 & 201.078 & 5.60 \\
\hline 295.0468 & 226.84 & 201.96 & 198.38 & 197.64 & 197.97 & 197.34 & 3.92 & 003 & 5.61 \\
\hline 295.5458 & 227 & 201.15 & 198.29 & 197.56 & 197.91 & 197.3 & .36 & 877 & 5.63 \\
\hline 296.0465 & 226.88 & 202.09 & 198.17 & 197.5 & 197.86 & 197.27 & 212.82 & 200.595 & 5.63 \\
\hline 296.5472 & 226.64 & 201.48 & 198.08 & 197.39 & 197.86 & 197.23 & 212.45 & 200.622 & 5.58 \\
\hline 297.047 & 226.09 & 201.37 & 197.99 & 197.34 & 197.74 & 197.15 & 212.04 & 197.844 & 5.56 \\
\hline 297.546 & 227.38 & 201.86 & 197.9 & 197.25 & 197.7 & 197.2 & 211.75 & 200.97 & 5.57 \\
\hline 298.0458 & 226 & 201.8 & 197.9 & 197.31 & 197.79 & 197.33 & .97 & 952 & 5.60 \\
\hline 298.5465 & 226.36 & 201.41 & 197.92 & 197.31 & 197.76 & 197.26 & 212.05 & 200.631 & 5.60 \\
\hline 299.0473 & 225.98 & 202.07 & 197.94 & 197.31 & 197.76 & 197.22 & 212.23 & 200.634 & 5.60 \\
\hline 299.547 & 234.2 & 202.04 & 197.97 & 197.33 & 197.78 & 197.22 & 212.45 & 200.739 & 5.60 \\
\hline 300.046 & 240.96 & 204.13 & 197.97 & 197.31 & 197.74 & 197.2 & 212.5 & 200.664 & 5.62 \\
\hline 300.5458 & 244.62 & 203.4 & 197.96 & 197.29 & 197.72 & 197.17 & 212.65 & 200.679 & 5.63 \\
\hline 301.0475 & 247 & 204.37 & 197.96 & 197.28 & 197.74 & 197.19 & 212.45 & 200.652 & 5.59 \\
\hline 301.5473 & 247.02 & 204.58 & 197.94 & 197.28 & 197.72 & 197.19 & 212.47 & 200.706 & 5.55 \\
\hline 302.0462 & 245.34 & 204.13 & 197.99 & 197.33 & 197.76 & 197.21 & 212.84 & 200.838 & 5.56 \\
\hline 302.546 & 242.34 & 205.32 & 198.07 & 197.39 & 197.82 & 197.24 & 213.12 & 200.721 & 5.61 \\
\hline 303.0458 & 239.35 & 205.39 & 198.14 & 197.44 & 197.87 & 197.28 & 213.36 & 200.859 & 5.61 \\
\hline 303.5465 & 236.52 & 205.05 & 198.17 & 197.42 & 197.76 & 197.15 & 213.35 & 200.88 & 5.58 \\
\hline 304.0463 & 233.4 & 204.48 & 198.23 & 197.5 & 197.89 & 197.32 & 213.88 & 201.198 & 5.60 \\
\hline 304.5462 & 230.36 & 204.48 & 198.36 & 197.62 & 197.98 & 197.37 & 214.54 & 201.021 & 5.61 \\
\hline 305.047 & 229.38 & 203.33 & 198.44 & 197.71 & 198.03 & 197.39 & 214.84 & 201.063 & 5.62 \\
\hline 305.5468 & 230.58 & 203.62 & 198.48 & 197.75 & 198.02 & 197.35 & 214.9 & 200.577 & 5.59 \\
\hline
\end{tabular}


Table B6. Continued.

\begin{tabular}{|c|c|c|c|c|c|c|c|c|c|}
\hline $\begin{array}{l}\text { Time } \\
\text { min }\end{array}$ & $\begin{array}{l}\mathrm{T} 1 \\
{ }^{\circ} \mathrm{C}\end{array}$ & $\begin{array}{l}\mathrm{T} 2 \\
{ }^{\circ} \mathrm{C}\end{array}$ & $\begin{array}{l}\mathrm{T} 3 \\
{ }^{\circ} \mathrm{C}\end{array}$ & $\begin{array}{l}\mathrm{T} 4 \\
{ }^{\circ} \mathrm{C}\end{array}$ & $\begin{array}{l}\mathrm{T} 5 \\
{ }^{\circ} \mathrm{C}\end{array}$ & $\begin{array}{l}\mathrm{T} 6 \\
{ }^{\circ} \mathrm{C}\end{array}$ & $\begin{array}{l}\text { Pinj } \\
\text { psig }\end{array}$ & $\begin{array}{l}\text { Pout } \\
\text { psig }\end{array}$ & $\begin{array}{c}\mathrm{Vw} \\
\mathrm{cc} / \mathrm{min}\end{array}$ \\
\hline 306.0467 & 232.92 & 204.19 & 198.5 & 197.71 & 197.98 & 197.28 & 214.71 & 200.43 & 5.56 \\
\hline 306.5465 & 233.53 & 205.29 & 198.43 & 197.64 & 197.93 & 197.3 & 214.25 & 200.418 & 5.57 \\
\hline 307.0472 & 232.26 & 204.97 & 198.32 & 197.59 & 197.91 & 197.28 & 213.78 & 200.769 & 5.58 \\
\hline 307.546 & 232.35 & 204 & 198.29 & 197.54 & 197.89 & 197.28 & 213.48 & 200.661 & 5.61 \\
\hline 308.0458 & 232.93 & 203.89 & 198.22 & 197.5 & 197.86 & 197.27 & 213.23 & 200.601 & 5.63 \\
\hline 308.5467 & 233.52 & 203.2 & 198.13 & 197.38 & 197.72 & 197.09 & 212.63 & 199.542 & 5.60 \\
\hline 309.0473 & 233.21 & 203.21 & 198 & 197.29 & 197.7 & 197.14 & 212.11 & 200.577 & 5.64 \\
\hline 309.5472 & 233.57 & 202.1 & 197.93 & 197.27 & 197.72 & 197.23 & 212.03 & 201.057 & 5.65 \\
\hline 310.047 & 233.41 & 202.44 & 197.91 & 197.31 & 197.74 & 197.2 & 212.02 & 201.024 & 5.59 \\
\hline 310.5458 & 233.45 & 202.8 & 197.91 & 197.31 & 197.75 & 197.29 & 212.09 & 200.97 & 5.56 \\
\hline 311.0457 & 233.21 & 202.86 & 197.93 & 197.31 & 197.75 & 197.29 & 212.11 & 200.943 & 5.58 \\
\hline 311.5465 & 232.48 & 202.28 & 197.93 & 197.31 & 197.77 & 197.27 & 2.2 & 973 & 5.61 \\
\hline 312.0472 & 231.95 & 203.23 & 197.95 & 197.31 & 197.74 & 197.25 & 212.2 & 646 & 5.60 \\
\hline 312.5462 & 232.3 & 203.34 & 197.95 & 197.31 & 197.74 & 197.24 & 212.19 & 200.871 & 5.61 \\
\hline 313.046 & 231.73 & 203.43 & 197.95 & 197.33 & 197.77 & 197.27 & 212.39 & 200.952 & 5.62 \\
\hline 313.5458 & 230.5 & 202.98 & 198.01 & 197.36 & 197.79 & 197.31 & 212.47 & 096 & 5.63 \\
\hline 314. & 231.86 & 202.7 & 198.04 & 197.4 & 197.83 & 197.31 & 212.8 & 57 & 5.62 \\
\hline 314.5463 & 232.93 & 202.66 & 198.12 & 197.45 & 197.88 & 197.34 & 213.05 & 039 & 5.57 \\
\hline 315.047 & 231.2 & 202.32 & 198.15 & 197.49 & 197.9 & 197.35 & 213.28 & 200.895 & 5.55 \\
\hline 315.546 & 232.27 & 202.81 & 198.22 & 197.54 & 197.92 & 197.33 & 213.71 & 200.796 & 5.58 \\
\hline 316.0467 & 233.02 & 203.77 & 198.29 & 197.58 & 197.96 & 197.35 & 213.99 & 200.859 & 5.61 \\
\hline 316.5465 & 231.88 & 203.4 & 198.37 & 197.63 & 197.97 & 197.35 & .35 & 53 & 5.60 \\
\hline 317.0463 & 232.63 & 204.04 & 198.42 & 197.69 & 198.01 & 197.36 & .56 & 024 & 5.61 \\
\hline 317.5462 & 231.73 & 204.06 & 198.46 & 197.72 & 198.03 & 197.36 & 214.69 & 200.775 & 5.62 \\
\hline 318.046 & 233 & 203.79 & 198.46 & 197.67 & 197.92 & 197.18 & 214.54 & 200.283 & 5.65 \\
\hline 318.5458 & 233.4 & 203.95 & 198.4 & 197.65 & 197.97 & 197.37 & 214.23 & 201.228 & 5.60 \\
\hline 319.0465 & 233.45 & 204.63 & 198.37 & 197.65 & 197.99 & 197.38 & 214.14 & 200.781 & 5.57 \\
\hline 319.5463 & 233.58 & 203.88 & 198.33 & 197.6 & 197.94 & 197.29 & 213.9 & 403 & 5.58 \\
\hline 320.0462 & 233.79 & 203.49 & 198.26 & 197.52 & 197.87 & 197.22 & 213.49 & 200.148 & 5.61 \\
\hline 320.547 & 233.54 & 203.58 & 198.22 & 197.47 & 197.83 & 197.22 & 213.16 & 200.283 & 5.61 \\
\hline 321.0458 & 233.47 & 204.13 & 198.13 & 197.42 & 197.79 & 197.2 & 212.82 & 200.247 & 5.60 \\
\hline 321.5465 & 233.17 & 204.28 & 198.1 & 197.4 & 197.83 & 197.24 & 212.58 & 200.445 & 5.60 \\
\hline 322.0473 & 232.06 & 203.92 & 198.03 & 197.37 & 197.76 & 197.24 & 212.46 & 200.472 & 5.63 \\
\hline 322.5462 & 233.59 & 203.56 & 198.01 & 197.33 & 197.74 & 197.22 & 212.34 & 200.466 & 5.62 \\
\hline 323.046 & 234.65 & 203.04 & 197.96 & 197.31 & 197.72 & 197.22 & 212.13 & 200.493 & 5.57 \\
\hline 323.5458 & 234.4 & 203.25 & 197.94 & 197.26 & 197.58 & 197.04 & 212.09 & 200.526 & 5.57 \\
\hline 324.0467 & 233.99 & 204.22 & 197.88 & 197.24 & 197.69 & 197.19 & 211.77 & 200.034 & 5.58 \\
\hline 324.5465 & 233.67 & 203.99 & 197.92 & 197.27 & 197.74 & 197.29 & 211.99 & 200.967 & 5.61 \\
\hline 325.0462 & 233.72 & 203.99 & 197.92 & 197.31 & 197.76 & 197.33 & 212.3 & 200.88 & 5.60 \\
\hline 325.546 & 236.96 & 201.23 & 197.56 & 197.04 & 197.56 & 197.24 & 233.32 & 200.499 & 5.61 \\
\hline 326.0468 & 239.42 & 197.78 & 196.59 & 196.25 & 196.95 & 196.93 & 238.62 & 200.085 & 5.62 \\
\hline 326.5457 & 241.37 & 196.29 & 195.89 & 195.64 & 196.43 & 196.66 & 250.06 & 200.007 & 5.64 \\
\hline 327.0465 & 241.21 & 195.5 & 195.57 & 195.43 & 196.13 & 196.09 & 261.62 & 200.394 & 5.62 \\
\hline
\end{tabular}


Table B6. Continued.

\begin{tabular}{|c|c|c|c|c|c|c|c|c|c|}
\hline $\begin{array}{c}\text { Time } \\
\text { min }\end{array}$ & $\begin{array}{l}\mathrm{T} 1 \\
{ }^{\circ} \mathrm{C} \\
\end{array}$ & $\begin{array}{l}\mathrm{T} 2 \\
{ }^{\circ} \mathrm{C} \\
\end{array}$ & $\begin{array}{l}\mathrm{T} 3 \\
{ }^{\circ} \mathrm{C} \\
\end{array}$ & $\begin{array}{l}\mathrm{T} 4 \\
{ }^{\circ} \mathrm{C} \\
\end{array}$ & $\begin{array}{l}\mathrm{T5} \\
{ }^{\circ} \mathrm{C} \\
\end{array}$ & $\begin{array}{l}\mathrm{T} 6 \\
{ }^{\circ} \mathrm{C} \\
\end{array}$ & $\begin{array}{l}\text { Pinj } \\
\text { psig }\end{array}$ & $\begin{array}{l}\text { Pout } \\
\text { psig }\end{array}$ & $\begin{array}{c}\mathrm{Vw} \\
\mathrm{cc} / \mathrm{min}\end{array}$ \\
\hline 328.0462 & 242.09 & 195.14 & 195.29 & 194.68 & 194.77 & 193.94 & 284.05 & 200.712 & 5.56 \\
\hline 328.5458 & 243.67 & 195.05 & 195.23 & 194.43 & 194.41 & 192.92 & 297.65 & 201.006 & 5.59 \\
\hline 329.0457 & 245.41 & 194.86 & 195.22 & 194.3 & 194.27 & 192.06 & 271.87 & 201.048 & 5.62 \\
\hline 329.5465 & 246.55 & 194.5 & 195.16 & 194.25 & 194.19 & 191.47 & 299.89 & 200.928 & 5.60 \\
\hline 330.0463 & 247.34 & 193.94 & 195.05 & 194.12 & 194.07 & 190.99 & 325.24 & 200.943 & 5.61 \\
\hline 330.5462 & 224.93 & 193.07 & 193.48 & 192.76 & 192.13 & 189.8 & 349.74 & 199.626 & 5.61 \\
\hline 331.046 & 228.47 & 192.83 & 193.07 & 192.06 & 191.22 & 187.83 & 266.77 & 200.994 & 5.65 \\
\hline 331.5458 & 235.67 & 191.51 & 193.12 & 192.06 & 191.4 & 187.22 & 301.95 & 201.036 & 5.62 \\
\hline 332.0473 & 238.5 & 190.56 & 193.1 & 192.12 & 191.6 & 187.42 & 329.74 & 200.766 & 5.56 \\
\hline 332.5463 & 242.63 & 188.67 & 193.01 & 192.06 & 191.69 & 187.22 & 350.22 & 200.55 & 5.56 \\
\hline 333.0462 & 245.19 & 187.22 & 192.9 & 191.99 & 191.7 & 186.86 & 280.71 & .52 & 5.58 \\
\hline 333.546 & 247 & 185.82 & 192.8 & 191.94 & 191.67 & 186.59 & 307.12 & 538 & 5.60 \\
\hline 334.0458 & 248.04 & 184.89 & 192.67 & 191.85 & 191.6 & 186.38 & 327.3 & 577 & 5.60 \\
\hline 334.5475 & 248.38 & 184.14 & 192.56 & 191.76 & 191.49 & 186.18 & 342.86 & 200.583 & 5.61 \\
\hline 335.0463 & 247.85 & 183.56 & 192.46 & 191.67 & 191.42 & 186 & 355.35 & 200.58 & 5.62 \\
\hline 335.5462 & 246.81 & 182.9 & 192.33 & 191.56 & 191.27 & 185.81 & 367.84 & 589 & 5.64 \\
\hline 336.046 & 241.53 & 189.7 & 191.42 & 191.58 & 191.27 & 186.61 & .22 & 65 & 5.60 \\
\hline 336.5458 & 218.83 & 195.31 & 191.29 & 191.56 & 191.27 & 187.83 & 388.42 & 91 & 5.57 \\
\hline 337.0465 & 202.04 & 196.08 & 191.65 & 191.47 & 191.24 & 187.53 & 400.46 & 199.992 & 5.58 \\
\hline 337.5463 & 199.73 & 196.02 & 191.58 & 191.26 & 191.04 & 186.42 & 413.77 & 200.034 & 5.61 \\
\hline 338.0462 & 199.37 & 194.11 & 191.47 & 191.06 & 190.79 & 185.72 & 428.19 & 43 & 5.61 \\
\hline 338.547 & 197.91 & 192.07 & 191.35 & 190.88 & 190.56 & 185.23 & 2.17 & 25 & 5.61 \\
\hline 339.0458 & 195.92 & 190.52 & 191.24 & 190.7 & 190.35 & 184.84 & 4.42 & 25 & 5.62 \\
\hline 339.5465 & 193.77 & 188.84 & 191.1 & 190.54 & 190.11 & 184.45 & 464.82 & 200.091 & 5.63 \\
\hline 340.0463 & 190.49 & 187.14 & 190.96 & 190.36 & 189.92 & 184.1 & 475.62 & 200.064 & 5.61 \\
\hline 340.5462 & 186.96 & 185.72 & 190.81 & 190.22 & 189.7 & 183.84 & 487.48 & 200.037 & 5.56 \\
\hline 341.046 & 182.55 & 184.2 & 190.67 & 190.08 & 189.52 & 183.55 & 502.1 & 200.07 & 5.57 \\
\hline 341.5468 & 177.77 & 182.6 & 190.53 & 189.9 & 189.31 & 183.27 & 519.17 & 103 & 5.59 \\
\hline 342.0475 & 172.45 & 180.9 & 190.37 & 189.76 & 189.11 & 182.96 & 536.59 & 200.088 & 5.61 \\
\hline 342.5465 & 167.65 & 179.12 & 190.22 & 189.6 & 188.94 & 182.71 & 553.41 & 200.094 & 5.59 \\
\hline 343.0462 & 162.86 & 177.36 & 190.08 & 189.44 & 188.74 & 182.44 & 568.34 & 200.088 & 5.62 \\
\hline 343.546 & 158.28 & 175.62 & 189.94 & 189.3 & 188.56 & 182.17 & 579.27 & 200.133 & 5.61 \\
\hline 344.0458 & 153.61 & 173.79 & 189.8 & 189.14 & 188.37 & 181.91 & 586.66 & 200.106 & 5.63 \\
\hline 344.5457 & 149.06 & 171.92 & 189.64 & 188.98 & 188.17 & 181.66 & 591.64 & 200.121 & 5.61 \\
\hline 345.0465 & 144.72 & 170.16 & 189.5 & 188.82 & 187.97 & 181.41 & 595 & 200.121 & 5.57 \\
\hline 345.5463 & 140.64 & 168.32 & 189.34 & 188.66 & 187.81 & 181.14 & 586.13 & 200.148 & 5.56 \\
\hline 346.047 & 136.7 & 166.72 & 189.18 & 188.51 & 187.62 & 180.89 & 263.12 & 200.115 & 5.58 \\
\hline 346.5458 & 132.65 & 165.72 & 189.02 & 188.34 & 187.4 & 180.62 & 256.89 & 200.133 & 5.60 \\
\hline 347.0457 & 128.71 & 165.55 & 188.84 & 188.18 & 187.23 & 180.34 & 298.69 & 200.163 & 5.59 \\
\hline 347.5473 & 124.93 & 164.46 & 188.7 & 188.02 & 187.03 & 180.07 & 331.13 & 200.157 & 5.59 \\
\hline 348.0463 & 121.25 & 162.28 & 188.52 & 187.86 & 186.83 & 179.8 & 271.75 & 200.142 & 5.60 \\
\hline 348.547 & 117.69 & 161.21 & 188.4 & 187.7 & 186.66 & 179.57 & 314.51 & 200.208 & 5.63 \\
\hline 349.046 & 114.3 & 159.81 & 188.27 & 187.54 & 186.43 & 179.32 & 272.28 & 200.217 & 5.61 \\
\hline
\end{tabular}


Table B6. Continued.

\begin{tabular}{|c|c|c|c|c|c|c|c|c|c|}
\hline $\begin{array}{c}\text { Time } \\
\text { min }\end{array}$ & $\begin{array}{l}\mathrm{T} 1 \\
{ }^{\circ} \mathrm{C} \\
\end{array}$ & $\begin{array}{l}\mathrm{T} 2 \\
{ }^{\circ} \mathrm{C} \\
\end{array}$ & $\begin{array}{l}\mathrm{T} 3 \\
{ }^{\circ} \mathrm{C} \\
\end{array}$ & $\begin{array}{l}\mathrm{T} 4 \\
{ }^{\circ} \mathrm{C} \\
\end{array}$ & $\begin{array}{l}\mathrm{T5} \\
{ }^{\circ} \mathrm{C} \\
\end{array}$ & $\begin{array}{l}\mathrm{T} 6 \\
{ }^{\circ} \mathrm{C} \\
\end{array}$ & $\begin{array}{l}\text { Pinj } \\
\text { psig }\end{array}$ & $\begin{array}{l}\text { Pout } \\
\text { psig }\end{array}$ & $\begin{array}{c}\mathrm{Vw} \\
\mathrm{cc} / \mathrm{min}\end{array}$ \\
\hline 328.0462 & 242.09 & 195.14 & 195.29 & 194.68 & 194.77 & 193.94 & 284.05 & 200.712 & 5.56 \\
\hline 328.5458 & 243.67 & 195.05 & 195.23 & 194.43 & 194.41 & 192.92 & 297.65 & 201.006 & 5.59 \\
\hline 329.0457 & 245.41 & 194.86 & 195.22 & 194.3 & 194.27 & 192.06 & 271.87 & 201.048 & 5.62 \\
\hline 329.5465 & 246.55 & 194.5 & 195.16 & 194.25 & 194.19 & 191.47 & 299.89 & 200.928 & 5.60 \\
\hline 330.0463 & 247.34 & 193.94 & 195.05 & 194.12 & 194.07 & 190.99 & 325.24 & 200.943 & 5.61 \\
\hline 330.5462 & 224.93 & 193.07 & 193.48 & 192.76 & 192.13 & 189.8 & 349.74 & 199.626 & 5.61 \\
\hline 331.046 & 228.47 & 192.83 & 193.07 & 192.06 & 191.22 & 187.83 & 266.77 & 200.994 & 5.65 \\
\hline 331.5458 & 235.67 & 191.51 & 193.12 & 192.06 & 191.4 & 187.22 & 301.95 & 201.036 & 5.62 \\
\hline 332.0473 & 238.5 & 190.56 & 193.1 & 192.12 & 191.6 & 187.42 & 329.74 & 200.766 & 5.56 \\
\hline 332.5463 & 242.63 & 188.67 & 193.01 & 192.06 & 191.69 & 187.22 & 350.22 & 200.55 & 5.56 \\
\hline 333.0462 & 245.19 & 187.22 & 192.9 & 191.99 & 191.7 & 186.86 & 280.71 & .52 & 5.58 \\
\hline 333.546 & 247 & 185.82 & 192.8 & 191.94 & 191.67 & 186.59 & 307.12 & 538 & 5.60 \\
\hline 334.0458 & 248.04 & 184.89 & 192.67 & 191.85 & 191.6 & 186.38 & 327.3 & 577 & 5.60 \\
\hline 334.5475 & 248.38 & 184.14 & 192.56 & 191.76 & 191.49 & 186.18 & 342.86 & 200.583 & 5.61 \\
\hline 335.0463 & 247.85 & 183.56 & 192.46 & 191.67 & 191.42 & 186 & 355.35 & 200.58 & 5.62 \\
\hline 335.5462 & 246.81 & 182.9 & 192.33 & 191.56 & 191.27 & 185.81 & 367.84 & 589 & 5.64 \\
\hline 336.046 & 241.53 & 189.7 & 191.42 & 191.58 & 191.27 & 186.61 & .22 & 65 & 5.60 \\
\hline 336.5458 & 218.83 & 195.31 & 191.29 & 191.56 & 191.27 & 187.83 & 388.42 & 91 & 5.57 \\
\hline 337.0465 & 202.04 & 196.08 & 191.65 & 191.47 & 191.24 & 187.53 & 400.46 & 199.992 & 5.58 \\
\hline 337.5463 & 199.73 & 196.02 & 191.58 & 191.26 & 191.04 & 186.42 & 413.77 & 200.034 & 5.61 \\
\hline 338.0462 & 199.37 & 194.11 & 191.47 & 191.06 & 190.79 & 185.72 & 428.19 & 43 & 5.61 \\
\hline 338.547 & 197.91 & 192.07 & 191.35 & 190.88 & 190.56 & 185.23 & 2.17 & 25 & 5.61 \\
\hline 339.0458 & 195.92 & 190.52 & 191.24 & 190.7 & 190.35 & 184.84 & 4.42 & 25 & 5.62 \\
\hline 339.5465 & 193.77 & 188.84 & 191.1 & 190.54 & 190.11 & 184.45 & 464.82 & 200.091 & 5.63 \\
\hline 340.0463 & 190.49 & 187.14 & 190.96 & 190.36 & 189.92 & 184.1 & 475.62 & 200.064 & 5.61 \\
\hline 340.5462 & 186.96 & 185.72 & 190.81 & 190.22 & 189.7 & 183.84 & 487.48 & 200.037 & 5.56 \\
\hline 341.046 & 182.55 & 184.2 & 190.67 & 190.08 & 189.52 & 183.55 & 502.1 & 200.07 & 5.57 \\
\hline 341.5468 & 177.77 & 182.6 & 190.53 & 189.9 & 189.31 & 183.27 & 519.17 & 103 & 5.59 \\
\hline 342.0475 & 172.45 & 180.9 & 190.37 & 189.76 & 189.11 & 182.96 & 536.59 & 200.088 & 5.61 \\
\hline 342.5465 & 167.65 & 179.12 & 190.22 & 189.6 & 188.94 & 182.71 & 553.41 & 200.094 & 5.59 \\
\hline 343.0462 & 162.86 & 177.36 & 190.08 & 189.44 & 188.74 & 182.44 & 568.34 & 200.088 & 5.62 \\
\hline 343.546 & 158.28 & 175.62 & 189.94 & 189.3 & 188.56 & 182.17 & 579.27 & 200.133 & 5.61 \\
\hline 344.0458 & 153.61 & 173.79 & 189.8 & 189.14 & 188.37 & 181.91 & 586.66 & 200.106 & 5.63 \\
\hline 344.5457 & 149.06 & 171.92 & 189.64 & 188.98 & 188.17 & 181.66 & 591.64 & 200.121 & 5.61 \\
\hline 345.0465 & 144.72 & 170.16 & 189.5 & 188.82 & 187.97 & 181.41 & 595 & 200.121 & 5.57 \\
\hline 345.5463 & 140.64 & 168.32 & 189.34 & 188.66 & 187.81 & 181.14 & 586.13 & 200.148 & 5.56 \\
\hline 346.047 & 136.7 & 166.72 & 189.18 & 188.51 & 187.62 & 180.89 & 263.12 & 200.115 & 5.58 \\
\hline 346.5458 & 132.65 & 165.72 & 189.02 & 188.34 & 187.4 & 180.62 & 256.89 & 200.133 & 5.60 \\
\hline 347.0457 & 128.71 & 165.55 & 188.84 & 188.18 & 187.23 & 180.34 & 298.69 & 200.163 & 5.59 \\
\hline 347.5473 & 124.93 & 164.46 & 188.7 & 188.02 & 187.03 & 180.07 & 331.13 & 200.157 & 5.59 \\
\hline 348.0463 & 121.25 & 162.28 & 188.52 & 187.86 & 186.83 & 179.8 & 271.75 & 200.142 & 5.60 \\
\hline 348.547 & 117.69 & 161.21 & 188.4 & 187.7 & 186.66 & 179.57 & 314.51 & 200.208 & 5.63 \\
\hline 349.046 & 114.3 & 159.81 & 188.27 & 187.54 & 186.43 & 179.32 & 272.28 & 200.217 & 5.61 \\
\hline
\end{tabular}


Table B6. Continued.

\begin{tabular}{|c|c|c|c|c|c|c|c|c|c|}
\hline $\begin{array}{c}\text { Time } \\
\text { min }\end{array}$ & $\begin{array}{l}\mathrm{T} 1 \\
{ }^{\circ} \mathrm{C} \\
\end{array}$ & $\begin{array}{l}\mathrm{T} 2 \\
{ }^{\circ} \mathrm{C} \\
\end{array}$ & $\begin{array}{l}\mathrm{T} 3 \\
{ }^{\circ} \mathrm{C} \\
\end{array}$ & $\begin{array}{l}\mathrm{T} 4 \\
{ }^{\circ} \mathrm{C} \\
\end{array}$ & $\begin{array}{l}\mathrm{T} 5 \\
{ }^{\circ} \mathrm{C} \\
\end{array}$ & $\begin{array}{l}\mathrm{T} 6 \\
{ }^{\circ} \mathrm{C} \\
\end{array}$ & $\begin{array}{l}\text { Pinj } \\
\text { psig }\end{array}$ & $\begin{array}{l}\text { Pout } \\
\text { psig }\end{array}$ & $\begin{array}{c}\mathrm{Vw} \\
\mathrm{cc} / \mathrm{min}\end{array}$ \\
\hline 350.0473 & 107.87 & 155.71 & 188.04 & 187.22 & 186.07 & 178.84 & 266.21 & 200.244 & 5.56 \\
\hline 350.5463 & 104.68 & 154.92 & 187.9 & 187.06 & 185.88 & 178.59 & 298.67 & 200.193 & 5.57 \\
\hline 351.0462 & 102.02 & 153.6 & 187.8 & 186.9 & 185.7 & 178.34 & 263.81 & 200.208 & 5.60 \\
\hline 351.5468 & 99.22 & 153.01 & 187.67 & 186.74 & 185.52 & 178.11 & 230.34 & 200.184 & 5.59 \\
\hline 352.0467 & 96.48 & 152 & 187.55 & 186.57 & 185.31 & 177.88 & 270.61 & 200.22 & 5.59 \\
\hline 352.5475 & 93.62 & 150.77 & 187.43 & 186.41 & 185.13 & 177.63 & 295.28 & 200.211 & 5.61 \\
\hline 353.0473 & 91.08 & 149.24 & 187.27 & 186.25 & 184.94 & 177.38 & 246.91 & 200.223 & 5.61 \\
\hline 353.5462 & 88.6 & 148.28 & 187.09 & 186.07 & 184.74 & 177.11 & 272.05 & 200.247 & 5.62 \\
\hline 354.0505 & 86.08 & 147.16 & 186.95 & 185.91 & 184.55 & 176.85 & 242.39 & 200.238 & 5.57 \\
\hline 354.5467 & 83.53 & 145.69 & 186.81 & 185.75 & 184.37 & 176.6 & 264.83 & 200.25 & 5.55 \\
\hline 355.0475 & 81.55 & 143.84 & 186.67 & 185.59 & 184.18 & 176.35 & 249.57 & 217 & 5.56 \\
\hline 355.5463 & 79.54 & 142.68 & 186.55 & 185.44 & 183.98 & 176.1 & 241.29 & 211 & 5.60 \\
\hline 356.0472 & 88.04 & 136.58 & 186.34 & 185.22 & 183.77 & 175.87 & 203.94 & 337 & 5.60 \\
\hline 356.547 & 89.27 & 137.55 & 186.16 & 185.03 & 183.56 & 175.6 & 204.05 & 200.268 & 5.59 \\
\hline 357.0458 & 94.54 & 136.78 & 186.06 & 184.84 & 183.36 & 175.37 & 204.68 & 200.52 & 5.60 \\
\hline 357.5465 & 111.9 & 138.92 & 185.99 & 184.61 & 183.13 & 175.27 & 205.23 & 200.748 & 5.62 \\
\hline 358.0463 & 182.37 & 143.15 & 185.65 & 184.21 & 182.8 & 175.09 & 07 & 94 & 5.63 \\
\hline 358.5462 & 196.87 & 165.99 & 184.83 & 183.82 & 182.4 & 174.92 & 206.58 & 93 & 5.58 \\
\hline 359.046 & 199.45 & 191.64 & 183.54 & 183.75 & 182.32 & 175.3 & 207.98 & 201.444 & 5.58 \\
\hline 359.5458 & 200.55 & 195.5 & 183.27 & 183.7 & 182.18 & 175.46 & 206.83 & 201.048 & 5.57 \\
\hline 360.0467 & 209.79 & 196.01 & 183.31 & 183.58 & 182.02 & 175.23 & 205.72 & 200.622 & 5.60 \\
\hline 360.5473 & 219.94 & 196.03 & 183.42 & 183.41 & 181.81 & 174.82 & 205.3 & 556 & 5.61 \\
\hline 361.0462 & 231.97 & 196.12 & 183.41 & 183.21 & 181.62 & 174.45 & 205.35 & .55 & 5.61 \\
\hline 361.546 & 241.33 & 197.04 & 183.27 & 183.04 & 181.42 & 174.15 & 205.83 & 200.499 & 5.62 \\
\hline 362.0458 & 254.7 & 198.63 & 184.22 & 182.88 & 181.21 & 173.95 & 206.15 & 200.679 & 5.62 \\
\hline 362.5457 & 262.84 & 200.61 & 192.12 & 182.74 & 181.05 & 173.79 & 206.39 & 200.604 & 5.62 \\
\hline 363.0465 & 265.9 & 201.79 & 194.61 & 182.63 & 180.91 & 173.62 & 206.97 & 200.589 & 5.57 \\
\hline 363.5463 & 267.04 & 201.99 & 195.49 & 182.51 & 180.75 & 173.44 & 207.85 & 200.607 & 5.56 \\
\hline 364.047 & 265.89 & 202.43 & 196.02 & 182.46 & 180.63 & 173.36 & 208.44 & 200.775 & 5.56 \\
\hline 364.5458 & 263.96 & 202.79 & 196.38 & 182.47 & 180.53 & 173.27 & 208.81 & 200.853 & 5.60 \\
\hline 365.0457 & 260.78 & 202.78 & 196.67 & 182.58 & 180.51 & 173.2 & 209.15 & 200.895 & 5.58 \\
\hline 365.5465 & 257.31 & 203.17 & 196.87 & 182.71 & 180.46 & 173.12 & 209.45 & 200.979 & 5.59 \\
\hline 366.0472 & 254.1 & 203.29 & 197.07 & 182.8 & 180.47 & 173.05 & 209.92 & 201.108 & 5.62 \\
\hline 366.5462 & 249.58 & 203.16 & 197.29 & 182.88 & 180.42 & 173 & 210.51 & 201.138 & 5.61 \\
\hline 367.046 & 244.23 & 202.78 & 197.49 & 183.57 & 180.37 & 173 & 211.21 & 201.237 & 5.61 \\
\hline 367.5458 & 239.34 & 202.4 & 197.68 & 185.47 & 180.3 & 173.15 & 211.89 & 201.366 & 5.55 \\
\hline 368.0465 & 234.22 & 201.89 & 197.93 & 189.37 & 180.25 & 173.19 & 213.17 & 201.606 & 5.55 \\
\hline 368.5463 & 227.94 & 201.82 & 198.22 & 195.86 & 180.19 & 173.31 & 214.36 & 201.624 & 5.56 \\
\hline 369.047 & 222.64 & 200.91 & 198.44 & 197.37 & 180.14 & 173.44 & 215.34 & 202.107 & 5.59 \\
\hline 369.5468 & 220.12 & 201.28 & 198.64 & 197.86 & 180.05 & 173.58 & 216.01 & 202.092 & 5.59 \\
\hline 370.0467 & 220.9 & 201.28 & 198.74 & 198.04 & 179.98 & 173.71 & 216.15 & 201.675 & 5.60 \\
\hline 370.5465 & 222.42 & 200.8 & 198.54 & 197.85 & 179.92 & 173.93 & 214.55 & 202.359 & 5.60 \\
\hline 371.0463 & 223.55 & 200.7 & 198.39 & 197.8 & 179.9 & 173.99 & 214.16 & 201.591 & 5.60 \\
\hline
\end{tabular}


Table B6. Continued.

\begin{tabular}{|c|c|c|c|c|c|c|c|c|c|}
\hline $\begin{array}{c}\text { Time } \\
\text { min }\end{array}$ & $\begin{array}{l}\mathrm{T} 1 \\
{ }^{\circ} \mathrm{C} \\
\end{array}$ & $\begin{array}{l}\mathrm{T} 2 \\
{ }^{\circ} \mathrm{C} \\
\end{array}$ & $\begin{array}{l}\mathrm{T} 3 \\
{ }^{\circ} \mathrm{C} \\
\end{array}$ & $\begin{array}{l}\mathrm{T} 4 \\
{ }^{\circ} \mathrm{C} \\
\end{array}$ & $\begin{array}{l}\mathrm{T} 5 \\
{ }^{\circ} \mathrm{C} \\
\end{array}$ & $\begin{array}{l}\mathrm{T} 6 \\
{ }^{\circ} \mathrm{C} \\
\end{array}$ & $\begin{array}{l}\text { Pinj } \\
\text { psig }\end{array}$ & $\begin{array}{l}\text { Pout } \\
\text { psig }\end{array}$ & $\begin{array}{c}\mathrm{Vw} \\
\mathrm{cc} / \mathrm{min}\end{array}$ \\
\hline 372.046 & 224.01 & 201.32 & 198.17 & 197.63 & 194.91 & 174.06 & 213.12 & 200.925 & 5.58 \\
\hline 372.5458 & 224.41 & 201.02 & 198.03 & 197.49 & 197.11 & 174.04 & 212.18 & 201.609 & 5.54 \\
\hline 373.0465 & 226.01 & 200.43 & 197.91 & 197.44 & 197.6 & 174.01 & 212.03 & 201.753 & 5.54 \\
\hline 373.5463 & 225.98 & 200.24 & 197.89 & 197.46 & 197.7 & 173.98 & 212.06 & 201.693 & 5.56 \\
\hline 374.0462 & 225.5 & 200.3 & 197.92 & 197.47 & 197.74 & 173.95 & 212.13 & 201.396 & 5.60 \\
\hline 374.547 & 224.68 & 200.55 & 197.94 & 197.53 & 197.76 & 173.9 & 212.05 & 201.282 & 5.59 \\
\hline 375.0458 & 224.15 & 200.29 & 197.89 & 197.48 & 197.77 & 173.85 & 212.1 & 201.192 & 5.59 \\
\hline 375.5465 & 223.44 & 200.06 & 197.9 & 197.48 & 197.79 & 173.88 & 212.06 & 201.144 & 5.59 \\
\hline 376.0463 & 222.44 & 200.6 & 197.92 & 197.51 & 197.85 & 174.4 & 212.19 & 200.994 & 5.61 \\
\hline 376.5462 & 222.02 & 200.66 & 197.96 & 197.57 & 197.78 & 178.45 & 212.24 & 200.97 & 5.63 \\
\hline 377.046 & 221.84 & 200.17 & 197.91 & 197.45 & 197.7 & 186 & 1.75 & 201.246 & 5.58 \\
\hline 377.5468 & 221.58 & 200.21 & 197.86 & 197.45 & 197.83 & 191.7 & .85 & 468 & 5.55 \\
\hline 378.0467 & 221.69 & 200.5 & 197.87 & 197.47 & 197.85 & 195 & 1.81 & 399 & 5.57 \\
\hline 378.5463 & 219.46 & 201.2 & 197.94 & 197.53 & 197.89 & 196.28 & 212.11 & 201.147 & 5.60 \\
\hline 379.0462 & 220.99 & 200.71 & 197.95 & 197.56 & 197.92 & 196.86 & 212.43 & 201.285 & 5.60 \\
\hline 379.547 & 224.61 & 201.43 & 198.05 & 197.63 & 197.97 & 197.2 & 2.88 & 225 & 5.60 \\
\hline 380.0468 & 230.66 & 201.85 & 198.09 & 197.66 & 198.01 & 197.28 & .16 & 306 & 5.61 \\
\hline 380.5457 & 237.98 & 201.71 & 198.11 & 197.68 & 198.02 & 197.36 & 3.23 & 324 & 5.62 \\
\hline 381.0465 & 241.67 & 202.82 & 198.09 & 197.66 & 198.02 & 197.38 & 213.23 & 201.396 & 5.62 \\
\hline 381.5463 & 242.84 & 203.63 & 198.01 & 197.49 & 197.74 & 197.1 & 212.27 & 201.291 & 5.57 \\
\hline 382.0462 & 242.2 & 203.92 & 197.89 & 197.42 & 197.8 & 197.28 & 212.25 & 201.582 & 5.55 \\
\hline 382.5458 & 239.59 & 204.47 & 197.93 & 197.47 & 197.9 & 197.34 & .49 & 735 & 5.57 \\
\hline 383.0457 & 236.46 & 203.58 & 197.99 & 197.54 & 197.92 & 197.36 & 212.71 & 546 & 5.60 \\
\hline 383.5465 & 235.27 & 203.85 & 198.05 & 197.58 & 197.92 & 197.3 & 212.84 & .961 & 5.61 \\
\hline 384.0463 & 235.45 & 204.93 & 198.06 & 197.61 & 197.93 & 197.3 & 212.86 & .039 & 5.60 \\
\hline 384.547 & 235.87 & 204.49 & 198.04 & 197.56 & 197.92 & 197.27 & 212.72 & 200.979 & 5.60 \\
\hline 385.0468 & 235.71 & 204.56 & 198.03 & 197.56 & 197.89 & 197.26 & 212.88 & 763 & 5.65 \\
\hline 385.5458 & 234.66 & 204.68 & 198.03 & 197.57 & 197.84 & 197.26 & 2.78 & 895 & 5.62 \\
\hline 386.0465 & 233.89 & 204.02 & 198 & 197.54 & 197.9 & 197.25 & 212.49 & 200.76 & 5.56 \\
\hline 386.5463 & 233.88 & 203.61 & 197.97 & 197.52 & 197.9 & 197.26 & 212.42 & 200.724 & 5.57 \\
\hline 387.0462 & 233.13 & 203.21 & 197.92 & 197.44 & 197.82 & 197.19 & 211.93 & 200.928 & 5.59 \\
\hline 387.5468 & 233.01 & 203.34 & 197.91 & 197.46 & 197.89 & 197.32 & 212.11 & 200.964 & 5.61 \\
\hline 388.0458 & 232.59 & 203.84 & 197.9 & 197.47 & 197.95 & 197.36 & 212.07 & 201.432 & 5.62 \\
\hline 388.5475 & 232.68 & 204.29 & 197.94 & 197.51 & 197.96 & 197.37 & 212.19 & 201.192 & 5.60 \\
\hline 389.0463 & 231.97 & 203.8 & 197.94 & 197.51 & 197.91 & 197.33 & 212.18 & 201.138 & 5.62 \\
\hline 389.5462 & 231.94 & 203.66 & 197.93 & 197.5 & 197.97 & 197.36 & 212.03 & 201.123 & 5.64 \\
\hline 390.046 & 232.41 & 203.34 & 197.92 & 197.49 & 197.95 & 197.34 & 212.13 & 201.141 & 5.59 \\
\hline 390.5458 & 231.5 & 203.42 & 197.94 & 197.53 & 197.98 & 197.37 & 212 & 200.985 & 5.55 \\
\hline 391.0465 & 231.97 & 203.94 & 197.91 & 197.48 & 197.94 & 197.32 & 211.93 & 201.117 & 5.56 \\
\hline 391.5463 & 232.01 & 203.18 & 197.93 & 197.5 & 197.97 & 197.32 & 212.26 & 201.216 & 5.59 \\
\hline 392.0472 & 234.97 & 202.91 & 197.95 & 197.52 & 197.97 & 197.34 & 212.22 & 201.096 & 5.60 \\
\hline 392.546 & 237.59 & 202.72 & 197.94 & 197.49 & 197.99 & 197.35 & 212.12 & 201.285 & 5.60 \\
\hline 393.0458 & 237.88 & 204.76 & 197.92 & 197.49 & 197.96 & 197.33 & 212.05 & 201.027 & 5.62 \\
\hline
\end{tabular}


Table B6. Continued.

\begin{tabular}{|c|c|c|c|c|c|c|c|c|c|}
\hline $\begin{array}{c}\text { Time } \\
\text { min }\end{array}$ & $\begin{array}{l}\mathrm{T} 1 \\
{ }^{\circ} \mathrm{C} \\
\end{array}$ & $\begin{array}{l}\mathrm{T} 2 \\
{ }^{\circ} \mathrm{C} \\
\end{array}$ & $\begin{array}{l}\mathrm{T} 3 \\
{ }^{\circ} \mathrm{C} \\
\end{array}$ & $\begin{array}{l}\mathrm{T} 4 \\
{ }^{\circ} \mathrm{C} \\
\end{array}$ & $\begin{array}{l}\mathrm{T5} \\
{ }^{\circ} \mathrm{C} \\
\end{array}$ & $\begin{array}{l}\mathrm{T} 6 \\
{ }^{\circ} \mathrm{C} \\
\end{array}$ & $\begin{array}{l}\text { Pinj } \\
\text { psig }\end{array}$ & $\begin{array}{l}\text { Pout } \\
\text { psig }\end{array}$ & $\begin{array}{c}\mathrm{Vw} \\
\mathrm{cc} / \mathrm{min}\end{array}$ \\
\hline 394.0463 & 235.9 & 205.22 & 197.79 & 197.31 & 197.77 & 197.15 & 211.32 & 200.184 & 5.63 \\
\hline 394.5462 & 236.23 & 204.74 & 197.76 & 197.33 & 197.8 & 197.2 & 211.38 & 200.667 & 5.57 \\
\hline 395.046 & 237.08 & 206.29 & 197.78 & 197.35 & 197.85 & 197.26 & 211.51 & 200.889 & 5.57 \\
\hline 395.5458 & 236.54 & 204.88 & 197.84 & 197.41 & 197.91 & 197.3 & 211.92 & 201.288 & 5.57 \\
\hline 396.0475 & 235.61 & 203.64 & 197.88 & 197.45 & 197.95 & 197.34 & 211.98 & 201 & 5.60 \\
\hline 396.5463 & 235.28 & 204.6 & 197.92 & 197.49 & 197.97 & 197.36 & 212.18 & 201.078 & 5.60 \\
\hline 397.0462 & 234.75 & 204.94 & 197.94 & 197.53 & 197.99 & 197.37 & 212.23 & 201.165 & 5.60 \\
\hline 397.546 & 235.11 & 205.3 & 197.96 & 197.53 & 198.02 & 197.39 & 212.37 & 201.231 & 5.62 \\
\hline 398.0468 & 235.09 & 206.13 & 197.98 & 197.56 & 198.02 & 197.39 & 212.38 & 201.09 & 5.64 \\
\hline 398.5467 & 235.08 & 205.74 & 197.99 & 197.56 & 198.04 & 197.38 & 212.4 & 201.141 & 5.61 \\
\hline 399.0473 & 234.9 & 205.03 & 198.01 & 197.58 & 198.05 & 197.38 & 212.5 & 201 & 5.57 \\
\hline 399.5463 & 235.03 & 203.65 & 198.03 & 197.59 & 198.05 & 197.41 & 2.51 & 165 & 5.55 \\
\hline 400.0462 & 235.38 & 204.75 & 198.02 & 197.59 & 198.07 & 197.41 & 212.46 & 237 & 5.57 \\
\hline 400.5458 & 235.58 & 204.2 & 198.04 & 197.57 & 198.07 & 197.41 & 212.51 & 201.216 & 5.60 \\
\hline 401.0457 & 235.62 & 205.26 & 198.03 & 197.6 & 198.08 & 197.4 & 212.5 & 201.201 & 5.60 \\
\hline 401.5473 & 234.26 & 204.9 & 198.05 & 197.62 & 198.1 & 197.42 & 12.6 & 177 & 5.60 \\
\hline 402.0463 & 234.34 & 205.16 & 198.05 & 197.6 & 198.12 & 197.42 & .64 & 147 & 5.61 \\
\hline 402.547 & 234.25 & 203.84 & 198.05 & 197.61 & 198.16 & 197.41 & 212.6 & 162 & 5.61 \\
\hline 403.046 & 234.4 & 203.64 & 198.04 & 197.59 & 198.09 & 197.41 & 12.5 & 201.174 & 5.63 \\
\hline 403.5458 & 234.81 & 204.15 & 198.04 & 197.6 & 198.15 & 197.42 & 212.46 & 201.144 & 5.57 \\
\hline 404.0465 & 234.96 & 203.54 & 198.01 & 197.58 & 198.08 & 197.4 & 212.47 & 132 & 5.56 \\
\hline 404.5463 & 235.23 & 203.83 & 198.01 & 197.58 & 198.09 & 197.41 & 212.33 & 123 & 5.58 \\
\hline 405.0462 & 235.07 & 203.68 & 198 & 197.57 & 198.09 & 197.39 & 212.43 & 096 & 5.58 \\
\hline 405.5468 & 234.74 & 204.11 & 198 & 197.57 & 198.09 & 197.41 & 212.35 & .075 & 5.60 \\
\hline 406.0467 & 234.72 & 204.13 & 197.99 & 197.56 & 198.08 & 197.38 & 212.32 & .003 & 5.61 \\
\hline 406.5475 & 234.33 & 204.96 & 197.94 & 197.49 & 197.92 & 197.2 & 211.75 & 200.796 & 5.62 \\
\hline 407.0463 & 234.8 & 204.22 & 197.87 & 197.44 & 197.98 & 197.35 & 211.7 & 201.252 & 5.64 \\
\hline 407.547 & 235.36 & 204.55 & 197.87 & 197.48 & 198.02 & 197.35 & 211.95 & .024 & 5.61 \\
\hline 408.046 & 235.36 & 203.98 & 197.91 & 197.48 & 198 & 197.36 & 211.92 & 200.847 & 5.57 \\
\hline 408.5458 & 235.04 & 204.05 & 197.91 & 197.48 & 197.99 & 197.32 & 211.88 & 200.646 & 5.56 \\
\hline 409.0475 & 235.22 & 205.26 & 197.9 & 197.45 & 197.97 & 197.27 & 211.78 & 200.493 & 5.60 \\
\hline 409.5463 & 235.42 & 204.28 & 197.87 & 197.44 & 197.96 & 197.29 & 211.73 & 200.538 & 5.60 \\
\hline 410.0462 & 235.48 & 204.46 & 197.85 & 197.44 & 197.96 & 197.28 & 211.67 & 200.697 & 5.60 \\
\hline 410.546 & 235.3 & 204.96 & 197.87 & 197.42 & 197.96 & 197.28 & 211.89 & 200.577 & 5.61 \\
\hline 411.0467 & 234.59 & 205.63 & 197.87 & 197.5 & 197.98 & 197.32 & 211.77 & 200.562 & 5.64 \\
\hline 411.5465 & 234.39 & 204.84 & 197.88 & 197.46 & 198 & 197.32 & 211.86 & 200.583 & 5.64 \\
\hline 412.0463 & 234.9 & 205.35 & 197.88 & 197.41 & 197.82 & 197.18 & 211.52 & 200.541 & 5.58 \\
\hline 412.5462 & 235.01 & 205.08 & 197.83 & 197.4 & 197.92 & 197.26 & 211.49 & 201.06 & 5.57 \\
\hline 413.046 & 234.92 & 204.88 & 197.85 & 197.38 & 197.97 & 197.35 & 211.63 & 201.114 & 5.58 \\
\hline 413.5458 & 234.82 & 204.81 & 197.89 & 197.46 & 197.99 & 197.4 & 211.83 & 201.015 & 5.61 \\
\hline 414.0475 & 234.84 & 204.19 & 197.91 & 197.48 & 198.01 & 197.37 & 211.89 & 200.925 & 5.60 \\
\hline 414.5463 & 234.96 & 204.5 & 197.91 & 197.48 & 198.04 & 197.37 & 211.94 & 201.081 & 5.61 \\
\hline 415.0462 & 235.04 & 205.13 & 197.93 & 197.5 & 198.06 & 197.37 & 211.94 & 201.06 & 5.62 \\
\hline
\end{tabular}


Table B6. Continued.

\begin{tabular}{|c|c|c|c|c|c|c|c|c|c|}
\hline $\begin{array}{c}\text { Time } \\
\text { min }\end{array}$ & $\begin{array}{l}\mathrm{T} 1 \\
{ }^{\circ} \mathrm{C} \\
\end{array}$ & $\begin{array}{l}\mathrm{T} 2 \\
{ }^{\circ} \mathrm{C} \\
\end{array}$ & $\begin{array}{l}\mathrm{T} 3 \\
{ }^{\circ} \mathrm{C} \\
\end{array}$ & $\begin{array}{l}\mathrm{T} 4 \\
{ }^{\circ} \mathrm{C} \\
\end{array}$ & $\begin{array}{l}\mathrm{T} 5 \\
{ }^{\circ} \mathrm{C} \\
\end{array}$ & $\begin{array}{l}\mathrm{T} 6 \\
{ }^{\circ} \mathrm{C} \\
\end{array}$ & $\begin{array}{l}\text { Pinj } \\
\text { psig }\end{array}$ & $\begin{array}{l}\text { Pout } \\
\text { psig }\end{array}$ & $\begin{array}{c}\mathrm{Vw} \\
\mathrm{cc} / \mathrm{min}\end{array}$ \\
\hline 416.0458 & 234.88 & 204.22 & 197.93 & 197.52 & 198.06 & 197.38 & 212.03 & 200.901 & 5.59 \\
\hline 416.5467 & 234.92 & 203.65 & 197.92 & 197.51 & 198.14 & 197.37 & 211.99 & 200.862 & 5.56 \\
\hline 417.0465 & 234.85 & 204.76 & 197.94 & 197.53 & 198.05 & 197.37 & 212.01 & 200.766 & 5.55 \\
\hline 417.5472 & 234.76 & 206.02 & 197.92 & 197.51 & 198.05 & 197.39 & 212.02 & 200.856 & 5.58 \\
\hline 418.0462 & 235.09 & 206.09 & 197.93 & 197.51 & 198.07 & 197.35 & 211.97 & 200.865 & 5.59 \\
\hline 418.5468 & 235.13 & 206.02 & 197.93 & 197.5 & 198.05 & 197.35 & 211.96 & 200.826 & 5.60 \\
\hline 419.0457 & 235.74 & 205.11 & 197.93 & 197.5 & 198.04 & 197.34 & 1.91 & 200.607 & 5.62 \\
\hline 419.5465 & 235.47 & 205.31 & 197.91 & 197.5 & 198.11 & 197.3 & 211.87 & 200.379 & 5.62 \\
\hline 420.0463 & 235.24 & 206.44 & 197.88 & 197.47 & 198 & 197.27 & 211.66 & 200.4 & 5.64 \\
\hline 420.5462 & 235.26 & 206.49 & 197.86 & 197.43 & 197.97 & 197.25 & 211.57 & 200.424 & 5.61 \\
\hline 421.046 & 235.28 & 206.01 & 197.83 & 197.42 & 197.97 & 197.27 & .55 & 200.412 & 5.56 \\
\hline 421.5458 & 235.28 & 205.99 & 197.83 & 197.42 & 197.97 & 197.28 & 55 & 463 & 5.57 \\
\hline 422.0465 & 234.92 & 207.5 & 197.85 & 197.44 & 197.98 & 197.28 & .58 & 200.508 & 5.60 \\
\hline 422.5472 & 235.18 & 206.25 & 197.85 & 197.44 & 197.98 & 197.28 & 211.71 & 200.454 & 5.62 \\
\hline 423.047 & 235.52 & 205.32 & 197.85 & 197.44 & 197.98 & 197.28 & 211.6 & 200.475 & 5.61 \\
\hline 423.5468 & 236.13 & 206.04 & 197.84 & 197.44 & 197.98 & 197.28 & .68 & 367 & 5.63 \\
\hline 424.0467 & 235.72 & 205.29 & 197.84 & 197.44 & 198.02 & 197.28 & .72 & 469 & 5.65 \\
\hline 424.5465 & 235.72 & 204.84 & 197.86 & 197.45 & 198 & 197.29 & 1.67 & 200.508 & 5.62 \\
\hline 425.0463 & 235.6 & 204.95 & 197.86 & 197.43 & 197.99 & 197.29 & 211.65 & 200.511 & 5.59 \\
\hline 425.5462 & 235.54 & 206.08 & 197.84 & 197.45 & 198.01 & 197.29 & .69 & 200.481 & 5.60 \\
\hline 426.046 & 235.4 & 206.13 & 197.88 & 197.45 & 198.01 & 197.29 & 1.82 & 200.385 & 5.62 \\
\hline 426.5467 & 235.37 & 204.54 & 197.88 & 197.45 & 198.01 & 197.31 & .71 & 373 & 5.61 \\
\hline 427.0475 & 235.51 & 205.07 & 197.87 & 197.46 & 197.99 & 197.29 & 11.7 & 200.436 & 5.63 \\
\hline 427.5463 & 235.66 & 204.91 & 197.89 & 197.46 & 198.01 & 197.3 & 11.7 & 200.472 & 5.65 \\
\hline 428.0462 & 235.34 & 204.12 & 197.89 & 197.48 & 198.03 & 197.31 & 211.78 & 200.484 & 5.65 \\
\hline 428.546 & 235.32 & 204.25 & 197.89 & 197.48 & 198.01 & 197.3 & 211.76 & 200.397 & 5.59 \\
\hline 429.0458 & 235.54 & 205.39 & 197.89 & 197.46 & 198.03 & 197.3 & 1.75 & 200.478 & 5.59 \\
\hline 429.5465 & 235.63 & 204.55 & 197.87 & 197.48 & 198.03 & 197.28 & .74 & 200.43 & 5.61 \\
\hline 430.0463 & 235.43 & 205.14 & 197.89 & 197.48 & 198.04 & 197.28 & 211.78 & 200.382 & 5.62 \\
\hline 430.5462 & 235.29 & 205.16 & 197.89 & 197.48 & 198.11 & 197.3 & 211.78 & 200.481 & 5.60 \\
\hline 431.046 & 235.08 & 205.74 & 197.88 & 197.48 & 198.04 & 197.31 & 211.82 & 200.49 & 5.63 \\
\hline 431.5458 & 235.56 & 205.11 & 197.88 & 197.47 & 198.04 & 197.31 & 211.74 & 200.481 & 5.65 \\
\hline 432.0467 & 236.12 & 206.31 & 197.86 & 197.47 & 198.02 & 197.31 & 211.74 & 200.436 & 5.63 \\
\hline 432.5463 & 235.69 & 206.78 & 197.88 & 197.47 & 198.11 & 197.31 & 211.83 & 200.394 & 5.59 \\
\hline 433.0462 & 235.65 & 207.16 & 197.86 & 197.45 & 198.03 & 197.29 & 211.74 & 200.448 & 5.56 \\
\hline 433.546 & 235.55 & 207.01 & 197.9 & 197.47 & 198.03 & 197.31 & 211.69 & 200.424 & 5.63 \\
\hline 434.0458 & 236.09 & 205.33 & 197.87 & 197.47 & 198.03 & 197.29 & 211.7 & 200.373 & 5.60 \\
\hline 434.5457 & 235.62 & 205.08 & 197.87 & 197.47 & 198.04 & 197.31 & 211.66 & 200.301 & 5.62 \\
\hline 435.0465 & 236.14 & 205.89 & 197.87 & 197.45 & 198.01 & 197.29 & 211.6 & 200.412 & 5.65 \\
\hline 435.5472 & 235.87 & 205.78 & 197.85 & 197.46 & 198.01 & 197.26 & 211.61 & 199.737 & 5.65 \\
\hline 436.047 & 235.35 & 205.91 & 197.82 & 197.39 & 197.94 & 197.24 & 211.31 & 200.655 & 5.59 \\
\hline 436.5458 & 236.05 & 205.03 & 197.8 & 197.4 & 198 & 197.37 & 211.44 & 201.066 & 5.57 \\
\hline 437.0457 & 236.05 & 204.89 & 197.85 & 197.44 & 198.1 & 197.4 & 211.62 & 200.961 & 5.60 \\
\hline
\end{tabular}


Table B7-Temperature and production data for run 7

\begin{tabular}{|c|c|c|c|c|c|c|c|c|c|}
\hline $\begin{array}{l}\text { Time } \\
\text { min }\end{array}$ & $\begin{array}{l}\mathrm{T} 1 \\
{ }^{\circ} \mathrm{C}\end{array}$ & $\begin{array}{l}\mathrm{T} 2 \\
{ }^{\circ} \mathrm{C}\end{array}$ & $\begin{array}{l}\mathrm{T} 3 \\
{ }^{\circ} \mathrm{C}\end{array}$ & $\begin{array}{l}\mathrm{T} 4 \\
{ }^{\circ} \mathrm{C}\end{array}$ & $\begin{array}{l}\text { T5 } \\
{ }^{\circ} \mathrm{C}\end{array}$ & $\begin{array}{l}\mathrm{T} 6 \\
{ }^{\circ} \mathrm{C}\end{array}$ & $\begin{array}{l}\text { Pinj } \\
\text { psig }\end{array}$ & $\begin{array}{l}\text { Pout } \\
\text { psig }\end{array}$ & $\begin{array}{c}\mathrm{Vw} \\
\mathrm{cc} / \mathrm{min}\end{array}$ \\
\hline 0.547333 & 44.97 & 159.52 & 53.84 & 56.58 & 56.12 & 50.53 & 204.96 & 201.222 & 5.57 \\
\hline 1.047167 & 247.92 & 192.5 & 53.81 & 56.57 & 56.09 & 50.5 & 204.95 & 201.315 & 5.55 \\
\hline 1.547 & 248.83 & 196.07 & 53.82 & 56.6 & 56.06 & 50.49 & 205 & 201.255 & 5.55 \\
\hline 2.046833 & 248.64 & 196.73 & 53.83 & 56.59 & 56.09 & 50.52 & 204.99 & 201.324 & 5.57 \\
\hline 2.546667 & 247.84 & 197.02 & 53.84 & 56.6 & 56.1 & 50.55 & 205.13 & 201.339 & 5.59 \\
\hline 3.047333 & 246.33 & 196.83 & 53.87 & 56.63 & 56.15 & 50.58 & 203.88 & 200.292 & 5.61 \\
\hline 3.546333 & 244.11 & 196.63 & 53.9 & 56.64 & 56.18 & 50.59 & 204.03 & 200.397 & 5.60 \\
\hline 4.047 & 241.54 & 196.51 & 53.91 & 56.66 & 56.17 & 50.6 & 205.96 & 202.356 & 5.61 \\
\hline 4.546834 & 238.57 & 196.52 & 53.92 & 56.67 & 56.2 & 50.63 & 206.28 & 202.569 & 5.62 \\
\hline 5.046667 & 235.99 & 196.64 & 53.95 & 56.72 & 56.23 & 50.62 & 206.51 & 202.815 & 5.62 \\
\hline 5.5465 & 234.02 & 196.57 & 53.96 & 56.73 & 56.26 & 50.66 & 205.9 & 202.239 & 5.57 \\
\hline 6.047166 & 232.46 & 196.73 & 53.99 & 56.74 & 56.28 & 50.69 & 206.06 & 389 & 5.55 \\
\hline 6.547 & 231.16 & 196.74 & 54 & 56.75 & 56.27 & 50.66 & 206.22 & 437 & 5.56 \\
\hline 7.046 & 230.04 & 196.83 & 54 & 56.78 & 56.28 & 50.71 & 206.37 & 202.575 & 5.58 \\
\hline 7.546667 & 229.28 & 196.74 & 54.05 & 56.81 & 56.33 & 50.72 & 206.33 & 202.509 & 5.58 \\
\hline 8.048333 & 229.54 & 197.03 & 54.06 & 56.81 & 56.36 & 50.73 & 206.46 & 202.551 & 5.58 \\
\hline 8.547167 & 232.11 & 197.22 & 54.05 & 56.8 & 56.35 & 50.74 & 01 & 74 & 5.58 \\
\hline 9.047 & 234.57 & 197.28 & 54.06 & 56.76 & 56.32 & 50.75 & 04 & 101 & 5.58 \\
\hline 9.546833 & 234.85 & 197.38 & 54.07 & 56.78 & 56.33 & 50.76 & 20 & .08 & 5.62 \\
\hline 10.04667 & 234.42 & 197.66 & 54.11 & 56.79 & 56.36 & 50.73 & 206.15 & 202.14 & 5.64 \\
\hline 10.5475 & 234.06 & 197.56 & 54.16 & 56.8 & 56.35 & 50.75 & 206.35 & 202.074 & 5.58 \\
\hline 11.0 & 234.03 & 197.49 & 54.17 & 56.85 & 56.36 & 50.76 & 20 & 104 & 5.56 \\
\hline 11.5 & 233 & 197.5 & 54.24 & 56.82 & 56.33 & 50.77 & 49 & .11 & 5.57 \\
\hline 12.04683 & 23 & 197.55 & 54.3 & 56.85 & 56.39 & 50.78 & 206.52 & 202.044 & 5.59 \\
\hline 12.54667 & 233.21 & 197.34 & 54.39 & 56.84 & 56.4 & 50.77 & 206.74 & 202.092 & 5.60 \\
\hline 13.04833 & 233.11 & 197.47 & 54.47 & 56.85 & 56.47 & 50.8 & 206.84 & 202.11 & 5.59 \\
\hline 13.54733 & 233.32 & 197.52 & 54.57 & 56.84 & 56.42 & 50.81 & 206.98 & 125 & 5.58 \\
\hline 14.04617 & 232.86 & 197.74 & 54.72 & 56.84 & 56.43 & 50.82 & 207.31 & 092 & 5.61 \\
\hline 14.547 & 232.44 & 198.02 & 54.84 & 56.83 & 56.44 & 50.84 & 207.78 & 158 & 5.65 \\
\hline 15.04583 & 231.94 & 198.02 & 55.02 & 56.88 & 56.46 & 50.85 & 208.63 & 311 & 5.60 \\
\hline 15.5475 & 231.75 & 198.64 & 55.22 & 56.91 & 56.49 & 50.86 & 209.76 & 202.227 & 5.55 \\
\hline 16.04633 & 231.55 & 199.15 & 55.47 & 56.93 & 56.48 & 50.89 & 211.02 & 202.245 & 5.55 \\
\hline 16.54717 & & 199.98 & 55.73 & 56.89 & 56.53 & 50.89 & 211 & 254 & 5.57 \\
\hline 17.047 & 230 & 200.49 & 56.06 & 56.88 & 56.51 & 50.9 & 211.1 & 236 & 5.61 \\
\hline 17.54683 & 230.05 & 199.76 & 56.39 & 56.86 & 56.54 & 50.91 & 211.07 & 202.155 & 5.60 \\
\hline 18.04667 & 230.13 & 200.18 & 56.78 & 56.83 & 56.53 & 50.9 & 210.77 & 202.161 & 5.60 \\
\hline 18.54833 & 230.24 & 199.1 & 57.18 & 56.86 & 56.54 & 50.91 & 210.87 & 202.131 & 5.60 \\
\hline 19.04717 & 230.32 & 198.64 & 57.61 & 56.83 & 56.55 & 50.9 & 210.85 & 202.137 & 5.63 \\
\hline 19.54783 & 230.47 & 199.19 & 58.09 & 56.82 & 56.55 & 50.91 & 210.72 & 202.095 & 5.63 \\
\hline 20.04767 & 230.61 & 199.05 & 58.57 & 56.79 & 56.62 & 50.89 & 210.41 & 202.044 & 5.58 \\
\hline 20.54567 & 230.81 & 198.95 & 59.09 & 56.8 & 56.57 & 50.9 & 210.43 & 202.068 & 5.57 \\
\hline 21.0465 & 231.46 & 198.89 & 59.63 & 56.81 & 56.58 & 50.93 & 210.66 & 202.059 & 5.58 \\
\hline 21.54633 & 233.8 & 199.34 & 60.19 & 56.76 & 56.57 & 50.9 & 211.53 & 202.065 & 5.62 \\
\hline 22.04617 & 236.47 & 199.31 & 60.77 & 56.73 & 56.57 & 50.91 & 211.28 & 202.011 & 5.62 \\
\hline
\end{tabular}


Table B6. Continued.

\begin{tabular}{|c|c|c|c|c|c|c|c|c|c|}
\hline $\begin{array}{c}\text { Time } \\
\text { min }\end{array}$ & $\begin{array}{l}\mathrm{T} 1 \\
{ }^{\circ} \mathrm{C} \\
\end{array}$ & $\begin{array}{l}\mathrm{T} 2 \\
{ }^{\circ} \mathrm{C} \\
\end{array}$ & $\begin{array}{l}\mathrm{T} 3 \\
{ }^{\circ} \mathrm{C} \\
\end{array}$ & $\begin{array}{l}\mathrm{T} 4 \\
{ }^{\circ} \mathrm{C} \\
\end{array}$ & $\begin{array}{l}\mathrm{T5} \\
{ }^{\circ} \mathrm{C} \\
\end{array}$ & $\begin{array}{l}\mathrm{T} 6 \\
{ }^{\circ} \mathrm{C} \\
\end{array}$ & $\begin{array}{l}\text { Pinj } \\
\text { psig }\end{array}$ & $\begin{array}{l}\text { Pout } \\
\text { psig }\end{array}$ & $\begin{array}{c}\mathrm{Vw} \\
\mathrm{cc} / \mathrm{min}\end{array}$ \\
\hline 22.54683 & 237.09 & 199.46 & 61.42 & 56.71 & 56.56 & 50.91 & 211.6 & 201.993 & 5.62 \\
\hline 23.04667 & 236.9 & 199.07 & 62.07 & 56.7 & 56.59 & 50.9 & 211.82 & 202.035 & 5.63 \\
\hline 23.54833 & 236.69 & 199.51 & 62.74 & 56.71 & 56.62 & 50.93 & 212.15 & 201.963 & 5.63 \\
\hline 24.04717 & 236.79 & 199.45 & 63.46 & 56.7 & 56.62 & 50.92 & 212.25 & 201.96 & 5.59 \\
\hline 24.54617 & 236.88 & 199.38 & 64.21 & 56.69 & 56.67 & 50.94 & 212.71 & 201.939 & 5.57 \\
\hline 25.04683 & 236.8 & 199.44 & 64.97 & 56.66 & 56.68 & 50.93 & 212.83 & 201.921 & 5.56 \\
\hline 25.54667 & 236.74 & 199.9 & 65.75 & 56.65 & 56.72 & 50.92 & 213.03 & 201.93 & 5.59 \\
\hline 26.04833 & 236.8 & 200.12 & 66.61 & 56.63 & 56.75 & 50.95 & 213.4 & 201.951 & 5.61 \\
\hline 26.54817 & 236.97 & 200.61 & 67.49 & 56.62 & 56.79 & 50.95 & 213.77 & 201.885 & 5.59 \\
\hline 27.047 & 236.7 & 200.67 & 68.38 & 56.63 & 56.86 & 50.98 & 214.47 & 201.81 & 5.59 \\
\hline 27.54683 & 235.78 & 200.57 & 69.39 & 56.64 & 56.92 & 51.01 & 215.18 & 201.87 & 5.61 \\
\hline 28.04667 & 234.98 & 201.26 & 70.47 & 56.64 & 56.95 & 51.03 & 216.07 & 201.813 & 5.64 \\
\hline 28.54833 & 234.43 & 201.58 & 71.68 & 56.65 & 57.01 & 51.04 & 217.02 & 201.849 & 5.62 \\
\hline 29.04633 & 233.97 & 202.49 & 72.97 & 56.62 & 56.96 & 51.01 & 218.01 & 201.831 & 5.58 \\
\hline 29.54717 & 233.8 & 201.92 & 74.55 & 56.63 & 57.01 & 51.05 & 218.77 & 201.807 & 5.57 \\
\hline 30.046 & 234.15 & 201.62 & 76.29 & 56.61 & 57.01 & 51.06 & 218.91 & 201.786 & 5.59 \\
\hline 30.54667 & 234.19 & 201.36 & 78.19 & 56.6 & 57.04 & 51.05 & 218.94 & 201.717 & 5.62 \\
\hline 31.0465 & 234.39 & 201.35 & 80.2 & 56.59 & 57.06 & 51.17 & 218.89 & 201.669 & 5.60 \\
\hline 31.54733 & 234.52 & 200.89 & 82.27 & 56.56 & 57.11 & 51.37 & 218.77 & 201.582 & 5.61 \\
\hline 32.04617 & 234.91 & 200.91 & 84.26 & 56.6 & 57.13 & 51.45 & 218.71 & 201.6 & 5.63 \\
\hline 32.546 & 235.4 & 201.26 & 86.3 & 56.61 & 57.22 & 51.49 & 218.71 & 201.507 & 5.65 \\
\hline 33.04583 & 235.76 & 201.3 & 88.43 & 56.6 & 57.18 & 51.52 & 218.64 & 201.486 & 5.61 \\
\hline 33.5465 & 236.16 & 200.59 & 90.71 & 56.58 & 57.17 & 51.52 & 218.66 & 201.375 & 5.57 \\
\hline 34.04733 & 236.24 & 200.95 & 93.23 & 56.59 & 57.2 & 51.59 & 218.54 & 201.354 & 5.57 \\
\hline 34.54617 & 236.33 & 200.71 & 96.08 & 56.63 & 57.2 & 51.63 & 218.53 & 201.267 & 5.60 \\
\hline 35.047 & 236.46 & 200.82 & 99.28 & 56.64 & 57.23 & 51.7 & 218.55 & 201.213 & 5.62 \\
\hline 35.54683 & 236.44 & 200.81 & 102.86 & 56.68 & 57.27 & 51.8 & 218.94 & 201.168 & 5.59 \\
\hline 36.04667 & 236.53 & 201.25 & 106.74 & 56.69 & 57.32 & 51.84 & 219.13 & 201.096 & 5.61 \\
\hline 36.5465 & 236.52 & 201.29 & 110.9 & 56.73 & 57.34 & 51.91 & 219.57 & 201.006 & 5.62 \\
\hline 37.04617 & 236.2 & 201.06 & 115.47 & 56.8 & 57.37 & 51.99 & 220.05 & 200.91 & 5.63 \\
\hline 37.546 & 235.81 & 200.91 & 120.43 & 56.84 & 57.39 & 52.05 & 220.48 & 200.847 & 5.57 \\
\hline 38.04583 & 235.51 & 201.07 & 125.75 & 56.89 & 57.4 & 52.13 & 220.98 & 200.748 & 5.56 \\
\hline 38.54667 & 235.27 & 200.88 & 131.51 & 56.93 & 57.42 & 52.18 & 221.42 & 200.55 & 5.55 \\
\hline 39.04734 & 235 & 201.12 & 137.78 & 56.92 & 57.33 & 52.22 & 222.29 & 200.448 & 5.61 \\
\hline 39.54633 & 234.88 & 201.48 & 144.96 & 56.98 & 57.38 & 52.28 & 223.27 & 200.346 & 5.60 \\
\hline 40.04617 & 234.66 & 201.97 & 152.29 & 57.02 & 57.36 & 52.36 & 224.35 & 200.163 & 5.60 \\
\hline 40.54683 & 234.48 & 202.26 & 160.85 & 57.08 & 57.37 & 52.45 & 225.6 & 201.837 & 5.61 \\
\hline 41.04667 & 234.35 & 202.66 & 170.53 & 57.13 & 57.34 & 52.47 & 227.21 & 201.063 & 5.59 \\
\hline 41.54734 & 234.49 & 203.24 & 178.21 & 57.21 & 57.42 & 52.55 & 229.06 & 200.61 & 5.62 \\
\hline 42.04717 & 235.13 & 204 & 185.1 & 57.31 & 57.46 & 52.63 & 230.26 & 200.289 & 5.58 \\
\hline 42.54617 & 235.71 & 203.81 & 189.91 & 57.39 & 57.51 & 52.72 & 230.79 & 200.076 & 5.56 \\
\hline 43.046 & 236.09 & 204.15 & 192.9 & 57.51 & 57.53 & 52.8 & 231.17 & 201.981 & 5.56 \\
\hline 43.54667 & 236.25 & 203.87 & 194.95 & 57.63 & 57.52 & 52.88 & 231.46 & 201.492 & 5.57 \\
\hline 44.04734 & 236.63 & 204 & 196.53 & 57.71 & 57.58 & 52.96 & 231.56 & 201.891 & 5.61 \\
\hline
\end{tabular}


Table B6. Continued.

\begin{tabular}{|c|c|c|c|c|c|c|c|c|c|}
\hline $\begin{array}{c}\text { Time } \\
\text { min }\end{array}$ & $\begin{array}{l}\mathrm{T} 1 \\
{ }^{\circ} \mathrm{C} \\
\end{array}$ & $\begin{array}{l}\mathrm{T} 2 \\
{ }^{\circ} \mathrm{C} \\
\end{array}$ & $\begin{array}{l}\mathrm{T} 3 \\
{ }^{\circ} \mathrm{C} \\
\end{array}$ & $\begin{array}{l}\mathrm{T} 4 \\
{ }^{\circ} \mathrm{C} \\
\end{array}$ & $\begin{array}{l}\mathrm{T} 5 \\
{ }^{\circ} \mathrm{C} \\
\end{array}$ & $\begin{array}{l}\mathrm{T} 6 \\
{ }^{\circ} \mathrm{C} \\
\end{array}$ & $\begin{array}{l}\text { Pinj } \\
\text { psig }\end{array}$ & $\begin{array}{l}\text { Pout } \\
\text { psig }\end{array}$ & $\begin{array}{c}\mathrm{Vw} \\
\mathrm{cc} / \mathrm{min}\end{array}$ \\
\hline 44.54816 & 237.1 & 204.17 & 197.68 & 57.83 & 57.6 & 53.02 & 231.44 & 201.204 & 5.59 \\
\hline 45.04617 & 237.52 & 203.55 & 198.43 & 57.99 & 57.63 & 53.09 & 231.01 & 200.7 & 5.60 \\
\hline 45.546 & 237.78 & 203.55 & 199 & 58.13 & 57.63 & 53.19 & 230.59 & 200.064 & 5.60 \\
\hline 46.04583 & 238.09 & 203.63 & 199.47 & 58.32 & 57.66 & 53.23 & 229.86 & 199.452 & 5.63 \\
\hline 46.5475 & 238.54 & 203.02 & 199.76 & 58.54 & 57.64 & 53.29 & 229.11 & 201.459 & 5.61 \\
\hline 47.04633 & 238.96 & 203.01 & 199.95 & 58.81 & 57.63 & 53.33 & 228.62 & 200.625 & 5.57 \\
\hline 47.54617 & 238.98 & 202.51 & 200.1 & 59.15 & 57.69 & 53.36 & 228.05 & 199.818 & 5.55 \\
\hline 48.046 & 238.93 & 202.66 & 200.23 & 59.52 & 57.68 & 53.4 & 227.58 & 205.041 & 5.57 \\
\hline 48.54583 & 238.95 & 202.7 & 200.36 & 59.96 & 57.68 & 53.45 & 227.76 & 201.156 & 5.59 \\
\hline 49.0465 & 238.74 & 202.65 & 200.47 & 60.46 & 57.69 & 53.49 & 227.6 & 200.073 & 5.60 \\
\hline 49.54734 & 238.35 & 202.21 & 200.54 & 60.97 & 57.69 & 53.53 & 227.53 & 199.224 & 5.59 \\
\hline 50.04717 & 237.96 & 202.66 & 200.66 & 61.58 & 57.7 & 53.57 & 227.74 & 201.804 & 5.61 \\
\hline 50.546 & 237.75 & 202.72 & 200.77 & 62.18 & 57.72 & 53.6 & 228.2 & 200.547 & 5.61 \\
\hline 51.04683 & 237.45 & 202.8 & 200.92 & 62.82 & 57.71 & 53.62 & 228.63 & 199.524 & 5.61 \\
\hline 51.5475 & 237.37 & 202.94 & 201.05 & 63.53 & 57.73 & 53.68 & 228.98 & 699 & 62 \\
\hline 52.04633 & 237.09 & 203.22 & 201.18 & 64.27 & 57.77 & 53.75 & 229.64 & 197.955 & .58 \\
\hline 52.54617 & 236.63 & 203.15 & 201.36 & 65.05 & 57.76 & 53.81 & 230.53 & 197.277 & 5.58 \\
\hline 53.046 & 236.52 & 203.64 & 201.58 & 65.87 & 57.75 & 53.83 & 231.77 & 203.391 & 5.61 \\
\hline 53.54583 & 236.36 & 203.78 & 201.92 & 66.72 & 57.75 & 53.85 & 233.68 & 202.833 & 5.61 \\
\hline 54.04667 & 236.19 & 204.2 & 202.32 & 67.59 & 57.75 & 53.9 & 235.55 & 202.236 & 5.60 \\
\hline 54.5465 & 236.14 & 204.72 & 202.65 & 68.6 & 57.76 & 53.92 & 237.02 & 202.581 & 61 \\
\hline 55.04617 & 236.31 & 204.89 & 202.96 & 69.62 & 57.78 & 53.96 & 238.52 & 202.32 & 5.63 \\
\hline 55.546 & 236.51 & 205.09 & 203.21 & 70.64 & 57.77 & 54.03 & 239.72 & 201.921 & 5.63 \\
\hline 56.04583 & 236.66 & 205.04 & 203.43 & 71.78 & 57.77 & 54.07 & 240.54 & 201.543 & 5.58 \\
\hline 56.54667 & 237 & 205.44 & 203.56 & 72.99 & 57.78 & 54.15 & 241.01 & 201.117 & 5.56 \\
\hline 57.0465 & 237.31 & 205.46 & 203.67 & 74.19 & 57.76 & 54.17 & 241.4 & .787 & 5.57 \\
\hline 57.54633 & 237.39 & 205.41 & 203.7 & 75.51 & 57.79 & 54.22 & 241.42 & 200.382 & 5.60 \\
\hline 58.048 & 237.89 & 205.42 & 203.72 & 76.89 & 57.81 & 54.26 & 241.29 & 200.07 & 5.60 \\
\hline 58.54583 & 237.88 & 205.46 & 203.69 & 78.26 & 57.8 & 54.34 & 241.23 & 200.241 & 5.60 \\
\hline 59.04567 & 238.08 & 205.23 & 203.65 & 79.71 & 57.82 & 54.36 & 240.84 & 200.451 & 5.60 \\
\hline 59.54734 & 238.41 & 204.84 & 203.59 & 81.17 & 57.79 & 54.39 & 240.46 & 200.511 & 5.62 \\
\hline 60.04717 & 238.23 & 204.93 & 203.56 & 82.68 & 57.83 & 54.43 & 240.35 & 200.601 & 5.63 \\
\hline 60.547 & 238.07 & 204.92 & 203.52 & 84.25 & 57.83 & 54.45 & 240.11 & 200.352 & 5.59 \\
\hline 61.046 & 238.04 & 205.03 & 203.47 & 85.88 & 57.87 & 54.49 & 240.05 & 200.4 & 5.55 \\
\hline 61.54583 & 237.79 & 205.1 & 203.51 & 87.47 & 57.92 & 54.51 & 240.16 & 200.469 & 5.56 \\
\hline 62.0465 & 237.72 & 205.14 & 203.53 & 89.13 & 57.92 & 54.54 & 240.38 & 200.541 & 5.61 \\
\hline 62.54633 & 237.75 & 205 & 203.59 & 90.85 & 57.94 & 54.56 & 240.7 & 200.607 & 5.61 \\
\hline 63.047 & 237.62 & 205.4 & 203.65 & 92.59 & 57.93 & 54.58 & 241.23 & 200.664 & 5.58 \\
\hline 63.546 & 237.52 & 205.55 & 203.78 & 94.38 & 58.01 & 54.57 & 241.97 & 200.853 & 5.60 \\
\hline 64.04583 & 237.4 & 205.75 & 203.96 & 96.24 & 58.07 & 54.61 & 242.89 & 200.808 & 5.61 \\
\hline 64.5465 & 237.2 & 206.27 & 204.18 & 98.17 & 58.13 & 54.65 & 243.98 & 200.838 & 5.62 \\
\hline 65.04633 & 236.96 & 206.31 & 204.4 & 100.19 & 58.19 & 54.67 & 245.27 & 200.862 & 5.59 \\
\hline 65.54617 & 236.87 & 206.42 & 204.65 & 102.31 & 58.29 & 54.72 & 246.82 & 200.913 & 5.56 \\
\hline 66.04684 & 236.8 & 206.73 & 204.94 & 104.55 & 58.29 & 54.74 & 248.23 & 200.943 & 5.57 \\
\hline
\end{tabular}


Table B6. Continued.

\begin{tabular}{|c|c|c|c|c|c|c|c|c|c|}
\hline $\begin{array}{c}\text { Time } \\
\text { min }\end{array}$ & $\begin{array}{l}\mathrm{T} 1 \\
{ }^{\circ} \mathrm{C} \\
\end{array}$ & $\begin{array}{l}\mathrm{T} 2 \\
{ }^{\circ} \mathrm{C} \\
\end{array}$ & $\begin{array}{l}\mathrm{T} 3 \\
{ }^{\circ} \mathrm{C} \\
\end{array}$ & $\begin{array}{l}\mathrm{T} 4 \\
{ }^{\circ} \mathrm{C} \\
\end{array}$ & $\begin{array}{l}\mathrm{T} 5 \\
{ }^{\circ} \mathrm{C} \\
\end{array}$ & $\begin{array}{l}\mathrm{T} 6 \\
{ }^{\circ} \mathrm{C} \\
\end{array}$ & $\begin{array}{l}\text { Pinj } \\
\text { psig }\end{array}$ & $\begin{array}{l}\text { Pout } \\
\text { psig }\end{array}$ & $\begin{array}{c}\mathrm{Vw} \\
\mathrm{cc} / \mathrm{min}\end{array}$ \\
\hline 66.54583 & 236.75 & 206.97 & 205.23 & 106.96 & 58.44 & 54.78 & 249.62 & 200.784 & 5.58 \\
\hline 67.0475 & 236.85 & 207.27 & 205.48 & 109.51 & 58.54 & 54.8 & 250.9 & 200.931 & 5.61 \\
\hline 67.54633 & 236.99 & 207.15 & 205.68 & 112.2 & 58.64 & 54.84 & 251.94 & 200.943 & 5.63 \\
\hline 68.04617 & 237.21 & 207.51 & 205.87 & 115.04 & 58.74 & 54.85 & 252.85 & 200.946 & 5.60 \\
\hline 68.546 & 237.39 & 207.87 & 206.03 & 118.01 & 58.84 & 54.87 & 253.48 & 201.081 & 5.64 \\
\hline 69.04583 & 237.61 & 207.82 & 206.12 & 121.23 & 58.99 & 54.89 & 253.92 & 201.114 & 5.65 \\
\hline 69.5465 & 237.87 & 207.68 & 206.2 & 124.6 & 59.15 & 54.9 & 254.18 & 201.111 & 5.59 \\
\hline 70.04633 & 238.07 & 207.94 & 206.24 & 128.26 & 59.28 & 54.94 & 254.31 & 201.186 & 5.56 \\
\hline 70.54617 & 238.16 & 207.48 & 206.26 & 132.14 & 59.44 & 54.96 & 254.36 & 201.198 & 5.58 \\
\hline 71.046 & 238.38 & 207.71 & 206.26 & 136.24 & 59.58 & 54.98 & 254.27 & 201.294 & 5.61 \\
\hline 71.54684 & 238.61 & 207.66 & 206.26 & 140.52 & 59.75 & 55 & 254.23 & 315 & 5.60 \\
\hline 72.04666 & 238.81 & 207.32 & 206.25 & 144.97 & 59.91 & 55.03 & 254.42 & 339 & 5.61 \\
\hline 72.5465 & 238.39 & 207.38 & 206.29 & 149.84 & 60.08 & 55.03 & 254.47 & 18 & 5.62 \\
\hline 73.04617 & 238.01 & 207.45 & 206.31 & 154.89 & 60.25 & 55.03 & 254.75 & 138 & 5.63 \\
\hline 73.546 & 237.75 & 207.69 & 206.35 & 160.26 & 60.41 & 55.04 & 254.95 & 201.195 & 5.62 \\
\hline 74.04583 & 237.77 & 207.51 & 206.42 & 165.94 & 60.6 & 55.06 & 255.4 & 198 & 5.57 \\
\hline 74.54567 & 237.91 & 207.5 & 206.51 & 171.83 & 60.79 & 55.06 & 256.31 & 55 & 5.55 \\
\hline 75.0465 & 236.93 & 208.18 & 206.7 & 178.36 & 61 & 55.08 & 257.24 & 315 & 5.58 \\
\hline 75.54633 & 236.06 & 208.43 & 206.93 & 184.52 & 61.2 & 55.09 & 258.67 & 333 & 5.61 \\
\hline 76.04617 & 235.13 & 208.63 & 207.22 & 189.99 & 61.43 & 55.09 & 260.18 & 363 & 5.60 \\
\hline 76.54684 & 234.41 & 209 & 207.51 & 195.46 & 61.64 & 55.11 & 261.85 & .447 & 5.61 \\
\hline 77.04567 & 234.22 & 209.12 & 207.8 & 198.94 & 61.87 & 55.11 & 263.44 & 477 & 5.62 \\
\hline 77.5465 & 234.76 & 209.45 & 208.09 & 201.59 & 62.12 & 55.14 & 265.02 & 507 & 5.65 \\
\hline 78.04633 & 235.44 & 209.79 & 208.36 & 203.42 & 62.38 & 55.16 & 266.34 & 582 & 5.60 \\
\hline 78.54617 & 236.02 & 210.3 & 208.58 & 204.78 & 62.65 & 55.16 & 267.56 & 201.225 & 5.57 \\
\hline 79.046 & 236.53 & 210.44 & 208.78 & 205.77 & 62.94 & 55.18 & 268.4 & 201.312 & 5.58 \\
\hline 79.54583 & 237.01 & 210.55 & 208.89 & 206.51 & 63.24 & 55.21 & 268.97 & 201.372 & 5.61 \\
\hline 80.04733 & 237.53 & 210.32 & 208.98 & 206.94 & 63.59 & 55.23 & 269.16 & 429 & 5.61 \\
\hline 80.54633 & 237.95 & 210.24 & 208.95 & 207.25 & 63.91 & 55.23 & 269.09 & 441 & 5.60 \\
\hline 81.047 & 238.29 & 210.13 & 208.93 & 207.48 & 64.29 & 55.25 & 268.87 & .537 & 5.60 \\
\hline 81.546 & 238.65 & 209.9 & 208.88 & 207.61 & 64.64 & 55.24 & 268.49 & 201.561 & 5.63 \\
\hline 82.04666 & 238.9 & 209.9 & 208.81 & 207.65 & 65.03 & 55.24 & 268.02 & 201.594 & 5.62 \\
\hline 82.5475 & 238.91 & 209.78 & 208.74 & 207.68 & 65.45 & 55.3 & 267.75 & 201.633 & 5.57 \\
\hline 83.04633 & 238.61 & 209.85 & 208.69 & 207.72 & 65.87 & 55.28 & 267.35 & 201.621 & 5.57 \\
\hline 83.54617 & 238.57 & 209.64 & 208.64 & 207.69 & 66.27 & 55.28 & 267.06 & 201.75 & 5.58 \\
\hline 84.046 & 238.66 & 209.79 & 208.62 & 207.69 & 66.71 & 55.29 & 267.03 & 201.747 & 5.61 \\
\hline 84.54583 & 238.06 & 209.73 & 208.61 & 207.75 & 67.07 & 55.29 & 267.25 & 201.774 & 5.60 \\
\hline 85.0465 & 237.75 & 209.99 & 208.7 & 207.82 & 67.58 & 55.31 & 267.71 & 201.477 & 5.61 \\
\hline 85.54633 & 237.67 & 210.04 & 208.79 & 207.95 & 68.04 & 55.29 & 268.5 & 201.543 & 5.62 \\
\hline 86.04617 & 237.46 & 210.3 & 208.97 & 208.13 & 68.53 & 55.32 & 269.75 & 201.588 & 5.64 \\
\hline 86.546 & 236.63 & 210.51 & 209.26 & 208.42 & 69.02 & 55.36 & 271.42 & 201.711 & 5.62 \\
\hline 87.04583 & 235.95 & 211.14 & 209.57 & 208.74 & 69.57 & 55.38 & 273.27 & 201.726 & 5.57 \\
\hline 87.5465 & 236.05 & 211.54 & 209.89 & 209.07 & 70.12 & 55.38 & 275.13 & 201.741 & 5.56 \\
\hline 88.04733 & 236.59 & 211.49 & 210.2 & 209.39 & 70.73 & 55.4 & 276.8 & 201.762 & 5.59 \\
\hline
\end{tabular}


Table B6. Continued.

\begin{tabular}{|c|c|c|c|c|c|c|c|c|c|}
\hline $\begin{array}{c}\text { Time } \\
\text { min }\end{array}$ & $\begin{array}{l}\mathrm{T} 1 \\
{ }^{\circ} \mathrm{C} \\
\end{array}$ & $\begin{array}{l}\mathrm{T} 2 \\
{ }^{\circ} \mathrm{C}\end{array}$ & $\begin{array}{l}\mathrm{T} 3 \\
{ }^{\circ} \mathrm{C} \\
\end{array}$ & $\begin{array}{l}\mathrm{T} 4 \\
{ }^{\circ} \mathrm{C} \\
\end{array}$ & $\begin{array}{l}\mathrm{T5} \\
{ }^{\circ} \mathrm{C} \\
\end{array}$ & $\begin{array}{l}\mathrm{T} 6 \\
{ }^{\circ} \mathrm{C} \\
\end{array}$ & $\begin{array}{l}\text { Pinj } \\
\text { psig }\end{array}$ & $\begin{array}{l}\text { Pout } \\
\text { psig }\end{array}$ & $\begin{array}{c}\mathrm{Vw} \\
\mathrm{cc} / \mathrm{min}\end{array}$ \\
\hline 88.54617 & 236.82 & 211.69 & 210.45 & 209.66 & 71.39 & 55.44 & 278.01 & 201.828 & 5.62 \\
\hline 89.046 & 237.06 & 211.83 & 210.63 & 209.87 & 72.07 & 55.48 & 278.73 & 201.849 & 5.60 \\
\hline 89.54583 & 237.56 & 211.96 & 210.73 & 209.94 & 72.82 & 55.5 & 278.97 & 201.93 & 5.61 \\
\hline 90.04666 & 238.1 & 211.77 & 210.73 & 209.94 & 73.63 & 55.54 & 278.8 & 201.861 & 5.61 \\
\hline 90.5465 & 238.68 & 211.79 & 210.66 & 209.89 & 74.5 & 55.58 & 278.27 & 201.978 & 5.65 \\
\hline 91.04617 & 239.11 & 211.79 & 210.55 & 209.78 & 75.46 & 55.66 & 277.49 & 201.774 & 5.62 \\
\hline 91.546 & 239.06 & 211.34 & 210.39 & 209.64 & 76.5 & 55.67 & 276.68 & 201.867 & 5.56 \\
\hline 92.04684 & 238.9 & 211.42 & 210.24 & 209.5 & 77.57 & 55.74 & 275.77 & 201.912 & 5.56 \\
\hline 92.54567 & 238.9 & 211.56 & 210.08 & 209.35 & 78.75 & 55.8 & 274.76 & 201.711 & 5.58 \\
\hline 93.0465 & 239.24 & 211.07 & 209.88 & 209.17 & 79.99 & 55.86 & 273.71 & 201.81 & 5.60 \\
\hline 93.54716 & 239.53 & 211.12 & 209.72 & 208.99 & 81.3 & 55.92 & 272.84 & 201.831 & 5.60 \\
\hline 94.04617 & 239.23 & 211.01 & 209.6 & 208.87 & 82.66 & 55.98 & 272.27 & 201.846 & 5.61 \\
\hline 94.54684 & 238.07 & 210.89 & 209.49 & 208.78 & 84.02 & 56.04 & 271.83 & 201.927 & 5.62 \\
\hline 95.04567 & 237.07 & 210.95 & 209.48 & 208.75 & 85.44 & 56.12 & 271.75 & 201.966 & 5.64 \\
\hline 95.5465 & 236.33 & 210.7 & 209.48 & 208.73 & 86.96 & 56.16 & .92 & 579 & 5.60 \\
\hline 96.04633 & 235.73 & 210.84 & 209.57 & 208.84 & 88.43 & 56.24 & 272.67 & 627 & 5.57 \\
\hline 96.54617 & 235.25 & 211.19 & 209.74 & 209 & 90.02 & 56.28 & 273.87 & 834 & 5.58 \\
\hline 97.04684 & 235.23 & 211.38 & 209.95 & 209.2 & 91.64 & 56.34 & 275.16 & 201.768 & 5.61 \\
\hline 97.54583 & 235.71 & 211.73 & 210.17 & 209.4 & 93.41 & 56.41 & 276.3 & 201.972 & 5.61 \\
\hline 98.0465 & 236.15 & 211.89 & 210.33 & 209.58 & 95.18 & 56.49 & 276.99 & 201.885 & 5.61 \\
\hline 98.54633 & 236.76 & 212.03 & 210.41 & 209.67 & 97.19 & 56.57 & 277.14 & 246 & 5.62 \\
\hline 99.047 & 237.26 & 211.89 & 210.34 & 209.62 & 99.34 & 56.67 & 276.57 & 201.339 & 5.63 \\
\hline 99.546 & 237.66 & 211.75 & 210.25 & 209.53 & 101.64 & 56.8 & 275.83 & 201.441 & 5.61 \\
\hline 100.0467 & 238 & 211.56 & 210.11 & 209.37 & 104.1 & 56.92 & 274.75 & 201.654 & 5.56 \\
\hline 100.5465 & 238.56 & 211.17 & 209.88 & 209.18 & 106.8 & 57.05 & 273.37 & 201.603 & 5.57 \\
\hline 101.0463 & 238.86 & 211.17 & 209.61 & 208.9 & 109.68 & 57.21 & .77 & 621 & 5.59 \\
\hline 101.5462 & 239.01 & 210.62 & 209.33 & 208.63 & 112.82 & 57.38 & 270.13 & 201.54 & 5.61 \\
\hline 102.046 & 239.12 & 210.53 & 209.04 & 208.29 & 116.15 & 57.57 & 268.32 & 201.363 & 5.59 \\
\hline 102.5458 & 238.96 & 210.05 & 208.72 & 207.99 & 119.73 & 57.76 & 266.59 & 201.294 & 5.62 \\
\hline 103.0465 & 238.69 & 209.65 & 208.35 & 207.63 & 123.58 & 57.99 & 264.54 & 201.072 & 5.61 \\
\hline 103.5463 & 238.46 & 209.48 & 208.01 & 207.31 & 127.62 & 58.2 & 262.77 & 201.123 & 5.63 \\
\hline 104.0462 & 238.32 & 209.26 & 207.7 & 207.01 & 131.82 & 58.47 & 261.05 & 201.291 & 5.61 \\
\hline 104.546 & 238.11 & 208.87 & 207.4 & 206.72 & 136.18 & 58.76 & 259.6 & 201.297 & 5.57 \\
\hline 105.0477 & 237.89 & 208.91 & 207.15 & 206.47 & 140.73 & 59.08 & 258.31 & 201.357 & 5.56 \\
\hline 105.5465 & 237.82 & 208.66 & 206.96 & 206.24 & 145.43 & 59.4 & 257.23 & 201.339 & 5.58 \\
\hline 106.0473 & 237.57 & 208.61 & 206.76 & 206.08 & 150.47 & 59.79 & 256.46 & 201.306 & 5.60 \\
\hline 106.5472 & 237.38 & 208.45 & 206.66 & 205.94 & 155.57 & 60.2 & 255.94 & 201.426 & 5.59 \\
\hline 107.046 & 237.09 & 208.56 & 206.5 & 205.76 & 161.89 & 60.64 & 254.88 & 200.886 & 5.59 \\
\hline 107.5458 & 236.84 & 208.63 & 206.38 & 205.68 & 168.04 & 61.17 & 254.5 & 201.021 & 5.60 \\
\hline 108.0467 & 236.81 & 208.56 & 206.29 & 205.55 & 174.18 & 61.74 & 254 & 201.066 & 5.63 \\
\hline 108.5465 & 236.79 & 208.79 & 206.14 & 205.43 & 181.06 & 62.37 & 253.08 & 201.144 & 5.61 \\
\hline 109.0462 & 236.83 & 208.76 & 205.91 & 205.14 & 189.54 & 63.11 & 251.38 & 200.658 & 5.56 \\
\hline 109.546 & 236.87 & 208.37 & 205.58 & 204.84 & 194.74 & 63.92 & 249.59 & 200.772 & 5.56 \\
\hline 110.0468 & 237.03 & 207.67 & 205.2 & 204.48 & 198.61 & 64.89 & 247.51 & 200.844 & 5.57 \\
\hline
\end{tabular}


Table B6. Continued.

\begin{tabular}{|c|c|c|c|c|c|c|c|c|c|}
\hline $\begin{array}{c}\text { Time } \\
\text { min }\end{array}$ & $\begin{array}{l}\mathrm{T} 1 \\
{ }^{\circ} \mathrm{C} \\
\end{array}$ & $\begin{array}{l}\mathrm{T} 2 \\
{ }^{\circ} \mathrm{C} \\
\end{array}$ & $\begin{array}{l}\mathrm{T} 3 \\
{ }^{\circ} \mathrm{C} \\
\end{array}$ & $\begin{array}{l}\mathrm{T} 4 \\
{ }^{\circ} \mathrm{C} \\
\end{array}$ & $\begin{array}{l}\mathrm{T} 5 \\
{ }^{\circ} \mathrm{C} \\
\end{array}$ & $\begin{array}{l}\mathrm{T} 6 \\
{ }^{\circ} \mathrm{C} \\
\end{array}$ & $\begin{array}{l}\text { Pinj } \\
\text { psig }\end{array}$ & $\begin{array}{l}\text { Pout } \\
\text { psig }\end{array}$ & $\begin{array}{c}\mathrm{Vw} \\
\mathrm{cc} / \mathrm{min}\end{array}$ \\
\hline 110.5457 & 237.26 & 207.14 & 204.79 & 204.04 & 200.67 & 65.93 & 245.14 & 200.94 & 5.60 \\
\hline 111.0465 & 236.98 & 206.35 & 204.31 & 203.54 & 201.73 & 67.1 & 242.46 & 201.036 & 5.59 \\
\hline 111.5463 & 237.14 & 206.35 & 203.63 & 202.82 & 201.96 & 68.44 & 238.45 & 200.517 & 5.59 \\
\hline 112.0462 & 237.16 & 206.41 & 202.95 & 202.2 & 201.75 & 69.9 & 235.27 & 200.613 & 5.61 \\
\hline 112.5458 & 237.36 & 205.28 & 202.34 & 201.61 & 201.34 & 71.5 & 232.22 & 200.673 & 5.61 \\
\hline 113.0457 & 237.58 & 204.49 & 201.77 & 201.07 & 201.02 & 73.25 & 229.52 & 200.796 & 5.62 \\
\hline 113.5465 & 237.61 & 204.28 & 201.15 & 200.4 & 200.41 & 75.08 & 226.06 & 200.226 & 5.57 \\
\hline 114.0472 & 237.7 & 203.51 & 200.58 & 199.91 & 200.11 & 77.03 & 223.88 & 200.307 & 5.55 \\
\hline 114.5462 & 237.79 & 202.9 & 200.18 & 199.57 & 199.83 & 79.04 & 222.16 & 200.385 & 5.56 \\
\hline 115.0478 & 237.99 & 202.98 & 199.88 & 199.27 & 199.58 & 81.08 & 220.73 & 200.427 & 5.60 \\
\hline 115.5467 & 238.17 & 202.32 & 199.59 & 198.9 & 199.06 & 83.05 & 219.14 & 199.902 & 5.60 \\
\hline 116.0473 & 237.6 & 202.25 & 199.1 & 198.49 & 198.84 & 85.05 & 216.91 & 199.977 & 5.59 \\
\hline 116.5463 & 237.75 & 202.01 & 198.86 & 198.29 & 198.72 & 86.97 & 216.1 & 200.043 & 5.60 \\
\hline 117.047 & 237.89 & 201.3 & 198.72 & 198.18 & 198.61 & 88.84 & 215.58 & 200.121 & 5.62 \\
\hline 117.546 & 237.89 & 201.52 & 198.63 & 198.13 & 198.56 & 90.69 & 5.25 & 154 & 5.63 \\
\hline 118.0458 & 237.61 & 201.46 & 198.6 & 198.06 & 198.53 & 92.47 & .08 & 208 & 5.58 \\
\hline 118.5465 & 237.48 & 201.82 & 198.56 & 198.03 & 198.51 & 94.13 & 214.97 & .214 & 5.58 \\
\hline 119.0463 & 237.55 & 202.15 & 198.56 & 198.03 & 198.56 & 95.73 & 215.03 & 200.28 & 5.57 \\
\hline 119.5462 & 237.45 & 201.79 & 198.57 & 198.06 & 198.56 & 97.27 & 215.16 & 200.307 & 5.60 \\
\hline 468 & 237.07 & 201.81 & 198.6 & 198.01 & 198.37 & 98.79 & 4.92 & 836 & 5.61 \\
\hline 458 & 236.7 & 201.41 & 198.51 & 197.96 & 198.46 & 100.28 & .86 & 881 & 5.61 \\
\hline 121.0465 & 236.63 & 201.06 & 198.53 & 198.03 & 198.53 & 101.72 & 215.13 & 199.887 & 5.62 \\
\hline 121.5463 & 236.7 & 201.43 & 198.62 & 198.09 & 198.6 & 103.17 & 215.51 & 200.022 & 5.62 \\
\hline 122.0462 & 236.82 & 202.24 & 198.7 & 198.16 & 198.66 & 104.61 & 215.78 & 200.04 & 5.62 \\
\hline 122.546 & 237.02 & 202.21 & 198.75 & 198.21 & 198.71 & 106.05 & 215.94 & 200.082 & 5.57 \\
\hline 123.0458 & 236.94 & 201.85 & 198.77 & 198.25 & 198.73 & 107.5 & 216.11 & .112 & 5.56 \\
\hline 123.5465 & 236.81 & 201.94 & 198.7 & 198.07 & 198.41 & 108.97 & 215.07 & 199.62 & 5.56 \\
\hline 124.0463 & 236.6 & 202.55 & 198.54 & 198 & 198.49 & 110.54 & 214.84 & 199.668 & 5.60 \\
\hline 124.5462 & 236.83 & 202.08 & 198.52 & 198 & 198.5 & 112.16 & 214.82 & 199.695 & 5.58 \\
\hline 125.046 & 237.14 & 202.3 & 198.51 & 197.97 & 198.58 & 113.91 & 214.8 & 199.83 & 5.59 \\
\hline 125.5458 & 237.41 & 201.75 & 198.5 & 197.99 & 198.51 & 115.81 & 214.78 & 199.809 & 5.62 \\
\hline 126.0475 & 237.62 & 201.66 & 198.51 & 197.99 & 198.45 & 117.79 & 214.74 & 199.836 & 5.61 \\
\hline 126.5465 & 237.57 & 201.01 & 198.47 & 197.95 & 198.51 & 119.85 & 214.6 & 199.908 & 5.61 \\
\hline 127.0462 & 237.68 & 201.37 & 198.47 & 197.94 & 198.47 & 121.94 & 214.5 & 199.911 & 5.55 \\
\hline 127.547 & 237.7 & 201.96 & 198.19 & 197.54 & 197.95 & 124.78 & 212.46 & 199.44 & 5.55 \\
\hline 128.0458 & 237.52 & 201.75 & 197.97 & 197.45 & 197.95 & 128.78 & 212.13 & 199.491 & 5.56 \\
\hline 128.5457 & 237.54 & 201.02 & 197.92 & 197.42 & 197.94 & 133.11 & 211.96 & 199.551 & 5.59 \\
\hline 129.0465 & 237.43 & 201.75 & 197.9 & 197.38 & 197.94 & 137.33 & 211.87 & 199.644 & 5.59 \\
\hline 129.5472 & 237.57 & 201.45 & 197.89 & 197.37 & 197.92 & 141.33 & 211.82 & 199.638 & 5.60 \\
\hline 130.0462 & 237.5 & 202.6 & 197.89 & 197.39 & 197.92 & 144.93 & 211.75 & 199.638 & 5.60 \\
\hline 130.5458 & 237.67 & 202.08 & 197.87 & 197.37 & 197.92 & 148.14 & 211.7 & 199.704 & 5.60 \\
\hline 131.0457 & 237.88 & 201.54 & 197.85 & 197.33 & 197.92 & 151.24 & 211.73 & 199.728 & 5.63 \\
\hline 131.5465 & 237.35 & 201.45 & 197.69 & 197.01 & 197.4 & 156.43 & 210.08 & 199.233 & 5.58 \\
\hline 132.0472 & 236.97 & 201.49 & 197.48 & 196.96 & 197.5 & 161.61 & 209.8 & 199.308 & 5.54 \\
\hline
\end{tabular}


Table B6. Continued.

\begin{tabular}{|c|c|c|c|c|c|c|c|c|c|}
\hline $\begin{array}{c}\text { Time } \\
\text { min }\end{array}$ & $\begin{array}{l}\mathrm{T} 1 \\
{ }^{\circ} \mathrm{C} \\
\end{array}$ & $\begin{array}{l}\mathrm{T} 2 \\
{ }^{\circ} \mathrm{C}\end{array}$ & $\begin{array}{l}\mathrm{T} 3 \\
{ }^{\circ} \mathrm{C} \\
\end{array}$ & $\begin{array}{l}\mathrm{T} 4 \\
{ }^{\circ} \mathrm{C} \\
\end{array}$ & $\begin{array}{l}\mathrm{T5} \\
{ }^{\circ} \mathrm{C} \\
\end{array}$ & $\begin{array}{l}\mathrm{T} 6 \\
{ }^{\circ} \mathrm{C} \\
\end{array}$ & $\begin{array}{l}\text { Pinj } \\
\text { psig }\end{array}$ & $\begin{array}{c}\text { Pout } \\
\text { psig }\end{array}$ & $\begin{array}{c}\mathrm{Vw} \\
\mathrm{cc} / \mathrm{min}\end{array}$ \\
\hline 132.5462 & 237.22 & 201.35 & 197.46 & 196.94 & 197.5 & 164.93 & 209.83 & 199.392 & 5.54 \\
\hline 133.046 & 237.19 & 201.15 & 197.46 & 196.96 & 197.51 & 167.11 & 209.95 & 199.476 & 5.56 \\
\hline 133.5458 & 236.92 & 200.94 & 197.48 & 196.98 & 197.55 & 168.75 & 210.01 & 199.479 & 5.60 \\
\hline 134.0465 & 237.06 & 201.64 & 197.52 & 197 & 197.57 & 170.35 & 210.13 & 199.476 & 5.59 \\
\hline 134.5463 & 237.01 & 201.28 & 197.55 & 197.05 & 197.61 & 171.91 & 210.28 & 199.536 & 5.59 \\
\hline 135.047 & 237.33 & 201.01 & 197.57 & 197.07 & 197.64 & 173.57 & 210.48 & 199.542 & 5.59 \\
\hline 135.546 & 237.12 & 201.05 & 197.63 & 197.11 & 197.66 & 175.55 & 210.57 & 199.587 & 5.61 \\
\hline 136.0458 & 237.23 & 201.62 & 197.63 & 197.13 & 197.68 & 177.54 & 210.68 & 199.644 & 5.63 \\
\hline 136.5465 & 237.05 & 201.82 & 197.34 & 196.71 & 197.18 & 184.6 & 208.7 & 199.236 & 5.58 \\
\hline 137.0473 & 236.96 & 202 & 197.23 & 196.72 & 197.27 & 187.09 & 208.77 & 199.215 & 5.55 \\
\hline 137.547 & 237.01 & 201.23 & 197.25 & 196.75 & 197.31 & 188.9 & 208.94 & 199.26 & 5.57 \\
\hline 138.046 & 237.16 & 201.32 & 197.27 & 196.77 & 197.33 & 190.45 & 209.06 & 199.293 & 5.60 \\
\hline 138.5458 & 237.36 & 201.21 & 197.29 & 196.79 & 197.33 & 191.68 & 209.15 & 199.359 & 5.60 \\
\hline 139.0465 & 237.59 & 201.12 & 197.31 & 196.81 & 197.36 & 192.51 & 209.21 & 199.404 & 5.60 \\
\hline 139.5463 & 237.77 & 201.59 & 197.33 & 196.83 & 197.44 & 192.98 & .31 & 199.41 & 5.61 \\
\hline 140.0472 & 237.9 & 201.07 & 197.37 & 196.86 & 197.44 & 193.3 & .46 & 199.449 & 5.62 \\
\hline 140.547 & 238.04 & 200.79 & 197.38 & 196.88 & 197.47 & 193.55 & 209.58 & 199.5 & 5.62 \\
\hline 141.0458 & 238 & 201.41 & 197.28 & 196.69 & 197.15 & 194.98 & 208.51 & 199.032 & 5.57 \\
\hline 141.5465 & 237.83 & 200.98 & 197.15 & 196.63 & 197.2 & 195.18 & 208.4 & 199.059 & 5.55 \\
\hline 142.0463 & 237.99 & 201.04 & 197.15 & 196.63 & 197.22 & 195.32 & 3.46 & 199.119 & 5.57 \\
\hline 142.5472 & 238.15 & 200.82 & 197.17 & 196.65 & 197.24 & 195.51 & .52 & 199.158 & 5.60 \\
\hline 143.047 & 238.31 & 200.68 & 197.19 & 196.69 & 197.26 & 195.68 & 208.57 & 199.191 & 5.61 \\
\hline 143.5458 & 238.49 & 201.42 & 197.21 & 196.69 & 197.28 & 195.86 & 208.65 & 199.239 & 5.60 \\
\hline 144.0467 & 238.48 & 201.15 & 197.23 & 196.71 & 197.3 & 196.01 & 208.75 & 199.272 & 5.60 \\
\hline 144.5465 & 238.49 & 200.56 & 197.23 & 196.74 & 197.32 & 196.13 & 208.83 & 199.305 & 5.65 \\
\hline 145.048 & 238.1 & 201.44 & 197.28 & 196.78 & 197.35 & 196.21 & .97 & 335 & 5.62 \\
\hline 145.546 & 238.13 & 200.95 & 197.3 & 196.8 & 197.39 & 196.33 & 209.11 & 199.362 & 5.56 \\
\hline 146.0458 & 237.89 & 201.96 & 197.23 & 196.6 & 197.03 & 196.01 & 208.09 & 198.828 & 5.57 \\
\hline 146.5467 & 237.55 & 202.67 & 197.07 & 196.53 & 197.12 & 196.3 & 207.99 & 198.852 & 5.59 \\
\hline 147.0465 & 237.56 & 201.44 & 197.09 & 196.6 & 197.18 & 196.41 & 208.2 & 198.93 & 5.61 \\
\hline 147.5463 & 237.69 & 201.06 & 197.12 & 196.62 & 197.2 & 196.46 & 208.4 & 199.032 & 5.62 \\
\hline 148.0462 & 237.49 & 201.81 & 197.18 & 196.66 & 197.23 & 196.52 & 208.6 & 199.029 & 5.60 \\
\hline 148.5458 & 237.37 & 201.99 & 197.21 & 196.75 & 197.29 & 196.55 & 208.77 & 199.08 & 5.62 \\
\hline 149.0457 & 237.53 & 201.49 & 197.25 & 196.75 & 197.32 & 196.6 & 208.94 & 199.074 & 5.64 \\
\hline 149.5465 & 237.73 & 201.83 & 197.29 & 196.77 & 197.34 & 196.62 & 209.08 & 199.098 & 5.59 \\
\hline 150.0482 & 237.87 & 202.17 & 197.32 & 196.8 & 197.36 & 196.64 & 209.2 & 199.122 & 5.55 \\
\hline 150.5462 & 237.98 & 203.5 & 197.32 & 196.82 & 197.36 & 196.66 & 209.3 & 199.182 & 5.56 \\
\hline 151.046 & 238.05 & 202.25 & 197.36 & 196.86 & 197.39 & 196.68 & 209.38 & 199.155 & 5.59 \\
\hline 151.5458 & 238.02 & 201.44 & 197.38 & 196.86 & 197.4 & 196.72 & 209.48 & 199.212 & 5.60 \\
\hline 152.0465 & 237.87 & 201.75 & 197.24 & 196.63 & 197.09 & 196.36 & 208.3 & 198.654 & 5.60 \\
\hline 152.5472 & 237.89 & 202.21 & 197.13 & 196.59 & 197.16 & 196.54 & 208.31 & 198.711 & 5.62 \\
\hline 153.0462 & 237.73 & 201.5 & 197.16 & 196.65 & 197.22 & 196.55 & 208.47 & 198.768 & 5.62 \\
\hline 153.546 & 237.7 & 201 & 197.18 & 196.66 & 197.24 & 196.61 & 208.57 & 198.798 & 5.63 \\
\hline 154.0458 & 237.6 & 201.68 & 197.2 & 196.68 & 197.25 & 196.63 & 208.63 & 198.864 & 5.57 \\
\hline
\end{tabular}


Table B6. Continued.

\begin{tabular}{|c|c|c|c|c|c|c|c|c|c|}
\hline $\begin{array}{c}\text { Time } \\
\text { min }\end{array}$ & $\begin{array}{l}\mathrm{T} 1 \\
{ }^{\circ} \mathrm{C} \\
\end{array}$ & $\begin{array}{l}\mathrm{T} 2 \\
{ }^{\circ} \mathrm{C}\end{array}$ & $\begin{array}{l}\mathrm{T} 3 \\
{ }^{\circ} \mathrm{C} \\
\end{array}$ & $\begin{array}{l}\mathrm{T} 4 \\
{ }^{\circ} \mathrm{C} \\
\end{array}$ & $\begin{array}{l}\mathrm{T} 5 \\
{ }^{\circ} \mathrm{C} \\
\end{array}$ & $\begin{array}{l}\mathrm{T} 6 \\
{ }^{\circ} \mathrm{C} \\
\end{array}$ & $\begin{array}{l}\text { Pinj } \\
\text { psig }\end{array}$ & $\begin{array}{c}\text { Pout } \\
\text { psig }\end{array}$ & $\begin{array}{c}\mathrm{Vw} \\
\mathrm{cc} / \mathrm{min}\end{array}$ \\
\hline 154.5465 & 237.55 & 201.77 & 197.2 & 196.7 & 197.25 & 196.63 & 208.66 & 198.87 & 5.57 \\
\hline 155.0482 & 237.64 & 201.09 & 197.22 & 196.7 & 197.29 & 196.67 & 208.7 & 198.93 & 5.57 \\
\hline 155.5462 & 237.66 & 201.89 & 197.24 & 196.72 & 197.29 & 196.67 & 208.74 & 198.909 & 5.60 \\
\hline 156.046 & 237.73 & 201.54 & 197.26 & 196.76 & 197.31 & 196.72 & 208.76 & 199.05 & 5.60 \\
\hline 156.5458 & 237.77 & 200.36 & 197.24 & 196.76 & 197.33 & 196.72 & 208.79 & 198.942 & 5.60 \\
\hline 157.0465 & 237.82 & 201.14 & 197.26 & 196.75 & 197.35 & 196.74 & 208.85 & 198.963 & 5.62 \\
\hline 157.5463 & 237.84 & 201.16 & 197.28 & 196.76 & 197.35 & 196.72 & 208.92 & 198.996 & 5.64 \\
\hline 158.0462 & 237.84 & 200.93 & 197.29 & 196.78 & 197.37 & 196.74 & 208.98 & 199.017 & 5.61 \\
\hline 158.546 & 237.79 & 201.57 & 197.24 & 196.61 & 196.99 & 196.25 & 208.05 & 198.45 & 5.57 \\
\hline 159.0458 & 237.59 & 201.95 & 197.06 & 196.52 & 197.1 & 196.49 & 207.82 & 198.549 & 5.55 \\
\hline 159.5465 & 237.68 & 201.52 & 197.06 & 196.54 & 197.15 & 196.58 & 208.05 & 198.555 & 5.57 \\
\hline 160.0463 & 237.74 & 200.95 & 197.1 & 196.61 & 197.17 & 196.6 & 208.24 & 198.66 & 5.60 \\
\hline 160.5462 & 237.84 & 201.14 & 197.13 & 196.63 & 197.21 & 196.58 & 208.37 & 198.651 & 5.60 \\
\hline 161.046 & 237.99 & 202.18 & 197.17 & 196.65 & 197.22 & 196.61 & 208.5 & 198.681 & 5.60 \\
\hline 161. & 237.86 & 202.08 & 197.19 & 196.69 & 197.24 & 196.63 & 208.62 & 834 & 5.61 \\
\hline 162.0467 & 237.74 & 200.95 & 197.23 & 196.71 & 197.28 & 196.65 & 208.75 & 198.699 & 5.61 \\
\hline 162.5465 & 237.68 & 201.47 & 197.24 & 196.74 & 197.3 & 196.67 & 208.83 & 198.765 & 5.63 \\
\hline 163.0462 & 237.67 & 201.72 & 197.26 & 196.74 & 197.3 & 196.67 & 208.93 & 198.747 & 5.57 \\
\hline 163.547 & 237.67 & 201.26 & 197.3 & 196.78 & 197.32 & 196.69 & 209.01 & 198.804 & 5.56 \\
\hline 164.0458 & 237.63 & 201.63 & 197.28 & 196.78 & 197.33 & 196.69 & 209.11 & 198.774 & 5.58 \\
\hline 164 & 237.61 & 202.1 & 197.32 & 196.8 & 197.35 & 196.69 & 209.17 & 198.837 & .58 \\
\hline 165.0465 & 237.69 & 201.2 & 197.32 & 196.83 & 197.35 & 196.71 & 209.2 & 198.84 & 5.60 \\
\hline 165.5472 & 237.72 & 202.4 & 197.33 & 196.81 & 197.37 & 196.72 & 209.25 & 198.819 & 5.61 \\
\hline 166.0462 & 237.61 & 202.69 & 197.23 & 196.64 & 197.14 & 196.49 & 208.32 & 198.492 & 5.62 \\
\hline 166.5458 & 237.85 & 202.42 & 197.16 & 196.64 & 197.21 & 196.6 & 208.39 & 198.462 & 5.64 \\
\hline 167.0457 & 237.78 & 201.51 & 197.17 & 196.65 & 197.21 & 196.6 & 208.43 & 198.489 & 5.61 \\
\hline 167.5465 & 237.85 & 201.78 & 197.17 & 196.65 & 197.25 & 196.62 & 208.49 & 198.498 & 5.57 \\
\hline 168.0472 & 237.85 & 202.3 & 197.19 & 196.67 & 197.23 & 196.62 & 208.5 & 198.567 & 5.56 \\
\hline 168.5462 & 237.92 & 201.87 & 197.19 & 196.67 & 197.25 & 196.64 & 208.49 & 198.579 & 5.60 \\
\hline 169.0468 & 238.05 & 201.35 & 197.18 & 196.67 & 197.25 & 196.64 & 208.5 & 198.624 & 5.60 \\
\hline 169.5458 & 238.15 & 201.67 & 197.19 & 196.68 & 197.25 & 196.64 & 208.51 & 198.618 & 5.60 \\
\hline 170.0465 & 238.23 & 201.24 & 197.18 & 196.69 & 197.27 & 196.66 & 208.52 & 198.63 & 5.61 \\
\hline 170.5463 & 238.32 & 200.56 & 197.19 & 196.71 & 197.27 & 196.66 & 208.54 & 198.633 & 5.64 \\
\hline 171.0462 & 238.37 & 201.12 & 197.19 & 196.69 & 197.27 & 196.64 & 208.54 & 198.759 & 5.64 \\
\hline 171.5468 & 238.32 & 201.71 & 197.19 & 196.71 & 197.28 & 196.68 & 208.58 & 198.684 & 5.58 \\
\hline 172.0458 & 238.25 & 201.42 & 197.19 & 196.69 & 197.3 & 196.71 & 208.61 & 198.762 & 5.57 \\
\hline 172.5465 & 238.16 & 201.53 & 197.21 & 196.71 & 197.3 & 196.66 & 208.66 & 198.771 & 5.58 \\
\hline 173.0463 & 238.14 & 201.8 & 197.23 & 196.75 & 197.3 & 196.68 & 208.75 & 198.729 & 5.61 \\
\hline 173.5462 & 238 & 202.48 & 197.12 & 196.55 & 197.02 & 196.35 & 207.75 & 198.216 & 5.60 \\
\hline 174.046 & 237.94 & 202.82 & 197.05 & 196.55 & 197.14 & 196.52 & 207.88 & 198.279 & 5.61 \\
\hline 174.5458 & 238.03 & 202.01 & 197.09 & 196.59 & 197.14 & 196.57 & 208.17 & 198.306 & 5.62 \\
\hline 175.0475 & 238.14 & 201.58 & 197.14 & 196.59 & 197.18 & 196.59 & 208.33 & 198.33 & 5.62 \\
\hline 175.5463 & 238.21 & 203.3 & 197.14 & 196.66 & 197.23 & 196.59 & 208.46 & 198.387 & 5.59 \\
\hline 176.0462 & 238.23 & 203.07 & 197.18 & 196.68 & 197.25 & 196.61 & 208.56 & 198.438 & 5.56 \\
\hline
\end{tabular}


Table B6. Continued.

\begin{tabular}{|c|c|c|c|c|c|c|c|c|c|}
\hline $\begin{array}{c}\text { Time } \\
\text { min }\end{array}$ & $\begin{array}{l}\mathrm{T} 1 \\
{ }^{\circ} \mathrm{C} \\
\end{array}$ & $\begin{array}{l}\mathrm{T} 2 \\
{ }^{\circ} \mathrm{C} \\
\end{array}$ & $\begin{array}{l}\mathrm{T} 3 \\
{ }^{\circ} \mathrm{C} \\
\end{array}$ & $\begin{array}{l}\mathrm{T} 4 \\
{ }^{\circ} \mathrm{C} \\
\end{array}$ & $\begin{array}{l}\mathrm{T} 5 \\
{ }^{\circ} \mathrm{C} \\
\end{array}$ & $\begin{array}{l}\mathrm{T} 6 \\
{ }^{\circ} \mathrm{C} \\
\end{array}$ & $\begin{array}{l}\text { Pinj } \\
\text { psig }\end{array}$ & $\begin{array}{l}\text { Pout } \\
\text { psig }\end{array}$ & $\begin{array}{c}\mathrm{Vw} \\
\mathrm{cc} / \mathrm{min}\end{array}$ \\
\hline 176.546 & 238.23 & 201.8 & 197.22 & 196.7 & 197.25 & 196.61 & 208.67 & 198.435 & 5.55 \\
\hline 177.0458 & 238.25 & 202.25 & 197.22 & 196.71 & 197.27 & 196.62 & 208.74 & 198.468 & 5.58 \\
\hline 177.5465 & 238.36 & 202.28 & 197.23 & 196.73 & 197.29 & 196.64 & 208.8 & 198.465 & 5.59 \\
\hline 178.0463 & 238.45 & 201.68 & 197.25 & 196.75 & 197.31 & 196.64 & 208.86 & 198.504 & 5.60 \\
\hline 178.5462 & 238.48 & 202.52 & 197.25 & 196.75 & 197.31 & 196.64 & 208.89 & 198.492 & 5.62 \\
\hline 179.046 & 238.41 & 201.48 & 197.27 & 196.75 & 197.32 & 196.66 & 208.9 & 198.501 & 5.62 \\
\hline 179.5458 & 238.36 & 201.66 & 197.27 & 196.77 & 197.32 & 196.68 & 208.9 & 198.579 & 5.64 \\
\hline 180.0467 & 238.3 & 202.19 & 197.27 & 196.77 & 197.32 & 196.68 & 208.9 & 198.525 & 5.61 \\
\hline 180.5465 & 238.16 & 202.45 & 197.27 & 196.77 & 197.34 & 196.7 & 208.94 & 198.534 & 5.56 \\
\hline 181. & 238.12 & 202.21 & 197.29 & 196.75 & 197.34 & 196.7 & 208.92 & 576 & 5.57 \\
\hline 181.546 & 237.95 & 202.84 & 197.22 & 196.64 & 197.11 & 196.45 & 208.2 & 198.315 & 5.60 \\
\hline 182.0458 & 237.89 & 202.18 & 197.13 & 196.61 & 197.2 & 196.59 & 208.16 & 198.246 & 5.62 \\
\hline 182.5457 & 237.91 & 201.71 & 197.13 & 196.63 & 197.2 & 196.61 & 208.2 & 198.387 & 5.61 \\
\hline 183.0465 & 237.95 & 201.77 & 197.11 & 196.65 & 197.22 & 196.61 & 208.24 & 198.282 & 5.63 \\
\hline 63 & 238.04 & 201 & 197.13 & 196.66 & 197.22 & 196.61 & 25 & 12 & 5.65 \\
\hline 184 & 238.11 & 200 & 197.11 & 196.63 & 197.24 & 196.61 & & & .62 \\
\hline 458 & 238.11 & 201.25 & 197.15 & 196.63 & 197.24 & 196.63 & 20 & & 5.59 \\
\hline 185.0457 & 238.18 & 201.66 & 197.15 & 196.66 & 197.26 & 196.61 & 208.32 & 198.318 & 5.60 \\
\hline 185.5465 & 238.2 & 201.55 & 197.15 & 196.66 & 197.26 & 196.61 & 208.39 & 198.393 & 5.62 \\
\hline 163 & 238.14 & 201.07 & 197.17 & 196.68 & 197.27 & 196.65 & 20 & 396 & 5.61 \\
\hline 62 & 238.16 & 202 & 197.18 & 196.68 & 197.27 & 196.66 & 20 & 05 & 5.63 \\
\hline 046 & 238.18 & 202.14 & 197.2 & 196.7 & 197.27 & 196.65 & 208.59 & 399 & 5.65 \\
\hline 187.5458 & 238.22 & 201.93 & 197.22 & 196.72 & 197.31 & 196.7 & 208.62 & 198.471 & 5.65 \\
\hline 188.0465 & 238.25 & 202.63 & 197.22 & 196.72 & 197.33 & 196.67 & 208.72 & 198 & 5.59 \\
\hline 463 & 238.29 & 202.5 & 197.26 & 196.72 & 197.2 & 196.52 & 208.69 & 096 & 5.59 \\
\hline 189 & 238.27 & 202.97 & 197.2 & 196.65 & 197.22 & 196.61 & 208.42 & 207 & 5.61 \\
\hline 189.546 & 238.29 & 203.06 & 197.17 & 196.65 & 197.22 & 196.63 & 208.44 & 198.276 & 5.62 \\
\hline 190.0458 & 238.27 & 203.18 & 197.19 & 196.7 & 197.26 & 196.63 & 208.52 & 198.306 & 5.60 \\
\hline 190.5465 & 238.29 & 202.25 & 197.2 & 196.7 & 197.28 & 196.63 & 208.61 & 198.324 & 5.63 \\
\hline 191.0463 & 238.25 & 202.31 & 197.2 & 196.7 & 197.28 & 196.65 & 208.68 & 198.306 & 5.65 \\
\hline 191.5462 & 238.27 & 201.86 & 197.24 & 196.72 & 197.29 & 196.67 & 208.74 & 399 & 5.63 \\
\hline 046 & 238.22 & 201 & 197.24 & 196.74 & 197.29 & 196.67 & 208.78 & 354 & 5.59 \\
\hline 192.5458 & 238.27 & 201.93 & 197.26 & 196.76 & 197.31 & 196.69 & 208.8 & 198.405 & 5.56 \\
\hline 193.0465 & 238.17 & 203.02 & 197.26 & 196.78 & 197.33 & 196.69 & 208.83 & 198.405 & 5.63 \\
\hline 193.5463 & 238.06 & 203.13 & 197.26 & 196.76 & 197.33 & 196.69 & 208.86 & 198.405 & 5.60 \\
\hline 194.0462 & 238.06 & 201.59 & 197.26 & 196.76 & 197.33 & 196.69 & 208.87 & 198.408 & 5.62 \\
\hline 194.546 & 237.95 & 201.48 & 197.28 & 196.77 & 197.35 & 196.7 & 208.83 & 198.447 & 5.65 \\
\hline 195.0458 & 237.97 & 201.58 & 197.28 & 196.76 & 197.33 & 196.72 & 208.85 & 198.435 & 5.65 \\
\hline 195.5465 & 238.06 & 202.47 & 197.24 & 196.78 & 197.35 & 196.72 & 208.82 & 198.453 & 5.59 \\
\hline 196.0463 & 238.19 & 201.54 & 197.26 & 196.76 & 197.35 & 196.71 & 208.82 & 198.507 & 5.57 \\
\hline 196.5462 & 238.22 & 201.9 & 197.24 & 196.72 & 197.26 & 196.62 & 208.55 & 198.216 & 5.60 \\
\hline 346 & 238.19 & 202.26 & 197.21 & 196.69 & 197.24 & 196.65 & 208.46 & 198.318 & 5.61 \\
\hline 197.5458 & 238.26 & 202.51 & 197.17 & 196.65 & 197.24 & 196.65 & 208.43 & 198.3 & 5.59 \\
\hline 198.0467 & 238.28 & 202.94 & 197.17 & 196.67 & 197.26 & 196.67 & 208.42 & 198.327 & 5.60 \\
\hline
\end{tabular}


Table B6. Continued.

\begin{tabular}{|c|c|c|c|c|c|c|c|c|c|}
\hline $\begin{array}{c}\text { Time } \\
\text { min }\end{array}$ & $\begin{array}{l}\mathrm{T} 1 \\
{ }^{\circ} \mathrm{C} \\
\end{array}$ & $\begin{array}{l}\mathrm{T} 2 \\
{ }^{\circ} \mathrm{C} \\
\end{array}$ & $\begin{array}{l}\mathrm{T} 3 \\
{ }^{\circ} \mathrm{C} \\
\end{array}$ & $\begin{array}{l}\mathrm{T} 4 \\
{ }^{\circ} \mathrm{C} \\
\end{array}$ & $\begin{array}{l}\mathrm{T5} \\
{ }^{\circ} \mathrm{C} \\
\end{array}$ & $\begin{array}{l}\mathrm{T} 6 \\
{ }^{\circ} \mathrm{C} \\
\end{array}$ & $\begin{array}{l}\text { Pinj } \\
\text { psig }\end{array}$ & $\begin{array}{l}\text { Pout } \\
\text { psig }\end{array}$ & $\begin{array}{c}\mathrm{Vw} \\
\mathrm{cc} / \mathrm{min}\end{array}$ \\
\hline 198.5465 & 238.31 & 202.63 & 197.17 & 196.69 & 197.26 & 196.65 & 208.39 & 198.297 & 5.61 \\
\hline 199.0472 & 238.33 & 201.63 & 197.15 & 196.67 & 197.26 & 196.65 & 208.44 & 198.351 & 5.63 \\
\hline 199.547 & 238.28 & 201.43 & 197.17 & 196.69 & 197.26 & 196.67 & 208.44 & 198.369 & 5.59 \\
\hline 200.0458 & 238.26 & 201.83 & 197.17 & 196.69 & 197.28 & 196.67 & 208.47 & 198.411 & 5.57 \\
\hline 200.5467 & 238.28 & 202.08 & 197.17 & 196.69 & 197.28 & 196.67 & 208.49 & 198.414 & 5.56 \\
\hline 201.0465 & 238.24 & 202.56 & 197.19 & 196.69 & 197.28 & 196.67 & 208.58 & 198.45 & 5.61 \\
\hline 201.5463 & 238.15 & 203.3 & 197.21 & 196.71 & 197.3 & 196.69 & 208.64 & 198.402 & 5.59 \\
\hline 202.047 & 238.28 & 203.17 & 197.23 & 196.73 & 197.32 & 196.69 & 208.67 & 198.474 & 5.61 \\
\hline 202.5458 & 238.33 & 202.74 & 197.23 & 196.74 & 197.32 & 196.71 & 208.77 & 198.441 & 5.60 \\
\hline 203.0457 & 238.33 & 202.22 & 197.25 & 196.74 & 197.34 & 196.71 & 208.83 & 198.468 & 5.63 \\
\hline 203.5465 & 238.42 & 201.9 & 197.26 & 196.76 & 197.35 & 196.73 & 208.9 & 198.459 & 5.61 \\
\hline 204.0463 & 238.44 & 201.85 & 197.28 & 196.78 & 197.37 & 196.73 & 209 & 198.54 & 5.58 \\
\hline 204.5462 & 238.44 & 201.17 & 197.3 & 196.8 & 197.37 & 196.73 & 209.12 & 198.507 & 5.58 \\
\hline 205.046 & 238.41 & 202.15 & 197.28 & 196.73 & 197.3 & 196.64 & 208.8 & 198.192 & 5.60 \\
\hline 205.5458 & 238.39 & 202.78 & 197.25 & 196.74 & 197.3 & 196.67 & 208.84 & 198.282 & 5.60 \\
\hline 206.0465 & 238.34 & 202.78 & 197.27 & 196.77 & 197.32 & 196.68 & 208.92 & 315 & 5.61 \\
\hline 206.5463 & 238.23 & 202.6 & 197.28 & 196.77 & 197.36 & 196.69 & 208.96 & 24 & 5.60 \\
\hline 207.0462 & 238.16 & 203.87 & 197.28 & 196.78 & 197.39 & 196.69 & 209 & 198 & 5.61 \\
\hline 207.5468 & 238.14 & 203.89 & 197.3 & 196.8 & 197.36 & 196.75 & 209.04 & 198.372 & 5.62 \\
\hline 208.0458 & 238.03 & 202.14 & 197.3 & 196.8 & 197.38 & 196.71 & 209.04 & 198.39 & 5.57 \\
\hline 208.5465 & 238.28 & 202.14 & 197.32 & 196.8 & 197.38 & 196.71 & 209.07 & 198.408 & 5.56 \\
\hline 209.0473 & 238.28 & 201.53 & 197.3 & 196.82 & 197.38 & 196.73 & 209.04 & 396 & 5.56 \\
\hline 209.5462 & 238.3 & 202.6 & 197.3 & 196.8 & 197.38 & 196.73 & 209.03 & 198.432 & 5.60 \\
\hline 210.046 & 238.28 & 203.19 & 197.3 & 196.8 & 197.34 & 196.73 & 209.02 & 198.456 & 5.59 \\
\hline 210.5458 & 238.66 & 203.75 & 197.29 & 196.79 & 197.36 & 196.73 & 209.01 & 198.441 & 5.59 \\
\hline 211.0465 & 239.16 & 202.44 & 197.29 & 196.79 & 197.38 & 196.73 & 209 & 198.507 & 5.59 \\
\hline 211.5463 & 239.23 & 203.14 & 197.31 & 196.79 & 197.38 & 196.75 & 208.95 & 198.54 & 5.61 \\
\hline 212.0462 & 238.91 & 201.37 & 197.29 & 196.79 & 197.38 & 196.75 & 208.92 & 198.519 & 5.62 \\
\hline 212.546 & 238.7 & 201.76 & 197.29 & 196.8 & 197.38 & 196.73 & 208.9 & 198.495 & 5.58 \\
\hline 213.0458 & 238.55 & 202.28 & 197.29 & 196.79 & 197.34 & 196.77 & 208.9 & 198.558 & 5.56 \\
\hline 213.5465 & 238.48 & 202.93 & 197.23 & 196.72 & 197.29 & 196.64 & 208.57 & 198.24 & 5.56 \\
\hline 214.0463 & 238.5 & 201.95 & 197.2 & 196.7 & 197.29 & 196.68 & 208.53 & 198.267 & 5.59 \\
\hline 214.5462 & 238.5 & 201.89 & 197.22 & 196.72 & 197.33 & 196.68 & 208.58 & 198.306 & 5.60 \\
\hline 215.0488 & 238.48 & 201.77 & 197.22 & 196.72 & 197.31 & 196.68 & 208.61 & 198.342 & 5.58 \\
\hline 215.5458 & 238.43 & 203.14 & 197.22 & 196.7 & 197.33 & 196.7 & 208.66 & 198.345 & 5.60 \\
\hline 216.0467 & 238.56 & 203.02 & 197.24 & 196.72 & 197.33 & 196.7 & 208.73 & 198.408 & 5.62 \\
\hline 216.5465 & 238.79 & 202.61 & 197.24 & 196.75 & 197.35 & 196.7 & 208.8 & 198.396 & 5.64 \\
\hline 217.0462 & 238.97 & 201.68 & 197.25 & 196.75 & 197.36 & 196.72 & 208.86 & 198.405 & 5.59 \\
\hline 217.546 & 238.81 & 202.5 & 197.27 & 196.79 & 197.38 & 196.72 & 208.93 & 198.408 & 5.56 \\
\hline 218.0458 & 238.61 & 203.11 & 197.29 & 196.79 & 197.38 & 196.74 & 209 & 198.447 & 5.57 \\
\hline 218.5457 & 238.4 & 202.97 & 197.29 & 196.81 & 197.35 & 196.74 & 209.1 & 198.525 & 5.59 \\
\hline 219.0465 & 238.11 & 202.7 & 197.31 & 196.84 & 197.45 & 196.74 & 209.18 & 198.423 & 5.59 \\
\hline 219.5463 & 238.15 & 203.17 & 197.35 & 196.85 & 197.42 & 196.76 & 209.25 & 198.501 & 5.59 \\
\hline
\end{tabular}


Table B6. Continued.

\begin{tabular}{|c|c|c|c|c|c|c|c|c|c|}
\hline $\begin{array}{c}\text { Time } \\
\text { min }\end{array}$ & $\begin{array}{l}\mathrm{T} 1 \\
{ }^{\circ} \mathrm{C} \\
\end{array}$ & $\begin{array}{l}\mathrm{T} 2 \\
{ }^{\circ} \mathrm{C}\end{array}$ & $\begin{array}{l}\mathrm{T} 3 \\
{ }^{\circ} \mathrm{C} \\
\end{array}$ & $\begin{array}{l}\mathrm{T} 4 \\
{ }^{\circ} \mathrm{C} \\
\end{array}$ & $\begin{array}{l}\mathrm{T5} \\
{ }^{\circ} \mathrm{C} \\
\end{array}$ & $\begin{array}{l}\mathrm{T} 6 \\
{ }^{\circ} \mathrm{C} \\
\end{array}$ & $\begin{array}{l}\text { Pinj } \\
\text { psig }\end{array}$ & $\begin{array}{l}\text { Pout } \\
\text { psig }\end{array}$ & $\begin{array}{c}\mathrm{Vw} \\
\mathrm{cc} / \mathrm{min}\end{array}$ \\
\hline 220.0462 & 238.16 & 202.63 & 197.35 & 196.85 & 197.44 & 196.77 & 209.33 & 198.477 & 5.59 \\
\hline 220.5458 & 238.16 & 202.52 & 197.37 & 196.85 & 197.44 & 196.77 & 209.38 & 198.519 & 5.63 \\
\hline 221.0457 & 238.13 & 201.63 & 197.4 & 196.88 & 197.44 & 196.78 & 209.46 & 198.624 & 5.63 \\
\hline 221.5465 & 238.26 & 202.38 & 197.38 & 196.9 & 197.46 & 196.78 & 209.48 & 198.615 & 5.58 \\
\hline 222.0463 & 238.38 & 203.11 & 197.39 & 196.9 & 197.51 & 196.79 & 209.51 & 198.552 & 5.57 \\
\hline 222.5462 & 238.38 & 202.74 & 197.39 & 196.88 & 197.48 & 196.78 & 209.48 & 198.525 & 5.58 \\
\hline 223.046 & 238.24 & 202.97 & 197.39 & 196.9 & 197.47 & 196.79 & 209.48 & 198.543 & 5.60 \\
\hline 223.5458 & 238.13 & 202.04 & 197.35 & 196.8 & 197.33 & 196.64 & 208.98 & 198.225 & 5.59 \\
\hline 224.0465 & 238.04 & 202.26 & 197.28 & 196.78 & 197.35 & 196.67 & 208.91 & 198.285 & 5.58 \\
\hline 224.5463 & 238.08 & 201.63 & 197.28 & 196.8 & 197.35 & 196.69 & 91 & 198.282 & 5.61 \\
\hline 225.0462 & 238.13 & 200.83 & 197.26 & 196.76 & 197.37 & 196.71 & 208.87 & 198.285 & 5.64 \\
\hline 225.546 & 238.22 & 201.54 & 197.26 & 196.76 & 197.37 & 196.71 & 208.86 & 198.321 & 5.62 \\
\hline 226.0467 & 238.28 & 201.54 & 197.25 & 196.76 & 197.35 & 196.71 & 208.81 & 198.342 & 5.57 \\
\hline 226.5465 & 238.37 & 202.1 & 197.25 & 196.76 & 197.35 & 196.73 & 208.78 & 198.384 & 5.57 \\
\hline 227. & 238.49 & 202.53 & 197.25 & 196.75 & 197.35 & 196.71 & 75 & 348 & 5.59 \\
\hline 227.547 & 238.55 & 203.03 & 197.25 & 196.73 & 197.35 & 196.71 & 208.72 & 198.495 & 5.61 \\
\hline 228.046 & 238.6 & 203.16 & 197.25 & 196.74 & 197.35 & 196.71 & 208.68 & 198.366 & 5.61 \\
\hline 228.5467 & 238.53 & 202.37 & 197.23 & 196.75 & 197.35 & 196.71 & 208.69 & 198.378 & 5.61 \\
\hline 229.0465 & 238.55 & 201.92 & 197.21 & 196.73 & 197.34 & 196.73 & 208.69 & 198.423 & 5.65 \\
\hline 229.5463 & 238.75 & 202.28 & 197.23 & 196.75 & 197.36 & 196.71 & .71 & 198.414 & 5.65 \\
\hline 230.0462 & 239.05 & 201.35 & 197.23 & 196.78 & 197.36 & 196.71 & 208.75 & 198.42 & .58 \\
\hline 230.546 & 238.87 & 201.22 & 197.25 & 196.8 & 197.37 & 196.73 & 208.84 & 198.435 & 5.57 \\
\hline 231.0458 & 238.62 & 201.6 & 197.27 & 196.79 & 197.38 & 196.73 & 208.89 & 198.465 & 5.59 \\
\hline 231.5465 & 238.78 & 201.92 & 197.29 & 196.8 & 197.41 & 196.75 & 209 & 198.447 & 5.61 \\
\hline 232.0463 & 238.77 & 203.07 & 197.3 & 196.82 & 197.43 & 196.75 & 9.09 & 198.516 & 5.61 \\
\hline 232 & 238.53 & 201.49 & 197.3 & 196.84 & 197.41 & 196.77 & .18 & 198.441 & 5.62 \\
\hline 233.047 & 238.62 & 201.15 & 197.34 & 196.86 & 197.43 & 196.77 & 209.27 & 198.51 & 5.62 \\
\hline 233.5458 & 238.64 & 201.39 & 197.34 & 196.82 & 197.36 & 196.66 & 209.12 & 198.198 & 5.62 \\
\hline 234.0467 & 238.64 & 201.87 & 197.32 & 196.8 & 197.38 & 196.7 & 209.13 & 198.453 & 5.59 \\
\hline 234.5465 & 238.39 & 201.62 & 197.34 & 196.84 & 197.39 & 196.71 & 209.18 & 198.393 & 5.55 \\
\hline 235.0462 & 238.34 & 201.6 & 197.32 & 196.82 & 197.36 & 196.7 & 209.2 & 198.438 & 5.58 \\
\hline 235.546 & 238.14 & 201.66 & 197.34 & 196.84 & 197.41 & 196.73 & 209.25 & 198.441 & 5.61 \\
\hline 236.0458 & 238.36 & 202.41 & 197.36 & 196.86 & 197.41 & 196.73 & 209.24 & 198.327 & 5.61 \\
\hline 236.5457 & 239.02 & 203.63 & 197.34 & 196.84 & 197.41 & 196.73 & 209.22 & 198.324 & 5.60 \\
\hline 237.0465 & 239.38 & 203.11 & 197.34 & 196.84 & 197.41 & 196.73 & 209.22 & 198.333 & 5.60 \\
\hline 237.5463 & 239.58 & 202.72 & 197.33 & 196.84 & 197.42 & 196.74 & 209.14 & 198.381 & 5.63 \\
\hline 238.0462 & 239.63 & 203.07 & 197.33 & 196.81 & 197.4 & 196.72 & 209.1 & 198.369 & 5.59 \\
\hline 238.5458 & 239.65 & 202.25 & 197.31 & 196.82 & 197.4 & 196.74 & 209.03 & 198.423 & 5.56 \\
\hline 239.0457 & 239.72 & 202.11 & 197.29 & 196.8 & 197.38 & 196.73 & 208.95 & 198.363 & 5.56 \\
\hline 239.5465 & 239.6 & 202.14 & 197.29 & 196.79 & 197.38 & 196.74 & 208.87 & 198.375 & 5.59 \\
\hline 240.0463 & 239.61 & 201 & 197.25 & 196.79 & 197.38 & 196.75 & 208.79 & 198.414 & 5.59 \\
\hline 240.5462 & 239.65 & 201.64 & 197.25 & 196.75 & 197.38 & 196.74 & 208.76 & 198.399 & 5.58 \\
\hline 241.046 & 239.9 & 202.43 & 197.26 & 196.75 & 197.36 & 196.75 & 208.72 & 198.432 & 5.57 \\
\hline 241.5458 & 239.88 & 203.18 & 197.26 & 196.75 & 197.38 & 196.75 & 208.72 & 198.483 & 5.55 \\
\hline
\end{tabular}


Table B6. Continued.

\begin{tabular}{|c|c|c|c|c|c|c|c|c|c|}
\hline $\begin{array}{c}\text { Time } \\
\text { min }\end{array}$ & $\begin{array}{l}\mathrm{T} 1 \\
{ }^{\circ} \mathrm{C}\end{array}$ & $\begin{array}{l}\mathrm{T} 2 \\
{ }^{\circ} \mathrm{C}\end{array}$ & $\begin{array}{l}\mathrm{T} 3 \\
{ }^{\circ} \mathrm{C}\end{array}$ & $\begin{array}{l}\mathrm{T} 4 \\
{ }^{\circ} \mathrm{C}\end{array}$ & $\begin{array}{l}\mathrm{T} 5 \\
{ }^{\circ} \mathrm{C}\end{array}$ & $\begin{array}{l}\mathrm{T} 6 \\
{ }^{\circ} \mathrm{C}\end{array}$ & $\begin{array}{l}\text { Pinj } \\
\text { psig }\end{array}$ & $\begin{array}{l}\text { Pout } \\
\text { psig }\end{array}$ & $\begin{array}{c}\mathrm{Vw} \\
\mathrm{cc} / \mathrm{min}\end{array}$ \\
\hline 242.0465 & 239.01 & 203.02 & 197.26 & 196.77 & 197.38 & 196.77 & 208.76 & 198.579 & 5.55 \\
\hline 242.5463 & 238.54 & 202.06 & 197.26 & 196.74 & 197.31 & 196.67 & 208.59 & 198.222 & 5.57 \\
\hline 243.047 & 238.5 & 202.22 & 197.22 & 196.74 & 197.33 & 196.7 & 208.58 & 198.207 & 5.59 \\
\hline 243.546 & 238.27 & 202.27 & 197.22 & 196.74 & 197.33 & 196.69 & 208.64 & 198.27 & 5.61 \\
\hline 244.0458 & 238.27 & 201.52 & 197.24 & 196.74 & 197.35 & 196.72 & 208.71 & 198.297 & 5.60 \\
\hline 244.5475 & 238.52 & 201.97 & 197.26 & 196.77 & 197.35 & 196.74 & 208.82 & 198.333 & 5.61 \\
\hline 245.0463 & 238.63 & 201.63 & 197.26 & 196.78 & 197.37 & 196.72 & 208.91 & 198.279 & 5.62 \\
\hline 245.5462 & 238.61 & 201.47 & 197.29 & 196.81 & 197.4 & 196.74 & 209.04 & 198.414 & 5.62 \\
\hline 246.046 & 238.79 & 201.47 & 197.29 & 196.81 & 197.4 & 196.74 & 209.14 & 198.468 & 5.57 \\
\hline 246.5458 & 238.74 & 203.63 & 197.33 & 196.83 & 197.42 & 196.74 & 209.23 & 198.465 & 5.55 \\
\hline 247.0465 & 238.92 & 202.4 & 197.33 & 196.88 & 197.39 & 196.74 & 209.31 & 198.492 & 5.56 \\
\hline 247.5463 & 238.83 & 203.28 & 197.39 & 196.87 & 197.44 & 196.76 & 209.37 & 198.384 & 5.58 \\
\hline 248.0462 & 238.83 & 203.94 & 197.37 & 196.88 & 197.44 & 196.76 & 209.38 & 198.366 & 5.58 \\
\hline 248.546 & 239.21 & 203.55 & 197.39 & 196.88 & 197.46 & 196.78 & 209.4 & 198.408 & 5.58 \\
\hline 249.0458 & 239.49 & 203.28 & 197.37 & 196.89 & 197.44 & 196.76 & 209.4 & 198.42 & 5.58 \\
\hline 249 & 239.53 & 203.03 & 197.39 & 196.88 & 197.46 & 196.78 & 209.37 & 426 & 5.58 \\
\hline 250. & 239.55 & 202.63 & 197.37 & 196.88 & 197.46 & 196.76 & 209.34 & 198.414 & 5.62 \\
\hline 250.5462 & 239.57 & 202.92 & 197.35 & 196.83 & 197.37 & 196.71 & 209.09 & 198.186 & 5.64 \\
\hline 251.046 & 239.49 & 202.9 & 197.28 & 196.8 & 197.35 & 196.71 & 208.98 & 198.261 & 5.58 \\
\hline 251.5458 & 239.46 & 203.01 & 197.28 & 196.8 & 197.37 & 196.71 & 208.89 & 198.216 & 5.56 \\
\hline 252. & 239.39 & 203.1 & 197.26 & 196.78 & 197.35 & 196.73 & 208.83 & 282 & 5.57 \\
\hline 252.5465 & 239.5 & 202.78 & 197.26 & 196.74 & 197.32 & 196.71 & 208.74 & 198.312 & 5.59 \\
\hline 253.0472 & 239.44 & 202.31 & 197.24 & 196.74 & 197.41 & 196.71 & 208.68 & 198.312 & 5.60 \\
\hline 253.546 & 239.46 & 202.53 & 197.23 & 196.74 & 197.35 & 196.73 & 208.62 & 198.285 & 5.59 \\
\hline 254.0458 & 239.43 & 203.66 & 197.21 & 196.75 & 197.35 & 196.73 & 208.56 & 198.327 & 5.58 \\
\hline 254.5457 & 239.71 & 202.19 & 197.19 & 196.73 & 197.34 & 196.73 & 208.53 & 198.318 & 5.61 \\
\hline 255 & 239.78 & 202.15 & 197.21 & 196.75 & 197.34 & 196.71 & 20 & 372 & 5.65 \\
\hline 255.5463 & 238.93 & 201.92 & 197.21 & 196.75 & 197.34 & 196.75 & 208.6 & 198.345 & 5.60 \\
\hline 256.0462 & 238.5 & 202.26 & 197.21 & 196.75 & 197.35 & 196.75 & 208.64 & 198.354 & 5.55 \\
\hline 256.5468 & 238.41 & 202.66 & 197.23 & 196.75 & 197.37 & 196.75 & 208.71 & 198.417 & 5.55 \\
\hline 257.0467 & 238.5 & 203.18 & 197.27 & 196.77 & 197.37 & 196.75 & 208.77 & 198.435 & 5.57 \\
\hline 257.5473 & 238.55 & 203.09 & 197.27 & 196.78 & 197.39 & 196.75 & 208.9 & 198.414 & 5.61 \\
\hline 258.0463 & 238.28 & 202.12 & 197.3 & 196.82 & 197.41 & 196.75 & 209 & 198.441 & 5.60 \\
\hline 258.5462 & 238.18 & 201.28 & 197.32 & 196.86 & 197.43 & 196.77 & 209.09 & 198.432 & 5.60 \\
\hline 259.046 & 238.14 & 202.03 & 197.34 & 196.86 & 197.43 & 196.77 & 209.23 & 198.408 & 5.60 \\
\hline 259.5458 & 238.21 & 202.43 & 197.36 & 196.87 & 197.45 & 196.78 & 209.36 & 198.465 & 5.63 \\
\hline 260.0465 & 238.41 & 201.87 & 197.39 & 196.89 & 197.48 & 196.79 & 209.46 & 198.396 & 5.63 \\
\hline 260.5463 & 238.79 & 201.91 & 197.38 & 196.86 & 197.38 & 196.68 & 209.2 & 198.183 & 5.58 \\
\hline 261.0462 & 238.61 & 202.11 & 197.34 & 196.84 & 197.4 & 196.73 & 209.2 & 198.174 & 5.57 \\
\hline 261.546 & 238.73 & 203.18 & 197.36 & 196.82 & 197.36 & 196.72 & 209.21 & 198.198 & 5.58 \\
\hline 262.0458 & 239.02 & 203.25 & 197.34 & 196.84 & 197.42 & 196.72 & 209.21 & 198.258 & 5.62 \\
\hline 262.5475 & 239.04 & 203.86 & 197.34 & 196.84 & 197.45 & 196.73 & 209.17 & 198.276 & 5.62 \\
\hline
\end{tabular}


Table B6. Continued.

\begin{tabular}{|c|c|c|c|c|c|c|c|c|c|}
\hline $\begin{array}{c}\text { Time } \\
\text { min }\end{array}$ & $\begin{array}{l}\mathrm{T} 1 \\
{ }^{\circ} \mathrm{C} \\
\end{array}$ & $\begin{array}{l}\mathrm{T} 2 \\
{ }^{\circ} \mathrm{C} \\
\end{array}$ & $\begin{array}{l}\mathrm{T} 3 \\
{ }^{\circ} \mathrm{C} \\
\end{array}$ & $\begin{array}{l}\mathrm{T} 4 \\
{ }^{\circ} \mathrm{C} \\
\end{array}$ & $\begin{array}{l}\mathrm{T} 5 \\
{ }^{\circ} \mathrm{C} \\
\end{array}$ & $\begin{array}{l}\mathrm{T} 6 \\
{ }^{\circ} \mathrm{C} \\
\end{array}$ & $\begin{array}{l}\text { Pinj } \\
\text { psig }\end{array}$ & $\begin{array}{l}\text { Pout } \\
\text { psig }\end{array}$ & $\begin{array}{c}\mathrm{Vw} \\
\mathrm{cc} / \mathrm{min}\end{array}$ \\
\hline 263.0463 & 238.7 & 203.63 & 197.34 & 196.84 & 197.42 & 196.75 & 209.12 & 198.267 & 5.62 \\
\hline 263.5462 & 238.27 & 204.79 & 197.33 & 196.84 & 197.42 & 196.74 & 209.08 & 198.279 & 5.63 \\
\hline 264.046 & 238.11 & 204.9 & 197.33 & 196.79 & 197.42 & 196.75 & 209 & 198.333 & 5.63 \\
\hline 264.5458 & 238.18 & 203.47 & 197.31 & 196.83 & 197.34 & 196.74 & 208.9 & 198.333 & 5.59 \\
\hline 265.0465 & 238.25 & 202.82 & 197.29 & 196.79 & 197.38 & 196.75 & 208.82 & 198.306 & 5.57 \\
\hline 265.5463 & 238.32 & 202.47 & 197.25 & 196.79 & 197.38 & 196.75 & 208.75 & 198.474 & 5.56 \\
\hline 266.0472 & 238.38 & 202.81 & 197.26 & 196.77 & 197.36 & 196.74 & 208.67 & 198.408 & 5.59 \\
\hline 266.546 & 238.47 & 203 & 197.24 & 196.77 & 197.38 & 196.74 & 208.59 & 198.48 & 5.61 \\
\hline 267.0458 & 238.58 & 204.38 & 197.22 & 196.75 & 197.36 & 196.75 & 208.54 & 198.324 & 5.59 \\
\hline 267.5465 & 238.63 & 203.36 & 197.22 & 196.76 & 197.36 & 196.77 & .56 & 198.357 & 5.59 \\
\hline 268.0463 & 238.73 & 201.88 & 197.22 & 196.77 & 197.36 & 196.75 & 208.59 & 198.357 & 5.61 \\
\hline 268.5462 & 238.83 & 201.71 & 197.22 & 196.72 & 197.33 & 196.68 & 208.48 & 198.153 & 5.64 \\
\hline 269.046 & 238.83 & 202 & 197.18 & 196.72 & 197.31 & 196.7 & 208.49 & 198.126 & 5.62 \\
\hline 269.5468 & 238.77 & 203.02 & 197.22 & 196.72 & 197.33 & 196.72 & 208.58 & 198.171 & 5.58 \\
\hline 270.0467 & 238.74 & 202.88 & 197.22 & 196.74 & 197.35 & 196.72 & 65 & 198.189 & 5.57 \\
\hline 270.5465 & 238.7 & 203.25 & 197.24 & 196.75 & 197.35 & 196.7 & 208.74 & 198.225 & 5.59 \\
\hline 271.0462 & 238.61 & 202.97 & 197.26 & 196.81 & 197.33 & 196.74 & 208.87 & 198.225 & 5.62 \\
\hline 271.547 & 238.61 & 202.43 & 197.28 & 196.79 & 197.4 & 196.74 & 208.97 & 198.21 & 5.60 \\
\hline 272.0468 & 238.59 & 202.61 & 197.31 & 196.83 & 197.42 & 196.76 & 209.09 & 198.213 & 5.61 \\
\hline 272.5467 & 238.61 & 202.06 & 197.33 & 196.85 & 197.42 & 196.74 & 209.21 & 198.255 & 5.63 \\
\hline 273.0465 & 238.54 & 201.97 & 197.37 & 196.86 & 197.44 & 196.74 & 209.3 & 98.246 & 5.65 \\
\hline 273.5463 & 238.58 & 203.27 & 197.37 & 196.86 & 197.45 & 196.76 & 209.33 & 198.249 & 5.61 \\
\hline 274.0462 & 238.59 & 203.61 & 197.37 & 196.88 & 197.45 & 196.76 & 209.35 & 198.318 & 5.57 \\
\hline 274.5458 & 238.59 & 203.42 & 197.37 & 196.88 & 197.49 & 196.76 & 209.37 & 198.288 & 5.57 \\
\hline 275.0475 & 238.58 & 203.15 & 197.37 & 196.9 & 197.47 & 196.78 & 209.3 & 198.276 & 5.60 \\
\hline 275.5473 & 238.56 & 203.79 & 197.37 & 196.86 & 197.46 & 196.77 & 209.24 & 327 & 5.62 \\
\hline 276.0472 & 238.59 & 203.1 & 197.37 & 196.86 & 197.44 & 196.77 & 209.17 & 198.282 & 5.59 \\
\hline 276.5462 & 238.6 & 202.45 & 197.33 & 196.83 & 197.44 & 196.76 & 209.1 & 198.321 & 5.61 \\
\hline 277.046 & 238.65 & 202.81 & 197.31 & 196.85 & 197.44 & 196.77 & 209.01 & 198.285 & 5.62 \\
\hline 277.5467 & 238.7 & 203.19 & 197.31 & 196.83 & 197.42 & 196.78 & 208.93 & 198.345 & 5.63 \\
\hline 278.0465 & 238.78 & 203.35 & 197.28 & 196.81 & 197.4 & 196.76 & 208.85 & 198.357 & 5.57 \\
\hline 278.5463 & 238.78 & 202.44 & 197.26 & 196.79 & 197.39 & 196.76 & 208.8 & 198.339 & 5.56 \\
\hline 279.0462 & 238.79 & 202.18 & 197.26 & 196.76 & 197.31 & 196.67 & 208.5 & 198.024 & 5.55 \\
\hline 279.5468 & 238.85 & 202.02 & 197.21 & 196.72 & 197.31 & 196.69 & 208.36 & 198.09 & 5.61 \\
\hline 280.0458 & 238.9 & 201.68 & 197.15 & 196.69 & 197.28 & 196.69 & 208.3 & 198.069 & 5.60 \\
\hline 280.5465 & 238.94 & 202.01 & 197.15 & 196.67 & 197.28 & 196.67 & 208.29 & 198.006 & 5.60 \\
\hline 281.0463 & 238.96 & 202.88 & 197.15 & 196.69 & 197.3 & 196.67 & 208.31 & 198.135 & 5.61 \\
\hline 281.547 & 238.97 & 203.44 & 197.15 & 196.69 & 197.3 & 196.71 & 208.33 & 198.147 & 5.59 \\
\hline 282.046 & 239.01 & 203.12 & 197.19 & 196.71 & 197.33 & 196.69 & 208.39 & 198.087 & 5.62 \\
\hline 282.5458 & 238.97 & 204.19 & 197.17 & 196.71 & 197.3 & 196.71 & 208.46 & 198.066 & 5.58 \\
\hline 283.0465 & 238.83 & 203.17 & 197.21 & 196.71 & 197.32 & 196.69 & 208.57 & 198.162 & 5.56 \\
\hline 283.5463 & 238.99 & 203.47 & 197.23 & 196.76 & 197.35 & 196.69 & 208.71 & 198.06 & 5.56 \\
\hline 284.0462 & 238.69 & 202.65 & 197.26 & 196.78 & 197.37 & 196.69 & 208.8 & 198.108 & 5.57 \\
\hline
\end{tabular}


Table B6. Continued.

\begin{tabular}{|c|c|c|c|c|c|c|c|c|c|}
\hline $\begin{array}{c}\text { Time } \\
\text { min }\end{array}$ & $\begin{array}{l}\mathrm{T} 1 \\
{ }^{\circ} \mathrm{C}\end{array}$ & $\begin{array}{l}\mathrm{T} 2 \\
{ }^{\circ} \mathrm{C}\end{array}$ & $\begin{array}{l}\mathrm{T} 3 \\
{ }^{\circ} \mathrm{C}\end{array}$ & $\begin{array}{l}\mathrm{T} 4 \\
{ }^{\circ} \mathrm{C}\end{array}$ & $\begin{array}{l}\mathrm{T} 5 \\
{ }^{\circ} \mathrm{C}\end{array}$ & $\begin{array}{l}\mathrm{T} 6 \\
{ }^{\circ} \mathrm{C}\end{array}$ & $\begin{array}{l}\text { Pinj } \\
\text { psig }\end{array}$ & $\begin{array}{l}\text { Pout } \\
\text { psig }\end{array}$ & $\begin{array}{c}\mathrm{Vw} \\
\mathrm{cc} / \mathrm{min}\end{array}$ \\
\hline 284.546 & 238.56 & 202.19 & 197.28 & 196.8 & 197.39 & 196.73 & 208.87 & 198.126 & 5.61 \\
\hline 285.0458 & 238.58 & 203.4 & 197.3 & 196.8 & 197.39 & 196.71 & 208.99 & 198.126 & 5.59 \\
\hline 285.5465 & 238.64 & 204.05 & 197.3 & 196.8 & 197.39 & 196.73 & 209.05 & 198.093 & 5.60 \\
\hline 286.0463 & 238.69 & 203.05 & 197.34 & 196.83 & 197.41 & 196.73 & 209.13 & 198.063 & 5.60 \\
\hline 286.5472 & 238.71 & 202.92 & 197.34 & 196.85 & 197.41 & 196.73 & 209.2 & 198.144 & 5.63 \\
\hline 287.046 & 238.74 & 202.23 & 197.34 & 196.85 & 197.43 & 196.69 & 209.21 & 198.156 & 5.61 \\
\hline 287.5458 & 238.66 & 201.85 & 197.34 & 196.85 & 197.41 & 196.73 & 209.22 & 198.168 & 5.57 \\
\hline 288.0467 & 238.62 & 202.65 & 197.35 & 196.85 & 197.42 & 196.73 & 209.18 & 198.138 & 5.55 \\
\hline 288.5465 & 238.57 & 203.42 & 197.35 & 196.85 & 197.41 & 196.73 & 209.14 & 198.171 & 5.57 \\
\hline 289.0462 & 238.57 & 203.53 & 197.32 & 196.83 & 197.41 & 196.73 & 209.08 & 198.174 & 5.59 \\
\hline 289.546 & 238.62 & 202.39 & 197.32 & 196.82 & 197.39 & 196.73 & 208.99 & 198.174 & 5.60 \\
\hline 290.0458 & 238.64 & 201.71 & 197.3 & 196.82 & 197.39 & 196.69 & 95 & 186 & 5.59 \\
\hline 290.5457 & 238.64 & 202.49 & 197.3 & 196.8 & 197.39 & 196.73 & 208.85 & 216 & 5.61 \\
\hline 291.0465 & 238.73 & 202.49 & 197.27 & 196.78 & 197.39 & 196.73 & 208.77 & 198.186 & 5.61 \\
\hline 291.5463 & 238.73 & 203.05 & 197.25 & 196.76 & 197.37 & 196.73 & 208.68 & 198.192 & 5.61 \\
\hline 292.0462 & 238.73 & 202.96 & 197.25 & 196.76 & 197.36 & 196.73 & 208.61 & 327 & 5.62 \\
\hline 292.5458 & 238.73 & 203.19 & 197.23 & 196.77 & 197.36 & 196.73 & 20 & 303 & 5.58 \\
\hline 293.0457 & 238.78 & 202.87 & 197.21 & 196.73 & 197.34 & 196.71 & 208.51 & 222 & 5.58 \\
\hline 293.5465 & 238.8 & 203.07 & 197.21 & 196.75 & 197.36 & 196.73 & 208.5 & 198.171 & 5.61 \\
\hline 294.0463 & 238.86 & 203.12 & 197.2 & 196.73 & 197.36 & 196.73 & 208.53 & 198.177 & 5.61 \\
\hline 294.5462 & 238.86 & 202.14 & 197.21 & 196.75 & 197.36 & 196.75 & 208.56 & 198.246 & 5.60 \\
\hline 295.046 & 238.84 & 201.17 & 197.23 & 196.77 & 197.38 & 196.73 & 208.65 & 198.21 & 5.61 \\
\hline 295.5458 & 238.86 & 201.03 & 197.23 & 196.77 & 197.36 & 196.66 & 208.66 & 198.159 & 5.63 \\
\hline 296.0473 & 238.84 & 203.16 & 197.23 & 196.75 & 197.34 & 196.71 & 208.61 & 198.168 & 5.63 \\
\hline 296.5472 & 238.82 & 203.77 & 197.23 & 196.75 & 197.34 & 196.7 & 208.68 & 198.228 & 5.58 \\
\hline 297.0462 & 238.84 & 203.2 & 197.25 & 196.79 & 197.36 & 196.71 & 208.77 & 198.114 & 5.56 \\
\hline 297.546 & 238.91 & 202.82 & 197.27 & 196.77 & 197.38 & 196.71 & 208.89 & 198.114 & 5.57 \\
\hline 298.0467 & 238.91 & 202.5 & 197.29 & 196.8 & 197.4 & 196.73 & 208.99 & 198.066 & 5.60 \\
\hline 298.5465 & 238.88 & 201.96 & 197.31 & 196.82 & 197.38 & 196.73 & 209.05 & 198.168 & 5.60 \\
\hline 299.0463 & 238.81 & 201.43 & 197.32 & 196.84 & 197.41 & 196.72 & 209.14 & 198.153 & 5.60 \\
\hline 299.5462 & 238.72 & 201.75 & 197.34 & 196.86 & 197.45 & 196.75 & 209.18 & 198.147 & 5.60 \\
\hline 300.046 & 238.68 & 202.5 & 197.36 & 196.84 & 197.45 & 196.75 & 209.23 & 198.312 & 5.62 \\
\hline 300.5458 & 238.59 & 203.43 & 197.36 & 196.88 & 197.43 & 196.77 & 209.26 & 198.195 & 5.63 \\
\hline 301.0465 & 238.54 & 204.43 & 197.34 & 196.86 & 197.45 & 196.75 & 209.21 & 198.186 & 5.59 \\
\hline 301.5463 & 238.5 & 203.74 & 197.35 & 196.86 & 197.43 & 196.75 & 209.18 & 198.186 & 5.55 \\
\hline 302.0462 & 238.5 & 202.63 & 197.35 & 196.86 & 197.43 & 196.77 & 209.1 & 198.168 & 5.56 \\
\hline 302.547 & 238.54 & 203.49 & 197.31 & 196.86 & 197.45 & 196.75 & 209.05 & 198.201 & 5.61 \\
\hline 303.0458 & 238.57 & 202.48 & 197.31 & 196.86 & 197.42 & 196.75 & 208.95 & 198.204 & 5.61 \\
\hline 303.5465 & 238.59 & 202.02 & 197.31 & 196.84 & 197.42 & 196.75 & 208.85 & 198.192 & 5.58 \\
\hline 304.0473 & 238.65 & 202.73 & 197.27 & 196.79 & 197.42 & 196.75 & 208.77 & 198.297 & 5.60 \\
\hline 304.5462 & 238.68 & 203.15 & 197.26 & 196.79 & 197.38 & 196.74 & 208.69 & 198.276 & 5.61 \\
\hline 305.046 & 238.75 & 202.93 & 197.24 & 196.77 & 197.38 & 196.75 & 208.62 & 198.345 & 5.62 \\
\hline
\end{tabular}


Table B6. Continued.

\begin{tabular}{|c|c|c|c|c|c|c|c|c|c|}
\hline $\begin{array}{c}\text { Time } \\
\text { min }\end{array}$ & $\begin{array}{l}\mathrm{T} 1 \\
{ }^{\circ} \mathrm{C}\end{array}$ & $\begin{array}{l}\mathrm{T} 2 \\
{ }^{\circ} \mathrm{C}\end{array}$ & $\begin{array}{l}\mathrm{T} 3 \\
{ }^{\circ} \mathrm{C}\end{array}$ & $\begin{array}{l}\mathrm{T} 4 \\
{ }^{\circ} \mathrm{C}\end{array}$ & $\begin{array}{l}\mathrm{T} 5 \\
{ }^{\circ} \mathrm{C}\end{array}$ & $\begin{array}{l}\mathrm{T} 6 \\
{ }^{\circ} \mathrm{C}\end{array}$ & $\begin{array}{l}\text { Pinj } \\
\text { psig }\end{array}$ & $\begin{array}{l}\text { Pout } \\
\text { psig }\end{array}$ & $\begin{array}{c}\mathrm{Vw} \\
\mathrm{cc} / \mathrm{min}\end{array}$ \\
\hline 306.0467 & 238.84 & 201.82 & 197.22 & 196.76 & 197.36 & 196.74 & 208.52 & 198.273 & 5.56 \\
\hline 306.5465 & 238.84 & 202.14 & 197.22 & 196.77 & 197.36 & 196.76 & 208.51 & 198.291 & 5.57 \\
\hline 307.0462 & 238.84 & 202.59 & 197.22 & 196.75 & 197.36 & 196.76 & 208.53 & 198.336 & 5.58 \\
\hline 307.546 & 238.81 & 202.68 & 197.22 & 196.7 & 197.29 & 196.61 & 208.4 & 197.907 & 5.61 \\
\hline 308.0458 & 238.77 & 202.74 & 197.17 & 196.68 & 197.28 & 196.68 & 208.35 & 198.006 & 5.63 \\
\hline 308.5467 & 238.74 & 202.41 & 197.19 & 196.7 & 197.31 & 196.68 & 208.4 & 197.982 & 5.60 \\
\hline 309.0465 & 238.79 & 201.86 & 197.2 & 196.74 & 197.33 & 196.68 & 208.49 & 198.015 & 5.64 \\
\hline 309.5463 & 238.83 & 202.59 & 197.22 & 196.74 & 197.33 & 196.72 & 208.57 & 198.066 & 5.65 \\
\hline 310.0462 & 238.86 & 203.08 & 197.24 & 196.75 & 197.35 & 196.7 & 208.67 & 198.093 & 5.59 \\
\hline 310.5458 & 238.86 & 202.79 & 197.24 & 196.77 & 197.37 & 196.72 & 208.78 & 198.081 & 5.56 \\
\hline 311.0457 & 238.79 & 203.2 & 197.27 & 196.77 & 197.38 & 196.7 & 208.87 & 129 & 5.58 \\
\hline 311.5465 & 238.72 & 202.04 & 197.29 & 196.81 & 197.4 & 196.74 & 208.96 & 996 & 5.61 \\
\hline 312.0463 & 238.63 & 202.86 & 197.31 & 196.83 & 197.4 & 196.72 & 209.03 & 198 & 5.60 \\
\hline 312.5462 & 238.56 & 202.47 & 197.33 & 196.83 & 197.43 & 196.74 & 209.07 & 198.081 & 5.61 \\
\hline 313.0468 & 238.52 & 203.2 & 197.33 & 196.81 & 197.44 & 196.72 & 209.06 & 198.222 & 5.62 \\
\hline 313.5458 & 238.47 & 202.27 & 197.33 & 196.84 & 197.42 & 196.72 & 209.04 & 198 & 5.63 \\
\hline 314.0465 & 238.49 & 202 & 197.33 & 196.83 & 197.42 & 196.74 & 209 & 05 & 5.62 \\
\hline 314.5463 & 238.49 & 202.75 & 197.31 & 196.81 & 197.4 & 196.74 & 208.95 & 117 & 5.57 \\
\hline 315.0462 & 238.51 & 202.56 & 197.29 & 196.83 & 197.4 & 196.74 & 208.85 & 198.105 & 5.55 \\
\hline 315.546 & 238.52 & 203.22 & 197.28 & 196.79 & 197.35 & 196.74 & 208.79 & 198.117 & 5.58 \\
\hline 316.0458 & 238.6 & 202.58 & 197.24 & 196.78 & 197.38 & 196.72 & 208.67 & 198.168 & 5.61 \\
\hline 316.5465 & 238.68 & 202 & 197.24 & 196.76 & 197.37 & 196.74 & 208.57 & 87 & 5.60 \\
\hline 317.0463 & 238.79 & 202.07 & 197.22 & 196.74 & 197.35 & 196.72 & 208.47 & 213 & 5.61 \\
\hline 317.5462 & 238.85 & 202.51 & 197.19 & 196.72 & 197.35 & 196.72 & 208.4 & 198.132 & 5.62 \\
\hline 318.046 & 238.88 & 202.02 & 197.17 & 196.7 & 197.33 & 196.74 & 208.33 & 198.177 & 5.65 \\
\hline 318.5458 & 238.9 & 202.27 & 197.17 & 196.72 & 197.37 & 196.74 & 208.32 & 198.135 & 5.60 \\
\hline 319.0465 & 238.86 & 202.95 & 197.19 & 196.7 & 197.37 & 196.74 & 208.34 & 198.18 & 5.57 \\
\hline 319.5473 & 238.88 & 202.95 & 197.19 & 196.72 & 197.35 & 196.74 & 208.38 & 198.144 & 5.58 \\
\hline 320.0462 & 238.92 & 202.92 & 197.17 & 196.72 & 197.31 & 196.67 & 208.34 & 197.967 & 5.61 \\
\hline 320.546 & 238.88 & 204.04 & 197.17 & 196.7 & 197.29 & 196.7 & 208.36 & 198.105 & 5.61 \\
\hline 321.0458 & 238.81 & 204.28 & 197.19 & 196.7 & 197.31 & 196.69 & 208.42 & 198.045 & 5.60 \\
\hline 321.5465 & 238.81 & 203.74 & 197.2 & 196.74 & 197.33 & 196.7 & 208.5 & 197.994 & 5.60 \\
\hline 322.0463 & 238.77 & 204.65 & 197.22 & 196.74 & 197.31 & 196.69 & 208.62 & 198.036 & 5.63 \\
\hline 322.5462 & 238.77 & 203.85 & 197.24 & 196.78 & 197.35 & 196.7 & 208.72 & 198.054 & 5.62 \\
\hline 323.046 & 238.79 & 204.15 & 197.26 & 196.79 & 197.38 & 196.72 & 208.88 & 198.03 & 5.57 \\
\hline 323.5468 & 238.76 & 202.95 & 197.29 & 196.81 & 197.38 & 196.72 & 208.96 & 198.012 & 5.57 \\
\hline 324.0467 & 238.69 & 202.29 & 197.31 & 196.81 & 197.4 & 196.72 & 209.01 & 198.096 & 5.58 \\
\hline 324.5465 & 238.69 & 203.22 & 197.3 & 196.85 & 197.4 & 196.72 & 209.08 & 198.084 & 5.61 \\
\hline 325.0462 & 238.67 & 203.53 & 197.33 & 196.83 & 197.42 & 196.72 & 209.1 & 198.099 & 5.60 \\
\hline 325.546 & 238.63 & 202.52 & 197.33 & 196.85 & 197.42 & 196.74 & 209.09 & 198.033 & 5.61 \\
\hline 326.0458 & 238.63 & 203.22 & 197.31 & 196.85 & 197.42 & 196.74 & 209.04 & 198.075 & 5.62 \\
\hline 326.5457 & 238.58 & 203.78 & 197.31 & 196.83 & 197.42 & 196.72 & 209.01 & 198.096 & 5.64 \\
\hline 327.0465 & 238.62 & 203.44 & 197.31 & 196.81 & 197.42 & 196.72 & 208.91 & 198.144 & 5.62 \\
\hline
\end{tabular}


Table B6. Continued.

\begin{tabular}{|c|c|c|c|c|c|c|c|c|c|}
\hline $\begin{array}{c}\text { Time } \\
\text { min }\end{array}$ & $\begin{array}{l}\mathrm{T} 1 \\
{ }^{\circ} \mathrm{C} \\
\end{array}$ & $\begin{array}{l}\mathrm{T} 2 \\
{ }^{\circ} \mathrm{C} \\
\end{array}$ & $\begin{array}{l}\mathrm{T} 3 \\
{ }^{\circ} \mathrm{C} \\
\end{array}$ & $\begin{array}{l}\mathrm{T} 4 \\
{ }^{\circ} \mathrm{C} \\
\end{array}$ & $\begin{array}{l}\mathrm{T5} \\
{ }^{\circ} \mathrm{C} \\
\end{array}$ & $\begin{array}{l}\mathrm{T} 6 \\
{ }^{\circ} \mathrm{C} \\
\end{array}$ & $\begin{array}{l}\text { Pinj } \\
\text { psig }\end{array}$ & $\begin{array}{l}\text { Pout } \\
\text { psig }\end{array}$ & $\begin{array}{c}\mathrm{Vw} \\
\mathrm{cc} / \mathrm{min}\end{array}$ \\
\hline 327.5463 & 238.6 & 204.42 & 197.3 & 196.79 & 197.38 & 196.76 & 208.84 & 198.123 & 5.57 \\
\hline 328.0462 & 238.62 & 204.69 & 197.28 & 196.8 & 197.4 & 196.74 & 208.77 & 198.141 & 5.56 \\
\hline 328.5458 & 238.67 & 204.37 & 197.26 & 196.78 & 197.4 & 196.74 & 208.65 & 198.144 & 5.59 \\
\hline 329.0457 & 238.72 & 204.07 & 197.24 & 196.76 & 197.35 & 196.69 & 208.48 & 198.072 & 5.62 \\
\hline 329.5465 & 238.78 & 202.92 & 197.19 & 196.72 & 197.31 & 196.69 & 208.34 & 198.048 & 5.60 \\
\hline 330.0463 & 238.81 & 203.11 & 197.17 & 196.7 & 197.3 & 196.71 & 208.24 & 197.973 & 5.61 \\
\hline 330.5462 & 238.79 & 202.42 & 197.15 & 196.69 & 197.26 & 196.69 & 208.23 & 198.018 & 5.61 \\
\hline 331.046 & 238.83 & 201.5 & 197.14 & 196.69 & 197.3 & 196.69 & 208.18 & 198.018 & 5.65 \\
\hline 331.5458 & 238.83 & 201.93 & 197.13 & 196.69 & 197.31 & 196.69 & 208.2 & 198.066 & 5.62 \\
\hline 332.0465 & 238.87 & 201.58 & 197.15 & 196.71 & 197.31 & 196.71 & 208.22 & 198.006 & 5.56 \\
\hline 332.5463 & 238.9 & 202.87 & 197.15 & 196.71 & 197.31 & 196.71 & 208.26 & 45 & 5.56 \\
\hline 333.0462 & 238.85 & 202.77 & 197.17 & 196.71 & 197.31 & 196.71 & 208.33 & 42 & 5.58 \\
\hline 333.546 & 238.79 & 203.8 & 197.15 & 196.72 & 197.37 & 196.7 & 208.43 & 198 & 5.60 \\
\hline 334.0458 & 238.79 & 202.44 & 197.21 & 196.74 & 197.33 & 196.72 & 208.55 & 198.054 & 5.60 \\
\hline 334.5465 & 238.81 & 202.17 & 197.23 & 196.76 & 197.35 & 196.71 & 208.67 & 198.078 & 5.61 \\
\hline 335.0473 & 238.81 & 202.22 & 197.26 & 196.79 & 197.38 & 196.71 & 208.77 & 84 & 5.62 \\
\hline 335. & 238.78 & 201.81 & 197.28 & 196.79 & 197.4 & 196.74 & 89 & 11 & 5.64 \\
\hline 336.046 & 238.7 & 201.75 & 197.3 & 196.81 & 197.42 & 196.72 & 209.01 & 333 & 5.60 \\
\hline 336.5458 & 238.65 & 203.08 & 197.31 & 196.83 & 197.44 & 196.74 & 209.12 & 198.084 & 5.57 \\
\hline 337.0465 & 238.58 & 203.49 & 197.35 & 196.85 & 197.48 & 196.76 & 209.16 & 198.144 & 5.58 \\
\hline 337.5463 & 238.53 & 204.08 & 197.35 & 196.87 & 197.44 & 196.76 & 209.2 & 198.102 & 5.61 \\
\hline 338.0462 & 238.45 & 204.53 & 197.37 & 196.87 & 197.44 & 196.71 & 209.14 & 943 & 5.61 \\
\hline 338.546 & 238.44 & 203.74 & 197.33 & 196.85 & 197.42 & 196.7 & 209.02 & 197.961 & 5.61 \\
\hline 339.0458 & 238.44 & 203.06 & 197.31 & 196.81 & 197.39 & 196.71 & 208.92 & 198.027 & 5.62 \\
\hline 339.5465 & 238.49 & 203.6 & 197.3 & 196.81 & 197.44 & 196.72 & 208.82 & 197.982 & 5.63 \\
\hline 340.0463 & 238.49 & 203.67 & 197.26 & 196.78 & 197.38 & 196.69 & 208.71 & 197.991 & 5.61 \\
\hline 340.5462 & 238.63 & 203.29 & 197.24 & 196.78 & 197.35 & 196.7 & 208.59 & 197.952 & 5.56 \\
\hline 341.047 & 238.7 & 204.19 & 197.22 & 196.74 & 197.35 & 196.7 & 208.52 & 015 & 5.57 \\
\hline 341.5458 & 238.88 & 202.02 & 197.21 & 196.72 & 197.33 & 196.7 & 208.39 & 198.006 & 5.59 \\
\hline 342.0467 & 239.05 & 201.86 & 197.15 & 196.72 & 197.33 & 196.69 & 208.27 & 198.006 & 5.61 \\
\hline 342.5465 & 239.24 & 202.24 & 197.15 & 196.7 & 197.31 & 196.69 & 208.16 & 198 & 5.59 \\
\hline 343.0462 & 239.4 & 203.21 & 197.14 & 196.67 & 197.31 & 196.69 & 208.09 & 198.036 & 5.62 \\
\hline 343.546 & 239.51 & 203.06 & 197.12 & 196.65 & 197.3 & 196.71 & 208.08 & 198.012 & 5.61 \\
\hline 344.0458 & 239.69 & 203.44 & 197.12 & 196.67 & 197.28 & 196.71 & 208.08 & 198.036 & 5.63 \\
\hline 344.5457 & 239.83 & 203.4 & 197.12 & 196.67 & 197.3 & 196.71 & 208.12 & 198.06 & 5.61 \\
\hline 345.0465 & 239.98 & 202.01 & 197.12 & 196.67 & 197.3 & 196.71 & 208.17 & 198.027 & 5.57 \\
\hline 345.5463 & 240.05 & 202.01 & 197.15 & 196.65 & 197.31 & 196.72 & 208.26 & 198.057 & 5.56 \\
\hline 346.047 & 239.98 & 203.56 & 197.17 & 196.72 & 197.33 & 196.76 & 208.38 & 198.084 & 5.58 \\
\hline 346.5458 & 239.48 & 203.94 & 197.21 & 196.74 & 197.31 & 196.67 & 208.45 & 197.943 & 5.60 \\
\hline 347.0457 & 239.01 & 202.99 & 197.21 & 196.72 & 197.31 & 196.69 & 208.54 & 197.937 & 5.59 \\
\hline 347.5465 & 238.94 & 202.11 & 197.24 & 196.76 & 197.33 & 196.69 & 208.64 & 197.904 & 5.59 \\
\hline 348.0463 & 239.08 & 201.58 & 197.26 & 196.78 & 197.37 & 196.69 & 208.8 & 197.949 & 5.60 \\
\hline 348.5462 & 238.7 & 202.24 & 197.28 & 196.79 & 197.38 & 196.7 & 208.92 & 198.024 & 5.63 \\
\hline
\end{tabular}


Table B6. Continued.

\begin{tabular}{|c|c|c|c|c|c|c|c|c|c|}
\hline $\begin{array}{c}\text { Time } \\
\text { min }\end{array}$ & $\begin{array}{l}\mathrm{T} 1 \\
{ }^{\circ} \mathrm{C} \\
\end{array}$ & $\begin{array}{l}\mathrm{T} 2 \\
{ }^{\circ} \mathrm{C} \\
\end{array}$ & $\begin{array}{l}\mathrm{T} 3 \\
{ }^{\circ} \mathrm{C} \\
\end{array}$ & $\begin{array}{l}\mathrm{T} 4 \\
{ }^{\circ} \mathrm{C} \\
\end{array}$ & $\begin{array}{l}\mathrm{T5} \\
{ }^{\circ} \mathrm{C} \\
\end{array}$ & $\begin{array}{l}\mathrm{T} 6 \\
{ }^{\circ} \mathrm{C} \\
\end{array}$ & $\begin{array}{l}\text { Pinj } \\
\text { psig }\end{array}$ & $\begin{array}{l}\text { Pout } \\
\text { psig }\end{array}$ & $\begin{array}{c}\mathrm{Vw} \\
\mathrm{cc} / \mathrm{min}\end{array}$ \\
\hline 349.5458 & 238.96 & 202.31 & 197.31 & 196.83 & 197.42 & 196.72 & 209.09 & 197.988 & 5.56 \\
\hline 350.0465 & 239.22 & 203.31 & 197.33 & 196.83 & 197.4 & 196.72 & 209.06 & 198.096 & 5.56 \\
\hline 350.5463 & 239.39 & 203.35 & 197.33 & 196.85 & 197.42 & 196.71 & 209.03 & 197.985 & 5.57 \\
\hline 351.0462 & 239.37 & 203.1 & 197.31 & 196.83 & 197.4 & 196.71 & 208.97 & 198.051 & 5.60 \\
\hline 351.546 & 239.28 & 202.87 & 197.3 & 196.81 & 197.35 & 196.72 & 208.92 & 198.03 & 5.59 \\
\hline 352.0458 & 239.15 & 202.22 & 197.3 & 196.81 & 197.4 & 196.72 & 208.82 & 198.033 & 5.59 \\
\hline 352.5465 & 239.05 & 202.92 & 197.26 & 196.78 & 197.39 & 196.74 & 208.72 & 198.024 & 5.61 \\
\hline 353.0463 & 238.99 & 203.26 & 197.24 & 196.78 & 197.39 & 196.71 & 208.6 & 198.039 & 5.61 \\
\hline 353.5462 & 239.01 & 204.6 & 197.23 & 196.76 & 197.37 & 196.72 & 208.51 & 198.03 & 5.62 \\
\hline 354.046 & 239.06 & 204.66 & 197.21 & 196.74 & 197.35 & 196.72 & 208.37 & 198.069 & 5.57 \\
\hline 354.5458 & 239.14 & 203.71 & 197.17 & 196.71 & 197.33 & 196.7 & 208.23 & 198.075 & 5.55 \\
\hline 355.0465 & 239.28 & 202.72 & 197.14 & 196.71 & 197.33 & 196.71 & 208.16 & 3.09 & 5.56 \\
\hline 355.5463 & 239.49 & 202.26 & 197.14 & 196.67 & 197.31 & 196.74 & 208.1 & 198.162 & 5.60 \\
\hline 356.0472 & 239.67 & 202.63 & 197.14 & 196.69 & 197.3 & 196.71 & 208.09 & 198.195 & 5.60 \\
\hline 356.546 & 239.78 & 202.52 & 197.12 & 196.65 & 197.26 & 196.65 & 208.03 & 197.865 & 5.59 \\
\hline 357.0458 & 239.71 & 203.31 & 197.1 & 196.65 & 197.28 & 196.67 & 208.02 & 919 & 5.60 \\
\hline 357.5465 & 239.12 & 202.96 & 197.1 & 196.65 & 197.28 & 196.69 & 20 & 34 & 5.62 \\
\hline 358.0473 & 238.67 & 202.92 & 197.14 & 196.67 & 197.28 & 196.69 & 208.18 & 197 & 5.63 \\
\hline 358.5462 & 238.67 & 202.13 & 197.14 & 196.69 & 197.3 & 196.67 & 208.32 & 197.949 & 5.58 \\
\hline 359.046 & 238.78 & 201.38 & 197.19 & 196.73 & 197.33 & 196.71 & 208.46 & 197.982 & 5.58 \\
\hline 359.5458 & 238.78 & 201.65 & 197.21 & 196.76 & 197.35 & 196.71 & 208.59 & 197.943 & 5.57 \\
\hline 360.0467 & 238.83 & 201.86 & 197.24 & 196.78 & 197.35 & 196.71 & 208.74 & 33 & 5.60 \\
\hline 360.5473 & 238.92 & 202.44 & 197.26 & 196.8 & 197.39 & 196.71 & 208.9 & 197.979 & 5.61 \\
\hline 361.0462 & 238.98 & 202.65 & 197.32 & 196.82 & 197.42 & 196.73 & 209.02 & 198.033 & 5.61 \\
\hline 361.546 & 238.85 & 203.05 & 197.33 & 196.85 & 197.44 & 196.73 & 209.12 & 198.03 & 5.62 \\
\hline 362.0458 & 238.71 & 204.07 & 197.35 & 196.85 & 197.44 & 196.74 & 209.2 & 198.027 & 5.62 \\
\hline 362.5457 & 238.85 & 203.1 & 197.33 & 196.89 & 197.44 & 196.74 & 209.16 & 198.042 & 5.62 \\
\hline 363.0465 & 239.03 & 203.49 & 197.35 & 196.87 & 197.39 & 196.76 & 209.08 & 069 & 5.57 \\
\hline 363.5472 & 239.12 & 203.1 & 197.32 & 196.85 & 197.41 & 196.69 & 208.98 & 197.922 & 5.56 \\
\hline 364.0462 & 239.07 & 202.15 & 197.3 & 196.81 & 197.39 & 196.69 & 208.81 & 197.889 & 5.56 \\
\hline 364.5458 & 238.99 & 201.94 & 197.26 & 196.78 & 197.37 & 196.69 & 208.66 & 197.928 & 5.60 \\
\hline 365.0457 & 238.98 & 203.17 & 197.24 & 196.76 & 197.35 & 196.69 & 208.52 & 197.958 & 5.58 \\
\hline 365.5465 & 238.96 & 203.06 & 197.23 & 196.72 & 197.33 & 196.69 & 208.42 & 197.901 & 5.59 \\
\hline 366.0472 & 238.81 & 202.58 & 197.19 & 196.74 & 197.33 & 196.71 & 208.33 & 197.937 & 5.62 \\
\hline 366.5462 & 238.67 & 201.63 & 197.17 & 196.71 & 197.32 & 196.69 & 208.19 & 197.934 & 5.61 \\
\hline 367.046 & 238.6 & 201.72 & 197.15 & 196.69 & 197.3 & 196.71 & 208.09 & 198.054 & 5.61 \\
\hline 367.5458 & 238.58 & 201.27 & 197.12 & 196.65 & 197.3 & 196.69 & 207.97 & 197.958 & 5.55 \\
\hline 368.0465 & 238.72 & 202.35 & 197.1 & 196.65 & 197.28 & 196.71 & 207.93 & 197.961 & 5.55 \\
\hline 368.5463 & 238.9 & 202.35 & 197.1 & 196.64 & 197.28 & 196.69 & 207.95 & 198.018 & 5.56 \\
\hline 369.0462 & 239.03 & 201.95 & 197.1 & 196.64 & 197.24 & 196.73 & 207.95 & 197.964 & 5.59 \\
\hline 369.546 & 239.21 & 201.79 & 197.1 & 196.65 & 197.28 & 196.71 & 208.02 & 197.997 & 5.59 \\
\hline 370.0458 & 239.39 & 201.79 & 197.1 & 196.65 & 197.3 & 196.69 & 208.12 & 198.018 & 5.60 \\
\hline 370.5465 & 239.57 & 202.35 & 197.14 & 196.69 & 197.32 & 196.71 & 208.24 & 198.021 & 5.60 \\
\hline
\end{tabular}


Table B6. Continued.

\begin{tabular}{|c|c|c|c|c|c|c|c|c|c|}
\hline $\begin{array}{c}\text { Time } \\
\text { min }\end{array}$ & $\begin{array}{l}\mathrm{T} 1 \\
{ }^{\circ} \mathrm{C} \\
\end{array}$ & $\begin{array}{l}\mathrm{T} 2 \\
{ }^{\circ} \mathrm{C} \\
\end{array}$ & $\begin{array}{l}\mathrm{T} 3 \\
{ }^{\circ} \mathrm{C} \\
\end{array}$ & $\begin{array}{l}\mathrm{T} 4 \\
{ }^{\circ} \mathrm{C} \\
\end{array}$ & $\begin{array}{l}\mathrm{T5} \\
{ }^{\circ} \mathrm{C} \\
\end{array}$ & $\begin{array}{l}\mathrm{T} 6 \\
{ }^{\circ} \mathrm{C} \\
\end{array}$ & $\begin{array}{l}\text { Pinj } \\
\text { psig }\end{array}$ & $\begin{array}{l}\text { Pout } \\
\text { psig }\end{array}$ & $\begin{array}{c}\mathrm{Vw} \\
\mathrm{cc} / \mathrm{min}\end{array}$ \\
\hline 371.0463 & 239.53 & 202.47 & 197.17 & 196.71 & 197.33 & 196.71 & 208.35 & 198.015 & 5.60 \\
\hline 371.5462 & 239.26 & 202.1 & 197.19 & 196.73 & 197.35 & 196.73 & 208.48 & 198.003 & 5.63 \\
\hline 372.0468 & 239.1 & 201.92 & 197.21 & 196.76 & 197.35 & 196.74 & 208.65 & 198.039 & 5.58 \\
\hline 372.5458 & 238.83 & 202.44 & 197.26 & 196.8 & 197.39 & 196.74 & 208.81 & 198.024 & 5.54 \\
\hline 373.0465 & 238.67 & 202.47 & 197.3 & 196.82 & 197.39 & 196.69 & 208.87 & 197.841 & 5.54 \\
\hline 373.5463 & 238.51 & 203.06 & 197.28 & 196.82 & 197.37 & 196.71 & 208.91 & 197.952 & 5.56 \\
\hline 374.0462 & 238.51 & 203.05 & 197.3 & 196.82 & 197.39 & 196.71 & 208.95 & 197.871 & 5.60 \\
\hline 374.546 & 238.38 & 202.98 & 197.32 & 196.83 & 197.39 & 196.73 & 208.97 & 197.916 & 5.59 \\
\hline 375.0467 & 238.58 & 202.76 & 197.32 & 196.81 & 197.41 & 196.71 & 208.93 & 197.871 & 5.59 \\
\hline 375.5465 & 238.85 & 203.44 & 197.3 & 196.82 & 197.39 & 196.71 & 208.9 & 197.871 & 5.59 \\
\hline 376.0473 & 238.87 & 204.87 & 197.3 & 196.81 & 197.39 & 196.71 & 208.82 & 197.919 & 5.61 \\
\hline 376.5472 & 238.8 & 203.78 & 197.28 & 196.8 & 197.37 & 196.69 & 208.73 & .94 & 5.63 \\
\hline 377.046 & 238.64 & 202.19 & 197.24 & 196.78 & 197.37 & 196.71 & 208.65 & 197.949 & 5.58 \\
\hline 377.5458 & 238.58 & 202.89 & 197.23 & 196.76 & 197.35 & 196.69 & 208.54 & 197.961 & 5.55 \\
\hline 378.0467 & 238.58 & 203.06 & 197.21 & 196.74 & 197.33 & 196.69 & 208.41 & 197.961 & 5.57 \\
\hline 378.5463 & 238.67 & 202.65 & 197.19 & 196.73 & 197.33 & 196.69 & 208.31 & 94 & 5.60 \\
\hline 379.0472 & 238.74 & 202.29 & 197.17 & 196.71 & 197.33 & 196.71 & 20 & 09 & 5.60 \\
\hline 379.546 & 238.81 & 202.67 & 197.15 & 196.69 & 197.3 & 196.71 & 208.09 & 85 & 5.60 \\
\hline 380.0458 & 238.9 & 202.31 & 197.14 & 196.69 & 197.33 & 196.71 & 208.02 & 198.018 & 5.61 \\
\hline 380.5457 & 239.1 & 202.88 & 197.12 & 196.65 & 197.28 & 196.71 & 208 & 198.009 & 5.62 \\
\hline 381.0465 & 239.24 & 202.76 & 197.12 & 196.67 & 197.28 & 196.71 & 207.99 & 198.03 & 5.62 \\
\hline 381.5463 & 239.44 & 202.92 & 197.12 & 196.65 & 197.3 & 196.71 & 208.02 & 39 & 5.57 \\
\hline 382.0462 & 239.69 & 202.81 & 197.12 & 196.67 & 197.3 & 196.69 & 208.08 & 772 & 5.55 \\
\hline 382.5458 & 239.84 & 203.64 & 197.14 & 196.69 & 197.32 & 196.71 & 208.18 & 198.003 & 5.57 \\
\hline 383.0457 & 239.84 & 203.91 & 197.15 & 196.67 & 197.32 & 196.71 & 208.31 & 198.111 & 5.60 \\
\hline 383.5465 & 239.75 & 202.83 & 197.17 & 196.74 & 197.24 & 196.6 & 208.36 & 197.784 & 5.61 \\
\hline 384.0472 & 239.42 & 202.46 & 197.19 & 196.69 & 197.3 & 196.65 & 208.36 & 197.715 & 5.60 \\
\hline 384.5462 & 238.96 & 202.2 & 197.19 & 196.73 & 197.32 & 196.69 & 208.44 & 197.76 & 5.60 \\
\hline 385.046 & 238.62 & 202.19 & 197.19 & 196.72 & 197.33 & 196.67 & 208.54 & 197.781 & 5.65 \\
\hline 385.5458 & 238.49 & 201.95 & 197.23 & 196.76 & 197.33 & 196.67 & 208.67 & 197.808 & 5.62 \\
\hline 386.0465 & 238.62 & 202.53 & 197.24 & 196.78 & 197.35 & 196.67 & 208.73 & 197.811 & 5.56 \\
\hline 386.5463 & 238.92 & 203.92 & 197.28 & 196.78 & 197.37 & 196.69 & 208.8 & 198.012 & 5.57 \\
\hline 387.0462 & 239.1 & 203.21 & 197.26 & 196.8 & 197.35 & 196.71 & 208.79 & 197.952 & 5.59 \\
\hline 387.546 & 239.15 & 202.69 & 197.26 & 196.78 & 197.37 & 196.69 & 208.74 & 197.979 & 5.61 \\
\hline 388.0458 & 239.08 & 202.81 & 197.26 & 196.78 & 197.35 & 196.67 & 208.66 & 197.952 & 5.62 \\
\hline 388.5465 & 238.96 & 203.49 & 197.26 & 196.78 & 197.35 & 196.69 & 208.61 & 197.877 & 5.60 \\
\hline 389.0473 & 238.83 & 203.38 & 197.23 & 196.74 & 197.33 & 196.69 & 208.51 & 198.036 & 5.62 \\
\hline 389.5462 & 238.78 & 204.15 & 197.22 & 196.74 & 197.33 & 196.69 & 208.43 & 197.886 & 5.64 \\
\hline 390.046 & 238.78 & 202.7 & 197.19 & 196.72 & 197.33 & 196.67 & 208.3 & 197.865 & 5.59 \\
\hline 390.5458 & 238.78 & 202.97 & 197.15 & 196.71 & 197.31 & 196.69 & 208.18 & 197.892 & 5.55 \\
\hline 391.0465 & 238.81 & 202.6 & 197.15 & 196.69 & 197.29 & 196.7 & 208.07 & 197.907 & 5.56 \\
\hline 391.5463 & 238.85 & 202.86 & 197.1 & 196.69 & 197.28 & 196.69 & 207.97 & 197.997 & 5.59 \\
\hline 392.0462 & 238.99 & 202.76 & 197.12 & 196.65 & 197.28 & 196.69 & 207.9 & 197.862 & 5.60 \\
\hline
\end{tabular}


Table B6. Continued.

\begin{tabular}{|c|c|c|c|c|c|c|c|c|c|}
\hline $\begin{array}{c}\text { Time } \\
\text { min }\end{array}$ & $\begin{array}{l}\mathrm{T} 1 \\
{ }^{\circ} \mathrm{C} \\
\end{array}$ & $\begin{array}{l}\mathrm{T} 2 \\
{ }^{\circ} \mathrm{C} \\
\end{array}$ & $\begin{array}{l}\mathrm{T} 3 \\
{ }^{\circ} \mathrm{C} \\
\end{array}$ & $\begin{array}{l}\mathrm{T} 4 \\
{ }^{\circ} \mathrm{C} \\
\end{array}$ & $\begin{array}{l}\mathrm{T5} \\
{ }^{\circ} \mathrm{C} \\
\end{array}$ & $\begin{array}{l}\mathrm{T} 6 \\
{ }^{\circ} \mathrm{C} \\
\end{array}$ & $\begin{array}{l}\text { Pinj } \\
\text { psig }\end{array}$ & $\begin{array}{l}\text { Pout } \\
\text { psig }\end{array}$ & $\begin{array}{c}\mathrm{Vw} \\
\mathrm{cc} / \mathrm{min}\end{array}$ \\
\hline 393.0458 & 239.14 & 202.2 & 197.08 & 196.63 & 197.26 & 196.71 & 207.89 & 198.057 & 5.62 \\
\hline 393.5465 & 239.21 & 202.22 & 197.1 & 196.63 & 197.26 & 196.67 & 207.92 & 198.03 & 5.62 \\
\hline 394.0473 & 239.24 & 202.11 & 197.12 & 196.65 & 197.28 & 196.67 & 207.99 & 197.886 & 5.63 \\
\hline 394.5462 & 239.26 & 203.79 & 197.12 & 196.65 & 197.26 & 196.65 & 207.99 & 197.736 & 5.57 \\
\hline 395.0497 & 239.3 & 203.56 & 197.12 & 196.65 & 197.26 & 196.65 & 208.06 & 197.73 & 5.57 \\
\hline 395.5458 & 239.31 & 205.1 & 197.14 & 196.67 & 197.26 & 196.67 & 208.17 & 197.796 & 5.57 \\
\hline 396.0467 & 239.06 & 204.24 & 197.15 & 196.7 & 197.31 & 196.67 & 208.29 & 197.799 & 5.60 \\
\hline 396.5463 & 238.76 & 204.1 & 197.19 & 196.72 & 197.33 & 196.69 & 208.41 & 197.766 & 5.60 \\
\hline 397.0462 & 238.56 & 204.55 & 197.2 & 196.74 & 197.35 & 196.67 & 208.53 & 197.811 & 5.60 \\
\hline 397.546 & 238.51 & 204.14 & 197.24 & 196.78 & 197.35 & 196.7 & 208.65 & 197.802 & 5.62 \\
\hline 398.0458 & 238.6 & 203.56 & 197.28 & 196.78 & 197.42 & 196.67 & 208.76 & 197.892 & 5.64 \\
\hline 398.5457 & 238.79 & 203.55 & 197.28 & 196.79 & 197.39 & 196.72 & 208.8 & 817 & 5.61 \\
\hline 399.0465 & 238.92 & 204.51 & 197.26 & 196.79 & 197.37 & 196.7 & 208.8 & 197.838 & 5.57 \\
\hline 399.5463 & 238.88 & 203.55 & 197.28 & 196.79 & 197.39 & 196.7 & 208.77 & 197.811 & 5.55 \\
\hline 400.0462 & 238.72 & 201.93 & 197.28 & 196.79 & 197.39 & 196.71 & 208.75 & 197.862 & 5.57 \\
\hline 400.5458 & 238.63 & 202.83 & 197.26 & 196.79 & 197.37 & 196.7 & 208.66 & 886 & 5.60 \\
\hline 401.0467 & 238.63 & 204.08 & 197.24 & 196.76 & 197.37 & 196.7 & 20 & 356 & 5.60 \\
\hline 401.5465 & 238.7 & 202.17 & 197.22 & 196.76 & 197.35 & 196.69 & 208.5 & 197.847 & 5.60 \\
\hline 402.0463 & 238.69 & 202.13 & 197.21 & 196.74 & 197.33 & 196.69 & 208.39 & 197.874 & 5.61 \\
\hline 402.5462 & 238.76 & 202.77 & 197.19 & 196.72 & 197.33 & 196.72 & 208.28 & 197.925 & 5.61 \\
\hline 403.046 & 238.81 & 202.9 & 197.17 & 196.7 & 197.31 & 196.7 & 208.18 & 197.868 & 5.63 \\
\hline 403.5458 & 238.83 & 203.74 & 197.13 & 196.69 & 197.31 & 196.69 & 208.08 & 871 & 5.57 \\
\hline 404.0465 & 238.92 & 203.04 & 197.12 & 196.67 & 197.3 & 196.69 & 207.99 & 197.907 & 5.56 \\
\hline 404.5463 & 238.94 & 202.97 & 197.1 & 196.65 & 197.28 & 196.69 & 207.98 & 197.922 & 5.58 \\
\hline 405.0462 & 238.96 & 202.42 & 197.12 & 196.65 & 197.3 & 196.69 & 207.96 & 197.928 & 5.58 \\
\hline 405.546 & 238.94 & 202.88 & 197.12 & 196.63 & 197.28 & 196.69 & 207.97 & 197.913 & 5.60 \\
\hline 406.0458 & 238.96 & 202.87 & 197.12 & 196.67 & 197.28 & 196.71 & 208.01 & 197.91 & 5.61 \\
\hline 406.5465 & 238.94 & 202.13 & 197.12 & 196.67 & 197.28 & 196.65 & 208.08 & 197.694 & 5.62 \\
\hline 407.0463 & 238.83 & 202.63 & 197.14 & 196.65 & 197.26 & 196.65 & 208.06 & 197.655 & 5.64 \\
\hline 407.5462 & 238.74 & 202.06 & 197.14 & 196.67 & 197.28 & 196.65 & 208.14 & 197.877 & 5.61 \\
\hline 408.046 & 238.71 & 202.61 & 197.15 & 196.69 & 197.28 & 196.65 & 208.25 & 197.745 & 5.57 \\
\hline 408.5458 & 238.65 & 202.13 & 197.17 & 196.71 & 197.32 & 196.65 & 208.33 & 197.76 & 5.56 \\
\hline 409.0465 & 238.62 & 203.8 & 197.19 & 196.72 & 197.32 & 196.67 & 208.47 & 197.862 & 5.60 \\
\hline 409.5463 & 238.56 & 202.92 & 197.21 & 196.74 & 197.28 & 196.67 & 208.57 & 197.82 & 5.60 \\
\hline 410.0462 & 238.44 & 203.49 & 197.24 & 196.78 & 197.35 & 196.69 & 208.67 & 197.823 & 5.60 \\
\hline 410.546 & 238.38 & 202.4 & 197.26 & 196.78 & 197.37 & 196.69 & 208.7 & 197.847 & 5.61 \\
\hline 411.0458 & 238.29 & 203.31 & 197.26 & 196.79 & 197.37 & 196.71 & 208.75 & 197.868 & 5.64 \\
\hline 411.5465 & 238.42 & 202.45 & 197.28 & 196.79 & 197.39 & 196.69 & 208.71 & 197.796 & 5.64 \\
\hline 412.0473 & 238.55 & 203.12 & 197.28 & 196.8 & 197.39 & 196.69 & 208.68 & 197.766 & 5.58 \\
\hline 412.5472 & 238.72 & 202.9 & 197.26 & 196.78 & 197.37 & 196.69 & 208.61 & 197.754 & 5.57 \\
\hline 413.046 & 238.85 & 203.53 & 197.22 & 196.74 & 197.33 & 196.69 & 208.53 & 197.805 & 5.58 \\
\hline 413.5458 & 238.92 & 203.4 & 197.22 & 196.76 & 197.33 & 196.7 & 208.43 & 197.823 & 5.61 \\
\hline 414.0467 & 239.05 & 202.72 & 197.21 & 196.74 & 197.33 & 196.69 & 208.35 & 197.883 & 5.60 \\
\hline
\end{tabular}




\section{APPENDIX C}

\section{PRODUCTION DATA}

\section{Table C1-Production data for Run 1}

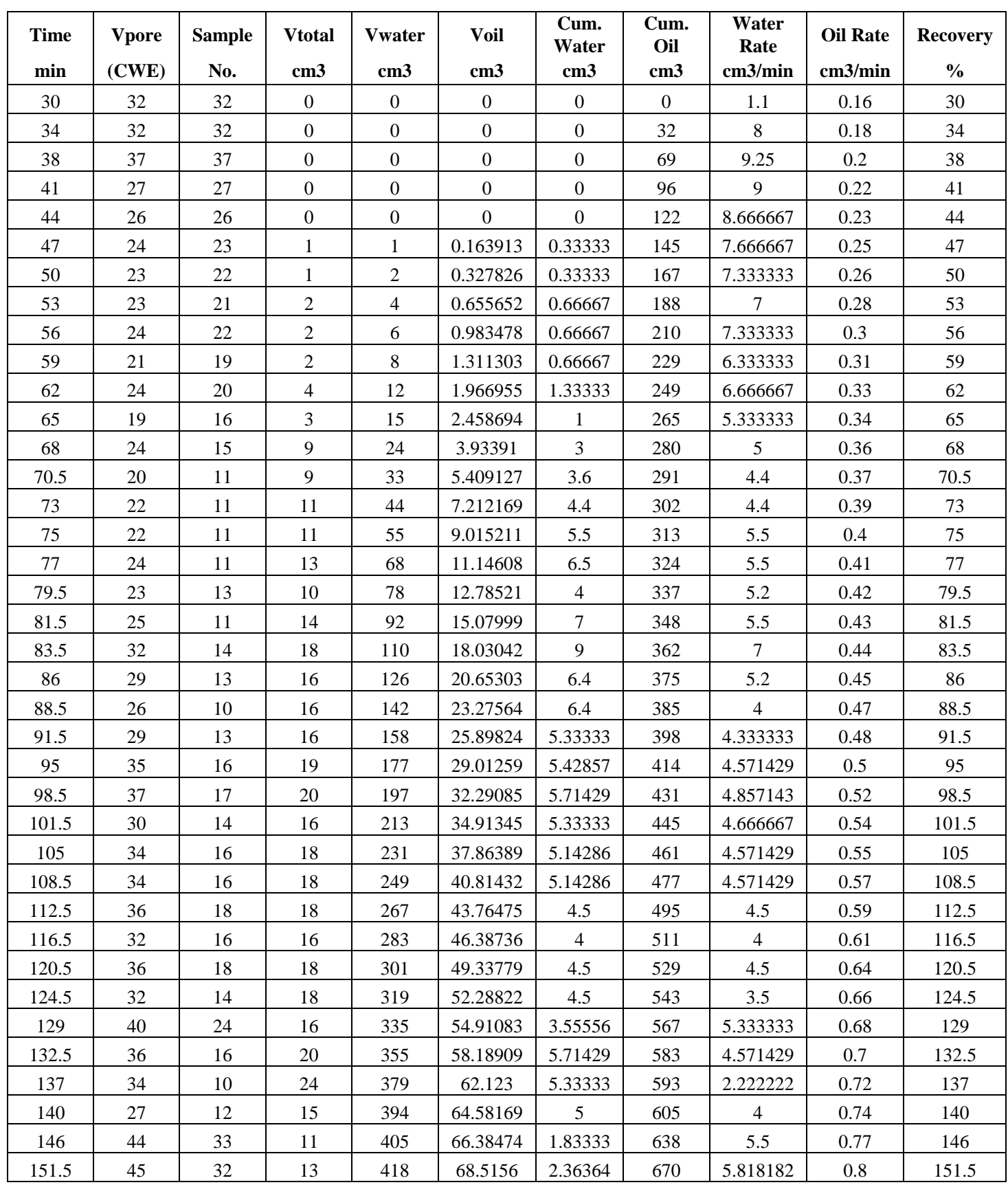


Table C1-Continued

\begin{tabular}{|c|c|c|c|c|c|c|c|c|c|c|}
\hline $\begin{array}{l}\text { Time } \\
\text { min }\end{array}$ & $\begin{array}{l}\text { Vpore } \\
\text { (CWE) }\end{array}$ & $\begin{array}{c}\text { Sample } \\
\text { No. } \\
\end{array}$ & $\begin{array}{c}\text { Vtotal } \\
\text { cm3 }\end{array}$ & $\begin{array}{c}\text { Vwater } \\
\text { cm3 }\end{array}$ & $\begin{array}{l}\text { Voil } \\
\text { cm3 }\end{array}$ & $\begin{array}{c}\text { Cum. } \\
\text { Water } \\
\text { cm3 }\end{array}$ & $\begin{array}{c}\text { Cum. } \\
\text { Oil } \\
\text { cm3 }\end{array}$ & $\begin{array}{c}\text { Water } \\
\text { Rate } \\
\text { cm3/min }\end{array}$ & $\begin{array}{l}\text { Oil Rate } \\
\mathrm{cm} 3 / \mathrm{min}\end{array}$ & $\begin{array}{c}\text { Recovery } \\
\% \\
\end{array}$ \\
\hline 158 & 40 & 35 & 5 & 423 & 69.33517 & 0.76923 & 705 & 5.384615 & 0.83 & 158 \\
\hline 163.5 & 33 & 30 & 3 & 426 & 69.82691 & 0.54545 & 735 & 5.454545 & 0.86 & 163.5 \\
\hline 169 & 35 & 32 & 3 & 429 & 70.31865 & 0.54545 & 767 & 5.818182 & 0.89 & 169 \\
\hline 174 & 31 & 28 & 3 & 432 & 70.81039 & 0.6 & 795 & 5.6 & 0.92 & 174 \\
\hline 180 & 36 & 33 & 3 & 435 & 71.30212 & 0.5 & 828 & 5.5 & 0.95 & 180 \\
\hline 186 & 33 & 31 & 2 & 437 & 71.62995 & 0.33333 & 859 & 5.166667 & 0.98 & 186 \\
\hline 191.5 & 37 & 35 & 2 & 439 & 71.95778 & 0.36364 & 894 & 6.363636 & 1.01 & 191.5 \\
\hline 196.5 & 41 & 38 & 3 & 442 & 72.44951 & 0.6 & 932 & 7.6 & 1.04 & 196.5 \\
\hline 203 & 50 & 46 & 4 & 446 & 73.10517 & 0.61538 & 978 & 7.076923 & 1.07 & 203 \\
\hline 210 & 23 & 20 & 3 & 449 & 73.59691 & 0.42857 & 998 & 2.857143 & 1.11 & 210 \\
\hline 217 & 36 & 34 & 2 & 451 & 73.92473 & 0.28571 & 1032 & 4.857143 & 1.15 & 217 \\
\hline 224 & 41 & 39 & 2 & 453 & 74.25256 & 0.28571 & 1071 & 5.571429 & 1.18 & 224 \\
\hline 230 & 39 & 36 & 3 & 456 & 74.7443 & 0.5 & 1107 & 6 & 1.21 & 230 \\
\hline 235 & 32 & 30 & 2 & 458 & 75.07212 & 0.4 & 1137 & 6 & 1.24 & 235 \\
\hline 240 & 30 & 28 & 2 & 460 & 75.39995 & 0.4 & 1165 & 5.6 & 1.27 & 240 \\
\hline
\end{tabular}


Table C2-Production Data for Run 2

\begin{tabular}{|c|c|c|c|c|c|c|c|c|c|}
\hline $\begin{array}{l}\text { Time } \\
\text { (Min) }\end{array}$ & Vtotal(cm3) & Vwater(cm3) & $\begin{array}{c}\text { Voil } \\
\text { (cm3) }\end{array}$ & $\begin{array}{l}\text { Cum } \\
\text { oil } \\
\text { (cm3) }\end{array}$ & $\begin{array}{c}\text { Recovery } \\
\% O O I P\end{array}$ & $\begin{array}{c}\text { Oil rate } \\
\text { (cc/min) }\end{array}$ & $\begin{array}{l}\text { Cum } \\
\text { water } \\
(\mathrm{cm} 3)\end{array}$ & $\begin{array}{l}\text { Water } \\
\text { Rate } \\
\text { (cc/min) }\end{array}$ & $\begin{array}{c}\text { Vpore } \\
\text { (CWE) }\end{array}$ \\
\hline 32 & 41 & 41 & 0 & 0 & 0 & 0 & 0 & 1.28125 & 0.17 \\
\hline 36 & 47 & 47 & 0 & 0 & 0 & 0 & 41 & 10.25 & 0.19 \\
\hline 40 & 30 & 30 & 0 & 0 & 0 & 0 & 88 & 11.75 & 0.21 \\
\hline 44 & 40 & 40 & 0 & 0 & 0 & 0 & 118 & 7.5 & 0.23 \\
\hline 48 & 35 & 34 & 1 & 1 & 0.163913 & 0.25 & 158 & 10 & 0.26 \\
\hline 57 & 26 & 25 & 1 & 2 & 0.327826 & 0.11111 & 192 & 3.7777778 & 0.30 \\
\hline 62 & 36 & 34 & 2 & 4 & 0.655652 & 0.4 & 217 & 5 & 0.33 \\
\hline 67 & 37 & 30 & 7 & 11 & 1.803042 & 1.4 & 251 & 6.8 & 0.36 \\
\hline 69 & 31 & 18 & 13 & 24 & 3.93391 & 6.5 & 281 & 15 & 0.37 \\
\hline 72 & 35 & 20 & 15 & 39 & 6.392604 & 5 & 299 & 6 & 0.38 \\
\hline 74 & 34 & 15 & 19 & 58 & 9.50695 & 9.5 & 319 & 10 & 0.39 \\
\hline 77 & 35 & 15 & 20 & 78 & 12.78521 & 6.66667 & 334 & 5 & 0.41 \\
\hline 80 & 31 & 11 & 20 & 98 & 16.06347 & 6.66667 & 349 & 5 & 0.43 \\
\hline 83 & 31 & 13 & 18 & 116 & 19.0139 & 6 & 360 & 3.66666667 & 0.44 \\
\hline 86 & 31 & 14 & 17 & 133 & 21.80042 & 5.66667 & 373 & 4.3333333 & 0.46 \\
\hline 88 & 28 & 10 & 18 & 151 & 24.75085 & 9 & 387 & 7 & 0.47 \\
\hline 91 & 31 & 11 & 20 & 171 & 28.02911 & 6.66667 & 397 & 3.3333333 & 0.48 \\
\hline 94 & 31 & 11 & 20 & 191 & 31.30737 & 6.66667 & 408 & 3.6666667 & 0.50 \\
\hline 97 & 31 & 11 & 20 & 211 & 34.58563 & 6.66667 & 419 & 3.66666667 & 0.52 \\
\hline 100 & 34 & 15 & 19 & 230 & 37.69997 & 6.33333 & 430 & 3.6666667 & 0.53 \\
\hline 102 & 35 & 16 & 19 & 249 & 40.81432 & 9.5 & 445 & 7.5 & 0.54 \\
\hline 105 & 32 & 14 & 18 & 267 & 43.76475 & 6 & 461 & 5.3333333 & 0.56 \\
\hline 107 & 32 & 16 & 16 & 283 & 46.38736 & 8 & 475 & 7 & 0.57 \\
\hline 110 & 26 & 12 & 14 & 297 & 48.68214 & 4.66667 & 491 & 5.3333333 & 0.59 \\
\hline 114 & 33 & 16 & 17 & 314 & 51.46866 & 4.25 & 503 & 3 & 0.61 \\
\hline 117.5 & 33 & 14 & 19 & 333 & 54.58301 & 5.42857 & 519 & 4.5714286 & 0.63 \\
\hline 121 & 36 & 18 & 18 & 351 & 57.53344 & 5.14286 & 533 & 4 & 0.64 \\
\hline 125.5 & 38 & 20 & 18 & 369 & 60.48387 & 4 & 551 & 4 & 0.67 \\
\hline 130 & 39 & 20 & 19 & 388 & 63.59822 & 4.22222 & 571 & 4.4444444 & 0.69 \\
\hline 135 & 41 & 24 & 17 & 405 & 66.38474 & 3.4 & 591 & 4 & 0.72 \\
\hline 140 & 40 & 30 & 10 & 415 & 68.02387 & 2 & 615 & 4.8 & 0.74 \\
\hline 146 & 44 & 34 & 10 & 425 & 69.663 & 1.66667 & 645 & 5 & 0.78 \\
\hline 153.5 & 30 & 26 & 4 & 429 & 70.31865 & 0.53333 & 679 & 4.5333333 & 0.82 \\
\hline 158 & 41 & 38 & 3 & 432 & 70.81039 & 0.66667 & 705 & 5.7777778 & 0.84 \\
\hline 167 & 45 & 41 & 4 & 436 & 71.46604 & 0.44444 & 743 & 4.2222222 & 0.89 \\
\hline 176 & 48 & 40 & 8 & 444 & 72.77734 & 0.88889 & 784 & 4.5555556 & 0.94 \\
\hline 180 & 40 & 36 & 4 & 448 & 73.43299 & 1 & 824 & 10 & 0.96 \\
\hline 186 & 35 & 34 & 1 & 449 & 73.59691 & 0.16667 & 860 & 6 & 0.99 \\
\hline 195 & 35 & 33 & 2 & 451 & 73.92473 & 0.22222 & 894 & 3.7777778 & 1.04 \\
\hline 200 & 45 & 43 & 2 & 453 & 74.25256 & 0.4 & 927 & 6.6 & 1.06 \\
\hline 208 & 43 & 41 & 2 & 455 & 74.58038 & 0.25 & 970 & 5.375 & 1.11 \\
\hline 215 & 42 & 40 & 2 & 457 & 74.90821 & 0.28571 & 1011 & 5.8571429 & 1.14 \\
\hline 224 & 37 & 35 & 2 & 459 & 75.23603 & 0.22222 & 1051 & 4.4444444 & 1.19 \\
\hline 233 & 39 & 38 & 1 & 460 & 75.39995 & 0.11111 & 1086 & 3.8888889 & 1.24 \\
\hline 240 & 37 & 35 & 2 & 462 & 75.72777 & 0.28571 & 1124 & 5.4285714 & 1.28 \\
\hline
\end{tabular}


Table C3-Production data for Run 3

\begin{tabular}{|c|c|c|c|c|c|c|c|c|c|}
\hline $\begin{array}{l}\text { Time } \\
\text { (Min) }\end{array}$ & $\begin{array}{l}\text { Vtotal } \\
(\mathrm{cm} 3)\end{array}$ & $\begin{array}{l}\text { Vwater } \\
(\mathrm{cm} 3)\end{array}$ & $\begin{array}{c}\text { Voil } \\
\text { (cm3) }\end{array}$ & $\begin{array}{c}\text { Cum } \\
\text { oil } \\
\text { (cm3) }\end{array}$ & $\begin{array}{c}\text { Recovery } \\
\% O O I P\end{array}$ & $\begin{array}{c}\text { Oil rate } \\
\text { (cc/min) }\end{array}$ & $\begin{array}{c}\text { Cum water } \\
\text { (cm3) }\end{array}$ & $\begin{array}{c}\text { Water } \\
\text { Rate } \\
\text { (cc/min) }\end{array}$ & Vpore(CWE) \\
\hline 61 & 44 & 0 & 44 & 0 & 0 & 0 & 44 & 0.7213115 & 0.29 \\
\hline 65 & 40 & 0 & 40 & 0 & 0 & 0 & 88 & 11 & 0.31 \\
\hline 70 & 41 & 2 & 39 & 2 & 0.601775 & 0.4 & 128 & 8 & 0.33 \\
\hline 78 & 39 & 2 & 37 & 4 & 1.20355 & 0.25 & 167 & 4.875 & 0.37 \\
\hline 83 & 43 & 3 & 40 & 7 & 2.106213 & 0.6 & 204 & 7.4 & 0.40 \\
\hline 87 & 42 & 3 & 39 & 10 & 3.008876 & 0.75 & 244 & 10 & 0.42 \\
\hline 92 & 40 & 2 & 38 & 12 & 3.610651 & 0.4 & 283 & 7.8 & 0.44 \\
\hline 96 & 35 & 8 & 27 & 20 & 6.017752 & 2 & 321 & 9.5 & 0.46 \\
\hline 100 & 41 & 12 & 29 & 32 & 9.628404 & 3 & 348 & 6.75 & 0.48 \\
\hline 105 & 68 & 20 & 48 & 52 & 15.64616 & 4 & 377 & 5.8 & 0.50 \\
\hline 110 & 38 & 18 & 20 & 70 & 21.06213 & 3.6 & 425 & 9.6 & 0.52 \\
\hline 115 & 35 & 9 & 26 & 79 & 23.77012 & 1.8 & 445 & 4 & 0.55 \\
\hline 119 & 33 & 16 & 17 & 95 & 28.58432 & 4 & 471 & 6.5 & 0.57 \\
\hline 123 & 39 & 11 & 28 & 106 & 31.89409 & 2.75 & 488 & 4.25 & 0.59 \\
\hline 129 & 41 & 4 & 37 & 110 & 33.09764 & 0.66667 & 516 & 4.66666667 & 0.62 \\
\hline 135 & 39 & 11 & 28 & 121 & 36.4074 & 1.83333 & 553 & 6.1666667 & 0.64 \\
\hline 142 & 15 & 13 & 2 & 134 & 40.31894 & 1.85714 & 581 & 4 & 0.68 \\
\hline 154 & 45 & 3 & 42 & 137 & 41.2216 & 0.25 & 583 & 0.1666667 & 0.73 \\
\hline 160 & 48 & 3 & 45 & 140 & 42.12427 & 0.5 & 625 & 7 & 0.76 \\
\hline 164 & 41 & 5 & 36 & 145 & 43.6287 & 1.25 & 670 & 11.25 & 0.78 \\
\hline 170 & 43 & 3 & 40 & 148 & 44.53137 & 0.5 & 706 & 6 & 0.81 \\
\hline 177 & 40 & 3 & 37 & 151 & 45.43403 & 0.42857 & 746 & 5.7142857 & 0.84 \\
\hline 184 & 36 & 2 & 34 & 153 & 46.03581 & 0.28571 & 783 & 5.2857143 & 0.88 \\
\hline 191 & 36 & 3 & 33 & 156 & 46.93847 & 0.42857 & 817 & 4.8571429 & 0.91 \\
\hline 205 & 43 & 4 & 39 & 160 & 48.14202 & 0.28571 & 850 & 2.3571429 & 0.98 \\
\hline 212 & 47 & 2 & 45 & 162 & 48.74379 & 0.28571 & 889 & 5.5714286 & 1.01 \\
\hline 218 & 34 & 2 & 32 & 164 & 49.34557 & 0.33333 & 934 & 7.5 & 1.04 \\
\hline 224 & 44 & 2 & 42 & 166 & 49.94734 & 0.33333 & 966 & 5.3333333 & 1.07 \\
\hline 230 & 45 & 3 & 42 & 169 & 50.85001 & 0.5 & 1008 & 7 & 1.10 \\
\hline 236 & 49 & 3 & 46 & 172 & 51.75267 & 0.5 & 1050 & 7 & 1.13 \\
\hline 240 & 40 & 2 & 38 & 174 & 52.35445 & 0.5 & 1096 & 11.5 & 1.14 \\
\hline
\end{tabular}


Table C4-Production data for Run 4

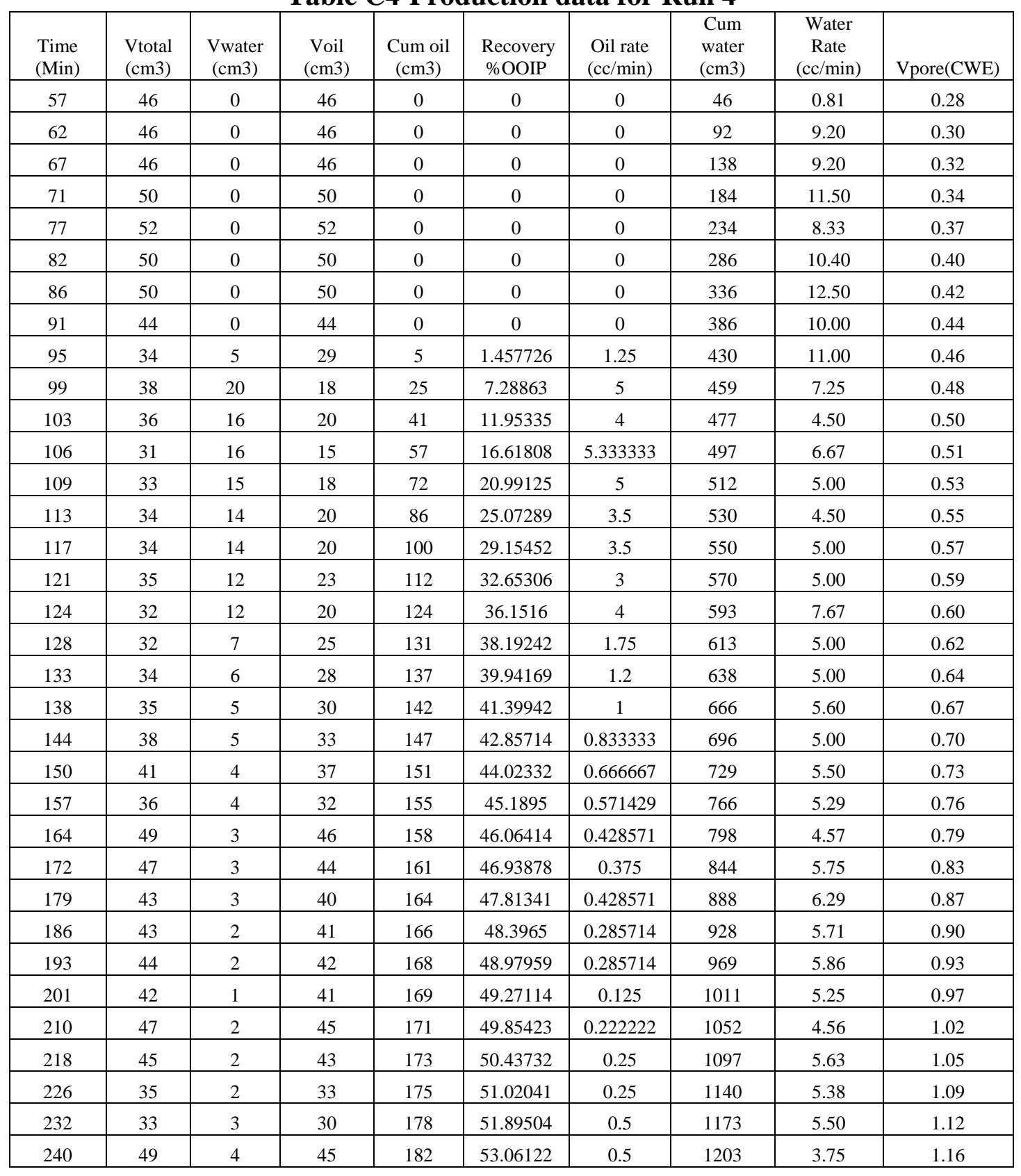


Table C5-Production data for Run 5

\begin{tabular}{|c|c|c|c|c|c|c|c|c|c|}
\hline $\begin{array}{l}\text { Time } \\
\text { (Min) }\end{array}$ & $\begin{array}{l}\text { Vtotal } \\
(\mathrm{cm} 3)\end{array}$ & $\begin{array}{c}\text { Voil } \\
\text { (cm3) }\end{array}$ & $\begin{array}{c}\text { Vwater } \\
\text { (cm3) }\end{array}$ & $\begin{array}{c}\text { Cum oil } \\
(\mathrm{cm} 3)\end{array}$ & $\begin{array}{c}\text { Recovery } \\
\% \text { OIP }\end{array}$ & $\begin{array}{c}\text { Oil rate } \\
\text { (cc/min) }\end{array}$ & $\begin{array}{l}\text { Cum } \\
\text { water } \\
\text { (cm3) } \\
\end{array}$ & $\begin{array}{c}\text { Water } \\
\text { Rate } \\
\text { (cc/min) } \\
\end{array}$ & Vpore(CWE) \\
\hline 55 & 55 & 0 & 55 & 0 & 0 & 0 & 55 & 1.6666667 & 0.26 \\
\hline 60 & 52 & 0 & 52 & 0 & 0 & 0 & 110 & 11 & 0.29 \\
\hline 66 & 58 & 0 & 58 & 0 & 0 & 0 & 162 & 8.6666667 & 0.32 \\
\hline 72 & 56 & 0 & 56 & 0 & 0 & 0 & 220 & 9.6666667 & 0.34 \\
\hline 79 & 62 & 0 & 62 & 0 & 0 & 0 & 276 & 8 & 0.38 \\
\hline 84 & 32 & 12 & 20 & 12 & 3.560831 & 2.4 & 338 & 12.4 & 0.40 \\
\hline 87 & 42 & 6 & 36 & 18 & 5.341246 & 2 & 358 & 6.6666667 & 0.42 \\
\hline 91 & 45 & 4 & 41 & 22 & 6.52819 & 1 & 394 & 9 & 0.43 \\
\hline 94 & 35 & 14 & 21 & 36 & 10.68249 & 4.6666667 & 435 & 13.666667 & 0.45 \\
\hline 98 & 37 & 18 & 19 & 54 & 16.02374 & 4.5 & 456 & 5.25 & 0.47 \\
\hline 103 & 35 & 17 & 18 & 71 & 21.06825 & 3.4 & 475 & 3.8 & 0.49 \\
\hline 106 & 35 & 13 & 22 & 84 & 24.92582 & 4.333333 & 493 & 6 & 0.51 \\
\hline 111 & 40 & 17 & 23 & 101 & 29.97033 & 3.4 & 515 & 4.4 & 0.53 \\
\hline 115 & 37 & 13 & 24 & 114 & 33.82789 & 3.25 & 538 & 5.75 & 0.55 \\
\hline 120 & 44 & 15 & 29 & 129 & 38.27893 & 3 & 562 & 4.8 & 0.57 \\
\hline 125 & 42 & 12 & 30 & 141 & 41.83976 & 2.4 & 591 & 5.8 & 0.60 \\
\hline 130 & 40 & 15 & 25 & 156 & 46.2908 & 3 & 621 & 6 & 0.62 \\
\hline 135 & 19 & 3 & 16 & 159 & 47.18101 & 0.6 & 646 & 5 & 0.64 \\
\hline 144 & 40 & 9 & 31 & 168 & 49.85163 & 1 & 662 & 1.7777778 & 0.69 \\
\hline 152 & 45 & 5 & 40 & 173 & 51.33531 & 0.625 & 693 & 3.875 & 0.73 \\
\hline 159 & 40 & 3 & 37 & 176 & 52.22552 & 0.428571 & 733 & 5.7142857 & 0.76 \\
\hline 165 & 35 & 2 & 33 & 178 & 52.81899 & 0.333333 & 770 & 6.1666667 & 0.79 \\
\hline 171 & 44 & 3 & 41 & 181 & 53.7092 & 0.5 & 803 & 5.5 & 0.82 \\
\hline 180 & 36 & 2 & 34 & 183 & 54.30267 & 0.222222 & 844 & 4.5555556 & 0.86 \\
\hline 188 & 45 & 2 & 43 & 185 & 54.89614 & 0.25 & 878 & 4.25 & 0.90 \\
\hline 196 & 40 & 2 & 38 & 187 & 55.48961 & 0.25 & 921 & 5.375 & 0.94 \\
\hline 203 & 44 & 2 & 42 & 189 & 56.08309 & 0.285714 & 959 & 5.4285714 & 0.97 \\
\hline 210 & 41 & 3 & 38 & 192 & 56.97329 & 0.428571 & 1001 & 6 & 1.00 \\
\hline 218 & 49 & 2 & 47 & 194 & 57.56677 & 0.25 & 1039 & 4.75 & 1.04 \\
\hline 225 & 40 & 2 & 38 & 196 & 58.16024 & 0.285714 & 1086 & 6.7142857 & 1.07 \\
\hline 233 & 15 & 1 & 14 & 197 & 58.45697 & 0.125 & 1124 & 4.75 & 1.11 \\
\hline 240 & 48 & 2 & 46 & 199 & 59.05045 & 0.285714 & 1138 & 2 & 1.15 \\
\hline
\end{tabular}


Table C6-Production data for Run 6

\begin{tabular}{|c|c|c|c|c|c|c|c|c|c|c|}
\hline $\begin{array}{l}\text { Time } \\
\min \end{array}$ & $\begin{array}{l}\text { Vpore } \\
\text { (CWE) }\end{array}$ & $\begin{array}{c}\text { Sample } \\
\text { No. }\end{array}$ & $\begin{array}{c}\text { Vtotal } \\
\text { cm3 }\end{array}$ & $\begin{array}{c}\text { Vwater } \\
\text { cm3 }\end{array}$ & $\begin{array}{l}\text { Voil } \\
\text { cm3 }\end{array}$ & $\begin{array}{c}\text { Cum. } \\
\text { Water } \\
\text { cm3 }\end{array}$ & $\begin{array}{c}\text { Cum. } \\
\text { Oil } \\
\text { cm3 }\end{array}$ & $\begin{array}{c}\text { Water } \\
\text { Rate } \\
\text { cm3/min }\end{array}$ & $\begin{array}{l}\text { Oil Rate } \\
\text { cm3/min }\end{array}$ & $\begin{array}{c}\text { Recovery } \\
\%\end{array}$ \\
\hline 39 & 35 & 0 & 35 & 0 & 0 & 0 & 35 & 0.8974359 & 0.19 & 39 \\
\hline 43 & 45 & 0 & 45 & 0 & 0 & 0 & 70 & 8.75 & 0.21 & 43 \\
\hline 48 & 32 & 1 & 31 & 1 & 0.1683502 & 0.2 & 115 & 9 & 0.23 & 48 \\
\hline 53 & 33 & 0 & 33 & 1 & 0.1683502 & 0 & 146 & 6.2 & 0.26 & 53 \\
\hline 56 & 27 & 1 & 26 & 2 & 0.3367003 & 0.3333333 & 179 & 11 & 0.27 & 56 \\
\hline 60 & 32 & 1 & 31 & 3 & 0.5050505 & 0.25 & 205 & 6.5 & 0.29 & 60 \\
\hline 65 & 32 & 2 & 30 & 5 & 0.8417508 & 0.4 & 236 & 6.2 & 0.32 & 65 \\
\hline 69 & 33 & 2 & 31 & 7 & 1.1784512 & 0.5 & 266 & 7.5 & 0.34 & 69 \\
\hline 73 & 38 & 2 & 36 & 9 & 1.5151515 & 0.5 & 297 & 7.75 & 0.36 & 73 \\
\hline 78 & 32 & 2 & 30 & 11 & 1.8518519 & 0.4 & 333 & 7.2 & 0.38 & 78 \\
\hline 83 & 32 & 2 & 30 & 13 & 2.1885522 & 0.4 & 363 & 6 & 0.4 & 83 \\
\hline 88 & 33 & 3 & 30 & 16 & 2.6936027 & 0.6 & 393 & 6 & 0.43 & 88 \\
\hline 93 & 14 & 8 & 6 & 24 & 4.040404 & 1.6 & 423 & 6 & 0.45 & 93 \\
\hline 96 & 36 & 15 & 21 & 39 & 6.5656566 & 5 & 429 & 2 & 0.47 & 96 \\
\hline 98 & 28 & 14 & 14 & 53 & 8.9225589 & 7 & 450 & 10.5 & 0.48 & 98 \\
\hline 100 & 28 & 14 & 14 & 67 & 11.279461 & 7 & 464 & 7 & 0.49 & 100 \\
\hline 102 & 34 & 12 & 22 & 79 & 13.299663 & 6 & 478 & 7 & 0.5 & 102 \\
\hline 105 & 30 & 15 & 15 & 94 & 15.824916 & 5 & 500 & 7.3333333 & 0.51 & 105 \\
\hline 108 & 30 & 20 & 10 & 114 & 19.191919 & 6.6666667 & 515 & 5 & 0.53 & 108 \\
\hline 111 & 31 & 14 & 17 & 128 & 21.548822 & 4.6666667 & 525 & 3.3333333 & 0.54 & 111 \\
\hline 115 & 25 & 12 & 13 & 140 & 23.569024 & 3 & 542 & 4.25 & 0.56 & 115 \\
\hline 119 & 34 & 30 & 4 & 170 & 28.619529 & 7.5 & 555 & 3.25 & 0.58 & 119 \\
\hline 124 & 37 & 29 & 8 & 199 & 33.501684 & 5.8 & 559 & 0.8 & 0.6 & 124 \\
\hline 128 & 40 & 34 & 6 & 233 & 39.225589 & 8.5 & 567 & 2 & 0.62 & 128 \\
\hline 132 & 38 & 33 & 5 & 266 & 44.781145 & 8.25 & 573 & 1.5 & 0.64 & 132 \\
\hline 136 & 39 & 23 & 16 & 289 & 48.653199 & 5.75 & 578 & 1.25 & 0.66 & 136 \\
\hline 140 & 21 & 10 & 11 & 299 & 50.3367 & 2.5 & 594 & 4 & 0.68 & 140 \\
\hline 144 & 39 & 13 & 26 & 312 & 52.525253 & 3.25 & 605 & 2.75 & 0.7 & 144 \\
\hline 148 & 39 & 37 & 2 & 349 & 58.754209 & 9.25 & 631 & 6.5 & 0.72 & 148 \\
\hline 153 & 40 & 19 & 21 & 368 & 61.952862 & 3.8 & 633 & 0.4 & 0.75 & 153 \\
\hline 157 & 18 & 6 & 12 & 374 & 62.962963 & 1.5 & 654 & 5.25 & 0.76 & 157 \\
\hline 162 & 26 & 7 & 19 & 381 & 64.141414 & 1.4 & 666 & 2.4 & 0.79 & 162 \\
\hline 167 & 15 & 13 & 2 & 394 & 66.329966 & 2.6 & 685 & 3.8 & 0.81 & 167 \\
\hline 172 & 38 & 3 & 35 & 397 & 66.835017 & 0.6 & 687 & 0.4 & 0.84 & 172 \\
\hline 176 & 30 & 10 & 20 & 407 & 68.518519 & 2.5 & 722 & 8.75 & 0.86 & 176 \\
\hline 181 & 34 & 3 & 31 & 410 & 69.023569 & 0.6 & 742 & 4 & 0.88 & 181 \\
\hline 186 & 36 & 1 & 35 & 411 & 69.191919 & 0.2 & 773 & 6.2 & 0.91 & 186 \\
\hline 191 & 40 & 2 & 38 & 413 & 69.52862 & 0.4 & 808 & 7 & 0.93 & 191 \\
\hline
\end{tabular}


Table C6-Continued

\begin{tabular}{|c|c|c|c|c|c|c|c|c|c|c|}
\hline $\begin{array}{l}\text { Time } \\
\text { min }\end{array}$ & $\begin{array}{l}\text { Vpore } \\
\text { (CWE) }\end{array}$ & $\begin{array}{c}\text { Sample } \\
\text { No. } \\
\end{array}$ & $\begin{array}{c}\text { Vtotal } \\
\text { cm3 }\end{array}$ & $\begin{array}{c}\text { Vwater } \\
\text { cm3 } \\
\end{array}$ & $\begin{array}{l}\text { Voil } \\
\text { cm3 }\end{array}$ & $\begin{array}{c}\text { Cum. } \\
\text { Water } \\
\text { cm3 } \\
\end{array}$ & $\begin{array}{c}\text { Cum. } \\
\text { Oil } \\
\text { cm3 } \\
\end{array}$ & $\begin{array}{c}\text { Water } \\
\text { Rate } \\
\text { cm3/min } \\
\end{array}$ & $\begin{array}{l}\text { Oil Rate } \\
\text { cm3/min }\end{array}$ & $\begin{array}{c}\text { Recovery } \\
\% \\
\end{array}$ \\
\hline 195 & 38 & 7 & 31 & 420 & 70.707071 & 1.75 & 846 & 9.5 & 0.95 & 195 \\
\hline 200 & 41 & 2 & 39 & 422 & 71.043771 & 0.4 & 877 & 6.2 & 0.97 & 200 \\
\hline 205 & 41 & 5 & 36 & 427 & 71.885522 & 1 & 916 & 7.8 & 1 & 205 \\
\hline 210 & 41 & 1 & 40 & 428 & 72.053872 & 0.2 & 952 & 7.2 & 1.02 & 210 \\
\hline 215 & 39 & 2 & 37 & 430 & 72.390572 & 0.4 & 992 & 8 & 1.05 & 215 \\
\hline 220 & 37 & 4 & 33 & 434 & 73.063973 & 0.8 & 1029 & 7.4 & 1.07 & 220 \\
\hline 225 & 45 & 2 & 43 & 436 & 73.400673 & 0.4 & 1062 & 6.6 & 1.1 & 225 \\
\hline 230 & 15 & 2 & 13 & 438 & 73.737374 & 0.4 & 1105 & 8.6 & 1.12 & 230 \\
\hline 235 & 31 & 6 & 25 & 444 & 74.747475 & 1.2 & 1118 & 2.6 & 1.14 & 235 \\
\hline 240 & 35 & 1 & 34 & 445 & 74.915825 & 0.2 & 1143 & 5 & 1.17 & 240 \\
\hline 270 & 37 & 0 & 37 & 445 & 74.915825 & 0 & 1177 & 1.1333333 & 1.32 & 270 \\
\hline 276 & 37 & 0 & 37 & 445 & 74.915825 & 0 & 1214 & 6.1666667 & 1.34 & 276 \\
\hline 283 & 3 & 1 & 2 & 446 & 75.084175 & 0.1428571 & 1251 & 5.2857143 & 1.38 & 283 \\
\hline 289 & 35 & 1 & 34 & 447 & 75.252525 & 0.1666667 & 1253 & 0.3333333 & 1.41 & 289 \\
\hline 296 & 35.5 & 0.5 & 35 & 447.5 & 75.3367 & 0.0714286 & 1287 & 4.8571429 & 1.44 & 296 \\
\hline 303 & 39 & 1 & 38 & 448.5 & 75.505051 & 0.1428571 & 1322 & 5 & 1.48 & 303 \\
\hline 310 & 28 & 2 & 26 & 450.5 & 75.841751 & 0.2857143 & 1360 & 5.4285714 & 1.51 & 310 \\
\hline 316 & 35 & 2 & 33 & 452.5 & 76.178451 & 0.3333333 & 1386 & 4.3333333 & 1.54 & 316 \\
\hline 322 & 33 & 3 & 30 & 455.5 & 76.683502 & 0.5 & 1419 & 5.5 & 1.57 & 322 \\
\hline 329 & 37 & 2 & 35 & 457.5 & 77.020202 & 0.2857143 & 1449 & 4.2857143 & 1.6 & 329 \\
\hline 336 & 17 & 2 & 15 & 459.5 & 77.356902 & 0.2857143 & 1484 & 5 & 1.64 & 336 \\
\hline 341 & 33 & 2 & 31 & 461.5 & 77.693603 & 0.4 & 1499 & 3 & 1.66 & 341 \\
\hline 378 & 42 & 2 & 40 & 463.5 & 78.030303 & 0.0540541 & 1530 & 0.8378378 & 1.84 & 378 \\
\hline 385 & 43 & 2 & 41 & 465.5 & 78.367003 & 0.2857143 & 1570 & 5.7142857 & 1.88 & 385 \\
\hline 392 & 43 & 1 & 42 & 466.5 & 78.535354 & 0.1428571 & 1611 & 5.8571429 & 1.91 & 392 \\
\hline 398 & 41 & 2 & 39 & 468.5 & 78.872054 & 0.3333333 & 1653 & 7 & 1.94 & 398 \\
\hline 404 & 28 & 1 & 27 & 469.5 & 79.040404 & 0.1666667 & 1692 & 6.5 & 1.97 & 404 \\
\hline 410 & 43 & 2 & 41 & 471.5 & 79.377104 & 0.3333333 & 1719 & 4.5 & 2 & 410 \\
\hline 416 & 37 & 1 & 36 & 472.5 & 79.545455 & 0.1666667 & 1760 & 6.8333333 & 2.03 & 416 \\
\hline 423 & 31 & 2 & 29 & 474.5 & 79.882155 & 0.2857143 & 1796 & 5.1428571 & 2.06 & 423 \\
\hline 429 & 44 & 2 & 42 & 476.5 & 80.218855 & 0.3333333 & 1825 & 4.8333333 & 2.09 & 429 \\
\hline 435 & 39 & 1 & 38 & 477.5 & 80.387205 & 0.1666667 & 1867 & 7 & 2.12 & 435 \\
\hline 442 & 37 & 1 & 36 & 478.5 & 80.555556 & 0.1428571 & 1905 & 5.4285714 & 2.15 & 442 \\
\hline
\end{tabular}


Table C7-Production data for Run 7

\begin{tabular}{|c|c|c|c|c|c|c|c|c|c|c|}
\hline $\begin{array}{l}\text { Time } \\
\text { min }\end{array}$ & $\begin{array}{l}\text { Vpore } \\
\text { (CWE) }\end{array}$ & $\begin{array}{c}\text { Sample } \\
\text { No. }\end{array}$ & $\begin{array}{c}\text { Vtotal } \\
\text { cm3 }\end{array}$ & $\begin{array}{c}\text { Vwater } \\
\text { cm3 }\end{array}$ & $\begin{array}{l}\text { Voil } \\
\text { cm3 }\end{array}$ & $\begin{array}{l}\text { Cum. } \\
\text { Water } \\
\text { cm3 }\end{array}$ & $\begin{array}{c}\text { Cum. } \\
\text { Oil } \\
\text { cm3 }\end{array}$ & $\begin{array}{c}\text { Water } \\
\text { Rate } \\
\text { cm3/min }\end{array}$ & $\begin{array}{l}\text { Oil Rate } \\
\text { cm3/min }\end{array}$ & $\begin{array}{c}\text { Recovery } \\
\%\end{array}$ \\
\hline 44 & 35 & 0 & 35 & 0 & 0 & 0 & 35 & 0.8974359 & 0.21 & 44 \\
\hline 49 & 45 & 0 & 45 & 0 & 0 & 0 & 70 & 7 & 0.24 & 49 \\
\hline 54 & 31 & 0 & 31 & 0 & 0 & 0 & 115 & 9 & 0.26 & 54 \\
\hline 60 & 33 & 0 & 33 & 0 & 0 & 0 & 146 & 5.1666667 & 0.29 & 60 \\
\hline 67 & 26 & 0 & 26 & 0 & 0 & 0 & 179 & 4.7142857 & 0.33 & 67 \\
\hline 72 & 31 & 0 & 31 & 0 & 0 & 0 & 205 & 5.2 & 0.35 & 72 \\
\hline 78 & 30 & 0 & 30 & 0 & 0 & 0 & 236 & 5.1666667 & 0.38 & 78 \\
\hline 84 & 36 & 5 & 31 & 5 & 0.841751 & 0.833333 & 266 & 5 & 0.41 & 84 \\
\hline 90 & 47 & 17 & 30 & 22 & 3.703704 & 2.833333 & 297 & 5.1666667 & 0.44 & 90 \\
\hline 95 & 47 & 19 & 28 & 41 & 6.902357 & 3.8 & 327 & 6 & 0.46 & 95 \\
\hline 98 & 42 & 21 & 21 & 62 & 10.43771 & 7 & 355 & 9.3333333 & 0.48 & 98 \\
\hline 101 & 37 & 23 & 14 & 85 & 14.30976 & 7.6666667 & 376 & 7 & 0.49 & 101 \\
\hline 105 & 38 & 24 & 14 & 109 & 18.35017 & 6 & 390 & 3.5 & 0.51 & 105 \\
\hline 108 & 44 & 27 & 17 & 136 & 22.89562 & 9 & 404 & 4.66666667 & 0.53 & 108 \\
\hline 111 & 38 & 33 & 5 & 169 & 28.45118 & 11 & 421 & 5.66666667 & 0.54 & 111 \\
\hline 114 & 41 & 35 & 6 & 204 & 34.34343 & 11.66667 & 426 & 1.66666667 & 0.56 & 114 \\
\hline 117 & 42 & 34 & 8 & 238 & 40.06734 & 11.33333 & 432 & 2 & 0.57 & 117 \\
\hline 120 & 36 & 32 & 4 & 270 & 45.45455 & 10.66667 & 440 & 2.66666667 & 0.58 & 120 \\
\hline 123 & 37 & 28 & 9 & 298 & 50.16835 & 9.333333 & 444 & 1.3333333 & 0.6 & 123 \\
\hline 127 & 27 & 19 & 8 & 317 & 53.367 & 4.75 & 453 & 2.25 & 0.62 & 127 \\
\hline 131 & 33 & 19 & 14 & 336 & 56.56566 & 4.75 & 461 & 2 & 0.64 & 131 \\
\hline 136 & 33 & 17 & 16 & 353 & 59.42761 & 3.4 & 475 & 2.8 & 0.66 & 136 \\
\hline 140 & 32 & 15 & 17 & 368 & 61.95286 & 3.75 & 491 & 4 & 0.68 & 140 \\
\hline 145 & 33 & 13 & 20 & 381 & 64.14141 & 2.6 & 508 & 3.4 & 0.71 & 145 \\
\hline 152 & 37 & 11 & 26 & 392 & 65.99327 & 1.571429 & 528 & 2.8571429 & 0.74 & 152 \\
\hline 158 & 39 & 9 & 30 & 401 & 67.50842 & 1.5 & 554 & 4.3333333 & 0.77 & 158 \\
\hline 166 & 35 & 10 & 25 & 411 & 69.19192 & 1.25 & 584 & 3.75 & 0.81 & 166 \\
\hline 173 & 36 & 8 & 28 & 419 & 70.53872 & 1.142857 & 609 & 3.5714286 & 0.84 & 173 \\
\hline 180 & 32 & 5 & 27 & 424 & 71.38047 & 0.714286 & 637 & 4 & 0.88 & 180 \\
\hline 187 & 36 & 4 & 32 & 428 & 72.05387 & 0.571429 & 664 & 3.8571429 & 0.91 & 187 \\
\hline 195 & 34 & 3 & 31 & 431 & 72.55892 & 0.375 & 696 & 4 & 0.95 & 195 \\
\hline 204 & 33 & 3 & 30 & 434 & 73.06397 & 0.333333 & 727 & 3.4444444 & 0.99 & 204 \\
\hline 213 & 30 & 2 & 28 & 436 & 73.40067 & 0.222222 & 757 & 3.3333333 & 1.04 & 213 \\
\hline 223 & 36 & 2 & 35 & 438 & 73.73737 & 0.2 & 785 & 2.8 & 1.09 & 223 \\
\hline 233 & 33 & 2 & 32 & 440 & 74.07407 & 0.2 & 820 & 3.5 & 1.14 & 233 \\
\hline 242 & 32 & 1 & 31 & 441 & 74.24242 & 0.111111 & 852 & 3.5555556 & 1.18 & 242 \\
\hline 250 & 37 & 1 & 35 & 442 & 74.41077 & 0.125 & 883 & 3.875 & 1.22 & 250 \\
\hline 260 & 40 & 1 & 38 & 443 & 74.57912 & 0.1 & 918 & 3.5 & 1.27 & 260 \\
\hline
\end{tabular}


Table C7-Continued

\begin{tabular}{|c|c|c|c|c|c|c|c|c|c|c|}
\hline $\begin{array}{l}\text { Time } \\
\text { min }\end{array}$ & $\begin{array}{l}\text { Vpore } \\
\text { (CWE) }\end{array}$ & $\begin{array}{c}\text { Sample } \\
\text { No. } \\
\end{array}$ & $\begin{array}{c}\text { Vtotal } \\
\text { cm3 }\end{array}$ & $\begin{array}{c}\text { Vwater } \\
\text { cm3 }\end{array}$ & $\begin{array}{l}\text { Voil } \\
\text { cm3 }\end{array}$ & $\begin{array}{c}\text { Cum. } \\
\text { Water } \\
\text { cm3 } \\
\end{array}$ & $\begin{array}{c}\text { Cum. } \\
\text { Oil } \\
\text { cm3 }\end{array}$ & $\begin{array}{c}\text { Water } \\
\text { Rate } \\
\text { cm3/min }\end{array}$ & $\begin{array}{l}\text { Oil Rate } \\
\text { cm3/min }\end{array}$ & $\begin{array}{c}\text { Recovery } \\
\% \\
\end{array}$ \\
\hline 268 & 33 & 2 & 31 & 445 & 74.91582 & 0.25 & 956 & 4.75 & 1.31 & 268 \\
\hline 278 & 40 & 2 & 39 & 447 & 75.25253 & 0.2 & 987 & 3.1 & 1.35 & 278 \\
\hline 290 & 37 & 2 & 36 & 449 & 75.58923 & 0.166667 & 1026 & 3.25 & 1.41 & 290 \\
\hline 304 & 38 & 1 & 37 & 450 & 75.75758 & 0.071429 & 1062 & 2.5714286 & 1.48 & 304 \\
\hline 315 & 38 & 1 & 37 & 451 & 75.92593 & 0.090909 & 1099 & 3.3636364 & 1.53 & 315 \\
\hline 328 & 14 & 1 & 13 & 452 & 76.09428 & 0.076923 & 1136 & 2.8461538 & 1.6 & 328 \\
\hline 342 & 35 & 1 & 34 & 453 & 76.26263 & 0.071429 & 1149 & 0.9285714 & 1.67 & 342 \\
\hline 356 & 39 & 1 & 37 & 454 & 76.43098 & 0.071429 & 1183 & 2.4285714 & 1.73 & 356 \\
\hline 367 & 38 & 1 & 37 & 455 & 76.59933 & 0.090909 & 1220 & 3.3636364 & 1.79 & 367 \\
\hline 373 & 3 & 2 & 2 & 457 & 76.93603 & 0.333333 & 1257 & 6.1666667 & 1.82 & 373 \\
\hline 388 & 34 & 1 & 33 & 458 & 77.10438 & 0.0666667 & 1259 & 0.1333333 & 1.89 & 388 \\
\hline 403 & 38 & 1 & 36 & 459 & 77.27273 & 0.0666667 & 1292 & 2.2 & 1.96 & 403 \\
\hline 413 & 27 & 1 & 26 & 460 & 77.44108 & 0.1 & 1328 & 3.6 & 2.01 & 413 \\
\hline 422 & 36 & 2 & 35 & 462 & 77.77778 & 0.222222 & 1354 & 2.8888889 & 2.06 & 422 \\
\hline 435 & 31 & 1 & 31 & 463 & 77.94613 & 0.076923 & 1389 & 2.6923077 & 2.12 & 435 \\
\hline 440 & 40 & 1 & 40 & 464 & 78.11448 & 0.2 & 1420 & 6.2 & 2.14 & 440 \\
\hline 268 & 33 & 2 & 31 & 445 & 74.91582 & 0.25 & 956 & 4.75 & 1.31 & 268 \\
\hline 278 & 40 & 2 & 39 & 447 & 75.25253 & 0.2 & 987 & 3.1 & 1.35 & 278 \\
\hline 290 & 37 & 2 & 36 & 449 & 75.58923 & 0.166667 & 1026 & 3.25 & 1.41 & 290 \\
\hline 304 & 38 & 1 & 37 & 450 & 75.75758 & 0.071429 & 1062 & 2.5714286 & 1.48 & 304 \\
\hline 315 & 38 & 1 & 37 & 451 & 75.92593 & 0.090909 & 1099 & 3.3636364 & 1.53 & 315 \\
\hline 328 & 14 & 1 & 13 & 452 & 76.09428 & 0.076923 & 1136 & 2.8461538 & 1.6 & 328 \\
\hline 342 & 35 & 1 & 34 & 453 & 76.26263 & 0.071429 & 1149 & 0.9285714 & 1.67 & 342 \\
\hline 356 & 39 & 1 & 37 & 454 & 76.43098 & 0.071429 & 1183 & 2.4285714 & 1.73 & 356 \\
\hline 367 & 38 & 1 & 37 & 455 & 76.59933 & 0.090909 & 1220 & 3.3636364 & 1.79 & 367 \\
\hline 373 & 3 & 2 & 2 & 457 & 76.93603 & 0.333333 & 1257 & 6.1666667 & 1.82 & 373 \\
\hline 388 & 34 & 1 & 33 & 458 & 77.10438 & 0.0666667 & 1259 & 0.1333333 & 1.89 & 388 \\
\hline 403 & 38 & 1 & 36 & 459 & 77.27273 & 0.0666667 & 1292 & 2.2 & 1.96 & 403 \\
\hline 413 & 27 & 1 & 26 & 460 & 77.44108 & 0.1 & 1328 & 3.6 & 2.01 & 413 \\
\hline 422 & 36 & 2 & 35 & 462 & 77.77778 & 0.222222 & 1354 & 2.8888889 & 2.06 & 422 \\
\hline 435 & 31 & 1 & 31 & 463 & 77.94613 & 0.076923 & 1389 & 2.6923077 & 2.12 & 435 \\
\hline 440 & 40 & 1 & 40 & 464 & 78.11448 & 0.2 & 1420 & 6.2 & 2.14 & 440 \\
\hline
\end{tabular}




\section{VITA}

Name:

Permanent Address:

Education:

Member:

Email Address:
Rajiv Menon Madhavan

3116 TAMU, Richardson Building

Texas A\&M University, College Station, Texas, 77843

B.E. (Hons.). Chemical Engineering

Vellore Institute of Technology, India 2006

M.S. Petroleum Engineering

Texas A\&M University, College Station 2009

Society of Petroleum Engineers

mrajivmenon@gmail.com 